Thomas Treiber

\title{
Pfade in die Krise
}

VGR 


\section{V\&R Academic}

Open-Access-Publikation im Sinne der CC-Lizenz BY 4.0

(c) 2018, VधR unipress $\mathrm{GmbH}$, Göttingen 
Wittener Schriften zu Familienunternehmen

Band 24

Herausgegeben von

Arist von Schlippe und Tom Rüsen

im Wittener Institut für Familienunternehmen (WIFU)

an der Universität Witten/Herdecke 


\section{Thomas Treiber}

\section{Pfade in die Krise}

Der Einfluss des Faktors Familie auf die Genese und Dynamik strategischer Pfadabhängigkeit in Familienunternehmen

Mit 28 Abbildungen

V\& R unipress 
Bibliografische Information der Deutschen Nationalbibliothek

Die Deutsche Nationalbibliothek verzeichnet diese Publikation in der Deutschen Nationalbibliografie; detaillierte bibliografische Daten sind im Internet über http://dnb.d-nb.de abrufbar.

ISSN 2198-526X

ISBN 978-3-7370-0835-8

Weitere Ausgaben und Online-Angebote sind erhältlich unter: www.v-r.de

○ 2018, V\&R unipress GmbH, Robert-Bosch-Breite 6, D-37079 Göttingen / www.v-r.de Dieses Werk ist als Open-Access-Publikation im Sinne der Creative-Commons-Lizenz BY International 4.0 (»Namensnennung «) unter dem DOI 10.14220/9783737008358 abzurufen. Um eine Kopie dieser Lizenz zu sehen, besuchen Sie https://creativecommons.org/licenses/by/4.0/. Jede Verwertung in anderen als den durch diese Lizenz zugelassenen Fällen bedarf der vorherigen schriftlichen Einwilligung des Verlages. 


\section{Inhalt}

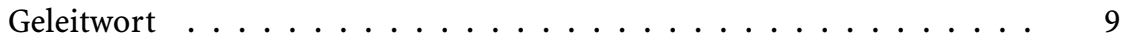

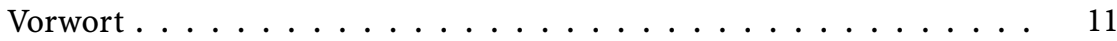

Abbildungsverzeichnis . . . . . . . . . . . . . . . . 15

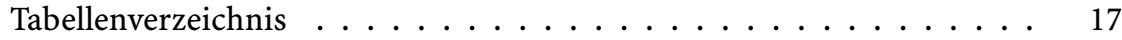

Abkürzungsverzeichnis . . . . . . . . . . . . . . . . 19

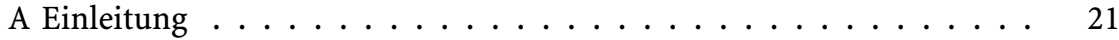

1 Problemstellungen ...................... 21

2 Ordnungsrahmen und theoretisch orientierte Forschungsfrage . . . 26

3 Qualitativ-zirkuläre Forschungsstrategie und Zielsetzung . . . . . . 31

4 Gang der Arbeit . . . . . . . . . . . . . . . 36

B Theoretischer Bezugsrahmen: Der Prozess strategischer Pfade im

Kontext familiär beeinflusster Unternehmen . . . . . . . . . . . . . . 39

1 Prozess: Strategische Pfade als spezifische Prozesse der Krisenentstehung . . . . . . . . . . . . . . . . . . . . . . . 39

1.1 Unternehmenskrisen und deren Entstehung . . . . . . . . . . 40

1.1.1 Begriff und prozessualer Charakter der

Unternehmenskrise . . . . . . . . . . . . 41

1.1.2 Forschung zu Unternehmenskrisen - Entwicklung und

Defizite .................... 44

1.1.3 Krisenursachen und Prozess der Krisenentstehung . . . . 48

1.2 Der strategische Wandel als permanente Herausforderung des Managements . . . . . . . . . . . . . . . 54

1.2.1 Das soziale System Organisation in seiner bestandskritischen dynamischen Umwelt . . . . . . . . 55 
1.2.2 Strategischer Wandel und dessen Management . . . . . . . 59

1.3 Das Ausbleiben des Wandels aufgrund strategischer

Pfadabhängigkeit . . . . . . . . . . . . . . . 64

1.3.1 Kurze Entwicklungsgeschichte der Pfadtheorie . . . . . . 66

1.3.2 Phasen der Pfadgenese und deren Komponenten . . . . . 71

1.3.2.1 Phase I: »Historische Vorprägung« - die

Historizität strategischer Entscheidungsprozesse . 73

1.3.2.2 Phase II: "Pfadformation« - das Wirken selbstverstärkender Mechanismen . . . . . . . 76

1.3.2.3 Phase III: Lock-in und Pfadabhängigkeit . . . . . 83

1.3.3 Das definitorische Verständnis strategischer Pfade in der

Organisation ................ 86

1.3.4 Theoretische Abgrenzung der organisationalen

Pfadtheorie . . . . . . . . . . . . . 87

1.3.5 Die vernachlässigte Bedeutung des Kontexts in der

organisationalen Pfadforschung . . . . . . . . . 92

2 Kontext: der Einfluss des Faktors Familie auf Unternehmen . . . . . 97

2.1 Die Familie: fehlende Variable der Managementforschung . . . 98

2.2 Das Verhältnis der sozialen Systeme Familie und Unternehmen . 108

2.2.1 Divergierende Systemlogiken . . . . . . . . . . . . 109

2.2.2 Strukturelle Kopplung und Ko-Evolution der Systeme . . . 115

2.2.3 Der Einfluss der Familie über die Kanäle »Macht»,

»Generation« und »Kultur» . . . . . . . . . . . . . . . 122

2.3 Die strukturellen Merkmale des familiären Einflusses in der

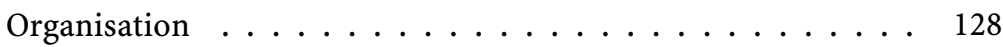

2.3.1 »Command«: Entscheidungsautonomie, Zentralisierung

und Personenorientierung . . . . . . . . . . 132

2.3.2 »Continuity«: Generationale Orientierung und Kontinuität 145

2.3.3 »Community« und »Connections«: Kulturelle Merkmale . 152

C Empirische Untersuchung: Strategische Pfade in die Krise . . . . . . . 163

1 Vorgehen bei Durchführung der empirischen Studie . . . . . . . . 164

1.1 Einstieg ins Feld und Prozess des Samplings . . . . . . . . . . 164

1.1.1 Explorative Vorstudie . . . . . . . . . . . . . . . . . . . 164

1.1.2 Sampling: Die Fallgruppen der monozentrischen und

polyzentrischen Konfiguration . . . . . . . . . . . . . 168

1.2 Prozess und Methoden der Datenerhebung und -auswertung . . 170

1.2.1 Methoden der Datenerhebung . . . . . . . . . . . . 171

1.2.2 Ablauf der Fallanalysen und Methoden der

Datenauswertung ................ 
2 Fallstudien: Strategische Pfade in die Krise . . . . . . . . . . . . . 178

2.1 Fallstudie zur Fallgruppe I: Anton Schlecker e.K. . . . . . . . . . 178

2.1.1 Historische Entwicklung des Unternehmens und seiner

Branchenumwelt .............. . 179

2.1.2 Pfadanalyse . . . . . . . . . . . . . . . 188

2.1.2.1 Analyse der strategischen Persistenz . . . . . 188

2.1.2.1.1 Gesamtunternehmensstrategie: Wachstum durch Multiplikation . . . . . . . . . . . 188

2.1.2.1.2 Entwicklung von Geschäftsmodell und

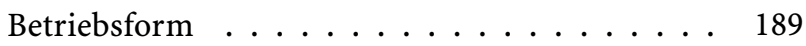

2.1.2.2 Pfaddiagnose . . . . . . . . . . . . 198

2.1.2.2.1 Selbstverstärkende Mechanismen . . . . . . . . 198

2.1.2.2.2 Lock-in . . . . . . . . . . . . . 208

2.2 Fallstudie zur Fallgruppe II: Berentzen-Gruppe AG . . . . . . . 218

2.2.1 Historische Entwicklung des Unternehmens und dessen

Branchenumwelt ............... . . 219

2.2.2 Pfadanalyse . . . . . . . . . . . . . . . 230

2.2.2.1 Analyse der strategischen Persistenz . . . . . . 230

2.2.2.1.1 Organisationale Entwicklungsrichtung: "Wachsen oder weichen« . . . . . . . . . . . 230

2.2.2.1.2 Entwicklung der Geschäftsfelder in strategischer Gesamtkonfiguration ........... . . . . . . 236

2.2.2.2 Pfaddiagnose . . . . . . . . . . . . 247

2.2.2.2.1 Der Konflikt als sich selbst verstärkender sozio-emotionaler Mechanismus . . . . . . . . . . 248

2.2.2.2.2 Lock-in . . . . . . . . . . . . . . 269

3 Zwischenfazit zur empirischen Untersuchung . . . . . . . . . . 272

D Der Einfluss des Kontextfaktors Familie im Prozess der Pfadgenese . . 275

1 Die Phase der historischen Vorprägung: Die Historizität des strategischen Entscheidungsraumes und die prägende Wirkung organisationaler Imprints . . . . . . . . . . . . . . . . . 276

1.1 Imprints aus der externen Umwelt der Organisation . . . . . . 279

1.2 Sozio-emotionales Vermögen . . . . . . . . . . . . . . . . 285

1.3 Kultur der Organisation . . . . . . . . . . . . . . . 293

1.4 Image der Organisation und deren Marken . . . . . . . . . . . 302

1.5 Eigentumsstruktur und familial-organisationale Konfiguration . 307

2 Der strategische Entscheidungsprozess in der Phase der Pfadformation . . . . . . . . . . . . . . . . . 316

2.1 Macht und Immunität der dominanten Koalitionen . . . . . . . 319 
2.1.1 Macht, Entscheidungsautonomie und Selbstkontrolle der dominanten Koalition . . . . . . . . . . . 320

2.1.2 Die immunisierende Wirkung organisationaler Kulturen und Strukturen . . . . . . . . . . . . . . . . 335

2.2 Die Bedeutung von Diversität und Konflikt für die ausbleibende strategische Reaktion . . . . . . . . . . . . . . . 346

2.2.1 Diversität und Konflikt in der monozentrischen Konfiguration der Macht . . . . . . . . . . . . . 350

2.2.1.1 Homogenität der dominanten Koalition . . . . . . 351

2.2.1.2 Mangelnder Sachkonflikt in monozentrischen Konfigurationen . . . . . . . . . . . . . 359

2.2.2 Diversität und Konflikt in der polyzentrischen Konfiguration der Macht . . . . . . . . . . . . 370

2.2.2.1 Heterogenität der dominanten Koalition . . . . 370

2.2.2.2 Sach- und Beziehungskonflikte in polyzentrischen Konfigurationen . . . . . . . 379

3 Fazit: Der Einfluss des Faktors Familie auf die Genese

der strategischen Pfade . . . . . . . . . . . . . . . . . . . 392

E Schlussbetrachtung und Diskussion . . . . . . . . . . . . 395

1 Zusammenfassung . . . . . . . . . . . . . . . 395

2 Kritische Würdigung . . . . . . . . . . . . . . . . . . 402

2.1 Zuverlässigkeit der Ergebnisse und Limitationen der Arbeit . . 403

2.2 Beitrag der Arbeit und weiterführende Diskussion . . . . . . . 409

F Anhang . . . . . . . . . . . . . . . . . . . . . 415

1 Kurzchroniken der Unternehmensentwicklungen . . . . . . . . . 415

1.1 Fallgruppe 1: Anton Schlecker e.K. und Dirk Rossmann GmbH . 415

1.2 Fallgruppe 2: Berentzen-Gruppe AG und Eckes AG . . . . . . 417

2 Quellen referenzierter Daten . . . . . . . . . . . . . . 420

3 Sonstiges . . . . . . . . . . . . . . . . . . . . 428

Literaturverzeichnis . . . . . . . . . . . . . . . . . . . . . . 429 


\section{Geleitwort}

Die Strategie, die der Art und Weise, wie ein Unternehmen jeweils seine Aufgaben verfolgt, zugrundliegt, gehört zum Kern unternehmerischer Tätigkeit. Wann immer ein Unternehmen mit seinen Aktivitäten in seinem jeweiligen Umfeld erfolgreich Fuß gefasst und seine Überlebensfähigkeit über einen längeren Zeitraum unter Beweis gestellt hat, verfügt es über eine Strategie, wenn diese manchmal auch nur implizit ist. Gar nicht so selten wird eine Strategie nämlich nicht im Rahmen eines expliziten Entscheidungsprozesses innerhalb der Unternehmensführung erarbeitet, sondern entsteht in der Alltagspraxis, angelehnt an groben orientierenden Leitlinien des Eigentümers oder der Eigentümer. Mehr oder weniger spiegeln sich darin die Grundüberzeugungen und mentalen Modelle der jeweiligen Entscheidungsträger. Gerade in Familienunternehmen finden sich solche Formen der Zukunftsorientierung, die sich von den in öffentlichen Unternehmen entwickelten Prozessen unterscheiden. Nicht selten ist es hier die unternehmerische Intuition des Gründers/Eigentümers oder, in späteren Generationen, die der Schlüsselfiguren aus der Familie, die als charakteristisches Entscheidungsmuster erkennbar wird.

Wenn es darum geht, die vielfach zu beobachtenden enormen Erfolge von Familienunternehmen zu erklären, wird gern auf derartige Muster verwiesen: die Fähigkeit, intuitiv und damit schnell, entschieden und erfolgreich auf Markterfordernisse zu reagieren, gilt als großes Plus dieser Unternehmensform. Doch diese Art der Strategieentwicklung ist nicht frei von Ambivalenz. Denn eine einmal auf diese Weise gefundene und erfolgreiche Strategie und die ihr folgenden eingespielten Muster des Umgangs mit kritischen Existenzfragen bergen die Gefahr einer spezifischen Selektivität in der Wahrnehmung der Unternehmensumwelt in sich, die mit Begriffen der Selbstberuhigung, ja Selbstimmunisierung, und damit der Pfadabhängigkeit beschrieben werden können: Das eigene Führungshandeln wird immer weniger selbstkritisch betrachtet, beunruhigende Entwicklungen auf dem Markt ignoriert. Einmal gewählte und lange erfolgreich beschrittene Wege der Entscheidungsfindung werden immer wieder begangen. Auch das Umfeld, die Familie, die Mitarbeiter, spielt hier lange mit, man schweigt im 
Zweifelsfall lieber, um nicht als "Kassandra" angesehen zu werden. So kann es geschehen, dass heikle Themen eher nicht thematisiert werden und Kulturen der Vermeidung von Konflikten und des gemeinsamen Nichthinschauens entstehen.

Die Frage, wie es dazu kommen kann, dass Unternehmen, gerade Familienunternehmen einmal eingeschlagene Wege unbeirrt weiter verfolgen, auch wenn sich die Umwelt so sehr verändert hat, dass die fortgesetzte Reproduktion dieser strategischen Muster existenzbedrohend ist, ist bislang noch nicht zufriedenstellend beantwortet. Zum einen liegt noch wenig wissenschaftlich abgesichertes Wissen über die oft langwierigen Prozesse der Entstehung strategischer Engführungen vor: was sind die Indikatoren, die den Betroffenen und ihren Beobachtern (z.B. Beratern) helfen, die Gefahren rechtzeitig zu erkennen? Zum anderen gibt es wenige Befunde, die den Kontext, innerhalb dessen sich solche gefährliche Pfadabhängigkeiten entwickeln, mit einbeziehen, bei Familienunternehmen ist dieser besondere Kontext die Familie. Auf dieser Kritik an der "ahistorischen" wie "aprozessualen" Managementforschung zu Unternehmenskrisen, die daher bislang auch nur wenige fundierte Erklärungsansätze zu bieten hat, setzt die vorliegende Studie auf.

Die Relevanz dieses Thema ist unbestreitbar: es ist von existenzieller Bedeutung, ein Instrumentarium zur Verfügung zu haben, das der Führung erlaubt, spezifische Selbstbeobachtung auf Pfadabhängigkeit hin vorzunehmen. Denn praktisch jedes Unternehmen ist wiederholt mit der Notwendigkeit strategischer Neuausrichtung konfrontiert, und man weiß, welche enormen Werte dabei auf dem Spiel stehen, ganz zu schweigen von der Bedeutung für oft Tausende von Mitarbeitern und deren Familien. Das vorliegende Buch erfüllt genau diesen Zweck. Ich bin sicher, es wird bei Betroffenen wie bei beratend und wissenschaftlich Tätigen gleichermaßen auf große Resonanz stoßen. Die Lektüre kann ich ohne jeden Vorbehalt empfehlen!

Witten, im September 2017

Arist v. Schlippe 


\section{Vorwort}

Vorliegende Studie widmet sich mit strategischen Pfaden spezifischen strategischen Entwicklungswegen, die von den Entscheidungsträgern der untersuchten Unternehmen zu einem frühen Zeitpunkt der organisationalen Evolution eingeschlagen wurden und denen dann über viele Jahre - teils Jahrzehnte hinweg gefolgt wurde. Die Entscheidungen zum Beschreiten dieser Wege waren zum Teil der historischen Prägung der Unternehmen geschuldet, zum Teil zufallsabhängig und kontingent. Es hätte lange Zeit auch andere Entwicklungsmöglichkeiten gegeben. Nachdem jedoch die erste Wegstrecke zurückgelegt worden war, fiel es den Entscheidungsträgern - unabhängig von den ursprünglichen Ursachen und Motiven - aufgrund einer spezifischen sich selbst verstärkenden Prozessdynamik immer schwerer vom einmal eingeschlagenen Weg wieder abzukehren je länger sie ihm folgten. So wurde der Pfad immer weiter gegangen - bis er die Unternehmen schlussendlich in existenzbedrohende Krisen führte.

Solchen strategischen Pfaden wird sich in der vorliegenden Arbeit theoretisch und empirisch angenähert. Bevor sich diesem Phänomen zugewandt wird, sei jedoch an dieser Stelle zunächst jenen Menschen gedankt, die den Entstehungsprozess dieser Arbeit lange vor dessen eigentlichen Anfang bedingten und prägten, sowie den Wegbegleitern Dank ausgesprochen, die mir halfen, den Forschungsprozess in fortgeschrittenen Stadien offen für neue Perspektiven und Impulse zu halten und ihn somit vor einer unheilvollen Pfadabhängigkeit bewahrten.

Die »historische Prägung" dieser Arbeit nimmt ihren Anfang bei meinen Eltern und Großeltern. Jeder knüpft mit seinen Handlungen an Bestehendes an und wird von diesem geformt. Daher verdanke ich viel meiner Familie, die mir nicht nur Wärme und vielfältige Unterstützung zukommen ließ, sondern mir auch zahlreiche Möglichkeiten eröffnete, um meinen eigenen Weg zu gehen. Ihnen sei daher in tiefer Dankbarkeit diese Arbeit gewidmet.

Großer Dank gilt aber auch meinen alten Freunden aus Heidelberg, die mich seit Kindestagen an begleiten. Die Diversität ihrer Persönlichkeiten und Per- 
spektiven auf die Welt hat mich sehr bereichert und der damit einhergehende kritische Austausch meinen Blick geweitet.

In der akademischen Welt gilt mein erster Dank Prof. Dr. Lutz Kruschwitz und Prof. Dr. Jürgen Wolters. Ohne den Einsatz, den sie mir in einer schwierigen Phase zu Beginn meines Studiums zukommen ließen, hätte diese Arbeit gar nicht erst ihren Anfang nehmen können. Für ihr persönliches Engagement werde ich ihnen immer verbunden sein.

Eher zufällig begann ich die Tätigkeit als studentische Hilfskraft beim Forschungsprojekt »Von Generation zu Generation - der Nachfolgeprozess in Familienunternehmen«. Rückblickend betrachtet war - in der Terminologie der Pfadtheorie gesprochen - diese Mitarbeit wohl aber das »critical juncture « für vorliegende Studie, denn Prof. Dr. Martin Kohli, Dr. Isabell Stamm und Nicole Schmiade machten mich nicht nur theoretisch mit dem Forschungsgegenstand des Familienunternehmens vertraut, sondern weckten durch vielfältige Einblicke in ihre Forschungsarbeit vor allem mein Interesse am wissenschaftlichen Arbeiten.

Den Umstand, dass diese Arbeit zu Ende geführt, dabei aber nicht pfadabhängig wurde, verdanke ich einer Reihe von Menschen, die meine Arbeit in den letzten Jahren unterstützten. Prof. Dr. Arist von Schlippe und Prof. Dr. Andreas Hack danke ich dafür, dass sie meiner Forschungsidee von Beginn an offen und interessiert gegenüberstanden und mir einen größtmöglichen Handlungsspielraum in ihrer Verfolgung einräumten. Das entgegengebrachte Vertrauen wirkte sehr motivierend.

Prof. Dr. Georg Schreyögg an der Freien Universität Berlin gebührt Dank für die wohlwollende Begleitung und konstruktive Kritik, die er dieser Arbeit zuteil werden ließ. Als externer Doktorand gerät man ohne den täglichen Austausch mit Kollegen leicht in Gefahr sich geistig zu versteifen und wenngleich es letztlich natürlich dem Promovenden obliegt, einen passenden Weg durch die Komplexität der wissenschaftlichen Aufgabe zu finden, so bewahrten mich die Hinweise Prof. Dr. Schreyöggs doch zumindest des Öfteren davor, weniger zielführende Richtungen weiter zu verfolgen. Vor dem kognitiven »Lock-in" schützten auch die Diskussionen mit anderen Promovierenden in den Doktorandenkolloquien an der Universität Witten/Herdecke und an der FU Berlin. Deshalb sei an dieser Stelle auch der Vielzahl der dort angetroffenen guten Gesprächspartner gedankt.

Finanziell wurde dieses Forschungsvorhaben großzügig unterstützt von der Konrad-Adenauer-Stiftung, die mir durch zahlreiche interessante Seminare oder Auslandsaufenthalte zudem auch eine fruchtbare ideelle Förderung außerhalb der engen thematischen Grenzen des eigenen Forschungsprojekts zukommen ließ.

Gedankt sei natürlich auch den Interviewpartnern, die mir im Zuge der 
empirischen Untersuchung, tiefe Einblicke in ihre Unternehmen und Gedankenwelten gewährten und so diese Arbeit überhaupt erst ermöglichten.

Trotz dieser Unterstützung, die dem Forschungsvorhaben von vielen Seiten zukam, verlief der Entstehungsprozess dieser Arbeit selbstverständlich aber nicht ohne Tiefen. Dass die Arbeit zum Ende des Forschungsprozesses nicht doch noch in einer Krise mündete, verdanke ich an erster Stelle meiner Familie, die mich nicht erst während der Promotion mit aller Kraft - und jeder auf seine Weise - unterstützt und immer an mich geglaubt hat. Insbesondere meiner wie stets über die Maßen hilfsbereiten Mutter und meiner Schwester, die viel Zeit und Geduld in die Überarbeitung des Manuskripts investierten. Wertvolle Unterstützung in dieser kritischen Phase erfuhr ich auch von Dr. Sybille Strobel, bei der ich mich für einen konstruktiv-kritischen Blick und einen äußerst fruchtbaren Austausch bedanken möchte.

Das letzte Wort des Danks gebührt allerdings meiner Lebensgefährtin Tanja. Für ihre Nachsicht mit mir in den vergangenen Jahren, ihren Beistand und dafür, dass sie so ist, wie sie ist.

London, im Juli 2016

Thomas Treiber 
Open-Access-Publikation im Sinne der CC-Lizenz BY 4.0

(C) 2018, VeR unipress GmbH, Göttingen 


\section{Abbildungsverzeichnis}

Abbildung 1: Ordnungsrahmen der Forschungsarbeit 30

Abbildung 2: Teilschritte des zirkulären Forschungsprozesses $\quad 35$

Abbildung 3: Idealtypischer Phasenverlauf der Krisenentstehung 53

Abbildung 4: Phasenmodell der organisationalen Pfadgenese nach Sydow et al. (2009) 72

Abbildung 5: $\quad$ Formen kausaler Verknüpfungen von Mechanismen 78

Abbildung 6: Strukturelle Kopplung von Familie und Unternehmen 120

Abbildung 7: Einfluss der Familie im Verlauf der Ko-Evolution und Ausprägung organisationaler Strukturmerkmale 132

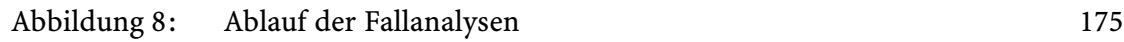

Abbildung 9: Entwicklung der Filialzahlen der führenden Drogeriemarktunternehmen Deutschlands $\quad 184$

Abbildung 10: Wachstumsdynamik der Wettbewerber der Anton Schlecker e.K. 185

Abbildung 11: Einordnung der Wachstumsstrategie der Anton Schlecker e.K. in Produkt/Markt-Matrix 197

Abbildung 12: Sich selbst verstärkende Wachstumsdynamik der Anton Schlecker e.K. und komplementärer Betriebstyp 207

Abbildung 13: Entwicklung des Umsatzes pro Filiale Anton Schlecker e.K. und dm-Drogeriemarkt 209

Abbildung 14: Vergleich der Flächenproduktivität der führenden Drogeriemarktunternehmen Deutschlands im Jahr $2010 \quad 210$

Abbildung 15: Umkehrung der Wachstumsspirale der Anton Schlecker e.K. 215

Abbildung 16: Gewinnentwicklung der Anton Schlecker e.K. in den Jahren 1999-2011 217

Abbildung 17: Entwicklung der Aktie der Berentzen-Gruppe AG im Zeitraum 1995-2015

Abbildung 18: Entwicklung Pro-Kopf-Konsum Spirituosen in D 1960-2012 / Umsatz- und Gewinnentwicklung der Berentzen-Gruppe AG 234

Abbildung 19: Sparten und Geschäftsbereiche der Berentzen-Gruppe AG 236

Abbildung 20: Berentzen-Gruppe AG: Entwicklung des Umsatzes der Sparte alkoholfreie Getränke 1983-2015

Abbildung 21: Die Geschäftsfelder der Berentzen-Gruppe AG im Rahmen der Produkt/Markt-Matrix 
Abbildung 22: Dualität von Handlungsdruck und Handlungsspielraum im Krisenprozess

Abbildung 23: Zirkulärer Prozess der Konflikteskalation

Abbildung 24: Ausbildung eines Entwicklungskorridors durch Imprints in der Phase der historischen Vorprägung

Abbildung 25: Unternehmensimage der fünf führenden Drogeriemärkte Deutschlands im Jahr 2009

Abbildung 26: Struktur des Eigentums der Berentzen-Gruppe AG 316

Abbildung 27: Die Phase der Pfadformation im Prozess der Pfadgenese

Abbildung 28: Wirkungszusammenhang organisationaler Variablen in den Entscheidungsprozessen der Phase der Pfadformation 


\section{Tabellenverzeichnis}

Tabelle 1: Divergierende Systemlogiken von Familie und Unternehmen

Tabelle 2: Strukturelle Merkmale des familiären Einflusses der Kategorie "Command"

Tabelle 3: Strukturelle Merkmale des familiären Einflusses der Kategorie "Continuity"

Tabelle 4: Strukturelle Merkmale des familiären Einflusses der Kategorien "Community« und »Connections"

Tabelle 5: Narrative Experteninterviews im Rahmen der explorativen Vorstudie 166

Tabelle 6: Fallauswahl des komparativen Samplings

Tabelle 7: Übersicht über narrative biografische Fallinterviews

Tabelle 8: Übersicht zu Daten aus internen und externen Dokumenten

Tabelle 9: Stufen der Konflikteskalation nach Glasl (2013) 
Open-Access-Publikation im Sinne der CC-Lizenz BY 4.0

(C) 2018, VeR unipress GmbH, Göttingen 


\section{Abkürzungsverzeichnis}

\begin{tabular}{ll} 
AMJ & Academy of Management Journal \\
AMR & Academy of Management Review \\
ASQ & Administrative Science Quarterly \\
AU & Österreich \\
BE & Belgien \\
CEO & Chief Executive Officer \\
CFO & Chief Financial Officer \\
COO & Chief Operating Officer \\
D & Deutschland \\
DK & Dänemark \\
EG & Europäische Gemeinschaft \\
EHI & EHI Retail Institute e.V. (vormals: EuroHandelsinstitut e.V.) \\
ESP & Spanien \\
ET\&P & Entrepreneurship Theory and Practice \\
FBN & Family Business Network \\
FR & Frankreich \\
GfK & Gesellschaft für Konsumforschung \\
HUN & Ungarn \\
KMU & Kleine- und mittlere Unternehmen \\
1 & Liter \\
LUX & Luxemburg \\
JOM & Journal of Management \\
JMS & Journal of Management Studies \\
LEH & Lebensmitteleinzelhandel \\
Mio. & Millionen \\
Mrd. & Milliarden \\
NL & Niederlande \\
ORGAN SCI & Organisation Science \\
ORGAN STUD & Organisation Studies \\
SB & Selbstbedienung \\
TMT & Topmanagement-Team \\
UE & Upper-Echelon \\
USA & United States of America \\
& \\
\hline & \\
\hline
\end{tabular}


Open-Access-Publikation im Sinne der CC-Lizenz BY 4.0

(C) 2018, VeR unipress GmbH, Göttingen 


\section{A Einleitung}

\section{Problemstellungen}

Die Insolvenz der Anton Schlecker e.K. im Jahr 2012 war eine der größten Insolvenzen der deutschen Nachkriegsgeschichte. Ein Unternehmen mit ehemals 7 Mrd. Euro Umsatz und 14.000 Filialen verschwand innerhalb kürzester Zeit vollständig vom Markt. Beinahe 50.000 Mitarbeiter verloren ihren Arbeitsplatz.

Für viele Beobachter kam die Insolvenz des ehemaligen Marktführers jedoch nicht gänzlich überraschend. Während die Konkurrenz von dm oder Rossmann im Laufe der Jahre ein deutliches Trading-Up vollzogen hatte und die Kunden nun mit freundlicher Ladengestaltung, einem großen Sortiment und kleinen Preisen in die Geschäfte lockte, hatte sich das Geschäftsmodell der Anton Schlecker e.K. kaum verändert: Die Filialen in abgelegenen Lagen waren ebenso klein wie das Sortiment, die Gänge schmal und das Licht kam aus der Neonröhre. Einen Vorteil bot Schlecker seinen Kunden jedoch - es gab fast überall eine Schlecker-Filiale. Denn anstatt das Geschäftsmodell grundlegend zu überarbeiten, investierte das Unternehmen einen Großteil seiner Ressourcen in die fortgesetzte Expansion mit dem althergebrachten Betriebstyp. Selbst als ab 2004 die Umsätze auf den bestehenden Flächen massiv einbrachen, wurden jedes Jahr noch Hunderte neue Filialen eröffnet, ohne dass alte, unrentable Verkaufsstellen geschlossen worden wären. Doch warum wurde so lange Zeit an dieser Wachstumsstrategie festgehalten, anstatt das Geschäftsmodell grundlegend zu überarbeiten? Warum erfolgte der notwendige strategische Wandel nicht, obwohl die Umwelt sich veränderte?

Hinsichtlich der hier zu beobachtenden strategischen Persistenz ist die Anton Schlecker e.K. allerdings kein Einzelfall. Unternehmen wie der traditionsreiche Autobauer Karmann, das Wirtschaftswunderunternehmen Grundig oder KarstadtQuelle zeugen ebenfalls von über Jahrzehnte hinweg ausgebliebenem Wandel - und dessen Konsequenzen. Denn diese Persistenzen führten die Unternehmen in die Krise und beraubten sie schlussendlich auch ihrer 
Existenz. Vor dem Hintergrund solcher Beobachtungen stellt sich die grundlegende Forschungsfrage dieser Arbeit:

Warum verfolgen manche Unternehmen dauerhaft und unverändert spezifische Strategien, obgleich die fortgesetzte Reproduktion dieser strategischen Muster existenzbedrohend ist?

(Empirisch abgeleitete Fragestellung)

Von dieser Fragestellung ausgehend, soll sich im Rahmen dieser Arbeit auf die Suche nach Antworten begeben werden.

\section{Allgemeine Problemstellung: Unternehmenskrisen aufgrund strategischer Persistenzen}

Auf dieser Suche schlägt die Arbeit zunächst einen Weg ein, der seinen Ausgangspunkt beim manifestierten Resultat solcher strategischer Persistenzen nimmt: dem Phänomen der Unternehmenskrise.

Die Wahrnehmung und Reflexion krisenhafter Situationen ist in vielen Bereichen der postmodernen Gesellschaft zu einem ständigen Begleiter geworden. Dennoch ist es insbesondere unser hochgradig komplexes und vernetztes ökonomisches System, welches im Großen wie im Kleinen von einer besonderen Krisenhaftigkeit gekennzeichnet ist, stellt in unserer wettbewerbsorientierten ökonomischen Ordnung der Ernstfall des Unternehmens doch den Normalzustand des Systems dar. ${ }^{1}$ Beinahe zwangsläufig führt die Dynamik unserer ökonomischen Umwelt, welche aus der Interaktion von Milliarden miteinander verbundener Akteure erwächst, zu krisenhaften Entwicklungen all jener Unternehmen, die auf den Wandel ihrer Umwelt nicht angemessen reagieren können. Diese permanente Gefährdung der ökonomischen Existenz durch eine herausfordernde Umwelt ist zwar kein grundlegend neues gesellschaftliches Phänomen. Im Zuge einer zunehmend integrierten und globalisierten Weltwirtschaft, eines sich beschleunigenden technologischen Wandels und sich beständig verändernder gesellschaftlicher Bedürfnisse hat sich die Bedrohungslage in den letzten Jahrzehnten jedoch nochmals verschärft.

Die Unternehmen als Treiber wie Getriebene der Entwicklung können diesen Wandel gestalten; ihre Entwicklung ist durch die Umwelt nicht schicksalhaft determiniert. $^{2}$ Gestalten und wandeln müssen sie sich aber, wollen sie ihre

1 Vgl. Staehle \& Albach (1984: 15). Sind die vielen »Krisen im Kleinen« auf Unternehmensebene doch nach Schumpeter (1950) der effektivste Weg, um die Krise des gesamten ökonomischen Systems zu vermeiden.

2 In dieser Arbeit wird somit grundlegend dem Gedanken der "strategic choice« nach Child (1972) oder Schendel \& Hofer (1979) gefolgt. Vgl. hierzu auch: Zajac et al. (2000); zu den organisationstheoretischen Grundlagen Lawrence \& Lorsch (1967) und Thompson (1967). 
Passung mit der Umwelt nicht verlieren und in der sich immer schneller verändernden und existenziell herausfordernden Umwelt bestehen.

Organisationale Entwicklungen wie jene der Anton Schlecker e.K. verweisen jedoch darauf, dass dies nicht jedem Unternehmen dauerhaft gelingt. Der Unternehmenskrisenforschung gemäß gerät gar jedes fünfte Unternehmen in einem Zeitraum von zehn Jahren in eine existenzbedrohende Krise; und nur ungefähr einem Drittel dieser Unternehmen gelingt deren erfolgreiche Bewältigung. ${ }^{3}$ Das Scheitern jahrzehntelanger Marktführer wie Schlecker oder Nokia stellt somit lediglich die sichtbare Spitze des Eisbergs dar.

Die Ursachen für dieses Scheitern werden von vielen verantwortlichen Entscheidungsträgern ex post in einer ungünstigen und nicht $\mathrm{zu}$ beeinflussenden Entwicklung der Umwelt gesucht. Der Tenor der Krisenursachenforschung lautet im Gegensatz hierzu jedoch, dass den meisten Unternehmenskrisen eben keine exogenen Schocks wie Konjunkturkrisen zugrunde liegen, sondern im Kern meist Kombinationen innerorganisationaler Problemfaktoren, welche dazu führen, dass das Unternehmen sich im Verlauf der Zeit nicht mehr adäquat den Veränderungen seiner Umwelt anpassen kann. ${ }^{4}$ Die meisten Krisen brechen nicht schicksalhaft über das Unternehmen herein, sondern sind vielmehr meist die Manifestation eines gescheiterten evolutionären Wandlungsprozesses.

Im Lichte dieser Erkenntnis stellt sich die von dieser Arbeit aufgeworfene Frage nach den Ursachen des ausbleibenden Wandels - eine Forschungsfrage, die angesichts der Bedeutung strategischer Persistenzen für das häufige Auftreten von Unternehmenskrisen eine gewichtige Problemstellung des ökonomischen Geschehens adressiert. ${ }^{5}$ Hier bedarf es weiterer Arbeit durch die Managementforschung.

Theoretische Problemstellung: Defizite der bisherigen Unternehmenskrisenforschung Der wissenschaftliche Handlungsbedarf erscheint dabei umso dringlicher, wenn man den bisherigen Stand der Managementforschung zu Unternehmenskrisen und deren strukturelle Defizite reflektiert.

3 Vgl. Chowdhury \& Lang (1996: 172); Hambrick \& Schecter (1983: 237); Pandit (2000: 32 f.).

4 Vgl. Arogyaswamy et al. (1995); Hambrick \& D’Aveni (1988: 10ff.); Schendel et al. (1976: 7); Weitzel \& Jonsson (1989: 10).

5 Dies gilt, selbst wenn man wie Staehle \& Albach (1984: 14f.) oder Witte (1981: 12) die teils reinigende Kraft von Unternehmenskrisen für die Gesamtökonomie reflektiert. Denn weder die auf das Einzelunternehmen bezogene Managementforschung noch die durch sie beratenen Unternehmer können und wollen ex-ante zwischen existenzberechtigten und nicht existenzberechtigten Unternehmen trennen. Die finale Selektion sollte dem Markt überlassen werden. 
»Sound empirical work begins with a strong grounding in relevant literature, identifies a research gap and proposes research questions that address that gap."

(Eisenhardt \& Graebner, 2007) ${ }^{6}$

Lange Zeit folgte auch die Managementforschung dem wehenden Banner des Wachstumsparadigmas und vernachlässigte auf der Suche nach Erfolgsfaktoren und Wettbewerbsvorteilen das Scheitern von Unternehmen beinahe vollkommen. Erst in den letzten dreißig Jahren fand die Unternehmenskrise nach und nach ihren Platz im betriebswirtschaftswissenschaftlichen Diskurs. ${ }^{7}$ Allerdings weist die existierende Forschung neben einem allgemeinen Theoriedefizit in ihrer grundsätzlichen thematischen Ausrichtung bis zum heutigen Tage große strukturelle Ungleichgewichte und Defizite auf. Dies wird deutlich, wenn man die bestehenden Beiträge nach dem klassischen Ordnungsschema der strategischen Prozessforschung nach Pettigrew (1987) kategorisiert, welches die drei Dimensionen des Prozesses der Strategiebildung, der Inhalte von Strategien und des Kontexts von Strategien unterscheidet. ${ }^{8}$

Hierbei wird ersichtlich, dass sich der mit Abstand größte Teil der Managementforschung zu Unternehmenskrisen ausschließlich mit den Möglichkeiten der Krisenbewältigung auseinandersetzt. Unter Begriffen wie Sanierung, Restrukturierung oder Turnaround widmen sich die meisten Beiträge in meist stark präskriptiver Weise und aus recht technischer, häufig finanzwissenschaftlicher oder juristischer Perspektive in erster Linie dem Inhalt (»content«) von Strategien der Krisenbewältigung.

Vor dem Hintergrund dieser ausgeprägten Fokussierung auf den Inhalt scheinbar erfolgsversprechender Maßnahmen offenbart die bisherige Forschung zu Unternehmenskrisen jedoch ihre strukturellen Lücken, wenn man mit der diese Arbeit leitenden Frage nach den tieferliegenden Ursachen der Krise an die bestehende Literatur herantritt. Hier werden zwei Defizite rasch deutlich: zum einen die beinahe konsequente Vernachlässigung des Prozesses der Krisenentstehung (»process«) und dessen spezifischer Dynamiken, zum anderen die ungenügende Integration von Kontextvariablen (»context») wie der Organisationskultur oder die Zusammensetzung des Managements. ${ }^{9}$ In ihren Rah-

6 Eisenhardt \& Graebner (2007: 26).

7 Staehle \& Albach (1984: 9ff.) mussten so noch eindringlich fordern, dass die Unternehmenskrise endlich als permanentes Problem der Betriebswirtschaftslehre wahrgenommen werden müsse. Zur aktuellen Stellung der Unternehmenskrise in der Managementforschung vgl. Schreyögg \& Ostermann (2014: 120).

8 Vgl. Pettigrew (1987: 657); Pettigrew (1987); auch Mintzberg \& Waters (1990). Zur Rezeption Bresser (2010: 15).

9 Vgl. die Reviews Pandit (2000) und Trahms et al. (2013) zu Turnaround bzw. »organizational decline«. So halt Pandit (2000: 32f.) fest: »Whilst questions relating to the content of turnaround strategies are reasonably frequent, those relating to the context and process of turnaround are rare." 
men ist jedoch sowohl der Prozess der Krisenentstehung als auch jener der Krisenbewältigung stets eingebettet. ${ }^{10}$

Ein systematischer Bezug auf die zugrunde liegenden prozessualen Ursachenzusammenhänge und situativen Kontextbedingungen erscheint allerdings nicht zuletzt für die nachhaltige Bewältigung der Krise unabdingbar. ${ }^{11}$ Ohne einen theoretisch fundierten Einbezug der Prozessualität der Krisenentstehung laufen Handlungsempfehlungen nämlich leicht Gefahr, wichtige Interdependenzen und Entwicklungsdynamiken zu übersehen. Die auf solchen Empfehlungen basierenden Entscheidungen müssen jedoch stets auch die historischen Entscheidungen adressieren, die in die Krise geführt haben.

Dessen ungeachtet finden sich insbesondere in der dominanten angloamerikanischen Literatur zu Unternehmenskrisen allerdings immer noch recht häufig idealisierte und ahistorische Konzeptionen des strategischen Entscheidungs- und Handlungsraumes. Dieser Raum erscheint nicht selten - losgelöst vom Ballast der Vergangenheit - als Ort voller Möglichkeiten, in dem wie am Reißbrett neue strategische Optionen geplant und der strategische Wandel nur noch implementiert werden muss. Durch diese Vernachlässigung der historischen Vorprägung fällt die Unternehmenskrisenforschung hier aber weit hinter die Erkenntnisse der allgemeinen strategischen Prozessforschung zurück, welche seit Langem auf die Bedeutung historischer Entscheidungen für gegenwärtige organisationale Entscheidungsprozesse aufmerksam macht. ${ }^{12}$ Nicht zuletzt im Kontrast zu diesem etablierten Forschungsfeld erscheint die Managementforschung zu Unternehmenskrisen häufig ebenso ahistorisch wie aprozessual.

Über diese mangelnde Berücksichtigung der Prozessualität von Krisen hinaus vernachlässigt ein großer Teil der bisherigen Unternehmenskrisenforschung auch die kulturellen, sozialen und psychologischen Variablen des organisationalen Kontexts. ${ }^{13}$ Potenziell relevante organisationale Kontextvariablen wie die Organisationskultur, die demografische Zusammensetzung des Topmanagements oder die Eigentümerstruktur werden nicht hinreichend in die Analyse einbezogen. Es sind jedoch gerade solche Variablen, die in hohem Maße die

10 Vgl. Arogyaswamy et al. (1995: 494); Hambrick \& D’Aveni (1988: 20); Pandit (2000); Trahms et al. (2013).

11 Vgl. Hofer (1980); Slatter (1984); Starbuck et al. (1978).

12 Vgl. zur strategischen Prozessforschung: Quinn (1980); Eisenhardt \& Zbaracki (1992). Pettigrew (1977: 79): »Yesterday's strategies will provide some of the pathways to and input's for today's strategies [...] the consequences of the implementation of today's strategies will provide part of the context for tomorrow's strategies."

13 Vgl. Pandit (2000: 20); Trahms et al. (2013: 1290). Obgleich Beiträge wie Hambrick \& D’Aveni (1988: 20) oder Nystrom \& Starbuck (1984) zum Beispiel seit Langem eine verstärkte Integration des Kontexts anmahnen. 
Struktur und Dynamik von strategischen Veränderungsprozessen beeinflussen und den strategischen Wandel ebenso behindern wie befördern können. ${ }^{14}$

Angesichts dieser beiden strukturellen Forschungslücken tritt neben die allgemeine Problemstellung der Vielzahl von Unternehmenskrisen aufgrund strategischer Persistenzen somit auch eine theoretische Problemstellung: die zu enge Fokussierung der Managementforschung auf den Inhalt scheinbar erfolgversprechender Maßnahmen der Krisenbewältigung und die damit einhergehende Vernachlässigung der zugrunde liegenden prozessualen Dynamiken und Kontextfaktoren.

Vor dem Hintergrund dieser Defizite wundert es nicht, wenn die Unternehmenskrisenforschung im Hinblick auf die Frage nach den Ursachen strategischer Persistenz höchstens vereinzelte Hinweise, schlussendlich aber kaum konsistente und theoretisch fundierte Erklärungsansätze anbietet. ${ }^{15}$

Zur Untersuchung dieser Problemstellung scheint daher eine alternative theoretische Perspektive außerhalb der Unternehmenskrisenforschung geboten, welche es vermag, die prozessuale Historizität der Entscheidungsprozesse zu erfassen und den kontextuellen Rahmen zu berücksichtigen.

\section{Ordnungsrahmen und theoretisch orientierte Forschungsfrage}

Prozess und Kontext - diese beiden von der Managementforschung zu Unternehmenskrisen vernachlässigten Dimensionen strategischer Phänomene sollen im Fortgang der Arbeit adressiert werden. ${ }^{16}$ Sie spannen den Ordnungsrahmen auf, um die Ursachen ausbleibenden strategischen Wandels zu ergründen.

»Theoretically sound and practically useful research on change should explore the contexts, content and process of change together with their interconnectedness through time."

(Pettigrew, 1990) $)^{17}$

In diesem Rahmen möchte die vorliegende Arbeit einen spezifischen Prozess der Entstehung strategischer Persistenzen und daraus resultierender Krisen in einem spezifischen Kontext untersuchen.

14 Vgl. Finkelstein et al. (2009: 83ff.); Wiersema \& Bantel (1992: $91 \mathrm{ff}$.$) .$

15 Vgl. zu dieser Einschätzung Krystek \& Moldenhauer (2007: 50ff.); Schreyögg \& Ostermann (2014).

16 Vgl. Bresser (2010): »Eine der wenigen Übereinstimmungen in der Strategieliteratur betrifft die fundamentalen Dimensionen, die berücksichtigt werden müssen, um Strategiephänomene zu erforschen [...] der Prozess der Strategiebildung, der Inhalt von Strategien und deren Kontext."

17 Pettigrew (1990: 268). 


\section{Prozess: Strategische Pfade als spezifische Prozesse der Krisenentstehung}

Die Gründe für existenzbedrohende strategische Persistenzen können vielfältig sein. Die Praxis verweist auf Phänomene wie den »unternehmerischen Tunnelblick«, eine organisationsinterne »Betriebsblindheit« oder das Festhalten an Erfolgsmustern der Vergangenheit. Die Managementforschung macht zum Beispiel mit dem »Ikarus-Paradox « darauf aufmerksam, dass die Faktoren, die einst den Erfolg des Unternehmens begründeten, auch zu dessen Niedergang führen können. ${ }^{18}$ Sie zeigt aber auch die Risiken politischer Konstellationen auf, welche den Wandel hemmen können. Die Organisationstheorie adressiert die »structural inertia« von Routinen und Praktiken oder organisationale Widerstände gegen Wandel. ${ }^{19}$

In einigen Fällen liegt dem Scheitern von Unternehmen jedoch eine spezifische prozessuale Entwicklungsdynamik zugrunde, die zu einer besonders kritischen Art von strategischer Persistenz führt. So können zunächst scheinbar unbedeutend erscheinende Ereignisse oder Entscheidungen Mechanismen in Gang setzen, welche sich in der Folge durch positive Rückkopplungen selbst immer weiter verstärken. Im Zuge der Sogwirkung solcher selbstverstärkender Mechanismen kann die Reproduktion eines bestimmten strategischen Musters im Entscheidungsprozess immer weiter begünstigt werden, so lange, bis die Wirkkraft der Mechanismen so hoch ist, dass die Organisation den eingeschlagenen strategischen Entwicklungsweg nicht mehr verlassen kann. Das Unternehmen ist auf einem bestimmten strategischen Pfad "gefangen«. Seine weitere strategische Entwicklung ist pfadabhängig. ${ }^{20}$

Aus diesem Zustand erwächst für das Unternehmen rasch eine existenzbedrohende Krisensituation, wenn sich die Wege der stets dynamischen Umwelt und des Unternehmens zu trennen beginnen. Die Pfadabhängigkeit führt das Unternehmen dann direkt in die Krise. ${ }^{21}$

Solche strategischen Pfade sollen im Rahmen dieser Arbeit als eine bestimmte Ursache strategischer Persistenz und als spezifischer Prozess der Krisenentstehung untersucht werden. Denn strategische Pfade sind nicht nur von nachgewiesener empirischer Relevanz, sondern stellen vor dem Hintergrund des aufgezeigten theoretischen und prozessualen Defizits der bisherigen Unter-

18 Vgl. Miller (1992).

19 Vgl. Hannan \& Freeman (1984). Zum Festhalten an Erfolgsmustern vgl. auch Luhmann (2000: 360). Aus der Managementforschung: Miller (1992); zu politischen Prozessen Pettigrew (1987) oder die »Architecture of Simplicity« von Miller (1993). In der Organisationstheorie vor allem Hannan \& Freeman (1984), aber auch Staw (1976); Stinchcombe (1965). Zu den organisationalen Widerständen gegen Wandel grundlegend Lewin (1947).

20 Vgl. Sydow et al. (2009). Zur organisationalen Pfadtheorie ausführlich Kapitel B.1.3.

21 Die Reduktion der Handlungsspielräume des Managements durch die Sogkraft des selbstverstärkenden Mechanismus ist bereits dann als äußert kritisch zu betrachten, wenn sich der etablierte strategische Pfad noch im Einklang mit der Umwelt des Unternehmens befindet. 
nehmenskrisenforschung auch einen geeigneten analytisch-theoretischen $\mathrm{Zu}$ gang zum Forschungsfeld »Unternehmenskrisen aufgrund strategischer Persistenz« dar. ${ }^{22}$ Ist es doch eine charakteristische Leistung der organisationalen Pfadtheorie über ihr zentrales Explanans der selbstverstärkenden Mechanismen und des daraus resultierenden Zustands des »Lock-in« die Prozesshaftigkeit der Krise zu erfassen. ${ }^{23}$ Auf diesem Wege vermag sie es, die Dynamik des Krisenprozesses von der Entstehung der Krise über deren zunehmende Verschärfung, bis hin zu den Möglichkeiten ihrer Bewältigung einer theoretisch konsistenten Analyse zuzuführen. Im Vergleich zu vielen anderen Erklärungsansätzen postuliert die organisationale Pfadtheorie nicht nur, dass ein kausaler Zusammenhang zwischen einem Faktor und dem Explanandum der strategischen Persistenz besteht, sondern erfasst durch ihre prozessendogene Erklärung auch trennscharf und prozessbegleitend wie die Kausalität besteht und dynamisch wirkt. $^{24}$

Jüngere Arbeiten zu strategischer Pfadabhängigkeit wie jene von Koch (2011) weisen jedoch zu Recht darauf hin, dass es zu einem tiefer gehenden Verständnis solcher Prozesse und deren organisationalen Charakters der Integration einer weiteren Dimension bedarf: des spezifischen Kontexts von Struktur und Kultur, in dem sich die strategischen Pfade entfalten. ${ }^{25}$

\section{Kontext: Der Einfluss des Kontextfaktors Familie}

Kontextuell geprägte Variablen wie die Organisationskultur oder Macht wurden jedoch bislang nicht nur von der Unternehmenskrisenforschung, sondern auch von der organisationalen Pfadtheorie vernachlässigt. So spricht Koch (2011) von der »unexplored role of context « in Bezug auf die Genese strategischer Pfade und fordert eine stärkere Reflexion der organisationalen Einbettung strategischer Pfade. ${ }^{26}$ Dieser Forderung soll im Rahmen dieser Arbeit gefolgt werden.

Dabei wird die Bedeutung kontextueller Variablen wie Macht jedoch nicht wie teilweise geschehen - rein abstrakt untersucht. ${ }^{27}$ Vielmehr soll der Einfluss solcher kontextueller Variablen auf die Genese und Dynamik strategischer Pfade im Wirkungsrahmen eines spezifischen Kontextfaktors untersucht werden: dem Faktor Familie in der Sphäre von Familienunternehmen.

22 Vgl. für empirische Studien zur strategischen Pfadabhängigkeit Holtmann (2008); Koch (2011); Schüßler (2008) und zu deren Bedeutung für die Entstehung von Krisen Rindfleisch (2011); Scholle (2013); Tobias (2012).

23 Im Folgenden soll die eingedeutschte sprachliche Schreibweise "Lock-in« verwendet werden.

24 Vgl. Sydow et al. (2009: 696) und die kurze Theoriediskussion in Kapitel B.1.3.4.

25 Vgl. zu dieser Forderung Koch (2011: 341f.); Scholle (2013: 422); Sydow et al. (2009: 701).

26 Vgl. Koch (2011: 359).

27 Vgl. zu solchen Versuchen zum Beispiel Koch et al. (2009); Petermann (2010). 
Entgegen aller Prognosen und jahrzehntelanger Nachrufe der Managementliteratur können heutzutage noch immer ungefähr zwei Drittel aller Unternehmen in Deutschland als Familienunternehmen begriffen werden. ${ }^{28}$ Doch von der etablierten Organisations- und Strategietheorie wurde der Faktor Familie in der Analyse organisationaler Zusammenhänge trotz dessen ungebrochener empirischer Relevanz bis vor wenigen Jahren beinahe vollständig ignoriert. ${ }^{29}$

Auch die Thematik des Familienunternehmens in der Unternehmenskrise fand bislang nicht die Beachtung, die der empirischen Bedeutung dieser Form ökonomischer Aktivität gerecht würde. ${ }^{30}$ Es existieren zwar Beiträge zur verwandten Thematik der Krise in KMU, und auch innerhalb des Feldes der Familienunternehmensforschung wurden mittlerweile einige Beiträge $\mathrm{zu}$ bestimmten Aspekten der Krise publiziert. ${ }^{31}$ Die etablierte Managementforschung hat sich dieser Thematik jedoch noch nicht mit der gebotenen empirischen und theoretischen Fundierung gewidmet.

Hier klafft somit zweifelsohne eine Forschungslücke. Denn die bisherige Forschung zu Familienunternehmen legt die Vermutung nahe, dass organisationale Spezifika von Familienunternehmen - wie die erhöhte Entscheidungsautonomie der Entscheidungsträger oder die Bedeutung des sozio-emotionalen Vermögens - eine simple und vollständige Übertragung der Erkenntnisse der Management- und Unternehmenskrisenforschung auch hinsichtlich dieser Thematik verbieten. ${ }^{32}$

Mittlerweile wurde jedoch sowohl von der Unternehmenskrisenforschung als auch von der organisationalen Pfadforschung erkannt, dass mit diesen organisationalen und strategischen Strukturmerkmalen auch die Notwendigkeit einer gesonderten Analyse einhergeht. So weist zum Beispiel Schüßler (2008) in ihrer Studie zu strategischen Persistenzen in der Textilindustrie auf die Bedeutung

28 Vgl. Berle \& Means (1932) und Chandler (1962) zur These der aussterbenden ökonomischen Form Familienunternehmen; zur dennoch anhaltenden empirischen Prävalenz internationale Studien wie Faccio \& Lang (2002) oder La Porta et al. (1999); für Deutschland zum Beispiel Klein (2010).

29 Hierbei sind das seit Anfang der 1980er Jahre existierende, aber lange Zeit weitgehend isolierte Feld der Familienunternehmensforschung und die allgemeine, etablierte Managementforschung zu unterscheiden.

30 Vgl. zu dieser Einschätzung Cater \& Schwab (2008: 31); Rüsen (2009: 12); Trahms et al. (2013: 1293).

31 Vgl. im internationalen Diskurs Cater \& Schwab (2008). In der deutschen Forschung zu Familienunternehmen vor allem umfassend Rüsen (2009); Rüsen \& von Schlippe (2007) oder Großmann (2014). Vgl. auch Rosenbauer (1994); Wiechers (2006). Zu Krisen in KMU: Boyle \& Desai (1991); Chowdhury \& Lang (1996); Robbins \& Pearce (1993).

32 Vgl. zu diesen Spezifika Miller \& LeBreton-Miller (2005); zudem siehe vor allem Kapitel B.2.3.3 
familieninduzierter Konflikte für die Genese strategischer Pfade hin. ${ }^{33}$ Sie beobachtete familieninterne Auseinandersetzungen, die aufgrund »festgefahrener Eigentümerstrukturen « zur Blockade von Entscheidungsprozessen führten und somit maßgeblich zur Entstehung der strategischen Pfadabhängigkeit beitrugen. Über die Nennung solcher Indizien hinaus wurde der Einfluss des Faktors Familie auf die Entstehung strategischer Pfade bisher allerdings nicht weiter analysiert. Vor dem Hintergrund der vorliegenden Indizien erscheint dies aber als ein fruchtbares Feld. ${ }^{34}$

Mit dem Prozess des strategischen Pfades und dem Kontextfaktor der Familie ist der grundlegende Rahmen der Arbeit aufgespannt. Ausgehend von der empirischen Forschungsfrage, warum einige Unternehmen fortwährend strategische Muster reproduzieren, obgleich diese strategische Persistenz die Unternehmen in die Unternehmenskrise führt, untersucht die vorliegende Arbeit strategische Pfade als spezifische Ursache solcher Persistenzen in Familienunternehmen.

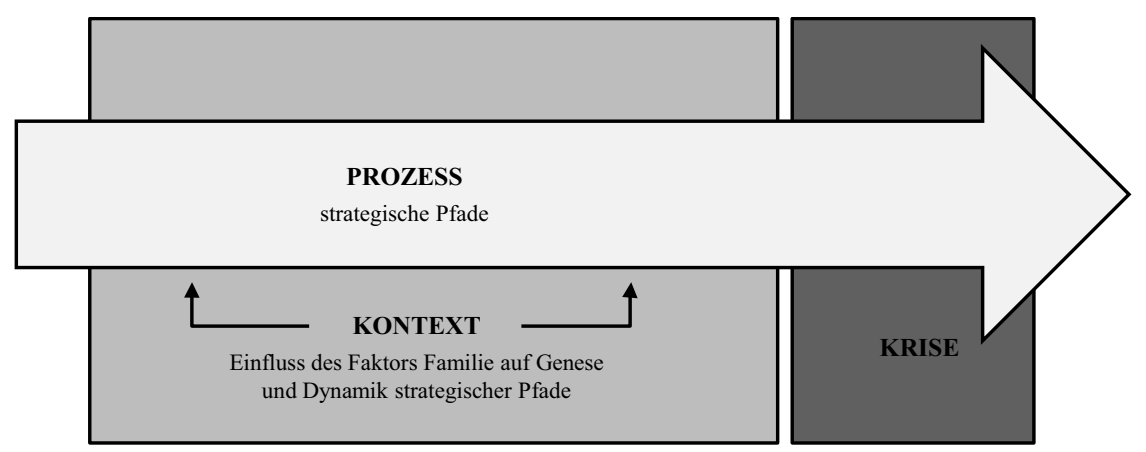

Abbildung 1: Ordnungsrahmen der Forschungsarbeit

(Quelle: Eigene Darstellung).

Innerhalb dieses theoretischen Bezugsrahmens kann die allgemeine Fragestellung zur Ursache strategischer Persistenz nun zur zentralen, theoretisch orientierten Forschungsfrage ausformuliert und spezifiziert werden $:^{35}$

33 Vgl. für die Unternehmenskrisenforschung Trahms et al. (2013: 1293); für die Pfadforschung Schüßler (2008).

34 Zugleich begegnet der Rekurs auf die organisationale Pfadtheorie im Rahmen dieser Arbeit dem immer wieder beklagten Theoriedefizit der Familienunternehmensforschung. Vgl. Zahra \& Sharma (2004: 340).

35 Die eingangs aufgeworfene, an der Stelle aber noch allgemein gehaltene Frage nach den Ursachen häufig beobachteter strategischer Persistenzen kann somit als das ursprünglich interessierende Phänomen und als vorläufige Fragestellung verstanden werden, die im Verlauf des Forschungsprojekts durch die Theorie der Pfadabhängigkeit und Fokussierung 
Inwiefern und wie beeinflusst die Wirkkraft des Kontextfaktors Familie die Genese und Dynamik strategischer Pfade?

(Theoretisch orientierte Forschungsfrage)

Dieser zentralen Forschungsfrage wurde im Rahmen einer empirischen Untersuchung nachgegangen, deren Erkenntnisse im Zuge der vorliegenden Arbeit ausgeführt werden sollen.

\section{Qualitativ-zirkuläre Forschungsstrategie und Zielsetzung}

Angesichts des explorativen Charakters der Forschungsfrage, des geringen Forschungsstandes zu strategischen Persistenzen in Familienunternehmen sowie der tiefgreifenden organisationalen Einbettung möglicher relevanter organisationaler Variablen erscheint zur Leitung der empirischen Untersuchung eine qualitative Forschungsstrategie angemessen. ${ }^{36}$

Denn das Ziel der Arbeit ist nicht die Überprüfung bereits formulierter theoretischer Zusammenhänge, sondern die Entdeckung neuer Zusammenhänge in der Empirie und deren Integration in das theoretische Gerüst der Pfadtheorie. Ein offenes, qualitatives Vorgehen ermöglicht es, den organisationalen Kontext in familiär geprägten Unternehmen beleuchten zu können. Darüber hinaus scheint eine qualitative Strategie aber auch am besten dazu geeignet, die zu rekonstruierende, komplexe Prozessdynamik des Forschungsgegenstands der strategischen Entscheidungsprozesse erfassen zu können. Eine solche tiefgehende Rekonstruktion ist für die Analyse strategischer Pfade unerlässlich, denn zur Erklärung der Entstehung strategischer Persistenz durch strategische Pfadabhängigkeit reicht es nicht aus, eine Korrelation zwischen den Ursachen und dem Explanandum der strategischen Persistenz festzustellen. Vielmehr muss auch rekonstruierend untersucht werden können, wie dieser Zusammenhang das Resultat bewirkte. Hierzu müssen die ökonomischen und sozialen Mechanismen rekonstruiert werden, die im Prozessverlauf die Ursachen mit den Wirkungen verbinden. ${ }^{37}$

Einer solchen rekonstruierenden Untersuchung erschien einzig eine qualitative Forschungsarbeit dienlich, welche sich an den grundlegenden Prinzipien qualitativer Sozialforschung - wie der Offenheit gegenüber dem Forschungs-

auf den Kontextfaktor Familie mit der obigen Forschungsfrage theoretisch spezifiziert wurde.

$36 \mathrm{Zu}$ den Anwendungskontexten qualitativer Forschung in Bezug auf das Erkenntnisinteresse vgl. Flick (2007: 22 ff.); Przyborski \& Wohlrab-Sahr (2008: 15ff.).

37 Vgl. zur Forderung der organisationalen Pfadforschung nach rekonstruierenden Längsschnittuntersuchungen Sydow et al. (2009: 704). 
gegenstand, der Gegenstandsangemessenheit oder der Zirkularität des Forschungsprozesses - orientiert. $^{38}$

Diese Prinzipien gründen insbesondere im wegweisenden methodologischen Ansatz der Grounded Theory nach Glaser und Strauss (1967), welcher auch in der strategischen Prozessforschung weithin rezipiert wird. ${ }^{39}$ Allerdings sollte sich eine Forschungsarbeit nur dann in die Tradition dieser vielzitierten Forschungsstrategie stellen, wenn deren Programm stringent und auch im Detail konsequent verfolgt wird. Das Durchführen qualitativer Interviews mit offenen Fragestellungen und deren anschließende Kodierung allein ist noch keine gegenstandsbegründete Theoriebildung.

Da bereits die Zielstellung dieser Arbeit - die Exploration der Bedeutung des Kontextfaktors Familie im Rahmen des bestehenden Theoriegefüges der Pfadtheorie - von der primären Zielstellung der gegenstandsbegründeten, offenen Theoriegenerierung durch die Grounded Theory abweicht, ist auch der vorliegende Beitrag keine Forschungsarbeit im eigentlichen Sinne von Glaser und Strauss (1967). Nichtsdestotrotz war deren Forschungsstrategie, neben den methodologischen Ansätzen der strategischen Prozessforschung und des Fallstudienansatzes, der wichtigste methodologische Orientierungspunkt des dieser Arbeit zugrunde liegenden Forschungsprozesses. ${ }^{40}$

Im Zuge dieses Forschungsprozesses wurde versucht; sich durch einen rekonstruierenden Zugang und interpretierende Beobachtungen den Prozessen der sozialen Welt zu nähern. Das abstrakte Ziel des Forschungsprozesses war dabei die Herausarbeitung der für die Erklärung des Forschungsgegenstands notwendigen Konzepte aus der sozialen Welt und ein näherungsweises Verstehen der sozialen Prozesse. ${ }^{41}$

Zur Begegnung der Subjektivität sozialer Konstruktionen beruhte der Forschungsprozess auf einer systematischen Konzeption des Erkenntnisgewinns; sowie der Wahl gegenstandsangemessener Untersuchungs- und Auswertungsmethoden und deren Triangulation. Zugleich wurde sich stets an den Gütekriterien von Validität, Reliabilität und Objektivität orientiert, die entsprechend dem epistemologischen Hintergrund qualitativer Sozialforschung verstanden wurden. $\mathrm{Zu}$ deren Wahrung wurden entsprechende Schritte unternommen. ${ }^{42}$

38 Vgl. Flick (2007: 26ff.); Strauss (1991).

39 Zu den Grundlagen der Grounded Theory vgl. Glaser \& Strauss (1967) und Strauss (1991). Zur Rezeption in der strategischen Prozessforschung vgl. Burgelman (2011); Eisenhardt (1989); Pettigrew (1990).

40 Für eine Übersicht über Methodologie und Praxis der strategischen Prozessforschung vgl. Pettigrew (2012: 1316ff.). Vgl. auch die methodologischen Hinweise bei Burgelman (2011); Eisenhardt (1989); Pettigrew (1990). Zum Fallstudienansatz Yin (2009).

41 Vgl. Weber (1972:1), gemäß dem die Sozialwissenschaft soziales Handeln deutend verstehen und in seinem Ablauf ursächlich erklären möchte.

42 Vgl. zu diesen Kriterien der qualitativen Forschung als Qualitätsstandards Przyborski \& 
Über diese Schritte zur Qualitätssicherung hinaus orientierte sich die Forschungsstrategie an zwei etablierten Prinzipien der qualitativen Sozialforschung: dem Prinzip der Offenheit und der aus ihr erwachsenden Zirkularität des Forschungsprozesses.

Das Prinzip der Offenheit ist das grundlegende Prinzip jedweder qualitativen Forschung. ${ }^{43}$ Zur Exploration neuer Zusammenhänge gilt es, den Trichter der Wahrnehmung im Forschungsprozess stets möglichst weit zu öffnen, sodass auch Unbekanntes, Irritierendes und Störendes in den Erkenntnisprozess einfließen kann. Insbesondere wenn im Rahmen eines bereits etablierten theoretischen Konstrukts wie der organisationalen Pfadtheorie geforscht wird, gilt es durch reflektierte Einstellung und methodische Unterstützung vor allem eine umfassende theoretische Vorstrukturierung zu vermeiden. ${ }^{44}$ Obgleich stets die theoretische Anschlussfähigkeit gewahrt werden muss, gilt es zu verhindern, dass nur jene Konstrukte und Zusammenhänge verwendet werden, die bereits formuliert wurden. Daher wurde zum Beispiel auf eine ex-ante Formulierung von Hypothesen verzichtet. ${ }^{45}$

"Start with an interesting question, not a fancy hypothesis. Hypotheses close me down, questions open me up.«

(Mintzberg, 2005)

Gegenstandsbezogene Theorien werden vor der empirischen Untersuchung zur Schärfung des Erkenntnisinteresses und der Weitung der Perspektive zwar einbezogen, die Verwendung ihrer Kategorien aber stets kritisch reflektiert. ${ }^{46}$

Weiteren Ausdruck findet das Prinzip der Offenheit in einer für die Managementforschung typischen, grundsätzlichen Offenheit gegenüber den Erkenntnissen anderer Forschungsfelder und Disziplinen, sowie natürlich vor allem in der Wahl offener Erhebungs- und Auswertungsmethoden wie des narrativen Interviews nach Schütze (1978). ${ }^{47}$ Diese Methoden werden in Teil C ausführlicher dargestellt.

Wohlrab-Sahr (2008: 35ff.) bzw. zu »Strategien der Qualitätssicherung» Flick (2007: 508). Vgl. auch Yin (2009: 33).

43 Vgl. Glaser \& Strauss (1967: 37); Lamnek (2005: 21 f.); Mayring (2002: 25). Eine besondere Zuspitzung erfährt dieses Prinzip in der »theoretischen Offenheit» nach Hoffmann-Riem (1980: 343).

44 Vgl. Lamnek (2005: 22); Przyborski \& Wohlrab-Sahr (2008: 44). Glaser \& Strauss (1967: 1 ff.) forderten ursprünglich gar den Verzicht auf die vorherige Lektüre von theoretischen Arbeiten zum Forschungsfeld. Theoretische Konstrukte sollten am Ende, nicht am Anfang des Forschungsprozesses stehen. Das primäre Ziel der Sozialforschung sei nicht Theorietest, sondern Theoriegenerierung. Aus dieser Haltung floss das allgemeine Prinzip der Offenheit.

45 Vgl. Lamnek (2005: 21); Mintzberg (2005: 361).

46 Vgl. Flick (2007: 72ff.); Przyborski \& Wohlrab-Sahr (2008: 44).

47 Zur Tradition der Betriebswirtschaftslehre als eklekzistischer Wissenschaft und der Mana- 
Doch müssen nicht nur die Methoden, sondern vor allem auch der Forschungsprozess an sich offen sein für Irritationen. Deshalb wurde der Forschungsprozess der vorliegenden Arbeit nicht linear, sondern zirkulär konzipiert.

Im Rahmen quantitativer Forschungsprozesses werden die einzelnen Aufgaben - von der Hypothesenbildung, über deren Test, bis hin zu den daraus abgeleiteten Schlussfolgerungen - nacheinander abgearbeitet. Im Gegensatz hierzu sollte sich der Prozess qualitativer Sozialforschung in vielen kleinen Zyklen und Rückkopplungen dem Forschungsgegenstand und schlussendlich dem Erkenntnisgewinn annähern. ${ }^{48}$ Datenerhebung und -auswertung sollten nicht sequenziell abgearbeitet werden, sondern zeitlich wie inhaltlich eng miteinander verzahnt werden und insbesondere auch rückwirksam sein. Die gewählten Methoden und das festgelegte Sampling sollten ebenso wie die Erkenntnisse so lange wie möglich als vorläufig betrachtet werden und in Referenz auf den Forschungsgegenstand permanent reflektiert und angepasst werden. ${ }^{49}$

Das Anliegen, diesen beiden grundlegenden Prinzipien der qualitativen Sozialforschung gerecht $\mathrm{zu}$ werden, resultierte in folgendem dieser Arbeit zugrunde liegenden Forschungsprozess (siehe Abbildung 2).

Ausgehend von der existierenden theoretischen Literatur der Unternehmenskrisen-, Familienunternehmens-, und Pfadforschung wurde zum Einstieg ins Feld zunächst eine explorative Vorstudie durchgeführt. So sollte die grundsätzliche Relevanz selbstverstärkender Mechanismen und Pfadabhängigkeiten im Kontext familiär beeinflusster Unternehmen geprüft und erste bedeutsame organisationale Variablen identifiziert werden. Darüber hinaus diente die explorative Vorstudie insbesondere aber auch der Gewinnung einer gegenstandsangemessenen Samplestruktur.

Auf der Grundlage der in der explorativen Vorstudie gewonnenen Daten wurden anschließend in Rückkopplung mit der theoretischen Literatur zwei Fallgruppen gebildet, welche aufgrund der Indizien der Vorstudie und der relevanten Literatur als besonders sensitiv für die Genese strategischer Pfadabhängigkeit gelten konnten. ${ }^{50}$ Im nächsten Schritt wurden aus diesen beiden Fallgruppen dann im Zuge eines "purposive sampling“ gemäß Patton (2001) zwei typische empirische Repräsentanten ausgewählt und einer Fallstudie un-

gementlehre als »Problemlösungs- und Integrationswissenschaft« Steinmann \& Schreyögg (2005: 42).

48 Grundlegend zum Prozessmodell qualitativer Forschung Flick (2007: 122); Strauss (1991). Vgl. für die strategische Prozessforschung Eisenhardt (1989).

49 Tauchten zum Beispiel in Interviews relevant erscheinende Variablen auf, so wurden diese in den folgenden Interviews weiter verfolgt, später teils aber auch wieder verworfen. Kriterium war stets die gegenstandsangemessene Relevanz, nicht der vorher festgelegte und einzuhaltende Standard.

50 Zum Sampling und den beiden Fallgruppen ausführlicher Kapitel C.1.1.2. 


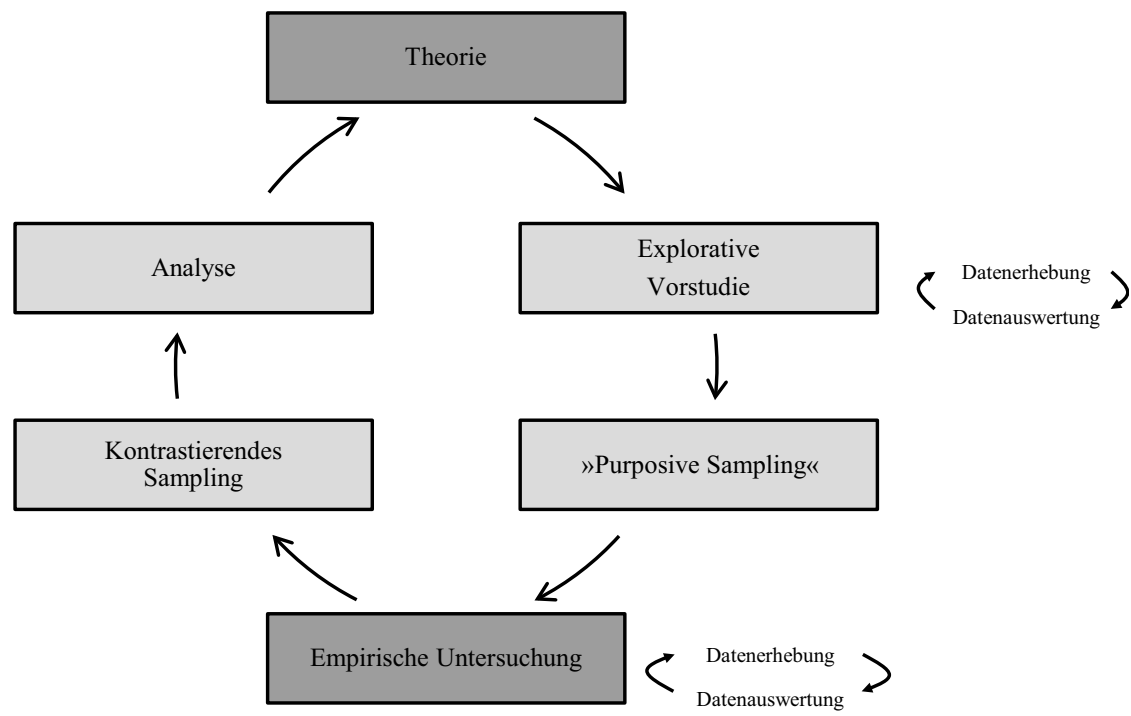

Abbildung 2: Teilschritte des zirkulären Forschungsprozesses

(Quelle: Eigene Darstellung).

terzogen: die Anton Schlecker e.K. und die Berentzen-Gruppe AG. ${ }^{51}$ Das Fallstudiendesign erschien dabei besonders geeignet, um die kausalen Verknüpfungen der Pfadgenese zu identifizieren. ${ }^{52}$

Nach dem Durchlauf mehrerer Schleifen aus Datenerhebung und Datenauswertung erfolgte die erste Formulierung analytischer Zusammenhänge. Allerdings wurde nach einer Rückkopplung mit weiteren, bislang nicht einbezogenen Fällen festgestellt, dass es zur weiteren Validierung der vermuteten Zusammenhänge des Einbezugs weiterer Fälle bedarf. Dementsprechend wurde ganz im Sinne des "theoretical sampling " nach Glaser \& Strauss (1967), wonach die Entscheidungen hinsichtlich Auswahl, Zusammensetzung und Fallzahl des Samplings erst im Prozess der Datenerhebung und -auswertung getroffen werden sollen, mit der Dirk Rossmann GmbH und der Eckes AG zwei weitere Fälle in die Untersuchung einbezogen. ${ }^{53}$ Diese beiden Fälle entstammen denselben Branchen wie die zuerst ausgewählten Fälle und wiesen auch eine ähnliche strategische Ausgangssituation auf. Vor allem aber zeigen sie hinsichtlich vieler vermeintlich für die Pfadgenese bedeutsamer organisationaler Variablen die

51 Vgl. Patton (2001). Als typischer Fall soll hier nach Flick (2007: 165) ein Fall verstanden werden, in dem die Entwicklung für diese Fallgruppe besonders charakteristisch ist.

52 Vgl. George \& Bennett (2005: 22): »A final advantage of case studies is their ability to accommodate complex causal relations such as equifinality, complex interactions effects, and path dependency."

53 Vgl. Glaser \& Strauss (1967: 53); Flick (2007: 158ff.). 
gleichen Ausprägungen wie die schlussendlich pfadabhängigen Unternehmen. Aus den Entscheidungsprozessen dieser Unternehmen erwuchs jedoch keine strategische Persistenz, sondern ein bemerkenswerter strategischer Wandel. Eine komparative Analyse versprach daher einen zusätzlichen Erkenntnisgewinn.

Die im Zuge dieser kontrastierenden Analyse identifizierten Zusammenhänge und gewonnenen Erkenntnisse wurden dann anschließend in Rückkopplung mit der bestehenden Forschungsliteratur diskutiert und in die bestehende Managementforschung $\mathrm{zu}$ organisationaler Pfadabhängigkeit und strategischen Entscheidungsprozessen integriert.

Auf der Grundlage dieses Vorgehens möchte die vorliegende Arbeit durch die Analyse eines spezifischen Prozesses der Krisenentstehung in einem spezifischen Kontext in erster Linie zum besseren Verständnis solch abgegrenzter Krisenphänomene beitragen. Durch die Erarbeitung einer theoretischen Plattform zur Reflexion soll aber auch Problemerkennungswissen generiert werden, welches Akteuren der unternehmerischen und beratenden Praxis in vergleichbaren Situationen zu einer besseren Analyse der eigenen Situation zu helfen vermag und eine Stoßrichtung zur Lösung dieser organisationalen Probleme aufzeigen kann.

\section{$4 \quad$ Gang der Arbeit}

Mit der Skizzierung des weiteren Gangs der Arbeit schließt nun der TEIL A. In dessen Verlauf wurde eingangs in die Problemstellung der Unternehmenskrise aufgrund ausbleibenden strategischen Wandels eingeführt und die noch allgemein gehaltene Frage aufgeworfen, warum manche Unternehmen fortdauernd etablierte strategische Muster reproduzieren, obgleich diese strategische Persistenz ihre Existenz bedrohen kann. Anschließend wurde festgehalten, dass die bisherige Unternehmenskrisenforschung auf die Frage nach den Ursachen so bedingter Krisen kaum erhellende Antworten anbietet. Insbesondere, weil die bisherige Forschung strukturell sowohl den Prozess der Krisenentstehung, als auch die den Krisenprozess rahmenden Kontextvariablen vernachlässigt.

Vor dem Hintergrund dieser beiden strukturellen Forschungsdefizite sollen mit den strategischen Pfaden ein bestimmter Prozess der Krisenentstehung und mit dem Faktor Familie ein spezifischer Kontextfaktor untersucht werden. In dem so aufgespannten Rahmen fragt die vorliegende Arbeit mit Blick auf die zentrale Forschungsfrage nach dem Einfluss des Faktors Familie auf die Genese und Dynamik strategischer Pfade.

Im darauf folgenden TEIL B wird der durch den Prozess der Pfadgenese und den Kontextfaktor Familie aufgespannte theoretische Bezugsrahmen ausführ- 
licher dargestellt. Somit soll eine tief verankerte theoretische Grundlage geschaffen werden, auf der dann im Fortgang der Arbeit aufgebaut werden kann.

Das erste Kapitel (1) widmet sich zunächst der Dimension des Prozesses und führt die strategischen Pfade als spezifische Prozesse der Krisenentstehung näher ein. Ihren Ausgangspunkt findet diese Einführung im ersten Unterkapitel im Phänomen der Unternehmenskrise. Von diesem empirisch beobachtbaren Phänomen ausgehend, soll dann - analog zum Aufbau der gesamten Arbeit Schritt für Schritt und theoretisch orientiert zu den tieferliegenden spezifischen Ursachenkomplexen vorgedrungen werden.

Die im ersten Unterkapitel (1.1) herausgearbeitete Erkenntnis, dass Unternehmenskrisen einen prozessualen Charakter besitzen und meist nicht aus exogenen Schocks resultieren, sondern aus endogenen Problemkonstellationen, die den notwendigen Wandel verhindern, lenkt den Blick anschließend auf die permanente Managementaufgabe des strategischen Wandels. Diese wird im zweiten Unterkapitel (1.2) behandelt. Dabei kann die existenzielle Notwendigkeit eines umweltadäquaten strategischen Wandels aus systemtheoretischer Perspektive auch theoretisch herausgearbeitet werden.

An dieser Stelle der Arbeit sollte jedoch bereits deutlich geworden sein, dass vielen Unternehmen der notwendige strategische Wandel nicht gelingt. Daher wird im dritten Unterkapitel (1.3) mit der organisationalen Pfadtheorie eine spezifische Prozessperspektive auf das Entstehen strategischer Persistenz eingeführt. Dieser theoretische Ansatz vermag anhand des Konstrukts der selbstverstärkenden Mechanismen und der daraus erwachsenden strategischen Pfade in einigen spezifischen Fällen das Ausbleiben des Wandels und das Entstehen daraus resultierender Krisen zu erklären. Im Zuge dieses Unterkapitels wird vor allem das dreiphasige Modell der Pfadgenese nach Sydow et al. (2009) skizziert und in diesem Rahmen mit der Historizität von Prozessen, den selbstverstärkenden Mechanismen und dem »lock-in« die Kernkonzepte der Pfadtheorie ausgeführt.

Das zweite Kapitel der theoretischen Einleitung (2) widmet sich dann der Dimension des Kontexts. Mit der Familie wird jener Kontextfaktor eingeführt, dessen potenzieller Einfluss auf die Genese und Dynamik strategischer Pfade im Zentrum dieser Arbeit steht.

Nachdem im ersten Unterkapitel (2.1) die Vernachlässigung dieses Faktors durch die Managementforschung geschildert wurde, werden in Anknüpfung an die systemtheoretische Perspektive im zweiten Unterkapitel (2.2) Unternehmen und Familie als zwei getrennt operierende, aber strukturell gekoppelte soziale Systeme konzipiert. Im dritten Unterkapitel (2.3) werden daran anschließend die aus dieser strukturellen Kopplung und Ko-Evolution der beiden Systeme erwachsenden Strukturmerkmale familiär beeinflusster Unternehmen skizziert, welche auch für die Pfadgenese potenziell bedeutsam sein könnten. 
Mit TEIL C beginnt der empirische Teil der Arbeit. Hierbei wird im ersten Kapitel (1) vorweg die Vorgehensweise der empirischen Untersuchung geschildert. So wird im ersten Unterkapitel (1.1) der Einstieg ins Feld durch die explorative Vorstudie skizziert, deren Erkenntnisse das weitere Vorgehen und insbesondere das Sampling der Hauptstudie maßgeblich anleiteten. Im zweiten Unterkapitel (1.2) werden des Weiteren die verwendeten Methoden der Datenerhebung und Datenauswertung, sowie der grundlegende Ablauf der Fallanalysen präsentiert.

Mit dem zweiten Kapitel (2) wird sich dann ausführlich den untersuchten empirischen Fällen gewidmet. Im ersten Unterkapitel (2.1) wird zunächst die strategische Entwicklung der Anton Schlecker e.K. in der gebotenen Ausführlichkeit dargestellt und einer zweistufigen Pfadanalyse zugeführt. Hierbei wird im ersten Schritt der Analyse zunächst die strategische Persistenz des Unternehmens geprüft, bevor hieran anknüpfend im zweiten Schritt untersucht wird, inwieweit diese strategische Persistenz durch die Komponenten der organisationalen Pfadtheorie erklärt werden kann. Analog zu diesem Vorgehen erfolgt im zweiten Unterkapitel (2.2) die Pfadanalyse der Berentzen-Gruppe AG.

Auf der bis zu diesem Punkt erarbeiteten theoretischen und empirischen Grundlage wird dann in TEIL D analysiert, inwieweit und wie der Kontextfaktor Familie in den untersuchten Fällen zur Genese und Dynamik strategischer Pfade beitrug.

In diesem Zuge wird im ersten Kapitel (1) untersucht, welche historischen Einflüsse den strategischen Entscheidungsraum zu Beginn der untersuchten strategischen Entwicklung in der Phase der historischen Vorprägung bedingten und welche Rolle der Kontextfaktor Familie bei dieser Prägung spielte.

Das zweite Kapitel (2) widmet sich der zweiten Phase der Pfadformation und untersucht in diesem Rahmen den Einfluss des Faktors Familie in dieser frühen Phase der Pfadgenese. Dabei werden jene organisationalen Variablen in ihren Ausprägungen skizziert, deren Wirkungszusammenhänge maßgeblich zur Genese der strategischen Pfade bei der Anton Schlecker e.K. und der BerentzenGruppe beitrugen: die Macht über und im Unternehmen, der Grad an Diversität innerhalb der dominanten Koalition und das Niveau an Sach- und Beziehungskonflikt. Hierbei wird jeweils erörtert, welchen Einfluss der Faktor Familie auf die Ausprägungen und Auswirkungen dieser Variablen des strategischen Entscheidungsprozesses besaß.

Der TEIL E schließt die Arbeit dann mit einer Zusammenfassung der Erkenntnisse (1), und einer kritischen Diskussion (3) der Grenzen, aber auch des Beitrags der vorliegenden Forschungsarbeit Zugleich wird ein Ausblick auf weiterführende Forschungsfragen gegeben. 


\section{B Theoretischer Bezugsrahmen: Der Prozess strategischer Pfade im Kontext familiär beeinflusster Unternehmen}

\section{Prozess: Strategische Pfade als spezifische Prozesse der Krisenentstehung}

Die vorliegende Arbeit nimmt ihren Ausgangspunkt in empirisch häufig beobachtbaren ausbleibenden strategischen Veränderungsprozessen, welche nicht selten zur Entstehung von existenzbedrohenden Unternehmenskrisen führen. Vor dem Hintergrund dieser Problemstellung fragt die Studie nach möglichen Gründen für solche strategischen Persistenzen.

Auf der Suche nach Antworten soll im folgenden Kapitel die prozessuale Dimension solcher Phänomene adressiert, und mit dem Konzept der strategischen Pfade ein spezifischer Prozess der Krisenentstehung vorgestellt werden. Hiermit wird ein erster theoretischer Erklärungsansatz auf die obige Frage nach den Ursachen strategischer Persistenz angeboten.

Die Arbeit schlägt dabei einen Weg ein, der im ersten Unterkapitel seinen Ausgangspunkt bei der Problemstellung der Unternehmenskrise nimmt. Durch eine Reflexion des aktuellen Forschungsstandes werden der prozessuale Charakter von Krisen und die Endogenität der Krisenursachen herausgearbeitet. Es wird gezeigt werden, dass den meisten Unternehmenskrisen nicht kurzfristig auftretende, exogene Schocks zugrunde liegen. Vielmehr sind meist sehr lange Prozesse zu beobachten, in denen die Unternehmen aufgrund endogener Problemkonstellationen und des daraus resultierenden ausbleibenden Wandels nach und nach ihre strategische Passung mit der Umwelt verlieren.

Dass eine solche umweltadäquate strategische Passung jedoch eine existenzielle Notwendigkeit darstellt, kann im zweiten Unterkapitel durch die Einnahme einer systemtheoretischen Perspektive verdeutlicht werden. Aus dieser Perspektive kann das bestandskritische Verhältnis zwischen der Organisation Unternehmen und dessen dynamischer Umwelt herausgearbeitet werden. Nicht zuletzt im Lichte dieser Theorie erscheint der strategische Wandel als permanente Herausforderung des strategischen Managements. 
Die Häufigkeit von Unternehmenskrisen verdeutlicht jedoch eindrücklich, dass die erfolgreiche Bearbeitung dieses existenziellen Erfordernisses nicht immer gelingt. Dies führt zu der Frage nach den Ursachen des ausbleibenden strategischen Wandels. Vor diesem Hintergrund wird mit der Theorie der organisationalen Pfadabhängigkeit im dritten Unterkapitel ein spezifischer Erklärungsansatz solcher Persistenzen eingeführt. Dieser vermag in spezifischen Fällen zu erklären, warum der existenzielle strategische Wandel in manchen Unternehmen über einen längeren Zeitraum hinweg nicht erfolgt.

Zur Schaffung eines fundierten Verständnisses dieser theoretischen Perspektive wird zunächst kurz die Entwicklungsgeschichte der Pfadtheorie skizziert, bevor im Anschluss hieran ein Phasenmodell der organisationalen Pfadgenese nach Sydow et al. (2009) eingeführt wird. Im Zuge der Skizzierung der Phasen der Pfadgenese werden auch die zentralen pfadkonstituierenden Elemente wie Historizität, Lock-in und vor allem die selbstverstärkenden Mechanismen dargestellt. Anhand dieser Elemente können strategische Pfade anschließend einer Definition zugeführt, und von anderen Ursachen strategischer Persistenz abgegrenzt werden. Auf dem so geschärften Verständnis aufbauend, wird abschließend nach der Bedeutung des organisationalen Kontexts für die Genese und Dynamik solcher strategischen Pfade gefragt. Auf diesem Wege wird das Theoriegebäude der Pfadtheorie für den im zweiten theoretischen Kapitel einzuführenden Kontextfaktor Familie geöffnet.

\subsection{Unternehmenskrisen und deren Entstehung}

Die vorliegende Arbeit fragt nach möglichen Ursachen krisenverursachender strategischer Persistenz und widmet sich in diesem Zuge mit den strategischen Pfaden einem spezifischen Prozess der Krisenentstehung. Die theoretische Annäherung an diese Thematik setzt in diesem ersten Unterkapitel an dem empirisch beobachtbaren Phänomen Unternehmenskrise als der »Spitze des Eisbergs« an. Von dieser Manifestation des ausbleibenden Wandels ausgehend, wird sich zu den tieferliegenden Ursachenkomplexen vorgearbeitet.

Im ersten Abschnitt wird durch eine begriffliche Annäherung an die Unternehmenskrise zunächst vor allem deren prozessualer Charakter herausgearbeitet. Anschließend werden im zweiten Abschnitt anhand eines kurzen Überblicks über die bisherige Forschung zu Unternehmenskrisen ausführlicher jene beiden Defizite der bisherigen Unternehmenskrisenforschung aufgezeigt, die im Zuge dieser Arbeit adressiert werden sollen: die Vernachlässigung prozessualer Entwicklungsdynamiken der Krise und die Kontextfaktoren, die diese Prozesse rahmen und beeinflussen. Im dritten Abschnitt wird dann mit einer kurzen Übersicht zu den Erkenntnissen der Krisenursachenforschung die Relevanz des 
Ausbleibens strategischer Veränderungsprozesse als Krisenursache herausgearbeitet.

Somit soll in diesem Unterkapitel das Bewusstsein für die Bedeutung jener Prozesse geschärft werden, die der sich später manifestierenden Krise zugrunde liegen, ihr zeitlich aber meist weit vorausgehen.

\subsubsection{Begriff und prozessualer Charakter der Unternehmenskrise}

Die etymologische Wurzel des heutigen Krisenbegriffs liegt im Altgriechischen. Dort bezeichnet die "krisis« eine schwerwiegende Entscheidung oder einen entscheidenden Wendepunkt. ${ }^{54}$ Auf dieses Charakteristikum der Wegscheide verweist auch das heutige Begriffsverständnis: Unter einer Krise wird nicht nur die Phase einer sich entwickelnden Gefährdung, sondern zugleich auch der situative Höhe- und Wendepunkt dieser Entwicklung verstanden. ${ }^{55} \mathrm{Da}$ im Prozess der Krise allerdings meist nicht nur unklar ist, ob dieser Wendepunkt bereits erreicht worden ist, sondern auch, ob mit dieser Wende eine Wendung zum Guten oder zum Schlechten verbunden ist, weisen Krisen einen inhärent ambivalenten Charakter auf.

Ein spezifischer, auf die Krise des einzelnen Unternehmens bezogener Krisenbegriff etablierte sich in der Betriebswirtschaftswissenschaft erst Anfang der 1980er Jahre. ${ }^{56}$ Krystek (1987) bestimmte Unternehmenskrisen begrifflich als

»ungeplante und ungewollte Prozesse von begrenzter Dauer und Beeinflussbarkeit sowie mit ambivalenten Ausgang. Sie sind in der Lage, den Fortbestand der gesamten Unternehmung substanziell und nachhaltig zu gefährden oder sogar unmöglich zu machen. Dies geschieht durch die Beeinträchtigung bestimmter dominanter Ziele, deren Gefährdung oder gar Nichterreichung gleichbedeutend ist mit einer nachhaltigen Existenzgefährdung oder Existenzvernichtung der Unternehmung als selbstständig und aktiv am Wirtschaftsprozess teilnehmende Einheit mit ihren bis dahin gültigen Zweck- und Zielsetzungen«.

(Krystek, 1987) $)^{57}$

Vier Elemente dieser Definition sind für die vorliegende Arbeit von besonderer Relevanz. Zentral ist der Gedanke, dass eine Krise nicht nur eine Situation

54 Vgl. Duden (2014).

55 Vgl. Duden (2014). Im Chinesischen wird die Krise als »weiji«危机 bezeichnet, Chance mit »jihui«机会. Beiden gemeinsam ist also das Zeichen »ji《机, das unter anderem »Gelegenheit bedeutet«. »Wie« dagegen heißt »Gefahr«, sodass in »weiji» die Bedrohung, aber auch ein Element der Wende zum Besseren enthalten ist. Vgl. hierzu Pons (2016).

56 Vgl. Krystek \& Moldenhauer (2007: 25). Vorher fand der Krisenbegriff in der Wirtschaftswissenschaft meist nur zur Beschreibung konjunktureller Phänomene Verwendung.

57 Krystek (1987: 9). Zur Rezeption dieser weitverbreiteten Definition vgl. Gless (1996: 16); Rüsen \& von Schlippe (2007: 311 f.). Für eine Übersicht zu weiteren Definitionen s. Schulenburg (2009: 29). 
darstellt, sondern vor allem einen Prozess beschreibt. Zwischen dem Beginn einer Krise und deren schlussendlicher Manifestation liegt meist eine gewisse Zeitspanne, innerhalb derer das Unternehmen ausgehend von einem Zustand »normaler« organisationaler Entwicklung einen Prozess des Niedergangs erlebt, welcher dann schlussendlich entweder mit dessen Gesundung oder dem Verlust der organisationalen Existenz endet. ${ }^{58}$

Ein solcher Krisenprozess sei des Weiteren "ungeplant und ungewollt" Attribute, welche viele Autoren mit den Merkmalen des Außergewöhnlichen und vor allem der damit einhergehenden »Unerwartetheit« und Unvorhersehbarkeit gleichsetzen. Diese vermeintlichen Charakteristika von Unternehmenskrisen sind jedoch zu Recht äußerst umstritten, stellt sich doch in vielen Fällen die Frage, ob die Krise tatsächlich so unerwartet und plötzlich über die Organisation hereinbrach wie von den Beteiligten im Nachhinein häufig beschrieben oder ob sie - wenn überhaupt - nur vor dem Hintergrund spezifischer Wahrnehmungsdefizite überraschend auftrat. ${ }^{59}$ Die in der vorliegenden Arbeit untersuchten Prozesse der Krisenentstehung verweisen deutlich auf letzteren Umstand.

Ob nun überraschend oder vorhersehbar - ist die Unternehmenskrise eingetreten, besitzt sie einen ambivalenten Charakter. Sie ist meist nicht Bedrohung oder Chance, sondern häufig Bedrohung UND Chance zugleich. ${ }^{60}$ Auf der einen Seite bedroht sie das Unternehmen in seiner Existenz, auf der anderen Seite kann gerade diese Bedrohung dazu führen, dass der seit Lange festgefahrene strategische Fokus des Unternehmens neu ausgerichtet und althergebrachte Interpretations- und Verhaltensweisen aufgebrochen werden. Krisen tragen so in vielen Fällen das Potenzial zur Überwindung der durch sie selbst verschärften Situation bereits in sich. Sie schaffen durch Destruktion die Möglichkeit zur Rekonstruktion. In jedem Falle ist durch die Unternehmenskrise aber zunächst einmal die Existenz des Unternehmens bedroht. ${ }^{61}$ Allerdings nicht erst wenn die Insolvenztatbestände Zahlungsunfähigkeit und Überschuldung erreicht sind,

58 Vgl. Hambrick \& D’Aveni (1988); Weitzel \& Jonsson (1989).

59 Vgl. Schreyögg \& Ostermann (2014: 12f.). Der Aspekt der Unvorhersehbarkeit hängt wie jener Frage der Wahrscheinlichkeit des Auftretens der Krise stark von deren Definition ab. Verwendet man wie Pearson \& Clair (1998) einen sehr breiten Krisenbegriff, der auch technologische Unfälle wie Atomkatastrophen inkludiert, so können Krisen als unvorhersehbar und wenig wahrscheinlich verstanden werden. Weick (1988: 305) beschreibt Krisen entsprechend als »low probality events.« Dies trifft für Unternehmenskrisen im hier definierten Sinne nicht zu. An diesen beiden Punkten zeigt sich deutlich die Bedeutung einer klaren Begriffsführung.

60 Vgl. Nystrom \& Starbuck (1984: 55); Pearson \& Clair (1998: 67).

61 Vgl. Staehle \& Albach (1984: 14). Die Notwendigkeit der Existenzbedrohung für die Definition als Krise war jedoch lange Zeit umstritten, vgl. Gless (1996: 45f.); Pandit (2000: 34ff.); Schreyögg \& Ostermann (2014: 121). 
sondern bereits dann, wenn die langfristige Sicherung des Erfolgspotenzials der Unternehmung aus Ressourcen und Kompetenzen gefährdet erscheint.

Über die obige Definition hinaus, scheinen für das Verständnis von Unternehmenskrisen zwei weitere Attribute bedeutsam. ${ }^{62}$ Zum einen ist die Ambiguität des Phänomens zu nennen. Die der Unternehmenskrise zugrunde liegenden Ursachen und Wirkungszusammenhänge sind häufig sehr komplex, da meist multilokal und mehrschichtig. Zum anderen sind die meisten Krisen durch eine Dualität von Zeit- und Handlungsdruck gekennzeichnet, die sich aus der verstärkenden Dynamik von Krisen ergibt, aber erst in der Prozessperspektive deutlich wird.

\section{Begriffliche Abgrenzung}

Das so umrissene Verständnis von Unternehmenskrisen als existenzbedrohende Prozesse unterliegt den weiteren Überlegungen. An dieser Stelle soll es aber auch noch der kurzen Abgrenzung des Begriffs der Unternehmenskrise von verwandten Begriffen dienen, die zwar ebenfalls negativ zu bewertende Situationen adressieren, aber teils stark von der Unternehmenskrise abweichende Phänomene beschreiben.

Dem Unternehmenskrisenverständnis dieser Arbeit am nächsten kommen die Phänomene, welche in der Organisationsforschung unter den prozessorientierten Begriff des organisationalen Niedergangs (»organizational decline») subsumiert werden. ${ }^{63}$

Scharf abzugrenzen ist die Unternehmenskrise allerdings von Phänomenen wie Katastrophen (»disaster«), Risiken oder Konflikten. ${ }^{64}$ Hier werden meist vollkommen anders gelagerte Phänomene beschrieben, aber von der Managementforschung teilweise dennoch als Krisen tituliert. Der Forderung von Kovoor-Misra et al. (2001) nach einer präziseren Verwendung des Begriffs der Krise durch Klärung deren Eigenschaften und der Abgrenzung verwandter Konstrukte kann sich daher nur angeschlossen werden. ${ }^{65}$ Darüber hinaus greift die betriebswirtschaftliche Forschung aber noch auf eine Reihe weiterer Begrifflichkeiten zurück: So spricht die stärker finanzwirtschaftlich orientierte

62 Vgl. Hermann (1963); Kovoor-Misra et al. (2001); Pearson \& Clair (1998).

63 Vgl. zu dieser Forschungsrichtung Trahms et al. (2013); Weitzel \& Jonsson (1989) oder Whetten (1980).

64 Vgl. Krystek (1987: 28ff.); Pearson \& Clair (1998: 60ff.); Weick (1988: 316). Für eine Übersicht der durch den Begriff der Krise beschriebenen Phänomene, welche von Serverausfällen über die Explosionen von Gaspipelines bis hin zur Entführung von Managern reichen: Kovoor-Misra et al. (2001).

65 Vgl. Kovoor-Misra et al. (2001: 88). Eine unklare Begriffsführung führt ansonsten dazu, dass Beiträge wie jener von Schulenburg (2009: 10) ohne Kontextualisierung Ergebnisse der Forschung zu Katastrophen für Ausführungen zu Unternehmenskrisen im hier vorgestellten Sinne heranziehen. 
angelsächsische Literatur alternativ von einem "financial distress«, einer finanziellen Notlage aufgrund von Zahlungsschwierigkeiten. Spätestens mit einer solchen Liquiditätskrise ist meist auch die von der Managementforschung zur Krisenbewältigung adressierte »turnaround situation « erreicht. ${ }^{66}$ Diese verweist auf die letzten Stadien des unten dargestellten idealtypischen Verlaufs von Unternehmenskrisen.

$\mathrm{Zu}$ guter Letzt ist noch der Begriff der Insolvenz vom Krisenbegriff abzugrenzen. Er markiert für viele Unternehmen zwar das finale Stadium der Krise, steht aber für das Management des Unternehmens unter gänzlich anderen juristischen und finanziellen Vorzeichen. Die Insolvenz erfordert daher stets eine eigenständige Betrachtung. ${ }^{67}$

\subsubsection{Forschung zu Unternehmenskrisen - Entwicklung und Defizite}

Wie die Betriebswirtschaftslehre allgemein, wurde auch die Unternehmenskrisenforschung in ihrer Entwicklung stets stark von ihrem jeweiligen zeitgeschichtlichen und politisch-ökonomischen Kontext geprägt. Während bereits in Zeiten der Weltwirtschaftskrise betriebliche Krisenursachen analysiert wurden, rückte die Thematik der Unternehmenskrise in den Nachkriegsjahrzehnten im Zuge eines schier grenzenlosen Wachstums in der wirtschaftswissenschaftlichen Forschung für lange Zeit in den Hintergrund. ${ }^{68}$ Die Analyse der aus dem Wachstum resultierenden Herausforderungen dominierte das Feld. Hierüber wurde die Beschäftigung mit dem Niedergang von Organisationen und Unternehmen systematisch vernachlässigt. Erst unter dem Eindruck der Wirtschaftskrisen der 1970er- und frühen 1980er-Jahre wurde in der Betriebswirtschaftslehre wieder verstärkt die Forderung nach einer Beschäftigung mit dem organisationalen Niedergang laut. ${ }^{69}$

In der Folge wurde im angloamerikanisch geprägten Diskurs der Managementforschung die Krise von Organisationen und Unternehmen vor allem innerhalb zweier Strömungen zum Gegenstand: der Forschung zum »organizational decline« und der Turnaround-Forschung.

66 Vgl. zum »financial distress« Luther (2008); Platt \& Platt (2002); Sudarsanam \& Lai (2001). Zur »turnaround situation« Hambrick (1985); Pandit (2000: 32); Schendel et al. (1976: 3). Teilweise ist von einer »turnaround situation« jedoch bereits dann die Rede, wenn der Geschäftserfolg über mehrere Perioden unterhalb eines minimal zu akzeptierenden Niveaus liegt.

67 Vgl. für die Pfadforschung Rindfleisch (2011); Tobias (2012).

68 Vgl. für frühe Arbeiten Flege-Althoff (1930); Hasenack (1932); Isaac (1932); Le'Coutre (1926).

69 Vgl. Staehle \& Albach (1984: 14); Whetten (1980); Cameron et al. (1987). Bedeutsam war die VHB-Konferenz im Jahr 1979 zum Thema "Unternehmenskrisen«. Vgl. hierzu den Tagungsband Bratschitsch \& Schnellinger (1981). 
Erstere widmete sich vor allem der prozessualen Darstellung des Niedergangs von Organisationen. ${ }^{70}$ Sie fokussierte jedoch tendenziell eher Organisationen wie Universitäten und seltener Unternehmen. Zudem wurde die aus dem Niedergang resultierende Krisensituation nur sporadisch in die Analyse miteinbezogen. Die Möglichkeiten zur Bewältigung der Krise blieben weitgehend vollkommen ausgespart.

Strategien und Maßnahmen der Krisenbewältigung wurden hingegen von der stärker unternehmens- und handlungsorientierten Turnaround-Forschung diskutiert. Deren Beiträge stellen heute den Großteil der internationalen Managementliteratur zur Thematik der Unternehmenskrise. Diese Strömung erlebte Anfang der 1980er-Jahre eine starke Zunahme an Forschungsaktivität, nicht zuletzt, weil die Beiträge die hohe empirische Bedeutung von Unternehmenskrisen mit quantitative Studien herausarbeiten konnten. ${ }^{71}$

Die meisten Beiträge fokussierten jedoch ausschließlich die inhaltliche Stoßrichtung (»content») möglicher Maßnahmen der Krisenbewältigung und zeichneten sich darüber hinaus auch durch einen stark präskriptiven Charakter aus. ${ }^{72}$ Ob dieser Fokussierung auf erfolgsträchtige Maßnahmen der Krisenbewältigung blendete diese Strömung - quasi spiegelverkehrt zur Forschung des "organisational decline« - in ihren Handlungsempfehlungen allerdings häufig den vorausgegangenen Prozess (»process«) der Krisenentstehung aus. Die mit dieser Vernachlässigung der prozessualen Entwicklungsdynamik einhergehende Ahistorizität prägt die Turnaround-Forschung bis zum heutigen Tage. ${ }^{73}$ Obgleich insbesondere die empirische Strategieprozessforschung die Bedeutung historischer Entscheidungen für gegenwärtige strategische Entscheidungsprozesse seit Längerem erkannt hat, lässt ein Großteil der Beiträge zur Bewältigung von Unternehmenskrisen diese organisationale Vorprägung bislang immer noch außer Acht. Der Leitvorstellung der "rational choice« folgend, werden die Möglichkeiten des strategischen Wandels vielmehr häufig in einem grundsätzlich offenen Möglichkeitsraum präsentiert. Dieser erscheint losgelöst von der Entwicklungsgeschichte des Unternehmens und wird auch zu selten mit den zugrunde liegenden spezifischen Krisenursachen verknüpft. Eine solch ahistorische Betrachtung läuft jedoch leicht Gefahr, wichtige Interdependenzen

70 Vgl. zum »organizational decline« Argenti (1976); Cameron et al. (1987); Whetten (1980).

71 Vgl. für einen frühen Beitrag Schendel et al. (1976), auf welchen einflussreiche Studien wie Hofer (1980) oder Hambrick \& Schecter (1983) folgten.

72 Vgl. die Titel der Beiträge Slatter (1984) »Corporate Recovery - Successful Turnaround Strategies and their Implementation« oder O’Neill (1986) »Turnaround and recovery: what strategy do you need?«. Zu dieser Einschätzung auch die Reviews von Pearce \& Robbins (1993) und Pandit (2000).

73 Vgl. Trahms et al. (2013: 1280ff.). So verweist im Rahmen der Managementforschung zu Unternehmenskrisen laut Pandit (2000: 40) nur der Beitrag Slatter (1984) auf die Bedeutung vorhergegangener Strategien für die strategischen Maßnahmen der Krisenbewältigung. 
zu übersehen, die den strategischen Handlungsraum maßgeblich bedingen können.

Das zweite Defizit der Turnaround-Forschung, und somit des Großteils der gesamten Managementforschung zum Thema Unternehmenskrise, ist die Vernachlässigung des organisationalen Kontexts. ${ }^{74}$ Die Motive und Charakteristika der handelnden Entscheidungsträger, die Struktur der Organisation, oder die in ihr tief verankerten kulturellen Muster bedingen sowohl den Prozess der Krisenentstehung, als auch die Möglichkeiten zur Krisenbewältigung. Trotzdem wurden sie bislang nur selten in die Betrachtungen integriert.

Erst seit wenigen Jahren widmen sich einige Beiträge verstärkt zum Beispiel der Rolle der Stakeholder im Krisenprozess. ${ }^{75}$ Unter besonderer Fokussierung der Rolle der Eigentümer, erachten aktuelle Forschungsbeiträge hier nicht zuletzt den Einfluss von Eigentümerfamilien als für den Krisenprozess besonders bedeutsam. So weisen beispielsweise Cater \& Schwab (2008) darauf hin, dass systemische "familiäre" Charakteristika wie die interne Orientierung der Entscheidungsträger oder die langfristige Perspektive der Eigentümerfamilie auf das Unternehmen die Umsetzung von Turnaround-Strategien maßgeblich beeinflussen können. ${ }^{76}$ Trahms et al. (2013) argumentieren ähnlich und nehmen nach einem Review der aktuellen Forschung die Rolle von Eigentümerfamilien entsprechend in ihren Katalog zukünftiger Forschungsfelder der Unternehmenskrisenforschung auf. ${ }^{77}$ Mit der Untersuchung des Einflusses des Kontextfaktors Familie auf die Genese und Dynamik strategischer Pfade adressiert die vorliegende Arbeit somit nicht nur abstrakt zwei grundlegende Defizite eines Großteils der bisherigen internationalen Managementforschung zu Unternehmenskrisen, sondern knüpft auch inhaltlich an eine aktuelle Forschungsströmung an.

Mit dem Fokus auf die Prozessualität der Krisenentstehung schließt die vorliegende Arbeit indirekt zudem an eine Forschungstradition der deutschen Unternehmenskrisenforschung an. Denn diese thematisiert das Phänomen der Unternehmenskrise im Vergleich zur international dominanten angloamerikanischen Managementforschung seit jeher ganzheitlicher und prozessorientierter. ${ }^{78}$ So versuchen einige Beiträge die Krisenursachen und die Möglichkeiten der Krisenbewältigung zu verknüpfen - und adressieren so implizit das Defizit der englischsprachigen Forschung. Da sie allerdings auf den fortgeschrittenen internationalen Diskurs keinen Bezug nimmt und kaum empirische Forschung

74 Vgl. Lohrke et al. (2004: 63ff.); Nystrom \& Starbuck (1984: 53ff.); Pandit (2000: 40).

75 Vgl. Buschmann (2006); Pajunen (2006).

76 Vgl. Cater \& Schwab (2008).

77 Trahms et al. (2013: 1293): »Research has identified family and management ownership as particularly relevant to turnaround situations."

78 Vgl. Krummenacher (1981); Krystek (1987); Müller (1986). 
anbietet, ist die deutsche Unternehmenskrisenforschung in diesen Bemühungen weitgehend isoliert. ${ }^{79}$

Neben solchen Beiträgen der Unternehmenskrisenforschung, sind auch in Deutschland praxisorientierte juristische oder finanzwissenschaftliche Studien zu Sanierung oder Restrukturierung sehr dominant. ${ }^{80}$ Ohne ihren Beitrag für die Praxis schmälern zu wollen, ist jedoch anzumerken, dass sie es aufgrund ihrer meist statischen Betrachtung nicht vermögen, die Ursachen der Krise, deren Auswirkungen und die Möglichkeiten der Krisenbewältigung konsistent oder gar theoretisch miteinander zu verknüpfen.

Eine solche theoretische Verknüpfung ist wiederum jedoch stets der Anspruch der klassischen deutschen Unternehmenskrisenforschung gewesen ein Anspruch, welchen sie über die Entwicklung einer wie auch immer gearteten »Theorie der Unternehmenskrise« einzulösen gedenkt. ${ }^{81}$ Von einer solchen allumfassenden Krisentheorie erhofft sich die Unternehmenskrisenforschung nicht nur allgemeingültige Erkenntnisse, sondern auch homogene Handlungsempfehlungen. Diese Hoffnung erscheint aber, angesichts der Multikausalität und Vielschichtigkeit empirisch beobachtbarer Krisenphänomene ebenso illusionär wie die Zielstellung einer "Theorie der Unternehmenskrise" an sich. Während der angloamerikanische Diskurs also an einer zu starken Fragmentierung zwischen den Beiträgen des »organizational decline« zu prozessualen Dynamiken und den rein auf den Inhalt der Krisenbewältigung bezogenen Handlungsempfehlungen der Turnaround-Forschung leidet, scheint die deutsche Forschung in ihrer Suche nach der »Theorie der Unternehmenskrise« an ihrem ganzheitlichen Anspruch zu scheitern.

Vor dem Hintergrund dieser Defizite erscheint es sinnvoller, auf der Suche nach Antworten auf die Frage nach den Gründen für krisenverursachende strategische Persistenzen der eigentlichen Unternehmenskrisenforschung den Rücken zu kehren und sich etablierten Theorien der Managementforschung zuzuwenden. ${ }^{82}$ Ein solcher Anschluss erscheint erfolgsversprechender, um tieferliegende spezifische Ursachenkomplexe theoretisch orientiert und konsistent zu ergründen. Zugleich dient ein solcher Anschluss auch dazu, das oft beklagte

79 So nimmt der in Deutschland vielzitierte Krystek (1987); Krystek (2007) beispielsweise an keiner Stelle auf die bedeutenden Arbeiten von Schendel (1976); Schendel et al. (1976); Hofer (1980); Hambrick (1985) Bezug.

80 Diese meist populärwissenschaftlichen deutschen Arbeiten verfügen allerdings nicht über die gleiche Qualität wie die angloamerikanischen Beiträge zu »bankruptcy« oder »financial distress«. Vgl. Altman (1968); Altman \& Hotchkiss (2006).

81 Vgl. Krystek (1987);Witte (1981: 13). Für einen aktuellen Überblick über die deutsche Literatur zu dieser Diskussion vgl. Schulenburg (2009: $3 \mathrm{ff}$.).

82 Andere Forschungsarbeiten zu Unternehmenskrisen profitieren vielleicht von einem stärkeren Einbezug von Theorien der Finanzwissenschaft, Soziologie oder Psychologie. 
»Theoriedefizit" der Unternehmenskrisenforschung zu adressieren. ${ }^{83} \mathrm{Ihm}$ folgen auch jüngere Beiträge der Unternehmenskrisenforschung, die zur Untersuchung von Krisenursachen beispielsweise auf die evolutionstheoretische Perspektive zurückgreifen. ${ }^{84}$

Im dritten Unterkapitel wird daher mit der Theorie der organisationalen Pfadabhängigkeit die in dieser Arbeit eingenommene theoretische Perspektive auf Unternehmenskrisen eingeführt und mit den strategischen Pfaden ein spezifischer Prozessen der Krisenentstehung skizziert. Zuvor soll im folgenden Abschnitt jedoch zunächst noch die Bedeutung jener strategischen Persistenzen als Krisenursache herausgearbeitet werden, für die mit der organisationalen Pfadtheorie anschließend eine erste Erklärung angeboten wird.

\subsubsection{Krisenursachen und Prozess der Krisenentstehung}

Die vorliegende Arbeit fragt nach möglichen Ursachen für ausbleibenden strategischen Wandel. Im folgenden Abschnitt soll die Bedeutung solcher strategischer Persistenzen für den Prozess der Krisenentstehung nochmals verdeutlicht werden.

Es wurde eingangs bereits festgehalten, dass Unternehmenskrisen ein meist multikausales, mehrstufiges und multilokales organisationales Phänomen darstellen. In der Regel existieren mehrere Krisenherde, die auf verschiedenen organisationalen Ebenen und an mehreren Entstehungsorten zu verorten sind. Sie erzeugen so komplexe und interdependente Ursache-Wirkungs-Zusammenhänge. ${ }^{85}$ Allein aufgrund dieser Interdependenzen erscheint weniger die Identifikation einzelner Ursachen geboten, als vielmehr die Analyse des Krisenentstehungsprozesses in seiner ganzen Dynamik und Komplexität.

Die klassische Krisenursachenforschung versucht jedoch genau Ersteres. Die von ihr identifizierten Ursachen für die Entstehung von Unternehmenskrisen lassen sich dabei grundsätzlich in exogene und endogene Ursachen kategorisieren. ${ }^{86}$

83 Vgl. Rindfleisch (2011: 36); Schulenburg (2009: 16).

84 Vgl. Schulenburg (2009); Welsch (2010), grundlegend Hannan \& Freeman (1977); Hannan \& Freeman (1984).

85 Vgl. Böckenförde (1991: 22ff.); Buschmann (2006: 16); Krystek (1987: 34). Auf diese Komplexität sind wohl auch die teils widersprüchlichen Ergebnisse mancher Studien der Krisenursachenforschung zurückzuführen. Diese vermögen es häufig nicht, Ursachen und Symptome trennscharf zu differenzieren oder die verbindenden Mechanismen zu benennen.

86 Diese Differenzierung geht bis auf Flege-Althoff (1930) zurück und findet sich u. a. in Böckenförde (1991: 27) und Pearce \& Robbins (2008: 122). 


\section{Endogene und exogene Krisenursachen}

Unter organisationsexogenen Krisenursachen sollen jene möglichen Ursachen verstanden werden, die sich dem unmittelbaren Einflusspotenzial der Organisation grundsätzlich entziehen. Hinsichtlich solcher Ursachen kann zunächst festgehalten werden, dass negative gesamtökonomische konjunkturelle Entwicklungen Krisen auf der Ebene des einzelnen Unternehmens nur äußerst selten originär und unmittelbar verursachen. ${ }^{87}$ Wenn die negative konjunkturelle Lage überhaupt Einfluss auf die Krisenentstehung nimmt, dann indem sie latent bereits seit Längerem vorhandene krisenhafte Entwicklungen innerhalb der Organisation zusätzlich verschärft oder zum Vorschein bringt.

Von größerer Relevanz als Krisenursache im engeren Sinne sind dynamische Veränderungen des Branchenumfelds, wie zum Beispiel »disruptive innovations ${ }^{88}{ }^{88}$ Solche technologischen Umbrüche, aber auch Veränderungen in der rechtlichen Rahmensetzung oder der Wettbewerbsstruktur, können die Wertschöpfung in der Branche grundlegend neu strukturieren und so auf der Ebene des einzelnen Unternehmens krisenhafte Entwicklungen auslösen.

Doch auch ein solch dynamisches Branchenumfeld gerät für die Unternehmen nur dann zu einer existenziellen Bedrohung, wenn es das Unternehmen nicht vermag, adäquat und rechtzeitig auf diesen Wandel in seiner Umwelt zu reagieren. So machen Hambrick \& D'Aveni (1988) in ihrer Vergleichsstudie zwischen gescheiterten und überlebenden Unternehmen derselben Branche darauf aufmerksam, dass beide Gruppen die gleichen exogen bedingten Branchenkrisen erfuhren. Die schlussendlich gescheiterten Unternehmen seien jedoch bereits vor Ausbruch der Krise strategisch schlechter positioniert gewesen und hätten zusätzlich über weniger Reserven (»slack«) verfügt. ${ }^{89}$ Die Branchenkrise bedeutete für die gescheiterten Unternehmen lediglich das Ende einer länger anhaltenden Abwärtsspirale, welche letztlich das Unternehmensschicksal besiegelte.

»For the strong, smog is an uncomfortable nuisance that may have subtle health effects. For the weak, though, smog can kill.«

(Hambrick \& D’Aveni, 1988) $^{90}$

So kann für das Gros der Krisenursachenforschung festgehalten werden, dass die wenigsten Unternehmen allein wegen exogener Entwicklungen scheitern. Die

87 Vgl. Böckenförde (1991: 34); Krystek (1987: 51); Pearce \& Robbins (2008: 122).

88 Vgl. Christensen (2013); Tushman \& Anderson (1986). Solche Veränderungen werden insbesondere von der Forschung zu »organizational decline« oder "discontinuos change« untersucht. Vgl. Grinyer et al. (1990: 53); Krystek \& Moldenhauer (2007: 51); Pearce \& Robbins (2008: 122).

89 Vgl. Hambrick \& D’Aveni (1988).

90 Hambrick \& D’Aveni (1988: 17). 
wirklichen Ursachen liegen in den meisten Fällen tiefer und sind im endogenen, innerbetrieblichen Einflussbereich zu suchen. ${ }^{91}$ Diese Erkenntnis lenkt die Analyse auf unternehmensinterne Prozesse und endogene Problemkonstellationen.

Die meisten Forschungsarbeiten erachten hier die Unternehmensleitung als den zentralen krisenverursachenden Faktor. ${ }^{92}$ In der Tat sind es in Organisationen, die über Entscheidungen operieren, schlussendlich auch die Fehlentscheidungen oder Versäumnisse der Entscheidungsträger auf funktional-operativer und vor allem strategischer Ebene, die das Unternehmen in die Krise führen. Damit der sehr allgemeine Hinweis auf die Bedeutung der Unternehmensleitung für die Entstehung von Unternehmenskrisen allerdings nicht zur Leerformel gerät, müssen diese Fehler und Versäumnisse zum einen genau spezifiziert und zum anderen in Referenz zu einem Bezugspunkt gesetzt werden.

Hier kommt auf der Ebene strategischer Entscheidungen nur die Umwelt der Organisation als letzte Instanz infrage. Die Umwelt fordert die Unternehmen mit ihrer Dynamik heraus und misst schlussendlich auch die Gültigkeit der organisationalen Reaktion. So ist es entsprechend die Kombination aus Umweltdynamik und endogenen Problemkonstellation, die laut Krisenursachenforschung für das Entstehen der meisten Krisen verantwortlich ist. In den meisten Fällen führt eine Kombination von innerbetrieblichen Problemen dazu, dass das Unternehmen sich nicht mehr adäquat den Veränderungen der Umwelt anpassen kann und in die Krise gerät. ${ }^{93}$ So betonen Schendel et al. (1976):

$»[\ldots]$ an interaction of poor managerial decisions coupled with or in response to unfavorable environmental events lies behind most of the cases."

(Schendel et al., 1976) ${ }^{94}$

Die Forschung zu Unternehmenskrisen verweist somit ausdrücklich auf die Relevanz endogen bedingter strategischer Persistenzen als bedeutsame Krisenursache.

\section{Modelle der Krisenentstehung}

Auf die kritische Beziehung zwischen dynamischen Umweltentwicklungen und organisationsinternen Problemkonstellationen verweisen auch zahlreiche theoretische Modelle der Krisenentstehung außerhalb der eigentlichen Unter-

91 Vgl. Arogyaswamy et al. (1995); Krystek (1987: 51); Schendel et al. (1976: 7).

92 Vgl. Bibeault (1982: 53); Nystrom \& Starbuck (1984: 57f.); Schendel et al. (1976: 6); Slatter (1984: 53). Für eine Übersicht gleichlautender deutscher Arbeiten Schulenburg (2009).

93 Vgl. Arogyaswamy et al. (1995: 507). Ausführlich Cameron et al. (1987); Hambrick \& D'Aveni (1988); Whetten (1987).

94 Schendel et al. (1976: 7). 
nehmenskrisenforschung. Diese Beiträge werden von Schreyögg \& Ostermann (2014) auch als »Krisentheorien « bezeichnet. ${ }^{95}$

Sowohl der "frame-breaking-change" in der Umwelt bei Tushman \& Anderson (1986) als auch die entwicklungsstadienspezifischen, revolutionären Perioden bei Greiner (1972) können als zwangsläufig notwendige Transformations- und Adaptionsherausforderungen verstanden werden, die - wenn sie aufgrund interner Probleme nicht bewältigt werden können - zu existenzbedrohenden Unternehmenskrisen führen können. ${ }^{96}$

Auf die Endogenität, Vielschichtigkeit und lange Entwicklungszeit solcher Problemkonstellationen verweisen insbesondere auch die sogenannten Kumulationstheorien. ${ }^{97}$ Deren Vertreter machen darauf aufmerksam, dass sich die Ursachen von Krisen meist in einer langen Vorlaufzeit - und oft unterhalb der Aufmerksamkeitsschwelle der Organisation - langsam aufbauen, sich nach und nach verfestigen und verstärken, um sich schlussendlich in der manifesten Unternehmenskrise niederzuschlagen. Schreyögg \& Ostermann (2014) zufolge wurden gerade die Gefahren solch schleichender Entwicklungen von der Managementforschung lange Zeit vernachlässigt. ${ }^{98}$ Dieses Defizit erscheint umso bedeutsamer, wenn man der Überlegung folgt, dass die Überwindung solcher sich über viele Jahre aufgetürmter, vielschichtiger und interdependenter Problemkonstellationen doch in vielen Fällen schwieriger sein dürfte als die Adressierung spezifizierbarer Probleme im Rahmen von abrupt auftretenden konjunkturell bedingten Krisen.

Auf die Relevanz solcher längerfristigen Entwicklungsprozesse und kritischer endogener Konstellationen verweist auch die Forschung zum »organizational decline«. So gehen Roux-Dufort (2007) davon aus, dass die meisten Krisen aufgrund einer Kumulation vieler kleiner Anomalien auf der einen Seite und einer zunehmenden Ignoranz gegenüber diesen Anomalien auf der anderen Seite entstehen. Aus dieser Perspektive haben Krisen ebenfalls eine lange organisationale »Reifezeit«, bevor sie durch ein auslösendes Ereignis aufbrechen. ${ }^{99}$

95 Vgl. für eine Übersicht zu diesen verschiedenen Ansätzen Schreyögg \& Ostermann (2014: 482).

96 Vgl. Tushman \& Anderson (1986); Greiner (1972); auch Quinn \& Cameron (1983) oder Argenti (1976).

97 Vgl. Hwang \& Lichtenthal (2000); zu ähnlichen Ansätzen Schreyögg \& Ostermann (2014: 129).

98 Vgl. Schreyögg \& Ostermann (2014: 130). Tichy \& Devanna (1986) verwendet zur Verdeutlichung der Gefahren des graduellen Wandels der Umwelt die vielzitierte Metapher des "boiling frog". Während ein Frosch, den man in kochendes Wasser werfe, sofort wieder herausspringe, bemerke ein bereits im Wasser sitzender Frosch die Erwärmung erst dann, wenn es zu spät sei.

99 Ähnlich u. a. Nystrom \& Starbuck (1984); Starbuck et al. (1978); Cameron et al. (1987). Die meisten dieser Arbeiten machen für eine solche Ignoranz sowohl individuelle wie kollektive Wahrnehmungsverzerrungen verantwortlich. 
Die für die Einordnung und das Verständnis dieser Arbeit sehr bedeutsame Feststellung einer langen Vorlaufzeit von Krisen und eines langen Krisenprozesses bestätigen Hambrick \& D’Aveni (1988) empirisch. In ihrer Untersuchung des organisationalen Niedergangs und schlussendlichen Konkurses von 62 großen US-amerikanischen Unternehmen im Zeitraum von 1972 bis 1982 zeigen sie, dass die Krisen häufig nur die Konsequenz eines teils zehnjährigen Niedergangs waren. ${ }^{100}$

Diese empirischen Ergebnisse verdeutlichen wie die anderen Beiträge, auf die in diesem Abschnitt verwiesen wurde, dass Unternehmenskrisen selten ein plötzlich über das Unternehmen hereinbrechendes Ereignis, sondern meist ein langandauernder Prozess sind, dessen Ursachen innerhalb der Organisation zu suchen sind. Zugleich verweisen sie auf die, vorliegende Forschungsarbeit leitende empirische Fragestellung, warum einige Unternehmen über einen längeren Zeitraum nicht entscheidend gegensteuern, sondern weiterhin an existenzbedrohenden Strategien festhalten.

\section{Phasen der Krisenentstehung: von der Strategiekrise zur Liquiditätskrise}

Der nun herausgearbeitete lang andauernde Prozess der Krisenentstehung im Sinne einer »downward spiral« lässt sich abschließend idealtypisch zeitlich und inhaltlich-theoretisch in spezifische Phasen mit generalisierbaren Eigenschaften differenzieren. ${ }^{101}$

So geraten in der Phase der Strategiekrise die relativen Wettbewerbsvorteile oder die zugrunde liegenden Ressourcen nach und nach, aber substanziell in Gefahr zu geraten. In dieser frühen Phase der Krisenentstehung ist der Handlungsspielraum für das Unternehmen zwar weiterhin groß und die Existenzbedrohung für das Unternehmen eher abstrakter Natur. ${ }^{102}$ Werden aber keine adäquaten korrigierenden Maßnahmen ergriffen, besteht die Möglichkeit, dass sich eine solche strategische Krise auch bald im (nachlassenden) Erfolg des Unternehmens widerspiegelt. Da eine solche strategische Inaktivität im ökonomischen Geschehen regelmäßig zu beobachten ist, sprechen Weitzel \&

100 Vgl. Hambrick \& D’Aveni (1988: 20): »[...] these bankruptcies were culminations of often ten-year declines".

101 Vgl. zur »downward spiral« Hambrick \& D’Aveni (1988). In der Literatur werden hierbei der Faktor Zeit, sowie die Intensität der destruktiven Wirkungen als fundamentale Klassifikationskriterien herangezogen. Vgl. Krystek (1987: 2); Weitzel \& Jonsson (1989: 97ff.); Hambrick \& D'Aveni (1988: 14). Für eine Übersicht zu Phasenkonzepten der deutschen Unternehmenskrisenforschung: Schulenburg (2009: 44f.). Die vorliegende Arbeit orientiert sich an der oft rezipierten Phasenklassifikation nach Müller (1986: $25 \mathrm{ff}$.); die auch die von Krystek (1987: 29 ff.) angeführte Unterscheidung zwischen potenziellen, latenten und akuten Krisen einschließt.

102 Vgl. Cezanne (1999: 12); Krystek (1987: 29 ff.); Müller (1986: 25 ff.). 
Jonsson (1989) in Bezug auf die Strategiekrise von der »inaction« und »blinded stage ${ }^{103}$

Die Phase der Strategiekrise und die zu beobachtende strategische Inaktivität stehen im Mittelpunkt der vorliegenden Arbeit. Diese ausbleibende strategische Reaktion wird an späterer Stelle durch die organisationale Pfadtheorie und den Einfluss der Familie zu erklären versucht. ${ }^{104}$

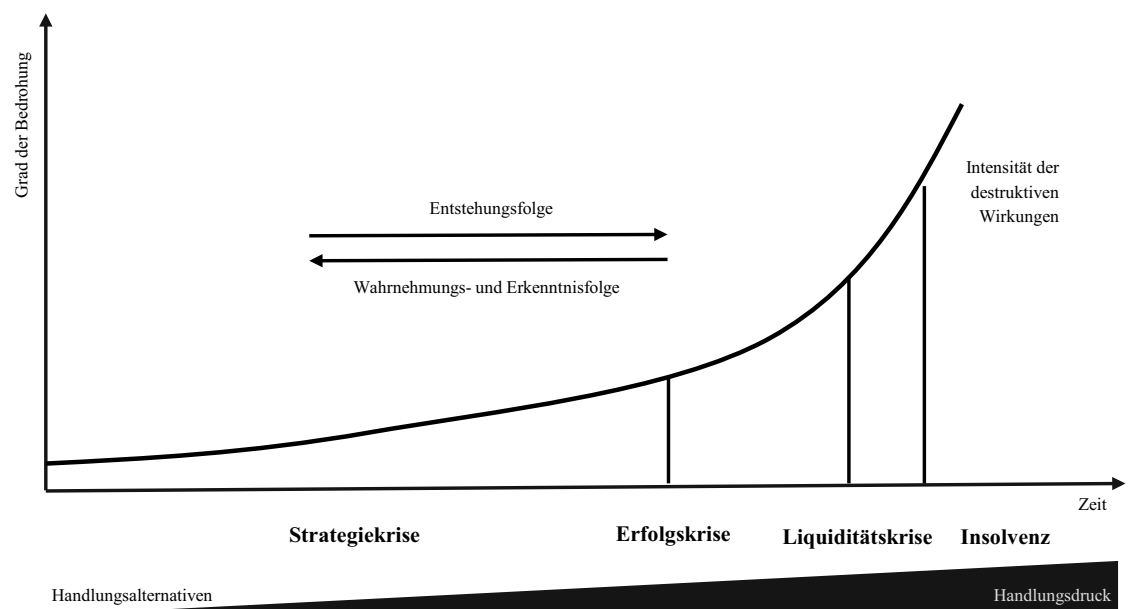

Abbildung 3: Idealtypischer Phasenverlauf der Krisenentstehung (Quelle: Eigene Darstellung; in Anlehnung an Müller [1982]).

Aus dieser Inaktivität gewinnt der anschließende Krisenprozess auch seine sich zuspitzende Dramatik. Denn werden die strategischen Probleme über einen längeren Zeitraum nicht konsequent oder adäquat adressiert, so schlägt sich die strategische Krise ab einem gewissen Zeitpunkt auch im Erfolg der Unternehmung wieder. Im Zuge der nun beginnenden Erfolgskrise werden die finanziellen Ziele hinsichtlich Umsatz, Gewinn, oder Rentabilität zunehmend verfehlt. Darüber hinaus beginnen aber beispielsweise auch die Stakeholder ihre Beziehung zum Unternehmen zu überdenken und anzupassen. ${ }^{105}$

Aufgrund dieser krisenbedingten Entwicklungen verringern sich die Handlungsspielräume des Unternehmens immer weiter, während gleichzeitig die

103 Weitzel \& Jonsson (1989: 94). Weitzel führt die ausbleibende strategische Reaktion vor allem auf vielfältige Wahrnehmungsdefizite der in das Prozessgeschehen involvierten Entscheidungsträger zurück.

104 Die vorliegende Arbeit folgt somit auch einer alten Forderung der Unternehmenskrisenforschung sich stärker den »actions and contexts of failures" Hambrick \& D’Aveni (1988: 20) vor Ausbruch der eigentlichen Krise zu widmen.

105 Vgl. Cezanne (1999: 12); Müller (1986). 
existenzielle Bedrohung stetig zunimmt. Bewirkt der so zunehmende Druck auf das Management immer noch nicht die erforderlichen Reaktionen, so gewinnt der Krisenprozess ab einem gewissen Punkt eben jene eine Dynamik, die Hambrick \& D'Aveni (1988) als »downward spiral« bezeichnen. ${ }^{106}$ Der Begriff verweist auf einen Prozess, in dem Schwäche so lange zu weiterer Schwäche führt, bis sich die finanziellen Spielräume derart verengt haben, dass Zahlungsunfähigkeit oder Überschuldung unmittelbar drohen. Das Unternehmen hat nun die Phase der Liquiditätskrise erreicht. ${ }^{107}$

Kann die Liquidität binnen kurzer Frist nicht entscheidend verbessert werden, droht die Insolvenz als die extremste Form der Unternehmenskrise. Das Unternehmen hat sich in der Auseinandersetzung mit seiner Umwelt und den innerorganisationalen Herausforderungen nicht bewährt und büßt in den meisten Fällen seine Existenz ein.

\subsection{Der strategische Wandel als permanente Herausforderung des Managements}

Im vorigen Kapitel wurde herausgearbeitet, dass existenzbedrohende Krisen selten schicksalshaft über ein Unternehmen hereinbrechen. Krisen liegen in der Regel vielmehr endogene Problemkonstellationen zugrunde, die sich im Zuge längerer Prozesse aufgebaut haben und aufgrund derer die Organisationen schlussendlich nicht mehr adäquat auf die Dynamik der herausfordernden und sich verändernden Umwelt reagieren können.

Diese Vorstellung einer unablässigen Auseinandersetzung von Organisationen mit einer bestandskritischen Umwelt rückte insbesondere über die sozialwissenschaftlich orientierten Ansätze der Systemtheorie wie jene von Parsons (1952) und Luhmann (1984) in den Fokus der Managementtheorie. ${ }^{108}$ Diese Beiträge verstanden Stabilität nicht länger als den »Wesenskern des Seienden«, sondern problematisierten den Erhalt von Organisationen in der Umwelt als

106 Hambrick \& D’Aveni (1988); vgl. auch Staw et al. (1981). Paradoxerweise ist die Erfolgskrise häufig der Zeitpunkt, an dem Veränderungen am ehesten vorgenommen werden können. Unter moderatem Stress können unter Umständen nun auch Tabuthemen angesprochen und divergierende Interessen dem übergeordneten Ziel der Existenzsicherung untergeordnet werden. Vgl. Weitzel \& Jonsson (1989: 103).

107 Vgl. Cezanne (1999: 13); Müller (1986: 54).

108 Vgl. Schreyögg (2006: 303). Erste Beachtung fand diese auf die Umwelt gerichtete Perspektive aber bereits durch die kybernetische Systemtheorie, zum Beispiel durch Ashby (1956). Zur sozialwissenschaftlichen Systemtheorie vgl. Parsons (1952); Luhmann (1984). Vgl. Martens \& Ortmann (2006) für eine Einführung in die organisationstheoretische Systemtheorie und Steinmann \& Schreyögg (2005: 68 ff.) für die Implikationen der systemtheoretischen Perspektive auf das Management von Organisationen. 
permanente organisationale Herausforderung im Spannungsfeld von Konstanz und Veränderung, Stabilität und Wandel. ${ }^{109}$ Sie arbeiteten so, theoretisch besonders prägnant, jene Notwendigkeit des organisationalen und strategischen Wandels heraus, auf die bereits im obigen Kapitel aufmerksam gemacht wurde.

Die Systemtheorie soll jedoch nicht nur wegen dieser theoretischen Herausarbeitung des Erfordernisses organisationalen Wandels als die organisationstheoretische Perspektive gewählt werden. Die Wahl fällt vor allem deshalb auf die Systemtheorie, weil diese im Sinne eines institutionellen Organisationsverständnisses das über die ökonomische Ratio und den wirtschaftlichen Nutzen hinausgehende sozial-organisationale »Ganze« in den Blick zu nehmen vermag: die informellen Prozesse, die vermeintlich dysfunktionalen Elemente der Organisation oder die Emergenz organisationaler Strukturen und Strategien. ${ }^{110}$ Eine derart weite theoretische Perspektive ist nicht zuletzt für die später erfolgende, verständnisfördernde Konzeption des Forschungsgegenstandes Familienunternehmen von Bedeutung. Denn aus einer klassischen organisationstheoretischen, ökonomisch-rationalen Sichtweise würde ein großer Teil der aus dem Zusammenspiel von Familie und Unternehmen erwachsenden Spezifika ausschließlich irrational und dysfunktional anmuten. Die systemtheoretische Konzeption kann hingegen aufzeigen, dass diese Spezifika lediglich auch einer zweiten Rationalität folgen.

Wenden wir uns zur theoretischen Herausarbeitung der Notwendigkeit eines steten strategischen Wandels an dieser Stelle jedoch zunächst dem Ausgangspunkt allen systemtheoretischen Denkens zu: der Umwelt. ${ }^{111}$

\subsubsection{Das soziale System Organisation in seiner bestandskritischen dynamischen Umwelt}

\section{Die Notwendigkeit einer Differenz zur komplexen Umwelt}

Die Komplexität der Umwelt stellt Organisationen als Systeme vor die existenzielle Herausforderung, eine spezifische Art und Weise der Bearbeitung dieser

109 Zum »Wesenskern des Seienden« vgl. Luhmann (1995: 157); zudem vgl. Steinmann \& Schreyögg (2005).

110 Vgl. Steinmann \& Schreyögg (2005: 108); Luhmann (2000: 26ff.).

111 Luhmann (2002: 66ff.): "Die Theorie beginnt [...] mit der Differenz von System und Umwelt.« Der Fokus auf die System/Umwelt-Differenz ersetzt in der neueren soziologischen Systemtheorie das Verständnis von Systemen als Ganzheit, zusammengesetzt aus seinen einzelnen Teilen. Diese Perspektive verwies zwar gewinnbringend auf Interdependenzen, gerät ob der starken Fokussierung auf das Innere des Systems und dessen Aufbau häufig jedoch zu statisch. Die neuere Systemtheorie lenkt den Blick hingegen in dynamischer Perspektive auf die Grenzziehung und Grenzerhaltung des Systems und somit deren Wandel. 
chaotischen Umwelt zu finden. Sie stellt dem System gewissermaßen die Existenzfrage - ist die Umwelt als jener unendlich große Teil der Welt, der nicht zur Organisation gehört, doch derart vielgestaltig und unübersichtlich, dass ein Handeln ohne eine handlungsorientierende Ordnung in dieser Überfülle an Möglichkeiten unmöglich wäre. ${ }^{112}$ Hierzu bedarf es stabiler Strukturen, ohne die die Organisation in der Flut an Möglichkeiten und Unordnung vergehen würde.

Der grundlegende Schritt zum Aufbau organisationaler Ordnung ist die Errichtung und Aufrechterhaltung einer Grenze zur Umwelt durch die Herstellung und Reproduktion einer Differenz zwischen dem System und dem Rest der Welt. ${ }^{113}$ Ohne eine solche Grenze zur Umwelt wäre das System eine Teilmenge von allem und würde in der chaotischen Welt aufgehen. Die System/UmweltDifferenz schützt die Organisation somit als eine Art Damm vor diesem Zerrinnen und dem anstürmenden Chaos der Umwelt.

Da soziale Systeme im Gegensatz zu biologischen Systemen aber keine natürlichen Grenzen aufweisen, müssen sie die Leistung der Grenzziehung selbst erbringen und Grenzarbeit betreiben. ${ }^{114}$ Somit können sie durch die Wahl bestimmter Handlungsbereiche allerdings auch selbst bestimmen, was für sie Umwelt ist und was nicht. Ob die Umwelt diese Grenzziehungen auf Dauer akzeptiert und die Organisation diese Grenzen und sich selbst reproduzieren kann, bleibt jedoch stets fraglich.

\section{Mittel und Zweck: Reduktion von Komplexität}

Die Grenzbildung durch die Herstellung einer System/Umwelt-Differenz zielt im Kern auf die Reduktion von Komplexität durch Organisation. ${ }^{115}$ In einem arbeitsteiligen Prozess versuchen Organisationen die komplexe Umwelt auf ein für die Subsysteme bearbeitbares Maß zu reduzieren. Es gilt die Überfülle an Möglichkeiten in der Welt in einer spezifischen Form zu strukturieren und einzugrenzen, um zumindest diesen kleinen Teil als spezifische Umwelt und Handlungsbereich bearbeitbar zu machen.

Das Ergebnis dieser Anstrengungen ist eine Situation, die durch ein Komplexitätsgefälle gekennzeichnet ist: Die Komplexität innerhalb der Organisation ist geringer als die Komplexität außerhalb der Organisation. Innerhalb der organisationalen Grenzen findet sich somit nun eine ansatzweise zu überblickende Situation, in der versucht werden kann zu handeln. Die Reduktion von Kom-

112 Vgl. Luhmann (1984: 34ff.); zum Komplexitätsbegriff Child (1972: 3); Simon (2011).

113 Vgl. Luhmann (2000: 36ff.); auch Martens \& Ortmann (2006: 427ff.).

114 Vgl. Luhmann (2000: 52ff.). Für eine Übersicht zu der Debatte um die Grenzen von Organisationen vgl. Schreyögg et al. (2003: 304).

115 Vgl. Luhmann (2000: 34); auch Martens \& Ortmann (2006: 429); Steinmann \& Schreyögg (2005: 69f.). 
plexität ist somit ebenso Leistung von Systemen wie Nutzen der Systembildung. ${ }^{116}$

\section{Der Zwang zur Reduktion von Komplexität zwingt zur Selektion}

Zum Aufbau dieser Differenz und Form von Ordnung nutzen Systeme die sich aus der Interaktion mit der Umwelt zufällig ergebenden angebotenen Möglichkeiten. ${ }^{117}$ Dabei ist allerdings eine Selbstbeschränkung unabdingbar. Die Komplexität der Welt zwingt das System zur Reduktion von Komplexität über Selektion. Unternehmen müssen ihr Leistungsangebot auf ein bestimmtes Sortiment und auf bestimmte Märkte begrenzen. Sie müssen jene Bereiche der Umwelt selegieren, die sie beobachten möchten. Sie müssen die Themen festlegen, mit denen sie sich beschäftigen möchten. Durch diese Einschränkung der Handlungsmöglichkeiten gewinnt das System letztlich Handlungsspielraum. Zugleich werden Unternehmen durch diese Selektionen und Differenzbildungen zur Umwelt für andere Systeme in der Umwelt aber auch erst erkenn- und somit unterscheidbar. ${ }^{118}$

Durch die selegierende Leistung erschaffen sich Systeme ihre eigene Umwelt. Allerdings ist mit der Reduktion von Komplexität innerhalb des Systems die Komplexität außerhalb des Systems nach wie vor gegeben. Die Umstellung von der Komplexität der Welt auf den Modus der Systemkomplexität ist lediglich eine Problemverschiebung. ${ }^{119}$ Denn Reduktion von Komplexität bedeutet zwangsläufig Vereinfachung. Richtet die Organisation den Fokus ihrer Aufmerksamkeit durch Selektion auf eine bestimmte Thematik, bleiben aufgrund der Knappheit an Ressourcen andere Aspekte der Umwelt im Dunkeln. Wird sich für Alternative A entschieden, kann meist nicht gleichzeitig auch B gewählt werden. Es werden nur noch bestimmte Teile der Welt wahrgenommen, nur noch bestimmte Fragen an diese gestellt, teils nur noch bestimmte Antworten zugelassen. Diese Selektion ist zwingend notwendig, sie erzeugt durch die Schaffung virtueller Sicherheiten aber zwangsläufig auch neue Unsicherheit.

Nicht zuletzt, weil Selektion notwendigerweise immer kontingent ist. ${ }^{120}$ Das Bild der Umwelt, welches die Organisation durch Selektion entwirft, kann immer so, aber auch anders gezeichnet werden. Vorgenommene Selektionen können

116 Vgl. Steinmann \& Schreyögg (2005: 140). Luhmann (2000: 10): „Die Prämisse von Organisationen ist das Unbekanntsein der Zukunft und der Erfolg von Organisationen liegt in der Behandlung dieser Ungewissheit."

117 Vgl. Luhmann (2000: 31); Simon (2011: 25f.).

118 Durch die Grenzbildung wird für die Umwelt nachvollziehbar, dass Unternehmen A Pumpen für den asiatischen Markt produziert, während Unternehmen B Friseurdienstleistungen in einem bestimmten mecklenburgischen Dorf D anbietet.

119 Vgl. Steinmann \& Schreyögg (2005: $140 \mathrm{ff}$.); Simon (2011: $23 \mathrm{ff}$.).

120 Vgl. Luhmann (1984: 141); Steinmann \& Schreyögg (2005: 140f.). Martens \& Ortmann (2006: 427) verstehen Kontingenz als das »so-und-auch-anders-möglich-Sein«. 
sich später als Fehlselektion erweisen, aus ungeahnter und unbearbeiteter Richtung können plötzlich existenzgefährdende Risiken erwachsen. Die Umwelt bleibt auch oder gerade nach der Reduktion von Komplexität durch Selektion eine ständige Bedrohung. Unsicherheit wird zu einem ständigen Begleiter der Organisation.

\section{Der Wandel als permanente Herausforderung der dynamischen Umwelt}

Unsicherheit ist die Organisation vor allem deshalb ausgesetzt, weil die Umwelt in hohem Maße dynamisch und ständig in Veränderung begriffen ist. ${ }^{121}$ Immer wieder ergeben sich innerhalb der unendlichen Menge an potenziellen Anschlüssen der Umwelt neue Verbindungen, die vorher kaum geahnt, geschweige denn vorhergesagt werden konnten. Innerhalb kürzester Zeit entstehen neue Technologien. Langsam, aber stetig wandeln sich Branchen.

Je nach selegiertem Fokus werden diese Veränderungen wahrgenommen oder eben nicht. Je nach Selektion des Handlungsbereichs werden diese Veränderungen bearbeitet - oder nicht weiter beachtet. Diese Nichtbeachtung von Umweltaspekten ist kein Defizit per se, sondern eine grundsätzliche organisationale Leistung, welche das System entlastet. Da die Umwelt etablierten Lösungsmustern des Systems allerdings jederzeit die Grundlage entziehen und die Existenzberechtigung ehemals erfolgreicher Selektionen infrage stellen kann, können falsch selektierte Umweltbeobachtungen oder eine zu weitreichende Ignoranz der Umwelt die Existenz des Systems gefährden.

In jedem Falle stört die Umwelt über diese Veränderungen und Irritationen das System; fordert es permanent heraus - und treibt über solche Störungen auch dessen Evolution an. ${ }^{122}$ Durch die unablässigen Irritationen und Perturbationen hält die Umwelt die Entwicklung des Systems in Gang, fordert beständig organisationalen Wandel beständig ein. Auf diesem Wege wird die Umwelt zum Motor für eine Veränderung des Systems auf der Grundlage des Systems zu einer neuen Grundlage des Systems. Eine Entwicklung, die Baecker (2002) als »evolutionären Drift« beschreibt. ${ }^{123}$

Vor dem Hintergrund dieser theoretischen Erkenntnis darf der Wandel der Organisation somit nicht als eine Episode missverstanden werden, nach deren Bewältigung sich das System - in der Vorstellung einer organisationalen Homöostase - wieder in einem stabilen Gleichgewicht einrichten kann. Obgleich revolutionärer Wandel in bestimmten Situationen wünschenswert und auch durchsetzbar ist, ist organisationaler Wandel im Allgemeinen keine abgrenz-

121 Child (1972: 3) nennt drei Subdimensionen der Umweltdynamik: die Häufigkeit der Veränderungen der Umweltelemente (Stabilität), das Ausmaß ihrer Veränderungen (Intensität) und ihre Regelhaftigkeit. Vgl. zur Dynamik der Umwelt auch Schreyögg (2006: 311ff.).

122 Vgl. Luhmann (1984: 70); Simon (2011: 26); Steinmann \& Schreyögg (2005: 142).

123 Vgl. Baecker (2002: 90); auch Simon (2011: 40). 
bare und für sich definier-, analysier- und lösbare Sequenz. ${ }^{124}$ Gerade in Zeiten umgreifender gesellschaftlicher und technologischer Veränderungen kann ein Verständnis des organisationalen Wandels als zeitlicher und sachlicher Sonderfall vor der Realität nicht bestehen. Der Wandel der dynamischen Umwelt stellt für die Bestandserhaltung des Systems vielmehr eine permanente Herausforderung dar, die die Organisation bewältigen muss, um zu überleben. ${ }^{125}$

Dabei soll keineswegs einer naiven Wandelgläubigkeit gefolgt oder die existenzielle Notwendigkeit von Stabilität negiert werden. Auch der Wandel birgt Gefahren. Er ist meist kostenintensiv und stört die etablierten Abläufe der Organisation. Seine Risiken dürfen nicht vernachlässigt werden. ${ }^{126}$ Doch letztlich gilt: der Wandel mag riskant sein, die Bewahrung des Status quo ist in einer dynamischen Umwelt auf Dauer riskanter. ${ }^{127}$

\subsubsection{Strategischer Wandel und dessen Management}

Die skizzierte systemtheoretische Konzeption der Beziehung zwischen dem sozialen System und seiner bestandskritischen und sich ständig verändernden Umwelt verweist somit eindrücklich auf die Unverzichtbarkeit eines adäquaten organisationalen Wandels. Nur durch beständig neu definierte Systemgrenzen kann die stets prekäre Reproduktion der Organisation aufrechterhalten werden. Diesen beständigen Wandel durch eigene Beobachtungen und durch veränderte Selektionen zu gestalten, ist in der Organisationsform Unternehmen die permanente Aufgabe des Managements. ${ }^{128}$

\section{Organisationaler und strategischer Wandel}

Das Management sollte dafür Sorge tragen, dass im Unternehmen verschiedenste Dinge dem Wandel der Zeit angepasst sind. So sollte die Arbeitsumgebung ebenso den gegenwärtigen gesellschaftlichen Erwartungen entsprechen wie die Fertigungstechnologie dem aktuellen Stand der Technik. Ausgehend von der empirischen Forschungsfrage, warum in einigen Unternehmen dauerhaft an Strategien festgehalten wird, obwohl die fortgesetzte Reproduktion dieser

124 Vgl. zur Diskussion um inkrementellen und revolutionären strategischen Wandel Bresser (2010: 160ff.). Die episodenhafte Konzeption kommt insbesondere im Lewinschen Dreiklang (Lewin (1951) aus »unfreezing - moving - freezing« klar zum Ausdruck Diesem Denken folgen aber auch die oben eingeführten jüngeren Phasenkonzeptionen, als auch Ansätze der Organisationsentwicklung wie Bowers (1977)); Beckhard (1969) oder Palazzoli (1984). Vgl. im Gegensatz hierzu Schreyögg (2006: 497 ff.) zur evolutionären Wandelperspektive der Studien zum Organisationalen Lernen.

125 Vgl. Luhmann (1991 (1968): 39ff.); Schreyögg (2006: 542 f.).

126 Vgl. Luhmann (2002: 330 ff.); Miller \& Friesen (1980).

127 Vgl. Luhmann (2000: 333). Zu den Gefahren des Wandels vgl. u. a. Amburgey et al. (1990).

128 Vgl. Steinmann \& Schreyögg (2005: 149). 
strategischen Muster die Unternehmen in ihrer Existenz bedroht, widmet sich die vorliegende Arbeit im Folgenden jedoch nicht dem Wandel der Arbeitsorganisation oder Fertigungstechnologie, sondern dem Wandel von Strategien und dessen Ausbleiben. ${ }^{129}$

In diesem Zuge fokussiert sie aber nicht nur formulierte und explizierte Strategien, sondern in erster Linie strategische Entscheidungsprozesse, die Wandel und Persistenz schlussendlich stets zugrunde liegen. Diese Prozesse stellen die fokale Untersuchungseinheit dieser Arbeit dar - beruhen strategischer Wandel und Persistenz im Grunde doch auf einer kontinuierlichen und niemals endenden Aneinanderreihung von Problemlösungen und "strategischer « Entscheidungen, die im Sinne eines Musters aus Aktion und Reflektion in dynamischer Perspektive Wandel erzeugen - oder nicht. ${ }^{130}$ In Anlehnung an die strategische Prozessforschung sollen in diesem Rahmen somit vor allem das tatsächliche strategische Entscheidungsverhalten in Organisationen und dessen Determinanten untersucht werden. ${ }^{131}$

In Anlehnung an Mintzberg et al. (1976) und Eisenhardt \& Zbaracki (1992) soll eine Entscheidung dann als "strategisch" verstanden werden, wenn sie "important, in terms of the actions taken, the resources committed, or the precedents set « ist. ${ }^{132}$ Im Fokus stehen damit im Kern all jene Entscheidungen von Entscheidungsträgern, die kritisch sind für das langfristige Überleben des Unternehmens. ${ }^{133}$

Diese abstrakte Zielrichtung der Bestandssicherung wird in der Strategietheorie häufig in das Ziel der Herstellung eines "strategic fits" im Sinne von Child (1972) zwischen den Chancen und Risiken der Umwelt und den Stärken und Schwächen des Unternehmens übersetzt. Die "strategische« Perspektive

129 Vgl. für eine Übersicht zur Forschung zum »strategic change« Welge \& Al-Laham (2012: $160 \mathrm{ff}$.). Es sollte im Fortgang der Arbeit jedoch deutlich werden, dass mit dieser Fokussierung keineswegs eine Vernachlässigung des organisationalen Kontexts einhergeht, denn während bestimmte Formen organisationalen Wandels ohne eine Änderung der strategischen Ausrichtung des Unternehmens erfolgen können, müssen strategische Wandelprozesse stets in den existierenden Rahmen der Organisation gestellt werden.

130 Vgl. Koch (2011); Mintzberg (1987). Dies wird insbesondere in einer systemtheoretischen Managementperspektive deutlich. Vgl. Schreyögg et al. (2003); Luhmann (1991 (1968)); Luhmann (2000).

131 Vgl. zur strategischen Prozessforschung Pettigrew (1973); Mintzberg \& Waters (1985). Dieser Strömung der Strategietheorie liegen insbesondere die Arbeiten von Simon (1957); March \& Simon (1958) und Cyert \& March (1963) zugrunde, durch die auch in der Managementforschung eine Abkehr von der neo-klassischen rationalen Wahl eingeleitet wurde.

132 Vgl. Mintzberg et al. (1976: 246); Eisenhardt \& Zbaracki (1992: 17).

133 Vgl. Barney (2002: 17). 
besitzt somit einen inhärenten Umweltbezug und verweist qua natura auf die Notwendigkeit des strategischen Wandels. ${ }^{134}$

»The process of continuously adapting to the changes in a firm's environment is called strategic management."

(Schendel \& Hofer, 1979) $)^{135}$

Vor diesem theoretischen Hintergrund sollen unter dem Terminus strategischer Wandel - im weiten Mintzbergschen Verständnis - all jene Maßnahmen und Entscheidungen subsumiert werden, die im Zuge strategischer Entscheidungsprozesse auf eine umweltadäquate Veränderung von Strategien abzielen.

\section{Die Dimensionen des Managementprozesses des strategischen Wandels}

Der Wandel und Persistenz zugrunde liegende Management- und Entscheidungsprozess darf aber keineswegs nur als Entwurf eines strategischen Plans und Versuch dessen Implementierung verstanden werden. Vielmehr muss er als ein Zyklus aus Selektion, Kompensation und Entwicklung begriffen werden, der sich auf der Grundlage der klassischen Managementfunktionen Planung, Organisation, Personal, Führung und Kontrolle vollzieht. ${ }^{136}$ Nur ein solch modernes, systemtheoretisch orientiertes Managementverständnis - welches den anderen Managementfunktionen einen gleichberechtigten Platz neben der ehemals dominanten Planung einräumt und diese nicht lediglich als Erfüllungsgehilfen dieser Funktion betrachtet - versetzt den Beobachter in die Lage, organisationale Fehlentwicklungen nicht nur als ein Problem fehlerhafter Planung oder »Störungen« im Zuge der Planimplementierung zu verstehen, sondern die spezifische Eigenlogik und Dynamik der strategischen Entwicklung zu erfassen.

In diesem Sinne rückt in der Analyse des ausbleibenden strategischen Wandels und der zugrunde liegenden Entscheidungsprozesse zunächst die Managementdimension der Organisation in den Vordergrund. Ist diese doch eben nicht nur manifestierter Wille der Planung (»structure follows strategy«), sondern die Planung vor allem auch Ausfluss der organisationalen Struktur, historischen Prägung und Kultur (»strategy follows structure $)^{137}$

134 Vgl. Child (1972). Die ständige Auseinandersetzung mit einer sich kontinuierlich verändernden Umwelt kann nach Welge \& Al-Laham (2012: 18) oder Bresser (2010: 160f.) als eine der wichtigsten Wurzeln dieser Disziplin betrachtet werden.

135 Vgl. Schendel \& Hofer (1979: 10).

136 Vgl. für Kritik am klassischen plandeterminierten Verständnis Schreyögg (1984); für eine kurze Darstellung des systemtheoretisch orientierten Managementverständnisses Steinmann \& Schreyögg (2005: 139ff.). Ausführlicher die Pioniere Ulrich (1968) und Kirsch (1992).

137 Vgl. Chandler (1962); Hall \& Saias (1980). Zur Diskussion um die Kausalrichtung vgl. 
Nicht zuletzt die neuere Systemtheorie macht mit ihrem Verständnis von Selbstreferenzialität und Autopoiesis darauf aufmerksam, dass Systeme sich in ihren Operationen letztendlich immer nur auf sich selbst beziehen können. ${ }^{138}$ Aus dieser Perspektive müssen den Operationen von Organisationen stets jene Strukturen zugrunde liegen, welche sie durch vorherige Operationen selbst erzeugt haben und die im Anschluss wiederum die weiteren Operationen des Systems, dessen Wahrnehmungen und Handlungen prägen. Entscheidungen können immer nur auf der Grundlage der spezifischen organisationalen Entscheidungsprämissen wie der etablierten Kommunikationswege oder Kultur getroffen werden. Somit gilt: »history matters «. ${ }^{139}$

Auf der einen Seite dienen solche Prämissen und Strukturen durch die Kanalisierung der Informations- und Kommunikationsströme der Reduktion der Umweltkomplexität auf ein bearbeitbares Maß und ermöglichen Handeln überhaupt erst. Auf der anderen Seite erhöht diese Vorstrukturierung aber wiederum die Eigenkomplexität innerhalb des Systems und führt zur Herausbildung einer organisationalen Eigenlogik. Nur vor dem Hintergrund dieser organisationalen Eigenlogik wird verständlich, ob eine strategische Alternative wie der Verkauf des traditionellen Kerngeschäfts im strategischen Entscheidungsprozess als potenzielle Alternative im sozialen System überhaupt gedacht oder kommuniziert werden kann. Über diese Eigenkomplexität verweist die organisationale Strukturierung somit zugleich auf die Schranken des strategischen Managements. ${ }^{140}$

Über diese Aspekte der Organisation hinaus gilt es in der Analyse strategischer Entscheidungsprozesse auch Fragen der die Organisation ergänzenden Dimension der Führung zu beachten. ${ }^{141}$ Da natürlich nicht alle Fragen und organisationalen Probleme des Managementprozesses ex ante antizipiert und durch formelle Regeln oder kulturelle Normen vorwegnehmend durch Orga-

Bresser (2010: 139f.); zur immer stärkeren Verbindung von Organisation und Strategie in der Managementforschung Schreyögg (1999).

138 Spätestens Luhmann (1984) kehrt vom Modell offener Systeme ab und leitet in Bezug auf die Art und Weise der Reproduktion sozialer Systeme den Paradigmenwechsel zum Modell der selbstreferenziellen Systeme und seiner autopoietischen Variation nach Maturana \& Varela (1987) ein. Auf diesem Wege führt er über Konzepte wie Autopoiesis, Selbstreferenzialität, strukturelle Kopplung (s.u.) oder Beobachtung das neue Systemverständnis des operativ geschlossenen Systems ein.

139 Schreyögg et al. (2011: 81); vgl. auch Luhmann (2000: 222ff.). Zu den entscheidbaren Entscheidungsprämissen gehören Kommunikationswege, Entscheidungsprogramme und Entscheidungen über Personen. Zu den größtenteils von der Organisation unentscheidbaren zählen die Kultur der Organisation oder kognitiven Routinen.

140 Steinmann \& Schreyögg (2005: 143). Diese Fokussierung des Eigensinnes sozialer Systeme ist im Luhmann'schen Theoriegebäude von großer Bedeutung; vgl. hierzu Martens \& Ortmann (2006: 434f.).

141 Vgl. Steinmann \& Schreyögg (2005: 153). 
nisation koordiniert werden können, müssen auch Führungsstile oder Gruppenprozessvariablen wie die Kohäsion oder das Niveau des Konfliktes in ihrer Bedeutung für den strategischen Entscheidungsprozess beobachtet werden.

Des Weiteren gilt es der Dimension des Personals gebührende Aufmerksamkeit zu widmen. Ist das Spiel von Eintritt und Austritt von Organisationsmitgliedern doch originäre Quelle des Wandels und somit einer der potenziell wichtigsten Motoren der Systementwicklung. ${ }^{142}$ Der Eintritt neuer Mitglieder in die Organisation ist geradezu dafür prädestiniert eine bestehende Ordnung durch neue Ideen und Perspektiven zu bereichern, konstruktive Konflikte anzuregen, etablierte Denkmuster zu durchbrechen und somit das System zu öffnen. Vor dem Hintergrund dieser Bedeutung stellt sich jedoch umgekehrt die Frage, wie sich ein ausbleibender personeller Wandel und vielleicht auch eine niedrige Diversität im Management auf den Entscheidungsprozess auswirken.

Die Integration der Dimensionen Organisation, Führung und Personal in die analytische Rekonstruktion der Genese und Dynamik der strategischen Muster soll jedoch keineswegs eine Vernachlässigung der Analyse der formalen strategischen Planung zur Folge haben. ${ }^{143}$ Im Zuge der Untersuchung strategischer Persistenzen und der ihnen zugrunde liegenden Entscheidungsprozesse kommt der Analyse von Zielen, formulierten Strategien, Maßnahmenbündeln und entsprechender strategischer Kommunikation höchste Bedeutung zu.

Dies gilt auch für die Zwillingsfunktion der Planung, die strategischen Kontrolle. Da Planung auf Selektionen basiert und mit diesen Selektionen unter Ambiguität stets das Risiko der Fehlselektion einhergeht, ist Planung in der Konsequenz stets riskant und »beobachtungsbedürftige Systemaktivität «. ${ }^{144}$ Daher gilt es bei der Analyse der Entscheidungsprozesse auch die Formen der Beobachtung und Selbstbeobachtung des Systems zu berücksichtigen, die diesem Risiko zum Beispiel durch die Einrichtung von Kontroll- und Aufsichtsorganen begegnen sollen.

142 Vgl. Steinmann \& Schreyögg (2005: 151); Luhmann (2000: 279 ff.).

143 Vgl. zur dominanten Stellung der Planung die klassische Strategiedefinition nach Chandler (1962: 23), für den Strategie bedeutet: »The determination of the basic long-term goals and objectives of an enterprise, and the adoption of courses of action and the allocation of resources necessary for carrying out these goals.« Vgl. auch Barney (2002: 11). Diese (ehemals) dominante Stellung kommt auch in älteren Zeitschriftentiteln wie »Long Range Planning" zum Ausdruck.

144 Vgl. Steinmann \& Schreyögg (2005: 151), auch Schreyögg \& Steinmann (1987). 


\subsection{Das Ausbleiben des Wandels aufgrund strategischer Pfadabhängigkeit}

Im Zuge der ersten beiden Abschnitte dieses Kapitels wurde die Bedeutung endogen bedingter strategischer Persistenzen für den Prozess der Krisenentstehung herausgearbeitet und der strategische Wandel als eine permanente Aufgabe des Managements präsentiert.

Im ökonomischen Geschehen lassen sich jedoch unzählige Fälle beobachten, in welchen der für das Fortbestehen der Organisation existenzielle Wandel trotz einer sich verändernden Umwelt unterblieben ist. Solche Beobachtungen führten zu der dieser Arbeit zugrunde liegenden allgemeinen Frage nach der fortgesetzten Reproduktion existenzbedrohender strategischer Muster.

Wie eingangs bereits festgestellt wurde, können die Antworten auf diese Frage je nach beobachtetem Phänomen und theoretischer Perspektive unterschiedlich ausfallen. Sie können von »escalating commitment" bis hin zur "structural inertia « reichen. ${ }^{145}$ Trotz der unbestreitbaren Relevanz entsprechender Beiträge wählt die vorliegende Arbeit einen anderen Ansatz zur Erklärung solcher strategischer Persistenzen. Vor dem Hintergrund der oben skizzierten Vernachlässigung der Prozessdimension durch die bisherige Managementforschung zu Unternehmenskrisen wird mit der Theorie der organisationalen Pfadabhängigkeit gemäß Arthur (1989), David (1985) und Sydow et al. (2009) eine stark prozessorientierte Perspektive gewählt, um die Entstehung strategischer Persistenzen zu erklären und das Defizit der bisherigen Forschung zu adressieren. ${ }^{146}$

Die Theorie der organisationalen Pfadabhängigkeit basiert dabei auf dem grundlegenden Gedanken, dass der Ablauf von Prozessen durch zeitlich weit zurückreichende Ereignisse stark beeinflusst oder gar determiniert werden kann. Somit gilt wiederum: »history matters «. ${ }^{147}$ Vor langer Zeit getroffene Entscheidungen bedingen gegenwärtige und zukünftige Entscheidungsprozesse. $^{148}$

Allerdings ist nicht jeder historisch geprägte Entscheidungsverlauf pfadab-

145 Zum Begriff »escalating commitment « vgl. Staw (1976), zu jenem der »structural inertia» Hannan \& Freeman (1984). Vgl. zu solchen Persistenzen auch die Beiträge der Managementforschung wie Miller (1992); zur »Architecture of Simplicity« Miller (1993): Pettigrew (1987) zur Bedeutung politischer Prozesse. In der Organisationstheorie neben Hannan \& Freeman (1984) und Staw (1976) auch Stinchcombe (1965).

146 Vgl. Arthur (1989); David (1985); Sydow et al. (2009). Für einen aktuellen Überblick zur Pfadtheorie und Pfadforschung s. Beyer (2015).

147 Schreyögg et al. (2011: 81); vgl. auch Beyer (2015: 149). Durch diese Betonung der prozessualen Historizität greift sie den oben eingeführten grundlegenden Gedanken der strategischen Prozessforschung und des systemtheoretisch orientierten Managementverständnisses der historisch-organisationalen Verankerung von Entscheidungsprozessen auf.

148 Vgl. Sydow et al. (2009: 690); Sewell (1996). Teece et al. (1997: 522) merken entsprechend an: »bygones are rarely bygones." 
hängig im Sinne der organisationalen Pfadtheorie. ${ }^{149}$ Der Verweis auf die Historizität von Prozessen ist zwar notwendiges, aber kein hinreichendes Argument zur Beschreibung eines pfadabhängigen Prozesses. Wäre die Beobachtung von Historizität bereits ausreichend um von Pfadabhängigkeit sprechen zu können, so müssten in der Konsequenz beinahe alle Prozesse des sozialen Raumes als pfadabhängig verstanden werden.

Die Pfadtheorie geht über die reine Betonung der Historizität von Prozessen allerdings weit hinaus und fokussiert mit dem Konstrukt der selbstverstärkenden Mechanismen ein spezifisches Explanans für das Explanandum der Persistenz strategischer Muster.

Ihr zufolge können solche selbstverstärkenden Mechanismen, durch bestimmte Ereignisse oder Entscheidungen ausgelöst, im Zuge der Wirkkraft positiver Rückkopplungen zu einer derartigen Verstärkung des etablierten strategischen Musters führen, dass der strategische Entwicklungsweg ab einem gewissen Zeitpunkt nicht mehr verlassen werden kann. Selbst dann nicht, wenn sich in der Zwischenzeit in der organisationalen Umwelt vorteilhaftere Wege eröffnet haben. Das Unternehmen befindet sich dann in einem Zustand des strategischen Lock-in und hat beinahe jeden strategischen Handlungsspielraum verloren - es ist pfadabhängig. Dies ist ein gefährlicher Zustand in einer sich permanent wandelnden Welt.

Insbesondere über das Konstrukt der selbstverstärkenden Mechanismen vermag es die organisationale Pfadtheorie somit eine spezifische Persistenz strategischer Muster und somit eine bestimmte Form der Krisengenese aus dem Prozess ihrer Entstehung heraus zu erklären. Wo andere Konzepte organisationaler Trägheit und strategischer Persistenz lediglich das Phänomen treffend beschreiben, hinsichtlich der Ursache-Wirkungs-Zusammenhänge aber teils recht diffus bleiben, kann die Pfadtheorie mit dem Konstrukt der selbstverstärkenden Mechanismen prozessendogen und prozessbegleitend Ursachen und Wirkungen im Prozess der Entstehung verknüpfen. ${ }^{150}$ Die Theorie der organisationalen Pfadabhängigkeit wurde daher als gegenstandsbezogene theoretische Perspektive auf die Entstehung strategischer Persistenzen und Unternehmenskrisen gewählt. Sie soll im folgenden Unterkapitel näher vorgestellt und spezifiziert werden.

Im Zuge dieses Unterfangens wird im ersten Abschnitt im Rahmen einer kurzen Entwicklungsgeschichte der Pfadtheorie an die grundlegenden theoretischen Charakteristika dieser theoretischen Perspektive herangeführt. Mit der Skizzierung der theoretischen Wurzeln soll ein grundlegendes Verständnis für

149 Vgl. Sydow et al. (2009: 689).

150 Vgl. zu dieser Einschätzung Schreyögg et al. (2003: 259) und die kontrastierende Theoriediskussion bei Sydow et al. (2009: 696ff.). 
die Pfadtheorie geschaffen werden. Hierauf aufbauend wird dann im zweiten Abschnitt das Modell organisationaler Pfadabhängigkeit nach Sydow et al. (2009) eingeführt, welches die drei Kernkonzepte der Pfadtheorie - Historizität, selbstverstärkende Mechanismen und den Zustand des Lock-in - in eine logische Phasenabfolge einordnet. ${ }^{151}$ Im Zuge der Einführung dieses Modells werden die drei Kernkonzepte zugleich ausführlich skizziert. Auf der Grundlage des so geschärften theoretischen Verständnisses können dann im dritten Abschnitt strategische Pfade als das zentrale zu untersuchende theoretische Konstrukt dieser Arbeit definiert werden, um es im vierten Abschnitt anschließend von verwandten theoretischen Konzepten strategischer Persistenz abzugrenzen. Doch so groß der Erklärungsbeitrag der Pfadtheorie auch sein mag - diese theoretische Perspektive hat ebenfalls Lücken. Daher wird im fünften Abschnitt die in den Beiträgen zur organisationalen Pfadforschung bislang weitgehend "unexploded role of context" für die Genese und Dynamik strategischer Pfade kritisch analysiert. ${ }^{152}$ Auf diesem Wege wird das Tor der Pfadtheorie für den im zweiten theoretischen Kapitel einzuführenden Kontextfaktor Familie geöffnet.

\subsubsection{Kurze Entwicklungsgeschichte der Pfadtheorie}

Im Verlauf der letzten beiden Jahrzehnte entwickelte sich das Konzept der Pfadabhängigkeit zu einem der meist-verwendeten Erklärungsansätze in der ökonomischen und sozialwissenschaftlichen Forschung. ${ }^{153}$ Der grundlegende Gedanke des Pfadabhängigkeitskonzepts - die Vorstellung, dass der Verlauf von Entwicklungen durch zeitlich weit zurückliegende Ereignisse stark beeinflusst oder gar vollständig determiniert werden kann - wurde gleichermaßen in den Wirtschaftswissenschaften, der Politik- und Geschichtswissenschaft, der Soziologie oder auch in der Geografie zur Erklärung von rigiden Entwicklungsverläufen herangezogen. ${ }^{154}$

151 Vgl. Schreyögg et al. (2003), die diese drei Konstrukte als die grundlegenden Komponenten der organisationalen Pfadforschung erachten. Autoren der klassischen ökonomischen Pfadforschung wie Arthur (1989: 121f.) thematisieren im Rahmen der Erörterung pfadabhängiger Entwicklungsverläufe noch Eigenschaften wie Nichtvorhersagbarkeit; Nonergodizität oder Inflexibilität; vgl. auch Pierson (2000: 253). Diese Eigenschaften finden jedoch ebenfalls Eingang in das unten darzustellende Phasenmodell.

152 Vgl. Koch (2011: 341).

153 Vgl. Beyer (2010: 1). Nach Sydow et al. (2009: 659) widmeten sich im Zeitraum 1995-2008 beinahe $5 \%$ aller Artikel der führenden Managementjournals ASQ, ORGAN SCI, ORGAN STUD der Pfadabhängigkeit. Vergne \& Durand (2010) sprechen gar davon, dass knapp $10 \%$ aller in den führenden Managementzeitschriften veröffentlichten Beiträge im Zeitraum von 2003-2007 dieses Konzept direkt referenzierten.

154 Vgl. für die Politikwissenschaft Pierson (2000); Mahoney (2000). Für die Geografie: Krugman (1991). Zur Entwicklung von Institutionen North (1990); Acemoglu \& Robinson (2012). Für eine Übersicht: Beyer (2010: 150). 
Im Zuge dieser Verbreitung wurde die Pfadtheorie jedoch ein Stückweit Opfer ihres eigenen Erfolgs. Brachte die zunehmende Verwendung in unterschiedlichen Disziplinen im Verlauf der Zeit doch derart viele inhaltlichen Erweiterungen und Variationen des ursprünglichen Konzepts mit sich, dass heute in der Folge leider nicht mehr immer vollkommen klar ist, was mit dem Terminus Pfadabhängigkeit eigentlich zum Ausdruck gebracht werden soll. So titulieren beispielsweise Baron et al. (1999) die von ihnen beobachteten Phänomene als pfadabhängig, greifen dabei aber nicht auf die Literatur der Pfadtheorie zurück, sondern auf die Konzepte und Beiträge des Imprinting-Ansatzes. Pierson (2000) spricht entsprechend von einem "concept stretching ", aus dem häufig eine eher metaphorische denn wirkliche theoretische Begriffsführung resultiere. ${ }^{155}$ So kann konstatiert werden:

"Quite often, path dependence is defined as little more than the vague notion that shistory matters` or that, the past influences the future."

(Mahoney, 2000) $)^{156}$

Vor dem Hintergrund dieser begrifflichen und auch theoretischen Diffusität scheint eine Klärung des Begriffs der Pfadabhängigkeit und des theoretischen Konzepts dringend geboten. Zur Schaffung eines klaren konzeptionellen Verständnisses organisationaler Pfadabhängigkeit sollen daher im Folgenden zunächst die ökonomischen Wurzeln der Theorie beleuchtet werden, aus denen später die organisationstheoretische Adaption erwuchs.

\section{Die klassische ökonomische Pfadtheorie}

Ihren Ursprung nahm die Theorie der Pfadabhängigkeit in den Arbeiten des Wirtschaftshistorikers David (1985) und des Ökonomen Arthur (1989) zur Verbreitung und Durchsetzung von Technologien in Märkten. ${ }^{157}$ Hierbei zeigte David (1985) in einer Kritik an den neoklassischen Gleichgewichts- und Effizienzannahmen am Beispiel des QWERTY-Tastaturlayouts, wie sich aufgrund selbstverstärkender Mechanismen am Markt eine grundsätzlich inferiore technologische Lösung gegen effizientere Alternativen durchsetzen und zu einem bis zum heutigen Tage ungebrochenen De-facto-Standard avancieren konnte. ${ }^{158}$

155 Vgl. Sartori (1970); Baron et al. (1999: 527). Zur Einschätzung der konzeptionellen Überdehnung: Pierson (2000: 252) und Sartori (1970); zudem Sydow et al. (2009: 689); Beyer (2015: 149ff.); Ackermann (2003: 278).

156 Mahoney (2000: 507). Als ein Beispiel für eine solche metaphorische Begriffsführung vgl. in der Forschung zu Familienunternehmen zum Beispiel Sharma \& Manikutty (2005: 293ff.).

157 Vgl. David (1985); Arthur (1989); auch Arthur (1994). Vgl. zur zentralen Bedeutung dieser Beiträge Sydow et al. (2009: 690) oder Beyer (2010: 1).

158 Vgl. David (1985); auch David (1986); Schreyögg et al. (2003: 246f.). Dieser Schreibmaschinentastaturtyp wird entsprechend der Anordnung der ersten sechs Buchstaben auf der ursprünglichen englischsprachigen Tastatur benannt. Andere Wissenschaftler untersuch- 
Die Ursprünge dieses QWERTY-Layouts gehen dabei auf die Überlegungen des amerikanischen Erfinders Christopher Scholes zurück, der nach mehrjährigen Versuchen 1873 die damals übliche alphabetische Reihung der Buchstaben zugunsten einer Anordnung aufgab, bei der häufig nacheinander getippte Buchstabenkombinationen möglichst weit auseinanderlagen. ${ }^{159}$ Mit dieser Maßnahme sollte das damals übliche, ständige Verhaken der Typenhebel verhindert werden.

Aufgrund technologischer Fortentwicklungen war dieser Vorteil jedoch bereits wenige Jahre später weitgehend obsolet. Nach den Annahmen der klassischen Ökonomie hätten daher nun effizientere, an der Ergonomie des Schreibens und nicht an technischen Zwängen orientierte Layouts die QWERTYTastatur verdrängen müssen. ${ }^{160}$ Doch obgleich Alternativen wie die anfangs von Apple propagierte Dvorak-Tastatur ein um bis zu 40 \% schnelleres Schreiben versprechen, blieb diese Substitution bis zum heutigen Tage aus. Die QWETYTastatur ist und bleibt trotz dieser technologischen Ineffizienz der milliardenfach genutzte de-facto Standard und integraler Bestandteil unseres modernen Alltags. ${ }^{161}$

Warum wich die technologische Entwicklung trotz scheinbar überlegener technologischer Alternativen nicht von diesem Entwicklungspfad ab? Hinsichtlich dieser Frage verwies David (1985) auf die Wirkkraft positiver Rückkopplungseffekte. ${ }^{162}$

Diese positiven Rückkopplungen nahmen ihren Ausgang in der Entwicklung des bis heute gebräuchlichem Zehnfinger-Schreibsystems des "touch-typing", welches kurze Zeit nach der Einführung der QWERTY-Tastatur eher zufälliger Weise auf diesem Layout, und nicht auf einer der vielen hundert Alternativen entwickelt wurde. Dieser Vorgang war zunächst wenig bedeutend und nur wenige Marktteilnehmer nahmen von ihn überhaupt Notiz. Allerdings setzte er einen sich selbstverstärkenden Prozess in Gang, der beinahe 150 Jahre später noch immer einen bedeutenden Teil unserer technologischen Umgebung prägt.

Dieser selbstverstärkende Prozess setzte damit ein, dass die Nutzer der QWERTY-Tastatur durch die neue Schreibtechnik und das seltener auftretende Verhaken nun zunächst etwas schneller schreiben konnten als mit vergleich-

ten im Anschluss an David die Verbreitung von Videosystemstandards. Vgl. Cusumano et al. (1992: 51 ff.); Liebowitz \& Margolis (1995: 208f.). Zur pfadabhängigen Verbreitung von Computersoftware vgl. Foray (1997: $737 \mathrm{ff}$.).

159 Vgl. zur historischen Entwicklung der QWERTY-Tastatur Schreyögg et al. (2003: 264f.).

160 Spätestens mit der Einführung von Kugelkopfschreibmaschinen nach dem Zweiten Weltkrieg oder dem Aufkommen von elektronischen Computern war dieses mechanische Problem hinfällig.

161 Vgl. David (1985: 332). Zur Diskussion um die Effizienz der Layouts vgl. Liebowitz \& Margolis (1995).

162 Vgl. David (1985: 41 ff.). 
baren Alternativen. Der Vorteil war gering, führte aber dazu, dass in der Folgezeit mehr und mehr Schreibkräfte das Tippen auf der QWERTY-Tastatur erlernten. Von größerer Bedeutung für die folgende technologische Entwicklung als der kleine, stets umkehrbare Effizienzvorteil, war nun jedoch, dass angesichts des wachsenden Angebots an entsprechend ausgebildeten und produktiven Arbeitskräften für die Arbeitgeber die Anschaffung von QWERTY-Schreibmaschinen und in der Folge wiederum die Einstellung entsprechend ausgebildeter Schreibkräfte immer attraktiver wurde. Die in diesem Zuge steigende Nachfrage nach solchen Schreibkräften erhöhte dann wiederum die Nachfrage nach einer Ausbildung auf der QWERTY-Tastatur und in der Folge schnellte die Anzahl der verkauften QWERTY-Schreibmaschinen ebenso rasch in die Höhe wie die Anzahl der auf QWERTY ausgebildeten Schreibkräfte. Nur wenige Jahre später erlernten alle neu auszubildenden Schreibkräfte nur noch dieses komplementäre Paket und die meisten anderen Layouts verschwanden vom Markt. Trotz ihrer vermeintlich höheren Schreibleistung wurden sie aufgrund ihrer geringen Verbreitung nicht mehr nachgefragt. ${ }^{163}$ Im Gegensatz hierzu stieg mit der zunehmenden Verbreitung von QWERTY für alle Marktteilnehmer der Nutzen dieser Technologie. Trotz einer sich früh abzeichnenden technologischen Unterlegenheit war es für jeden Einzelnen in der Folge individuell rational sich für das QWERTY-Layout zu entscheiden.

Mit der von ihm anhand dieses Beispiels aufgezeigten Marktdurchdringung einer ineffizienten Technologie erbrachte David (1985) durch die Verknüpfung bereits vorher diskutierter Konzepte wie Historizität oder Skalenerträge zu einer neuartigen Form von Prozessen somit den Nachweis, dass sich im Spiel der freien Marktkräfte schlussendlich nicht immer unbedingt die effizienteste Technologie durchsetzt. ${ }^{164}$ Diese Feststellung des Marktversagens, welche durch Arthur (1989) in ersten pfadtheoretischen Modellierungen mathematisch formalisiert und spezifiziert wurde, provozierte die neoklassische Ökonomie. ${ }^{165}$ Eine Provokation, die wohl ursächlich für die große Aufmerksamkeit und Verbreitung gewesen sein dürfte, welche die Pfadtheorie in der Folgezeit erfuhr.

\section{Übertragung des Pfadkonzepts in andere Disziplinen}

North (1990) zog das Konzept der Pfadabhängigkeit zur Erklärung unterschiedlicher institutioneller Ausgestaltungen und deren Evolution in verschiedenen Gesellschaften heran. Der Fokus dieses und ähnlicher Beiträge des historischen Institutionalismus liegen vor allem auf zeitlich sehr weit zurücklie-

163 Vgl. David (1985: 332ff.). Der Grund für die zunehmende Verbreitung war somit im Kern die wahrgenommene oder antizipierte zunehmende Verbreitung der Technologie.

164 Vgl. David (1985: 336): „Competition in the absence of perfect futures markets drove the industry prematurely into standardization on the wrong system «.

165 Vgl. Arthur (1989); Arthur (1994). 
genden Ereignissen, die als frühe kritische Weichenstellungen im Sinne von Collier \& Collier (1991) als »critical junctures« eine Entwicklung initiieren, von der später nur noch schwer abgewichen werden kann und welche die Ausgestaltung von Institutionen prägt, insbesondere, weil die Akteure sich bei der Gestaltung von Institutionen stets im Orientierungsrahmen der bestehenden Institutionen bewegen und an diese anknüpfen müssen. ${ }^{166}$ Der Fokus dieses und ähnlicher Beiträge liegt somit jedoch weniger auf der Erklärung der Reproduktion des immer Gleichen, sondern stärker auf der Untersuchung der Verlaufsform eines inkrementellen, aber pfadabhängigen evolutionären Wandels. ${ }^{167}$

Die sozialwissenschaftliche Adaptionen des Pfadabhängigkeitskonzepts wie jene von Pierson (2000) oder Mahoney (2000) richten den Blick auf Faktoren wie Macht und deren Verteilung, welche die Entstehung von Pfadabhängigkeit begünstigen könnten. ${ }^{168}$ Über die Diskussion der Relevanz solcher kontextueller Variablen hinaus, an die weiter unten im Zuge der Erörterung der Bedeutung des Kontexts angeknüpft werden soll, verweist Mahoney (2000) zum Beispiel mit der Legitimation auch auf weitere Mechanismen der Reproduktion. Darüber hinaus führte er mit "reaktiven Sequenzen" gar eine erweiterte Form von Pfadabhängigkeit ein. ${ }^{169}$ Diese reaktiven Sequenzen sind dabei als Ereignisketten konzipiert, bei denen jedes Ereignis in der Kette prozessual mit den vorhergehenden kausal verknüpft ist, so dass es "pfadabhängig" aus den vorigen hervorgeht und Teil eines Prozesses aus Ereignis und Reaktion ist. Bei dieser Form von »Pfadabhängigkeit" steht die Erklärung eines bestimmten Entwicklungsverlaufs, welcher nicht selbstverstärkend sein muss, im Vordergrund, nicht mehr, wie im klassischen, ursprünglichen Sinn der Theorie, die persistente Reproduktion eines bestimmten Musters oder einer technologischen Lösung.

Es sollte somit deutlich werden, dass diese und weitere, ähnliche Beiträge das Pfadabhängigkeitskonzept nicht nur ausdehnen oder variieren, sondern im Grunde grundsätzlich verschiedene Formen von »Pfadabhängigkeit« beschreiben. Diese Formen haben außer der Historizität des Prozesses relativ wenig mit dem ursprünglichen Konzept nach David (1985) und Arthur (1989) gemein, welches die selbstverstärkenden Effekte in den theoretischen Mittelpunkt stellt. ${ }^{170}$ Angesichts solcher einschneidender Veränderungen weist Pierson

166 Vgl. North (1990); Collier \& Collier (1991); Thelen (1999). Hall et al. (2001) zu den »Varieties of Capitalism«.

167 Vgl. North (1990: 95ff.); North (1993: 17f.) zu dessen Theorie des institutionellen Wandels. 168 Vgl. Mahoney (2000); Pierson (2000). Zur Übersicht Beyer (2005: 10f.).

169 Institutionen werden demnach auch dann reproduziert, wenn mächtige Akteure sich zu dieser Reproduktion verpflichtet fühlen oder die Institution als "gegeben« annehmen. Vgl. Beyer (2005: 12); Mahoney (2000: 509) zu den reaktiven Sequenzen.

170 Mahoney (2000: 509f.) unterscheidet auch explizit zwischen auf Selbstverstärkung beruhender Pfadabhängigkeit und reaktiven Sequenzen. Ähnlich auch Arrow (2003). 
(2000) wohl vollkommen zu Recht auf die Gefahr einer zu weiten Ausdehnung und inflationären Verwendung des Pfadabhängigkeitsbegriffs hin. ${ }^{171}$

Die konzeptionelle Überdehnung veranlasste die organisationale Pfadforschung nach Schreyögg et al. (2003) im Zuge der Übertragung des Konzeptes in den organisationalen Rahmen dazu, klar zwischen Pfadabhängigkeit im engeren und weiteren Sinne zu unterscheiden. ${ }^{172}$ Sich von der bloßen Beobachtung der Historizität von Entscheidungsprozessen klar abgrenzend, kehrte sie im Sinne eines engen Verständnisses von Pfadabhängigkeit gewissermaßen zu den Wurzeln des Konzepts zurück und stellte wieder die selbstverstärkenden Mechanismen ins Zentrum der Überlegungen. ${ }^{173}$

In diesem Zuge modellierten Sydow et al. (2009) die aus solchen Mechanismen resultierende Persistenz organisationaler Entwicklungen und arbeiteten dabei im Kontrast zur historisierenden metaphorischen Verwendung des Pfadabhängigkeitsbegriffs trennscharf die spezifische, auf selbstverstärkenden Mechanismen beruhende Dynamik pfadabhängiger Prozesse heraus. ${ }^{174}$ Die diesem engen, spezifizierten Verständnis zugrunde liegenden Konstrukte der Historizität, der selbstverstärkenden Mechanismen und des Lock-ins sollen im Folgenden im Rahmen des Drei-Phasen-Modells Sydow et al. (2009) näher beleuchtet werden.

\subsubsection{Phasen der Pfadgenese und deren Komponenten}

Zur theoretischen Konzeptualisierung der organisationalen Pfadgenese schlagen Sydow et al. (2009) ein mittlerweile weit rezipiertes Modell vor, welches drei Entwicklungsphasen umfasst. ${ }^{175}$ Die Autoren bringen in diesem Modell die drei Pfadkomponenten Historizität, selbstverstärkende Mechanismen und Lock-in hinsichtlich ihrer spezifischen temporären Wirkkraft auf den emergierenden Pfad in eine entwicklungsdynamisch logische Phasenfolge. ${ }^{176}$ Denn nicht jedes

171 Vgl. Pierson (2000: 252), der diesbezüglich von »concecpt stretching « spricht.

172 Vgl. Sydow et al. (2009: 689). Für ein breites Verständnis vgl. Barnett \& Burgelman (1996), die Pfade als Ereignisfolgen konzipieren und Teece et al. (1997), die im Grunde alle historisch geprägten Prozesse als pfadabhängig bezeichnen und Pfadabhängigkeiten auf Grund von selbstverstärkenden Mechanismen als Sonderfall der allgemeinen Konzeption begreifen.

173 Vgl. Sydow et al. (2009: 698); Beyer (2015: 160).

174 Sydow et al. (2009) knüpfen im Zuge dieser Modellierung dabei ebenso an die oben skizzierten institutionenökonomischen North (1990), Collier \& Collier (1991) wie sozialwissenschaftlichen Pierson (2000), Mahoney (2000) Beiträge der Pfadforschung an. In Bezug auf die Historizität aber auch an verwandte Beiträge der Organisationsforschung wie Boeker (1989), Stinchcombe (1965); oder Teece et al. (1997).

175 Vgl. zunächst Schreyögg et al. (2003), dann Sydow et al. (2009: 705). Zur Rezeption des Modells vgl. Beyer (2015); Garud et al. (2010); Vergne \& Durand (2010).

176 Sydow et al. (2009) adressieren somit in ihrem Modell das Problem, dass die von Arthur 
dieser drei pfadkonstituierenden Elemente wirkt zur gleichen Zeit auf die Pfadgenese ein.

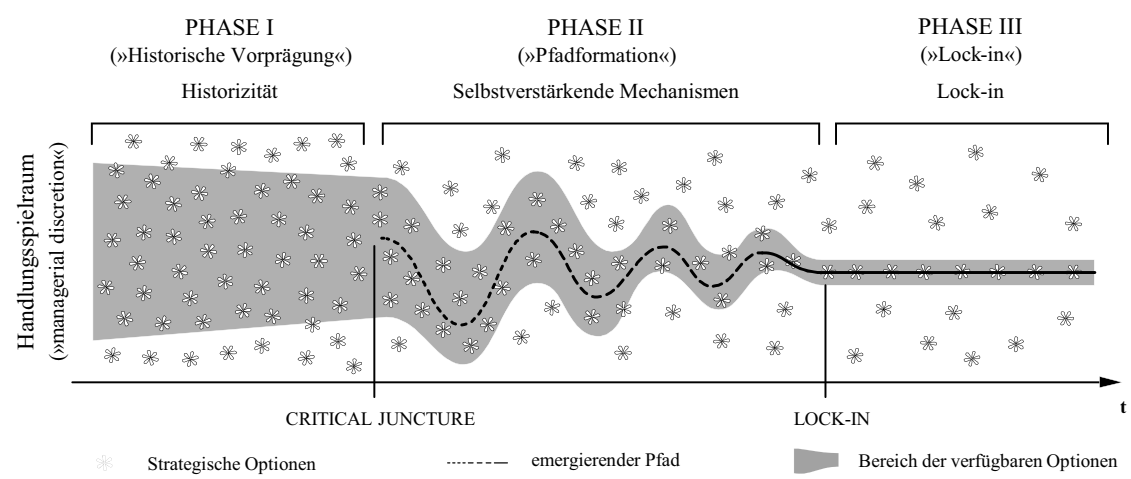

Abbildung 4: Phasenmodell der organisationalen Pfadgenese nach Sydow et al. (2009)

(Quelle: Eigene Darstellung in Anlehnung an Sydow et al. [2009: 692]).

So ist der strategische Entscheidungsprozess in der ersten Phase der »historischen Vorprägung " zwar wie in jedem organisationalen Kontext historisch geprägt, aber trotzdem noch durch einen relativ offenen Entscheidungsraum charakterisiert. In dieser Phase getroffene Entscheidungen oder bestimmte Ereignisse können allerdings unter Umständen eine sich selbstverstärkende Dynamik in Gang setzen und somit den Beginn der Phase der Pfadformation markieren. Diese Entscheidungen oder Ereignisse fungieren somit als »critical junctures«, durch welche die Organisation einen bestimmten strategischen Entwicklungsweg einzuschlagen beginnt. ${ }^{177}$

In der Phase der "Pfadformation« nimmt die Dynamik der selbstverstärkenden Mechanismen dann mehr und mehr zu. Dabei konstituiert oder stützt die Wirkkraft dieser Mechanismen zunehmend ein dominantes Muster aus Entscheidungen und Handlungen. Grundsätzlich ist ein Abweichen von diesem Muster zwar noch möglich, doch je wirkkräftiger die Mechanismen werden und je weiter sich das Muster reproduziert, desto schwieriger wird es für die Organisation den eingeschlagenen Pfad zu verlassen.

$\mathrm{Ab}$ einem gewissen Zeitpunkt sind die aus den selbstverstärkenden Mechanismen resultierenden Wirkkräfte dann so stark, dass es der Organisation bei-

(1994: 112f.) und Pierson (2000: 253) herausgearbeiteten Eigenschaften pfadabhängiger Prozesse der Nicht-Vorhersagbarkeit, Non-Ergodizität, Inflexibilität und Ineffizienz jeweils nicht den gesamten Prozess, sondern stets nur spezifische Abschnitte pfadabhängiger Prozesse charakterisieren. Vgl. Sydow et al. (2009: 691).

177 Vgl. Collier \& Collier (1991); auch Acemoglu \& Robinson (2012); Capoccia \& Kelemen (2007). 
nahe unmöglich ist, den eingeschlagenen Entwicklungsweg noch zu verlassen. Die Organisation befindet sich nun in der Phase des Lock-ins und kann im Prinzip nicht mehr vom gewachsenen und etablierten Muster abweichen, auch dann nicht, wenn sich der eingeschlagene Entwicklungsweg angesichts der sich wandelnden Umwelt als nicht mehr adäquat erweisen sollte. Selbst wenn in der Umwelt nun bessere Handlungsoptionen verfügbar sind - die Organisation kann diese aufgrund ihre Pfadabhängigkeit nicht mehr ergreifen. Sie ist auf einem bestimmten Entwicklungspfad gefangen und steuert mit hoher Wahrscheinlichkeit in die Krise.

Dieses Phasenmodell soll im Fortgang der Arbeit als ein spezifischer Prozess der Krisenentstehung zur Konzeption und Analyse der aus den strategischen Entscheidungsprozessen erwachsenden strategischen Pfade herangezogen werden.

Ob ihrer fundamentalen Bedeutung für den Prozess der Pfadgenese, werden in den folgenden Abschnitten die diesem Prozess zugrunde liegenden drei Pfadkomponenten Historizität, selbstverstärkende Mechanismen und Lock-in näher beleuchtet.

\subsubsection{Phase I: »Historische Vorprägung« - die Historizität strategischer Entscheidungsprozesse}

In der ersten Phase der Pfadgenese ist der strategische Handlungsraum der Organisation noch weit aufgespannt und umfasst eine Vielzahl verschiedenster Handlungsalternativen. ${ }^{178}$ Grenzenlos ist er allerdings nicht, denn über die natürlichen Begrenzungen des Wirtschaftens im Rahmen knapper Ressourcen hinaus, ist der strategische Entscheidungsraum stets auch historisch geprägt. ${ }^{179}$

»A firm's previous investments and its repertoire of routines (its history) constrain its future behavior."

(Teece et al., 1997) ${ }^{180}$

Vor langer Zeit getroffene Entscheidungen bedingen spätere Entscheidungsprozesse. Sie sind nicht immer reversibel, ihre Folgewirkungen halten an und begrenzen den Möglichkeitsraum für spätere Entscheidungen. Organisationen sind historische Maschinen, die in ihren Operationen auf historischen selbstproduzierten Strukturen und Prozessen aufbauen. ${ }^{181}$ Strategien sind eingebettet

178 Vgl. Sydow et al. (2009: 692f.). Ob und wohin sich ein späterer Pfad entwickelt, ist zu diesem Zeitpunkt nicht nur vollkommen unklar und unbestimmt, sondern angesichts der Spezifität des Phänomens auch unwahrscheinlich.

179 Die Historizität ist nach Schreyögg et al. (2003: 261) das »Basismerkmal« pfadabhängiger Prozesse. Sie wird in der obigen Grafik durch die grau schraffierte Fläche des Handlungskorridors markiert.

$180 \mathrm{Vgl}$. Teece et al. (1997: 522f.); auch Schreyögg et al. (2003: 268).

181 Vgl. von Foerster (1984: 60). Dies verdeutlichen insbesondere die systemtheoretische Perspektive und das daran orientierte modernes Managementverständnis. 
in die historisch gewachsene organisationale Struktur und Kultur (»strategy follows structure $\lll) .{ }^{182}$ Sie werden getragen von etablierten organisationalen Regeln und Praktiken.

Diese Einsicht erscheint naheliegend. In der von der Theorie der rationalen Entscheidung geprägten Managementforschung und Strategietheorie wurde sie aufgrund der Dominanz der Managementfunktion Planung und präskriptiver rational-entscheidungsorientierter Optimierungsmodelle jedoch lange Zeit nicht gebührend berücksichtigt. ${ }^{183}$ Vor diesem Hintergrund ist das Argument der Historizität weniger als neue originäre Erkenntnis, denn als methodologisches Argument zu verstehen, welches die Ahistorizität weiter Teile der Ökonomie und Managementforschung herausfordert. ${ }^{184}$

Erst neuere Ansätze der Strategie- und Organisationstheorie, insbesondere Beiträge der Strategieprozessforschung, versuchen die Historizität des tatsächlichen, begrenzt rationalen Entscheidungsverhaltens in ihre Theoriewelt zu integrieren. ${ }^{185}$ Sie knüpfen damit an die fundamentalen verhaltenswissenschaftlichen Beiträge wie Simon (1957) und Cyert \& March (1963) oder an die Systemtheorie an. Im Sinne des Prinzips "strategy follows structure" verweist die strategische Prozessforschung dabei auf die Begrenzung der Wahlmöglichkeiten der strategischen Entscheidungsträger durch vorhergehende organisationale Entscheidungen und Handlungen oder etablierte mentale Schemata und kollektive Orientierungsmuster. Ebenso wird die Beschränkung des Handlungsspielraums durch bestehende Machtstrukturen behandelt. ${ }^{186}$ Sind Strategien doch nicht selten auch das Ergebnis langwieriger Aushandlungsprozesse, die sich in einem historischen Prozess aus der Organisation herausgeschält haben. Da mit einer strategischen Neuausrichtung meist eine Reallokation der Macht verbunden ist, können Strategien in der Regel auch nicht reibungslos angepasst werden.

Die Strategieprozessforschung arbeitet somit deutlich jene emergente Komponente von Strategien heraus, die auch die Entstehung strategischer Pfade

182 Vgl. Chandler (1962); Hall \& Saias (1980).

183 Vgl. zur rational-entscheidungsorientierten Perspektive Ansoff (1970); Christensen et al. (1982); zu einer kritischen Diskussion Schreyögg (1984).

184 Vgl. Ackermann (2003: 228). Mittlerweile hat nach Schreyögg (1999: 388) diese Sichtweise auf organisational bedingte strategische Prozesse die Dominanz gegenüber den präskriptiven Planungsmodellen gewonnen.

185 Vgl. zur strategischen Prozessforschung Pettigrew (1973); Eisenhardt \& Bourgeois (1988); Mintzberg \& Waters (1985). Zu den verhaltenswissenschaftlichen Grundlagen Simon (1957); und Cyert \& March (1963).

186 Vgl. zur Bedeutung mentaler Schemata Miller et al. (1982); Prahalad \& Bettis (1986); Weick (1988). Zur Bedeutung politischer Prozesse und Machtstrukturen vgl. Mintzberg (1983); Eisenhardt \& Bourgeois (1988); Pettigrew (1973). 
prägt. ${ }^{187}$ Strategien besitzen eine Geschichte und diese Geschichte schränkt den strategischen Gestaltungsraum ein. Auf dieser für sich bereits gewinnbringenden, von der Forschung zu Unternehmenskrisen aber vielfach vernachlässigten Einsicht gründet die Theorie der organisationalen Pfadtheorie in ihrer Analyse organisationaler Phänomene. ${ }^{188}$ Darüber hinaus erfasst sie aber auch die Subdimension historischer Sequenzialität. Denn im Zuge der Genese strategischer Pfade wird deutlich: »[W]hen things happen within a sequence affects how they happen ${ }^{189}$

Das Charakteristikum der Historizität ist allerdings ebenso wenig ein Spezifikum wie eine hinreichende Beschreibung pfadabhängiger Prozesse. Wie oben bereits angemerkt wurde, kann bei näherer Betrachtung beinahe jedes soziale Ereignis auf ein anderes, zeitlich zurückliegendes Ereignis zurückgeführt werden. Die ganze Welt ist historisch und beinahe jedes Phänomen ist Teil eines historischen Entwicklungswegs, sodass sich das Problem des infiniten Regresses stellt. Wäre Historizität das einzige Kriterium für Pfadabhängigkeit, so wären in der Konsequenz beinahe alle sozialen Prozesse pfadabhängig. ${ }^{190}$

Pfadabhängige Prozesse sind jedoch mehr als einfach "nur " historisch geprägte Prozesse: Historizität ist notwendige, aber keineswegs hinreichende Eigenschaft, soll ein Prozess als pfadabhängig beschrieben werden. Ein pfadabhängiger Prozess beruht im hier gewählten engen Verständnis nicht lediglich auf "past-dependence«, sondern vor allem auf der spezifischen Entwicklungsdynamik der selbstverstärkenden Mechanismen. ${ }^{191}$

Ausgelöst werden diese Mechanismen von einer bestimmten Entscheidung, einer Handlung oder einem Ereignis. Diese Stimuli lösen eine spezifische Reaktion aus und können ex post als »critical juncture« der späteren strategischen Entwicklung verstanden werden. ${ }^{192}$

Die klassische ökonomische Pfadtheorie konzeptualisierte solche Auslöser als unbewusste, meist nicht weiter beachtete »small events « im Sinne von Arthur (1994), die aber weitreichende Konsequenzen haben können. ${ }^{193}$ Die organisationale Pfadforschung weist allerdings zu Recht darauf hin, dass im organisationalen Rahmen von Hierarchie und Macht solche Entscheidungen oder

187 Vgl. Mintzberg \& Waters (1985); Schreyögg et al. (2003: 8f.).

188 Vgl. Teece et al. (1997: 533); Sydow et al. (2009: 690).

189 Vgl. Mahoney (2000: 511); Pierson (2000: 253): „Earlier parts of a sequence matter much more than later parts, an event that happens stoo late may have no effect, although it might have ben of great consequence if the timing had been different."

190 Dies wäre die Konsequenz der von Teece et al. (1997) vorgeschlagenen weiten Begriffsfassung.

191 Vgl. Antonelli (1997: 662).

192 Vgl. Collier \& Collier (1991); auch Capoccia \& Kelemen (2007).

193 Sydow et al. (2009: 693) verweisen hier auf die Nähe zur Komplexitäts- und Chaostheorie und die populäre Metapher des »butterfly effects". 
Handlungen häufig keineswegs so klein und unbewusst sind, wie von der klassischen Pfadtheorie für atomisierte Marktteilnehmer herausgearbeitet. Im organisationalen Kontext können auch bewusste strategische Entscheidungen oder »big events « im Sinne von Sydow et al. (2009) kritische Weichenstellungen darstellen. ${ }^{194}$ Dennoch wird das "critical juncture« von den Beteiligten meist nicht als einschneidendes Erlebnis oder schwerwiegende Entscheidung wahrgenommen, sondern als eine von vielen Entscheidungssequenzen im Zuge der organisationalen Entwicklung. Selbst für einen außenstehenden Beobachter ist das »critical juncture« analytisch meist nur ex post als solches identifizierbar.

Von entscheidender Bedeutung für das Verständnis von Pfadabhängigkeit ist aber, dass die Konsequenzen auch intentionaler Entscheidungen und Handlungen für die organisationalen Akteure letztlich unbeabsichtigt und weitgehend unvorhersehbar sind. Auch bei größeren und intentionalen Entscheidungen kann in dieser frühen Phase der Pfadformation noch keine eindeutige, linear determinierte Kausalkette vorliegen. Die schlussendliche Gestalt des in der Folge emergierenden Pfades ist noch weitgehend unbekannt, denn sie ergibt sich nicht aus einer einzelnen Entscheidung, sondern erwächst während der Phase der Pfadformation aus dem Prozess und dessen Dynamik. ${ }^{195}$

\subsubsection{Phase II: »Pfadformation« - das Wirken selbstverstärkender Mechanismen} In der zweiten Phase der Pfadgenese, der Phase der Pfadformation, beginnt sich der durch das "critical juncture« ausgelöste Mechanismus in einer spiralförmigen Dynamik nachhaltig selbst zu verstärken und gewinnt mehr und mehr an Kraft. Im Zuge dieser Dynamik entwickelt er eine Sogwirkung, die immer stärker die Reproduktion des verbundenen strategischen Musters und die Verfolgung des eingeschlagenen strategischen Entwicklungsweges fördert.

"At the heart of organizational path dependence: self-reinforcing mechanisms.»

(Sydow et al., 2009)

Als das Kernkonzept der organisationalen Pfadtheorie soll das Konstrukt der selbstverstärkenden Mechanismen im Folgenden kurz in seiner Wirkweise abgegrenzt und anhand einiger selbstverstärkender Effekte veranschaulicht werden. $^{196}$

194 Vgl. Sydow et al. (2009: 693). Vergleiche zum Beispiel die Darstellung der vertraglichen Vereinbarung von JVC und anderen Herstellern zur Etablierung des VHS-Standards in Konkurrenz zu Beta C und Video 2000 bei Cusumano et al. (1992) sowie den anschließenden pfadabhängigen Prozess.

195 Vgl. Sydow et al. (2009: 696). Diese unbekannte Gestalt ist auch ein wesentlicher Unterschied zu den unten darzustellenden Imprints bei Stinchcombe (1965), die in ihrer Form bereits erkennbar sind und in eben dieser Form von Anfang an auf den Prozess einwirken.

196 Vgl. Sydow et al. (2009: 698); auch Pierson (2004); Beyer (2010); Vergne \& Durand (2010). Für eine sehr umfängliche Übersicht zu selbstverstärkenden Mechanismen im Rahmen 


\section{Selbstverstärkende Mechanismen als spezifische kausale Verknüpfung}

Im Allgemeinen beschreibt ein Mechanismus das Zusammenwirken mehrerer miteinander verbundener Elemente. Dieses Zusammenspiel ist nicht singulär, sondern kann wiederholt beobachtet werden. Mechanismen sind »wiederkehrende Prozesse, die bestimmte Ausgangsbedingungen mit einem bestimmten Ergebnis verknüpfen $«{ }^{197}$ Ihnen liegt somit eine Form von Kausalität zugrunde. Dabei verweist der Mechanismus im Gegensatz zur Beobachtung statistischer Häufigkeiten oder Korrelationen jedoch nicht nur darauf, dass eine Verbindung zwischen verschiedenen Elementen besteht, sondern auch, wie diese Elemente miteinander verbunden sind. ${ }^{198}$

Ein entsprechender kausaler Zusammenhang kann linear, aber auch zirkulärrekursiv oder zirkulär-verstärkend sein. ${ }^{199}$ Dies bedeutet, dass das Element $\mathrm{X}$ linear zu Element $Y$ führen kann, ohne dass es zu einer Rückwirkung auf $X$ kommt. Element $\mathrm{Y}$ kann aber auch auf das es auslösende Element $\mathrm{X}$ zurückwirken, sodass ein zirkulärer Kreislauf entsteht. Führt eine solche Rückkopplung nun sogar zu einer Verstärkung des Elements $X$ und diese Verstärkung im Fortgang wiederum zu einer Verstärkung von $Y$, so kann von einem sich zirkulär-verstärkenden Mechanismus im Sinne einer Spiralwirkung gesprochen werden. In Bezug auf Entscheidungen steigt in diesem Sinne die Wahrscheinlichkeit, dass eine Entscheidung in tlgetroffen wird, wenn in to bereits die gleiche oder eine mit ihr verbundene Entscheidung getroffen wurde. ${ }^{200}$

Solche Fälle positiver Rückkopplungen werden in der Literatur als »selfreinforcement (Arthur, 1994), "vicious circles" (Masuch, 1985), »mechanisms of reproduction« (Collier \& Collier, 1991) oder »increasing returns« (Pierson, 2000) bezeichnet. ${ }^{201}$

Angesichts der Breite des Mechanismenbegriffs, unter den im Vergleich zu

empirischer Beiträge der technologischen, geografischen und organisationalen Pfadforschung vgl. Dobusch \& Schüßler (2013: 617ff.).

197 Vgl. Hedström \& Ylikoski (2010: 207); auch Hedström \& Swedberg (1998).

198 Vgl. Mayntz (2005: 208); auch Hedström \& Swedberg (1998); Hedström \& Ylikoski (2010).

199 Vgl. Holtmann (2008: 37f.); Mayntz (2005: 209).

200 Vgl. allgemeiner Ackermann (2003: 230): »[...] wenn die Zunahme einer bestimmten Variable zu einer weiteren Zunahme dieser Variablen führt.«

201 Vgl. Arthur (1994); Collier \& Collier (1991); Masuch (1985); Pierson (2000). Schon Arthur (1994: 112) stellt heraus: »Self-reinforcement goes under different labels [...]: increasing returns; cumulative causation; deviation-amplifying mutual causal processes; virtuous and vicious circles; threshold effects; and nonconvexity.« Vgl. hierzu auch Dobusch \& Kapeller (2013: 10). Die meisten Begriffe, insbesondere "positive Rückkopplung« und »Selbstverstärkung« können nach Dobusch \& Schüßler (2013:618) als Synonyme verstanden werden. Abgegrenzt werden müssen jedoch die spezifischeren »Increasing Returns«. Denn selbstverstärkende Effekte können, aber müssen nicht auf »Increasing Returns« basieren. Eine positive Rückkopplung kann auch bei konstanten oder abnehmenden Skalenerträgen stattfinden. Vgl. Mahoney (2000) und Pierson (2000); beziehungsweise Campbell-Kelly (2001), der diese Abgrenzung leider nicht vornimmt. 


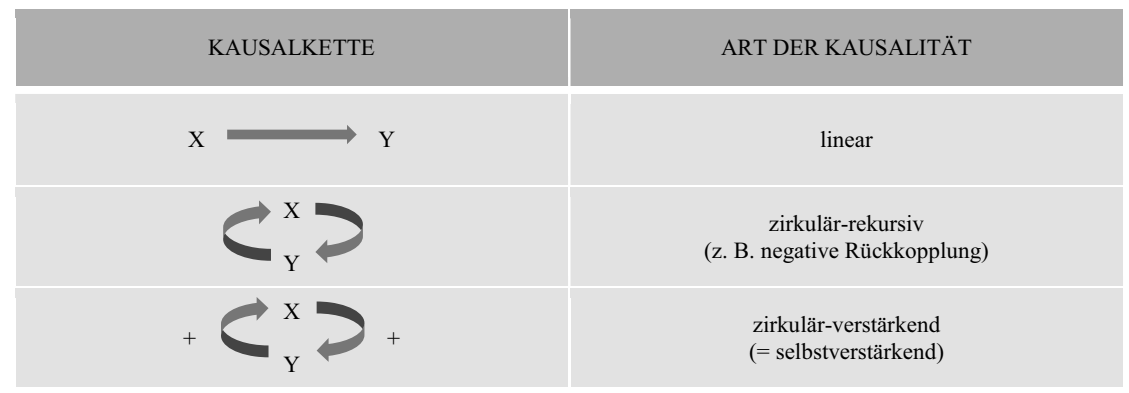

Abbildung 5: Formen kausaler Verknüpfungen von Mechanismen

(Quelle: Eigene Darstellung; vgl. Holtmann [2008: 38]).

»increasing returns « zum Beispiel auch sozio-emotionale Dynamiken subsumiert werden können, soll mit Schreyögg et al. (2003) jedoch bewusst weiterhin von selbstverstärkenden Mechanismen gesprochen werden. ${ }^{202}$

\section{Klassische ökonomische, nutzenorientierte Mechanismen}

Die zentrale Rolle selbstverstärkender Mechanismen für das Entstehen von Persistenz und Pfadabhängigkeit wurde anhand des oben skizzierten Beispiels des QWERTY-Tastaturlayouts zunächst von David (1985) herausgestellt. Hieran anknüpfend, wurde das Prinzip der Selbstverstärkung von Arthur (1989) weiter ausgearbeitet und mathematisch modelliert. In diesem Zuge wurde die Idee der Selbstverstärkung in utilitaristischer Perspektive auf »increasing returns« spezifiziert. Diese positiven Feedback-Effekte wurden in der Folge insbesondere von Arthur (1994) als entscheidende Eigenschaft der Selbstverstärkung hervorgehoben. ${ }^{203}$

Im Kontext von Technologien werden verschiedene Ursachenbereiche für die Entstehung von »increasing returns" genannt: neben den klassischen ökonomischen Konzepten der Skalenerträge und Erfahrungskurveneffekte zum Beispiel auch die insbesondere für die Verbreitung von Technologien bedeutsamen direkten und indirekten Netzexternalitäten. ${ }^{204}$

Direkte Netzexternalitäten liegen zum Beispiel dann vor, wenn der Nutzen einer Technologie direkt von deren Verbreitung abhängt. ${ }^{205}$ So werden bestimmte Technologien, zum Beispiel eine Social-Media-Plattform wie Facebook, umso attraktiver, je mehr Nutzer sie verwenden. Der Nutzen für einen einzigen Nutzer geht gegen Null, aber je mehr Nutzer diese Plattform benutzen, desto attraktiver wird sie wiederum für neue Nutzer, die sich daraufhin ebenfalls für

202 Vgl. Schreyögg et al. (2003: 269ff.).

203 Vgl. Arthur (1994); Arthur (1989); David (1985); David (1986).

204 Vgl. Ackermann (2003: 235); Arthur (1994: 116); Schreyögg et al. (2003: 262).

205 Vgl. David (1985: 334f.); Schreyögg et al. (2003: 262) und vor allem Katz \& Shapiro (1985). 
die weitverbreitete Plattform entscheiden. Somit liegt eine positive Rückkopplung vor: Die Zunahme der Variable Nutzer führt zu einer Zunahme der Variable Nutzer.

Indirekte Netzexternalitäten mit positiver Rückkopplung liegen hingegen zum Beispiel dann vor, wenn den Nutzern dieser Social-Media-Plattform auch Nutzen aus verwandten Produkten oder Dienstleistungen erwächst, deren Nutzen wiederum mit der Verbreitung der Plattform ansteigt. ${ }^{206}$

An diese Vorstellung selbstverstärkender Effekte knüpft die institutionelle Pfadforschung an und überträgt sie auf den institutionellen Kontext. Dabei weist sie insbesondere auf die Bedeutung von Koordinations- und Komplementaritätseffekten hin, welche zu fortschreitenden Kostenvorteilen bei den Interaktionspartnern und somit in der Folge zu deren Verstärkung beitragen. ${ }^{207}$

Die Koordinationseffekte wurden ursprünglich von North (1990) in den Pfaddiskurs eingebracht. ${ }^{208}$ Sie basieren auf den Vorteilen eines regelgeleiteten, aufeinander abgestimmten Verhaltens: Je mehr Akteure sich anhand einer bestimmten Institution, z. B. einer bestimmten Regel koordinieren, desto effizienter und kostengünstiger ist die Interaktion zwischen den Akteuren. ${ }^{209}$ Solche selbstverstärkenden Koordinationskostenvorteile können am Beispiel des Rechtsfahrgebotes illustriert werden. Prinzipiell ist das Fahren auf der rechten Fahrbahnseite nicht vorteilhafter als das Fahren auf der linken Seite und vice versa. Nachdem aber auch nur einige wenige Autofahrer mit dem rechtsseitigen Fahren begonnen hatten, war es für die hinzukommenden Autofahrer ebenfalls sinnvoll auf der rechten Seite zu fahren. Je mehr Autofahrer diese Regel beachten, desto größer ihr Nutzen der Unsicherheitsreduktion.

Sich selbst verstärkende Komplementaritätseffekte beruhen darauf, dass solche Regeln und Institutionen meist nicht isoliert existieren, sondern in ein interdependentes Netz eingewoben sind, in welchem sie sich komplementär ergänzen. Sich selbst verstärkende Effekte können sich in diesem Rahmen dann ergeben, wenn die Befolgung einer Regel durch deren Interdependenz mit einer anderen Regel attraktiver wird. ${ }^{210}$

206 Vgl. David (1985: 334f); Liebowitz \& Margolis (1995: 2); Schreyögg et al. (2003: 262). Ein anschauliches Beispiel für einen solchen indirekten Nutzen ist die zunehmende Möglichkeit, sich bei Software-Applikationen auf mobilen Endgeräten direkt mit einem bereits eingerichteten Facebook-Profil anzumelden, anstatt für jede Anwendung eigene Profile pflegen zu müssen.

207 Vgl. Ackermann (2003: 235ff.); Mahoney (2000: 515ff.); North (1990: 94); Pierson (2000: 254).

208 Vgl. North (1990: 23). Dieser verweist auch auf das skizzierte Beispiel des Rechtsfahrgebots.

209 Vgl. Ackermann (2003: 235); North (1990: 23); Sydow et al. (2009: 699).

210 Vgl. das Beispiel »individualistischer « und »kollektivistischer» Regelsysteme bei Ackermann (2003: 240f.). Diese Komplementaritätseffekte werden auch von der institutionellen Forschung zu den »Varieties of Capitalism» beobachtet, vgl. Hall (2001). 
Die Logik dieser Koordinations- und Komplementäreffekte ist somit analog zu jener der Netzexternalitäten, bei denen der Nutzen für den einzelnen Nutzer mit der Verbreitung der Technologie ansteigt. ${ }^{211}$ Während die Etablierung technischer Standards die Kosten der technischen Interaktion reduziert, können Regeln wie das Rechtsfahrgebot als etablierte und standardisierte Lösungen der sozialen Interaktion erachtet werden, die die Kosten der Unsicherheitsbewältigung reduziert.

\section{Sich selbstverstärkende Mechanismen im organisationalen Kontext}

Sydow et al. (2009) weisen darauf hin, dass diese ökonomisch geprägte Sicht auf utilitaristisches Verhalten und Kostenvorteile im organisationalen Kontext jedoch zu kurz greift. ${ }^{212}$

Eine selbstverstärkende Dynamik im oben skizzierten kausalen Sinn liegt zum Beispiel auch emotionalen Reaktionen wie Rache, kognitiven Verzerrungen, oder von Mikropolitik geprägten politischen Prozessen zugrunde. Solche Prozesse besitzen ebenfalls ein selbstverstärkendes Moment und können auch zu sich selbstverstärkenden Entscheidungsmustern führen. Nicht zuletzt auch deshalb, weil die Akteure im organisationalen Kontext sich in ihrer Entscheidungsfindung durch etablierte Strukturen und Kulturen gegenseitig noch viel stärker beeinflussen als im Rahmen des Marktgeschehens.

Die oben skizzierten klassischen, rein auf dem individuellen Nutzenprinzip basierenden Rückkopplungseffekte bieten für solch organisationalen Phänomene allerdings keinen Platz. Die Selbstverstärkung von Konflikten erklärt sich nicht aus dem individuellen Nutzen des Konflikts für die einzelnen Parteien. Um solche organisational aber ohne Zweifel bedeutsamen Momente der Selbstverstärkung dennoch in das theoretische Gerüst der Pfadtheorie integrieren zu können, plädieren Schreyögg et al. (2003) dafür, das Prinzip der positiven Rückkopplung aus seinem engen nutzentheoretischen Korsett zu befreien und zu einem allgemeinen Prinzip der Selbstverstärkung zu erweitern. ${ }^{213}$

Sie verweisen auf drei Bereiche, die sie vor dem Hintergrund der bestehenden Literatur der Managementforschung für das Entstehen von selbstverstärkenden Mechanismen im organisationalen Kontext als besonders relevant erachten:

211 Vgl. Ackermann (2003: 236); Dobusch \& Schüßler (2013: 621). Die externen Netzwerkeffekte können entsprechend als technologischer Sonderfall der allgemeineren Koordinations- und Komplementaritätseffekte verstanden werden.

212 Vgl. Sydow et al. (2009: 694); Schreyögg et al. (2003: 269 ff.).

213 Vgl. Schreyögg et al. (2003:269). Von diesem weiteren Verständnis scharf abzugrenzen sind jedoch darüber hinausgehende Mechanismen der institutionellen Reproduktion wie zum Beispiel Macht oder Legitimation; vgl. hierzu Pierson (2000); Mahoney (2000). Zur Übersicht: Beyer (2015: 153); Dobusch \& Kapeller (2013: 11). 
sozio-emotionale Prozesse, kognitive Prozesse und selbstreferentielle Rekursionsprozesse. ${ }^{214}$

Hinsichtlich der potenziellen Selbstverstärkung sozio-emotionaler Prozesse machen Schreyögg et al. (2003) beispielsweise auf die Dynamik von Emotionen aufmerksam. In diesem Zusammenhang wäre u.a. auf die selbstverstärkende Dynamik von Konfliktprozessen oder das über die Jahre zirkulär verstärkende "emotional attachment « zu einer Strategie zu verweisen. ${ }^{215}$

In Bezug auf kognitive Prozesse verweisen Schreyögg et al. (2003) auf kognitive Muster, welche die notwendig reduzierte Wahrnehmung und Interpretation der Umwelt prägen. ${ }^{216}$ In diesem Zusammenhang können sich Schemata herausbilden, die sich im Zeitverlauf verfestigen und verstärken. Einen solchen Wirkungszusammenhang beschreibt anschaulich die »Theorie X» von McGregor (1960): Habe ein Entscheidungsträger ein Menschenbild, in dem der Mensch faul, opportunistisch und nur durch monetäre Anreize zum Arbeiten zu bewegen erscheine, dann werde er in der Konsequenz bereits die organisationale Struktur oder das Anreizsystems entsprechend an seinen aus diesem Menschenbild abgeleiteten Verhaltenserwartungen ausrichten. Das Problem liegt nun darin, dass - selbst wenn das Bild nicht mit der Realität übereinstimmt übermäßige Kontrolle und autoritäres Auftreten im gegenwärtigen gesellschaftlichen Rahmen in der Folge wohl tatsächlich häufig zu Passivität und Opportunismus führen. Entsprechende Beobachtungen werden die Entscheidungsträger dann wiederum in ihrer negativen »Theorie X« über die Menschen bestätigen und in der Konsequenz zu einer weiteren Verschärfung der Kontrollmaßnahmen führen. Daraufhin fühlen sich die Mitarbeiter zunehmend drangsaliert und werden aufgrund der abnehmenden Motivation immer unproduktiver. Ein »Teufelskreis« adaptiver Erwartungen bildet sich heraus. ${ }^{217}$

Hinsichtlich selbstreferentieller Rekursionsprozesse finden sich insbesondere in der Managementforschung viele Beispiele, die auf eine Verstärkung und daraus resultierende Verfestigung sich einmal herausgebildeter Praktiken oder Strategien verweisen. ${ }^{218}$ So beschreibt unter anderem Leonard-Barton (1992) die Tendenz von Organisationen, ihre erfolgreichen Strategien und Handlungsmuster zu konservieren. Er warnt vor der Gefahr, dass aus "core competences"

214 Vgl. Schreyögg et al. (2003: 270).

215 Vgl. Sydow et al. (2009: 694); zum Begriff »emotional attachment« Burgelman (1994: 41 ff.).

216 Vgl. Schreyögg et al. (2003: 270); Sydow et al. (2009: 700 f.).

217 Vgl. McGregor (1960); Schreyögg (2006: 226ff.). Der selbstverstärkende Effekt solch adaptiver Erwartungen basiert auf dem grundlegenden Gedanken, dass viele Menschen eine bestimmte Erwartungshaltung besitzen, und ihr Verhalten vorwegnehmend bereits entsprechend anpassen. Dies kann zu dem vielbeschriebenen Phänomen der »self-fulfilling prophecy« führen.

218 Auch wenn diese Beiträge das Konzept der Pfadabhängigkeit nicht immer direkt referenzieren; vgl. Schreyögg et al. (2003: 270). 
im Verlauf der Zeit »core rigidities« werden können. ${ }^{219}$ Miller (1992) nennt das Ikarus-Paradox, nach dem das Verfolgen ehemals erfolgreicher Strategien ab einem gewissen Punkt zum Niedergang des Unternehmens führt, und die »architectur of simplicity« (Miller, 1993), innerhalb derer ein Set erfolgreicher Handlungsmuster immer weiter ausgebaut, strukturell und kognitiv verstärkt, in diesem Zuge aber auch übermäßig simplifiziert wird. ${ }^{220}$ Auch Burgelman (2002) macht in seinen Studien zur Entwicklung des Chipherstellers Intel auf die Bedeutung von positiv rückgekoppelten strategischen Mustern aufmerksam. ${ }^{221}$

Neben solchen Mechanismen darf die Dimension der Ressourcen für die fortwährende Reproduktion nicht vernachlässigt werden. Nicht selten werden Entscheidungen auch aufgrund bereits getätigter Investitionen und entsprechender "sunk costs" reproduziert. Von Pfadabhängigkeit im engeren Sinne kann in diesem Zusammenhang allerdings nur dann die Rede sein, wenn auch den Entscheidungsprozessen, die der Investition zugrunde liegen, ein selbstverstärkendes Moment innewohnte. ${ }^{222}$

Im Modell der Pfadgenese nach Sydow et al. (2009) führen solche selbstverstärkenden Mechanismen in der zweiten Phase der Pfadformation dazu, dass sich nach und nach ein strategisches Muster oder eine "organisationale Logik" zu entwickeln beginnt, welche von den selbstverstärkenden Effekten direkt präferiert oder getragen wird, und die mit der Zeit immer stärker zur Reproduktion tendiert. ${ }^{223}$

Während zu Beginn dieser Pfadgenese allerdings selbst kleinste Ereignisse oder vom Muster abweichende Entscheidungen dem Prozess noch eine andere Richtung geben oder ihn auch beenden können, wird ein Abweichen vom strategischen Entwicklungsweg desto schwieriger, je länger die selbstverstärkenden Mechanismen wirken und je häufiger das strategische Muster reproduziert wird. So beginnt das Regime der selbstverstärkenden Mechanismen die strategische Entwicklung immer stärker zu bestimmen und das etablierte dominante Muster aus »action and reflection « verfestigt sich fortwährend. ${ }^{224} \mathrm{Nach}$

219 Vgl. Leonard-Barton (1992). Ähnlich auch das Phänomen des »success breeds failure«; vgl. Starbuck et al. (1978); Tushman \& Anderson (1986).

220 Vgl. Miller (1992); Miller (1993: 116): »It turns into a monolithic, narrowly focused version of its former self, converting a formula for success into a path toward failure."

221 Vgl. Burgelman (2002:326):»A positive feedback process that increasingly ties the previous success of a company's strategy to its existing product-market environment, thereby making it difficult to change strategic direction."

222 Vgl. Schreyögg et al. (2003: 270); Arkes \& Blumer (1985); Williamson (1975). Zudem kann die Ressourcendimension natürlich die Determination des Entwicklungswegs verstärken. In solchen Fällen sind die »sunk costs« allerdings eher Ergebnis und Manifestation der Selbstverstärkung, als eigentliche Ursache der strategischen Persistenz.

223 Prahalad \& Bettis (1986); vgl. auch Koch (2011: 340); Sydow et al. (2009: 693f.).

224 Koch (2011: 340); vgl. Schreyögg et al. (2003: 272). 
und nach zeichnet sich der strategische Pfad ab und wird dominanter. Die strategischen Optionen werden weniger, der strategische Handlungsspielraum für die Akteure wird kleiner. Ein Abweichen vom bestimmenden Muster ist zwar immer noch möglich, gestaltet sich aber im Verlauf des Prozesses zunehmend schwieriger, bis hin zu einem Zustand, in dem der Prozess beinahe vollkommen determiniert ist: dem Lock-in.

\subsubsection{Phase III: Lock-in und Pfadabhängigkeit}

In dieser dritten und letzten Phase hat sich der Pfad nun vollends herausgebildet und befindet sich in seinem zunächst finalen Stadium. ${ }^{225}$ Die selbstverstärkenden Effekte haben zur Durchsetzung eines spezifischen strategischen Musters geführt, welches den strategischen Handlungsspielraum im Fortgang beinahe quasi-deterministisch bestimmt. Nahezu jedweder strategischer Handlungsspielraum ist verloren gegangen. Selbst wenn neue, attraktivere Entscheidungsoder Handlungsmöglichkeiten auftauchen, ist es der Organisation aufgrund von hohen "switching costs« oder »sunk costs« sowie der im Verlauf der Zeit immer tieferen Verankerung des Musters in der Organisation nicht möglich diese für sie besseren Alternativen zu ergreifen. ${ }^{226}$ Die Organisation ist auf einem bestimmten Pfad "gefangen «; sie ist pfadabhängig.

Allerdings darf für den organisationalen Rahmen keine direkte Analogie zur Pfadabhängigkeit im klassischen technologischen Kontext angenommen werden, denn während in Bezug auf Technologien, wie das Beispiel des QWERTYTastaturlayouts zeigt, tatsächlich lediglich eine einzige Wahlmöglichkeit verbleiben kann, ist ein solch vollständiger Determinismus im sozialen Kontext der Organisation nur schwer vorstellbar. Selbst wenn ressourcenbedingt nur noch sehr wenige Handlungsoptionen bestehen, existieren doch immer noch unterscheidbare Alternativen. Daher muss ein Lock-in im organisationalen Kontext eher als ein Korridor mit sehr stark eingeschränkten Handlungsmöglichkeiten verstanden werden. ${ }^{27}$ Strategische Pfade bringen eine extreme Verengung der Handlungsoptionen mit sich, aber niemals vollständige Vorbestimmtheit.

225 Vgl. Sydow et al. (2009: 693ff.). In Bezug auf Institutionen bezeichnet zum Beispiel Ackermann (2003: 225) den Lock-in als einen Zustand, in dem Regeln »ein großes Beharrungsvermögen aufweisen, so dass der spontane Prozess der Regelevolution den `Fehler nicht selbst korrigieren kann."

226 Dieser Lock-in kann je nach Art des Pfades und der selbstverstärkenden Mechanismen kognitiver oder ressourcenbedingter Natur sein. Sydow et al. (2009: 694) verweisen darauf, dass ein Lock-in meist eine Kombination aus der kognitiven, der normativen und der Ressourcendimension darstellt.

227 Vgl. Schreyögg et al. (2003: 272). Diesen eingeschränkten Handlungsspielraum soll der grau schattierte Korridor im Modell zum Ausdruck bringen, auf Grund dessen die außerhalb des Korridors liegenden Optionen nicht mehr erreichbar sind. Vgl. auch Sydow 
Wie groß der verbliebene Handlungsspielraum im Einzelfall noch ist, hängt von mehreren Faktoren ab: zum einen von dem spezifischen Muster, das sich in der Organisation entwickelt hat, zum anderen davon, wie der emergierte Pfad in die Organisation »eingebettet« ist. ${ }^{228}$ Darüber hinaus ist von Bedeutung, ob in der Phase des Lock-in die selbstverstärkenden Mechanismen noch wirksam sind. Denn es kann auch der Fall sein, dass sich die Mechanismen zum Beispiel aufgrund eines exogenen Schocks wieder abgeschwächt haben, die strategische Entwicklung aber aufgrund von entsprechend getätigten Ressourceninvestitionen und zu hoher »switching costs« trotzdem bereits vollends pfadabhängig ist.

Vor diesem Hintergrund wird deutlich, dass der Zustand der Pfadabhängigkeit im organisationalen Kontext nicht binär verstanden werden darf. Vielmehr muss er sowohl zeitlich in seiner Entstehung als auch hinsichtlich des Grades an Pfadabhängigkeit stets graduell begriffen werden. ${ }^{229}$

Im temporären Sinne geht die graduelle Genese bereits klar aus der grundlegenden prozessualen Konzeption hervor: Während zunächst noch eine Vielzahl an Wahlmöglichkeiten besteht, nimmt deren Anzahl im Verlauf des Prozesses nach und nach ab. Die Abhängigkeit der Organisation vom Pfad wird größer und ihre Entwicklung immer "pfadabhängiger".

Die graduelle Genese der Pfadabhängigkeit dürfte sich in den meisten Fällen aber auch in einer schrittweisen Ausbreitung des Pfades über verschiedene organisationale Ebenen hinweg zeigen. So mag zu Beginn des Prozesses nur eine bestimmte Gruppe von Entscheidungsträgern einer gewissen historischen Prägung oder mentalen Modellen unterliegen und entsprechend rigide Entscheidungen treffen. Diese können sich im Laufe ihrer fortwährenden Reproduktion und Selbstverstärkung dann jedoch so lange innerhalb der Organisation ausbreiten, bis im Zuge nicht mehr revidierbarer Entscheidungen schlussendlich die strategische Entwicklung des gesamten Unternehmens zunehmender Pfadabhängigkeit unterliegt.

Mit diesem graduellen Verständnis geht somit auch ein Verständnis von lokaler Pfadabhängigkeit einher. Zwar mag letztlich tatsächlich die gesamte Organisation einer pfadabhängigen Entwicklung folgen, doch ist lange Zeit eben nicht die gesamte Organisation von Imprints oder selbstverstärkenden Mechanismus betroffen, sondern beispielsweise nur eine bestimmte Gruppe von

et al. (2009: 695). Illustrative Beispiele liefern Burgelman (2008); Holtmann (2008); Koch (2011).

228 Vgl. Koch (2011: 341); Sydow et al. (2009: 695).

229 Vgl. Sydow et al. (2009); Vergne \& Durand (2010: 748); Scholle (2013: 422). Auf die unterschiedlichen Grade an Pfadabhängigkeit macht insbesondere Koch (2011: 342) aufmerksam. 
Entscheidungsträgern. Diese Vorstellung umschreibt Koch (2011) mit dem Bild des infizierten »organizational body ${ }^{230}$

Unabhängig vom spezifischen Grad der Pfadabhängigkeit ist die Verengung der Handlungsalternativen aus strategischer Sicht natürlich per se in hohem Maße problematisch. Ist eine Pluralität an Hanhandlungsoptionen doch grundsätzlich Voraussetzung für jedwedes strategisches Handeln. ${ }^{231}$

Ihre besondere Dramatik bezieht die strategische Pfadabhängigkeit jedoch aus dem Umstand der potenziellen Ineffizienz des persistenten strategischen Musters. Denn zum einen kann das nun etablierte Muster von vornherein auf einer ineffizienten Selektion beruhen, die sich aufgrund von selbstverstärkenden Mechanismen aber durchsetzte und aufrechterhalten wurde. Die QWERTYTastatur kann hier wiederum als Beispiel dienen. Im organisationalen Kontext häufiger zu beobachten ist jedoch, dass ein einstmals effizientes strategisches Muster im Zeitverlauf seinen »strategic fit« mit der Umwelt verliert, das Unternehmen aber aufgrund der selbstverstärkenden Mechanismen und des aus ihm resultierenden Lock-in nicht mehr auf diese Umweltdynamik reagieren kann, da die besseren Alternativen außerhalb des verengten Handlungskorridors liegen. ${ }^{232}$

Es ist diese aus dem Lock-in pfadabhängiger Prozess graduell erwachsende potenzielle Ineffizienz, welche die organisationale Pfadtheorie zu einer Theorie der Entstehung von Unternehmenskrisen prädestiniert. ${ }^{233}$ Reflektiert sie doch den im ersten Kapitel dieses Teils der theoretischen Heranführung skizzierten Umstand, dass Krisen nur selten auf kurzfristig auftretende, exogene Schocks zurückzuführen sind, sondern meist nach und nach aus längerfristigen Prozessen erwachsen, in denen endogene Problemkonstellationen dazu führen, dass die Unternehmen sich aufgrund von Persistenzen den Erfordernissen ihrer Umwelt nicht mehr adäquat anpassen können.

$\mathrm{Zu}$ dieser strategischen Persistenz können in der Phase des Lock-in schlussendlich auch eine ganze Reihe pfadstabilisierender Faktoren beitragen, wie der machtvolle Einfluss der Eigentümer auf den Entscheidungsprozess oder der po-

230 Koch (2011: 338).

231 Vgl. Child (1972); Prozessmodelle wie Hunger \& Wheelen (2008: 22f.).

232 Thematisiert man die Ineffizienz eines bestimmten strategischen Musters, so stellt sich natürlich immer die Frage nach dem Referenzpunkt in Vergleich zu dem das Muster als ineffizient beurteilt werden kann. Diese Frage kann aber wohl nur im Kontext des jeweiligen Pfades sinnvoll beantwortet werden.

233 Vgl. Beiträge zu organisationaler Pfadabhängigkeit als Krisenursache wie Holtmann (2008); Rindfleisch (2011); Tobias (2012); die aber in diesem weiten Forschungsfeld mit dem empirischen Nachweis von organisationaler Pfadabhängigkeit und der Wirkung der Insolenz auf den Bruch von Pfaden gänzlich andere Forschungsfragen und -ziele als die vorliegende Arbeit verfolgen. 
litische Rahmen der Organisation. ${ }^{234}$ Solche einseitig linear, nicht selbstverstärkend wirkenden Stabilitätsfaktoren sind zwar nicht ursächlich für das Entstehen der Pfadabhängigkeit, können aber in der Phase des Lock-in durchaus stabilisierend auf den strategischen Pfad einwirken.

Der Hinweis auf solche pfadstabilisierenden Faktoren führt ebenso wie die Historizität des Entscheidungsprozesses zur Frage nach der Bedeutung von Kontextvariablen für die Genese und Dynamik strategischer Pfade. Bevor diese für die vorliegende Arbeit äußerst bedeutsame Frage diskutiert wird, sei jedoch zunächst noch definitorisch festgehalten, was vor dem Hintergrund des nun entwickelten pfadtheoretischen Verständnisses nachfolgend unter einem organisationalen und strategischen Pfad verstanden wird.

\subsubsection{Das definitorische Verständnis strategischer Pfade in der Organisation}

In den obigen Abschnitten wurde zunächst die Entwicklung der Pfadtheorie von ihren klassischen ökonomischen Wurzeln über die Institutionenforschung bis zur Anwendung im organisationalen Kontext skizziert. Anschließend wurden die drei pfadkonstituierenden Kernkonzepte Historizität, selbstverstärkenden Mechanismen und Lock-in im organisationalen Kontext diskutiert. Auf diesem pfadtheoretischen Verständnis aufbauend, können organisationale Pfade nun im engeren pfadtheoretischen Sinne zusammenfassend definiert werden als

$»[\ldots]$ process that (1) is triggered by a critical event leading to a critical juncture; (2) is governed by a regime of positive, self-reinforcing feedback constituting a specific pattern of social practices, which gains more and more predominance against alternatives and (3) leads, at least potentially, into an organizational lock-in, understood as a corridor of limited scope of action that is strategically inefficient."

(Sydow et al., 2009) ${ }^{235}$

Diese Definition organisationaler Pfade kann und wird in der organisationalen Pfadforschung sowohl auf individuell-psychologische Pfade von Managern,

234 Vgl. vor allem Holtmann (2008: 145f.).

235 Vgl. Sydow et al. (2009: 704). Während in vielen anderen Forschungsfeldern häufig die Vielzahl und Widersprüchlichkeit der Definitionen beklagt wird, besitzt in Bezug auf die Definition von Pfadabhängigkeit die folgende Aussage von Pierson (2000: 252) noch immer Gültigkeit: »Analysts are increasingly inclined to invoke the concept of path dependence, but clear definitions are rare." Insbesondere mangelt es an präzisen Definitionen im hier eingeführten engen pfadtheoretischen Verständnis. Definitionsangebote im weiten, diffusen Sinn gibt es im Vergleich hierzu mehrere. Zum Beispiel Sewell (1996: 262f.): »[T]hat what happened at an earlier point in time will affect the possible outcomes of a sequence of events occurring at a later point in time." 
einzelne Organisationen, als auch auf die pfadabhängige Entwicklung ganzer Branchen angewandt. ${ }^{236}$

Vorliegende Arbeit fokussiert spezifisch strategische Entscheidungsprozesse im organisationalen Rahmen und untersucht die aus diesen Entscheidungsprozessen resultierenden strategischen Pfade. Vor dem Hintergrund dieses Fokus der vorliegenden Arbeit auf pfadbedingte strategische Persistenzen können strategische Pfade in Anlehnung an Koch (2011) noch spezifischer definiert werden:

Strategische Pfade sind aus einer spezifischen prozessualen Dynamik resultierende strategische Muster (Phase II und III) im Sinne von "patterns of action and reflection «; welche durch selbstverstärkende Mechanismen konstituiert (Phase II) und / oder aufrechterhalten werden (Phase III). Diese wurden ursprünglich von einem »small« oder »big event « oder einer Entscheidung ausgelöst, und führen zu einem erheblichen Verlust an strategischer Wahlmöglichkeit. ${ }^{237}$

(Definitorisches Verständnis strategischer Pfade)

Strategischen Pfaden liegt nach diesem Verständnis somit jene Prozessualität zugrunde, die als charakteristisch für die Entstehung der meisten Unternehmenskrisen zu betrachten ist, aber von der Managementforschung zu Unternehmenskrisen häufig vernachlässigt wird.

Der Begriff der Pfadabhängigkeit zielt hingegen sowohl auf die sich entwickelnde Eigenschaft solcher Prozesse, als auch auf deren schlussendliches Resultat im Sinne des Lock-in ab.

\subsubsection{Theoretische Abgrenzung der organisationalen Pfadtheorie}

Auf der erarbeiteten theoretischen Grundlage kann die pfadtheoretische Perspektive nun klar von anderen Theorien organisationaler Trägheit und strategischer Persistenz abgegrenzt werden. Dies dient sowohl dem Verständnis für den Wesenskern der Pfadtheorie, als auch der Verdeutlichung der Vorteilhaftigkeit der Pfadtheorie für die Untersuchung der Forschungsfrage.

Die Kontrastierung erfolgt anhand von drei weit verbreiteten Konzepten der Organisations- und Managementforschung, die eng mit der Pfadtheorie verwandt sind und auf den ersten Blick recht ähnliche Phänomene beschreiben: dem Konzept des »imprinting " (Stinchcombe, 1965), des »escalating commitment $\left(\right.$ Staw, 1976) und der »structural inertia» (Hannan \& Freeman, 1984). ${ }^{238}$

236 Vgl. zur organisationalen Pfadabhängigkeit auf dem individuellen Level Roedenbeck (2011); auf dem Level einzelner Organisationen Tobias (2012); Kunow (2013). Zur Pfadabhängigkeit von Branchen Scholle (2013); Schüßler (2008).

237 Vgl. Koch (2011: 341).

238 Vgl. Stinchcombe (1965); Staw (1976); Hannan \& Freeman (1984). Neben den hier skizzierten Konzepten wären zum Beispiel noch das Konzept des Organisatorischen Konser- 


\section{»Imprinting«}

Das Konzept des Imprinting scheint der Vorstellung von Pfadabhängigkeit zunächst relativ nahe zu kommen. ${ }^{239}$ Aus einer organisational-biografischen Perspektive verweist es auf die Bedeutung früher Entscheidungen, die als »Imprints« die spätere Entwicklung der Organisation prägen. So definieren Marquis \& Tilcsik (2013) Imprinting als

»a process whereby, during a brief period of susceptibility, a focal entity develops characteristics that reflect prominent features of the environment, and these characteristics continue to persist despite significant environmental changes in subsequent periods."

(Marquis \& Tilcsik, 2013) 240 $^{24}$

Aus dieser Perspektive wird vor allem die Phase der Gründung der Organisation fokussiert, in der grundlegende Entscheidungen zu den Eigentümerstrukturen, der Governance und anderen strategischen Rahmensetzungen getroffen werden. ${ }^{241}$ Der Imprinting-Ansatz betont hier vor allem die Bedeutung von Einflüssen der organisationalen Umwelt. Er thematisiert zudem die Relevanz individueller kognitiver Schemata und Kompetenzen wichtiger Akteure wie des Gründers, die in frühen Phasen der organisationalen Entwicklung eine stark prägende und nachhaltige Wirkkraft besitzen können. ${ }^{242}$

Das Konzept des Imprinting stellt somit ebenso wie die Pfadtheorie die Historizität von Entwicklungs- und Entscheidungsprozessen in das Zentrum der Betrachtungen. Der unterstellten Wirkkraft der Imprints liegt jedoch eine gänzlich andere Logik zugrunde. ${ }^{243}$ Imprints, wie zum Beispiel die Wahl eines

vatismus nach Kieser \& Hegele-Raih (1998); oder die Forschung zum Widerstand gegen Änderungen von Lewin (1947) zu nennen.

239 Vgl. zu dieser theoretischen Perspektive Stinchcombe (1965); auch Boeker (1989); Marquis \& Tilcsik (2013).

240 Vgl. zu dieser Definition Marquis \& Tilcsik (2013: 198ff.).

241 Vgl. Kimberly (1987: 223ff.), der vier Kategorien von Grundsatzentscheidungen differenziert: "governance decisions», »domain decisions», "expertise decisions" und »design decisions«. Solche Entscheidungen können auch zu einem späteren Zeitpunkt getroffen werden, maßgeblich ist für die Klassifikation als »Imprint«, dass sie die weitere Entwicklung des Unternehmens entscheidend prägen und zukünftigen Handlungsspielraum einschränken.

242 Vgl. Marquis \& Tilcsik (2013: 200ff.); Sydow et al. (2009: 696). Zum Einfluss des Gründers: Baron et al. (1999); Boeker (1989). Neben Entscheidungen aus der Gründungsphase können auch Ereignisse und Entscheidungen zu einem späteren Zeitpunkt den Organisationsverlauf entscheidend prägen. Wichtig ist, dass diese kritischen Ereignisse auf die weitere Unternehmensentwicklung ausstrahlen und die Veränderungsvarianz einschränken.

$243 \mathrm{Vgl}$. Sydow et al. (2009: 696): »[T] he replicated pattern in the imprinting approach is readymade at the beginning; it is a specific scheme that persists and continues to influence future processes. In contrast, the gestalt of an organizational path is not clear at all in the early stage; it is an unforeseeable product of later processes, which are initially unknown.«. Zur Abgrenzung seitens der Imprinting-Forschung vgl. Marquis \& Tilcsik (2013: 203): »Im- 
bestimmten Standortes, kommen zu Beginn des Prozesses zum Tragen, wirken von diesem Zeitpunkt an umgehend und kontinuierlich in dieser gegebenen Form linear auf den Entscheidungsprozess ein und beschränken auf diese Weise den Entscheidungsspielraum. Im Gegensatz dazu ist die Gestalt des durch eine Entscheidung ausgelösten Pfades zu Beginn des Prozesses weder erkenn- noch vorhersehbar. Der resultierende Pfad ist ein Produkt dieser Entscheidung und des dynamischen Prozesses. Während der Imprinting-Ansatz die fortdauernde Prägung organisationaler Prozesse durch bestimmte frühe Entscheidungen erfasst, fokussiert die Pfadtheorie den Prozess der Pfadgenese. Im Unterschied hierzu thematisiert der Imprinting-Ansatz nicht näher, wie die durch das Imprint geprägten Entscheidungen in dynamischer Perspektive nun genau mit einander verbunden sind. ${ }^{244}$ Dort wo in der Pfadtheorie das Konzept des selbstverstärkenden Mechanismus ansetzt, verbleibt hier in vielen Beiträgen eine Art »black box».

Nichtsdestotrotz sind von Beginn an wirksame Imprints auch für pfadabhängige Prozesse von Bedeutung. Sie werden daher als historisch gegebene Rahmenbedingungen oder Restriktionen in der Phase der "historischen Vorprägung« in das Modell integriert und auch im Zuge der vorliegenden Arbeit analysiert.

\section{»Escalating Commitment«}

Angesichts des Stellenwertes der eskalierenden Dynamik im Konzept des »Escalating Commitment" scheint dieses der Pfadtheorie noch näher als der Imprinting-Ansatz $\mathrm{zu}$ sein. ${ }^{245}$ Es fokussiert Entscheidungsprozesse, in denen trotz negativer Rückmeldungen Entscheidungsträger aus verschiedenen Gründen an ihrer Entscheidung festhalten und eine bestimmte organisationale Lösung beständig wiederholen. ${ }^{246}$

Allerdings lässt sich auch hier ein entscheidender Unterschied zur Pfadtheorie ausmachen: Pfadabhängige Prozesse entfalten sich meist über einen sehr langen Zeitraum, teilweise über Jahrzehnte, und am Anfang dieser Prozesse ist weder klar, dass ein solcher Pfad entsteht, noch dass er zu Ineffizienz führen kann. Vielmehr führen die positiven Rückkopplungen im Zuge der Pfadgenese häufig sogar zu zunächst positiven Resultaten.

Im Gegensatz hierzu fokussiert der Ansatz des »Escalating Commitment»

printing as we define it is clearly distinct from path dependence in a number of ways. Unlike path dependence, imprinting involves (1) prominent environmental conditions rather than historical accidents, (2) short sensitive periods rather than long-term event chains, and

(3) the stability of stamped in features rather than the increasing dominance of a pattern."

244 Vgl. Stinchcombe (1965); zu dieser Einschätzung Sydow et al. (2009: 696).

245 Vgl. Staw (1976); auch Bazerman et al. (1984); Ross \& Staw (1993).

246 Ein Verhalten, das an die Redewendung "gutes Geld schlechtem Geld hinterherwerfen" erinnern mag. 
Entscheidungssituationen oder Prozesse von vergleichsweise kurzer Zeitdauer. In diesen Situationen ist relativ rasch offensichtlich, dass die Entscheidung negative Konsequenzen hat, aber das Entscheidungsmuster wird aufgrund der Angst vor Gesichtsverlust oder Selbst-Rechtfertigungen dennoch weiter reproduziert. ${ }^{247}$ Das Konzept des »Escalating Commitment « bezieht sich somit eher auf pathologisches Entscheidungsverhalten.

\section{»Structural inertia«}

Unter den vielen Erklärungsansätzen organisationaler und strategischer Persistenz ist das Konzept der »Structural Inertia« wohl das bekannteste. ${ }^{248}$

Grundsätzlich geht die Populationsökologie dabei davon aus, dass verschiedene Organisationen zur Herstellung eines möglichst großen »fits « mit ihrer Umwelt spezifische Möglichkeiten der Variation testen. Hat eine Organisation in diesem Zuge schlussendlich eine erfolgreiche Variation gefunden - was nicht jeder Organisation gelingt - so gilt es diese aus populationsökologischer Perspektive unbedingt dauerhaft zu sichern und vor einer weiteren Mutation zu schützen, da die Umwelt der Stakeholder aus Kunden oder Lieferanten Berechenbarkeit und Verlässlichkeit präferierten. ${ }^{249}$

Vor dem Hintergrund dieser Nachfrage nach existenziell notwendiger Stabilität beginnen Organisationen feste Strukturen aufzubauen, Routinen zu etablieren und ihre Prozesse zu standardisieren.

Im Verlauf der Zeit verfestigt sich diese Stabilität jedoch beinahe zwangsläufig zu einer Hyperstabilität, die organisationale Trägheit mit sich bringt. So bedroht das Streben nach Existenzsicherung letztlich wiederum das Überleben der Organisation. ${ }^{250}$

Diese Vorstellung der organisationalen Trägheit weist Ähnlichkeiten zum schlussendlichen Zustand der Pfadabhängigkeit auf. Allerdings ist aus evolutionstheoretischer Perspektive vor allem der intentionale Aufbau von Stabilität für das Entstehen von organisationaler Trägheit verantwortlich, während die

247 Vgl. Ross \& Staw (1993); Staw (1976: 41); auch die Kontrastierung zur Pfadtheorie bei Sydow et al. (2009: 696). Zu den Ursachen der eskalierenden Entscheidungsbindung Staw (1976: 24): »No doubt, these forms of selfjustification could both be viewed as face-saving activities, with the distinction of an internal versus external orientation on the part of the decision maker. However, while the first form of selfjustification may be based on a general human need to be consistent and correct, the second form may relate to individual desires for social approval.»

248 Vgl. grundlegend Hannan \& Freeman (1977); Hannan \& Freeman (1984).

249 Vgl. Hannan \& Freeman (1984). Für eine Übersicht zu weiteren Faktoren, die organisationale Trägheit begünstigen wie »sunk costs«, politische Prozesse, oder die Komplementarität von Techniken und Strukturen, vgl. Kieser \& Woywode (2006: 312f.).

250 Vgl. hierzu die evolutionstheoretischen Arbeiten von Welsch (2010) und Schulenburg (2009) zur Entstehung von Unternehmenskrisen. 
Pfadtheorie auf die spezifische Prozessdynamik der nicht-intentionalen, selbstverstärkenden Mechanismen abstellt. Wo die Pfadtheorie den Fokus auf eine spezielle Prozessdynamik legt, beschreibt das Konzept der »Structural Intertia« eher einen relativ weit verbreiteten Zustand, mit dem im Verlauf seiner Evolution beinahe jedes Unternehmen zu kämpfen hat. ${ }^{251}$

\section{Erklärungsbeitrag der Pfadtheorie}

Bereits dieser kurze Theorievergleich sollte offenbaren, dass die Theorie der Pfadabhängigkeit als zeit- und dynamikorientierte theoretische Perspektive im Vergleich zu anderen theoretischen Erklärungsansätzen in besonderer Weise in der Lage ist, die verschiedenen Phasen der Entstehung strategischer Persistenz prozessendogen erklären zu können. Wo andere Ansätze die Bedeutung früher Ereignisse oder den schlussendlichen Zustand der organisationalen Trägheit fokussieren, stellt die Pfadtheorie vor allem auf die Dynamik des dazwischen liegenden Prozesses ab.

In dieser dynamischen Prozessperspektive erfasst sie den prozessualen Charakter von Unternehmenskrisen und vermag somit für einen spezifischen Prozess der Krisenentstehung zu erklären, welche Ursachen unter welchen Umständen in eine Krise führen und - über das zentrale Konzept der selbstverstärkenden Mechanismen - vor allem wie dies geschieht. ${ }^{252}$ Die organisationale Pfadtheorie adressiert somit eines der oben skizzierten Defizite der Managementforschung zu Unternehmenskrisen, welche in ihrer Ahistorizität solche spezifischen Ursachen und Entwicklungsdynamiken eben häufig vernachlässigt.

So vielversprechend der Erklärungsbeitrag der Pfadtheorie für die Unternehmenskrisenforschung erscheint - auch diese theoretische Perspektive hat ihre Defizite und Forschungslücken. Hinsichtlich solcher bislang unzureichend bearbeiteter Felder sticht vor allem die Vernachlässigung der Bedeutung des organisationalen Kontexts für die Genese und Dynamik strategischer Pfade hervor. Daher soll im folgenden Abschnitt die potenzielle Bedeutung von Kontextvariablen wie Macht oder der Organisationskultur diskutiert werden.

251 Vgl. Dobusch \& Schüßler (2013: 619); Sydow et al. (2009: 697).

252 Vgl. den ersten Abschnitt dieses Kapitels zum prozessualen Verständnis von Unternehmenskrisen; zum Beispiel die Krisendefinition von Krystek (1987: 6) oder die Beiträge zum Verlauf des »organizational decline» wie Weitzel \& Jonsson (1989: 91). 


\subsubsection{Die vernachlässigte Bedeutung des Kontexts in der organisationalen Pfadforschung}

Es wurde bereits mehrfach darauf hinwiesen, dass strategische Entscheidungsund Veränderungsprozesse nicht losgelöst von ihrem organisationalen Kontext betrachtet werden können. So können die Struktur und Kultur der Organisation den strategischen Wandel ebenso begrenzen wie ermöglichen. ${ }^{253}$

Die bisherige Forschung zu strategischen Pfaden thematisierte die Bedeutung solcher organisational-kontextuellen Variablen für die Pfadgenese bislang jedoch nur am Rande.

»Regarding the available literature on path dependence in the field of economics and institutional, political, and organizational theory, it is quite unclear how context affects path dependence.«

(Koch, 2011) $)^{254}$

Koch (2011) spricht daher folgerichtig von der "unexplored role of context « im Rahmen der organisationalen Pfadtheorie. ${ }^{255}$

Aufgrund zahlreicher empirischer Indizien für die Relevanz solcher Kontextvariablen hinsichtlich der Genese und Dynamik strategischer Pfade, gilt es diesen jedoch verstärkte Aufmerksamkeit zu widmen.

Anleihen können im Zuge dieses Vorhabens insbesondere bei der soziologischen und politikwissenschaftlichen Pfadforschung genommen werden. So thematisieren insbesondere Pierson (2000) und Mahoney (2000) die Bedeutung von Kontextvariablen wie Macht, Komplexität oder Ambiguität. ${ }^{256}$

Pierson (2000) sieht Organisationen und politische Institutionen dabei aufgrund solcher organisationaler Kontextvariablen im Vergleich zum Markt als besonders anfällig für das Entstehen von Pfadabhängigkeiten. ${ }^{257} \mathrm{Da}$ die Akteure in Organisationen in einer weit weniger transparenten Umgebung operieren würden, seien sie auch nicht im gleichen Maße den korrigierenden, für Fehlentwicklungen sensiblen Kräften des Marktes ausgesetzt. Ohne die volle Wirkkraft dieses Marktdrucks könnten sich in der Folge in Organisationen Stabilitäts- und Kontinuitätstendenzen eher entfalten und verfestigen.

In dieser Argumentationslinie führt er organisationale Faktoren an, die das Entstehen von Pfadabhängigkeit im institutionellen Kontext signifikant erhö-

253 Vgl. Pettigrew (1987); Teece et al. (1997: 522); Van de Ven \& Poole (1995).

254 Vgl. Koch (2011: 341).

255 Vgl. Koch (2011: 338ff.); auch Sydow et al. (2009: 701).

256 Vgl. Mahoney (2000); Pierson (2000). In der klassischen ökonomischen Pfadtheorie Arthur (1989) wird der Kontext in Form von fixen Prämissen als gegeben vorausgesetzt, ohne dass er näher ausgeführt wird.

257 Vgl. Pierson (2000: 260); auch Mahoney (2000: 7); Sydow et al. (2009: 701). 
hen. Er nennt hier die hohe Bedeutung kollektiven, interdependenten Handelns, sowie vor allem die Macht und deren Verteilung in der Organisation.

Sydow et al. (2009) betonen ebenfalls die Bedeutung des organisationalen Kontexts für die Genese pfadabhängiger Prozesse. Sie weisen aber zu Recht darauf hin, dass Kontextvariablen wie Macht keineswegs als selbstverstärkende Mechanismen (miss-)verstanden werden dürfen. ${ }^{258}$

Entsprechend grenzt auch Ackermann (2003) Macht als Ursache für institutionelle Inflexibilität kategorisch von Pfadabhängigkeit aufgrund positiver Rückkopplungen $\mathrm{ab.}^{259}$ Für ihn ist Pfadabhängigkeit im klassischen pfadtheoretischen Sinn das nicht-intendierte Ergebnis einer Vielzahl von Handlungen. Dieser Vorstellung stellt er Institutionen als die Ergebnisse bewusster Gestaltung gegenüber, welche durch das Mittel der Macht aufrechterhalten werden. Gleichwohl gesteht er aber zu, dass in der Realität meist auch machtvolle Einflüsse und Machtstrukturen von Bedeutung für die Pfadgenese aufgrund positiver Rückkopplungen sind. Diesen Faktor möchte er zugunsten der theoretischen Stringenz allerdings nur bei der Untersuchung von Pfadabhängigkeiten im weiten, lediglich historisierenden Sinne einbeziehen. ${ }^{260}$

Einem solchen theoretischen Purismus soll hier jedoch nicht gefolgt werden. Wenn pfadbedingte Persistenz »in der Realität « tatsächlich aus einer Mischung aus nicht-intendierten und intendierten Kräften erwächst, so erscheint es geboten, kontextuelle Variablen wie Macht nicht länger aus dem theoretischen Rahmen auszuklammern. Vielmehr sollten solche Kontextvariablen empirisch und theoretisch viel stärker in die theoretische Perspektive der Pfadtheorie integriert werden. Denn zwischen dem machtvollen, rigiden Beharren eines einzelnen Akteurs auf einer strategischen Lösung und deren vollkommen ungeplanter und unkoordinierter Entstehung aus der Interaktion atomisierter Akteure heraus, bestehen theoretische Zwischenräume, in denen es das Verhältnis zwischen Macht und positiven Rückkopplungen auszuloten gilt.

Die stärkere Berücksichtigung der Kontextvariable Macht erscheint aber nicht zuletzt auch deshalb sinnvoll, weil das Pierson'sche Argument von Macht als pfadstabilisierender Variable auch umgekehrt werden kann: Im klassischen, ökonomischen pfadtheoretischen Rahmen beruht die Wirkkraft der selbstverstärkenden Effekte auf der Marktlogik der dezentralen Entscheidungsfindung

258 Vgl. Sydow et al. (2009: 701). Denn teilweise wird Macht gar als pfadtreibende Kraft betrachtet, die Teil einer positiven Rückkopplungsschleife sei und somit direkt zur Entstehung von Pfadabhängigkeit beitrage. Vgl. hierzu Pierson (2000: 259); Koch (2011: 341).

259 Ackermann (2003: 232f.).

260 Ackermann (2003: 233) bemerktAckermann (2003)Ackermann (2003): »Wer im Sinne des Pfadabhängigkeit-als-Geschichte Paradigmas erklären möchte, warum die Dinge innerhalb einer Organisation so laufen und nicht anders, der wird historische Ereignisfolgen $\mathrm{zu}$ beschreiben haben, bei denen Macht in aller Regel eine ganz entscheidende Rolle spielt.» 
gleichberechtigt machtloser Akteure. ${ }^{261}$ Diese folgen dem Prinzip der individuellen Nutzenmaximierung und halten so das Rad des selbstverstärkenden Mechanismus in Gang. Doch selbst, wenn sie dies anstreben würden, hätten sie auch nicht das nötige Einflusspotenzial auf die Entwicklung, um dieses Rad zu stoppen. Die von der klassischen Pfadtheorie beschriebenen Pfade erwachsen daher rein aus dem kumulierten Zusammenwirken einer Vielzahl individueller Entscheidungen machtloser Akteure, die so gut wie keine Möglichkeiten besitzen, die Entwicklung und das Ergebnis des Prozesses zu beeinflussen. So scheiterten im Hinblick auf das Beispiel der QWERTY-Tastatur selbst vergleichsweise machtvolle Akteure wie Apple in ihren Versuchen eine effizientere technologische Alternative am Markt einzuführen. Die Organisation hatte keine Chance, das individuell rationale Handeln der vielen Milliarden Marktteilnehmer entscheidend zu beeinflussen. Im Vergleich zu deren kumuliertem Wirken war die Organisation relativ machtlos.

Es ist diese Machtlosigkeit der atomisierten Marktteilnehmer, aufgrund derer die Komponenten der Pfadtheorie und vor allem die selbstverstärkenden Mechanismen im Kontext von Technologien und Märkten die Genese der Pfade umfassend erklären. ${ }^{262} \mathrm{Da}$ es keine akkumulierte, potenzielle Gegenmacht gibt, können diese Mechanismen sich im machtlosen Raum des Marktes immer weiter ausbreiten und ihr vollständiges Wirkungspotenzial entfalten.

Im Gegensatz hierzu existiert in Organisationen jedoch nicht zuletzt Macht aufgrund von Hierarchie. Die machtvolle Steuerung von Einzelhandlungen über Hierarchie kann geradezu als eine fundamentale Grundlage von Organisationen betrachtet werden. ${ }^{263}$ Sie trägt im Rahmen der formalen Organisation maßgeblich dazu bei, dass individuelle Handlungen aufeinander abgestimmt werden, strukturell verstetigt und zu einem großen organisationalen Ganzen verbunden werden können.

Aufgrund dieses Machtpotenzials werden Macht und die Entscheidungen machtvoller Entscheidungsträger auf der Ebene der einzelnen Organisation aber zu erklärungsbedürftigen Variablen. Machtvolle organisationale Akteure könnten durchaus - im Unterschied zu den machtlosen atomisierten Akteuren im klassischen theoretischen Rahmen des Marktes - ihre Macht potenziell nutzen, um durch einen starken Einfluss den auf die einzelne Organisation bezogenen Zirkel der Selbstverstärkung in der Phase der Pfadformation zu brechen, solange der selbstverstärkende Mechanismus noch nicht seine volle

261 Vgl. Arthur (1994); Arthur (1989); David (1985).

$262 \mathrm{Da}$ es für jeden Akteur individuell vorteilhaft ist an der erwachsenden technologischen Lösung zu partizipieren und kein Akteur die Macht besitzt, um diesen Anreizrahmen grundlegend zu ändern, setzt sich die technologische Lösung somit immer weiter durch, bis sie schließlich zum beinahe unumstößlichen de-facto Standard avanciert.

263 Vgl. Steinmann \& Schreyögg (2005: 51 ff.). 
Wirkkraft erreicht hat. Auf diesem Wege könnte Macht unter Umständen gar die Entstehung von emergierenden Pfaden in der frühen Phase der Pfadformation verhindern.

Aufgrund dieses Potenzials, entscheidenden Einfluss auf die Genese und Dynamik des strategischen Pfades zu nehmen, gebührt Macht und anderen Variablen des organisationalen Kontexts mehr Aufmerksamkeit seitens der organisationalen Pfadforschung.

"In consequence, Pierson's insights should encourage further research to explore the contextual conditions enhancing (or hindering) the unfolding of selfreinforcing mechanisms and subsequent constitution of organizational paths."

(Sydow et al., 2009) ${ }^{264}$

Von den meisten Beiträgen der organisationalen Pfadforschung wird zum Beispiel dieses Konkurrenzverhältnis zwischen selbstverstärkenden Mechanismen und machtvollen Einflüssen bislang ignoriert. ${ }^{265}$ Anstatt die Komplexität des organisationalen Kontexts in die Analyse zu integrieren, klammern die meisten Beiträge Kontextvariablen wie Macht im Sinne der Argumentation von Ackermann (2003) zugunsten der theoretischen Reinheit aus und fokussieren ausschließlich die isolierte Wirkung der selbstverstärkenden Mechanismen. ${ }^{266}$ Dieses Defizit erscheint umso bedeutsamer, wenn man bedenkt, dass die wenigen Autoren, die den organisationalen Kontext in ihre Analyse integrieren, ausdrücklich auf die Bedeutung von Macht, sowie formalen und informalen Strukturen für die Genese der in der Empirie untersuchten strategischen Pfade verweisen. $^{267}$

Diese Beiträge der organisationalen Pfadtheorie streifen am Rande der Untersuchungen nicht nur die Bedeutung des Topmanagements oder der Eigentümer für die Entstehung und potenzielle Vermeidung von Pfadabhängigkeiten. Sie verweisen auch auf die potenzielle Relevanz jenes Kontextfaktors, dessen Bedeutung für die Genese und Dynamik von strategischen Pfaden in dieser Arbeit untersucht werden soll: die Familie.

So macht Holtmann (2008) in seiner Untersuchung der Pfadabhängigkeit des Bertelsmann Buchclubs auf die Macht der Eigentümerfamilie als pfadstabilisierenden Faktor aufmerksam, während Scholle (2013) in seiner abschließenden

264 Sydow et al. (2009: 701).

265 Vgl. zu dieser Problematik grundlegend die Arbeit von Petermann (2010), der das Verhältnis von Hierarchie und positiven Rückkopplungen in Organisationen mathematisch modelliert. Vgl. auch Koch et al. (2009).

266 Vgl. Ackermann (2003).

267 So stellt Koch (2011) die Bedeutung von Macht und formalen wie informalen Strukturen zur "Inscription" der selbstverstärkenden Mechanismen und strategischen Muster in der Entwicklung von deutschen Qualitätszeitungen heraus. Vgl. auch Dietrich (1997); Scholle (2013). 
Reflektion auf die prägende Rolle und den unternehmerischen Einfluss der Gründerfamilie hinwiest. ${ }^{268}$ Doch vor allem in der empirischen Studie von Schüßler (2008) finden sich in der Untersuchung der pfadabhängigen Entwicklung der deutschen Textilindustrie in vielen Fällen Indizien für die Bedeutung familiärer Strukturen für die Entstehung strategischer Persistenzen auf der Ebene der einzelnen Unternehmen. ${ }^{269}$ Die Autorin verweist beispielsweise auf "festgefahrene Eigentümerstrukturen «, die im Zuge von Konflikten dazu führten, dass »zukunftsorientierte Entscheidungen blockiert» worden seien. Sie erwähnt zudem das »Festhalten an alten Erfolgsrezepten« und Formen von "Betriebsblindheit", die auf familiäre Traditionen zurückzuführen seien. ${ }^{270}$ Darüber hinaus macht sie auf die Relevanz »regionaler Verwurzelung«, »sozialer Bindungen « und der Reputation der Unternehmensfamilien für das Festhalten an etablierten Geschäftsmodellen und Strategien aufmerksam.

Diese Studie verweist somit am Rande auf die Bedeutung des familiären Einflusses auf jene sozio-emotionalen Dynamiken und selbstreferentiellen Rekursionsprozesse, die Schreyögg et al. (2003) im Allgemeinen als besonderes relevant für das Entstehen von organisationaler und strategischer Pfadabhängigkeit ansehen. ${ }^{271}$ Auf der anderen Seite werden solche Dynamiken aber gerade auch von der Forschung zu Familienunternehmen als bedeutsam für die organisationale Entwicklung solcher Unternehmen erachtet. ${ }^{272}$

Nicht zuletzt aufgrund dieser empirischen Hinweise der organisationalen Pfadforschung einerseits und der theoretischen Schnittmenge zwischen den Forschungsfeldern andererseits, erscheint die weitgehendere Untersuchung der Bedeutung des Kontextfaktors Familie für die Genese strategischer Pfade und daraus resultierender Unternehmenskrisen als ein fruchtbares Feld.

Zur Adressierung des kontextuellen Defizits der Unternehmenskrisenforschung und der Pfadtheorie soll sich im folgenden Kapitel daher diesem Kontextfaktor Familie angenähert werden.

268 Vgl. Holtmann (2008: 235ff.), der den stark emotional geprägten pfadstabilisierenden Einfluss der Gründerfamilie herausarbeitet: »So lange ich diesen Laden führe, so lange ich verantwortlich bin, wird an dieser Königsidee [dem Buchclub] nichts geändert«; vgl. ähnlich auch Scholle (2013: 422).

269 Vgl. Schüßler (2008: 137): »Bei dem Unternehmen in Familienhand wurde die Tradition der Eigenproduktion fortgesetzt, bei dem Unternehmen mit einem Eigentümerwechsel kam es zu einem Bruch. Schüßler (2008: 143): »In vielen Fällen spielten Familienstrukturen eine Rolle.«

270 Die Auflösung der beobachteten Konflikte sei sowohl auf Grund der Kapitalsituation, als auch der emotionalen Bindung der Eigentümer an das Unternehmen und aneinander nicht möglich; vgl. Schüßler (2008: $143 \mathrm{ff}$.).

271 Vgl. Schreyögg et al. (2003: 270).

272 Vgl. Harvey \& Evans (1994); von Schlippe \& Kellermanns (2008). 


\section{Kontext: der Einfluss des Faktors Familie auf Unternehmen}

Im ersten Kapitel der Heranführung an den theoretischen Bezugsrahmen wurde das theoretische Konstrukt des strategischen Pfades als ein spezifischer Prozess der Entstehung von Unternehmenskrisen eingeführt. Auf diesem Wege wurde eine erste theoretische Erklärung auf die Frage nach den Ursachen strategischer Persistenzen angeboten.

Zugleich wurde festgestellt, dass für ein tiefer gehendes Verständnis der Genese und Dynamik solcher Pfade der bislang weitgehend vernachlässigten Rolle des organisationalen Kontexts mehr Aufmerksamkeit gewidmet werden muss. ${ }^{273}$ Insbesondere zur gegenstandsangemessenen Rekonstruktion pfadabhängiger Entwicklungsverläufe bedarf es einer verstärkten analytischen Integration des organisationalen Rahmens, in dem sich diese Prozesse entfalten.

Vor diesem Hintergrund soll der Fokus der weiteren Arbeit nun auf einen spezifischen Kontextfaktor gerichtet werden, welcher den organisationalen Rahmen vieler Unternehmen entscheidend prägt: die Familie. Denn Hinweise der Pfadforschung legen nahe, dass die vom Faktor Familie geprägten Variablen der organisationalen Struktur und Kultur auch für die Genese und Dynamik strategischer Pfade von erheblicher Bedeutung sein könnten. ${ }^{274}$

Im Zuge der Annäherung an diesen Kontextfaktor wird im ersten Unterkapitel die empirische Bedeutung des familiären Einflusses auf Unternehmen der Behandlung dieser ökonomischen Größe durch die Managementforschung gegenübergestellt.

Im zweiten Unterkapitel wird zur Schaffung eines grundlegenden Verständnisses der Verbindung zwischen Familie und Unternehmen dieses Verhältnis dann systemtheoretisch konzipiert. Dabei werden im ersten Schritt die divergierenden Funktionslogiken der beiden Systeme Familie und Unternehmen kontrastiert, die sich im Zuge des historischen Prozesses der funktionalen Differenzierung stark auseinander entwickelt haben. Im zweiten Schritt wird dargelegt, wie diese beiden derart unterschiedlich determinierten Systeme in der Sphäre von Familienunternehmen zusammenfinden und sich gegenseitig intensiv und permanent irritieren. Diese Form der gegenseitigen Irritation wird

273 Koch (2011: 338ff.); vgl. Sydow et al. (2009). Auf diesen Umstand verweist indirekt auch die systemtheoretisch orientierte Konzeption des Managementprozesses nach Steinmann \& Schreyögg (2005) oder die Forschung zu strategischen Entscheidungs- und Veränderungsprozessen wie Pettigrew (1987); Eisenhardt \& Zbaracki (1992).

274 Vgl. die in Abschnitt B.1.3.5 skizzierten empirischen Hinweise von Holtmann (2008: $235 \mathrm{ff}$.); Scholle (2013: 422); Schüßler (2008: 134ff.). Darüber hinaus auch die Hinweise zu den Ursachenbereichen selbstverstärkender Mechanismen durch Schreyögg et al. (2003), welche auf Phänomene wie sozio-emotionale Dynamiken hinsichtlich ihrer Bedeutung für die Pfadgenese aufmerksam machen. 
dann aus systemtheoretischer Perspektive als strukturelle Kopplung konzipiert, auf deren Grundlage sich die beiden Systeme im Zeitverlauf ko-evolutionär entwickeln.

Auf diesem Verständnis aufbauend, werden im dritten Unterkapitel ausführlich jene aus der strukturellen Kopplung und Ko-Evolution der beiden Systeme erwachsenden strukturellen Merkmale des familiären Einflusses herausgearbeitet, die von der bisherigen Forschung zu Familienunternehmen als bedeutsam für strategische Entscheidungsprozesse erachtet werden. Diese Strukturmerkmale sollen dann im Fortgang der Arbeit als Indikatoren des familiären Einflusses dienen und in ihrer Wirkung auf die Genese und Dynamik strategischer Pfade in Familienunternehmen analysiert werden.

\subsection{Die Familie: fehlende Variable der Managementforschung}

\section{Vernachlässigung des Faktors Familie durch die etablierte Managementforschung} Mintzberg \& Waters (1982) untersuchten sechs Jahrzehnte der strategischen Entwicklung und Strategieformation der Steinberg Inc., einer großen kanadischen Supermarktkette im Besitz des Gründers Sam Steinberg. Im Zuge der Analyse kamen die Autoren zu dem Schluss, dass das Unternehmen insbesondere aufgrund seiner unbürokratischen, unternehmerischen Strategieentwicklung auch in Zukunft prosperieren würde. ${ }^{275}$

Doch bereits wenige Jahre später war das Unternehmen insolvent - eine unvorhergesehene Entwicklung, die Mintzberg \& Waters (1990) zu folgendem Nachwort in einer zweiten Veröffentlichung ihres ursprünglichen Artikels veranlasste:

»The world of Steinberg's changed rather significantly after Steinberg's death in 1978. [...] Control of voting shares of the company meanwhile passed to Sam Steinberg's wife and so remained concentrated. But when his three daughters exercised their rights, difficulties arose between them."

$\mathrm{Zu}$ den konkreten strategischen Problemen merken die Autoren an:

»Two factors evident in our original article help to explain Steinberg's problems: [...] [T] he first is the generous wage settlements he made, which much later came back to haunt the company. His boys were striking and that was just not acceptable to someone who saw the firm as the extension of his family [...] The second was his effort to sustain

275 Vgl. Mintzberg \& Waters (1982: 498); auch Dyer (2003: 401f.). 
absolute legal control of the firm not only during his lifetime, but beyond. Such control had to disperse at some point, and when it did there was no mechanism to hold the factions together."

(Mintzberg \& Waters, 1990) ${ }^{276}$

Dass die führenden Managementforscher von der dramatischen Entwicklung des Unternehmens überrascht worden waren, ist somit auf ihre ursprüngliche Vernachlässigung des Faktors Familie zurückzuführen. Mintzberg \& Waters (1982) hatten in ihrer Analyse die Bedeutung familiärer Strukturen und Dynamiken nicht hinreichend berücksichtigt, die schlussendlich jedoch maßgeblich die strategischen Entscheidungsprozesse prägten und das Unternehmen in die Krise führten.

Die Familie ist jedoch nicht nur im Beitrag von Mintzberg \& Waters (1982) ein fehlender Faktor. ${ }^{277}$ Vielmehr besitzt die Vernachlässigung dieser ökonomischen Größe durch die Managementtheorie eine lange Tradition, die nicht zuletzt aus der Entwicklungs- und Ideengeschichte des professionellen Managements und der entsprechenden Forschung resultiert. ${ }^{278}$

Dieses rein professionelle Management von Unternehmen durch angestellte Manager hatte sich im Zuge der Entstehung der industriellen Großunternehmung herausgebildet. ${ }^{279}$ Infolge zunehmender Größe und Komplexität der Organisationen war der Koordinationsbedarf, dem die Unternehmensführung gerecht werden musste, stark gestiegen und konnte immer seltener von den Eigentümern allein bewältigt werden. In der Folge verbreitete sich die Beschäftigung rein professioneller Führungskräfte. Dies wiederum führte zu einer immer häufigeren Trennung von Eigentum und der direkten, operativen Verfügungsgewalt über dieses Eigentum.

Diese von Berle \& Means (1932) beschriebene Entwicklung veranlasste Burnham (1948) zur Postulierung der These von der "managerial revolution" und der aufkommenden $»$ Herrschaft der Manager $«{ }^{280}$ Bei solchen Beschreibungen und Prognosen beließ es die Wirtschaftswissenschaft jedoch nicht.

276 Vgl. Mintzberg \& Waters (1990).

277 Vgl. Dyer (2003: 401), der die Famile als "missing variable» der Managementforschung bezeichnet. Zu dieser Einschätzung gelangen aber auch Miller \& LeBreton-Miller (2005: 4f.) oder Schulze et al. (2001): »[F]amily firms have been virtually overlooked in the mainstream economic and management journals«. Deren Urteil beruht auf einem Review führender Managementzeitschriften wie AMJ, AMR, ASQ, ET\&P, JOM, JMS oder OS.

278 Vgl. für eine Übersicht zur Entwicklung der Managementtheorie Steinmann \& Schreyögg (2005: 33ff.). Litz (1997) beleuchtet die Gründe für die fehlende Berücksichtigung der Familie durch die Managementforschung.

279 Vgl. Chandler (1994: 1 ff.); Kocka (1975: 80ff.); Chandler (1994); Steinmann \& Schreyögg (2005: 33).

280 Vgl. Berle \& Means (1932); vor allem auch Burnham (1948) zum Regime der Manager. Burch (1972) für eine frühe Kritik an deren Prognosen. 
Vielmehr wurde die von professionellen Managern geführte Großunternehmung zum weltweiten, normativen Idealbild der Managementtheorie. ${ }^{281}$

In diesem Rahmen begann auch eine frühe Debatte um die Performance und zukünftige Bedeutung von Unternehmen, in denen Eigentum und Verfügungsgewalt noch in den Händen einer Familie konzentriert waren. Dabei verwies die Managementliteratur der 1960er- und 1970er-Jahre vor allem auf die Schwierigkeiten von Familienunternehmen, den mit dem Wachstum der Unternehmen einhergehenden Herausforderungen adäquat zu begegnen. Es wurde davon ausgegangen, dass die Eigenkapitalgeberfunktion der Familie ein Wachstumshindernis darstelle und sich die zunehmend komplexen Konzerne auch nur schwer innerhalb familiärer Strukturen führen ließen. ${ }^{282}$

Auf der Grundlage dieser Annahmen postulierte Chandler (1990) eine allgemeine lineare Entwicklung vom traditionellen Familienunternehmen zum großen managergeführten und kapitalmarktorientierten Konzern mit weit verstreuten Eigentumsverhältnissen. ${ }^{283}$

In dem auf der Trennung von Eigentum und Verfügungsgewalt beruhendem organisationsstrukturellen Entwurf »moderner « Unternehmensführung war für den Eigentümer grundsätzlich kein Platz in der Leitung des Unternehmens mehr vorgesehen. Als der perfekte Komplementär des rein professionellen, spezialisierten Managements erschien vielmehr der anonyme, renditeorientierte Aktionär. ${ }^{284}$ Insbesondere die Möglichkeit der Einflussnahme durch eine starke, unter Umständen gar emotional verbundene Eigentümerfamilie wurde zunehmend als Bedrohung und "Störfaktor « für die weitere Professionalisierung und Rationalisierung der Unternehmensführung wahrgenommen. Der damaligen modernen Managementtheorie diente diese vermeintlich überholte Form ökonomischer Aktivität gar häufig als Negativbeispiel zur kontrastierenden Illustration der neuen und zeitgemäßen Managementpraktiken. ${ }^{285}$

So wurden Unternehmensfamilien und die durch sie konstituierten Familienunternehmen zunehmend als Anachronismen der modernen Ökonomie be-

281 Vgl. Chandler (1990: 383 ff.); Perrow (1972). Zu klassischen Beispielen avancierten so die Beiträge der Manager Sloan (1964) und Drucker (1995).

282 Vgl. Chandler (1980: 13f.): "No family or financial institution was large enough to staff the managerial hierarchies required to administer modern multiunit enterprises." Chandler (1994: 304): »But only with the end of family control shortly after the war did the company put in place an administrative structure and develop the organizational capabilities needed to maintain a strong position in global markets."Vgl. auch Colli et al. (2003: 7).

283 Vgl. Chandler (1990); auch Colli (2003: 13); Morikawa (2001: 179): „[F]amily enterprises are intrinsically limited in their future prospects."

284 Vgl. Berle \& Means (1932: 64); Colli (2003: 50ff.); Penroese (1959: 19).

285 Vgl. Penroese (1959: 19); Perrow (1972: 8ff.):»Particularism means that irrelevant criteria (e.g., only relatives of the boss have a chance at top positions), in contrast to universalistic criteria (competence is all that counts), are employed in choosing employees." 
trachtet. Da man davon ausging, dass sich Familienunternehmen in Konkurrenz zu modernen, kapitalmarktorientierten Unternehmen auf kurz oder lang nicht behaupten würden, schien es, als hätten Familienunternehmen nur noch die Wahl zwischen einem schwierigen Prozess der Anpassung an die Moderne oder einem langsamen, aber unweigerlichen Ausscheiden aus dem Markt. So gingen Albach \& Freund (1989) noch Ende der 1980er-Jahre davon aus, dass Familienunternehmen in Kürze aus dem ökonomischen Geschehen verschwunden sein würden und es nur noch »Unternehmen an sich « geben würde. ${ }^{286}$

In solchen Thesen spiegelte sich der Glauben der Managementtheorie an eine einheitliche, objektivierbare Rationalität der Unternehmensführung. ${ }^{287}$ Gesucht wurden objektiv »optimale« Entscheidungen. Wer diese letztendlich trifft und umsetzt, war aufgrund der vermeintlichen Optimalität lange Zeit nebensächlich. Da im Rahmen dieses Gedankengebäudes das Persönliche, das Informelle und Emotionale keinen Platz mehr hatten, fand auf der Suche nach der »rational choice» auch die Familie als Faktor der Unternehmensführung in den Forschungsbeiträgen und Lehrbüchern der Managementtheorie keine Erwähnung mehr. ${ }^{288}$ Wenn sie doch einmal am Rande erwähnt wurde, dann eben lediglich als potenzielle »Störquelle« oder Hort emotionaler Irrationalität.

Diese Vernachlässigung des Faktors Familie stand jedoch stets in starkem Kontrast zur anhaltenden ökonomischen Präsenz von Familienunternehmen in den entwickelten Ökonomien. So zeigen internationale Erhebungen wie La Porta et al. (1999) oder Shleifer \& Vishny (1986) nicht nur, dass die von Berle \& Means (1932) beschriebene "managerial corporation « mit ihrer atomisierten Eigentümerstruktur lediglich eine Unternehmensform von vielen ist. Sie kommen auch zu dem Schluss, dass ungefähr 60-80\% aller Unternehmen weltweit als Familienunternehmen betrachtet werden könnten. ${ }^{289}$ Die These Chandlers von den aussterbenden Familienunternehmen wurde von der empirischen Realität somit zweifelsfrei widerlegt: Der Faktor Familie ist auch im 21. Jahrhundert ein integraler Bestandteil unseres gesamtökonomischen Systems.

286 Vgl. Albach \& Freund (1989: 266f.); Dyer (2003: 403).

287 Vgl. Schreyögg (1984); Simon (2012: 8).

288 Vgl. Schulze et al. (2001: 99). Handler (1989: 257) verweist auf vereinzelte Ausnahmen wie Donnelley (1964); Levinson (1971). Zur Vernachlässigung in deutschen Lehrbüchern vgl. Hopfenbeck (1989); Staehle (1980); Ulrich \& Fluri (1992 (1975)).

289 Vgl. für Deutschland FBN (2008): $79 \%$; Klein (2010): 58 \%; international die renommierten finanzwissenschaftlichen Beiträge La Porta et al. (1999); Shleifer \& Vishny (1986). GomezMejia et al. (2001) zufolge tragen Familienunternehmen 40-60 \% zum BIP der USA bei und beschäftigen $80 \%$ aller Arbeitskräfte. Anderson \& Reeb (2003) zeigen in ihrer vielbeachteten Studie, dass ein Drittel aller Unternehmen im Standard\% Poor's 500 Index familienkontrolliert sind und diese Unternehmen hinsichtlich der Performance die rein von professionellen Managern geführten Unternehmen übertreffen. 


\section{Das Feld der Familienunternehmensforschung}

Da die etablierte Managementforschung diesen Ausschnitt des ökonomischen Geschehens über Jahrzehnte hinweg weitgehend ignorierte, begannen sich $a b$ Anfang der 1970er-Jahre Wissenschaftler und professionell Interessierte außerhalb dieser Disziplin mit familienunternehmensspezifischen Thematiken wie der Nachfolgefrage oder konfliktreichen sozialen Beziehungen im Unternehmenskontext näher auseinanderzusetzen. ${ }^{290}$

Von zunächst stark praxisorientierten Beiträgen ausgehend, institutionalisierte sich zu Beginn der 1980er-Jahre die Familienunternehmensforschung gar als eigenständiges Forschungsfeld. ${ }^{291}$ Zur Legitimation der Forschungsbemühungen wurde vor allem versucht, die Spezifität des Unternehmenstypus des Familienunternehmens herauszuarbeiten. ${ }^{292}$

In den 1990er-Jahren wurde der Forschung zu Familienunternehmen dann wachsende Aufmerksamkeit zuteil. ${ }^{293}$ Allerdings nach wie vor nur im weitgehend abgeschotteten Feld der Familienunternehmensforschung, die lange Zeit isoliert von der allgemeinen Managementforschung und selbstreferenziell in einem in sich geschlossenen Wissenschaftssystem operierte. Die etablierte Managementforschung beachtete den Faktor Familie weiterhin kaum.

Ein Grund für diese Isolation der Familienunternehmensforschung war sicherlich der Umstand, dass die wissenschaftliche Qualität der Beiträge zu Familienunternehmen nicht immer mit der steigenden Quantität Schritt halten konnte. So waren viele Beiträge trotz mangelhafter theoretischer und empirischer Grundlage sehr normativ und besaßen eine ausgeprägte Tendenz zur Herausstellung der vermeintlich positiven oder überlegenen Eigenschaften von Familienunternehmen. ${ }^{294}$ Hierbei mag sicherlich die professionelle Nähe der Forschung zur beratenden Praxis und der daraus resultierenden Praxisorientierung der Beiträge eine Rolle gespielt haben. ${ }^{295}$

In jedem Fall war die empirische Evidenz der Familienunternehmensfor-

290 Vgl. Brockhaus (1994: 25ff.). Zur biografischen oder professionellen Nähe der ersten Autoren zum Forschungsgegenstand und dem hieraus abgeleiteten Interesse an der Etablierung dieses Forschungsfelds auch Gersick et al. (1997: 4f.); Handler (1989: 258); Perez \& Colli (2013: 15).

291 Vgl. Bird et al. (2002); Perez \& Colli (2013: 19); Sharma et al. (2012).

292 Vgl. zu dieser Einschätzung Casillas \& Acedo (2007: 150); Sharma et al. (2007: 19). Vgl. auch wegweisende Arbeiten wie Dyer (1986); Lansberg (1988); Handler (1989); Ward (1987).

293 Vgl. Bird et al. (2002: 388); Sharma (2004: 1).

294 Vgl. Litz (1997: 56): »[F]amily businesses remain largely omitted from rigorous empirical and conceptual study [...] this has resulted in the terrain being staked by a more anecdotally driven and prescriptively oriented practitioner community.« Ähnlich auch Sharma et al. (2012: 11).

295 Vgl. Brockhaus (1994: 25ff.). So generierten Sharma et al. (2012: 8) zufolge die Protagonisten des Feldes die Daten lange Zeit aus den eigenen Beratungen. 
schung noch zu häufig anekdotischer Natur und es wurde zu selten auf etablierte Theorien der Organisations- oder Strategietheorie zurückgriffen und an deren Erkenntnisse angeknüpft. ${ }^{296}$

Diese empirische und theoretische Kluft trug lange Zeit dazu bei, dass dem Faktor Familie nicht der Sprung aus dem Feld der Familienunternehmensforschung in die etablierte Managementforschung gelang.

\section{Die problematische Entität des Familienunternehmens}

Das größte Hindernis für den ausbleibenden Anschluss der Familienunternehmensforschung an die allgemeine Managementforschung und die Integration des Faktors Familie in deren Theoriegebäude war jedoch wahrscheinlich die Unklarheit darüber, was denn überhaupt unter einem Familienunternehmen zu verstehen und entsprechend zu untersuchen sei.

So wären einer im deutschsprachigen Raum häufig zitierten Studie des Institutes für Mittelstandsforschung zufolge $95 \%$ aller Unternehmen Familienunternehmen. ${ }^{297}$ Wenn jedoch tatsächlich nahezu jedes Unternehmen ein Familienunternehmen wäre - welche strukturellen Merkmale würden diese angeblich spezifische Form ökonomischer Aktivität dann unterscheidbar charakterisieren und damit für die Managementforschung interessant und $\mathrm{zu}$ gänglich machen?

Jahrzehntelang galt es als die vordringlichste Aufgabe der Familienunternehmensforschung solche idiosynkratrischen Charakteristika zu benennen, definitorisch zu bündeln und somit Familienunternehmen zu anderen ökonomischen Formen klar abzugrenzen. Doch ebenso häufig wie die Forderung nach einer stringenten Definition von Familienunternehmen gestellt wurde, musste auch konstatiert werden, dass es eine solche allgemein anerkannte Definition nicht gab. ${ }^{298}$ Während Ward (1987) unter einem Familienunternehmen ein Unternehmen verstand, in dem die Führung des Unternehmens bereits einmal innerhalb der Familie übergeben wurde, subsumierten Donckels \& Fröhlich (1991) unter diesen Begriff jedes Unternehmen, in dem 60 \% des Eigenkapitals von Familienmitgliedern gehalten werden. ${ }^{299}$ Eine Vielzahl weiterer, sich teil-

296 Vgl. Bird et al. (2002: 343); Dyer (2003: 416); IfM (2006); Wortman Jr. (1994: 3 ff.).

297 Vgl. IfM (2006). Der zugrunde liegenden Definition zufolge sind alle jene Unternehmen, bei denen die Eigentums- und Leitungsrechte in der Person des Unternehmers oder dessen Familie vereint sind, Familienunternehmen. Nach dieser Definition wäre aber auch der ledige Inhaber eines Internetcafés ein Familienunternehmer.

298 In der ersten Ausgabe des Leitjournals FBR fragten Lansberg et al. (1988): [W] hat is a family business?« Von Handler (1989) über Chua et al. (1999: 19) bis Klein et al. (2005:321) merken zahlreiche Beiträge aber an, dass es auf die so gestellte Frage bislang keine allgemein anerkannte Antwort gibt.

299 Vgl. Ward (1987: 252); Donckels \& Fröhlich (1991). 
weise gar widersprechender Definitionen veranlassten Klein et al. (2005) daher zum Postulat des "Family Business Definition Dilemma«. ${ }^{300}$

Dabei bestand das Dilemma für die Familienunternehmensforschung vor allem darin, dass die definitorische Unklarheit aufgrund der Vielzahl unterschiedlichster Definitionsansätze schwerwiegende Konsequenzen für die Reliabilität und Validität ihrer Forschung hatte. So kamen zum Beispiel Westhead \& Cowling (1998) in einer Meta-Analyse zu dem Ergebnis, dass je nachdem, auf welche von sieben identifizierten Definitionen eine Studie zurückgriff, der Anteil von Familienunternehmen im Sample von $15 \%$ bis zu 78,5\% streute. In der Folge wären natürlich auch die Ergebnisse der Studien, wäre eine andere Definition gewählt worden, anders ausgefallen. ${ }^{301}$

Die aus den definitorischen Schwächen resultierende geringe Validität und Vergleichbarkeit der empirischen Forschung trug maßgeblich zur lange währenden Isolation der Familienunternehmensforschung bei. ${ }^{302}$ Die unzähligen Definitionsansätze waren allerdings weniger originäre Ursache dieser Problematik als vielmehr selbst Symptom eines tiefer liegenden Problems dieses Forschungsfelds: dem unbeirrten Versuch, die Bedeutung der ökonomischen Größe Familie über die dichotome Unterscheidung von Familienunternehmen und Nicht-Familienunternehmen konzipieren, untersuchen und analysieren zu wollen. ${ }^{303}$

Den vielen Definitionsansätzen entsprechend, erfolgten solche binären Differenzierungen dabei anhand der unterschiedlichsten Merkmale und Kriterien: So bedürfe es eines bestimmten Eigentumsanteils der Familie, der Beteiligung von einem oder mehreren Familienmitgliedern an der Unternehmensleitung oder eines bereits erfolgten generationalen Transfers, um von einem Familienunternehmen sprechen zu können. ${ }^{304}$ Dabei erachteten manche Beiträge eines dieser Kriterien als entscheidend, während andere solche Kriterien in unterschiedlichster Weise und verschiedener Ausprägung kombinierten. Eines war den Ansätzen jedoch gemein: Es galt stets Familienunternehmen über solche Kriterien eindeutig von Nicht-Familienunternehmen abzugrenzen. ${ }^{305}$

So erstrebenswert eine solch eindeutige, vermeintlich stringente Definitionen

300 Vgl. Klein et al. (2005: 322); auch Chua et al. (1999: 21); Handler (1989: 260).

301 Vgl. Westhead \& Cowling (1998), auch Dyer (2003: 411). Zur gleichen Einschätzung gelangte bereits Handler (1989: 262).

302 Vgl. Chua et al. (1999: 20); Klein et al. (2005: 321); Sharma (2004: 4f).

303 Vgl. Astrachan et al. (2002: 52f); Chua et al. (1999: 19f.); Dyer (2003).

304 Vgl. hinsichtlich des Kriteriums Eigentum der Familie am Unternehmen Anderson \& Reeb (2003); Littunen \& Hyrsky (2000). Zu Eigentum und Management-Involvement Handler (1989). Zum Kriterium intendierter oder durchgeführter Eigentumstransfer in der Familie: Ward (1987); Chua et al. (1999). Für eine Übersicht über verschiedene Definitionsmöglichkeiten vgl. Chua et al. (1999: 20f.); Handler (1989: 260); Litz (1995).

305 Vgl. Handler (1989: 259); Sharma \& Salvato (2013: 40). 
auch erscheinen mag - die Suche nach einer solchen exakten inhaltlichen und begrifflichen Bestimmung scheiterte stets an der empirischen Komplexität des Phänomens Familienunternehmen. Indem sie zum Beispiel einen fixen Eigentumsanteil benannten, warfen viele dieser Versuche auch mehr Unklarheiten auf als sie beseitigten, und führten schlussendlich häufig zu einer künstlich anmutenden binären Trennung von Familienunternehmen und Nicht-Familienunternehmen. ${ }^{306}$

»[T]here is no clear demarcation between family and nonfamily businesses and [...] artificially dichotomizing family vs. nonfamily firms when no such clear-cut dichotomy exists creates more problems than it attempts to solve."

(Astrachan et al., 2002) (307 $^{307}$

Zudem warfen diese vermeintlich stringenten Definitionen anhand einzelner, messbarer Komponenten die Frage auf, ob denn der Umstand, dass sich ein bestimmter Eigentumsanteil in Händen einer Familie befinde, überhaupt die "Essenz« des familiären Einflusses ausmache und erkennen ließe. Diese äußere sich doch eher in organisationalen Spezifika wie dem Streben nach Kontinuität oder bestimmten kulturellen Mustern. ${ }^{308}$

Vor dem Hintergrund dieser definitorischen und konzeptionellen Probleme ist die Forschung zu Familienunternehmen im Laufe der letzten fünfzehn Jahre immer stärker zu einer anderen Form der Annäherung an das Phänomen der Verbindung von Familie und Unternehmen übergegangen: Anstatt weiterhin die scharf abgegrenzte Entität des Familienunternehmens dem Nicht-Familienunternehmen gegenüberzustellen, um die Besonderheiten dieser Form ökonomischer Aktivität zu untersuchen, fokussiert sie nun stärker den spezifischen Einfluss, den der Faktor Familie über formelle und informelle Mechanismen auf Unternehmen besitzen kann, und dessen Auswirkung auf Struktur und Kultur der Organisation:

"A relevant issue, therefore, is not whether a business is family or nonfamily, but the extent and manner of family involvement in and influence on the enterprise."

(Astrachan et al., 2002) 309 $^{309}$

306 Vgl. Chua et al. (1999: 22f.); Astrachan et al. (2002: 46). So ist auch ein katarischer Staatsfonds als Familienunternehmen zu definieren, wenn man nur einen Eigentumsanteil der Familie festlegt. Setzt man wie Ward (1987) einen erfolgten generationalen Transfer voraus, so wäre das Gründerunternehmen um den klassischen Patriarchen kein Familienunternehmen.

307 Astrachan et al. (2002: 46).

308 Vgl. Chua et al. (1999); Litz (1995). Auch Gersick et al. (1997: 29) fragen kritisch:»[I]t is family ownership that defines the family business? « Viele Autoren verneinen mittlerweile die Frage und sehen Eigentum als hinreichende, aber nicht notwendige Bedingung.

309 Astrachan et al. (2002: 47); vgl. auch Chua et al. (1999: 19ff.); Dyer (2003: 408ff.); Habbershon \& Williams (1999). Klein et al. (2005: 323): »Rather than dichotomizing enter- 
Dabei gehen jüngere Beiträge der Forschung zu Familienunternehmen grundsätzlich von einem graduellen Verständnis des familiären Einflusses aus. ${ }^{310}$ Dieser Auffassung zufolge, kann der Einfluss der Familie auf das Unternehmen in Abhängigkeit von der Form und Intensität der Verbindung zwischen Familie und Unternehmen mehr oder weniger stark ausgeprägt sein. Ein niedriger Eigentumsanteil führt zu einem geringeren Einfluss auf das Unternehmen, ein höherer Eigentumsanteil zu einem stärkeren Einfluss.

In der Konsequenz plädieren Chua et al. (2004) dafür, Familienunternehmen nicht mehr als abgeschlossene Entitäten, sondern Familienunternehmen und Nicht-Familienunternehmen lediglich als gedankliche Endpunkte eines Kontinuums des familiären Einflusses aufzufassen:

»[W]hether a business is a firm may be a matter of degree. Put differently, 'pure and nonfamily businesses may simply represent the extremes of a continuum."

(Chua et al., 2004) $)^{311}$

Ein Unternehmen ist nicht entweder Familienunternehmen oder Nicht-Familienunternehmen, sondern mehr oder weniger familiär beeinflusst. Im Sinne dieser Vorstellung gehen Beiträge wie Habbershon \& Williams (1999) mit ihrem Konzept der "familiness « davon aus, dass der familiäre Einfluss in Abhängigkeit je nach Stärke zu mehr oder weniger stark ausgeprägten Strukturmerkmalen führe. ${ }^{312}$ Auf diesem Wege wird versucht die organisationale Spezifität familiär beeinflusster Unternehmen zu erfassen sowie darzulegen, warum und wie das organisationale Verhalten familiär beeinflusster Unternehmen von Unternehmen abweicht, in denen kein oder nur ein sehr geringer familialer Einfluss festzustellen ist. ${ }^{313}$

Diese veränderte Herangehensweise an das empirische Phänomen Familienunternehmen erleichtert die Integration des Faktors Familie in bestehende

prises based on statistical methods, or arbitrarily, we ask: what makes a business a family business?»

310 Vgl. die frühe Forderung von Westhead \& Cowling (1998: 50):»Researchers [...] should consider developing a Ifamilyness s scale along which each business can be placed." Chua et al. (2004) Chua (2004: 39); Habbershon et al. (2003: 463); Klein et al. (2005: 334); Litz (1997: 56).

311 Vgl. Chua et al. (2004: 39); auch Astrachan et al. (2002):»From the One Definition Toward a Continuum of Family Business«; Dyer (2003: 408ff.); Habbershon \& Williams (1999).

312 Vgl. Habbershon \& Williams (1999); auch Chua et al. (2004: 39); Habbershon et al. (2003: 463): »The unified systems performance model does not make an a priori judgment about the degree or nature of the family factor influence [...] allows the researcher to identify and describe the family factor influence as antecedents to the resources and capabilities and link them to specific advantages and performance of the firm«. Weiter auch: König et al. (2013: $421 \mathrm{ff}$.); Miller \& LeBreton-Miller (2005); Sirmon \& Hitt (2003: $341 \mathrm{ff}$.); Wimmer et al. (2005).

313 Chua et al. (1999): »Defining the Family Business by Behavior«. 
theoretische Gerüste und trägt sicherlich einen Teil zu dessen fortschreitender Integration in die etablierte Managementforschung bei.

Zunehmende Integration des Faktors Familie in die etablierte Managementforschung Nicht zuletzt aufgrund dieser veränderten Betrachtungsweise erfuhr die ökonomische Größe der Familie ab der Jahrtausendwende dann auch zunehmende Aufmerksamkeit durch die Managementforschung. Dafür dürften Studien wie La Porta et al. (1999) von großer Bedeutung gewesen sein, die in renommierten Zeitschriften auf die weltweite Verbreitung und Bedeutung dieser Form ökonomischer Aktivität hinwiesen. ${ }^{314}$

Habbershon \& Williams (1999) oder Schulze et al. (2001) gehörten zu den ersten Autoren, welche die theoretische Lücke zwischen der Familienunternehmens- und der Managementforschung zu schließen begannen, indem sie in ihren Arbeiten an etablierte Theorien wie den ressourcenbasierten Ansatz oder die Agenturtheorie anknüpften. ${ }^{315}$

Schlussendlich dürfte für die zunehmende Hinwendung der Managementforschung zum Faktor Familie aber auch nicht unerheblich gewesen sein, dass sich nach der Jahrtausendwende das Bild von Familienunternehmen in der Öffentlichkeit stark zu wandeln begann. Denn jene Unternehmen, die vormals als ökonomische Anachronismen und Rückzugsgebiete autokratischer Patriarchen gegolten hatten, wurden seit dem Scheitern der "New Economy« und der sich zuspitzenden Finanzkrise zunehmend als »hidden champions" und Garanten eines langfristig orientierten und nachhaltigen Wirtschaftens wahrgenommen. ${ }^{316}$

All diese Faktoren dürften der Forschung zum Faktor Familie in der etablierten Managementforschung zu mehr Legitimität verholfen haben. In jedem Fall öffnete diese um die Jahrtausendwende die Tür für den Faktor Familie und in den anerkanntesten wissenschaftlichen Zeitschriften der Finanzwissenschaften oder der Managementforschung werden seitdem verstärkt Beiträge publiziert, die auf den Faktor Familie aufmerksam machen. ${ }^{317}$ Durch dessen

314 Vgl. Anderson \& Reeb (2003); La Porta et al. (1999); Shleifer \& Vishny (1997).

315 Vgl. zu dieser Entwicklung Sharma et al. (2012: 11); Chrisman et al. (2005: 559ff.). Zum ressourcenbasierten Ansatz vgl. grundlegend Barney (1991); zur Anwendung auf Familienunternehmen Habbershon (1999); Zahra et al. (2004). Zur Agententheorie vgl. grundlegend Jensen \& Meckling (1976); zu Familienunternehmen Gomez-Mejia et al. (2001); Schulze et al. (2001); Schulze et al. (2003).

316 Vgl. Simon (2007). So der Geschäftsführer von Tengelmann, Karl-Erivan Haub, laut FNP (2011) im Jahr 2011: »Imagemäßig werden wir Familienunternehmer heute nicht mehr belächelt, sondern zum Teil schon wieder bewundert«. Entsprechend wurde häufig eine "Renaissance des Familienunternehmens« beschrieben.

317 Vgl. Lee et al. (2003); Anderson et al. (2003); Anderson \& Reeb (2003); Anderson \& Reeb (2004); Gomez-Mejia et al. (2001); Gomez-Mejia et al. (2003); Lee et al. (2003); Schulze 
Integration verspricht man sich kontextualisierte und somit robustere Forschungsergebnisse. ${ }^{318}$

Die meisten dieser Beiträge knüpften dabei an die graduelle Konzeption des familiären Einflusses auf Unternehmen an. Sie fokussieren weniger die Entität des Familienunternehmens als vielmehr den mehr oder weniger starken Einfluss, den eine Familie zum Beispiel auf Variablen des strategischen Entscheidungs- und Veränderungsprozesses nehmen kann, und die Konsequenzen für zu erklärende Phänomene wie den organisationalen Wandel. ${ }^{319}$

Vor dem Hintergrund der Forschungsfrage nach dem Einfluss des Faktors Familie auf die Genese und Dynamik strategischer Pfadabhängigkeit, soll im Rahmen dieser Arbeit an diese Konzeption des graduellen familiären Einflusses angeknüpft werden, der über bestimmte Kanäle die Ausprägung bestimmter organisationaler Variablen beeinflussen kann.

Dieses grundlegende Verständnis wird im nächsten Unterkapitel aus systemtheoretischer Perspektive theoretisch genauer konzipiert und verständnisfördernd präzisiert.

\subsection{Das Verhältnis der sozialen Systeme Familie und Unternehmen}

Im obigen Unterkapitel wurde die Familie als ein lange Zeit von der Managementforschung vernachlässigter Faktor eingeführt. Nachfolgende Betrachtungen nähern sich dem Kontextfaktor Familie über eine systemtheoretisch orientierte Konzeption weiter an, die im Sinne der jüngeren Forschung weniger die Entität Familienunternehmen in den Blick fasst als vielmehr den Einfluss des Systems Familie auf das Unternehmen.

Im ersten Schritt werden die beiden grundsätzlich divergierenden Systemlogiken von Familie und Unternehmen dargestellt, die sich im Zuge des gesellschaftlichen Modernisierungsprozesses der funktionalen Differenzierung (auseinander-)entwickelt haben. Im zweiten Schritt wird erläutert, auf welche

et al. (2001); Schulze et al. (2003). So auch Finkelstein et al. (2009): »Clearly, a number of research opportunities exist in fleshing out the implications, costs, and benefits of longterm family control«.

318 Vgl. Dyer (2003: 405):»[T] he use of the family as a variable can help researchers build more robust, contextual theories«; auch Gomez-Mejia et al. (2001: 81); König et al. (2013: 501): "Our goal has been to advance our knowledge of when and how established companies adopt discontinuous technologies by integrating a new factor into the equation: the impact of the family system on the business system."

319 Vgl. Hatum \& Pettigrew (2006: 122 ff.) zum Einfluss des Faktors Familie auf die »organisationale Flexibilität«; König et al. (2013) zum Einfluss auf die Adoption von »discontinous technologies«; Sirmon et al. (2008) zur Bedeutung des familiären Einflusses auf strategische Reaktionen bei Gefahren von Imitationen. 
Weise diese beiden funktional differenzierten Systeme in der Sphäre von Familienunternehmen dennoch auf engstem Raum miteinander verschränkt sind und sich gegenseitig in ihrer Entwicklung beeinflussen.

\subsubsection{Divergierende Systemlogiken}

\section{Familie und Unternehmen im Prozess der funktionalen Differenzierung}

Im Begriff des Familienunternehmens gehen zwei soziale Systeme eine Verbindung ein, die in der Lebenswelt der meisten Menschen unserer modernen Gesellschaft keine Entsprechung mehr findet. Doch Unternehmen und Familie bilden heutzutage nicht nur zwei in fast jeder Hinsicht getrennte Lebenswelten. Sie sind vor allem auch zwei funktional differenzierte Systeme. ${ }^{320}$

Über viele Jahrhunderte war die sogenannte »Haushaltsfamilie mit Produktionsfunktion « die dominante Erscheinungsform von Familien. ${ }^{321}$ Beispielhaft kann hier der Typus des "ganzen Hauses « angeführt werden, welcher neben der Offenheit seiner räumlichen Grenzen insbesondere durch die Einheit von Haushalt und landwirtschaftlichem, gewerblichem oder handwerklichem Betrieb gekennzeichnet war. Dieser Betrieb stand im Zentrum des "familiären" Lebens und prägte über Jahrhunderte eine von heutigen Familientypen stark abweichende Form von »Familie ${ }^{322}$

Schon das lateinische »familia « zielt nicht auf die enge Verbindung von Eltern und Kindern $\mathrm{ab}$, die charakteristisch für das heutige Familienverständnis ist. Vielmehr verwies dieser Begriff auf einen Haushalt, der sowohl die Angestellten und Sklaven als vor allem auch das Vermögen dieser Gemeinschaft umfasste. ${ }^{323}$ Auf den Gesetzmäßigkeiten des Wirtschaftens einer solchen Haushaltsgemeinschaft (»oikos«) basiert wiederum der deutsche Begriff der Ökonomie. ${ }^{324}$

Die beiden Wörter "familia» und »oikos« tragen dem Umstand Rechnung, dass Familien in vorindustrieller Zeit nicht die Orte der Intimität und Regeneration im heutigen Sinne waren, sondern in erster Linie funktional diffuse ökonomische Überlebenseinheiten darstellten, die von der wirtschaftlichen

320 Vgl. zum Prozess der funktionalen Differenzierung als Leitprozess der Moderne Luhmann (1977: 29ff.); diesem vorausgehend Parsons (1952) und Weber (1972).

321 Vgl. Nave-Herz (2014: 3); Mitterauer \& Sieder (1977: $38 \mathrm{ff}$.$) .$

322 Vgl. Brunner (1968: 104ff.); Nave-Herz (2014: 4f.).

323 Von lat. famulus »Diener« oder auch »Haussklave«; vgl. Pons (2016). Einen spezifischen und exklusiven Begriff für die Familie im heutigen Verständnis gab es bis zum Beginn des 18. Jahrhunderts in der deutschen Sprache nicht. Vgl. Gestrich (2010: 4f.).

324 Vgl. Brunner (1968: 106): »Die Ökonomik als Lehre vom `Oikos« umfasst eben die Gesamtheit der menschlichen Beziehungen und Tätigkeiten im Hause.» 
Versorgung ihrer Mitglieder bis hin zur Kranken- und Altenpflege die vielfältigsten Funktionen zu erfüllen hatten. ${ }^{325}$

Doch im Zuge der Industrialisierung und der Entwicklung neuer gesamtgesellschaftlicher Leitideen setzte mit dem Prozess der funktionalen Differenzierung und Spezialisierung zu Beginn des 18. Jahrhunderts eine Entwicklung ein, welche die Gesellschaft im Allgemeinen und die Familie im Besonderen grundlegend verändern sollte. Denn in Reaktion auf die gestiegene Komplexität der Umwelt setzten zur Reduktion dieser Komplexität gesellschaftliche Differenzierungen in Form von Systembildungsprozessen ein: Die Gesellschaft spaltete sich sukzessive in arbeitsteilig spezialisierte Systeme der Nutzenproduktion auf. ${ }^{326}$

Im Verlauf dieses Prozesses der funktionalen Differenzierung wurden nach und nach viele der ursprünglichen Funktionen der Familie von anderen, spezielleren Systemen übernommen. So übernahmen im ökonomischen Kontext größere Betriebe und Organisationen als neue ökonomische Einheiten mehr und mehr die Produktion und Bereitstellung von knappen Waren ${ }^{327}$ Ein großer Teil der Arbeit wurde außer Haus verlagert.

Mit der damit verbundenen räumlichen Differenzierung der Sphären des Familienlebens und der Erwerbsarbeit ging eine zunehmende Differenzierung der psychischen Ebenen einher. Es entstanden immer schärfer auseinanderstrebende Sphären des Lebens, welche von spezifischen Sinn- und Sachlogiken geleitet wurden, über andere Rationalitäten operierten und als Systeme gänzlich unterschiedliche Funktionen erfüllen zu begannen. ${ }^{328}$

Die Organisation Unternehmen unterlag in diesem Zuge immer stärker einer ökonomischen Zweckrationalität, wohingegen sich die Familie zunehmend auf die emotionale Bedürfnisbefriedigung der Familienmitglieder konzentrierte. ${ }^{329}$ Für die Familie brachte die funktionale Differenzierung somit nicht nur einen Funktionsverlust, sondern vor allem auch eine funktionale Spezialisierung mit sich.

Diese Spezialisierung vollzog sich im Rahmen eines gesellschaftlichen Intimisierungs- und Emotionalisierungsprozesses, der durch die Hinwendung zum

325 Vgl. Nave-Herz (2014: 3). Die Familie war des Weiteren verantwortlich für Reproduktion, Sozialisation der Nachkommen, soziale Platzierung oder die Weitergabe von religiösen Riten.

326 Vgl. Esser (2000: 64); Luhmann (1977: 29ff.); Nave-Herz (2014: 2).

327 Vgl. Kocka (1975); Steinmann \& Schreyögg (2005: 5).

328 Vgl. Kieser (2006: 46); Simon et al. (2005: $30 \mathrm{ff}$.). Zur Rationalisierung auf der Ebene von Institutionen vgl. Weber (1972). Mit Habermas (1981: 192 ff.) kann von Lebenswelten gesprochen werden.

329 Vgl. Kieser (2006: 53ff.); Luhmann (1990: 207). Nave-Herz (2014: 11) zur Beschreibung der Familie. Weber (1972: $125 \mathrm{ff}$.) zu den idealtypischen Merkmalen der rational geordneten, bürokratischen Organisation. 
Individuum im Zuge von Aufklärung und Romantik, sowie der sozialen Bedeutungsverschiebung von Adel und Bauerntum hin zum Bürgertum Mitte des 18. Jahrhunderts eingesetzt hatte. Infolge dieses Prozesses erhielt die Familie eine zuvor unbekannte eigene Sinnzuschreibung. Im Familiensystem sollte der Mensch nun als "ganze Person« mit individueller Persönlichkeit und Eigenart wahrgenommen und aufgenommen werden. ${ }^{330}$ In der Familie sollten Glück, Liebe und Intimität erwartbar sein. Intimität und Personenorientierung erfordert jedoch relativ klare Grenzen, sodass die Familie sich zu einem relativ geschlossenen System mit einer festen Binnenstruktur, klaren Grenzen und einem exklusiven und exkludierenden Charakter entwickelte. ${ }^{331}$

Mit ihrer Orientierung an der einzelnen Person und dem Versprechen von Nähe und Vertrautheit stand und steht die moderne Familie nun aber in Kontrast zur zweckrationalen Arbeitswelt mit ihrem unpersönlichen, organisierten und bürokratischen Aufbau. ${ }^{332}$ Denn die organisierten Unternehmen haben nach der beschriebenen historischen Trennung der Sphären eine gänzlich andere, am Effizienzkriterium orientierte Logik entwickelt, die ihrem spezifischen funktionalen Auftrag entspricht.

Somit hatten sich im Verlauf der gesellschaftlichen Spezialisierung aus dem einstmals "ganzen Haus" mit der Familie und dem Unternehmen zwei sehr unterschiedliche, von divergenten Systemlogiken geprägte Systeme entwickelt. ${ }^{333}$ Da es im Kern diese unterschiedlichen Systemlogiken und ihr Zusammenspiel in der Sphäre von Familienunternehmen sind, aus denen viele der für die Untersuchung der Pfadgenese potenziell bedeutsamen Strukturmerkmale erwachsen, sollen einige Funktionslogiken von Familie und Unternehmen skizziert werden.

\section{Die divergenten Systemlogiken von Familie und Unternehmen}

Ein erster Zugang zu den verschiedenen Systemlogiken liegt in den spezifischen Problemen, die Unternehmen und Familie bearbeiten müssen, um zu überleben: Unternehmen sind im Rahmen ihrer Umweltbearbeitung dabei in erster Linie sachorientiert, wohingegen Familien die einzelne Person und deren Bedürfnissen fokussieren müssen, um zu bestehen. ${ }^{334}$

330 Vgl. Endruweit (2014: 77); Luhmann (1990: 200 ff.); Nave-Herz (2014: 12).

331 Vgl. Nave-Herz (2014: 11). Zu den Besonderheiten von Familie aus einer strikt systemtheoretischen Perspektive vgl. Luhmann (1990: 196ff.).

332 Vgl. zu den Merkmalen der bürokratischen Organisation Kieser (2006: 53f.); Weber (1972: $551 \mathrm{ff}$.).

333 Vgl. zu den divergierenden Systemlogiken vor allem Simon (2002: 18ff.). An die Familie wurde dabei nach Nave-Herz (2014: 12f.) die Erwartung gerichtet, dass es Belastungen der Arbeitswelt im Zuge ihrer »Spannungsausgleichsfunktion« kompensieren könne. Hierzu kritisch Luhmann (1990: 209f.).

334 Vgl. Luhmann (1990: 207f.); Schreyögg (2006: 8); Simon (2012: 20). 


\begin{tabular}{|c|c|c|}
\hline & UNTERNEHMEN & FAMILIE \\
\hline Existenzbedingung & Zahlungsfähigkeit & emotionaler Zusammenhalt \\
\hline $\begin{array}{l}\text { funktionale } \\
\text { Orientierung }\end{array}$ & Aufgabe und Sache & "ganze Person« \\
\hline \multirow{3}{*}{ Kommunikation } & $\begin{array}{l}\text { folgt überwiegend sachlichen } \\
\text { Notwendigkeiten }\end{array}$ & $\begin{array}{l}\text { folgt Eigenarten und } \\
\text { Bedürfnissen der } \\
\text { Familienmitglieder }\end{array}$ \\
\hline & teils geplant & spontan \\
\hline & $\begin{array}{l}\text { niedriges Maß an investierten } \\
\text { Gefühlen, affektive Neutralität }\end{array}$ & $\begin{array}{l}\text { hohes Maß an Emotionalität, } \\
\text { »enthemmte Kommunikation« }\end{array}$ \\
\hline \multirow[b]{2}{*}{ Zugehörigkeit } & inklusiver Charakter & exklusiver Charakter \\
\hline & $\begin{array}{c}\text { Zugang erfolgt aufgrund einer } \\
\text { bewussten beidseitigen } \\
\text { Entscheidung }\end{array}$ & $\begin{array}{c}\text { Zugang erfolgt durch Geburt } \\
\text { oder aufgrund von Emotionen } \\
\text { (Liebe) }\end{array}$ \\
\hline $\begin{array}{c}\text { Austauschbarkeit } \\
\text { von Personen }\end{array}$ & austauschbar & nicht austauschbar \\
\hline $\begin{array}{c}\text { Kündbarkeit der } \\
\text { Beziehung }\end{array}$ & kündbar & unkündbar \\
\hline Wert eines Mitglieds & $\begin{array}{c}\text { beruht auf der von Mitglied zu } \\
\text { erfüllenden Aufgabe }\end{array}$ & $\begin{array}{c}\text { beruht auf der Tatsache, dass } \\
\text { es Mitglied ist }\end{array}$ \\
\hline Verhaltenserwartung & Egoismus (Selbstorientierung) & $\begin{array}{c}\text { Altruismus } \\
\text { (Kollektivorientierung) } \\
\end{array}$ \\
\hline $\begin{array}{l}\text { Rollen und } \\
\text { Funktionen }\end{array}$ & meist fest definiert. (Spezifität) & $\begin{array}{l}\text { nicht formal definiert } \\
\text { (Diffusivität) }\end{array}$ \\
\hline Bilanzierung & $\begin{array}{l}\text { kurzfristige und interpersonell } \\
\text { überprüfbare monetäre } \\
\text { Bilanzierung }\end{array}$ & $\begin{array}{l}\text { langfristige und subjektive, } \\
\text { emotionale Bilanzierung }\end{array}$ \\
\hline Gerechtigkeit & $\begin{array}{c}\text { orientiert sich am } \\
\text { Leistungsprinzip Unterschiede } \\
\text { werden betont }\end{array}$ & $\begin{array}{l}\text { orientiert sich an der } \\
\text { Gleichheit von Ansprüchen, } \\
\text { Pflichten, Liebe }\end{array}$ \\
\hline
\end{tabular}

Tabelle 1: Divergierende Systemlogiken von Familie und Unternehmen (Quelle: Eigene, verkürzte Darstellung nach Simon et al. [2005]).

Unternehmen hören auf zu existieren, wenn auf längere Sicht weniger Geld eingenommen als ausgegeben wird. Ihre Existenzberechtigung ist ökonomisch definiert. ${ }^{335}$ Ihre Sachaufgabe besteht im Angebot und Verkauf von Produkten oder Dienstleistungen auf Märkten. Auf diesem Wege müssen ausreichende Zahlungen generiert werden, um über das Medium Geld den Fortbestand der Beziehungen der Mitarbeiter und des Unternehmens zu sichern. Daher werden in Unternehmen letztlich die meisten Sachverhalte mit Blick auf Gewinn und Verlust, Kostendeckung und Profitabilität betrachtet.

335 Vgl. die von der Unternehmenskrisenforschung herausgestellten dominanten Ziele nach Krystek (1987) bei deren Verfehlung eine Unternehmenskrise entsteht. 
Der Verkauf von Produkten und die Generierung von Zahlungen kann auf lange Sicht aber nur gewährleistet werden, wenn bestimmte Aufgaben erfüllt werden. Abstrakt gesehen müssen Prozesse etabliert, strukturiert und gesichert werden. Konkret müssen Waren beschafft oder Produkte am Markt verkauft werden. Die Mitarbeiter arbeiten als Funktionsträger zur Lösung solcher Sachprobleme mit. Sie sind somit zuvorderst Mittel zum Erreichen des spezifischen Zwecks der Organisation. ${ }^{336}$

Familien müssen sich im Gegensatz hierzu vollkommen anderen Problemen widmen. Im Zentrum ihrer Umweltbearbeitung steht nicht die Erfüllung spezifischer Sachaufgaben und Familien lösen sich auch nicht zwangsläufig auf, wenn ökonomische Verluste erzielt werden. Ihre Überlebensbedingung ist nicht ökonomisch definiert, sondern emotional. ${ }^{337}$ Eine intakte Familie, deren Mitglieder sich noch eng miteinander verbunden fühlen, hört nicht auf zu existieren, wenn das Einkommen der Familienmitglieder versiegt. Umgekehrt kann eine vermögende Familie aber zerbrechen, wenn der emotionale Zusammenhalt und dessen Bindungskraft schwinden, da eine Kommunikation unmöglich wird, die sich an den physischen, psychischen und sozialen Bedürfnissen der Familienmitglieder orientiert und nicht lediglich an deren Aufgabenerfüllung. ${ }^{338}$

Eine solche permanente Aufgabenerfüllung ist aber wiederum zentral für das Überleben eines Unternehmens. Denn dessen Stabilität und Dauerhaftigkeit hängen nicht zuletzt davon ab, dass die Organisationsmitglieder, die die Aufgaben ausführen, austauschbar sind. Organisationen werden daher tendenziell ad rem, nicht ad personam strukturiert. Es werden feste Rollen und Positionen definiert und anschließend immer wieder neu besetzt. ${ }^{339}$ Aus dieser Logik heraus ist das System Unternehmen auch in besonderem Maße auf offene Grenzen angewiesen und durch einen inklusiven Charakter gekennzeichnet. ${ }^{340}$ Die gewünschte Austauschbarkeit wird hierbei durch das Medium Geld erleichtert, welches Beziehungen auch kurzfristig festigen oder terminieren kann. Zugleich dreht sich die Kommunikation in Unternehmen zunächst um die Sache und

336 Vgl. Schreyögg (2006: 8).Simon (2012: 33).

337 Vgl. Nave-Herz (2014: 12); Simon et al. (2005:35).

338 Vgl. Luhmann (1990: 290): „Die Familie etabliert sich als der Ort, an dem das Gesamtverhalten, das als Person Bezugspunkt für Kommunikation werden kann, behandelt, erlebt, sichtbar gemacht, überwacht, betreut, gestützt werden kann. [...] Die Funktion der Familie ist somit nach wie vor die gesellschaftliche Inklusion der Vollperson [...] Die Familie lebt von der Erwartung, dass man hier für alles, was einen angeht, ein Recht auf Gehör, aber auch eine Pflicht hat, Rede und Antwort zu stehen."

339 Vgl. Schreyögg (2006: 125). Dies ist bedeutsam für Stabilität wie Wandel der Organisation.

340 Vgl. zur Grenzziehung zwischen Organisation und Umwelt Schreyögg (2006: 303 ff.). Zur Bedeutung offener Grenzen im Zuge der Managementfunktion des Personaleinsatzes Steinmann \& Schreyögg (2005: 785). 
Aufgabe, was eine Integration erleichtert. ${ }^{341}$ Die Austauschbarkeit in professionellen Beziehungen schafft eine funktionale Distanz zu anderen Organisationsmitgliedern, zur Arbeit und auch zur Organisation an sich.

Im Gegensatz hierzu behandelt die an den Bedürfnissen der Familienmitglieder orientierte Kommunikation der Familie Intimes und Vertrauliches. Zudem ist sie »enthemmt«. Das bedeutet, dass alles, was eine Person betrifft, zum Gegenstand der Kommunikation gemacht werden kann. ${ }^{342}$ Ein derart offener kommunikativer Raum erfordert ein Mindestmaß an Diskretion, Privatheit und Abgrenzung gegenüber der Umwelt, damit innerhalb schützender Grenzen die intime Kommunikation gepflegt werden kann. Vor dem Hintergrund ihrer spezifischen Funktionslogik weisen Familien somit einen inhärent exklusiven Charakter auf. ${ }^{343}$

"[...] [I]n modernen Familien [...] ist diese Kommunikationsverdichtung mitsamt ihren Konstruktionen, die sich wie ein Netz über die Familie legt, eine allgemeine Erfahrung. Die Familie übertreibt Gesellschaft. Dies Übertreiben von Kommunikation durch Beobachten des Beobachtens sichert einerseits die Grenzen des Familiensystems. Die Familie ist und bleibt [...] auf Diskretion ihrer Mitglieder angewiesen." (Luhmann, 1990) $)^{344}$

Entsprechend schwierig gestaltet sich meist die Frage der Zugehörigkeit zur Familie. Der Zugang zu einer bestehenden Familie ist entweder schicksalshaft oder fällt recht schwer. Ist man jedoch erst einmal Mitglied einer Familie, ist wiederum der Austritt nicht einfach.

»Das System Familie, in dem derart personenorientiert kommuniziert wird, ist, wie bereits gesagt, hochempfindlich gegen eine Veränderung der Personen.«

(Luhmann, 1990) ${ }^{345}$

Langjährige familiäre Beziehungen können gar in einer gewissen Art und Weise als unkündbar verstanden werden. ${ }^{346}$ Scheidet aber doch ein Mitglied aus dem Familienverbund aus, so reagieren Familien hochsensitiv auf diese Veränderung.

Wer in einer solch personenorientierten, quasi unkündbaren Beziehung lebt,

341 Vgl. Simon et al. (2005: 38) und die idealtypische Kontrastfigur der bürokratischen Unternehmung bei Kieser (2006: $53 \mathrm{f}$.).

342 Vgl. Luhmann (1990: 203 ff.).

343 Vgl. Nave-Herz (2014: 11). Der im Zuge der funktionalen Differenzierung zunehmend exklusive Charakter von Familien spiegelte sich räumlich im Aufkommen des modernen Hauses mit der räumlichen Trennung der Kernfamilie vom Rest der Hausbewohner wider.

344 Vgl. Luhmann (1990: 215); zur Privatheit in Familien auch die kurze Diskussion bei Burkart (2008: $147 \mathrm{ff}$.).

345 Vgl. Luhmann (1990: 213); auch Burkart (2008: 143).

346 Vgl. Simon (2002: 23). Ausführlich Claude (1981). Vgl. auch das Urteil XII ZB 607/12 zur Verpflichtung zum Elternunterhalt trotz lange Jahre ausbleibenden sozialen Kontakts. 
wird zwangsläufig auch emotional verstrickt oder gebunden. ${ }^{347}$ Denn zum einen verspricht und bietet die Familie Schutz, Vertrauen und Liebe. Die Fokussierung auf die einzelnen Personen und deren emotionale Bedürfnisbefriedigung ist ja gerade eine der zentralen Funktionen heutiger Familien. Doch dort, wo solch komplexe Erwartungen und Ansprüche bestehen, können die involvierten Personen auch in besonderer Weise enttäuscht werden. Solche enttäuschten Erwartungen führen zu emotionalen Reaktionen und diesen Emotionen wird im enthemmten Kommunikationsraum auch meist freien Lauf gelassen. Nicht zuletzt auch, weil aufgrund der versprochenen Dauerhaftigkeit und der impliziten Unkündbarkeit der Beziehungen - im Gegensatz zum Unternehmen - nicht mit einem Ausschluss gerechnet werden muss. Ob dieser hohen Emotionalität sind Familien äußerst konfliktreiche Systeme. ${ }^{348}$

\subsubsection{Strukturelle Kopplung und Ko-Evolution der Systeme}

Im Laufe der letzten beiden Jahrhunderte haben sich demnach im Zuge des gesellschaftlichen Wandels mit Unternehmen und Familie zwei funktional spezialisierte Systeme mit sehr divergenten Funktionslogiken entwickelt, die für die meisten Menschen zwei vollkommen getrennte Lebenswelten bilden. In der kommunikativen Sphäre von Familienunternehmen sind diese beiden so verschiedenen Systeme allerdings noch immer noch aufs Engste miteinander verbunden. Form und Dynamik dieser spezifischen Verbindung erschließen sich aus systemtheoretischer Perspektive.

Systemtheoretisch orientierte Betrachtungen von Familienunternehmen haben in der Familienunternehmensforschung eine lange Tradition, an die auch jüngere Beiträge der allgemeinen Managementforschung anknüpfen. ${ }^{349}$ Für viele Bereiche der Forschung zu Familienunternehmen kann die Systemtheorie gar als die dominante theoretische Perspektive erachtet werden. ${ }^{350}$

Da in der Forschung zu Familienunternehmen jedoch auf sehr unterschiedliche Varianten der in ihrer Evolution vielstufigen Systemtheorie zurückgegriffen wird, reicht ein bloßer Verweis auf eine systemtheoretische Konzeption des Verhältnisses von Familie und Unternehmen nicht aus. Da mit diesen Va-

347 Vgl. Simon (2002: 23); von Schlippe \& Kellermanns (2008: 43).

348 Vgl. Luhmann (1990: 208); von Schlippe (1995).

349 Vgl. Habbershon et al. (2003); König et al. (2013); Simon (2002); Stafford et al. (1999: 198). Für einen Überblick über die systemtheoretische Forschung zu Familienunternehmen: von Schlippe \& Frank (2013). Zu kritischen Aspekten der systemtheoretischen Perspektive: Stamm (2013: 8).

350 Vgl. von Schlippe \& Frank (2013); Stafford et al. (1999: 197):»[T]he prevailing theoretical orientation is a systems paradigm.» Lediglich im Diskurs zur Governance von Familienunternehmen sind die Agenturtheorie oder der Stewardship-Ansatz bedeutender. Vgl. hierzu die Übersicht bei Siebels \& zu Knyphausen-Aufseß (2012). 
rianten sehr unterschiedliche Vorstellungen der Verbindung zwischen den beiden Systemen einhergehen, muss diese theoretische Perspektive im Folgenden kurz weiter spezifiziert werden.

\section{Familienunternehmen als die Summe aus Familie und Unternehmen?}

Grundsätzlich machen alle systemtheoretischen Beiträge zu Familienunternehmen darauf aufmerksam, dass die Komplexität von Familienunternehmen und deren Spezifität nicht erfasst werden könne, wenn lediglich das System Unternehmen betrachtet werde. Hierzu bedürfe es mindestens noch der differenzierten Integration des Systems Familie. ${ }^{351}$

Zur weitergehenden Konzeption von Familienunternehmen greifen die meisten systemtheoretisch orientierten Beiträge aber meist implizit, seltener explizit, auf ältere systemtheoretische Ansätze zurück. Sie konzipieren Familienunternehmen entsprechend als ein System, welches sich aus mehreren Subsystemen und deren Eigenschaften zusammensetzt. ${ }^{352}$ So verstehen beispielsweise Habbershon et al. (2003) das Familienunternehmen als eine Art MetaSystem, das sich aus verschiedenen Subeinheiten zusammensetzt.

"A social system model must, therefore, show how the systemic influences of the system are a product of the continuous interaction of the parts [...]. The family business social system is a >metasystem ‘ comprised of three broad subsystem components: (1) the controlling family unit $[\ldots],(2)$ the business entity $[\ldots],(3)$ the individual family member [...]."

(Habbershon et al., 2003) ${ }^{353}$

Auch jene Beiträge, die wie Tagiuri \& Davis (1996) ihre Überlegungen zur Struktur von Familienunternehmen mit zwei oder drei sich überschneidenden Kreisen illustrieren, folgen der Vorstellung, dass Familienunternehmen aus mehreren, sich überlappenden Subsystemen bestehen. ${ }^{354}$ Kerngedanke ist, dass im Familienunternehmen - als der Schnittmenge der beiden Kreise - die divergenten Systemlogiken der beiden Subsysteme aufeinanderprallen und für die handelnden Akteure Widersprüchlichkeiten und Paradoxien erzeugen. Denn für die handelnden Akteure in Familienunternehmen stünden diese widerstreben-

351 Vgl. Gersick et al. (1997: 4f.); Tagiuri \& Davis (1996: 201). Diese Beiträge halten zur Erfassung der Komplexität aber gar eine Differenzierung der Systeme Unternehmen und Eigentum geboten.

352 Vgl. von Schlippe \& Frank (2013: 84f.): »Although there is no one theory of the family firm as yet, family businesses are usually conceptualized as systems comprising different subsystems." Die meisten der systemtheoretisch orientierten Beiträge knüpfen zumindest implizit an die klassische Definition von Hall \& Fagen (1956: 18) an, der zufolge ein System ein »set of elements" ist. Sie folgen damit eher der Theorie offener Systeme. Vgl. hierzu in der Familienunternehmensforschung Pieper \& Klein (2007).

353 Vgl. Habbershon et al. (2003: 454f.); zu diesem Ansatz auch Chrisman et al. (2005: 567). 354 Vgl. Tagiuri \& Davis (1996); auch Gersick et al. (1997). 
den Systemlogiken häufig gleichberechtigt nebeneinander stehen und könnten meist auch nicht dauerhaft in eine sequenzielle oder hierarchische Ordnung gebracht werden. ${ }^{355}$ So unterlägen Personal- oder Standortentscheidungen nicht nur dem Kosten- oder Professionalisierungsdruck der Branche, sondern seien auch vom Streben der Familie nach Identität, kollektiver Orientierung und nicht zuletzt der eigenen materiellen Versorgung geprägt. Derart bedingte intra- und interpersonellen Dilemmata und Zielkongruenzen seien nicht zuletzt Quell vieler Konflikte. ${ }^{356}$

Die meisten der systemtheoretisch orientierten Beiträge versuchen somit Phänomene des Meta-Systems Familienunternehmens anhand der Interaktion der Subsysteme zu erklären und konzipieren das Ganze größer als die Summe seiner Teile. ${ }^{357} \mathrm{Im}$ Vergleich zu anderen, nicht systemtheoretischen Perspektiven gelingt es ihnen auf diesem Wege, in besonders erhellender Weise die Bedeutung der Interdependenz von Familie und Unternehmen zu erfassen. ${ }^{358}$ So erscheint zum Beispiel das Phänomen der erhöhten Emotionalität aus dieser Perspektive, welche die oben skizzierten Funktionslogiken beider Systeme gleichermaßen integriert, in einem anderen Licht: Während die Emotionalität im Rahmen einer rein ökonomischen Rationalität lediglich irrational erscheint, ist sie nach Einbezug der notwendigen Funktionsweise von Familiensystemen durchaus als funktional im Sinne des Familiensystems zu deuten.

\section{Familie und Unternehmen als operational geschlossene, aber strukturell gekoppelte} Systeme

Der Versuch, die Komplexität des übergeordneten Systems Familienunternehmen durch die Interaktionen untergeordneter, ebenfalls komplexer Systeme zu erklären, führt in der Konsequenz jedoch eher zu einer Steigerung der theoretischen Komplexität als zu deren Reduktion. ${ }^{359}$ Dies gilt insbesondere, wenn

355 Vgl. Simon (2012: 19). Diese Problematik schließt vage Entscheidungsprämissen wie die von Birley et al. (1999) eingebrachte Ordnung von »family rules«, »family out« oder "family business jugglers « nicht aus.

356 Vgl. Gersick et al. (1997: 5); Simon (2012: 28ff.); Tagiuri \& Davis (1996).

357 Vgl. von Schlippe \& Frank (2013: 387); Stafford et al. (1999: $201 \mathrm{ff}$.). Beispielhaft für solche Konzeptionen Pieper \& Klein (2007: 304); Whiteside \& Brown (1991).

358 Vgl. Habbershon et al. (2003: 155); Distelberg \& Sorenson (2009: 66).

359 Vgl. von Schlippe \& Frank (2013:387): »[...] the behaviour of the larger system is somehow to be explained by the coordination of the smaller ones. This may, however, easily lead to theoretical dead ends." Dies gilt erst recht, wenn man reflektiert, dass die Theorie der offenen Systeme Personen mit in die Analyse einbezieht. So werden Phänomene in Familienunternehmen auf die Familie zurückgeführt, deren Phänomene wiederum auf die Interaktion der komplexen Persönlichkeiten usw. Es besteht die Gefahr eines infiniten Regresses. 
theoretisch offen bleibt, wie genau die einzelnen Sub-Systeme miteinander verbunden sind und interagieren. ${ }^{360}$

$\mathrm{Da}$ - im Sinne der jüngeren Forschung zu Familienunternehmen - die vorliegende Arbeit genau diese Verbindung und den Einfluss der Familie auf das Unternehmen untersucht und weniger das große Ganze des Meta-Systems Familienunternehmen, soll zur Beleuchtung dieser entscheidenden Verbindung von Familie und Unternehmen und ihrer Konsequenzen wiederum die Perspektive der neueren Systemtheorie nach Luhmann (1984) eingenommen werden, die dieser Arbeit an verschiedenen Stellen als orientierende Meta-Theorie dient. ${ }^{361}$ So hilft sie das grundlegende Verhältnis zwischen Familie und Unternehmen theoretisch klar und konsistent sowie vor allem verständnisfördernd $\mathrm{zu}$ ordnen.

Im Lichte dieses theoretischen Ansatzes wird zunächst in prägnanter Weise deutlich, dass Familie und Unternehmen nur begrifflich in der Entität des Familienunternehmens verschmelzen, denn aus dem theoretischen Blickwinkel der neueren Systemtheorie müssen Familie und Unternehmen grundlegend als zwei autonome, "operativ geschlossene" und getrennt operierende Systeme begriffen werden.$^{362}$ Die beiden Systeme können sich nicht überlappen, nicht im Familienunternehmen aufgehen und auch kein alles umfassendes Meta-System bilden. Sie sind füreinander stets Umwelt.

Diese »operationale Geschlossenheit « impliziert keineswegs, dass die beiden Systeme jeweils keinerlei Kontakte zur Umwelt - und damit vor allem auch zueinander - mehr hätten. Dies würde jedweder empirischen Erfahrung zuwiderlaufen. Vielmehr bringt das Konzept der operationalen Schließung zum Ausdruck, dass die Systeme ausschließlich auf der Grundlage ihrer eigenen Strukturen und Systemlogiken operieren können. ${ }^{363}$ Sie sind autonom in dem Sinne, dass sie nicht direkt von der Umwelt beeinflusst werden können, sondern alle Irritationen durch die system-spezifischen Strukturen prozessiert werden müssen.

Die Irritationen - als »Stein des Anstoßes« für die Reproduktion und Entwicklung der Systeme - stammen dessen ungeachtet stets aus der Umwelt. Diese

360 Vgl. zu dieser Einschätzung von Schlippe \& Frank (2013: 386f.); Wiechers (2006: $17 \mathrm{ff}$.).

361 Vgl. Luhmann (1984) und auch Abschnitt B.1.2.1. Zur Diskussion der neueren Systemtheorie als theoretische Perspektive auf Familienunternehmen vgl. Frank et al. (2010); von Schlippe \& Frank (2013); Wiechers (2006: $17 \mathrm{ff}$.).

362 Vgl. allgemein: Luhmann (2000: $51 \mathrm{f}$.); zum Verhältnis von Familie und Unternehmen aus dieser Perspektive Simon (2012: 17ff.); Wiechers (2006: 158).

363 Vgl. Luhmann (2000: 52): „Operative Schließung heißt in diesem Falle nicht, dass ein Organisationssystem keine Kontakte mit der [...] Umwelt unterhalten kann«. Vielmehr: »[...] Als Empfänger von Kommunikationen regeln die eigenen Strukturen der Organisation, durch welche Informationen man sich irritieren und zu eigener Informationsverarbeitung anregen lässt«. Zusammenfassend auch Simon (2011: 25). 
ist Motor für eine Veränderung des Systems auf der Grundlage des Systems zu einer neuen Grundlage des Systems. ${ }^{364}$

In der Umwelt der Systeme Familie und Unternehmen finden sich in der Sphäre von Familienunternehmen nun vor allem auch die Systeme Familie beziehungsweise Unternehmen vor, die sich unablässig beobachten und gegenseitig anstoßen. So irritiert die Familie das Unternehmen über ihre Beteiligung an der Unternehmensführung oder die Prägung der organisationalen Kultur. Reziprok irritiert das Unternehmen aber auch das Familiensystem. Denn der private Raum der Familie wird einerseits permanent durch die ökonomische Realität und Rationalität des Unternehmens gestört, das andererseits zugleich sinn- und identitätsstiftend die familiäre Kultur prägt. ${ }^{365}$ Trotz der operationalen Schließung und strukturellen Determiniertheit stehen Familie und Unternehmen also in einer intensiven und permanenten Beziehung zueinander. ${ }^{366}$

Diese Beziehung der permanenten, gegenseitigen Irritation kann mit der neueren Systemtheorie über das Konzept der strukturellen Kopplung theoretisch klar gefasst werden. Maturana \& Varela (1987) verstehen unter einer solchen strukturellen Kopplung im Allgemeinen eine Beziehung folgender Art:

"[Z]wei plastische Systeme werden aufgrund ihrer sequentiellen Interaktion dann strukturell gekoppelt, wenn ihre jeweiligen Strukturen sequentielle Änderungen erfahren, ohne dass die Identität des Systems zerstört wird. Die strukturelle Kopplung zweier unabhängiger strukturell plastischer Einheiten ist daher notwendige Folge ihrer Interaktionen und umso stärker, je mehr Interaktionen stattfinden."

(Maturana) $)^{367}$

Die hier eingenommene Perspektive der neueren Systemtheorie macht somit deutlich, dass Familie und Unternehmen sich als strukturell gekoppelte Systeme zwar intensiv irritieren, dabei aber immer nur auf der Grundlage ihrer jeweils spezifischen »Identität« operieren können. Diese erfährt Änderungen, geht aber niemals in einem übergeordneten System auf.

Diese Konzeption der Verbindung verdeutlicht, warum die vorliegende Arbeit zur Untersuchung der Fragestellung weniger die Entität des Familienunternehmens fokussiert als vielmehr die spezifische Beschaffenheit der Systeme, die

364 Vgl. Baecker (2002: 90); auch Simon (2011: 40).

365 Vgl. zur reziproken Beeinflussung mit einem besonderen Fokus auf die Beeinflussung der Familie durch das Unternehmen Stamm (2013: 86).

366 Im Folgenden wird hinsichtlich dieser Irritationen zur besseren Integration der Arbeit in die Managementforschung und bezüglich der Forschungsfrage meist von »Einfluss« gesprochen. Da die neuere Systemtheorie einen direkten Umwelteinfluss jedoch, wie dargestellt, kategorisch ausschließt, muss an dieser Stelle darauf hingewiesen werden, dass "Einfluss « im hier skizzierten Sinne als permanente Irritation zu verstehen ist.

367 Maturana (1982: $150 \mathrm{f}$.). 


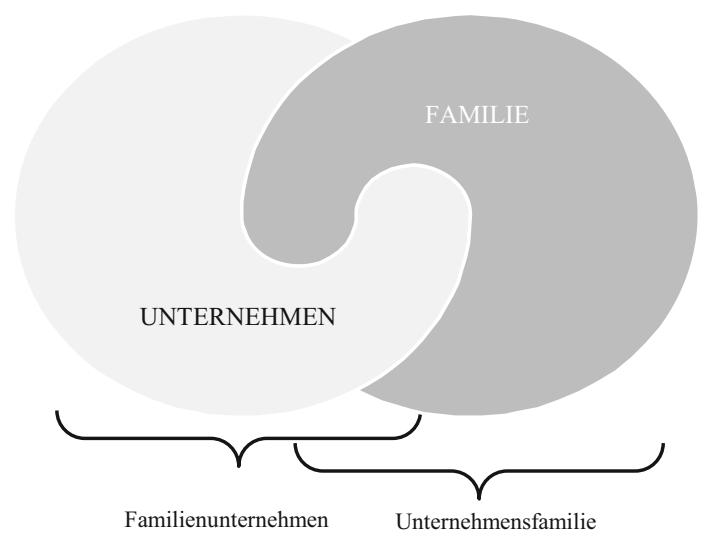

Abbildung 6: Strukturelle Kopplung von Familie und Unternehmen (Quelle: Eigene Darstellung; vgl. Wiechers [2006]).

Art und Weise der gegenseitigen Irritationen und vor allem die strukturellen Auswirkungen dieser Irritationen.

Diese Auswirkungen erwachsen in dynamischer Perspektive aus der KoEvolution der beiden strukturell gekoppelten Systeme. ${ }^{368}$ Unter einer solchen ko-evolutionären Entwicklung versteht die Biologie, aus welcher die Systemtheorie diesen Begriff entlehnt, einen evolutionären Prozess der wechselseitigen Anpassung zweier voneinander unabhängiger, aber stark interagierender und aufeinander einwirkender Arten. ${ }^{369}$ An diese Vorstellung anknüpfend, kann in den Sozialwissenschaften unter der Ko-Evolution zweier Systeme die gemeinsame und gleichzeitige Ausbildung von Strukturmerkmalen sozialer Gebilde verstanden werden.

»Die gleichzeitige und evolutionäre Veränderung in der Ko-Konstitution ist entsprechend eine Ko-Evolution. Dies gilt insbesondere für die gemeinsame und simultane Entwicklung von strukturellen Merkmalen der sozialen Gebilde [...].«

$(\text { Esser, 2002) })^{370}$

In Bezug auf das Verhältnis der Systeme Unternehmen und Familie bedeutet dies, dass die Systeme sich im Verlauf ihrer Entwicklung gegenseitig mit Irritationen und Komplexität konfrontieren, die sie dann auf der Grundlage ihrer jeweiligen Logiken und Strukturen verarbeiten. Die Irritationen können zwar

368 Vgl. von Schlippe \& Frank (2013: 391f.); Wiechers (2006).

369 Vgl. für die Biologie Futuyma (1983).

370 Vgl. Esser (2002: 359). Dieser merkt hierzu in Bezug auf das Beispiel der protestantischen Ethik nach Weber an. »wie dabei die institutionell und materiell geprägten Interessen ihre kulturellen Vorstellungen und Ideen beeinflussen und diese wiederum die Institutionen und Ideen und wie das alles in ein Gleichgewicht und schließlich in eine sich verselbstständigende Eigendynamik geraten kann«. 
nicht strukturdeterminierend in die Systeme eingreifen, beeinflussen aber langfristig gesehen die im System selbst produzierten Strukturen und lösen somit einen »structural drift « aus. ${ }^{371}$ Dieser Drift führt dann im Verlauf der KoEvolution der beiden strukturell gekoppelten Systeme Familie und Unternehmen in der Konsequenz zur simultanen Ausbildung spezifischer struktureller Merkmale. In der Familie bilden sich aufgrund der Irritationen des Unternehmens von diesem System geprägte Merkmale aus und, reziprok dazu, erwachsen im Unternehmen von der Familie geprägte Strukturmerkmale. Die Familie wird somit etwas unternehmensähnlicher und das Unternehmen in der Konsequenz der Ko-Evolution etwas familienähnlicher. ${ }^{372}$ Es entwickeln sich zwei Systeme, die in Referenz zum jeweils anderen System als Unternehmensfamilie und Familienunternehmen bezeichnet werden können.

\section{Definitorisches Verständnis von Familienunternehmen}

Weit über die systemtheoretische Forschung zu Familienunternehmen i.e.S. hinaus, wird die beschriebene dynamische wechselseitige Beziehung zwischen Familie und Unternehmen mittlerweile als das konstitutive Element der Forschung zu Familienunternehmen erachtet. ${ }^{373}$

Sie kann aber auch dazu dienen, die im Fortgang dieser Arbeit untersuchten Unternehmen einer theoretischen Arbeitsdefinition zuzuführen. So sollen im Sinne des in den letzten beiden Unterkapiteln dargelegten systemtheoretischen Verständnisses Familienunternehmen in dieser Arbeit folgendermaßen begriffen werden:

Als Familienunternehmen sollen jene Unternehmen bezeichnet werden, die mit einem Familiensystem mehr oder weniger intensiv verschränkt und strukturell gekoppelt sind, und sich mit diesem durch wechselseitige Irritationen über formelle und informelle Mechanismen ko-evolutionär entwickeln.

(Arbeitsdefinition)

Diese theoretische Definition ermöglicht eine für vorliegende Arbeit ausreichende theoretische Differenzierung gegenüber anderen verwandten Formen ökonomischer Aktivität wie dem »lone-founder «. ${ }^{374}$ Zudem lassen sich all jene in der empirischen Studie untersuchten Unternehmen auf dieser Grundlage eindeutig als familiär beeinflusste Unternehmen verstehen.

Das systemtheoretisch orientierte Verständnis des graduellen familiären

371 Vgl. Luhmann (2000: 397).

372 Vgl. von Schlippe \& Frank (2013: 391); Wimmer et al. (2005: 16ff.).

373 Vgl. Sharma (2004: 9): "The intertwinement and reciprocal relationship between the family and business systems is being recognized as the key feature distinguishing this field of study from others. " Auch Habbershon et al. (2003: 452); Sharma (2013: 7); Litz et al. (2012: 28).

374 Dieser Unterschied wird mittlerweile auch von der allgemeinen Managementforschung stark hervorgehoben; vgl. Cannella et al. (2015). 
Einflusses stellt vor allem eine geeignete Perspektive dar, um sich den Strukturmerkmalen von Familienunternehmen anzunähern, die aus der Ko-Evolution der beiden Systeme und dem Einfluss der Familie auf das Unternehmen erwachsen. Bevor diese strukturellen Merkmale ausführlich dargestellt werden, soll zunächst das in diesem Abschnitt dargelegte systemtheoretische Verhältnis zwischen Familie und Unternehmen auf spezifische Kanäle des familiären Einflusses heruntergebrochen und präzisiert werden.

\subsubsection{Der Einfluss der Familie über die Kanäle »Macht«, »Generation« und $\gg$ Kultur«}

Zur Erfassung des familiären Einflusses auf Unternehmen führten Astrachan et al. (2002) eine ausführliche Inhaltsanalyse verschiedenster Definitionsansätze der Familienunternehmensforschung durch und destillierten die wesentlichen Merkmale. Auf dieser Grundlage konzipierten sie den formellen und informellen Einfluss der Familie im Rahmen ihrer »F-PEC-Scale« anhand der drei Dimensionen "power«, "experience« und »culture.$^{375}$

In Anknüpfung an das graduelle Verständnis des familiären Einflusses machen die Autoren deutlich, dass das System Familie das System Unternehmen in verschiedener Weise und unterschiedlich stark irritieren kann, zum Beispiel indem die Familie über den Kanal der Macht ihre Eigentümerrechte ausübt, direkt über die Leitung des Unternehmens dessen strategische Entwicklung steuert oder durch informelle Mechanismen wie die Tradierung kultureller Muster das Wertegerüst und Normengefüge des Unternehmens prägt.

\section{Einflusskanal »Macht«}

Der Einflusskanal der Macht bezieht sich auf jenen Einfluss auf das Unternehmen und dessen Entscheidungsprozesse, welchen die Familie über das Eigentum am Unternehmen, die Führung des Unternehmens oder die Steuerung dessen Governance besitzen kann. ${ }^{376}$ Dabei soll Macht im Weberschen Sinne verstanden werden als »jede Chance, innerhalb einer sozialen Beziehung den eigenen Willen

375 Vgl. Astrachan et al. (2002); Klein et al. (2005). Schlussendlich soll so der gesamte familiäre Einfluss abgeschätzt oder gar, wie von Astrachan et al. (2002) vorgeschlagen, über die additiven und wechselseitig austauschbaren Kanäle anhand der Skala quantifiziert werden. Nach mehrmaligen Tests kann die Skala als ein zuverlässiges Instrument zur Messung oder Bewertung des Ausmaßes und der Qualität von familiärem Einfluss auf das Unternehmen betrachtet werden. Vgl. hierzu: Holt et al. (2010); Klein et al. (2005: 321 ff.); Rutherford et al. (2008). Im Folgenden sollen die Kanäle als »Macht«, "Generation« und »Kultur» bezeichnet werden.

376 Vgl. Astrachan et al. (2002: 48). 
auch gegen Widerstreben durchzusetzen, gleichwohl, worauf diese Chance beruht . $^{377}$

Fundament dieser Macht im und über das Unternehmen ist das Eigentum am Unternehmen. ${ }^{378}$ Das Eigenkapital der Familie übernimmt eine Haftungsfunktion und dient durch Kreditsicherung der Finanzierung und Existenzsicherung des Unternehmens. Mit dieser Unsicherheitsreduktion begründet es in seiner Herrschaftsfunktion den Leitungsanspruch der Familie und deren Autonomie als Eigentümer. Diese Autonomie befähigt die Familie, die Führung des Unternehmens selbst zu übernehmen oder diese an von ihr bestimmte angestellte Führungskräfte zu übergeben. ${ }^{379}$ Grundsätzlich ist die Stärke des Einflusses abhängig von der Höhe des Anteils, den die Familie am Eigenkapital des Unternehmens hält. Allerdings machen Anderson \& Reeb (2003) darauf aufmerksam, dass der faktische Einfluss der Familie häufig meist weit größer sei, als es der reine prozentuale Anteil am Eigentum vermuten ließe. ${ }^{380}$

Diese Beobachtung lässt auf die Bedeutung weiter gehender Einflussmöglichkeiten schließen: die Übernahme der Unternehmensführung durch die Familie, aber vor allem auch die Möglichkeiten der Einflussnahme über die Governance des Unternehmens. Denn die Struktur der Kontrolle und Aufsicht wird nicht nur maßgeblich von der Eigentümerfamilie gestaltet, sondern zumeist auch von dieser ausgeübt. Hierbei sind häufig spezifische Institutionen außerhalb des organisationsstrukturell-formellen Rahmens, wie Gesellschafterkreise oder Familienräte, von Bedeutung für den strategischen Entscheidungsprozess. ${ }^{381}$

Zwischen diesen Organen und Institutionen entstehen oft diffuse Beziehungsgeflechte, innerhalb derer eine Vielzahl verschiedener Akteure auf verschiedenen Positionen innerhalb und außerhalb der Organisation am strategischen Entscheidungsprozess beteiligt ist. Da die Grenzen in der Regel fließend sind, bietet es sich für die Analyse des familiären Einflusses an, nicht von formellen organisationalen Konstrukten wie dem Topmanagement-Team (TMT) auszugehen, sondern auf das Konzept der dominanten Koalition zu rekurrie-

377 Vgl. Weber (1972: 28). Ähnlich auch Dahl (1957: 202f.); vgl. Pfeffer (1981: 2 ff.).

378 So konstatiert Gutenberg (1976: 488): »In einem das Privateigentum an den Produktionsmitteln grundsätzlich anerkennenden Gesellschaftssystem verkörpern Eigentumsrechte Konzentration von Einfluss und Macht in den Betrieben.« Vgl. auch Felden \& Hack (2014: 154); Finkelstein (1992: 509).

379 Vgl. zu Funktionen des Eigenkapitals Gräfer et al. (2014: 64 ff.). Zur Bedeutung der Unsicherheitsreduktion als Grundlage von Macht vgl. Pfeffer (1981: 109ff.)

380 So ist der Anteil der Familie an Sitzen im Board in ihrer Studie der S\& P 500 Unternehmen 2,75 mal größer, als es der Eigenkapitalanteil nahelegen würde. Vgl. Anderson \& Reeb (2003: 1302).

381 Vgl. Felden \& Hack (2014: 287ff); Klein (2010: 140ff.); Koeberle-Schmidt (2008). 
ren. ${ }^{382}$ Dieser dominanten Koalition sollen all jene mehr oder weniger mächtigen Akteure zugerechnet werden, welche die organisationale Agenda kontrollieren und schlussendlich die strategischen Richtungsentscheidungen des Unternehmens treffen. Gewöhnlich gehören zu dieser Gruppierung die Geschäftsführung des Unternehmens, einige wichtige Führungskräfte der zweiten Hierarchieebene sowie die Protagonisten der Governance aus den Kontroll- und Aufsichtsorganen oder dem Gesellschafterkreis. In der Verantwortung dieser machtvollen Gruppe liegt es, Organisation und Umwelt auf Möglichkeiten und Probleme zu untersuchen, relevante Informationen $\mathrm{zu}$ interpretieren und entsprechende strategische Reaktionen und Initiativen auszulösen. Daher sind die Entscheidungsprozesse solcher »inner circle[s] (Finkelstein, 1992) von Familienunternehmen die zentrale Analyseeinheit vorliegender Arbeit. ${ }^{383}$

Über den unmittelbar aus dem Eigentum entspringenden Einfluss auf das Unternehmen hinaus ist das Eigentum aber auch das entscheidende Scharnier der strukturellen Kopplung und Ko-Evolution zwischen den beiden Systemen. ${ }^{384}$ Denn reziprok zum Einfluss der Familie auf das Unternehmen bildet das Eigentum am Unternehmen auch die Grundlage der materiellen Existenz der Familie. Zugleich bindet es die Familienmitglieder auf lange Sicht und intensiv aneinander und wird somit in dynamischer Perspektive zum entscheidenden Bindeglied zwischen der Entwicklung der Familie und der Entwicklung des Unternehmens. Diese Entwicklung wird aber nicht nur durch den Einflusskanal der Macht« irritiert, sondern auch durch jenen der »Generation«.

\section{Einflusskanal »Generation«}

Weniger offensichtlich als der machtvolle Einfluss der Familie und der um sie herum gruppierten dominanten Koalition, aber nicht minder bedeutsam sind

382 Vgl. Cyert \& March (1963); Hambrick \& Mason (1984: 196); Thompson (1967: 128):»[...] individuals who collectively have sufficient control of organizational resources to commit them in certain directions and to withhold them from others. « Zur Verwendung dieser Konzeption in der Familienunternehmensforschung vgl. Chua et al. (1999: 24). Zur Erörterung und Operationalisierung des Begriffs vgl. Carpenter et al. (2004: 759), denen zufolge er in der Managementforschung früher enger gefasst wurde. Heute umfasst er zum Beispiel auch Aufsichtsräte. Für Nielsen (2010: 306) liegt gerade in der Untersuchung der Interdependenz von CEO, TMT und Kontrollorgan ein großes Forschungspotenzial.

383 Vgl. Finkelstein (1992: 506); Wiersema \& Bantel (1992: 91f.) zu den Aufgaben der dominanten Koalition hinsichtlich des strategischen Wandels.

384 Vgl. Gersick et al. (1997: 7); Stamm (2013: 44). Das Eigentum wird in Anlehnung an Tagiuri \& Davis (1996) häufig gar als drittes System neben Unternehmen und Familie erachtet; vgl. von Schlippe \& Frank (2013: 394f.). Da das in vielen Unternehmen aber keineswegs als eigenständiges System beobachtet wird (vgl. Simon (2012: 24)), wird dieser Konzeption hier nicht gefolgt. 
die Irritationen, die hier im Rahmen des Einflusskanals der "Generation« skizziert werden. ${ }^{385}$

Unternehmensfamilien ist häufig eine typische Auffassung von Zeit inhärent, welche mit »Denken in Generationen « umschrieben werden kann. ${ }^{386}$ Über diesen transgenerationalen Kontinuitätsanspruch irritieren sie das Unternehmen und dessen Entscheidungsprozesse.

Auf die Bedeutung dieses familiären Strebens nach Dauerhaftigkeit und einer transgenerationalen Kontinuität von Eigentum und Kontrolle verweisen zahlreiche definitorische Ansätze zu Familienunternehmen. ${ }^{387}$ So ist für Chua et al. (1999) dieses Kontinuitätsstreben nicht nur Teil der »Essenz« von Familienunternehmen, sondern gar distinktes Definitionskriterium:

»The family business is a business governed and/or managed with the intention to shape and pursue the vision of the business held by a dominant coalition [...] in a manner that is potentially sustainable across generations."

(Chua et al., 1999) $)^{388}$

Jede Familie hat einen natürlichen transgenerationalen Bezug. In Unternehmensfamilien wird teilweise jedoch eine dynastische Perspektive auf die Familie und das Unternehmen gepflegt. ${ }^{389}$ Die Familienmitglieder reihen sich bewusst ein in eine Ahnenfolge, deren vergangene Generationen bis an die persönliche Identität der Entscheidungsträger heranrücken können. In diesem Rahmen wird das übernommene Unternehmen als ein Vermächtnis verstanden, welches es pflichtbewusst zu verwalten und für kommende Generationen $\mathrm{zu}$ bewahren gilt. ${ }^{390}$

»The concept of dynasty [...] suggests that the inheritance, in the form of the firm, is construed not as something that future generations are intended to consume, but as something that they are intended to preserve intact and transmit onward. Future generations are conceived as stewards of their inheritance, and are under a duty to conserve it, and if necessary enhance it, but without substantially changing its form.« (Casson, 1999) ${ }^{391}$

In vielen Unternehmensfamilien besteht zwischen den Generationen nicht nur über die familiäre Abstammungslinie eine Verbindung, sondern vor allem auch

385 Dieser Einflusskanal wird grundlegend anders als bei Astrachan et al. (2002) konzipiert, welche die aus vorherigen Generationenübergangen resultierende und kumulierte Erfahrung fokussieren.

386 Vgl. Jäkel-Wurzer (2010: 24); Stamm (2013: 88f.).

387 Vgl. Ward (1987: 252): »[A] business that will be passed on for the family's next generation to manage and control.« Vgl. auch Churchill \& Hatten (1997: 54); Handler (1989: 260).

388 Chua et al. (1999: 25); vgl. auch Kets de Vries (1993: 313).

389 Vgl. Casson (1999); Klein (2010: 68ff.).

390 Vgl. Handler (1994); Stamm (2013).

391 Casson (1999: 17). 
über ein gemeinsames, transgenerationales Projekt: dem Wunsch, über Generationen erfolgreich zu sein. Diese Ambition der Familie und ihrer Mitglieder wird im Momentum der Nachfolge häufig zu einem "shared dream « (Lansberg, 1999) der Generationen verwoben. ${ }^{392}$ In dieser Perspektive werden auch die der Unternehmensfamilie angehörigen Führungskräfte des Unternehmens weniger als vollkommen autonome Eigentümer betrachtet denn als Verwalter des dynastischen Erbes, die das Unternehmen zum Wohle des familiären Kollektivs in die Zukunft führen sollen.

Dieses transgenerationale Kontinuitätsstreben der Familie kann die Entwicklung des Unternehmens durch formelle wie informelle Mechanismen irritieren. So kann es die strategische Entwicklung durch eine langfristige strategische Zielplanung prägen, aber auch durch organisationale Mythen und Symbole, die Kontinuität und Verlässlichkeit zum Ausdruck bringen sollen.

Das transgenerationale Kontinuitätsstreben nimmt hinsichtlich des Einflusses der Familie somit eine Art temporäre und inhaltliche Scharnierfunktion ein: Es verbindet Historie und Zukunft, vergangenen, gegenwärtigen und zukünftigen Einfluss der Familie. Dabei ergibt es sich aus der bestehenden Macht der Familie im und über das Unternehmen, ist aber zugleich Grundlage für deren fortgesetzte Kontrolle über das Unternehmen und kulturelle Prägkraft.

\section{Einflusskanal »Kultur«}

Neben dem Einfluss über Macht und die Vermittlung eines spezifischen zeitlichen Horizontes auf das Unternehmen kann die Familie aufgrund ihrer zentralen Stellung das Unternehmen auch maßgeblich über kulturelle Muster prägen.

So beeinflusst der "Geist» (Kets de Vries, 1993) der Familie die im Unternehmen vorherrschenden Einstellungen, Werte und Normen. ${ }^{393}$ Es sind maßgeblich die Ziele oder auch die Sprache und die Narrationen der Familie, an denen sich nach und nach die organisationale Identität des Unternehmens und dessen Kultur ausrichtet. ${ }^{394}$

Unter einer solchen Kultur soll dabei ein spezifisches Set an gemeinsam geteilten Überzeugungen, Werten und Symbolen verstanden werden. ${ }^{395}$ Diese haben sich im historischen Entwicklungsverlauf der Organisation herausgeschält, wurden in Lernprozessen als gut und funktional erachtet und prägen

392 Vgl. Lansberg (1999); auch Gersick et al. (1997: 194); Simon et al. (2012: 134); Stamm (2013: 89).

393 Vgl. Kets de Vries (1993), auch Schein (1983) und für eine Übersicht Felden \& Hack (2014: $57 \mathrm{ff}$.$) .$

394 Vgl. Astrachan et al. (2002: 45); Sirmon \& Hitt (2003: 342f.).

395 Vgl. zu den folgenden allgemeinen Ausführungen zur Organisationskultur Schreyögg (2006: $448 \mathrm{ff}$.). 
nun das Verhalten der Organisationsmitglieder informell, aber nachhaltig. Als meist unreflektierte, aber von den Organisationsmitgliedern verinnerlichte Annahmen und Orientierungsmuster liegen sie dem Handeln in der Organisation zugrunde, strukturieren Wahrnehmungen und Interpretationen, und vermitteln durch die Bereitstellung eines Referenzsystems Sinn und Orientierung. ${ }^{396}$ Durch Praktiken, Rituale und Symbole werden sie vermittelt und neuen Organisationsmitgliedern nahegebracht.

Der Einfluss der Familie auf die Organisationskultur des Unternehmens nimmt ihren Anfang beim Gründer, der das soziale System Organisation begründet und kraft seiner Persönlichkeit und machtvollen Position gleichzeitig die Kultur der Organisation zu formen beginnt. Durch die implizite Vermittlung seiner persönlichen, meist recht stark akzentuierten Grundannahmen und Werte stiftet er Sinn und Orientierung. ${ }^{397}$ So hat er bereits klare Vorstellungen davon, ob die Entscheidungsprozesse hierarchisch oder eher demokratisch organisiert werden sollen, und wahrscheinlich hat er auch bereits bedeutsame Erfahrungen hinsichtlich der Frage gemacht, ob man »Fremden « vertrauen kann oder nicht. Entsprechend dieser Erfahrungen wird er Antworten auf die drängendsten Probleme der Umweltinteraktion und organisationalen Integration liefern. Dabei kommuniziert der Gründer seine Einstellungen und Werte über seine Handlungen, artikuliert diese explizit oder bringt sie durch Symbole zum Ausdruck. ${ }^{398}$ In jedem Fall legt er über solche informellen Einflüsse das Fundament der sich entwickelnden Organisationskultur.

Aufgrund seiner meist recht langen Verweildauer im Unternehmen kann der Gründer aber auch in der Folgezeit eine anhaltende kulturelle Wirkkraft ausüben. ${ }^{399}$ Über die persönliche Präsenz im Unternehmen hinaus ergibt sich die kulturelle Prägkraft des Gründers aber auch aus dessen Möglichkeit, die ersten Mitarbeiter des Unternehmens entsprechend seines persönlichen Wertegerüsts auszuwählen. Über diese kulturell komplementären Mitarbeiter verfestigt sich die Kultur weiter. ${ }^{400}$

Nichtsdestotrotz ist die Kultur einer Organisation niemals statisch, sondern

396 Schein (1985) zufolge beruhen solche Kulturen auf unsichtbaren und meist unreflektierten Basisannahmen, die Kern und Fundament der Organisationskultur bilden. Aus Annahmen über die Umwelt, die Natur des Menschen oder zwischenmenschlicher Beziehungen erwächst ein »Weltbild« und kulturelles Muster, welches wiederum in konkreten und bewussteren Wertvorstellungen und Normen seinen Niederschlag findet.

397 Vgl. Athanassiou et al. (2002: 140ff.); Dyer (1988: 39); Gersick et al. (1997: 149); Nelson (2003: $707 \mathrm{ff}$.$) ; Schein (1983: 13ff.).$

398 Vgl. die Übersicht der informellen Mechanismen bei Schein (1983: 22).

399 Vgl. Sharma (2004: 10).

400 Vgl. Berrone et al. (2010: 263); Eddleston (2008: 1056). 
stets dynamisch. ${ }^{401}$ Sie spiegelt stets den Prozess der komplexen Interaktion zwischen den vorhandenen Grundannahmen, Werten und Normen auf der einen Seite und dem Lernprozess der Organisation im Kontext ihrer Herausforderungen auf der anderen Seite wider.

In diesem dynamischen Prozess wirkt das System Familie - als soziale Institution, die seit Jahrtausenden verlässlich Werte und Normen von Generation zu Generation weiterreicht - nun häufig als Anker und stabilisierender Garant bestimmter kultureller Muster. ${ }^{402}$ So weist Schein (1983) darauf hin, dass Werte wie das Streben nach Unabhängigkeit in einem signifikant stärkeren Maße in der Organisation reproduziert werden, wenn Familienmitglieder im Unternehmen in leitender Position tätig bleiben. ${ }^{403} \mathrm{Da}$ familiäre Normen, sofern sie nicht grundsätzlich infrage gestellt werden, meist unbewusst von der nachfolgenden Generation im Zuge ihrer Sozialisation übernommen werden, trägt die Familie so zum Beispiel zur Pflege der kulturellen Wurzeln bei. Diese Übertragung von historisch gewachsenen Überzeugungen, Werten und Zielen durch die Familie führt zur Herausbildung von relativ stabilen kulturellen Mustern innerhalb des Unternehmens. ${ }^{404}$

\subsection{Die strukturellen Merkmale des familiären Einflusses in der Organisation}

In den beiden obigen Unterkapiteln wurde verdeutlicht, dass es für die Untersuchung des Einflusses des Faktors Familie auf organisationale und strategische Phänomene sinnvoller ist, weniger die alle Aspekte und Eigenschaften vereinende Gesamtheit des Familienunternehmens zu fokussieren als vielmehr den spezifischen Einfluss, welchen die Familie auf das Unternehmen ausübt. Dieses grundlegende Verständnis des Verhältnisses zwischen Familie und Unternehmen wurde entsprechend systemtheoretisch konzipiert und die Kanäle der wechselseitigen Irritationen wurden skizziert. So sollen Familie und Unternehmen im Fortgang der Arbeit als zwei autonome, operativ geschlossene soziale Systeme verstanden werden, die sich aber derart intensiv und permanent irritieren, dass man von einer strukturellen Kopplung und Ko-Evolution der Systeme sprechen kann.

401 Vgl. Schreyögg (2006: 451), der solche Kulturen als das Ergebnis historischer Lernprozesse beschreibt.

402 Vgl. Gersick et al. (1997: 149); Nave-Herz (2014: $3 \mathrm{ff}$.); Stamm (2013: 33).

403 Vgl. Schein (1983: 27); auch Grundström et al. (2012) gehen davon aus, dass in der Nachfolge des Familienunternehmens durch Familienangehörige ein Großteil der für Familienunternehmen spezifischen Werte tendenziell erhalten bleibt. Weiterführend: GarciaAlvarez et al. (2002: 189ff.); Sharma \& Salvato (2013: 43).

404 Vgl. Dyer (1988: 46); Hall et al. (2001: 195); Simon et al. (2012: 135). 
Durch die wechselseitige Irritationen über die Kanäle »Macht«, »Generation« und »Kultur« erwächst in Familien, welche mit einem Unternehmen strukturell gekoppelt sind, im Verlauf der Ko-Evolution eine Vielzahl struktureller Besonderheiten. ${ }^{405}$ So lassen sich in solchen Unternehmensfamilien häufig eigene Formen einer »unternehmerischen « Familienkultur beobachten, in der Leistung und Disziplin einen hohen Wert besitzen. Nicht selten können spezifische zeitliche Konstruktionen und ein transgenerationaler, beinahe dynastischer Kontinuitätsanspruch registriert werden. Des Weiteren führt das Eigentum am Unternehmen durch seine zentripetalen Bindungskräfte in entwicklungsdynamischer Perspektive aber auch zu sehr spezifischen Familienformen wie der quasi-dynastischen Großfamilie. ${ }^{406}$ Angesichts solcher, aus der strukturellen Kopplung mit dem Unternehmen erwachsenden Charakteristika bezeichnet Hildenbrand (2002) Unternehmensfamilien gar als Familien »eigener Art «. ${ }^{407}$

Die vorliegende Arbeit der Managementforschung fokussiert vor dem Hintergrund der leitenden Forschungsfrage nach dem Einfluss des Faktors Familie auf die Genese und Dynamik strategischer Pfade jedoch in erster Linie jene strukturellen Merkmale, welche die Ko-Evolution im Unternehmen hinterlässt. Diesen Strukturmerkmalen soll sich nun zum Abschluss dieses Kapitels gewidmet werden.

Dabei kann es nicht das Ziel dieses Unterkapitels sein, die Auswirkungen des familiären Einflusses auf das Unternehmen in all seinen Facetten und Schattierungen darzustellen. Vielmehr wird sich auf jene Merkmale konzentriert, die von der Forschung zu Familienunternehmen regelmäßig beobachtet wurden, als besonders bedeutsam für strategische Entscheidungsprozesse erachtet werden, und größtenteils auch in der explorativen Vorstudie identifiziert werden konnten. $^{408}$

In dem in dieser Arbeit gefolgten graduellen Verständnisses des familiären Einflusses dürfen diese Strukturmerkmale natürlich keineswegs als Kriterien zur binären Abgrenzung von Familien- und Nicht-Familienunternehmen

405 Vgl. zu diesen Charakteristika Simon (2002), vor allem auch Stamm (2013: 31). Die Besonderheiten von Unternehmensfamilien treten in der bisherigen Forschung allerdings stark hinter den Spezifika von Familienunternehmen zurück; vgl. Sharma et al. (2012: 10). Insbesondere die Erkenntnisse der etablierten Familienforschung müssten stärker integriert werden. Zur fehlenden Brücke zwischen der Forschung zu Familien und Familienunternehmen vgl. Dyer Jr. \& Dyer (2009); James et al. (2012).

406 Vgl. Gersick et al. (1997); Wiechers (2006: 189). Bereits Ward (1987) skizzierte eine idealtypische, am Eigentum orientierte Entwicklungslinie vom Gründerunternehmen über das von den Erben weitergeführte Unternehmen bis hin zum großen Unternehmen, das einer Familiendynastie gehört.

407 Vgl. Hildenbrand (2002).

408 Vgl. für Übersichten zu diesen Merkmalen Wimmer et al. (2005: $93 \mathrm{ff}$.), die systemtheoretisch orientierte Diskussion bei Wiechers (2006: $244 \mathrm{ff}$.), die psychologisch orientierte Darstellung bei Kets de Vries (1993: 314). 
missverstanden werden. ${ }^{409}$ Ein solches Vorgehen wäre allein schon deshalb kritisch, weil bestimmte Merkmale zwar spezifisch für den familiären Einfluss, aber keineswegs immer exklusiv auf diesen zurückführbar sind. So führt beispielsweise die Konzentration von Eigentum in den Händen der Familie zwar häufig auch zu einer Konzentration der Entscheidungsgewalt in der Leitung des Unternehmens. Dieses Merkmal der Zentralisierung kann in anderen organisationalen Kontexten aber auch in gänzlich anderen Faktoren begründet sein. Daher sollen diese Strukturmerkmale als spezifische Merkmalsausprägungen allgemeiner organisationaler Variablen - wie der Grad an Zentralisierung der Entscheidungsstrukturen - verstanden werden, deren regelmäßig beobachtbare Ausprägungen in bestimmten organisationalen Kontexten ebenso regelmäßig auf den familiären Einfluss zurückzuführen sind.

Zudem ist es stets von Ausmaß und Form des familiären Einflusses abhängig, wie dieser solche allgemeinen organisationalen Variablen im Einzelfall konkret ausprägt. Das bedeutet, dass die im Folgenden zu skizzierenden Manifestationen des familiären Einflusses nicht in jedem Familienunternehmen in gleicher Weise und zu gleichen Maßen beobachtet werden können. Vielmehr geht die vorliegende Arbeit in Anknüpfung an die Prämisse jüngerer Beiträge der Managementforschung zum Faktor Familie davon aus, dass ein marginaler Anstieg des familiären Einflusses zu einem Anstieg der zu beobachtenden Manifestationen dieses Einflusses insgesamt führt. ${ }^{410}$ Wie sich der Anstieg dieses familialen Einflusses im Einzelfall aber konkret niederschlägt, hängt jedoch generell von der spezifischen Art und Weise der gegenseitigen Irritation und strukturellen Kopplung zwischen Familie und Unternehmen ab. ${ }^{411}$ Ist ein Unternehmen in erster Linie über den Kanal »Macht" mit einer Familie verbunden, so wird sich dieser Umstand wahrscheinlich in einer stärker zentralisierten Organisationsstruktur niederschlagen. Ist ein Unternehmen aufgrund einer besonderen emotionalen, personalen oder räumlichen Nähe zur Eigentümerfamilie auch kulturell eng mit dieser verbunden, so werden sich in vielen Fällen auch besondere organisationskulturelle Phänomene wie die kollektive Wahrnehmung einer »Betriebsfamilie« beobachten lassen.

Die Annahme solcher charakteristischer Merkmalsausprägungen ist eine der

409 Vgl. Sharma (2004:5) zu den bisher erfolglos gebliebenen Versuchen solche separierenden Variablen zu finden; auch Astrachan et al. (2002: 46f.).

410 Die Arbeit schließt sich hier u. a. der grundlegenden Prämisse von König et al. (2013: 422) an, die ihren Überlegungen zum Einfluss der Familie voranstellen: »A key premise of our research is that continuity, command, community, and connections are reflective, covariant indicators of family influence. In other words, we assume that a marginal increase in family influence entails a marginal increase in all four domains, although this increase is not necessarily equally distributed."

$411 \mathrm{Vgl}$. Astrachan et al. (2002: $47 \mathrm{ff}$.); König et al. (2013: 422). 
fundamentalen legitimatorischen Grundlagen der Managementforschung zu Familienunternehmen. Weisen sie doch darauf hin, dass die Ergebnisse der allgemeinen Managementforschung in der Analyse organisationaler Zusammenhänge nicht unisono auf jene Unternehmen übertragen werden können, in deren Kontext eine Familie maßgeblich die Entscheidungsprozesse und kulturellen Muster des Unternehmens irritiert. Diese Einschränkung trifft vor allem auf die Analyse strategischer Entscheidungsprozesse zu - sind die Systeme Familie und Unternehmen an der Spitze der Organisation doch meist besonders eng gekoppelt und bilden so einen spezifischen Kontext des strategischen Entscheidungsprozesses. ${ }^{412}$

Die auf diesen besonderen Kontext verweisenden Merkmalsausprägungen sollen somit im Folgenden als reflexive Indikatoren des familiären Einflusses betrachtet werden und die Analyse des familiären Einflusses auf die Genese und Dynamik strategischer Pfade orientieren.

Doch dürfen diese strukturellen Merkmale des familiären Einflusses keineswegs als unmittelbare besondere Stärken oder Risiken von Familienunternehmen begriffen werden. Aus den Strukturmerkmalen des familiären Einflusses können nämlich sowohl besondere Stärken und Wettbewerbsvorteile, als auch spezifische Risiken erwachsen. So kann eine hohe Entscheidungsautonomie in einer stark zentralisierten Organisationsstruktur auf der einen Seite zu kurzen strategischen Reaktionszeiten führen. Auf der anderen Seite erhöht diese Machtkonzentration jedoch auch die riskante Abhängigkeit von den Kognitionen und Handlungen einzelner Entscheidungsträger. Angesichts dieser immanenten Polarisierung spricht die Familienunternehmensforschung von der "Janusköpfigkeit" solcher Strukturmerkmale, aus der eine große Nähe von spezifischen Chancen und Risiken familiär beeinflusster Unternehmen resultiert. Oftmals tragen die besonderen Stärken dieser Form ökonomischer Aktivität gar bereits den Kern ihrer Schwäche in sich, welche unter veränderten Rahmenbedingungen ihr negatives Potenzial entfalten können. ${ }^{413}$

Im Folgenden sollen diese Strukturmerkmale im klassifizierenden Rahmen des "4 C’s framework« nach Miller \& LeBreton-Miller (2005) skizziert werden, der die die Reflexionen des familiären Einflusses anhand von vier Dimensionen unterscheidet: "command «, »continuity», "community« und »connections «. ${ }^{414}$

412 Vgl. für einen Überblick zur Forschung zu strategischen Entscheidungsprozessen in familiär beeinflussten Unternehmen Ibrahim et al. (2008); Sharma et al. (1997); Zahra \& Sharma (2004).

413 Diese immanente Tendenz zu extremen Ausformungen schlägt sich häufig auch in der finanziellen Performance nieder. Vgl. Voigt (1990: 64). Wimmer et al. (2005: 95) verweisen mit Blick auf die »Janusköpfigkeit« darauf, dass Familienunternehmen entweder besonders "gut", oder besonders "schlecht" sind, aber selten bloßer Durchschnitt.

414 Vgl. Miller \& LeBreton-Miller (2005), die über diese Dimensionen die Merkmale besonders 


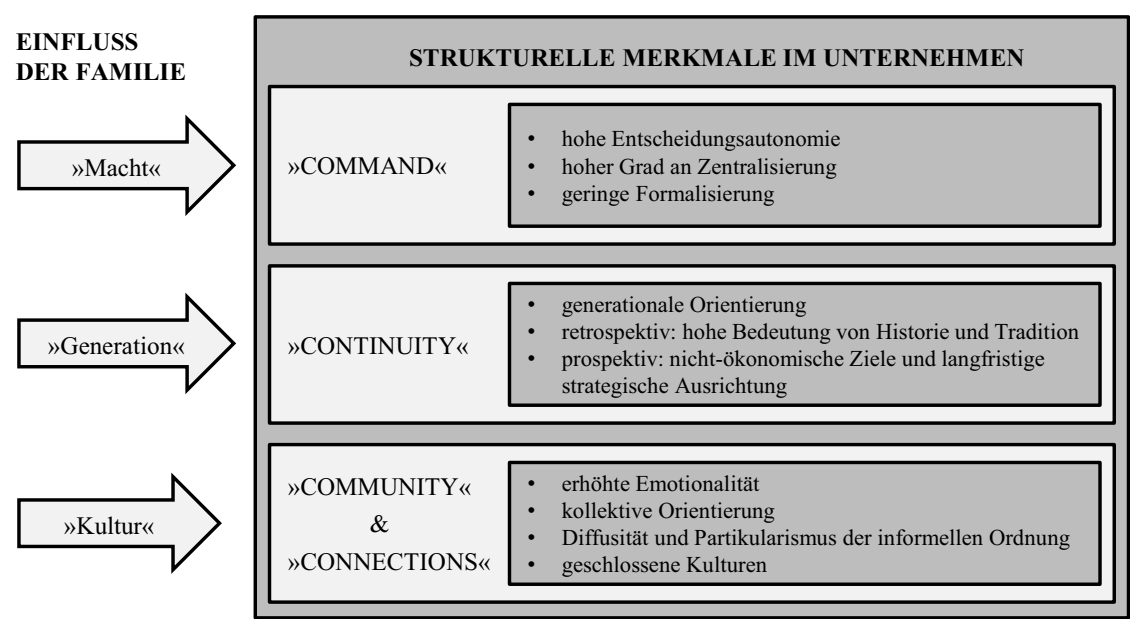

Abbildung 7: Einfluss der Familie im Verlauf der Ko-Evolution und Ausprägung organisationaler Strukturmerkmale

(Quelle: Eigene Darstellung).

\subsection{1 »Command «: Entscheidungsautonomie, Zentralisierung und Personenorientierung}

Von der bisherigen Forschung zu Familienunternehmen wird regelmäßig beobachtet und beschrieben, dass primär der aus dem konzentrierten Eigentum am Unternehmen resultierende familiäre Einfluss zu einer ausgeprägten Entscheidungsautonomie und hohen "managerial discretion « (Hambrick \& Finkelstein, 1987) der strategischen Entscheidungsträger der dominanten Koalition führt. Diese Facetten des familiären Einflusses fassen Miller \& LeBreton-Miller (2005) in ihrer Kategorie des "Command « zusammen. ${ }^{415}$

Die über diese Dimension adressierte Unabhängigkeit und Entscheidungs-

langlebiger und erfolgreicher Familienunternehmen beschreiben. Nicht zuletzt die augenscheinliche Nähe zu den gesammelten Definitionsmerkmalen und den Einflusskanälen nach Astrachan et al. (2002) verweisen aber darauf, dass diese Dimensionen auch zur Kategorisierung der Strukturmerkmale aller familiär beeinflussten Unternehmen herangezogen werden können. Vgl. zur Rezeption dieser Dimensionen beispielsweise König et al. (2013: 422).

415 Vgl. zur »managerial discretion« Hambrick \& Finkelstein (1987), aber auch das Modell der Pfadgenese nach Sydow et al. (2009) in Kapitel B.1.3.2, die über die »managerial discretion" den verbleibenden Handlungsspielraum der organisationalen Entscheidungsträger im Zuge der Pfadentstehung konzipieren. Zur erhöhten Entscheidungsautonomie der Entscheidungsträger in Familienunternehmen vgl. Miller \& LeBreton-Miller (2005: 47); auch Colli (2013: 86); Hatum \& Pettigrew (2006: 125); Kets de Vries (1993: 62). Zur Bedeutung des Eigentums, welches dieser Autonomie fundamental zugrunde liegt vgl. Finkelstein (1992: 509); Gutenberg (1976: 488). 
autonomie dominanter Koalitionen in Familienunternehmen kann anhand der mit steigendem familiärem Einfluss immer schwächer ausgeprägten Umweltfaktoren und organisationalen Faktoren aufgezeigt werden, welche die aus dem marktwirtschaftlichen Autonomieprinzip erwachsende Entscheidungsfreiheit der Eigentümer im Allgemeinen grundsätzlich beschränken. ${ }^{416}$

\section{Umweltfaktoren der »managerial discretion« und die Entscheidungsautonomie nach außen}

Unabhängig vom Typus des Unternehmens wird das Maß an Entscheidungsfreiheit grundsätzlich durch Umweltfaktoren wie das Wachstum der Branche, deren Wettbewerbsstruktur oder gesellschaftliche Normen bestimmt. ${ }^{417}$

Innerhalb dieses von der Umwelt gesetzten Rahmens erhöht in Familienunternehmen die Eigentümerfunktion der Familie jedoch maßgeblich den verbleibenden Umfang an Entscheidungsfreiheit der dominanten Koalition gegenüber der Umwelt, da das Privateigentum der Familie am Unternehmen die dominante Koalition doch weitestgehend vom Druck des Kapitalmarkts befreit und unabhängig von den Interessen des Kapitalmarkts und externen Governance-Mechanismen macht. Während in kapitalmarktorientierten Unternehmen der Handlungsspielraum des Managements durch Quartalsberichte, Wertverluste und die Gefahr feindlicher Übernahmen in erheblichem Maße eingeschränkt wird, nimmt die Reichweite und restriktive Wirkung solcher externen Governance-Mechanismen mit steigendem Eigenkapitalanteil der Familie stetig ab. ${ }^{418}$

In der Folge eröffnen sich der dominanten Koalition größere strategische Handlungsspielräume, die zum Beispiel die Einnahme längerer Zeitperspektiven oder eher intuitiv denn sachlich-rational begründete Entscheidungen ermöglichen. ${ }^{419}$

Abhängig von der Rechtsformwahl der Eigentümer können mit der Eigenkapitalgeberfunktion der Familie aber auch reduzierte Rechenschafts-, Informations- und Transparenzpflichten des Unternehmens einhergehen. ${ }^{420}$ Bestehen

416 Vgl. Hambrick \& Finkelstein (1987) und grundlegend zur Autonomie Gutenberg (1976: $460 \mathrm{ff}$.).

417 Vgl. Hambrick \& Finkelstein (1987: 398); auch Hannan \& Freeman (1984); Porter (1980) und die empirische Studie von Lieberson \& O'Connor (1972), die den Einfluss der Branche auf den Entscheidungsraum der Manager aufzeigt.

418 Vgl. zur Bedeutung dieser Governancemechanismen Fama \& Jensen (1983:313); Anderson \& Reeb (2004: 213f.).

419 Vgl. Carney (2005: 424). Kets de Vries (1993: 62) formuliert drastisch: "Compared with publicly held corporations, family firms are not the slaves of Wall Street, haunted by quarterly results."

420 Vgl. Carney (2005:255); Schulze et al. (2001: 106). Klein (2010: 144) zufolge wählen familiär beeinflusste Unternehmen überproportional häufig Rechtsformen wie die KG oder $\mathrm{GmbH}$ 
hier nur relativ wenige Pflichten, so erhöht dies die Autonomie der dominanten Koalition selbstverständlich weiter, denn mit verschärften Veröffentlichungspflichten gehen stets eine erhöhte öffentliche Aufmerksamkeit und Legitimationszwänge einher. In Familienunternehmen kann der familiäre Einfluss hingegen so groß sein, dass die Unternehmensfamilie in extremen Fällen zumindest rechtlich gesehen nur sich selbst Rechenschaft schuldig ist.

"Owner-managers are less subject to external constraints relating to accountability, disclosure, and transparency, in part because concentrated ownership allows owners to attenuate constraints to their discretion stemming from arm's length investors Thus, while the structure of authority under managerial and alliance governance is relatively diffuse, impersonal, and vested in the role [...] the ultimate authority in family governance is literally incorporated in the person of an owner-manager."

(Carney, 2005) ${ }^{421}$

Da mit steigendem Einfluss der Familie über die Eigenkapitalgeberfunktion somit häufig die handlungsbeschränkenden Kräfte des Kapitalmarktes und regulatorischer Vorgaben abnehmen, werden in stark familiär beeinflussten Unternehmen vor allem Fremdkapitalgeber und andere wichtige Stakeholder zu den entscheidenden Determinanten der Autonomie der Entscheidungsträger. Neben Banken sind hier wichtige Kunden oder Lieferanten zu nennen, welche die Autonomie der strategischen Entscheidungsträger erheblich beeinflussen können. ${ }^{422}$

In der Gestaltung dieser Beziehungen zu Fremdkapitalgebern und Stakeholdern spiegelt sich jedoch häufig ein meist stark ausgeprägtes Streben von Unternehmensfamilien nach der Wahrung von Unabhängigkeit und Autonomie wider. ${ }^{423}$ Existenzielle Unabhängigkeit ist in vielen Unternehmensfamilien mithin nicht nur das Motiv der Unternehmensgründung und ein etablierter zentraler Wert, sondern vielleicht sogar das übergeordnete Ziel des unternehmerischen Handelns der Familie. Von Schlippe (2000) beschreibt die Unabhängigkeit der Familie gar als die prägende Entscheidungsprämisse, an der die nachfolgenden strategischen Festlegungen und Bewertungen ausgerichtet werden. $^{424}$

mit hoher Kontrollmöglichkeit und wenig Rechenschaftspflichten. Auffällig ist zum Beispiel der hohe Anteil von Kommanditgesellschaften bei Unternehmen mit einem Umsatz von über 500 Mio. Euro.

421 Carney (2005: 255).

422 Vgl. allgemein Pfeffer \& Salancik (2003 (1978)).

423 Vgl. Donckels \& Fröhlich (1991:3); Gómez-Mejía et al. (2007: 106f.); Schein (1983: 26); von Schlippe (2009: 191); Wimmer et al. (2005: 158). Für eine wirtschaftshistorische Einschätzung: Kocka (1979: 101f.). Klein (2010: 114) weist daraufhin, dass viele Familienunternehmer bis weit in die 1970er-Jahre hinein das Ausscheiden aus der persönlichen Haftung insbesondere aufgrund dieses Kontrollstrebens ablehnten.

424 Vgl. von Schlippe (2009: 139); auch Felden \& Hack (2014: 101); Simon (2012: 56). 
Dieser These entsprechend wird immer wieder beschrieben, dass Unternehmensfamilien zur Wahrung der Unabhängigkeit auf Wachstumspotenziale verzichten, wenn diese mit einem hohen Kapitalbedarf verbunden sind. ${ }^{425}$ Gómez-Mejía et al. (2007) kommen in ihrer Studie gar zu dem Schluss, dass viele Unternehmensfamilien der Bewahrung des familiären Einflusses selbst dann die höchste Priorität zukommen lassen, wenn dies mit einem erheblichen finanziellen Risiko für das Unternehmen und letztlich für dessen Überleben einhergeht. Dabei stellen sie eine enge Korrelation des Autonomiestrebens mit der Höhe des beobachteten familiären Einflusses fest.

"We have shown that when family firms are faced with a strategic choice dilemma that involves (1) a high degree of certainty of improved financial gains and a better probability of survival, but loss of family control [...] and (2) a greater risk of declining performance and catastrophic business failure, but retention of family control (choosing to remain independent and not to join the coop), the clear winner is the the risk willing decision."

(Gómez-Mejía et al., 2007) (26 $^{426}$

Eben dieses Autonomiestreben schlägt sich häufig in der Gestaltung der Beziehungen zu Stakeholdern wie Banken nieder. So versuchen viele Unternehmensfamilien die Fremdkapitalfinanzierung des Unternehmens zu minimieren, um die mit dieser Finanzierungsform einhergehenden Rechenschaftspflichten und Kontrollverluste zu reduzieren. ${ }^{427}$

Dieses Streben von Unternehmensfamilien nach der Wahrung ihrer Autonomie lässt sich zum einen aus dem individuellen Bedarf der Familienmitglieder nach Wertschätzung und Selbstverwirklichung erklären. Die Möglichkeiten zur Befriedigung solcher Bedürfnisse sind meistens eng mit der Unabhängigkeit des Unternehmerdaseins verbunden und somit Teil des sozio-emotionalen Vermögens der Familie und ihrer Mitglieder am Unternehmen. ${ }^{428}$

Darüber hinaus lässt sich das Autonomiestreben der Familie aber wohl auch darauf zurückführen, dass für Unternehmensfamilien die Verbindung zwischen

425 Vgl. La Porta et al. (1999: 511); Simon (2012: 57).

426 Gómez-Mejía et al. (2007: 129); vgl. auch die empirischen Ergebnisse bei Cromie et al. (1995: 26). Zum Verzicht auf Wachstumsmöglichkeiten zum Beispiel Simon (2012: 57).

427 Vgl. Felden \& Hack (2014: 205); Schulze et al. (2001: 101); Sirmon \& Hitt (2003: $343 \mathrm{ff}$.). Sharma (2004: 21) zufolge ergeben empirische Untersuchungen ein klares Bild von der präferierten Rangordnung der Finanzierung. Die häufig beobachtbaren hohen Eigenkapitalquoten in Familienunternehmen können sicherlich als mittelbares Ergebnis dieses Bestrebens nach Unabhängigkeit und einer autonomiewahrenden Thesaurierungspolitik betrachtet werden. Vgl. hierzu Albach \& Freund (1989: 144); Wimmer et al. (2005: 159).

428 Vgl. zu diesen allgemeinen Bedürfnissen Maslow (1954: 388f); auch Steinmann \& Schreyögg (2005: 550 ff.). Zum sozio-emotionalen Vermögen und dessen Dimensionen GómezMejía et al. (2007); auch Berrone et al. (2012) und für einen Überblick Felden \& Hack (2014: $47 \mathrm{ff}$.$) .$ 
Familie und Unternehmen eben immer noch jene letzte ökonomische Überlebenseinheit ist, die der familiäre Haushalt in vorindustriellen Zeiten für den Großteil der Gesellschaft war. ${ }^{429}$ Für das mit der unternehmerischen Tätigkeit erreichte materielle und sozio-emotionale Wohlstandsniveau kann im Falle des Verlusts des Unternehmens keine entsprechende Kompensation erwartet werden. Über diese entscheidende, im Risiko kaum gestreute Quelle des materiellen sowie größtenteils auch sozio-emotionalen Wohlergehens möchten viele Familien natürlich eine möglichst starke Kontrolle ausüben. Demnach lässt sich insgesamt festhalten:

Ceteris paribus, je stärker der Einfluss der Familie auf ein Unternehmen, desto geringer die angestrebte Abhängigkeit von der Umwelt und entsprechend höher die Autonomie der dominanten Koalition.

(Annahme 1)

Organisationale Faktoren der »managerial discretion « und

Entscheidungsautonomie nach innen

Der familiäre Einfluss auf das Unternehmen stärkt nicht nur die Autonomie der dominanten Koalition gegenüber der Umwelt der Organisation. Vielmehr erhöht sich vielen Beiträgen der Familienunternehmensforschung zufolge mit steigendem familiärem Einfluss meist auch die Entscheidungsautonomie der Entscheidungsträger innerhalb der Organisation. ${ }^{430}$

Entscheidungsträger in stark familiär beeinflussten Unternehmen unterliegen demnach meist relativ geringen innerorganisationalen formalen und strukturellen Beschränkungen und können ohne langwierige und bürokratische Abstimmungsprozesse auch recht weitreichende Entscheidungen treffen. Viele Unternehmensfamilien und Entscheidungsträger der um sie gruppierten dominanten Koalitionen befinden sich daher insgesamt in einer außergewöhnlich machtvollen Position, um Kontrolle über die Organisation auszuüben und ihre Interessen zu verfolgen:

»[I]n summary, founding families are in exceptional control positions to pursue their interests."

(Anderson \& Reeb, 2004) ${ }^{431}$

Diese innerorganisationale Entscheidungsautonomie der dominanten Koalition erwächst ebenfalls prinzipiell aus der Eigenkapitalgeberfunktion der Familie, denn über diese Funktion werden den Eigentümern die Verfügungsrechte zugestanden, die Ressourcen des Unternehmens zu nutzen, in Form und Substanz

429 Vgl. zu dieser familiären Funktion Nave-Herz (2014: 3).

430 Vgl. Kets de Vries (1993: 62); Miller \& LeBreton-Miller (2005: 47).

431 Anderson \& Reeb (2003: 1304); vgl. auch Anderson \& Reeb (2004: 213); Carney (2005: 252). 
zu verändern, die Erträge aus dem Eigentum einzubehalten oder das Eigentum mitsamt aller Verfügungsrechte ganz oder teilweise veräußern zu können. ${ }^{432} \mathrm{Als}$ Eigentümer sind Unternehmensfamilien in der Wahrnehmung dieser Rechte innerhalb des gesetzlichen Rahmens dabei weitestgehend unbeschränkt und können auch rein eigennützige oder scheinbar irrationale Entscheidungen treffen. ${ }^{433}$

Über diese fundamentalen Verfügungsrechte der Eigentümer hinaus wird die Entscheidungsautonomie dominanter Koalitionen in Familienunternehmen vor allem auch dadurch erhöht, dass sich mit steigendem Einfluss der Familie häufig ein einziges Zentrum der betrieblichen Willensbildung entwickelt. Während in kapitalmarktorientierten Unternehmen mit dem rein professionellen Management ein zweites, weitgehend autonomes »Zentrum der betrieblichen Willensbildung " neben den Eigentümern existiert - was aber wiederum in ein fein austariertes, stark reguliertes und formalisiertes System der »checks and balances« eingewoben ist -, bilden Eigentum und Leitung in Familienunternehmen mit wachsendem Einfluss der Familie zunehmend eine Einheit. ${ }^{434}$ Auch wenn die Leitung des Unternehmens nicht ausschließlich in Familienhand liegt, kann die Familie über zahlreiche formelle wie informelle Mechanismen doch einen derart starken Einfluss auf das Management ausüben, dass aus diesem selten ein wirklich autonomes zweites Zentrum der Willensbildung erwächst. Insbesondere der strategische Entscheidungsprozess wird häufig aus dem Kreis der Eigentümer heraus gesteuert.

Bilden Eigentum und Leitung mit steigendem Einfluss der Familie zunehmend ein einziges Zentrum der betrieblichen Willensbildung, gerät immer stärker das Kontroll- und Aufsichtsorgan zum primären begrenzenden Faktor der innerorganisationalen Entscheidungsautonomie. Diesen Organen kommt dann auch die Aufgabe zu, die Unternehmen vor Formen des »owner opportu-

432 Vgl. für die Theorie der Verfügungsrechte Demsetz (1967).

433 Vgl. Carney (2005: 254); Fama \& Jensen (1983: 302). In der explorativen Vorstudie wurde der Satz, dass jeder Unternehmer befugt sei, sein Unternehmen selbst zugrunde zu richten, sinngemäß immer wieder geäußert. Ungeachtet der ethischen Fragwürdigkeit dieser Feststellung verweist sie doch auf eine Differenz zum Entscheidungsraum selbst des einflussreichsten angestellten Entscheidungsträgers.

434 Zum »Zentrum der Willensbildung« Gutenberg (1976: 488); vgl. auch Carney (2005: 250). Nach der Erhebung von Klein (2010: 130) werden $44 \%$ aller Familienunternehmen mit einem Jahresumsatz von über 1 Mio. Euro ausschließlich von Familienmitgliedern geführt. Nur in $14 \%$ der Unternehmen verzichtet die Familie vollkommen auf eine Beteiligung an der Geschäftsführung. Allerdings impliziert der Verweis auf die Existenz eines einzigen Zentrums der betrieblichen Willensbildung nicht, dass es innerhalb dieses einen Zentrums nicht mehrere, auch divergente Gruppierungen geben könnte. 
nism« (Schulze \& Lubatkin, 2001) oder »entrenchment« (Finkelstein \& D’Aveni, 1994) zu schützen. ${ }^{435}$

Teilweise führt das familiäre Streben nach Kontrolle und Unabhängigkeit dazu, dass in stark familiär beeinflussten Unternehmen manchmal immer noch keine Kontroll- und Aufsichtsorgane existieren. ${ }^{436}$

Doch selbst wenn entsprechende Gremien existieren, so zeigt sich in der Gestaltung dieser Organe häufig, dass die Unternehmensfamilien als Eigentümer die »internal rules of the game « bestimmen ${ }^{437}$ So führt der familiäre Einfluss dazu, dass nicht immer die notwendigen Regelungen und unabhängige Machtressourcen vorhanden sind, um die optimale Funktionsweise des Gremiums sicherzustellen. Selbst wenn entsprechende Regelungen existieren, so verhindert eine Vielzahl formeller und informeller Hindernisse eine tatsächliche Durchsetzung gegenüber den Eigentümerfamilien. Denn während kapitalmarktorientierte Unternehmen die Autonomie der Kontrollorgane durch eine Reihe an Regelungen zur maximalen Amtszeit von Vorstand und Aufsichtsräten oder zur Berufung von profilierten unabhängigen externen Persönlichkeiten erhöhen, gehören in stark familiär beeinflussten Unternehmen beispielsweise Entscheidungsträger der dominanten Koalition nicht selten Geschäftsführung und Kontrollorgan zugleich an. ${ }^{438}$ Diese Protagonisten können dann aufgrund der sie begünstigenden Informationsasymmetrien und der Autorität ihres Amtes einen großen Einfluss auf das Aufsichtsgremium ausüben und dessen Kontrolleffektivität maßgeblich verringern. ${ }^{439}$

Zudem steigt mit zunehmenden Einfluss der Familie auch die Möglichkeit der Eigentümerfamilien die Kontroll- und Aufsichtsorgane entsprechend eigener Charakteristika und Präferenzen zusammensetzen zu können. ${ }^{440}$ Hieraus resultieren immer noch häufig Kontroll- und Aufsichtsorgane mit vielen Familienangehörigen und nur relativ wenigen externen Mitgliedern, die zudem nicht

435 Vgl. Finkelstein \& D'aveni (1994: 1079ff.); Finkelstein et al. (2009: 31) zur UE-Perspektive auf diese Thematik; Schulze et al. (2001: 99ff.) und Jensen (1993: 847) zum agenturtheoretischen Blickwinkel.

436 Klein (2010: 133f.) zufolge verfügten 1995 knapp 80 \% der Nicht-Familienunternehmen, aber nur $35 \%$ der Familienunternehmen über Kontroll- und Aufsichtsorgane. Bemerkenswerterweise sinkt der Anteil von Familienunternehmen mit Kontrollorgan mit steigendem Eigenkapitalanteil der Familie.

437 Fama \& Jensen (1983: 304); vgl. auch Anderson \& Reeb (2004: 235).

438 Vgl. Klein (2010: 141); Schein (1983: 23); Schulze et al. (2001: 104). Zu solchen Regelungen in kapitalmarktorientierten Unternehmen vgl. Finkelstein et al. (2009).

439 Vgl. zu dieser »CEO duality« im Allgemeinen die Studie von Crossland \& Hambrick (2007); auch Fama \& Jensen (1983: 314f.); Hambrick (2007: 339); für einen Überblick Krause et al. (2014).

440 Diese Möglichkeit wird allgemein als wichtige Determinante des Einflusses des Managements auf dessen Kontrollorgan ausgemacht. Vgl. Finkelstein \& Hambrick (1989: 125); Westphal \& Zajac (1995: 60). 
selten in einem besonderen professionellen oder persönlichen Verhältnis zum Unternehmen oder dessen Protagonisten stehen. ${ }^{441}$

Hinsichtlich der Kontrolle der Entscheidungsträger lässt sich somit konstatieren, dass sich die Mechanismen deren Kontrolle mit steigendem familiärem Einfluss in einem sehr unterschiedlichen Rahmen vollziehen, der häufig die Entscheidungsautonomie der Entscheidungsträger erheblich erhöht. ${ }^{442}$

Ein weiterer Faktor, der die innerorganisationale Autonomie der dominanten Koalition in Familienunternehmen stärkt, ist das hohe Maß an Autorität und Legitimität, welches der Eigentümerfamilie in der Führung des Unternehmens zugesprochen wird. ${ }^{43}$ So wird den Eigentümerfamilien von den anderen Organisationsmitgliedern in den meisten Fällen das Recht eingeräumt, weitestgehend frei über ihr Eigentum verfügen zu können. Diese zum Beispiel aus der Legitimität der Tradition erwachsende Autorität ist nicht nur ein günstigerer Herrschaftsmechanismus als der ressourcenintensive Einsatz von Macht, sondern kann der Leitung des Unternehmens durch die Familie gar eine gewisse Immunität verleihen. ${ }^{44}$

Zusammengenommen führen all diese skizzierten Faktoren schlussendlich dazu, dass die dominanten Koalitionen in Familienunternehmen mit steigendem Einfluss der Familie auch innerhalb der Organisation meist recht autonom und ohne größere Widerstände entscheiden können.

"The unification of ownership and control concentrates and incorporates organizational authority in the person of an owner-manager or family. Consequently, these agents operate under fewer internal constraints as they may exempt themselves from the internal bureaucratic constraints that limit managerial authority in other modes of governance."

(Carney, 2005) $)^{445}$

Somit lässt sich als Annahme für die weitere Arbeit formulieren:

441 So sind nach Klein (2010: 140) nur in $32 \%$ der Unternehmen keine Familienmitglieder im Kontrollorgan tätig, während in 49 \% der Unternehmen die Familie mehr als die Hälfte der Mitglieder und in $21 \%$ gar alle Mitglieder stellt. Vgl. auch Schulze et al. (2001: 101). Zur Besetzung der Kontrollorgane mit »Insidern « auch: Nelson (2003: 722); Schulze et al. (2001: 104) ; Westphal \& Zajac (1995: $60 \mathrm{ff}$.$) .$

442 Vgl. Gomez-Mejia et al. (2001: 91): »The results presented here indicate that monitoring mechanisms operate differently under family and nonfamily principal-agent contracts." Vgl. auch Schein (1983: 26); Schulze et al. (2001: $108 \mathrm{ff}$.).

443 Vgl. Carney (2005: 251); allgemein Pfeffer (1981: 2).

444 Vgl. Gomez-Mejia et al. (2001: 83), die von einer »impliziten Immunität« sprechen, die Entscheidungsträger aus ihrem Status als Familienmitglieder beziehen. So sei zum Beispiel die Tätigkeit von Familienmitgliedern weitgehend von deren Performance und vom Erfolg des Unternehmens entkoppelt. Schein (1983: 24) spricht hier entsprechend von einer »secure position«.

445 Carney (2005: 254). 
Ceteris paribus, je stärker der Einfluss der Familie auf ein Unternehmen, desto schwächer sind die formellen und informellen Handlungsbeschränkungen innerhalb der Organisation und entsprechend höher sind die Entscheidungsautonomie und der Grad an Entscheidungsfreiheit.

(Annahme 2)

\section{Zentralisierung}

Der Einfluss des Kontextfaktors Familie führt neben der grundlegend hohen Entscheidungsautonomie zu weiteren spezifischen Ausprägungen allgemeiner organisationaler Variablen. ${ }^{446}$ So steigt hinsichtlich des Grades, in dem die Rechte der Entscheidungsfindung und der strategischen Kontrolle in der Organisation verstreut oder konzentriert sind, mit dem Einfluss der Familie häufig auch die Zentralisierung der formellen Entscheidungsgewalt und der Entscheidungsprozesse. ${ }^{447}$

In diesem Zusammenhang hält die Forschung $\mathrm{zu}$ Familienunternehmen immer wieder fest, dass die Steuerung und Kontrolle der strategischen Entscheidungsprozesse in vielen Familienunternehmen einer vergleichsweise geringen Anzahl von Entscheidungsträgern obliegt. ${ }^{448}$

So tendieren Wimmer et al. (2005) zufolge viele Eigentümer in Familienunternehmen dazu - teils entgegen ihren eigenen Vorsätzen - die wichtigsten Entscheidungskompetenzen in ihrer Hand zu konzentrieren. ${ }^{449}$ Der Umstand, dass vielen Entscheidungsträgern in Familienunternehmen die Dezentralisierung unternehmerischer Verantwortung bis hin zum operativen Aufgabenvollzug sehr schwer fällt, wurde auch in der explorativen Vorstudie immer wieder beobachtet und dürfte ein Grund für die von Cromie et al. (1995) beobachteten sehr kleinen Topmanagement-Teams sein. ${ }^{450}$

Dieser Scheu vor Delegation und Dezentralisierung der Verantwortung liegt

446 Vgl. zu bedeutenden Variablen der Erfassung der Organisationsstruktur Welge \& AlLaham (2012: 177).

447 Vgl. zur Zentralisierung: Fredrickson (1986: 282); Hatum \& Pettigrew (2006: 125); Nelson (2003: 722); Miller \& LeBreton-Miller (2005); Tagiuri \& Davis (1996: 201).

448 Vgl. Albach \& Freund (1989: 37); Westhead et al. (2001:373); auch die empirische Studie Cromie et al. (1995).

449 Vgl. Wimmer et al. (2005: 105), dem zufolge »bei mehr als 75 Prozent« aller Familienunternehmen eine tendenziell monokratische Spitze beobachtet werden kann.

450 Vgl. Cromie et al. (1995: 20). Ein Nachfolger eines Familienunternehmens äußerte zur Strukturierung in der vorhergehenden Generation: »er war der komplette Kopf. Bei ihm ist jeder einzelne Auftrag rein, er hat jedes Teil geplant und hat das eigentlich durchdacht und hat dann die fix und fertigen Auftragspapiere rausgegeben und die mussten es nur noch abarbeiten sozusagen, ja. Und das hat gut funktioniert. Und er hat immer gesagt, dass was er macht, das ist eigentlich die Arbeit von fünf Leuten, ja, im Unternehmen und ich habe es ihm nie geglaubt, ja, bis er raus ist und dann haben wir tatsächlich fünf Leute gebraucht, um das alles abzubilden.» 
häufig zugrunde, dass mit der Abgabe von Entscheidungskompetenzen direkt ein Teil der Kontrollmacht und somit eine wichtige Komponente des sozioemotionalen Vermögens am Unternehmen verloren geht. ${ }^{451}$ Neben den tatsächlichen Verlust von Entscheidungskompetenz tritt häufig aber auch die weitergehende Angst vor einem Kontrollverlust über das individuelle und familiäre Vermögen. Um Teile der Entscheidungsgewalt über dieses Vermögen an andere Führungskräfte abzutreten, muss daher in einem familiär-partikularistisch geprägten Gefüge erst ein besonderes Vertrauensverhältnis aufgebaut werden. $^{452}$

"Das heißt, es gab meine Eltern, dann gab es lange nichts und dann viele, viele Indianer. Das heißt, eigentlich die kompletten Führungsaufgaben und auch administrative Aufgaben zum größten Teil, lagen bei meinen Eltern ... und das war auch durchaus so gewollt, weil eben, auch Thema Entwicklung, weil meine Eltern dachten es ist gefährlich, wenn man sich gute Leute heranzüchtet, weil die können sich selbstständig machen und dann sich auch so eine Maschine in die Garage stellen. Und deshalb war Strategie meiner Eltern immer, lieber mit mittelmäßigeren Leuten arbeiten, deren Horizont beschränkt ist und wo diese Gefahr nicht auftritt. Und dann lieber selber mehr machen müssen. Das war so die Ausrichtung [...] weil wir Angst vor dem Kontrollverlust ja auch hatten.«

(Ehemaliger Familienunternehmer im Rahmen der explorativen Vorstudie)

Somit wird bezüglich der Konzentration von Entscheidungskompetenzen angenommen:

Ceteris paribus, je stärker der familiäre Einfluss, desto höher der Grad an Zentralisierung.

(Annahme 3)

\section{Geringe Formalisierung und Orientierung an Personen}

Hinsichtlich einer weiteren organisationalen Variable sinkt der bisherigen Forschung zu Familienunternehmen zufolge mit einem steigenden Einfluss der Familie auch der Grad der Formalisierung in der Bearbeitung strategisch relevanter Aufgaben. ${ }^{453}$

Unter dieser Formalisierung kann im Allgemeinen das Ausmaß verstanden werden, in dem die Organisation zur Koordination des Verhaltens der Organisationsmitglieder auf feste Regeln und Prozeduren zurückgreift. In Bezug auf die in dieser Arbeit fokussierten strategischen Prozesse betrifft dies auch das Ausmaß, in dem eine Organisation im Zuge ihrer Entscheidungsprozesse die

451 Vgl. Berrone et al. (2012); Felden \& Hack (2014: 47 ff.); Gómez-Mejía et al. (2007).

452 Vgl. Simon (2012: 61); Wimmer et al. (2005: 137ff.).

453 Vgl. Miller \& LeBreton-Miller (2005: 47); auch Daily \& Dollinger (1992: 124); Hatum et al. (2010: 266); König et al. (2013: 424). Zum Beispiel Sharma \& Manikutty (2005: 296): »Line managers' participation in strategic decisions tends to be less well defined in family firms." 
Selektion und Interpretation von Umweltveränderungen sowie die darauf erfolgenden strategische Reaktionen strukturiert. ${ }^{454}$

Hinsichtlich des Einflusses der Familie auf diese organisationale Variable beobachtet Ward (1988) in Familienunternehmen ein häufig wenig formalisiertes strategisches Management. ${ }^{455}$ In seiner Studie tendierten viele Entscheidungsträger in Familienunternehmen dazu, Fragen des strategischen Managements als zeitraubende und unnötige Aktivitäten zu betrachten, die sie von ihrer »eigentlichen" Arbeit abhielten. Vor diesem Hintergrund fällt es vielen Familienunternehmen auch schwer, feste Kommunikationssituationen zu kreieren, um beispielsweise die Umwelt auf Risiken zu prüfen oder Kritik und Konflikt strukturiert zu bearbeiten. Solche Strukturen gelten in stark familial beeinflussten Unternehmen nicht selten als »bürokratisch«. Stattdessen überwiegt häufig eine familiär geprägte, anlassbezogene Kommunikation zwischen wenigen Beteiligten.

»Das verbindliche Festlegen von Verantwortlichkeiten, der Respekt vor übertragenen Kompetenzen, das Einrichten und Pflegen von spezialisierten Kommunikationsanlässen, das offene Bewältigen von unvermeidlichen Konfliktsituationen, all das sind Fähigkeiten, die normalerweise in einer familialen Organisationskultur nicht ausgeprägt werden.«

(Wimmer et al., 2005) $)^{456}$

Daily \& Dollinger (1992) gemäß zeigt sich diese geringe Formalisierung organisationaler Strukturen und Prozesse zum Beispiel auch in tendenziell stärker informellen Monitoring- und Kontrollmechanismen, während Cromie et al. (1995) auf weniger klar definierte Managementrollen verweisen. ${ }^{457}$ In einem tendenziell diffuseren organisationalen Rahmen werden Entscheidungsprozesse seltener über präzise formulierte Vorgaben und konkrete schriftliche Anweisungen gesteuert, sondern eher über diffuse Erwartungsbündel, die in größerer Eigenverantwortung abgearbeitet werden können. Dies führt dazu dass in vielen Familienunternehmen eine Form der Aufgabenbewältigung und -koordination praktiziert wird, welche man als eine kollektive Orientierung an den vermeintlichen Absichten der obersten Entscheidungsträger umschreiben kann.

454 Vgl. Fredrickson (1986: 283); Hannan \& Freeman (1984: 151).

455 Vgl. Ward (1988: 114f.); auch Schulze et al. (2001: 104); Wimmer et al. (2005: 111). Die häufig wenig expliziten strategischen Entscheidungsprozesse werden indirekt auch durch das eingangs dieses Kapitels skizzierte Beispiel der Steinberg Inc. bei Mintzberg \& Waters (1982: 497f.) beschrieben: »That particular strategy was conceived in the entrepreneurial mode, the creativity and synthesis taking place informally and personally".

456 Wimmer et al. (2005: 143); vgl. auch Daily \& Dollinger (1992).

457 Vgl. Daily \& Dollinger (1992: 133); Cromie et al. (1995: 23), die auch darauf hinweisen, dass Familienunternehmen seltener auf formale Bewertungssysteme zurückgreifen. Vgl. hierzu auch Morris et al. (1997: 388), die ebenfalls postulieren:»Internal control systems tend to be more informal.» 
Diese in Familienunternehmen häufig beobachtete geringere Formalisierung kann auf verschiedene Ursachen zurückgeführt werden. So erwächst aus der stabilen Kapitalisierungsfunktion der Familie in Familienunternehmen häufig die Möglichkeit, eine langfristigere zeitliche Perspektive und strategische Ausrichtung zu verfolgen. Mit der Verfolgung einer solch langfristig orientierten strategischen Perspektive durch die Eigentümer - ein Merkmal familiär beeinflusster Unternehmen, welches weiter unten im Rahmen der Kategorie der »Continuity« ausführlicher erörtert wird - sinkt jedoch auch der Druck auf das Management zur permanenten Lieferung aktueller Kennzahlen und entsprechender Nachjustierungen der verfolgten Strategie. Es sind jedoch gerade auch solche organisationalen Aufgaben, die zum Beispiel in kapitalmarktorientierten Unternehmen aufgrund der Orientierung an der kurzfristigen Unternehmensentwicklung und eng gesetzter Rentabilitätsziele einen hohen Formalisierungsgrad erforderlich machen. ${ }^{458}$

Als ein weiterer Grund für die beobachtete geringere Formalisierung wird zudem der oben skizzierte Umstand angeführt, dass sich mit steigendem Einfluss der Familie in Familienunternehmen tendenziell ein einziges Zentrum der betrieblichen Willensfindung herausbildet. Da die Eigentümerfamilien daher häufig eng in die strategische Leitung ihres Unternehmens eingebunden sind, besteht für sie innerhalb dieser Strukturen nicht die gleiche Notwendigkeit einer formalisierten Kontrolle der strategischen Entscheidungsprozesse durch fest definierte Zustimmungspflichten oder ein dichtes Berichtswesen wie in den anonymen Eigentümerstrukturen kapitalmarktorientierter Unternehmen. Denn sie können diese Kontrolle wegen ihrer Nähe zum Unternehmen durch andere, unter Umständen sogar kostengünstigere Formen sicherstellen, zum Beispiel durch den Aufbau von transaktionskostensenkendem Vertrauen zu langjährigen Angestellten. ${ }^{459}$

Solche Formen der sozialen Kontrolle fügen sich besser in das von der Familie geprägte kulturelle Gefüge der »Community« ein, welches im nächsten Ab-

458 Vgl. König et al. (2013: 424); Miller \& LeBreton-Miller (2005: 33 ff.); Zahra et al. (2004: 367): »The time orientation of a firm's culture is reflected in its choice of control system. When organizations have a short-term orientation, they are likely to favor financial, rather than strategic controls."

459 Vgl. Jensen \& Meckling (1976: 312 ff.); auch Daily \& Dollinger (1992: 124); Schulze et al. (2001: 99): „Owner management should reduce agency costs because it naturally aligns the owner-managers' interests about growth opportunities and risk. This alignment reduces their incentive to be opportunistic, sparing firms the need to maintain scostly mechanisms for separating the management and control of decisionsı. Neuere Beiträge wie Fama \& Jensen (1983) oder Schulze et al. (2001) weisen aber auf andere Agenturkosten in eigentümergeführten Unternehmen hin. 
schnitt ausführlicher diskutiert wird. ${ }^{460}$ Denn in einem Umfeld, das tendenziell eher auf partikularistischen und stärker emotionsbasierten Beziehungen beruht, erweisen sich hochgradig formalisierte Abläufe häufig als nur wenig kompatibel, widersprechen elaborierten Regularien der Kommunikation doch der systemischen Logik von Familien. ${ }^{461}$

Die Kehrseite der geringen Formalisierung ist die stark ausgeprägte Orientierung an Personen hinsichtlich der Strukturierung von Einfluss, Verantwortlichkeit und Aufgaben. ${ }^{462}$ Diesbezüglich manifestiert sich der familiäre Einfluss am eindrücklichsten in der im Vergleich zu anderen ökonomischen Kontexten spezifischen personalen Kontinuität der Entscheidungsträger. So weisen der Eigentümerfamilie angehörige CEOs der Studie von McConaughy (2000) zufolge mit 17,6 Jahren eine deutlich längere Verweildauer im Unternehmen auf als entsprechende Führungskräfte in Nicht-Familienunternehmen, die durchschnittlich fünf bis sechs Jahre im Unternehmen verbleiben. ${ }^{463}$ Auf die aus dem familiären Einfluss erwachsende Möglichkeit, das Unternehmen vielleicht länger zu führen, als es aus sachlicher Sicht geboten erscheint, verweisen nicht zuletzt auch Shleifer \& Vishny (1997). ${ }^{464}$

\begin{tabular}{|c|c|c|}
\hline Strukturmerkmal & Facetten & Literatur \\
\hline $\begin{array}{l}\text { Hohe } \\
\text { Entscheidungsautonomie }\end{array}$ & $\begin{array}{c}\text { Geringere Wirkkraft } \\
\text { externer Governance- } \\
\text { Mechanismen; reduzierte } \\
\text { Rechenschaftspflichten; } \\
\text { niedriger Anteil } \\
\text { Fremdkapital; ein einziges } \\
\text { Zentrum der } \\
\text { Willensbildung; abhängige } \\
\text { Kontrollorgane }\end{array}$ & $\begin{array}{c}\text { Carney (2005); Gomez-Mejia } \\
\text { et al. (2001); Gómez-Mejía } \\
\text { et al. (2007); Schulze et al. } \\
(2001)\end{array}$ \\
\hline $\begin{array}{l}\text { Hoher Grad an } \\
\text { Zentralisierung }\end{array}$ & $\begin{array}{l}\text { Kleine TMT; geringe } \\
\text { Dezentralisierung von } \\
\text { Entscheidungskompetenz }\end{array}$ & $\begin{array}{l}\text { Cromie et al. (1995); } \\
\text { Westhead et al. (2001); } \\
\text { Wimmer et al. (2005) }\end{array}$ \\
\hline
\end{tabular}

460 Vgl. Miller \& LeBreton-Miller (2005); auch König et al. (2013: 424); Sirmon \& Hitt (2003: 345).

461 Vgl. Wiechers (2006: 204); Wimmer (2011: 143).

462 Vgl. Carney (2005: 255); Simon (2012: 93); Wimmer et al. (2005: 180).

463 Vgl. McConaughy (2000: 121ff.). Zu einem ähnlichen Ergebnis kommen auch Daily \& Dollinger (1992), die eine Verweildauer von 17 Jahren in Familienunternehmen, im Vergleich zu acht Jahren in nicht familiär beeinflussten Unternehmen feststellen, und GomezMejia et al. (2001), die ein höheres Maß an »entrenchment« beobachten.

464 Vgl. Shleifer \& Vishny (1997); auch Wiechers (2006: 306). 
(Fortsetzung)

\begin{tabular}{|c|c|c|}
\hline Strukturmerkmal & Facetten & Literatur \\
\hline $\begin{array}{c}\text { Geringe Formalisierung } \\
\text { und Orientierung an } \\
\text { Personen }\end{array}$ & $\begin{array}{c}\text { Informelle } \\
\text { Kontrollmechanismen; } \\
\text { Personale Kontinuität }\end{array}$ & $\begin{array}{c}\text { Daily \& Dollinger (1992); } \\
\text { Ward (1988); Schulze et al. } \\
\text { (2001) }\end{array}$ \\
\hline
\end{tabular}

Tabelle 2: Strukturelle Merkmale des familiären Einflusses der Kategorie »Command» (Quelle: Eigene Darstellung).

Die sehr lange Verweildauer der Entscheidungsträger im Unternehmen trifft allerdings nicht nur auf Familienangehörige, sondern vor allem auch auf familienfremde Führungskräfte zu. So arbeiten Topmanagement-Teams in familiär beeinflussten Unternehmen deutlich länger zusammen, als in nicht familiär beeinflussten Unternehmen. ${ }^{465}$ Die Teams setzen sich dabei häufig aus Personen zusammen, die der Eigentümerfamilie als besonders loyal, vertrauenswürdig und fleißig gelten. ${ }^{466}$

In dem Umstand, dass bestimmte familienfremde Führungskräfte Unternehmen teils über mehrere Jahrzehnte prägen, kommt wiederum die familiensystemische Orientierung an Personen, nicht formalen und anonymen Strukturen zum Ausdruck. Somit lässt sich als leitende Annahme festhalten:

Ceteris paribus, je stärker der Einfluss einer Familie auf ein Unternehmen, desto geringer ist der Grad an Formalisierung und desto stärker die Orientierung an Personen. (Annahme 4)

\subsection{2 »Continuity«: Generationale Orientierung und Kontinuität}

Das Strukturmerkmal der »Continuity« adressiert jene Facetten im Unternehmen, die das oben skizzierte familiäre Streben nach transgenerationaler Kontinuität widerspiegeln. ${ }^{467}$

Dieses familiäre Streben nach Kontinuität führt dazu, dass viele Familien-

465 Laut der Studie von Cromie et al. (1995: 20) arbeiteten 70 \% der TMT in Familienunternehmen mehr als sechs Jahre in der gleichen Konstellation zusammen, während dies bei Unternehmen ohne dominanten familiären Einfluss nur bei $40 \%$ der Fall war. Vgl. auch Westhead et al. (2001: 379).

466 Vgl. Gersick et al. (1997: 5); Wimmer et al. (2005: 151).

467 Vgl. Miller \& LeBreton-Miller (2005: 35 ff.). In dem Wunsch und der Möglichkeit, Eigentum und Kontrolle über das Unternehmen familienintern an die nächste Generation übergeben zu können, unterscheiden sich Familienunternehmen von verwandten ökonomischen Formen eigentümergeführten Unternehmen wie dem »lone-founder «. Vgl. hierzu Churchill \& Hatten (1997: 52): „What is usually meant by 'family business, , however, and the factor which most sharply differentiates family from owner-managed businesses, is either the occurrence or the anticipation that a younger family member has or will assume control of the business from an elder." 
unternehmen in besonderen zeitlichen Dimensionen operieren. ${ }^{468}$ So denken viele Entscheidungsträger in Familienunternehmen tendenziell eher in Generationen, denn in Quartalen, sowohl retrospektiv hinsichtlich der familiären Historie und Tradition, als auch prospektiv mit Blick auf die Zukunft.

\section{Retrospektiv: Bedeutung von Historie und Tradition}

In jeder Organisation werden durch verschiedene informelle Mechanismen Werte und Praktiken überliefert und zu bestimmten Anlässen die organisationale Historie referenziert. Zudem fühlen sich die meisten Entscheidungsträger mehr oder weniger mit den bestehenden materiellen und immateriellen Ressourcen ihres Unternehmens emotional verbunden. ${ }^{469}$

Mit steigendem Einfluss der Familie scheint dieser retrospektive Bezug auf die organisationale Vergangenheit besonders ausgeprägt $\mathrm{zu}$ sein und die emotionale Verbundenheit zum Bestehenden zuzunehmen. ${ }^{470}$ Die Forschung zu Familienunternehmen beobachtet und beschreibt hier häufig ein besonders hohes Bewusstsein für die organisationale Entwicklungsgeschichte und eine hohe Bedeutung der Tradition. Sie macht darauf aufmerksam, dass mit steigendem familiärem Einfluss auch im Unternehmen in hohem Maße auf Überzeugungen, Werte oder Handlungsmuster Bezug genommen wird, die von vorherigen Generationen tradiert wurden und spricht von einem spezifischen Konservatismus. ${ }^{41}$

So werden Prinzipien, Normen und Werte vergangener Generationen nicht nur unbewusst fortgelebt und praktiziert. Vielmehr wird das traditionelle Erbe häufig durch die Referenz auf überlieferte Symbole und kulturelle Artefakte, Erzählungen und Bräuche bewusst gepflegt und auf diesem Wege über sehr lange Zeiträume aufrechterhalten. ${ }^{472}$ Simon et al. (2005) stellten fest, dass sich in fast allen der zehn intensiv von ihnen untersuchten langlebigen Familienunternehmen teils noch nach hundert oder mehr Jahren eine Vielzahl spezifischer kultureller oder struktureller Aspekte zeigte, die eindeutig auf den Gründer oder andere frühere Generationen zurückverwiesen. ${ }^{473}$ Diese teils über Jahrhunderte

468 Vgl. Simon (2005: 37); Stamm (2013: 107).

469 Vgl. Burgelman \& Grove (1996); allgemein Steinmann \& Schreyögg (2005: 265).

470 Vgl. Dyer (1988: 41), der die zeitliche Orientierung des seiner Ansicht nach am weitest verbreiteten Kulturtyps so beschreibt: "Some paternalistic firms tend to be oriented to the past. Carrying on the founder's and family's legacy is the primary aim of the owning family. Thus, time-warn traditions are at the center of the culture.« Vgl. auch Gersick et al. (1997: 127); Micelotta \& Raynard (2011).

471 Vgl. Klein (2010); Stamm (2013: 89); Zahra et al. (2004: 364).

472 Vgl. Felden \& Hack (2014: 4); James (2006: 124).

473 Vgl. Simon et al. (2005: 14), die im Rahmen qualitativer Fallstudien langlebige Familienunternehmen wie die Merck KGaA (gegründet 1668), die Franz Haniel \& Cie. GmbH (gegründet 1756) und die Dr. August Oetker KG (gegründet 1891) untersuchten. 
praktizierten Führungsprinzipien oder Erbpraktiken wurden immer wieder explizit adressiert und dienten den Entscheidungsträgern als bedeutsame Handlungsorientierung in Entscheidungsprozessen.

Zugleich wird nach innen wie nach außen häufig die besondere Beziehung zum angestammten Standort des Unternehmens oder einer bestimmten Branche betont, und die Langlebigkeit des eigenen Unternehmens hervorgehoben. So zeigen Micelotta \& Raynard (2011) auf, wie in der Unternehmenskommunikation bewusst die Tradition des Familienunternehmens eingesetzt wird. ${ }^{474}$

Dieser enge Bezug zu Historie und Tradition kann gar so weit führen, dass Entscheidungsträger versuchen nicht nur das Unternehmen generell $\mathrm{zu}$ bewahren, sondern es gar in seiner ursprünglichen Form zu erhalten. ${ }^{475}$

Dieser retrospektive Rekurs wird maßgeblich von der Familie getragen, denn obgleich keine Organisation geschichtslos ist, ist es doch die fortwährende Präsenz der Familie, welche die Historie in Familienunternehmen in besonderer Weise lebendig hält. Sie ist in diesem organisationalen Kontext die primäre Trägerin der Geschichte. Diese vermittelt sie zum einen direkt im Unternehmen, zum anderen aber vor allem über persönliche und intime Beziehungen den nachfolgenden Familienmitgliedern, die früh mit den Traditionen des Unternehmens und der Familie vertraut gemacht und entsprechend sozialisiert werden. $^{476}$

"[...] weil die alle irgendwo diesen/ dieses Gen haben, und wo auch die Tradition, die Rückbesinnung auf die Großväter oder den Großvater, den manche vielleicht noch im Kopf hatten [...] Aber in Familienunternehmen wird das gepflegt. Da wird noch die Tradition die Erinnerung an den Urgroßvater (pocht wiederholt auf den Tisch), an den Gründer. Ich kenn das. Mein Großvater ist sozusagen/ ist der Urgründer von dem Laden, der da ist. Also, früher, ganz klar, traf man sich einmal im Jahr zu Allerseelen, wenn Ihnen das was sagt, also im November an den Gräbern. Ich hatte keinerlei Bezug zu ihm. Ich kannte ein Bild, aber die Tatsache, dass wir da als Kinder standen, gab irgendwie einen Zusammenhang. Und so was wird in Familienunternehmen stärker gepflegt. Kann ja auch stärker gepflegt werden, als in anonymen Kapitalgesellschaften, das ist völlig normal.»

(Ein auf Familienunternehmen spezialisierter Unternehmensberater)

474 Vgl. Micelotta \& Raynard (2011): »The visual and textual content of these websites underscore a desire to keep things suntouched،. This is visually represented by juxtaposing images of the past (e.g., the landscape, buildings, and family) with similar images in the present, thus suggesting an uninterrupted link between past and present. The textual content further reinforces this desire to preserve the past, by explicitly venerating the past and portraying it as a form of 'precious inheritance that needs to be protected and preserved."

475 Vgl. Chrisman et al. (2009: 739ff.); König et al. (2013: 425).

476 Vgl. zum Beispiel die Rolle der Familie als Trägerin protestantischer Werte bei Weber (1972). Gersick et al. (1997: 149): »[T] he family is perhaps the most reliable of all social structures for transmitting cultural values and practices across generations." Vgl. auch Felden \& Hack (2014: 4); Floris et al. (2013: 5). 
Zusammenfassend lässt sich somit festhalten, dass die fortwährende Präsenz der Familie im Kontext des Unternehmens und deren teils gar dynastisches Streben dazu führt, dass in Familienunternehmen in besonderer Weise gilt: »history matters«.

Ceteris paribus, je stärker der Einfluss einer Familie auf ein Unternehmen, desto höher ist die Bedeutung der Historie und Tradition für strategische Entscheidungen der Gegenwart.

(Annahme 4)

\section{Prospektiv: Langfristige strategische Ausrichtung und nicht-ökonomische Ziele}

Das transgenerationale Streben der Familie führt jedoch nicht nur zu einem besonderen Bezug zu Historie und Tradition, sondern verknüpft darüber hinaus Vergangenheit, Gegenwart und Zukunft in spezifischer Weise miteinander. ${ }^{477}$ Die Forschung zu Familienunternehmen macht hinsichtlich der prospektiven Komponente dieser zeitlichen Kontinuität vor allem darauf aufmerksam, dass die familiär beeinflussten Unternehmen mit steigendem Einfluss der Familie über zunehmend längere Zeithorizonte operieren und eine vergleichsweise langfristige strategische Ausrichtung verfolgen. ${ }^{478}$

Diese Langfristigkeit der strategischen Planung wird unmittelbar am Beispiel des Nachfolgeprozesses deutlich: Wenngleich juristische Fragen oftmals lange Zeit ungeklärt bleiben, beginnt der Prozess des generationalen Transfers von Eigentum und Unternehmensleitung doch häufig bereits in der Kindheit der potenziellen Nachfolger und erstreckt sich über mehrere Jahrzehnte. ${ }^{479}$ Eine derart langfristige Finanzierungs- und Personalplanung ist in anderen ökonomischen Kontexten wohl selten anzutreffen.

Häufig manifestiert sich der vergleichsweise langfristige Zeithorizont aber auch in einer anderen strategischen Gestaltung der Beziehung zu Mitarbeitern und weiteren Stakeholdern. Diese zielen nicht selten weniger auf kurzfristige Nutzenmaximierung, als auf langfristige Kontinuität ab. ${ }^{480}$

Des Weiteren verfolgen Anderson \& Reeb (2003) zufolge viele Familienunternehmen oft sehr langfristig orientierte Projekte, die teilweise erst nach einer

477 Vgl. Miller \& LeBreton-Miller (2005: 35); Stamm (2013: 89).

478 Vgl. Felden \& Hack (2014: 9); Schein (1983: 26); Simon et al. (2005: $146 \mathrm{ff}$.); Sirmon \& Hitt (2003: 343).

479 Vgl. Handler (1994: 133ff.); Klein (2010: 313); Sharma et al. (1997: 4). Auf den Umstand, dass Nachfolge nicht nur der Moment der Übertragung von Eigentumsrechten und operativer Führungsverantwortung ist, sondern ein teils lebenslanger Aushandlungsprozess, der bewusst oder unbewusst schon in der Kindheit der potenziellen Nachfolger eingeleitet wird, verweist auch Stamm (2013: 202).

480 Vgl. Kets de Vries (1993: 313); für den auch das soziale Engagement vor Ort ein Ausdruck dieser langfristigen Orientierung ist. Siehe hierzu die Ausführungen zu den Kategorien "Community« und »Connections». 
vergleichsweise langen Zeitspanne Profitabilität versprechen. ${ }^{481}$ Simon et al. (2005) sehen die von ihnen häufig in Familienunternehmen beobachteten stabilitätsorientierten Diversifikationsstrategien als Indiz dafür, dass für die Entscheidungsträger in Familienunternehmen langfristiges Wachstum und Stabilität von größerer Bedeutung sind als kurzfristige Renditemaximierung. ${ }^{482}$

Die Möglichkeit zu dieser langfristigen strategischen Ausrichtung erwächst prinzipiell aus der Verfügungsgewalt der Familie über das Eigentum und die Ressourcen des Unternehmens. Da die Entscheidungsträger im Unternehmen nicht dem ständigen Druck des Kapitalmarkts nach kurzfristigen maximalen Gewinnen, Wertsteigerungen und möglichst hohen Dividendenzahlungen unterliegen, können sie auch strategische Optionen verfolgen, die sich vermutlich erst nach vielen Jahren auszahlen. Zwischenzeitlich negative Entwicklungen können durch die Finanzierungsfunktion der Familie abgefangen werden. In ernsthaften Krisensituationen verzichten viele Eigentümerfamilien nicht nur auf Kapitalerträge, sondern nehmen teils auch über längere Zeiträume Verluste in Kauf. Sirmon \& Hitt (2003) sprechen daher vom "patient capital« der Unternehmensfamilien. ${ }^{483}$ Diese "Geduld « des eingesetzten Kapitals resultiert allerdings nicht aus einer grundlegenden Abkehr vom ökonomischen Gewinnstreben. Vielmehr geht die so ermöglichte langfristige strategische Orientierung vor allem darauf zurück, dass infolge der familiären Irritationen neben die ökonomischen auch eine Vielzahl von nicht-ökonomischen Zielen der Eigentümerfamilie treten. ${ }^{484}$

An erster Stelle stehen hier der tief verankerte Wunsch und das kollektive Ziel der Unternehmensfamilie, das Unternehmen und die familiäre Kontrolle über dieses Unternehmen für kommende Generationen zu erhalten. ${ }^{485}$

"Sie können natürlich Folgendes beobachten, Sie sagen einem Familienunternehmer, `Chef, dieses Jahr verdienen wir nix.« Da springt der nicht vor Freude Ihnen um den Hals. Aber das wird der wegstecken. Immer vorausgesetzt, da sind substanzielle Rücklagen gebildet und so weiter. Sie können dem alle möglichen Botschaften servieren, die den nicht glücklich machen, aber die den auch nicht um den Verstand bringen. Aber eins können Sie nicht, wenn Sie dem kommen und sagen: >Du fährst das Ding an die Wand, nächstes Jahr ist Feierabend. Und der sagt: `vor mir waren fünf, ich bin der sechste, und ich will es eigentlich an meine Kinder weiter geben`, ist die größte Horrorvorstellung im Leben eines Familienunternehmens, wenn wir ihn denn so definieren, und der ist dann der wie gesagt, der X-te in einer Kette. Und dann ist dessen

481 Vgl. Anderson \& Reeb (2003: 1305); auch Wimmer et al. (2005: 121).

482 Vgl. Simon et al. (2005: 149ff.).

483 Vgl. Sirmon \& Hitt (2003: 343); auch Kets de Vries (1993:313).

$484 \mathrm{Vgl}$. Berrone et al. (2012: 258ff.); Felden \& Hack (2014: $46 \mathrm{ff}$.$) .$

485 Vgl. Garcia-Alvarez et al. (2002: 190f.); Gómez-Mejía et al. (2007: 134); Ward (2004: 27): "After all, what is a family business about if it is not about the next generation?" 
einziger Alptraum, dass er derjenige ist, der im Geschichtsbuch der Familie, oder des Ortes oder der Nation steht als der, der es vergeigt hat."

(Unternehmensberater im Rahmen der explorativen Vorstudie)

Das dynastische Denken der Familie wird somit zur abstrakten Entscheidungsprämisse der strategischen Ausrichtung des Unternehmens, an der sich meist alle anderen strategischen Zielsetzungen ausrichten. ${ }^{486}$ So verzichten viele Unternehmensfamilien auf ein möglicherweise schnelleres Wachstum oder eine höhere Rendite, wenn damit eine nachhaltige Gefährdung ihrer unternehmerischen Autonomie durch die Aufnahme großer Mengen Fremdkapital oder gar die Ausgabe von Eigenkapital einhergehen würde. ${ }^{487}$ Großen Teilen der Forschung gelten Unternehmensfamilien daher als ausgesprochen risikoaverse Investoren. ${ }^{488}$ Da meist ein Großteil des Vermögens und somit auch des Investitionsrisikos in einem einzelnen Unternehmen gebündelt ist, tendierten Familien stärker zu risikomindernden Strategien wie einer verstärkten Diversifikation. ${ }^{489}$ Allerdings machen Gómez-Mejía et al. (2007) zu Recht darauf aufmerksam, dass Familienunternehmen nicht per se risikoavers seien, sondern ihre Einstellung zum Risiko von Strategien lediglich stark von den nicht-ökonomischen Zielstellungen moderiert werde. ${ }^{490}$ Sie zeigen, dass Unternehmensfamilien unter Umständen sogar äußerst riskante Strategien verfolgen, wenn damit eine Wahrung oder Steigerung ihrer Autonomie über das Familienunternehmen einhergeht. Neben dieses nicht-ökonomische Ziel der Autonomiewahrung treten eine Vielzahl anderer, durch die unternehmerische Tätigkeit befriedigter sozio-emotionaler Bedürfnisse und Ziele, deren Erreichung nicht durch kurzfristig lukrative, aber hochriskante Strategien gefährdet werden soll. ${ }^{491}$ So bietet die leitende Tätigkeit im Unternehmen die Möglichkeit, das

486 Vgl. Felden \& Hack (2014: 48), die das Unternehmen als »langfristiges, generationenübergreifendes Familieninvestment" begreifen. Auch die Definition von Stamm (2013: 124): »Aus der Perspektive eines institutionalisierten Lebenslaufes kann die Unternehmerfamilie als ein zeitlich stabiles Kollektiv verstanden werden, das über das Leben mehrerer Generationen hinweg gemeinschaftlich ein Projekt verfolgt«.

487 Vgl. Albach \& Freund (1989: 180); Sirmon \& Hitt (2003: 339); Wimmer et al. (2005: 158); Bereits Penroese (1959) vermerkt in ihrer Theorie zum Wachstum von Unternehmen, dass Eigentümerunternehmer häufig nicht das optimale Wachstum erzielen würden, weil sie »have been unwilling to $[\ldots]$ raise capital through procedures that would have reduced their control over the firms."

488 Vgl. Gómez-Mejía et al. (2007: 108); Kets de Vries (1993: 314).

489 Vgl. Gomez-Mejia et al. (2001: 84); La Porta et al. (1999: 511); Simon et al. (2005: 147).

490 Vgl. Gómez-Mejía et al. (2007); auch Felden \& Hack (2014: 47 ff.); Gimeno et al. (1997: $750 \mathrm{ff}$.$) zur Kontinuität des "psychic income from entrepreneurship«.$

491 Vgl. im Allgemeinen: Nelson (2003: 710); Smith \& Miner (1983: 333). Vgl. auch GómezMejía et al. (2007: 106), die unter sozio-emotionalem Vermögen die »non-financial aspects of the firm that meet the family's affective needs such as identity, the ability to exercise family influence, and the perpetuation of the family dynasty« verstehen. 
Umfeld durch Anweisungen und Sanktionen zu kontrollieren, persönliche Wünsche und Visionen zu verfolgen, und einen hervorgehobenen und aktiven Platz in der Gesellschaft einzunehmen, der öffentlichen Respekt und Status verschafft. ${ }^{492}$ Zudem verschafft das Unternehmen den Mitgliedern individuell wie kollektiv als Familie die Möglichkeit der Herausbildung einer stark akzentuierten Identität. ${ }^{493} \mathrm{Zu}$ nennen wären hier sicherlich aber auch die Möglichkeiten der Fortschreibung persönlicher und familiärer Werte im und durch das Familienunternehmen, die materiellen Möglichkeiten sich altruistisch gegenüber Familienmitgliedern zu zeigen, oder die verbindende Wirkung des Unternehmens auf die Familie.

\begin{tabular}{|c|c|c|}
\hline Strukturmerkmal & Facetten & Literatur \\
\hline $\begin{array}{c}\text { Retrospektiv: } \\
\text { Hohe Bedeutung von } \\
\text { Tradition und Historie }\end{array}$ & $\begin{array}{c}\text { Ausgeprägtes Bewusstsein } \\
\text { für Entwicklungsgeschichte; } \\
\text { Konservatismus; Mythen, } \\
\text { traditionelle Symbole; } \\
\text { emotionale Verbundenheit } \\
\text { zum Status quo }\end{array}$ & $\begin{array}{c}\text { Simon et al. (2005); Stamm } \\
\text { (2013) }\end{array}$ \\
\hline $\begin{array}{c}\text { Prospektiv: } \\
\text { Langfristige } \\
\text { strategische } \\
\begin{array}{c}\text { Ausrichtung und nicht- } \\
\text { ökonomische Ziele }\end{array}\end{array}$ & $\begin{array}{c}\text { Nachfolgeplanung; Stabilität } \\
\text { > Wachstum; Lange } \\
\text { Amortisationszeiten }\end{array}$ & $\begin{array}{c}\text { Berrone et al. (2012); Gómez- } \\
\text { Mejía et al. (2007); Simon } \\
\text { et al. (2005) }\end{array}$ \\
\hline
\end{tabular}

Tabelle 3: Strukturelle Merkmale des familiären Einflusses der Kategorie »Continuity» (Quelle: Eigene Darstellung).

Diese Bestandteile des sozio-emotionalen Vermögens, welches sich im Verlauf der Zeit durch investierte Energie und Lebenszeit fortwährend erhöht, können zum einen eine gegebenenfalls niedrigere Rendite kompensieren. ${ }^{494}$ Ein Niedergang des Unternehmens oder ein abnehmender familiärer Einfluss würden daher nicht nur hohe materielle, sondern auch große sozio-emotionale Verluste mit sich bringen. Daher legen Entscheidungsträger in Familienunternehmen in wichtigen Fragen neben den rein ökonomischen meist auch andere, aufgrund einer anderen Rationalität gleichberechtigte Maßstäbe an und inkorporieren diese nicht-ökonomischen Zielstellungen in die strategische Zielsetzung des Unternehmens. ${ }^{495}$ Somit kann festgehalten werden:

492 Vgl. Jensen \& Meckling (1976: 312), Wiechers (2006: 264).

493 Vgl. Stamm (2013: 255ff.) zur identitätsschaffenden und sozialdifferenzierenden Wirkung des Unternehmens.

494 Vgl. Gersick et al. (1997: 150); Sirmon \& Hitt (2003: 343).

495 Vgl. Chrisman et al. (2005: 363). Zudem Kets de Vries (1993: 59), der wiederum den Unternehmer Sam Steinberg zitiert: »That business was built specifically for my family. Anybody who wants to come in, there's an open place for him. If the business is destroyed as a result of that, so be it." 
Ceteris paribus, je stärker der Einfluss der Familie, desto bedeutsamer sind nichtökonomische Zielsetzungen und desto langfristiger ist die abstrakte strategische Orientierung des Unternehmens.

(Annahme 6)

\subsection{3 »Community« und »Connections«: Kulturelle Merkmale}

Die im Rahmen der Kategorie »Command « skizzierten Strukturmerkmale wie die erhöhte Zentralisierung und die geringe Formalisierung verweisen auf Besonderheiten der formalen Ordnung in Familienunternehmen. Allerdings prägt der Einfluss der Familie nicht nur die formalen Strukturen stark, sondern auch nachhaltig die informelle Ordnung der Unternehmen.

Grundsätzlich werden Familienunternehmen aufgrund der über einen langen Zeitraum kontinuierlich und somit nachhaltig wirkenden Prägung der Familie im Allgemeinen relativ starke Organisationskulturen zugeschrieben. ${ }^{496}$ Ihre kulturellen Muster sind meist recht prägnant, besitzen einen hohen Verbreitungsgrad und sind tief in der Organisation verankert.

Obgleich es natürlich nicht die eine Organisationskultur von Familienunternehmen gibt, lassen sich doch einige Facetten des familiären Einflusses ausmachen, die typenübergreifend den meisten Kulturen in Familienunternehmen gemeinsam sind: eine erhöhte Emotionalität, eine eher kollektive Orientierung, Diffusität und Partikularismus, wie auch eine Tendenz zur Geschlossenheit. ${ }^{497}$ Die Häufigkeit des Auftretens dieser kulturellen Merkmale und die Intensität ihrer Ausprägung korrelieren dabei eng mit dem Grad an familiärem Einfluss. ${ }^{498}$

"Though these perceptions have strong stereotypic components, it's possible to see that much of the stereotype is firmly based in reality if one examines a number of firstgeneration and family owned companies."

(Schein, 1983) $)^{499}$

496 Vgl. Denison et al. (2004: 68); Hall et al. (2001: 198); Miller \& LeBreton-Miller (2005: 39). $\mathrm{Zu}$ den Merkmalen starker Kulturen im Allgemeinen vgl. Schreyögg (2006: 464ff.).

497 Vgl. zu den Kulturtypen Dyer (1988). Zu den Merkmalen familiär geprägter Organisationskulturen Schein (1983: 26); Wimmer et al. (2005: 182). Vgl. zudem Miller \& LeBretonMiller (2005: 38ff.), die im Zuge ihrer Ausführungen zu den Merkmalen von »community» und "connections» an die Dimensionen von Gemeinschaft und Gesellschaft nach Parsons (1952: 67) anknüpfen: Diffusivität vs. Spezifität, Affektivität vs. affektive Neutralität, Partikularismus vs. Universalismus und Kollektivität vs. Selbstorientierung. Für einen Überblick zu Beiträgen zu Organisationskulturen in Familienunternehmen Fletcher et al. (2012).

498 Dyer (1988: 45f.) zufolge ist die paternalistische Kultur meist dort zu finden, wo der Einfluss der Familie am stärksten ist. Mit abnehmendem Einfluss nimmt die Verbreitung der Kultur des Professionalismus zu.

499 Schein (1983: 24). 


\section{Emotionalität}

Ein bedeutsamer Aspekt jeder Organisationskultur ist die Einstellung zum Umgang mit Emotionen im organisationalen Kontext. ${ }^{500}$ In Bezug auf diese Dimension der informellen Ordnung zwischenmenschlicher Beziehungen stellt sich zum Beispiel die Frage, inwiefern Emotionen offen gezeigt werden dürfen oder wie mit Konflikten umgegangen werden soll. Kulturelle Ausprägungen können hierbei zwischen den Polen einer hohen Affektivität und einer affektiven Neutralität verortet werden. ${ }^{501}$ Während Individuen in einem affektiven Umfeld dazu tendieren, sich emotional aufeinander einzulassen, ihre Gefühle zum Ausdruck zu bringen und auf die emotionalen Bedürfnisse des anderen einzugehen, geht affektive Neutralität mit einer strikten Vermeidung emotionaler Verstrickung und Expression von Gefühlen einher.

Hinsichtlich dieser kulturellen Dimension lässt sich feststellen, dass das Maß an akzeptierter und zum Ausdruck gebrachter Emotionalität in den zwischenmenschlichen Beziehungen und der Kommunikation in der Organisation zunimmt, je enger Familie und Unternehmen strukturell gekoppelt sind. ${ }^{502}$ So halten Tagiuri \& Davis (1996) fest, dass die Kommunikation in Familienunternehmen nicht nur durch ein hohes $\mathrm{Maß}$ an Emotionalität geprägt sei, sondern auch durch eine eher »familiäre « Sprache. ${ }^{503}$

Wenngleich die erhöhte Emotionalität grundlegend als ein Strukturmerkmal verstanden werden muss, welches auch die Expression von positiven Gefühle der Zuneigung oder Anerkennung begünstigt, wird sie in der Literatur zu Familienunternehmen jedoch vor allem im Zusammenhang mit dem Auftreten von Konflikten angeführt.

»Hatte mal einen Fall, ganz schwierige Aufsichtsratssitzungen... Wenn schwierige Fragen gestellt wurden, da flippte die Mutter eines Vorstands regelmäßig aus. Die Frau hat das nicht ertragen. Dafür hab ich ein gewisses Verständnis. Es ist halt nur unangenehm bis peinlich, wenn also ein erwachsener Mann, der das Unternehmen führen soll, sich am Ende hinter seine Mutti zurückzieht, die dann aus dem, aus dem Kostüm springt, und nur durch die Gegend kreischt, >du willst nur meinem Sohn die Zukunft nehmen`, und so weiter, und so weiter. Dann sitzt du halt da, und sagst, was soll ich jetzt antworten? [...] So ein Verhalten wäre in anderen Kontexten doch vollkommen tabu.« Unternehmensberater und ehemaliger Aufsichtsrat im Rahmen der explorativen (Vorstudie)

500 Vgl. allgemein Schein (2010: 105): »how much emotion to display«; auch Schreyögg (2006: 454).

501 Vgl. Parsons (1952: 58ff.).

502 Vgl. Cosier \& Harvey (1998: 75); Levinson (1971: 378ff.); von Schlippe \& Kellermanns (2008: 42).

503 Vgl. Tagiuri \& Davis (1996: 204f.): »It is an interesting paradox that in even the most embattled family company, there is often strong adherence to even the most painful family language." 
Wegen eben dieser erhöhten Emotionalität gelten Familienunternehmen als ein fruchtbares Feld für Beziehungskonflikte, deren Potenzial deutlich höher sei als in anderen ökonomischen Kontexten. ${ }^{504}$

Sie ergibt sich direkt aus der strukturellen Kopplung mit dem System Familie, das sich im Rahmen seiner eigenen Funktionslogik an den Bedürfnissen und Gefühlen der Mitglieder orientiert und durch seine Irritationen im Verlauf der Ko-Evolution das Unternehmen entsprechend "färbt«, da mit steigendem Einfluss der Familie die Muster der weitgehend regellosen und »enthemmten Kommunikation ${ }^{505}$ der Familie, in der Gefühle offen und ungefiltert zum Ausdruck gebracht werden dürfen, auf die Sphäre des Unternehmens übertragen werden. Daher lässt sich zusammenfassend festhalten:

Ceteris paribus, je stärker der familiäre Einfluss, desto höher das Maß an kulturell akzeptierter und zum Ausdruck gebrachter Emotionalität in der Kommunikation.

(Annahme 7)

\section{Kollektive Orientierung}

Eine weitere kulturelle Dimension, in der die strukturelle Kopplung des Unternehmens mit der Familie ihren Niederschlag findet, ist der Grad an kollektiver Orientierung. ${ }^{506}$ Diesbezüglich stellt sich die Frage, ob eher eine Orientierung an individuellen Interessen und Bedürfnissen, sowie ein entsprechend opportunistisches, individuell nutzenmaximierendes Verhalten erwartet, oder von einer Orientierung am Kollektiv ausgegangen wird.

Auf dieser Achse führt der Einfluss der Familie im Unternehmen zu einer zunehmend kollektivistischen Orientierung. ${ }^{507}$ In deren Rahmen wird erwartet, dass die Mitglieder verstärkt die Interessen der anderen Mitglieder oder des Kollektivs als Ganzes berücksichtigen.

Entsprechend wird das Verhalten von Organisationsmitgliedern in Familienunternehmen in einer Reihe an Beiträgen als in besonderer Weise altruistisch beschrieben. ${ }^{508}$ Diese Beiträge betonen, dass die Entscheidungen und Hand-

504 Zahlreiche Anekdoten und Sachverhalte wie der Niedergang der von Mintzberg \& Waters (1982) untersuchten Steinberg Inc, die nach dem Tod des Gründers am Streit der Erben zerbrach, lassen Harvey \& Evans (1994: 331) konstatieren: »Family businesses are fertile fields for conflict." Vgl. auch Eddleston \& Kellermanns (2007); Kets de Vries (1993); Levinson (1971); von Schlippe \& Kellermanns (2008).

505 Luhmann (1990: 203ff.).

506 Vgl. Parsons (1952: 60); Schein (1985: 150f.).

507 Vgl. Corbetta \& Salvato (2004: 356): »Family firms are often depicted as relying on mutual trust, intra-familial altruism in its purest sense (i.e., unselfish concern and devotion to others without expected return to oneself), and clan-based collegiality.« Vgl. auch Arregle et al. (2007); Sharma (2004: 16); Zahra et al. (2004: 365).

508 Vgl. Felden \& Hack (2014: 87); Stamm (2013: 351); insbesondere auch die Forschungsbeiträge, die Familienunternehmen aus der Perspektive der Stewardship-Theorie be- 
lungen von Organisationsmitgliedern weniger durch opportunistische und individuell nutzenmaximierende Kalküle geprägt seien, sondern durch kollektiv orientierte Motive. Tendenziell werden demnach Entscheidungen unter steigendem familiärem Einfluss so gestaltet, dass der individuelle Nutzen zunehmend auch vom Nutzen anderer Akteure bestimmt wird.

Zugleich wird davon ausgegangen, dass diese kollektive Orientierung im Verlauf der Zeit zur Etablierung einer kollektiven Identität beitrage, welche ein Gefühl von Zugehörigkeit und Gemeinschaft erzeuge. ${ }^{509}$ Diese starke Identifikation mit dem Unternehmen könne dann wiederum die Bindung der Organisationsmitglieder an die Organisation erhöhen und $\mathrm{zu}$ einer ausgeprägten Loyalität der Mitarbeiter zum Unternehmen führen, die sich in manchen Fällen auch auf die Eigentümerfamilie erstrecke. Im idealtypisch konzipierten Familienunternehmen konstituieren die Mitglieder um die Unternehmensfamilie herum gar eine Art »Pseudofamilie« oder »Betriebsfamilie» als Form einer erweiterten Familie. ${ }^{510}$

Diese in Familienunternehmen tendenziell erhöhte kollektive Orientierung ist integraler Bestandteil der oben skizzierten Funktionslogik des Systems Familie. ${ }^{511}$ In der Familie sind die Mitglieder meist bereit, bei Bedarf ihre eigenen Bedürfnisse für sehr lange Zeiträume hinter die der anderen Mitglieder zurückzustellen. Diese Erwartungshaltung wird im Zuge der Ko-Evolution teilweise auf das Unternehmen übertragen. Sie kann sich dort aber aufgrund der skizzierten personalen Kontinuität auch aus eigener Quelle herausbilden. Insgesamt lässt sich in jedem Falle festhalten:

Ceteris paribus, je stärker der Einfluss einer Familie auf ein Unternehmen, desto stärker ausgeprägt ist die erwartete und auch beobachtbare kollektive Orientierung in der Organisation.

(Annahme 8)

trachten; vgl. hier beispielsweise grundlegend Davis et al. (1997); für einen Überblick Felden \& Hack (2014: 89).

509 Vgl. Eddleston \& Kellermanns (2007: 479); Miller \& Breton-Miller (2006: 38); Stamm (2013: 105).

510 Vgl. Kets de Vries (1993: 62); Sirmon \& Hitt (2003: 339); Tan \& Fock (2001: 128). Manifestationen dieses Gefühls der Zusammengehörigkeit und der Identifikation mit dem Unternehmen seien sowohl eine geringere Mitarbeiterfluktuation in Familienunternehmen, als auch eine besondere Form der "Opferbereitschaft«. So seien Mitarbeiter in Familienunternehmen in Krisenzeiten tendenziell in stärkerem Maße bereit Einschnitte hinzunehmen, um das Überleben »ihres« Unternehmens zu sichern. Vgl. Wimmer et al. (2005: 182).

511 Vgl. Simon et al. (2005: 36). 


\section{Diffusität und Partikularismus der informellen Ordnung}

Eine weitere kulturelle Dimension, in welcher der familiäre Einfluss auf das Unternehmen verortet werden kann, ist die Reichweite und Präzision der Verpflichtungen innerhalb der sozialen Beziehungen im Unternehmen. ${ }^{512}$ Diese Beziehungen und Verpflichtungen können sehr eng gefasst und präzise definiert werden, oder aber sehr weitreichend und unbestimmt sein.

Hinsichtlich dieser kulturellen Dimension lässt sich regelmäßig beobachten, dass der familiäre Einfluss im Unternehmen zu eher diffusen Beziehungen in der Organisation führt. ${ }^{513}$ Natürlich basieren auch in Familienunternehmen die Arbeitsbeziehungen auf spezifizierten Verträgen. Doch im Rahmen des kollektiv orientierten Handlungsgefüges wird häufig erwartet und akzeptiert, dass eine Vielzahl an Ansprüchen gestellt werden kann, die über das vertraglich Geregelte weit hinausgehen. In diesem diffusen Erwartungsgefüge muss der Anspruchsteller in der Regel einen konkreten Anspruch nicht rechtfertigen. Hingegen muss im Zweifelsfall eher derjenige, der beispielsweise mit einer speziellen, nicht exakt im Arbeitsvertrag bestimmten Aufgabe betraut wird, rechtfertigen, warum er diesem Anspruch nicht nachkommen kann oder möchte.

Zugleich werden innerhalb solcher diffuser Beziehungsrahmen von den Vorgesetzten seltener explizite und detaillierte Anweisungen erteilt. Stattdessen werden häufig Erwartungen an bestimmte Personen in den Raum gestellt, die diese Personen dann ohne weitergehende definitorische Spezifikation im Sinne der vorgesetzten Entscheidungsträger zu erfüllen haben. ${ }^{514}$ Somit bleibt nicht nur der allgemeine Rahmen der Beziehungen diffus, sondern häufig auch die konkreten Leistungserwartungen, die eher im Sinne einer umfänglichen Gesamtaufgabe formuliert werden.

Diese in Familienunternehmen häufig beobachtbare Diffusität entstammt in erster Linie wiederum der oben skizzierten familiären Funktionslogik der Familie. ${ }^{515} \mathrm{Da}$ die in Familien anfallenden Aufgaben grundsätzlich sehr umfassend und ganzheitlich sind, werden innerhalb dieses Systems auch die Erwartungen weniger exakt spezifiziert. Diese diffuse Erwartungsstruktur wird im Verlauf der Ko-Evolution dann häufig von der Familie auf das Unternehmen übertragen.

Zur Diffusität der informellen Ordnung trägt zudem die Übertragung der

512 Vgl. Parsons (1952: 65f.); auch Schein (2010: 153): "[I]s the relationship very specific, dealing only with the exact reason for the relationship, as in a sales-customer-relationship, or diffuse, as in most?«»[...] do the participants in the relationship view each other in a very general >universalistic « way, based on stereotypes, as in most professional relationships, or do they perceive each other in a very >particularistic way as whole persons as in a spousal relationship or friendship?»

513 Vgl. Cromie et al. (1995: 23); Miller \& LeBreton-Miller (2005: 47).

514 Vgl. Wimmer et al. (2005: 110).

515 Vgl. Simon et al. (2005: 36). 
familiären Konsensfiktion auf das Unternehmen bei, sodass sachliche Verständigung - wie in Familien - als Nebenprodukt einer hohen Interaktionsdichte und Vertrautheit erwartet wird. ${ }^{516}$ Des Weiteren basiert, komplementär zu dieser vergleichsweise hohen Diffusität, die kulturelle Ordnung in Familienunternehmen mit steigendem familiärem Einfluss stärker auf partikularistischen Beziehungen und Standards. ${ }^{517}$ So knüpft zum Beispiel die Bewertung von Leistung weniger an universalistischen Normen an, sondern wird mehr im Rahmen spezifischer Beziehungsgefüge und Aushandlungsprozesse beurteilt. Die Beziehungen zwischen den Organisationsmitgliedern fußen mit wachsendem Einfluss der Familie immer weniger auf exakt spezifizierten und explizierten Verträgen, sondern stärker auf Vertrautheit und Vertrauen. ${ }^{518}$ Weniger aus altruistischen Motiven, sondern vor allem vor dem Hintergrund dieses partikularistischen Beziehungsgefüges wird die Qualität der wechselseitigen Beziehung zu den Mitarbeitern somit zu einer sehr relevanten Größe im Zielsystem familiär beeinflusster Unternehmen. ${ }^{519}$

Die innerhalb der Kategorie des "Command « beschriebenen organisationsstrukturellen Merkmale der zunehmenden Orientierung an Personen und der abnehmenden Formalisierung im Entscheidungsprozess bei steigendem familiärem Einfluss erwachsen ebenso aus diesen kulturellen Wurzeln des Partikularismus und der Diffusität der sozialen Beziehungen, wie sie in diese kulturelle Ordnung eingebettet sind - können diese strukturellen Prinzipien doch auch nur in diesem kulturellen Kontext praktiziert werden ${ }^{520}$ Zusammenfassend lässt sich also festhalten:

Ceteris paribus, je stärker der Einfluss einer Familie auf ein Unternehmen, desto diffuser und partikularistischer ist das Beziehungsgefüge der informellen Ordnung der Organisation.

(Annahme 9)

\section{Geschlossene Kulturen und Netzwerke}

Eine weitere kulturelle Dimension, die den familiären Einfluss reflektiert, ist die Beziehung von familiär geprägten Organisationen zu ihrer Umwelt. ${ }^{51}$

$516 \mathrm{Da}$ man in Familien zu wissen glaube, wie der andere denke und fühle, glaube man auch ohne großen Abstimmungsaufwand auf einer gemeinsamen Entscheidungsgrundlage operieren zu können. Vgl. Wimmer et al. (2005: 110f.).

517 Vgl. Carney (2005: 255); Miller \& LeBreton-Miller (2005: 45); Schein (1983: 25ff.).

518 Vgl. Corbetta \& Salvato (2004: 356); Gomez-Mejia et al. (2001: 82 ff.), Schein (1983: 25).

519 Vgl. Tagiuri \& Davis (1992: 46ff.), die die langfristige Mitarbeiterzufriedenheit als eines der wichtigsten Ziele der von ihnen untersuchten Familienunternehmen ausmachen. Vgl. auch Colli (2013: 103); Miller \& LeBreton-Miller (2005: 40); Wiechers (2006: 265).

520 Vgl. Miller \& LeBreton-Miller (2005: 40f.); auch Carney (2005: 253ff.), der von einem System der "Family Governance« spricht.

521 Vgl. Schein (2010: 73 ff.); Schreyögg (2006: 152ff.). 
Hinsichtlich der Frage, wie die Umwelt wahrgenommen wird und inwiefern sich die Organisation ihr gegenüber öffnen sollte, stellt die Forschung zu Familienunternehmen fest, dass ein zunehmender familiärer Einfluss häufig zu Manifestationen von Umweltabgewandtheit und exkludierender Abschottung führt. $^{522}$

»A popular anecdote tells of a management consultant beginning a seminar by asking attendees to describe their organizations as automobiles. One attendee responds by comparing his firm to a sports car, as his company is characterized by its innovative marketing efforts. Another respondent describes her firm as a station wagon because of the company's conservative history and staid product lines. A third individual likens his firm to an armoured car. When asked to explain the rationale for his selection, he responds that unlike the other two firms, his firm is a family business: no one gets in; no one gets out."

$(\text { Litz, 1997) })^{523}$

So lässt sich Hall et al. (2001) zufolge in vielen Familienunternehmen eine vergleichsweise starke Innen-außen-Differenzierung im Rahmen der organisationalen Grenzziehung beobachten. In Familienunternehmen komme der Frage "who is in and who is not in « eine besonders hohe Relevanz zu. ${ }^{524}$ Carney (2005) verweist in diesem Punkt auf die häufig referenzierte exkludierende »thick social wall«, die aus den partikularistischen Bindungen erwachse und vertrauenswürdige »Insider« von nicht vertrauenswürdigen »Außenseitern« scharf trenne.

»Many sociologists viewed family firms as a backward, premodern institution plagued with exclusionary values. In this view, the particularistic ties that bind families also create a sthick social wall that separates reliable insiders from untrustworthy outsiders."

(Carney, 2005) $)^{525}$

Diese starke kulturelle Differenzierung wurde auch in der explorativen Vorstudie immer wieder indiziert. ${ }^{526}$ Der Forschung zu Familienunternehmen

522 Vgl. Litz (1997: 60), der eine starke »preference for privacy« beschreibt; Miller \& LeBretonMiller (2005: 279); Litz (1997); Simon (2012: 55f); Ward (1987: 173). Hildenbrand (1992) spricht hier im Zuge der Untersuchung landwirtschaftlicher Unternehmensfamilien von "Hofindividualismus«.

523 Litz (1997: 58ff.).

524 Vgl. Hall et al. (2001: 201); allgemein Schein (2010: 87ff.). Ein Unternehmer im Rahmen der Vorstudie: „Familien und grade eben Familienunternehmen neigen dazu, sich ihre Welt zu konstruieren, die nichts mit der realen Welt zu tun hat, zumindest in Teilbereichen. Das heißt, wir sind wir, wir genügen uns auch teilweise. Das ist auch so, wenn der Blick nach Innen, wir beschäftigen uns am Schluss auch mit uns, nicht mehr mit den Märkten«; vgl. zur Filterfunktion von Organisationskulturen in Familienunternehmen Klein (2010); Hambrick \& D'Aveni (1988).

525 Carney (2005: 250).

526 Ein auf Familienunternehmen spezialisierter Unternehmensberater: »Während Familien- 
gemäß manifestiert sie sich besonders deutlich in der Personalpolitik. In diesem Zusammenhang wird eine große Vorsicht und Zurückhaltung gegenüber dem »Fremden« außerhalb der vertrauten Gemeinschaft beschrieben. Dies äußert sich zum Beispiel in frühen Entwicklungsstadien von Familienunternehmen im nur zögerlichen Zulassen des Eintritts familienfremder Führungskräfte ins Unternehmen. ${ }^{527}$ Da Führungskräfte dem privaten und intimen Bereich der Unternehmensfamilie häufig sehr nahe kommen, wird hier auch eine besondere kulturelle und emotionale "Nähe« in Bezug auf deren Werte oder Kommunikationsmuster erwartet. In diesem Zusammenhang ist auch die ausgeprägte Tendenz zur internen Personalrekrutierung zu stellen. ${ }^{528}$ Doch selbst dann wenn Führungskräfte aus der Umwelt des Systems rekrutiert werden - ein stark ausgeprägtes "Immunsystem" erschwert häufig die Integration unternehmensfremder Manager in das diffuse und partikularistische Beziehungsgefüge. ${ }^{529}$ Diesbezüglich zeigen sich in anderen ökonomischen Kontexten die Vorteile einer individualistischen und universalistisch orientierten kulturellen Ordnung, die die Austauschbarkeit der Personen erleichtert.

Die Scheu vor dem Fremden zeigt sich auch in der von Gómez-Mejía et al. (2007) beschriebenen Zurückhaltung ggü. der Akquisition spezialisierten Expertenwissens außerhalb des Unternehmens. ${ }^{530}$ Hier wird in vielen Fällen befürchtet, dass Externe einen zu tiefen Einblick in technologische, finanzielle oder vor allem auch sozial sensible Bereiche des Unternehmens erlangen können.

In diesem Zusammenhang muss auch eine äußerst zurückhaltende Informationspolitik betrachtet werden ${ }^{531}$ Insbesondere Finanzkennzahlen werden in vielen Familienunternehmen in ausnehmend vorsichtiger Weise gehandhabt und nicht zuletzt durch eine geeignete Rechtsformwahl gegenüber der organisationalen Umwelt abgeschottet. Insbesondere auch, weil die Unternehmens-

unternehmen ja doch im Kern, wenn sie konservativ sind, immer sagen, wir sind wir und die anderen sind unsere Feinde, generell.«

527 So fand Dyer (1988: 39) in stark familiär beeinflussten Kulturen ein ausgeprägtes Misstrauen der Familie gegenüber Außenstehenden. Vgl. auch Gersick et al. (1997: 168); Wimmer et al. (2005: 136). Klein (2010: 247) zufolge lassen viele Familien erst dann Fremdmanager im Unternehmen zu, wenn es durch äußere Gründe unvermeidbar erscheint.

528 Vgl. Simon et al. (2005: 73); Wimmer et al. (2005: 180). Langjährige Betriebszugehörigkeit und Loyalität werden hier zu wichtigen Kriterien der Karriere.

529 Vgl. Klein (2010: 246ff.); Wimmer et al. (2005: 181).

530 Vgl. Gómez-Mejía et al. (2007: 134).

531 Vgl. Klein (2010: 127); Wiechers (2006: 273). Nach der explorative Studie von Klein (1991) wollten $40 \%$ möglichst wenig oder gar keine Informationen an die Umwelt geben. Litz (1997: 60) schreibt: »This priority is understandable, however, insofar as disclosing firmspecific information is often, in the minds of family business members, virtually indistinguishable from disclosing family-specific information.» 
kennzahlen gegebenenfalls Rückschlüsse auf die finanzielle Situation der Familie zulassen, die aber als Privatsache betrachtet wird.

Doch die Innen-außen-Differenzierung erstreckt sich über die Grenzen der Organisation hinaus. Da viele Unternehmensfamilien langfristige Beziehungen zu Stakeholdern anonymen Transaktionen vorziehen - und durchaus bereit sind, eine möglicherweise entstehende Differenz zur kurzfristig ökonomisch bestmöglichen Lösung in Kauf zu nehmen -, verfügen die Unternehmen oft über ein sehr enges, stabiles Beziehungsgeflecht aus Kunden und Lieferanten und sind so in lokale oder regionale Netzwerke eingebettet. ${ }^{532} \mathrm{Da}$ der Aufbau solcher Netzwerke jedoch voraussetzungsreich ist und persönliche Beziehungen, Vertrauen und eine gewisse kulturelle Nähe erfordert, wird zunächst streng geprüft, wer als vertrauenswürdig und passend gelten kann. Auf dem Wege dieser relativen Definition von »innen « und "außen« wird auch die Problematik des dadurch häufig entstehenden Abhängigkeitsverhältnisses adressiert. Da die Netzwerkpartner nun zum inneren Kreis gerechnet werden, wird auch das Abhängigkeitsverhältnis oft nicht weiter problematisiert.

Solche Manifestationen von exkludierender Abschottung und einer stark akzentuierten Grenzziehung zwischen innen und außen sind in vielerlei Hinsicht die »dunkle Seite« (Miller \& LeBreton-Miller, 2005) der engen Gemeinschaft. ${ }^{533}$ Denn die starken Organisationskulturen, die auf kollektiv orientierten, partikularistischen und emotionalen Beziehungen beruhen, spiegeln sich diametral in den Beziehungen der Unternehmen zu ihrer Umwelt und führen zur Herausbildung von "geschlossenen Systemen «. ${ }^{534}$

\begin{tabular}{|c|c|c|}
\hline Strukturmerkmal & Facetten & Literatur \\
\hline $\begin{array}{c}\text { Erhöhte } \\
\text { Emotionalität }\end{array}$ & $\begin{array}{c}\text { "enthemmte Kommunikation«, } \\
\text { "familiäre« Sprache, Konflikte }\end{array}$ & $\begin{array}{c}\text { Kets de Vries (1993); von } \\
\text { Schlippe \& Kellermanns } \\
\text { (2008); }\end{array}$ \\
\hline $\begin{array}{c}\text { Kollektive } \\
\text { Orientierung }\end{array}$ & $\begin{array}{c}\text { Tendenz zu Altruismus, kollektive } \\
\text { Identität, }\end{array}$ & $\begin{array}{c}\text { Arregle et al. (2007); } \\
\text { Corbetta \& Salvato (2004); }\end{array}$ \\
\hline $\begin{array}{c}\text { Diffusität und } \\
\text { Partikularismus }\end{array}$ & $\begin{array}{c}\text { Unspezifizierte Erwartungsbündel, } \\
\text { Konsensfiktion, langfristig } \\
\text { orientierte Beziehungen }\end{array}$ & $\begin{array}{c}\text { Carney (2005); Miller \& } \\
\text { LeBreton-Miller (2005); } \\
\text { Wimmer et al. (2005) }\end{array}$ \\
\hline
\end{tabular}

532 Vgl. Colli (2013: 100); Kets de Vries (1993: 62); Simon (2012: 56).

533 Vgl. Miller \& LeBreton-Miller (2005: 279):»[T] he dark side to community is [...] that they can become cultlike, insular [...].»

534 Vgl. Simon (2012: 56); auch Schreyögg (2006: 477), der die Tendenz zur Abschließung, die Errichtung von Wandelbarrieren oder "Kulturdenken" als oftmals beobachtbare negative Effekte starker Kulturen benennt. 
(Fortsetzung)

\begin{tabular}{|c|c|c|}
\hline Strukturmerkmal & Facetten & Literatur \\
\hline $\begin{array}{c}\text { Geschlossene } \\
\text { Kulturen und } \\
\text { Netzwerke }\end{array}$ & $\begin{array}{c}\text { Differenzierung in »innen« und } \\
\text { "außen«, Zurückhaltung dem } \\
\text { "Fremden«, Abschottung zur } \\
\text { Umwelt hin }\end{array}$ & $\begin{array}{c}\text { Carney (2005); Litz (1997); } \\
\text { Simon (2012) }\end{array}$ \\
\hline
\end{tabular}

Tabelle 4: Strukturelle Merkmale des familiären Einflusses der Kategorien »Community» und "Connections"

(Quelle: Eigene Darstellung).

Zudem resultieren die exkludierenden Muster häufig auch daraus, dass die nach Unabhängigkeit strebende Familie versucht den Charakter als Familienunternehmen zu wahren.

Der Einfluss der Familie zeigt sich hier wiederum in der familiären Einfärbung der informellen Ordnung des Unternehmens im Zuge der Ko-Evolution. Durch die Erhöhung der Emotionalität oder die Präferenz für partikularistische Beziehungen trägt die Ordnung zur Entstehung exkludierender organisationaler Kulturen bei. Somit reflektiert sich im Unternehmen die Funktionsweise und Ordnung des Systems Familie, das sich nach Nave-Herz (2014) im Zuge der funktionalen Differenzierung eben gerade als spezialisiertes, aber relativ geschlossenes System mit einer festen Binnenstruktur, festen Grenzen und einem exklusiven und exkludierenden Charakter etablierte. ${ }^{535}$ Somit lässt sich abschließend festhalten:

Ceteris paribus, je stärker der familiäre Einfluss, desto stärker tendieren Unternehmen zur Ausprägung exkludierender Kulturen und Netzwerke.

(Annahme 10)

535 Vgl. Nave-Herz (2014: 11); auch Kocka (1979: 117 ff.), der darstellt wie die Unternehmer in der Zeit der Industrialisierung ihre Familien als einen geschützten, privaten Innenraum verstanden und diesen teils samt dem Unternehmen von der Umwelt abzugrenzen trachteten. 
Open-Access-Publikation im Sinne der CC-Lizenz BY 4.0

(C) 2018, VeR unipress GmbH, Göttingen 


\section{Empirische Untersuchung: Strategische Pfade in die Krise}

In Teil B wurde der theoretische Bezugsrahmen der Arbeit abgesteckt und somit die Grundlagen zur weitergehenden Untersuchung des Einflusses des Kontextfaktors Familie auf die Genese und Dynamik strategischer Pfade gelegt. Auf Basis dieses theoretischen Fundaments wird sich im folgenden Teil C nun der qualitativen Studie zugewandt, in deren Zuge der Forschungsfrage empirisch nachgegangen wurde.

Im ersten Kapitel wird zur besseren Nachvollziehbarkeit und Einordnung der Ergebnisse zunächst das grundsätzliche Vorgehen der empirischen Untersuchung skizziert.

Dem Verlauf der empirischen Untersuchung folgend werden im ersten Unterkapitel der Zweck und Ablauf der explorativen Vorstudie dargelegt, die den Einstieg ins Feld eröffnete. Anschließend wird das Sampling skizziert, welches maßgeblich von der Vorstudie geprägt wurde.

Das zweite Unterkapitel widmet sich dann Methodik und Methoden der empirischen Studie, mit denen die ausgewählten Fälle untersucht wurden. Hier werden zunächst die Methoden der Datenerhebung und -auswertung dargestellt, bevor im Anschluss der Ablauf der Fallanalysen umrissen wird.

Das zweite Kapitel wendet sich dann den beiden ausgewählten empirischen Fällen der Anton Schlecker e.K. und der Berentzen-Gruppe AG zu. In angesichts der Komplexität der Fragestellung gebotenen Ausführlichkeit werden die persistenten strategischen Entwicklungen dieser beiden Unternehmen im Kontext der Umweltdynamik analysiert.

Hierbei wird jeweils zunächst die historische organisational-strategische Entwicklung der beiden Unternehmen dargestellt. Anschließend wird durch eine Analyse relevanter strategischer Parameter in Gegenüberstellung zur Umweltdynamik im ersten Schritt der Pfadanalyse der Frage der strategischen Persistenz nachgegangen. Hieran anknüpfend wird anhand der pfadkonstituierenden Elemente der selbstverstärkenden Mechanismen und des Lock-in geprüft, ob gegebenenfalls nachgewiesene Persistenzen auf die Existenz von strategischen Pfaden zurückzuführen sind. 
Auf diesem Wege wird in Teil C die empirische Grundlage gelegt, um in Teil D zu untersuchen, inwieweit und gegebenenfalls wie der Faktor Familie einen Einfluss auf die Genese und Dynamik dieser Pfade hatte.

\section{Vorgehen bei Durchführung der empirischen Studie}

\subsection{Einstieg ins Feld und Prozess des Samplings}

Angesichts der bislang begrenzten Forschung zur Thematik strategischer Persistenzen in Familienunternehmen und des explorativen Charakters der Forschungsfrage wurde zum Einstieg ins Feld zunächst eine explorative Vorstudie durchgeführt, um den Prinzipien der Offenheit und Gegenstandsangemessenheit qualitativer Forschung zu entsprechen. ${ }^{536}$ Darüber hinaus wurde durch die im Zuge der Vorstudie gewonnenen ersten Erkenntnisse das weitere Vorgehen der empirischen Untersuchung bestimmt. So gab die explorative Vorstudie vor allem Orientierung für die Fallauswahl des Samplings.

\subsubsection{Explorative Vorstudie}

Mit der explorativen Vorstudie wurden mehrere Ziele verfolgt: Zunächst sollte grundlegend die Bedeutung von Pfadabhängigkeit für die Entstehung von strategischen Persistenzen und Unternehmenskrisen im Kontext von Familienunternehmen eruiert werden. ${ }^{537}$ Darüber hinaus sollten vor allem aber auch bereits Indizien für bedeutsame organisationale Variablen gesammelt werden.

Hinsichtlich des weiteren Vorgehens der empirischen Studie galt es des Weiteren für die Untersuchung der Forschungsfrage relevante Fallgruppen zu identifizieren.

Ein nicht zu vernachlässigender Aspekt der Vorstudie war zudem, dass über diese ein Zugang zum Feld von Familienunternehmen in Krisensituationen gefunden werden sollte. Schon Weitzel \& Jonsson (1989) machen darauf aufmerksam, dass sich Untersuchungen zu dieser Thematik erheblich schwieriger

536 Vgl. Hoffmann-Riem (1980); Flick (2002: 26f.).

537 Zur Vermeidung einer theoretischen Vorstrukturierung wurde vor der Durchführung der Vorstudie weitestgehend auf das Studium von theoretischer Literatur zur Pfadtheorie verzichtet. Nichtsdestotrotz entsteht im Zuge der Erarbeitung einer Forschungsfrage natürlich eine theoretische Vororientierung, die entgegen der frühen Postulate von Glaser \& Strauss (1967) nicht einfach zurückgestellt werden kann. Daher wurde in vorliegender Studie großer Wert darauf gelegt, diese Orientierung nicht über Gebühr an den Forschungsgegenstand heranzutragen. Stattdessen wurde stets ergebnisoffen darauf gesetzt, die vermutete Relevanz bestimmter Konstrukte wie der selbstverstärkenden Mechanismen oder Imprints in der Empirie »wiederzuentdecken". 
gestalten als beispielsweise die Forschung zu den Herausforderungen organisationalen Wachstums: Entscheidungsträgern in akuten Krisensituationen fehle meist die Zeit, ehemaligen Führungskräften in gescheiterten Unternehmen meist die persönliche Bereitschaft, um der Managementforschung Auskunft über den Niedergang ihres Unternehmens oder gar über eigene Fehler zu geben. ${ }^{538}$ Dies dürfte für Familienunternehmen eine besondere Gültigkeit beanspruchen, müssen die Interviewpartner hier doch nicht nur bereit sein, über funktionale Defizite des Unternehmens und sachliche Fehler zu sprechen, sondern darüber hinaus möglicherweise auch über krisenverursachende familiäre Dynamiken. Daher diente die explorative Vorstudie nicht nur der gegenstandsoffenen Annäherung an das Feld, sondern auch dazu, über Multiplikatoren und »Türöffner« in diesem Feld erste Kontakte und Vertrauen aufzubauen.

\section{Datenerhebung der explorativen Vorstudie}

Angesichts dieser Zielstellungen bot sich ein Einstieg in die Forschungsthematik durch qualitative Experteninterviews mit Gesprächspartnern an, die biografische oder professionelle Erfahrungen und Kontakte mit Familienunternehmen in Unternehmenskrisen besaßen.

Entgegen der gängigen Praxis wurden diese Experteninterviews allerdings nicht als Leitfadeninterviews konzipiert. Vielmehr orientierten sie sich am Ablaufschema des in C.1.2.1 näher darzustellenden offenen narrativen Interviews nach Schütze (1978). Die für die explorative Vorstudie gewählte Form der Datenerhebung kann daher als narratives Experteninterview bezeichnet werden. $^{539}$

Für die Datenerhebung wurden mit zehn Experten mit Bezug zu Unternehmenskrisen in Familienunternehmen narrative Experteninterviews durchgeführt. Der Fokus dieser Interviews lag auf eigenen professionellen Erlebnissen und der Rekonstruktion persönlich erlebter Krisenprozesse. ${ }^{540}$

538 Vgl. Weitzel \& Jonsson (1989); auch die pragmatischen Hinweise von Pettigrew (1990: 276).

539 Obgleich das narrative Interview heute meist in anderen Forschungskontexten verwendet wird, stellen solch prozessrekonstruierende Interviews mit Experten einen Anwendungsbereich dar, auf den Schütze selbst verwies. Denn er entwickelte diese Interviewform als Instrument im Rahmen von Feldstudien, in denen Entscheidungsträger zum Ablauf kommunaler Entscheidungsprozesse befragt wurden. Vgl. Schütze (1978: 2); Przyborski (2008: 219).

540 Denn das narrative Interview ist auch nur dort zur Verwendung geeignet, wo tatsächlich erlebte Geschichten erzählt und erlebte Prozesse rekonstruiert werden können. Vgl. Przyborski \& Wohlrab-Sahr (2008: 95) zu den Voraussetzungen zur Verwendung des narrativen Interviews. 


\begin{tabular}{|c|c|c|c|c|c|}
\hline Ziel & $\mathbf{n}$ & $\boldsymbol{\Sigma}(\mathbf{h})$ & $\emptyset$ & Quellen & Auswahlkriterien \\
\hline $\begin{array}{c}\text { explorative } \\
\text { Annäherung } \\
\text { an das Feld } \\
\text { Ableitung des } \\
\text { Samplings }\end{array}$ & 10 & $14: 09$ & $1: 25$ & $\begin{array}{c}\text { Berater, Coaches, } \\
\text { Mediatoren, Anwälte, } \\
\text { Insolvenzverwalter, leitende } \\
\text { Angestellte }\end{array}$ & $\begin{array}{c}\text { Bezug zu Familien- } \\
\text { unternehmen und } \\
\text { Unternehmenskrisen } \\
\text { eigene biografische } \\
\text { Erlebnisse }\end{array}$ \\
\hline
\end{tabular}

Tabelle 5: Narrative Experteninterviews im Rahmen der explorativen Vorstudie (Quelle: Eigene Darstellung).

Zur Gewinnung eines möglichst breiten Spektrums an Perspektiven und Erzählungen folgte die Auswahl der Interviewpartner der Leitlinie der maximalen Variation. So wurden Unternehmensberater, Mediatoren, ehemals leitende Angestellte in Familienunternehmen, Unternehmensfamilien beratende Rechtsanwälte und Insolvenzverwalter interviewt. Nach Durchführung und Auswertung der ersten Interviews wurden verstärkt kontrastierende Perspektiven einbezogen..$^{51}$

Die Durchführung der Experteninterviews begann mit einem bewusst sehr offen formulierten Stimulus. So wurden die Experten um die Erzählung eines persönlich erlebten Falls in seiner ganzen zeitlichen Spanne und Komplexität gebeten: von der Krisenentstehung bis hin zu den Versuchen der Krisenbewältigung. An die hierauf erfolgende Erzählung anknüpfend, wurde im zweiten Teil der Interviews versucht, interessante Sachverhalte dieser Darstellung durch gezielte Nachfragen zu vertiefen und die Schilderung weiterer empirischer Beispiele zu evozieren.

Erst zum Schluss wurden die Interviewpartner gebeten, über die bisherige Darstellung des empirischen Falls hinausgehende, abstrakte Zusammenhänge und Thesen darzulegen, sowie die Relevanz bestimmter theoretischer Konstrukte einzuschätzen.

Parallel zur Durchführung und Auswertung der Interviews wurden im Rahmen der explorativen Vorstudie mehrere ausführlichere Darstellungen von Krisenprozessen aus wirtschaftshistorischen Arbeiten und der Fach- und Tagespresse recherchiert und zur Annäherung an die Thematik und das Feld analysiert.

\section{Auswertung der Daten}

Die Auswertung der erhobenen Daten erfolgte im Sinne der grundlegend zirkulären Forschungsstrategie parallel zur weiteren Datenerhebung und leitete die

541 Auf die Auswertung eines Interviews mit einem Berater, der für eine auf große Familienkonzerne spezialisierte Beratung tätig war, folgte so z. B. ein Interview mit einem auf KMU spezialisierten Coach. 
Auswahl weiteren Materials an. Hierbei wurden die Interviewdaten zunächst transkribiert und anschließend offen und axial kodiert. ${ }^{542}$

Es zeigte sich bereits nach der Auswertung der ersten Interviews und der narrativ geschilderten Fälle, dass für die weitere Untersuchung des familiären Einflusses auf die Genese und Dynamik strategischer Pfade mehrere Typen der Verbindung zwischen Familie und Unternehmen unterschieden werden müssen. Als Leitlinie der Differenzierung zeichneten sich der idealtypische Lebenszyklus von Familienunternehmen und die zugrunde liegende Eigentumsstruktur als geeignet ab. ${ }^{543}$ Auf deren Bedeutung wurde mehrfach auch explizit von den Interviewpartnern hingewiesen:

"[...] dann ist sicherlich beim Familienunternehmen für Sie vielleicht auch wichtig, zu unterscheiden, ist es ein Familienunternehmen der ersten Generation? Ist es der Pionierunternehmer? Oder ist es schon ein Mehrgenerationenunternehmen? Das ist wichtig auch für die Krisentypologie beziehungsweise für die Anfälligkeit.»

(Ein auf Familienunternehmen spezialisierter Unternehmensberater im Rahmen der explorativen Vorstudie)

Diese Vorstellung einer idealtypischen Entwicklung von Eigentum, Familie und Unternehmen - vom gründergeführten Unternehmen bis zum komplexen Familienunternehmen - kann ob der zugrunde liegenden Wirkkräfte eine vergleichsweise hohe Geltungskraft beanspruchen. ${ }^{544}$ Denn insbesondere die Distribution des Eigentums entlang dominanter gesellschaftlicher Gerechtigkeitsnormen führt zur immer wieder beobachtbaren Ausbildung dieser typischen Entwicklungsstadien familiär beeinflusster Unternehmen. Daher werden diese Stadien in der Forschung zu Familienunternehmen immer wieder zur Differenzierung verschiedener Formen der Verbindung zwischen Familie und Unternehmen herangezogen. Analog zu vielen anderen Beiträgen der Forschung zu orientiert sich die empirische Untersuchung an diesen Stadien. ${ }^{54}$

542 Auf eine selektive Kodierung wurde in der Vorstudie verzichtet. Vgl. Przyborski \& Wohlrab-Sahr (2008: 204ff.).

543 Zur Vorstellung einer idealtypischen Entwicklung vom gründergeführten Unternehmen bis hin zum komplexen Familienunternehmen vgl. Ward (1987) und vor allem Gersick et al. (1997).

544 Herrschende Gerechtigkeitsnormen und Erbpraktiken, das biologische Wachstum der Unternehmensfamilie, sowie die Zwänge der Marktwirtschaft führen in der Summe mit einiger Sicherheit dazu, dass Familienunternehmen sich im Verlaufe von Generationen im Sinne dieser Vorstellung entwickeln. Vgl. Gersick et al. (1997: 15ff.); Simon et al. (2005: $39 \mathrm{ff}$.$) .$

545 Vgl. Hatum et al. (2010: 262) zur Bedeutung des jeweiligen Evolutionsstadiums für die Analyse der organisationalen Wandlungsfähigkeit. Ähnlich auch Goehler (1993); Rosenbauer (1994); Klein (2010: 279ff.). 


\subsubsection{Sampling: Die Fallgruppen der monozentrischen und polyzentrischen Konfiguration}

Den Indizien der explorativen Vorstudie zufolge sind jedoch weniger die zeitlichen Entwicklungsstadien an sich für die Genese und Dynamik strategischer Pfade im Kontext des familiären Einflusses von besonderer Bedeutung, sondern vielmehr zwei mit ihnen häufig verbundene besondere familiär-organisationale Konfigurationen des familiären Einflusses auf das Unternehmen. ${ }^{546}$ Daher wurden im Zuge des theoretischen Samplings zunächst folgende zwei Fallgruppen definiert:

(1) Familienunternehmen, in denen das familiäre Einflusspotenzial monozentrisch und asymmetrisch innerhalb einer relativ kleinen dominanten Koalition verteilt ist.

(2) Familienunternehmen, in denen das familiäre Einflusspotenzial polyzentrisch und annähernd symmetrisch innerhalb einer größeren dominanten Koalition verteilt ist.

(Fallgruppen der empirischen Untersuchung)

Erstere Konfiguration, in der eine im Vergleich zu anderen ökonomischen Organisationsformen bemerkenswert hohe Konzentration von Einflusspotenzial beobachtet werden kann, soll im Folgenden als monozentrische familial-organisationale Konfiguration bezeichnet werden. Sie findet sich empirisch häufig in Familienunternehmen der ersten und zweiten Generation.

Die zweite Konfiguration, mit ihrer ebenso familienunternehmensspezifischen symmetrischen Verteilung des Einflusspotenzials auf mehrere Subzentren innerhalb der dominanten Koalition, soll im Folgenden polyzentrisch genannt werden. Sie findet sich meist in Unternehmen, in denen bereits ein oder mehrere Generationenübergänge stattgefunden haben.

Eine an diesen beiden Polen ausgerichtete Untersuchung versprach einen großen Teil des oben umrissenen Spektrums des formalen und informalen Einflusses des Faktors Familie auf die Genese und Dynamik strategischer Pfade abzudecken. Darüber hinaus schienen sie in ausreichendem Maße dem qualitativ-methodologischen Kriterium der theoretischen Generalisierbarkeit zu genügen. ${ }^{547}$

546 Die Arbeit knüpft hiermit an das konfigurationale Denken der strategischen Prozessforschung an, welches der holistischen und aggregierten Untersuchung bestimmter Typen dient. Vgl. (Pettigrew 2001: 702); Mintzberg (1984). Zu Konfigurationen organisationaler Macht vgl. Fredrickson (1986).

547 Der Geltungsanspruch qualitativer Forschung beruht weniger auf der Anzahl der untersuchten Fälle als vielmehr auf der abstrakten Idee und dem theoretisch oder empirisch hergeleiteten logischen Zusammenhang, den die untersuchten Fälle repräsentieren und in dem die Erkenntnisse auch in anderen Fällen Gültigkeit beanspruchen können. Zur theoretischen Generalisierung ausführlich Przyborski \& Wohlrab-Sahr (2008: $311 \mathrm{ff}$.). 
Auf der Grundlage dieser beiden Fallgruppen wurden anschließend zwei empirische Fälle gesucht, die aufgrund von strategischer Pfadabhängigkeit in die Unternehmenskrise geraten waren. Nach dem Screening von sieben potenziell geeignet erscheinenden Fällen fiel die Wahl schließlich auf die Anton Schlecker e.K. als Repräsentant der ersten und auf die Berentzen-Gruppe AG als Repräsentant der zweiten Konfiguration. ${ }^{548}$ Anhand dieser beiden Fälle sollte im Zuge zweier Einzelfallstudien empirisch der Einfluss des Kontextfaktors Familie auf die Genese und Dynamik der strategischen Pfade analysiert werden. ${ }^{549}$

Bereits nach den ersten Auswertungsschritten wurde jedoch deutlich, dass dieses ursprüngliche Sampling erweitert werden musste, denn manche der für die Pfadgenese und -dynamik als relevant identifizierten organisationalen Variablen waren zwar in den singulären Fällen für die Entstehung der strategischen Persistenz von großer Bedeutung, aber in der Empirie wurden Fälle mit der gleichen Konfiguration und ähnlichen Ausprägungen dieser Variablen entdeckt, deren strategische Entwicklung von einem bemerkenswerten Wandel gekennzeichnet war.

Zur Validierung und schärferen Selektion der vermuteten Zusammenhänge wurden daher im Sinne des »theoretical samplings« (Glaser \& Strauss, 1967) dem zufolge die konkrete Auswahl des empirischen Materials grundsätzlich nicht vorab, sondern erst im Zuge des Prozesses der Datenerhebung und -auswertung erfolgen sollte - schrittweise weitere Fälle in das Sample aufgenommen. ${ }^{550}$

Dabei wurde das Sample zur Herausarbeitung und Validierung der für die Pfadgenese relevanten organisationalen Variablen zunächst um eine komparative Perspektive ergänzt. Gesucht wurden zwei Unternehmen innerhalb der jeweiligen Branchen, die zu Beginn der untersuchten strategischen Entwicklung sowohl die gleiche familial-organisationale Konfiguration als auch eine ähnliche strategische Ausgangslage aufgewiesen hatten. Gefunden wurden diese beiden Fälle mit der Dirk Rossmann $\mathrm{GmbH}$, dem ersten Drogeriemarktdiscounter in

548 Das Screening folgt den Empfehlungen von Pettigrew (1990: 275): »If the phenomena to be observed have to be contained within a single or relatively small number of cases then choose cases where the progress is transparently observable [...] sometimes social dramas and the public inquiries they produce offer social scientists the first opportunity to look inside a previously shielded social system."

549 Vgl. zur Fallstudie als Methode in der strategischen Prozessforschung Eisenhardt (1989: 536): »a research strategy that focuses on understanding the dynamics present in a single setting«; vor allem auch Eisenhardt \& Graebner (2007) oder Mintzberg (2005).

550 Der Gedanke ist hierbei, dass der Forschungsgegenstand sich dem Forscher nach dem Einstieg erst nach und nach erschließt. So soll nach einer Auswertung von Daten stets aufs Neue überlegt werden, von welchen zusätzlichen Daten oder Fällen neue Erkenntnisse zu erwarten sind. Die Auswahl wird dann abgeschlossen, wenn eine »theoretische Sättigung“ erreicht ist, d.h. von vergleichbaren neuen Fällen nichts entscheidend Neues mehr zu erwarten ist. Vgl. Flick (2002: 154ff.); auch Eisenhardt (1989). 
Deutschland, und der Eckes AG, dem ehemals größten deutschen Spirituosenhersteller.

\begin{tabular}{|c|c|c|c|}
\hline & \multicolumn{2}{|c|}{ strategische Persistenz } & strategischer Wandel \\
\hline monozentrisch & Anton Schlecker e.K. & 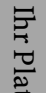 & Dirk Rossmann GmbH \\
\hline polyzentrisch & Berentzen-Gruppe AG & 吾 & Eckes AG \\
\hline
\end{tabular}

Tabelle 6: Fallauswahl des komparativen Samplings

(Quelle: Eigene Darstellung).

Obwohl diese beiden Unternehmen mit ihren pfadabhängig gewordenen Wettbewerbern eine Vielzahl von Ausprägungen organisationaler Variablen teilten, konnten sie auf die Dynamik der Umwelt reagieren und den strategischen Wandel erfolgreich gestalten. Somit ergab sich für die komparative Analyse die Frage, hinsichtlich welcher weiterer Variablen und Ausprägungen sich die Unternehmen in Bezug auf ihre Entscheidungsprozesse entscheidend unterschieden.

Einige der im Zuge dieser Vergleichsanalyse erarbeiteten und im Kapitel D präsentierten Zusammenhänge wurden anschließend an einem weiteren Fall, der Ihr Platz GmbH, konfigurationsübergreifend geprüft. ${ }^{551}$ Letztlich ergab sich aus dem »theoretical sampling « das in der obigen Tabelle dargestellte Sample der empirischen Studie.

\subsection{Prozess und Methoden der Datenerhebung und -auswertung}

Zur Untersuchung der ausgewählten Fälle wurde auf mehrere etablierte, aber auch einige innovative Methoden zur Erhebung und Auswertung qualitativer Daten zurückgegriffen.

551 Zur Wahrung der Übersichtlichkeit wird sich bei der Darstellung der empirischen Fälle aber auf die Anton Schlecker e.K., die Berentzen-Gruppe AG sowie deren beiden Vergleichsfälle konzentriert. 


\subsubsection{Methoden der Datenerhebung}

Hinsichtlich der Datenerhebung wurde eine Form der Methoden-Triangulation durchgeführt und die Methode des narrativen Interviews mit Verfahren der Dokumentenanalyse kombiniert. ${ }^{552}$

Kern der Datenerhebung waren offene Interviews, die der narrativen Interviewmethodik nach Schütze (1983) folgten. ${ }^{553}$ Diese Methodik basiert grundlegend auf der Annahme einer Homologie zwischen Erzählungskonstitution und Erfahrungskonstitution. So wird davon ausgegangen, dass sich im Gegensatz zu anderen Formen der Sachverhaltsdarstellungen, wie zum Beispiel der Beschreibung oder der Argumentation, in der Struktur der Erzählung die Struktur der Erfahrung widerspiegelt. ${ }^{554}$ Ursächlich für diese Strukturierung seien bestimmte »Zugzwänge des Erzählens«: der Detaillierungszwang, der Gestaltschließungszwang sowie der Relevanzfestlegungs- und Kondensierungszwang.

Die in der Methodenliteratur für den Einsatz dieser Methode angelegten Kriterien waren in Bezug auf die Untersuchung von strategischen Pfaden als spezifische Prozesse der Krisenentstehung erfüllt, denn zum einen liegt in Bezug auf den Prozess der Krisenentstehung und der schlussendlichen Unternehmenskrise eine manifeste autobiografische Erfahrung vor, welche in der Erzählung kognitiv reproduziert werden kann. ${ }^{55}$ Zum anderen liegt dieser Erfahrung eine Prozessstruktur zugrunde, welche der Erzähler rekonstruieren kann. Ob der intensiven Perzeption von Unternehmenskrisen und des ihnen inhärenten Prozesscharakters, scheint der Einsatz des narrativen Interviews zur Erhebung von Daten zu Unternehmenskrisen daher sehr geeignet. In der Literatur zur qualitativen Forschungsmethodik werden ähnliche Prozesse, wie zum Beispiel die "Betriebsstillegung", ebenfalls als Anwendungsbeispiele angeführt. ${ }^{556}$

Analog zu den Experteninterviews im Rahmen der explorativen Vorstudie wurden die biografisch-narrativen Interviews mit einem bewusst sehr offen formulierten Stimulus angestoßen. So wurden die biografischen Experten gebeten, zunächst ihre Zeit im Unternehmen gedanklich Revue passieren zu lassen

552 Vgl. zur Triangulation Flick (2007: 519f.).

553 Vgl. zur Methode des narrativen Interviews originär Schütze (1978); Schütze (1983). Für eine Diskussion Przyborski \& Wohlrab-Sahr (2008: 219ff.).

554 Vgl. hierzu ausführlich Bude (1985); Maindok (1996); Nassehi \& Saake (2002). Zur erzähltheoretischen Fundierung wie den Zugzwängen des Erzählens auch Flick (2007: 227 ff.); Przyborski \& Wohlrab-Sahr (2008: $221 \mathrm{ff}$.).

$555 \mathrm{Zu}$ den Kriterien für den Einsatz dieser Methode vgl. Przyborski \& Wohlrab-Sahr (2008: 95): »Entscheidend ist, dass der zu erforschende Gegenstand eine Prozessstruktur hat, die der Erzähler aus seiner persönlichen Perspektive rekonstruieren kann.»

556 Przyborski \& Wohlrab-Sahr (2008: 95): [E]ine Betriebsstillegung hat beispielsweise eine Prozessstruktur $[\ldots] .$. 
und dann zu erzählen wie das Unternehmen aus ihrer ganz persönlichen Perspektive in die Krise geriet. Somit wurde bewusst darauf verzichtet, dem Interviewpartner die Schablone der strategischen Pfadabhängigkeit anzubieten, oder bereits in der Frage einen vermuteten Einfluss des Faktors Familie zu suggerieren.

Im zweiten Teil der narrativen Interviews wurde dann durch gezieltes Nachfragen zu Besonderheiten des strategischen Entscheidungsprozesses versucht, interessante Sachverhalte dieser Darstellung zu vertiefen und die Schilderung entsprechender empirischer Beispiele zu evozieren.

Erst im dritten Teil wurden die Interviewpartner gebeten, die Relevanz von Konzepten einzuschätzen, die aus der theoretischen Literatur bekannt waren oder im Verlauf der empirischen Untersuchung bereits identifiziert worden waren.

Die narrativen Interviews dienten somit sowohl der Rekonstruktion der Prozesse der Krisenentstehung, als auch der Exploration des Einflusses des Faktors Familie auf den strategischen Entscheidungsprozess und die Genese und Dynamik der strategischen Pfade.

\begin{tabular}{|c|c|c|c|}
\hline Quellen & Kriterien des Einbezugs & $\mathrm{N}$ Interviews & $\mathrm{H}^{557}$ \\
\hline \multirow{2}{*}{$\begin{array}{c}\text { Mitglieder der } \\
\text { Unternehmensfamilie }\end{array}$} & \multirow{8}{*}{$\begin{array}{c}\text { inhaltliche Variation } \\
\text { chronologische Variation } \\
\text { bei Nicht- } \\
\text { Familienangehörigen: } \\
\text { Nähe zur } \\
\text { Unternehmensfamilie }\end{array}$} & \multicolumn{2}{|c|}{ Fallgruppe 1 (monozentrisch) } \\
\hline & & 7 & $11: 36$ \\
\hline \multirow{3}{*}{$\begin{array}{l}\text { Führungskräfte } \\
\text { ehemalige } \\
\text { Führungskräfte }\end{array}$} & & \multicolumn{2}{|c|}{ Fallgruppe 2 (polyzentrisch) } \\
\hline & & 12 & $12: 07$ \\
\hline & & \multicolumn{2}{|c|}{ konfigurationsübergreifend } \\
\hline \multirow{2}{*}{$\begin{array}{l}\text { Mitglieder der Kontroll- } \\
\text { und Aufsichtsorgane }\end{array}$} & & 4 & $5: 09$ \\
\hline & & \multicolumn{2}{|c|}{ gesamt } \\
\hline & & 23 & $28: 52$ \\
\hline
\end{tabular}

Tabelle 7: Übersicht über narrative biografische Fallinterviews (Quelle: Eigene Darstellung).

Insgesamt wurden mit 23 Interviewpartnern narrativ-biografische Interviews geführt. Wiederum galt das Kriterium der maximalen sowohl inhaltlichen als auch chronologischen Variation. ${ }^{558}$ Bei Nicht-Familienangehörigen war die Nähe zur Unternehmensfamilie ein weiteres Kriterium des Einbezugs.

Alle biografischen narrativen Interviews wurden persönlich vor Ort bei den

557 Die Dauer der Interviews betrug meist etwas mehr als eine Stunde, in einigen Fällen aber auch weit mehr als zwei Stunden oder nur knapp 30 Minuten.

558 Da strategische Entwicklungen zum Teil über einen Zeitraum von bis zu siebzig Jahren untersucht wurden, galt es nicht nur aktuelle Führungskräfte zu interviewen, sondern auch bereits vor Jahrzehnten aus dem Unternehmen ausgeschiedene ehemalige Entscheidungsträger. 
Gesprächspartnern durchgeführt und mit einem Aufnahmegerät aufgezeichnet. Anschließend wurden die Interviews nach den gängigen Regeln und Kriterien der qualitativen Methodenlehre transkribiert und so der weiteren Auswertung zugänglich gemacht.

Außer auf die Interviewdaten wurde insbesondere zur Rekonstruktion der historischen und strategischen Entwicklung der Fälle auf Daten aus Dokumenten wie Geschäftsberichten, Pressemitteilungen oder Investorenprospekte zurückgegriffen. ${ }^{559}$ In diesem Zuge wurden Daten vor allem aus Dokumenten wie Geschäftsberichten, Pressemitteilungen oder Artikel der Fachpresse erhoben. Dabei wurde großen Wert auf eine Variation der Quellen und einen quellenkritischen, kontextsensitiven Umgang mit diesen Daten gelegt.

\begin{tabular}{|c|c|c|c|}
\hline Ziel & Quellen & Einbezugskriterien & $\Sigma$ \\
\hline & Geschäftsberichte, & & \\
Extrahierung von (Prozess-)Daten & Pressemitteilungen, & & \\
zur historischen Entwicklung der & Artikel in & & \\
Fälle und der Branchen, & Fachzeitschriften und & vgl. Scott (1990), & \multirow{2}{*}{ 608 } \\
theoriegeleitete Pfaddiagnose & allgemeiner Presse, & Mayring (2010: 53) & \\
(deduktive Kategorienanwendung) & Branchendaten, & & \\
& unternehmensinterne & & \\
& Dokumente & & \\
\hline
\end{tabular}

Tabelle 8: Übersicht zu Daten aus internen und externen Dokumenten (Quelle: Eigene Darstellung).

Die aus den Dokumenten erhobenen Daten wurden ebenso wie die Interviews in der auf der qualitativen Forschungssoftware ATLAS.ti beruhenden Fallstudiendatenbank gespeichert und so der weiteren Analyse zugänglich gemacht.

\subsubsection{Ablauf der Fallanalysen und Methoden der Datenauswertung}

Die Analyse des Einflusses des Faktors Familie auf die Genese und Dynamik von strategischen Pfaden knüpft an die methodischen Vorarbeiten der organisationalen Pfadforschung von Holtmann (2008) und Koch (2011) zur Analyse von

559 Die Nutzung solcher Dokumente ist sowohl in der allgemeinen Managementforschung als auch in der Familienunternehmensforschung weit verbreitet. Vgl. Pettigrew (1990: 277); Berrone et al. (2012: 265); (Nordqvist et al. (2009). Selbstverständlich wäre es wünschenswert, wenn die Prozessrekonstruktion allein durch die Interviewdaten erfolgen würde. Dies ist im zeitlichen Rahmen einzelner Interviews für sich über Jahrzehnte erstreckende Prozesse allerdings kaum möglich. Zudem lässt die Analyse solcher Dokumente die Untersuchung von weit in der Vergangenheit liegenden Ereignissen zu, die sich der Erfahrung oder Erinnerung der Interviewpartner entziehen. 
strategischer Pfadabhängigkeit an. ${ }^{560}$ Sie basiert auf Globalanalysen vor dem Sampling und einer dreistufigen Pfadanalyse der ausgewählten Fälle.

\section{Globalanalysen}

Im Rahmen des Samplings wurden zunächst Globalanalysen durchgeführt, um die Eignung des Falls für die Untersuchung zu prüfen. ${ }^{561}$ Folgende Fragen wurden an die Fälle gestellt:

1. Ist ein zeitlich stabiles strategisches Muster erkennbar, welches auf konstanten Aktivitäten, Reflexionen und / oder Resultaten beruht?

2. Lassen sich Elemente selbstverstärkender Mechanismen ausmachen (bzw. kritische Ereignisse die darauf bezogen sind)?

3. Lassen sich in der Umwelt Veränderungen ausmachen, die das strategische Muster ab einem bestimmten Zeitpunkt als ökonomisch ineffizient erscheinen lassen?

4. Lassen sich ergebnislose oder gar kontraproduktive strategische Aktivitäten identifizieren, die darauf abzielen die strategische Situation zu ändern und somit zu verbessern?

5. Lässt sich ein signifikanter Einfluss des Systems Familie auf die Genese und Dynamik des strategischen Pfades im Unternehmen ausmachen?

(An die Daten gestellte Fragen im Zuge der Fallanalysen)

Zur Untersuchung dieser Fragen wurden externe Dokumente wie Medienberichte oder Marktforschungsstudien analysiert und mithilfe von Fallchroniken oder Zeitlinien ein erstes vorläufiges Bild von der organisational-strategischen Entwicklung gezeichnet. Ließ die Analyse dieser Daten zu viele der obigen Fragen offen wurde der Fall verworfen. Lagen hingegen gewichtige Indizien für die Existenz strategischer Pfade und eines signifikanten Einflusses des Faktors Familie vor, so konnte eine begründete Pfadhypothese aufgestellt werden. ${ }^{562}$

Für die entsprechend dem oben skizzierten Samplings ausgewählten Fälle der Anton Schlecker e.K. und der Berentzen-Gruppe AG wurde anschließend eine dreistufige pfadtheoretische Untersuchung in Anlehnung an das Vorgehen von Holtmann (2008) durchgeführt. ${ }^{563}$

560 Vgl. Holtmann (2008: $140 \mathrm{ff}$ )); Koch (2011: 346ff.).

561 Im Vordergrund dieser Analysen stand somit die Frage, ob sich sowohl die Existenz von Pfadabhängigkeit als auch ein signifikanter Einfluss der Familie begründet vermuten ließe.

562 Koch (2011: 346): »Assuming that a concrete and observable form of rigidity related to a specific pattern is constituted by a path-dependent process."

563 Vgl. Holtmann (2008: 64ff.) zur theoretischen Konzeption dieser Analyse und Holtmann (2008: 140ff.) zur Anwendung am Fall des Bertelsmann Buchclubs. 


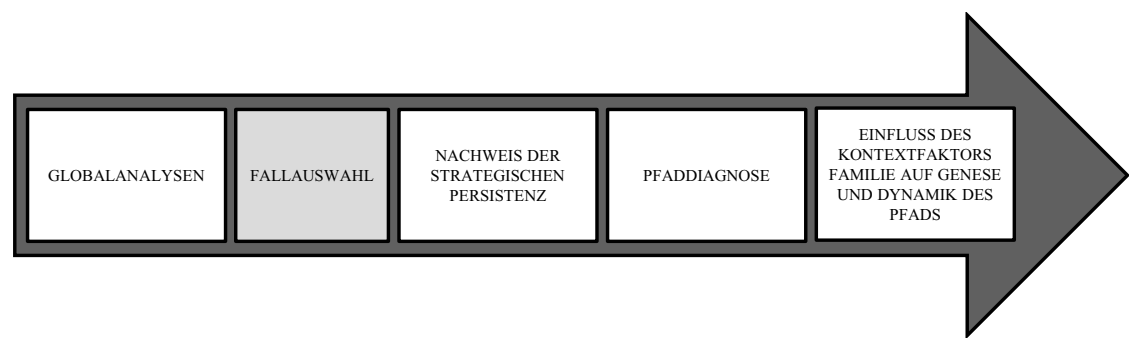

Abbildung 8: Ablauf der Fallanalysen

(Quelle: Eigene Darstellung).

\section{Schritt 1 der Pfadanalyse: Nachweis der strategischen Persistenz}

Da strategische Pfadabhängigkeit als eine spezifische Form strategischer Persistenz zu verstehen ist, wurde im ersten Schritt der Pfadanalyse der Frage nachgegangen, inwieweit die strategische Entwicklung der Fälle als persistent erachtet werden kann.

Zunächst wurden zentrale strategische Parameter wie zum Beispiel die Entwicklung der Gesamtunternehmensstrategien oder die Elemente des Geschäftsmodells im Detail auf Kontinuität geprüft. Anschließend wurden diese strategischen Parameter und deren Kontinuität mit der Entwicklung der Branchenumwelt aus Wettbewerbern oder Kundenbedürfnissen kontrastiert.

Zeigte sich in diesem Vergleich, dass ein strategisches Muster trotz einer sich dynamisch verändernden Umwelt unbeirrt und kontinuierlich weiter verfolgt wurde, so konnte eine strategische Persistenz attestiert werden. ${ }^{564}$

Bei diesem Analyseschritt wurde vor allem auf externe Dokumente wie Marktforschungsstudien und Artikel aus der Fachpresse zurückgegriffen, seltener auf interne Dokumente und Interviewdaten. ${ }^{565}$

Aus diesen sekundären Quellen wurden Prozessdaten zur chronologischen organisationalen und strategischen Entwicklung des Unternehmens und dessen Branchenumwelt extrahiert, welche anschließend unter Angabe von Merkmalen wie Jahr, Quelle oder Titel in eine EndNote-basierte Fallstudiendatenbank eingepflegt und chronologisch geordnet wurden. Anschließend wurden diese einzelnen Datensätze in Anlehnung an das Vorgehen der qualitativen Inhaltsanalyse nach Mayring (2010) generalisiert und reduziert. ${ }^{566}$

Die chronologisch geordnete und theoretisch strukturierte tatsächliche

564 Die Analyse der strategischen Persistenz fokussierte somit sowohl das Elemente "strategische Kontinuität«, als auch die Dynamik der Umwelt. Vgl. hierzu: Holtmann (2008: 65).

565 Die Interviewdaten wurden im Rahmen dieses Schrittes eher zur Validierung verwendet.

566 Vgl. Mayring (2010). Die meisten Kodes wurden induktiv aus dem Datenmaterial gewonnen, manche (z.B. die Phasen des Krisenprozesses) auch deduktiv eingeführt. Zudem wurden die Prozessdaten noch in ATLAS.ti kodiert. 
Entwicklung der Unternehmen wurde zur Rekonstruktion der »organisationalen Biographie« anschließend mit Hilfe eines aus der soziologischen Lebenslaufforschung nach Rosenthal \& Fischer-Rosenthal (2012) entlehnten Verfahrens analysiert: der sequenziellen retrospektiv-prospektiv kontrastierenden Analyse der organisational-strategischen Entwicklung. ${ }^{567}$

Zunächst wurden mehrere Prozessdaten zu einzelnen Phasen zugeordnet. Anschließend wurde das vorhandene Wissen um den tatsächlichen Verlauf der Entwicklung zurückgestellt und gefragt, was die einzelnen Prozessdaten wohl für die weitere Entwicklung des Unternehmens bedeuten könnten und welche Entwicklung ausgehend von der gerade betrachteten Phase nun wahrscheinlich wäre. ${ }^{568}$ So wurde versucht der methodologischen Aufforderung der Pfadforschung Genüge zu leisten, der zufolge Prozesse am besten in »Echtzeit» rekonstruiert werden. ${ }^{569}$

Diese Überlegungen resultierten in der Aufstellung sogenannter Entwicklungshypothesen, die anschließend mit der tatsächlichen organisational-strategischen Entwicklung verglichen wurden. Zeigte sich hierbei eine zunehmende Abweichung zwischen der Entwicklung der Umwelt und den potenziellen Möglichkeiten des Unternehmens auf der einen Seite und der tatsächlichen strategischen Entwicklung auf der anderen Seite, so wurde eine strategische Persistenz konstatiert. Hier setzte die Erörterung der Frage ein, warum die Entwicklung diesen Verlauf nahm und keinen anderen.

\section{Schritt 2 der Pfadanalyse: Pfaddiagnose}

Im zweiten Schritt der Pfadanalyse wurde untersucht, ob und inwieweit sich die identifizierte strategische Persistenz auf die Existenz eines strategischen Pfades zurückführen lässt.

Im Zuge dieser Pfaddiagnose wurde unter Heranziehung der Elemente der organisationalen Pfadtheorie analysiert, inwiefern die strategische Entwicklung und die Krisenentstehung durch pfadtheoretische Konstrukte wie Imprints, selbstverstärkende Mechanismen und Lock-ins erklärt werden kann.

So wurde zum Beispiel der Frage nachgegangen, in welchem Maß die strategische Entwicklung bereits früh historisch vorgeprägt und in ihrer Kontingenz

567 Vgl. Kimberly \& Bouchikhi (1995). Zur retrospektiv-prospektiv kontrastierenden Analyse in der Lebenslaufforschung Rosenthal \& Fischer-Rosenthal (2012).

568 So wurde z.B. gefragt welche Bedeutung ein von Sparsamkeit und Misstrauen geprägtes Umfeld für die Gründung eines jungen Unternehmers besitzt. Ist es wahrscheinlicher, dass ein solcher Unternehmer eine Parfümerie eröffnet, oder eher ein kostenzentriertes Einzelhandelsgeschäft?

569 Vgl. Garud et al. (2010: 770):»Methodologically, our path creation perspective suggests that it is important for a researcher to study processes in rreal times, i. e. place oneself at the time that events occurred even if one were looking at data gathered in the past." 
eingeschränkt war. Vor allem aber wurde geprüft, inwieweit die strategischen Muster durch etwaige selbstverstärkende Mechanismen gestützt wurden. Hierbei stand der strategische Entscheidungsprozess, der innerhalb der jeweiligen dominanten Koalitionen verlief, als zentrale »unit of analysis« im Zentrum des Interesses. ${ }^{570}$

In Bezug auf den aus den selbstverstärkenden Mechanismen resultierendem Lock-in wurde die Ineffizienz des »eingelockten« strategischen Musters nachgewiesen, vor allem aber dessen "Resistenz« und »Immunität« gegen Änderungsversuche von innen wie außen analysiert. ${ }^{571}$ Darüber hinaus wurde untersucht, auf welcher Ebene sich der Lock-in angesiedelt und entwickelt hatte. ${ }^{572}$

Für diesen zweiten Schritt der Pfadanalyse waren die Daten aus den narrativen biografischen Fallinterviews zentral. Die Interviewdaten wurden im Zuge der Auswertung entsprechend der gängigen Methodik der qualitativen Sozialforschung mithilfe der Analysesoftware ATLAS.ti offen, axial und selektiv kodiert. ${ }^{573}$ Auf diesem Wege wurde die Pfadhypothese getestet und Zusammenhänge zwischen den einzelnen Variablen identifiziert.

\section{Schritt 3 der Pfadanalyse: Einfluss des Faktors Familie}

Nachdem im ersten Schritt die strategische Persistenz nachgewiesen worden war und im zweiten Schritt diese Persistenz anhand der Elemente des strategischen Pfades erklärt werden konnte, wandte sich die Pfadanalyse mit dem dritten Schritt dem Faktor Familie zu. Es wurde gefragt, ob und - gegebenenfalls - wie der Einfluss des Kontextfaktors Familie zur Genese und Dynamik des strategischen Pfades beigetragen hatte.

Die Ergebnisse dieses Schrittes werden ausführlich in Kapitel D präsentiert. Zuvor wird im nächsten Kapitel aber die Pfadabhängigkeit der beiden Schwerpunktfälle der Anton Schlecker e.K. und der Berentzen-Gruppe AG aufgezeigt.

570 Wie in der Skizzierung des theoretischen Bezugsrahmens ausgeführt, umfasst das Konstrukt der dominanten Koalition in Bezug auf familiär beeinflusste Unternehmen die dominanten Entscheidungsträger der Systeme Unternehmen und Familie. Es adressiert somit den Umstand, dass im Zuge der Entscheidungsprozesse die Grenzen zwischen dem Management, dem Kreis der Gesellschafter oder weiteren Familienangehörigen sowie dem Kontrollorgan häufig fließend sind.

571 Der Nachweis der Ineffizienz des Pfades ist in der Pfadtheorie im Allgemeinen häufig schwer zu erbringen, gestaltete sich in dieser Untersuchung ob der manifestierten Unternehmenskrise jedoch problemlos.

572 Somit wurde der Frage nachgegangen, was nun genau zu welchem Zeitpunkt, pfadabhängig geworden war - »nur« die kognitiven Schemata der maßgeblichen Entscheidungsträger oder bereits die Ressourcenallokation des gesamten Unternehmens?

573 Vgl. Przyborski \& Wohlrab-Sahr (2008: 203ff.); Strauss (1991: $56 \mathrm{ff}$.). 


\section{Fallstudien: Strategische Pfade in die Krise}

Nachdem der theoretische Bezugsrahmen und das methodische Vorgehen dieser Arbeit erläutert wurden, wendet sich dieses Kapitel der Empirie zu und es werden im Folgenden die strategischen Entwicklungen der beiden primären Fallstudien der Anton Schlecker e.K. und der Berentzen-Gruppe AG untersucht. Im Sinne einer "rich description« (Mintzberg, 2005) werden zur Einführung jeweils zunächst die historischen Entwicklungen der Unternehmen im Kontext ihrer Umweltdynamik präsentiert. Besonderes Augenmerk gilt den Wettbewerbern, insbesondere den beiden Kontrastfällen der Dirk Rossmann GmbH und der Eckes AG.

"I need to be stimulated by rich description, stories best of all.» (Mintzberg, 2005) $)^{574}$

Auf diesem Verständnis der Fälle aufbauend wird dann im ersten Schritt der Pfadanalyse zunächst geprüft, inwiefern die Unternehmenskrisen der Anton Schlecker e.K. und der Berentzen-Gruppe AG auf strategische Persistenzen zurückgeführt werden können.

Im zweiten Schritt der Pfadanalyse wird aufgezeigt, dass diese Persistenzen maßgeblich auf die Existenz strategischer Pfade zurückgeführt werden können. Im Zentrum dieser Pfaddiagnosen stehen die pfadkonstituierenden Elemente der selbstverstärkenden Mechanismen und die aus ihnen erwachsende Situation des Lock-in.

\subsection{Fallstudie zur Fallgruppe I: Anton Schlecker e.K.}

Die Anton Schlecker e.K. war ein familiengeführtes Drogeriemarktunternehmen, welches ab 1975 schwerpunktmäßig in Deutschland Drogeriemärkte nach dem Discount-Prinzip betrieb. Das Unternehmen beschäftigte zeitweise mehr als 50.000 Mitarbeiter und erzielte mit seinen mehr als 14.000 Filialen im Jahr 2008 einen Umsatz von 7,4 Mrd. Euro. Nach massiven Umsatzeinbrüchen und daraus resultierenden finanziellen Problemen musste das Unternehmen im Jahr 2012 Insolvenz anmelden und wurde größtenteils zerschlagen.

574 Vgl. Mintzberg (2005: 362). Ziel der Darstellung ist eine Art »thick description«, die nicht nur einzelne Meilensteine der Unternehmensentwicklung aneinanderreiht, sondern diese in ihrem historischen Kontext präsentiert. Diesem Ziel soll auch die Einbindung der Interviewausschnitte und Zitate dienen. 


\subsubsection{Historische Entwicklung des Unternehmens und seiner Branchenumwelt}

\section{Vorspann}

Die Anfänge der ehemals größten Drogeriemarktkette Europas liegen in einer Metzgerei. Nachdem Anton Schlecker sen. 1950 aus der Kriegsgefangenschaft nach Ehingen auf die Schwäbische Alb zurückkehrt, eröffnet er zunächst einen, kurze Zeit später einen zweiten Fleischwarenladen. ${ }^{575}$ Bald darauf folgt eine dritte Filiale im Nachbarort.

1944 wird der älteste Sohn Anton Schlecker geboren, der einige Jahre später in die Fußstapfen des Vaters tritt und eine Ausbildung zum Metzger beginnt. 1965 steigt Anton Schlecker jun. als jüngster Metzgermeister Baden-Württembergs in den väterlichen Betrieb ein, der sich in der Zwischenzeit zu einer Fleischwarenfabrik mit 17 Metzgereien und 400 Mitarbeitern entwickelt hat. ${ }^{576}$

Die Familie Schlecker strebt jedoch nach mehr und so wird 1965 ein erster großer Verbrauchermarkt mit Selbstbedienung eröffnet. Der Erfolg dieses Marktes löst unter den Führungskräften eine regelrechte "Goldgräberstimmung « aus und rasch werden weitere Märkte im Umkreis eröffnet. Doch bereits kurze Zeit später erschüttern revolutionäre Entwicklungen die Handelswelt der schwäbischen Provinz: Das von den Brüdern Albrecht weniger Jahre zuvor entwickelte Discount-Konzept erreicht Ehingen und erschwert dem Unternehmen Schlecker zunehmend das Geschäft mit den SB-Verbrauchermärkten. ${ }^{577}$

Vor dem Hintergrund des großen Erfolgs im Lebensmitteleinzelhandel (LEH) wird nun in vielen Branchen des Handels darüber nachgedacht, das DiscountPrinzip zu übernehmen. So auch in der Drogeriebranche: Zu einem Zeitpunkt, zu dem es in Deutschland noch ca. 17.000 traditionelle, inhabergeführte Fachdrogerien gibt, eröffnet Dirk Roßmann in Antizipation des bevorstehenden Wegfalls der Preisbindung für Drogeriewaren 1972 in Hannover den ersten

575 Für eine Übersicht zu Quellen allgemein zugänglicher Daten zur historischen Entwicklung der Unternehmen wie deren Finanzkennzahlen siehe die jeweiligen Fallchroniken im Anhang. Eine separate Nennung jeder Quelle zu Finanzkennzahlen würde zu einer unübersichtlichen Darstellung führen.

576 Eine beachtenswerte Größe: Selbst im Jahr 2012 führten von 14.372 Betrieben des Fleischerhandwerks nur 27,0 \% eine weitere Filiale und nur ca. 1,5\% mehr als fünf Filialen. Vgl. S140809.

577 Kennzeichen des neuen Discount-Konzepts sind das bis weit in die 50er Jahre unbekannte Prinzip der Selbstbedienung (SB), ein relativ schmales und flaches Warensortiment, eine schlichte Präsentation der Waren, kein Kreditverkauf und keine Rabatte, dafür niedrige Preise, welche auf einer konsequenten, alle Funktionsbereiche umfassenden Kostensenkungsstrategie beruhen. Vgl. Brandes (2001: 19ff.). 
Drogerie-Discountmarkt Deutschlands. ${ }^{578}$ Ein Jahr später folgt Götz Werner mit dem ersten dm-Drogeriemarkt.

Diese Drogeriemärkte sind mit $190 \mathrm{~m}^{2}$ größer als die althergebrachten Drogerien, die meist eine Fläche von $60 \mathrm{~m}^{2}$ hatten, und setzen konsequent auf Selbstbedienung. Anstatt 10.000 Artikel werden nur noch 2.000 Artikel angeboten, die dafür aber im Preis deutlich reduziert sind. ${ }^{579}$

Dieses Konzept hat beim Kunden Erfolg und bald wird auch in Ehingen die im Vergleich zu Lebensmitteln sehr hohe Marge von Drogeriewaren aufmerksam registriert. ${ }^{580}$ Dort steht jedoch zunächst die Nachfolge in der Führung des Unternehmens an:

"Bisher war ich der Schlecker und das der Junior. Jetzt drehen wir es um. Ab sofort ist er der Schlecker, und ich mach nichts mehr."

(Anton Schlecker sen. 1974 vor seinen Mitarbeitern) ${ }^{581}$

1975 eröffnet dann auch das Unternehmen Schlecker seinen ersten Drogeriemarkt. Zur gleichen Zeit besitzt dm allerdings bereits über 20 Filialen, eröffnet ca. alle vier Wochen eine neue und wagt 1976 bereits den Schritt ins Ausland nach Österreich. Auch Rossmann hat sich trotz einiger finanzieller Schwierigkeiten in Norddeutschland etabliert. ${ }^{582}$

Hinsichtlich ihres am Discount-Prinzip orientierten Geschäftsmodells sind sich diese jungen Unternehmen in ihrer Gründungszeit recht ähnlich. Sie unterscheiden sich aber früh hinsichtlich ihrer organisationalen Konfiguration: Während Götz Werner sowohl zur Finanzierung des Unternehmens, als auch zur Unterstützung des Managements 50 \% der Unternehmensanteile an einen Partner abtritt und zugleich einen ehemaligen Aldi-Manager für das Topmanagement des Unternehmens rekrutiert, agieren Anton Schlecker und Dirk Roßmann in einer monozentrischen Konfiguration als dominante Protagonisten und Einzelunternehmer. Bei Rossmann besteht das Management aus engeren Bekannten Dirk Roßmanns, bei Schlecker aus den vom Vater übernommenen Führungskräften. Mit der Zeit nimmt jedoch vor allem Anton Schleckers Frau Christa, eine gelernte Fremdsprachensekretärin, an der Seite ihres Mannes eine führende Stellung im Unternehmen ein. ${ }^{583}$

578 Vgl. Bundesverband Drogerien (2014). Wegfall der Preisbindung für Drogeriewaren zum 01.01 .1974$.

579 Das Elnett Haarspray kostet bei dm nun bspw. 6,98 DM anstatt 9,90 DM; vgl. Werner (2013: $22 \mathrm{ff}$.$) .$

580 Einschätzung basiert auf Aussagen ehemaliger Führungskräfte Schleckers im Rahmen der narrativen Interviews.

581 Vgl. S120602HB.

582 Vgl. Michler (2009: 105f.); Werner (2013: 50).

583 Das Paar hatte sich 1970 kennengelernt und noch im gleichen Jahr geheiratet. Ein Jahr später wird Lars Schlecker geboren, 1973 Meike Schlecker. 
Ungeachtet solcher Unterschiede steht strategisch überall das Wachstum im Vordergrund - und am schnellsten wächst Schlecker: Zwei Jahre nach der Eröffnung der ersten Filiale wird 1977 die 100. Filiale eröffnet und nach der Übernahme der Biegert-Märkte im Jahr 1981 übernimmt Schlecker mit nun 470 Filialen und ca. 480 Mio. DM Umsatz die Marktführerschaft von dm. ${ }^{584}$ Das Unternehmen profitiert vom Sterben der klassischen Fachdrogerien ebenso, welches es zugleich befördert: Gab es 1975 noch ca. 15.00 Fachdrogerien, sind es 1981 nur noch ca. 8000. Dafür gibt es mittlerweile um die 1.500 Drogeriemärkte nach dem Discount-Konzept. ${ }^{585}$

In den folgenden zwanzig Jahren folgt eine beispiellose Expansion: 1984 eröffnet die Anton Schlecker e.K. die 1.000 Filiale und beginnt 1987 mit dem Eintritt in den österreichischen Markt die Expansion ins Ausland. ${ }^{586}$ Nach der Wiedervereinigung expandiert das Unternehmen umgehend in die neuen Bundesländer und 1992 verzeichnet die Anton Schlecker e.K. nach der Eröffnung von 900 neuen Filialen einen Umsatz von 4,2 Mrd. DM. 1993 ist die Anton Schlecker e.K. mit 4.200 Filialen und einem Marktanteil von ca. 40 \% im Drogeriewarenbereich der erste bundesweit vertretene Drogeriemarkt und nimmt immer stärker eine marktbeherrschende Stellung ein. ${ }^{587}$

Trotz dieser ungeheuren Expansion bleiben die Strukturen im Unternehmen und dessen Governance unverändert. Während Rossmann zur Finanzierung seines ebenfalls rasanten Wachstums bereits 1980 auf den Einstieg des Minderheitsgesellschafters Hannover Finanz angewiesen war und in diesem Zuge das Einzelunternehmen in eine $\mathrm{GmbH}$ umgewandelt wurde und einen Beirat erhielt, verzichtet die Familie Schlecker trotz steigender organisationaler Komplexität sowohl auf eine Umwandlung in eine Kapitalgesellschaft als auch auf die Einrichtung eines Kontroll- und Aufsichtsorgans. ${ }^{588}$ In der operativen Umsetzung ihrer Entscheidungen vertraut die Familie Schlecker weiterhin ausschließlich einem engen Kreis altgedienter Direktoren. Diese dominante Koalition setzt auch in den 1990er-Jahren auf eine ungebremste Wachstumsdynamik.

584 Werner (2013: 48) zur Gründungsphase von dm im Jahr 1974: »Jetzt kommt es darauf an, das Prinzip zu multiplizieren.« Vgl. S810522ZE zur Branchensituation im Jahr 1981: dm: 120 Filialen, 300 Mio. DM Umsatz; kd: 177 Filialen, 255 Mio. Umsatz.

585 Vgl. S810522ZE.

586 Es folgen 1989 die Niederlande, Luxemburg und Spanien. 1991 durch Übernahme der französischen Superdrug-Outlets Frankreich.

587 Vgl. S930426SP.

588 Die Hannover Finanz steigt mit einem Anteil von $10 \%$ bei Rossmann ein, den sie in den folgenden Jahren auf schlussendlich $38 \%$ im Jahr 2002 ausbaut. Die faktische strategische Entscheidungsmacht bleibt jedoch bei der um Dirk Roßmann gruppierten dominanten Koalition. Vgl. Michler (2009: 103f.). 


\section{Strategiekrise}

Im Verlauf der Zeit beginnt sich jedoch nach und nach die Wahrnehmung des Unternehmens durch die Öffentlichkeit zu ändern. Insbesondere die sich offenbarenden Missstände im Umgang mit den Mitarbeitern führen immer häufiger zu Negativschlagzeilen. ${ }^{589}$ Skandale um verunreinigte Lebensmittel und häufige Überfälle der schlecht gesicherten Filialen beschädigen das Image des Unternehmens und nehmen zunehmend negativen Einfluss auf die Geschäftstätigkeit.

Nicht zuletzt, weil sich ab Anfang der 1990er-Jahre im Handel ein Kulturwandel abzeichnet, der ein sich veränderndes Verbraucherverhalten zu reflektieren beginnt: Mehr und mehr Verbraucher wollen nicht mehr nur günstig konsumieren, sondern sich beim Einkaufen auch wohlfühlen. Sie verlangen weiterhin günstige Preise, wünschen aber nun auch ein differenziertes Sortiment, welches Bio-Produkte ebenso umfasst wie günstige Handelsmarken. ${ }^{590}$

Diese Entwicklungen greifen die Wettbewerber der Anton Schlecker e.K. mit einem Trading-Up des gesamten Leistungsprogramms auf. ${ }^{591}$ So beginnt insbesondere $\mathrm{dm}$ nach einem vorhergehenden organisationalen Wandel Veränderungen am Vertriebskonzept vorzunehmen: Das Sortiment wird breiter und tiefer, die Filialen entsprechend größer und es wird mehr Wert auf deren Gestaltung gelegt. 1995 verkündet das Unternehmen zudem, in Zukunft auf große Rabattaktionen zu verzichten und alle Preise von nun an mindestens vier Monate stabil zu halten. Auch die Dirk Rossmann GmbH, die Ende der 1980er-Jahre in eine erneute Liquiditätskrise geraten war, durch die Wiedervereinigung und die wahrgenommene Chance der raschen Expansion nach Osteuropa jedoch wieder auf Wachstumskurs gekommen war, vollzieht in weiten Teilen ein Trading-Up. Dieser Wandel der Branchenumwelt in den 1990er-Jahren kommt im neuen Slogan von dm zum Ausdruck: aus "Große Marken. Kleine Preise« wird »Hier bin ich Mensch, hier kauf ich ein«. ${ }^{592}$

Ungeachtet der unübersehbaren Umweltdynamik und zahlreicher Negativschlagzeilen expandiert die Anton Schlecker e.K. mit dem weitgehend unver-

589 Vgl. S930426SP zum Skandal um minderwertige Babynahrung der Schlecker-Handelsmarke, zu den Vorwürfen der Gewerkschaft bzgl. Tarifdumpings, Scheinarbeitsverhältnissen, oder der Verhinderung von Betriebsratswahlen. 1994 werden daraufhin zum ersten Mal die Geschäftsräume von Schlecker von der Staatsanwaltschaft durchsucht.

590 Vgl. zum veränderten Konsumentenverhalten Meffert (1994: 4).

591 Vgl. zum Trading-Up des Handels allgemein Meffert et al. (2015: 367). Vgl. Werner (2013: $162 \mathrm{ff}$.$) zu den Veränderungen bei dm, wo eine »dialogische Führung« als Kennzeichen der$ Organisationskultur postuliert wird, die zentralistische Organisationsstruktur zugunsten einer Matrix-Organisation abgeschafft wird, sich insgesamt von »Gleichheit» und »Standardisierung « ab- und Spezifizität und Regionalisierung zugewandt wird.

592 Vgl. Werner (2013: 166ff.). 
änderten Geschäftsmodell jedoch rasant weiter. ${ }^{593}$ So wächst das Unternehmen allein im Jahr 1997 um 1.000 weitere Filialen auf nun 7.500 Filialen, von denen sich 6.350 in Deutschland befinden. Die Umsätze steigen bis zum Jahr 2000 auf 5,13 Mrd. Euro. Im Vergleich hierzu kommen die Konkurrenten dm und Rossmann auf nur 2 Mrd. bzw. 763 Mio. Euro Umsatz.

Doch die große Zeit des Wachstums - mit jährlich zweistelligen Umsatzsteigerungen - und des Umbruchs beginnt für die Branche erst. ${ }^{594} 1999$ möchte das Osnabrücker Unternehmen Ihr Platz den zweiten Rang hinter dem Marktführer Schlecker erringen und übernimmt den Konkurrenten Drospa. dm wächst aus eigener Kraft um 14,5 \%. Die nur im Norden Deutschlands vertretene Dirk Rossmann GmbH und die in Süddeutschland präsente Müller GmbH \& Co. KG schließen eine Kooperation im Einkauf und bündeln hier ihre Kräfte. Der sich derart verschärfende Wettbewerb führt schlussendlich zu einer Konsolidierung. ${ }^{595}$ So kauft Rossmann im Jahr 200090 Idea-Märkte von der Rewe Group und verzeichnet $26 \%$ Umsatzwachstum. 2003 folgt die stufenweise Übernahme von $400 \mathrm{kd}$-Märkten.

Auf die zunehmende Konkurrenz reagiert Schlecker mit einer nochmaligen Steigerung des Wachstums: In den Jahren 1999 bis 2002 werden jedes Jahr ungefähr 1.000 neue Filialen eröffnet oder durch Übernahmen zugekauft. So steigt die Anzahl der Filialen von 9.000 im Jahr 1999 auf 13.300 im Jahr 2003. Trotz verschiedenster Versuche, im Ausland präsenter zu werden, gewinnt der deutsche Markt weiter an Bedeutung.

Gerade in Deutschland kommt es im Zuge der Einführung des Euro in der Drogeriemarktbranche jedoch zu einem zunehmenden Preiskampf mit teils deutlichen Preissenkungen. ${ }^{596}$ Da die Anton Schlecker e.K. diesen nicht immer folgen kann, versucht das Unternehmen durch eine Vielzahl von kleineren Maßnahmen die Umsatzentwicklung zu beleben: Es wird ein Mobilfunkportal gestartet, Autos über das Internet verkauft und eine Zusammenarbeit mit Blu-

5931998 erlässt das Amtsgericht Stuttgart gegen Anton Schlecker und seine Frau Christa einen Strafbefehl von jeweils zehn Monaten auf Bewährung wegen vielfachen Lohnbetrugs.

594 Vgl. S000410LZ: „Drogeriemarkt-Branche gibt weiter Gas, Wachstum hält unvermindert an [...] Gerangel auf den Plätzen [...] Drogeriemarkt-Szene steht vor dem Umbruch «; auch S031218LZ.

595 Vgl. S000420:»Mit der Übernahme der Douglas-Tochter Drospa läutete Ihr Platz eine neue Runde im internen Wettstreit der Drogeriemärkte ein. Experten waren sich schon seit Längerem einig, dass die Drogeriemarkt-Szene vor einem Umbruch steht. `Zehn Unternehmen wie in den 90er Jahren, wird es wohl auf mittlere Sicht nicht mehr gebenı, so ein Branchenbeobachter.« Tatsächlich wird die Zahl der Drogeriemarktunternehmen von zehn auf drei große bundesweite Wettbewerber im Jahr 2014 abnehmen.

596 So senkt dm seine Preise um ca. $4 \%$ bei 12.300 Artikeln, Aldi um $2 \%$. Odol Mundwasser kostet z.B. nun 4,15 Euro (8,12 DM) statt 8,35 DM. Schlecker verlangt 4,49 Euro, Lidl 4,27 Euro. 


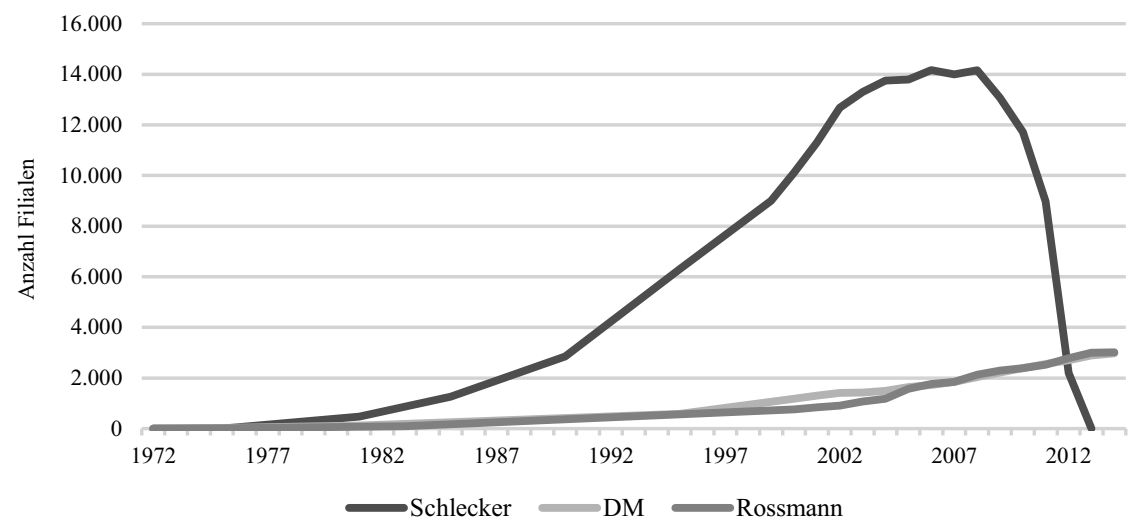

Abbildung 9: Entwicklung der Filialzahlen der führenden Drogeriemarktunternehmen Deutschlands

(Quelle: Eigene Darstellung, basierend auf einer Zusammenstellung von Daten des EHI).

menversendern begonnen. Marktbeobachter sprechen für das Jahr 2003 dennoch von flächenbereinigten Umsatzrückgängen von ca. $5 \%$.

»Schlecker zieht im direkten Wettbewerb mit dm oder Rossmann immer öfter den Kürzeren." Beide Kontrahenten verfügen über die wesentlich größeren Märkte und seien daher besser in der Lage, Drogeriemarktsortimente attraktiv feilzubieten. Zudem agieren sie preisaggressiver als Schlecker. Warenkorbanalysen rechnen einen Preisvorteil von bis zu 10 Prozent gegenüber den Ehingern aus."

(Die Lebensmittel Zeitung im Jahr 2003) (97 $^{59}$

Ende 2003 lösen die Dirk Rossmann GmbH und die Müller GmbH \& Co. KG ihre Einkaufskooperation wieder auf. Somit steht einer Expansion der Dirk Rossmann $\mathrm{GmbH}$ in den Süden Deutschlands nichts mehr entgegen. ${ }^{598}$

Zugleich verschärft sich bei der Anton Schlecker e.K. die Krise weiter : Anfang 2004 brechen Umsatz und Profitabilität nochmals drastisch ein. Das Unternehmen tritt von der Phase der Strategiekrise in die Phase der Erfolgskrise.

\section{Erfolgskrise}

Nichtsdestotrotz verkündet Schlecker zu Beginn des Jahres 2004, dass das Unternehmen sein Wachstum noch einmal steigern wolle. 2003 habe Schlecker lediglich 800 Märkte in Europa eröffnet, für das Jahr 2004 seien wieder 1000 neue Filialen geplant. Insbesondere die Expansion im Ausland soll forciert werden, unter anderem durch einen Markteinstieg in Osteuropa. ${ }^{599}$

597 Vgl. S031023LZ.

598 Vgl. S031218LZ: „Rossmann und Müller lösen ihre Einkaufskooperation auf [...] geht im Süden in die Offensive.«

599 Vgl. S040819LZ. 
Diese Expansion nach Osteuropa erfolgt dann allerdings erst mit einiger Verzögerung und in deutlich geringerem Umfang als angekündigt. Zudem werden zum ersten Mal in der Historie von Schlecker in größerem Umfang Filialen geschlossen.

Nun erhöhen dm und Rossmann allerdings die Geschwindigkeit ihrer Expansion. Von 2000 bis 2004 steigt die Anzahl der dm-Filialen um $40 \%$ und der Umsatz wächst im gleichen Zeitraum um $55 \%$ auf 3,1 Mrd. Euro. Rossmann steigert die Anzahl der Filialen gar um $54 \%$ und verschärft im Sommer 2004 durch eine harte Rabattaktion den Wettbewerb weiter.

Diesem Tempo können die restlichen Anbieter wie Ihr Platz, Müller oder Idea nicht mehr folgen -nicht zuletzt, weil nun dm auf die begonnene Expansion Rossmanns gen Süden reagiert und ab Oktober 2004 mit der Eröffnung des ersten dm-Marktes in Berlin seinerseits in den Norden Deutschlands strebt. In der Folge wird der Wettbewerb nun national ausgetragen. ${ }^{600}$

\begin{tabular}{|c|c|c|c|c|}
\hline & $\begin{array}{c}\text { Umsatz } \\
\text { (in Mio. Euro) }\end{array}$ & $\begin{array}{l}\text { Zuwachs } \\
\text { (in Prozent) }\end{array}$ & $\begin{array}{c}\text { Anzahl } \\
\text { der Filialen }\end{array}$ & $\begin{array}{c}\emptyset \text { Filialumsatz } \\
\text { (Mio. Euro / Jahr) }\end{array}$ \\
\hline \multicolumn{5}{|c|}{ ROSSMANN } \\
\hline 2006 & 2240 & $+13,7 \%$ & 1220 & 1,84 \\
\hline 2005 & 1970 & $+40,5 \%$ & 1160 & 1,70 \\
\hline 2004 & 1402 & $+14,6 \%$ & 817 & 1,72 \\
\hline 2003 & 1223 & $+19,7 \%$ & 770 & 1,59 \\
\hline 2002 & 1022 & $+10,1 \%$ & 670 & 1,53 \\
\hline 2001 & 928 & $+20,0 \%$ & 550 & 1,43 \\
\hline \multicolumn{5}{|l|}{$\mathrm{DM}$} \\
\hline $2005 / 06$ & 2695 & $+11,5 \%$ & 869 & 3,10 \\
\hline $2004 / 05$ & 2417 & $+8,9 \%$ & 799 & 3,02 \\
\hline $2003 / 04$ & 2220 & $+8,5 \%$ & 702 & 3,16 \\
\hline $2002 / 03$ & 2046 & $+10,3 \%$ & 660 & 3,10 \\
\hline $2001 / 02$ & 1855 & $+12,0 \%$ & 617 & 3,00 \\
\hline $2000 / 01$ & 1657 & $+18,0 \%$ & 564 & 2,93 \\
\hline
\end{tabular}

Abbildung 10: Wachstumsdynamik der Wettbewerber der Anton Schlecker e.K.

(Quelle: Eigene Darstellung auf der Grundlage von Daten der Dirk Rossmann GmbH und der dm-drogerie markt GmbH \& Co. KG).

600 Vgl. S041021LZ: "Drogeriemarkt-Filialist dm strebt neuen Ufern zu. Vornehmlich im Norden will das Unternehmen künftig Filialen eröffnen, mitten im Kerngebiet des Erzrivalen Rossmann. [...]. Keinen Hehl macht es daraus, dass die jüngste Expansionsoffensive von Rossmann ebenfalls zu der Entscheidung, nach Niedersachsen und in die Hauptstadt zu gehen, beigetragen habe." 
Der Leidtragende dieses zunehmenden Wettbewerbs ist die Anton Schlecker e.K. Diese muss insbesondere an den Standorten, an denen dm oder Rossmann nun neue Filialen eröffnen, deutliche Umsatzrückgänge hinnehmen. ${ }^{601}$ Mit seinen 14.166 Filialen in mittlerweile 17 Ländern und einem Umsatz von 6,6 Mrd. Euro erwirtschaftet der Marktführer nur noch einen buchhalterischen Gewinn von 6 Mio. Euro - den letzten seiner Unternehmensgeschichte. ${ }^{602}$

Unter der Offensive des LEH-Discounts im Frühjahr 2005 im Drogeriewarensortiment leidet aufgrund der Überschneidungen in der Käuferschaft wiederum vor allem Schlecker. ${ }^{603}$ In Bezug auf die Geschäftsmodelle greifen von »oben« nun Rossmann und $\mathrm{dm}$ an, von »unten« bedrohen Lidl und Aldi die Umsätze.

Während Schlecker sich versucht zu konsolidieren, verschärft sich in der Branche der Verdrängungswettbewerb weiter: Ihr Platz gerät in finanzielle Bedrängnis und meldet 2005 Insolvenz an. ${ }^{604}$

Zwei Jahre später wird das sanierte Unternehmen dann von der Anton Schlecker e.K. übernommen. Trotz dieser Übernahme berichten Marktforschungsinstitute von zweistelligen Umsatzrückgängen. ${ }^{605}$

"Die Lage verschlimmert sich für Schlecker. `In dem Maße, in dem DM und Rossmann ihr Filialnetz ausbauen, stößt Schleckers altes Konzept an seine Grenzen`, [...] Eröffnet nebenan eine DM-Filiale, hat Schlecker keine Chance. Dann schmelzen die Filialumsätze dahin wie Butter in der Pfanne."

(Ein Handelsexperte 2008 im Manager Magazin) ${ }^{606}$

In der Folge wird ein neues Vertriebskonzept vorgestellt: Schlecker XL. Mit diesem Konzept möchte Schlecker seine Waren zum ersten Mal auf Flächen um die $600 \mathrm{~m}^{2}$ vertreiben und somit wie die Konkurrenz verstärkt größere Märkte ins Auge fassen. Zunächst steigt aber der Verlust bei einem Umsatz von 7,4 Mrd. Euro im Jahr 2008 auf 50 Mio. Euro.

Zudem nimmt Anfang 2009 die negative Medienberichterstattung zu Schlecker nochmals zu. Das Unternehmen hatte in größerer Zahl Filialen geschlossen

601 Vgl. S040115LZ; S040220MM: „Schlecker zieht im direkten Wettbewerb immer häufiger den Kürzeren.«

602 Vgl. S041105HB; S051021HB zu den Marktanteilen in der Branche im Jahr 2004: Schlecker: $42 \%, \mathrm{dm}: 17,7 \%$, Rossmann: $14,5 \%$.

603 Vgl. S050210LZ: „Lidl geht in die Offensive. Mit Markenartikeln von Körperpflege bis Babynahrung greift der Discounter die Drogeriemärkte an«. Aldi zieht wenig später nach.

604 Vgl. IP050223LZ: »Drogeriemarkt im Umbruch [...]. Kaum jemand glaubt daran, dass die Osnabrücker auf lange Sicht ihre Eigenständigkeit bewahren können. Das Marktumfeld ist einfach zu schwierig geworden, als dass man mit einem in die Jahre gekommenen Konzept bestehen könnte."

605 Vgl. S060407LZ; S060819LZ: »[...] liefern die Marktforschungsinstitute alarmierende Zahlen."

606 Vgl. S080325MM; S120313WW. 
und der Stammbelegschaft gekündigt, teilweise jedoch dieselben Mitarbeiter zu deutlich schlechteren Konditionen über eine mit dem Unternehmen verbundene Zeitarbeitsfirma wieder eingestellt. Nicht zuletzt durch die verspätete und ausweichende Reaktion des Unternehmens verschärft sich das Negativimage dramatisch. Die GfK meldet im Gefolge dieses Imageschadens hohe kurzfristige Umsatzeinbußen. ${ }^{607}$

\section{Turnaround-Versuch}

Ab Juli 2010 leitet eine Unternehmensberatung gemeinsam mit der Familie Schlecker den ersten umfassenden Versuch eines Turnarounds ein. Die designierten Nachfolger Lars und Meike Schlecker präsentieren im Rahmen ihres ersten Auftrittes in der Öffentlichkeit im Oktober 2010 erste Grundzüge der mit der Beratung ausgearbeiteten Turnaround-Strategie »Fit for Future«: Durch mehr Licht, breitere Gänge und mehr Farbe sollen die Filialen attraktiver gestaltet werden und mit einem modernen Nahversorgerkonzept, regionalisierten Sortimenten und einer deutlichen Imagekorrektur soll das gesamte Unternehmen wieder auf Kurs kommen. Hierzu werden Investitionen in Höhe von 230 Mio. Euro für den Zeitraum der nächsten 18 Monate angekündigt. ${ }^{608} \mathrm{Im}$ Januar 2011 wird im Zuge des begonnenen strategischen und organisationalen Wandels durch die Etablierung einer Geschäftsführerebene auch die Organisationsstruktur reorganisiert. Ab sofort sind ein CFO und COO als Doppelspitze für die meisten operativen Aufgaben zuständig und berichten an die Familie.

\section{Liquiditätskrise und Insolvenz}

Bereits im Herbst 2011 wird jedoch die Liquiditätskrise des Unternehmens offenbar. Die hohen Verluste und die Kosten der Turnaround-Maßnahmen haben die einstmals hohen Eigenkapitalreserven aufgebraucht. In den Medien häufen sich Meldungen über Lücken in den Regalen. ${ }^{609}$

607 Nach zahlreichen Berichten in TV und Presse werden im November 2009 erste Anfragen zu Schlecker im Bundestag gestellt. Parteiübergreifend wird gefordert, dass ein solches Verhalten nicht geduldet werden dürfe. Die SPD-Spitze schreibt einen Brief an Anton Schlecker. Am 10. Januar 2010 spricht sich Bundesarbeitsministerin von der Leyen dafür aus, zu prüfen, ob bei Schlecker Missbrauch begangen wurde und kündigt eine Gesetzesinitiative an, die in der Öffentlichkeit als »lex Schlecker« bezeichnet wird. Erst daraufhin verkündet Schlecker keine neuen Verträge mit Meniar mehr abschließen zu wollen und Anton Schlecker gibt das erste Interview seit 15 Jahren.

608 Vgl. S101015LZ: „Schlecker erfindet sich neu." Mithilfe renommierter externer Partner wird versucht die gesamte Marke vom Logo bis zum Filialauftritt grundlegend zu modernisieren. Im Februar 2011 wird die erste neu konzipierte Filiale vorgestellt, welche in den Medien sehr positiv rezipiert wird.

609 Die vielen Lagerschließungen führen zu Problemen in der Logistik, infolge der zahlreichen Filialschließungen entsteht ein Personalüberhang, da die Mittel für Abfindungen aufgrund betriebsbedingter Kündigungen fehlen. 
Während nun zunehmend Filialen geschlossen werden und sich das Unternehmen aus den meisten Auslandsmärkten zurückzieht, sucht die Familie nach einem externen Investor. Da diese Versuche jedoch erfolgslos bleiben, wird am 23. Januar 2011 beim Amtsgericht Ulm ein Insolvenzantrag eingereicht. Nach erfolglosen Versuchen des Insolvenzverwalters und der Familie einen geeigneten Investor zu finden, oder anderweitig die Fortführung des Unternehmens zu sichern, muss der Insolvenzverwalter nach einer Sitzung des Gläubigerausschusses am 01. Juni 2011 in Berlin das Aus für die Anton Schlecker e.K. verkünden. ${ }^{610}$

\subsubsection{Pfadanalyse}

In den folgenden Abschnitten werden zunächst die Elemente des strategischen Musters und deren Persistenz im Kontrast zur Umweltweltdynamik dargestellt. Anschließend wird gezeigt, dass diese strategische Persistenz maßgeblich auf die Wirkkräfte selbstverstärkender Mechanismen und den daraus resultierenden Lock-in der strategischen Entwicklung zurückzuführen ist.

\subsubsection{Analyse der strategischen Persistenz}

Zur Untersuchung der strategischen Persistenz der Anton Schlecker e.K. wird die Entwicklung der Gesamtunternehmensstrategie und der zentralen Parameter des Geschäftsmodells in einer dynamischen Perspektive in Kontrast zur Entwicklung der Branchenumwelt gesetzt.

\subsection{Gesamtunternehmensstrategie: Wachstum durch Multiplikation}

Die Gesamtunternehmensstrategie eines Unternehmens zeigt die Richtung der allgemeinen organisationalen Entwicklung auf. Darüber hinaus legt sie fest, in welchen Geschäftsfeldern die Unternehmung tätig ist und wie die dem Unternehmen zur Verfügung stehenden Ressourcen allokiert werden. ${ }^{611}$

Bei der Anton Schlecker e.K. war die Gesamtunternehmensstrategie seit der Gründung des Unternehmens und insbesondere seit dem Einstieg in die Drogeriebranche ausschließlich auf Wachstum ausgerichtet. ${ }^{612}$ Die Steigerung der

610 Nur vereinzelte Filialen sind für die Wettbewerber von Interesse und werden übernommen. Im Laufe des Verfahrens summieren sich die Forderungen der Gläubiger bis 2013 auf eine Milliarde Euro.

611 Vgl. Steinmann \& Schreyögg (2005: 171); Welge \& Al-Laham (2012: 588).

612 Ein Interviewpartner verwies auf eine Aussage Anton Schleckers, der zufolge dieser und seine Frau Christa »Zunächst 10, dann 100 Filialen, dann 1.000 und dann 10.0000 Filialen eröffnen wollten«. Diese Fixierung auf die Anzahl der Filialen zeigte sich auch in den jährlich veröffentlichten Planvorgaben zur Eröffnung neuer Filialen. 
Filialanzahl und die Expansion des Filialnetzes wurden ein regelrecht sich selbst legitimierendes Ziel.

Das angestrebte Wachstum sollte dabei über Jahrzehnte hinweg allein durch die Multiplikation des Mitte der 1970er-Jahre konzipierten Betriebstyps des Drogeriediscounters erzielt werden:

"Anton Schlecker hat mir mal einen Artikel gegeben, der war aus einer wissenschaftlichen Zeitschrift aus dem Jahre, ich glaub '72 war das [...] und das war seine Philosophie nach der hat er gelebt. Da hieß es nämlich: Analysiere, konzipiere, multipliziere. Und das hat er gemacht. Er hat damals analysiert in den siebziger Jahren, da ist die Bindung, die Preisbindung gefallen. Er hat analysiert. Er hat konzipiert, nämlich ein Drogeriemarkt-Discountkonzept [...], und dann hat er multipliziert. Dann hat er multipliziert und multipliziert und hat nicht mehr aufgehört zu multiplizieren.» (Ehemalige Führungskraft der Anton Schlecker e.K.) ${ }^{613}$

Diese Wachstumsstrategie mit einem einzigen Betriebstyp wurde mehr als dreißig Jahre lang verfolgt. Sie wurde selbst dann noch fortgesetzt, als das Unternehmen um die Jahrtausendwende mit allein 10.550 Filialen in Deutschland nach Ansicht von Marktbeobachtern deutlich an Sättigungsgrenzen stieß und Umsatzeinbußen auf bestehenden Flächen hinnehmen musste.

So wurden zur Steigerung des Marktanteils jedes Jahr Hunderte neue Filialen eröffnet oder die Märkte anderer Unternehmen wie Sconti, Idea oder Ihr Platz übernommen.

»Wir führen unsere expansive und innovative Unternehmenspolitik weiter.»

(Anton Schlecker im Jahr 2003 gegenüber der Lebensmittel Zeitung) ${ }^{614}$

Erst ab dem Jahr 2004 wurde eine Konsolidierungsstrategie verfolgt. Eine signifikante Reduktion des Filialnetzes wurde gar erst kurz vor der Insolvenz vorgenommen.

Im Folgenden werden das Geschäftsmodell der Anton Schlecker e.K. und die mit ihm verbundene Betriebsform, auf der diese Wachstumsstrategie beruht, skizziert.

\subsection{Entwicklung von Geschäftsmodell und Betriebsform}

Unter einem Geschäftsmodell kann im Allgemeinen die Abbildung der grundlegenden geschäftlichen Aktivitäten eines Unternehmens verstanden werden, welche die Beziehungen zu Lieferanten, Kunden und Wettbewerbern einbezieht

613 Vgl. S041028LZ.

614 Vgl. S031023LZ: »Umsatzrückgänge signalisieren, dass die erfolgsverwöhnten Expansionsspezialisten Gefahr laufen, hierzulande an Grenzen zu stoßen. Unruhe kommt in der Schlecker-Zentrale dennoch nicht auf.« Vgl. auch die Einschätzung von Werner (2013: 239): "Anders als bei Schlecker war Wachstum für uns nie Selbstzweck." 
und die jeweilige Form der Erlösgenerierung und Finanzierung des Unternehmens berücksichtigt. ${ }^{615}$

Die Geschäftsmodelle des Handels basieren allesamt auf dem Umschlag fremdbezogener Güter, wobei die Handelsunternehmen eigene Leistungen erbringen müssen. Diese eigenen Leistungen können im Handelskontext wiederum über die sogenannte Betriebsform näher spezifiziert werden, welche die Ausrichtung in den einzelnen Funktionsbereichen festlegt und somit auch weitgehend die strategische Ausrichtung bestimmt. ${ }^{616}$

Im Sinne einer solchen Betriebsform war die Anton Schlecker e.K. - auch im Selbstverständnis des Unternehmens - stets ein Drogeriediscounter. ${ }^{617}$ Zur Prüfung der Persistenz der strategischen Entwicklung sollen daher im Folgenden die bei Schlecker beobachteten Ausprägungen der zentralen Elemente dieser Betriebsform in Vertrieb und Einkauf in Kontrast zu den Entwicklungen der Branchenumwelt gesetzt werden.

\section{Vertrieb: Sortimentspolitik}

Die Entscheidungen zur quantitativen und qualitativen Struktur des Sortiments im Rahmen der Sortimentspolitik nehmen im Handel eine dominierende Rolle ein, bestimmt die strukturelle Sortimentspolitik doch in weiten Teilen auch andere strategische Parameter wie die Standortpolitik. ${ }^{618}$

Die Anton Schlecker e.K. führte zunächst ein sehr schmales und gestrafftes Sortiment von ungefähr 2.000 Artikeln. Diese Größe war bei Markteintritt mit jener anderer Drogeriemärkte vergleichbar. Beispielsweise umfasste das Sortiment von dm zunächst 2.300 Artikel. ${ }^{619}$

Im Verlauf der Zeit erweiterten die meisten Wettbewerber jedoch Schritt für Schritt ihr Sortiment und führten ab den 1990er-Jahren 10.000 oder gar mehr als 12.500 Artikel ${ }^{620}$ Im Vergleich hierzu blieb das Sortiment von Schlecker mit im Schnitt konstant rund 4.000-4.500 Artikeln pro Verkaufsstellesehr schmal und gestrafft.

Auch auf die zunehmende Verbreiterung der Sortimente in der Drogeriemarktbrauche reagierte Schlecker erst spät. ${ }^{621}$ Obgleich im Food-Bereich und im

615 Vgl. zu diesem äußerst unscharf definierten Begriff Hungenberg (2011: 258f.).

616 Vgl. Mattmüller \& Tunder (2004: 39).

617 Vgl. S130621LZ; S120128WW: "Hart-Discount-Konzept an geringwertigen Standorten«; für eine systematische Einordnung in die Handelsbetriebslehre vgl. Mattmüller \& Tunder (2004: $40 \mathrm{ff}$.).

618 Vgl. zu dieser Einschätzung Mattmüller \& Tunder (2004: 189).

619 Vgl. zu Sortimentsbreite und Sortimentstiefe (Anzahl gleichartiger Artikel innerhalb einer Warengruppe, somit alternative Auswahlmöglichkeiten) Haller (2008); Mattmüller \& Tunder (2004); Mattmüller \& Tunder (2004: 192).

620 Vgl. Werner (2013: 205).

621 Vgl. zu den teils abenteuerlich anmutenden Experimenten S010412LZ: Beispielsweise 
Bereich der Non-Food Artikel teilweise experimentiert wurde, fanden insbesondere zwei wichtige Trends der Drogeriemarktbranche kaum Niederschlag in der Sortimentspolitik Schleckers: zum einen der Trend zu Bio-Produkten und Wellness-Artikeln, zum anderen die steigende Bedeutung von Handelsmarken. $^{622}$

dm hatte bereits Ende der 1980er-Jahre Alnatura- und Alverde-Produkte in sein Sortiment aufgenommen und mit starken Marken wie Balea das Vertrauen der Kunden zu erobern versucht. Ab Mitte der 1990er-Jahre stärkte dann auch Rossmann seine Handelsmarken. ${ }^{623}$ Im Gegensatz hierzu vernachlässigte Schlecker lange Zeit die Entwicklung eigener Handelsmarken, die in der Konsequenz weder hinsichtlich Image noch Qualität mit den Marken der Wettbewerber Schritt halten konnten und einen deutlich geringeren Umsatzanteil als bei den Wettbewerbern aufwiesen. ${ }^{624}$ Erst ab Anfang der 2000er-Jahre, als sich die Marken anderer Händler bereits etabliert hatten, begann Schlecker den Eigenmarkenbereich durch ansprechendere Verpackungsdesigns und höhere Qualität zu stärken. Nicht zuletzt aufgrund des insgesamt schlechtes Images von Schlecker konnten hier jedoch keine signifikanten Erfolge mehr verzeichnet werden.

Zusammenfassend lässt sich demnach festhalten, dass die strukturelle Sortimentspolitik über drei Jahrzehnte im Kern unverändert blieb. Insbesondere die vergleichsweise geringe Anzahl an Artikeln und die ausbleibende Differenzierung stachen im Verlauf der Branchenentwicklung zunehmend heraus.

\section{Vertrieb: Preispolitik}

Hinsichtlich der Preispolitik war die Preisführerschaft stets ein wichtiges strategisches Ziel der Anton Schlecker e.K., welches das Unternehmen über niedrige Kosten und vor allem günstige Konditionen im Einkauf erreichen wollte. ${ }^{625}$

Darauf waren Vertrieb und Einkauf hinsichtlich Standortpolitik, Filialge-

wurden Multimedia-Produkte oder Konzertkarten angeboten. 2002 Autos über das Internet verkauft. In der Folge war von einem »Warenhaus auf $100 \mathrm{~m}^{2}$ die Rede. Vgl. S071005HB; zum Food-Bereich S991227LZ; S040908MM.

622 Die Marktanteile der lange Zeit als »qualitativ geringwertig" wahrgenommenen Handelsmarken stiegen in den 90er Jahren durch verbessertes Marketing, höhere Qualität und positive Vergleichstests stetig an; vgl. Mattmüller \& Tunder (2004).

623 Vgl. Werner (2013: 81ff.); R160801WS.

624 Vgl. Werner (2013); S040220MM; S050523LZ: „Die Eigenmarkenrange unter dem Label AS hat weder vom Image noch von der Qualität das Niveau, das mittlerweile Wettbewerber wie dm oder Rossmann mit ihren Eigenmarken bieten können.« S120602HB: Auf die Frage: "Wie sehr vertrauen Sie den folgenden Unternehmen bzw. Marken?» erhielt dm als Spitzenreiter eine durchschnittliche Punktzahl von 67, Rossmann 53, Schlecker als Vorletzter 22.

625 Vgl. S930426SP: „Wir wollen der billigste Anbieter sein«, sagt Schlecker. »Das geht nun mal nur, wenn wir die Kosten so gering wie möglich halten.» 
staltung und Marketing ausgerichtet. Mit Verweis auf niedrig zu haltende Kostenstrukturen unterblieben so auch Investitionen in moderne Kassen- und Warenwirtschaftssysteme. ${ }^{626}$

Auf Kundenseite zielte das Vertriebskonzept Schleckers auf den sparsamen Kunden, den man mit günstigen Preisen und einem "Billig-Preisimage«, anstatt mit einem überlegenen Leistungsangebot für sich gewinnen wollte. In den Jahren nach dem Markeintritt waren günstige Preise tatsächlich lange ein Differenzierungsmerkmal Schleckers im Vergleich zur lokalen Konkurrenz der Drogeriefachhändler. Dieses Preisimage wollte Schlecker vor allem über gezielte, häufige und preisaggressive Aktionen demonstrieren. ${ }^{627}$

Mit dieser Aktionspolitik versuchte das Unternehmen zunehmend aber auch darüber hinwegzutäuschen, dass die großen Wettbewerber auf die Breite des Sortiments bezogen bereits in den 1990er-Jahren teils deutlich günstiger waren als Schlecker. 2003 errechneten Warenkorbanalysten dann sogar, dass das Sortiment von Schlecker im Schnitt ca. 10 \% teurer sei als jenes von dm oder Rossmann. ${ }^{628}$

Während dm auf der Grundlage einer sehr produktivitätsorientierten Kostenund Leistungsstruktur das Konzept der Dauerniedrigpreise einführte und das Image des insgesamt günstigsten Anbieters gewann, attackierte Rossmann das gleichförmige Aktionsgeschäft Schleckers durch immer aggressiveren Rabattaktionen ${ }^{629}$ Spätestens im Zuge des harten Preiskampfs ab Anfang der 2000er trat das hohe Preisniveau Schleckers offen zutage und ließ den »billigen« Marktauftritt des sehr harten Discount-Konzeptsimmer weniger stimmig erscheinen. ${ }^{630}$

»Tatsächlich hält mancher Preis im direkten Vergleich nicht, was der `billige` Eindruck, den die Filialen ausstrahlen, versprechen soll. Ein Preisvergleich ergab, dass Schlecker deutlich teurer war als andere. Ein Paket des Waschmittels `Persil Megaperls« schlug bei

626 Der Insolvenzverwalter äußerte sich verwundert darüber:»[...] dass Schlecker aus lauter Sparsamkeit nicht mal ein Warenwirtschaftssystem hatte."

627 Vgl. S000824LZ; S010823LZ.

628 Vgl. S031023LZ; auch Werner (2013: 232): „Schon damals war Schlecker immer der Teuerste."

629 Vgl. S020725LZ:»Bis zu 20-mal im Jahr steht immer der gleiche Artikel im Angebot« stellt ein Beobachter fest. „Das lockt keinen mehr zum Hamsterkauf.»

630 So senkte Preisführer dm 2002 seine Preise über das gesamte Sortiment um durchschnittlich $4 \%$; vgl. S020110LZ. Rossmann schwenkt ab 2004 zunehmend auf die dm-Preise ein; vgl. S040617LZ. Zugute kam Schlecker jedoch, dass das Image als »billiger« Drogeriemarkt noch währte, als die Konkurrenz schon deutlich günstiger war. Hier spielte sicherlich das konsistent »billig« wirkende Vertriebskonzept eine Rolle. 
DM und Rossmann mit 4,95 Euro zu Buche. Schlecker verlangte für dieselbe Menge einen Euro und vier Cent mehr.»

(Die FAZ zum Preisniveau Schleckers im Jahr 2007) ${ }^{631}$

Somit bestand eine immer größere Differenz zwischen dem extern kommunizierten und dem tatsächlichen Preisniveau. Doch ungeachtet der Tatsache, dass Schlecker schlussendlich gar rund 16-20\% teurer war als die relevanten Wettbewerber, wurde unverändert an einer Strategie der Preisführerschaft im Rahmen eines harten Discount-Konzepts festgehalten. ${ }^{632}$ Weder wurden nachhaltige Maßnahmen zur Senkung des Preisniveaus unternommen, wie zum Beispiel die Schließung unrentabler Filialen, noch wurde ein dem Preisniveau entsprechendes Trading-Up des Leistungsangebots vorgenommen.

\section{Vertrieb: Standortpolitik}

Die strategische Standortpolitik ist für das grundlegende Geschäftsmodell im Handel ähnlich bedeutsam wie die mit ihr eng verknüpfte Sortimentspolitik, insbesondere weil Standortfestlegungen durch spezifische Investitionen und meist äußerst eingeschränkte Ausweich- und Wechselmöglichkeiten häufig von sehr langer Dauer sind. ${ }^{633}$

Wichtige Determinanten der Standortpolitik sind die Größe der anzumietenden Fläche, die infrage kommenden Orte, die Lage der Standorte innerhalb dieser Orte, sowie die Dichte des Netzes und somit die Anzahl der Filialen. ${ }^{634}$

In Bezug auf die Fläche schwankte die durchschnittliche Größe einer Schlecker-Filiale jahrzehntelang um $200 \mathrm{~m}^{2}$. Dieses Beibehalten kleiner Flächen stand in immer stärkerem Gegensatz zur allgemeinen Entwicklung in der Drogeriewarenbranche, in der immer größere Flächen entwickelt wurden. Während die Wettbewerber ab Mitte der 1990er-Jahre Filialen von $500 \mathrm{~m}^{2}$ und ab der Jahrtausendwende auch Standorte von $900 \mathrm{~m}^{2}$ und mehr eröffneten, um das immer größer werdende Sortiment attraktiv zu präsentierten, beließ es Schlecker bei einer durchschnittlichen Flächengröße von $200 \mathrm{~m}^{2}{ }^{635}$

Solche Flächen fand das Unternehmen hauptsächlich in sehr kleinen Ortschaften um die 3.000 Einwohner, wo es die Standorte ehemaliger Fachdrogerien und Lebensmitteleinzelhändler übernahm. In größeren Städten zog es Schlecker nicht wie die Konkurrenz in die hochfrequentierten Lagen der In-

631 Vgl. S071004FA.

632 Vgl. S120324TA: „Acht Rollen Zewa-Klopapier - dm: 2,15 Euro, Schlecker: 3,49 Euro. Pflaster von Hansaplast - dm: 1,75 Euro, Schlecker: 3,15 Euro."

633 Vgl. Mattmüller \& Tunder (2004: 320f.).

634 Vgl. Mattmüller \& Tunder (2004: 45).

635 Vgl. S040115LZ, S030102LZ; S041026MM: »Auf der Internet-Seite ist zu lesen, dass das Unternehmen in allen Orten ab 1.500 Einwohnern Verkaufsräume ab einer Fläche von $120 \mathrm{~m}^{2}$ « zu mieten sucht." 
nenstadt oder in Shopping-Center, sondern in Gewerbe- und Wohngebiete. ${ }^{636}$ Von dieser Standortpolitik versprach sich Schlecker vor allem niedrige Mietkosten. ${ }^{637}$

An dieser Standortpolitik hielt die Anton Schlecker e.K. trotz damit verbundener höherer Personal- und Logistikkosten bis kurz vor der Insolvenz fest, selbst als das Unternehmen um die Jahrtausendwende mit mehr als 11.000 Standorten in Deutschland seine flächenmäßige Sättigungsgrenze erreichte und entsprechende Standorte zunehmend rar wurden. ${ }^{638}$ Anstatt das Geschäftsmodell neu auszurichten, wurden nun auch Ladenlokale ab $100 \mathrm{~m}^{2}$ in Ortschaften ab 1.500 Einwohnern akzeptiert, um die Expansion fortzusetzen. ${ }^{639} 2004$ wurde zwar ein Umdenken in der Standortpolitik kommuniziert, doch waren nach wie vor ungefähr zwei Drittel der in der Folgezeit neu eröffneten Filialen kleiner als $200 \mathrm{~m}^{2}{ }^{640}$ Erst 2008 erfolgte mit Schlecker XL der erste für die Öffentlichkeit sichtbare Versuch Flächen von $400-600 \mathrm{~m}^{2} \mathrm{zu}$ entwickeln. ${ }^{641}$

Somit lässt sich zusammenfassend festhalten, dass Schleckers Standortstrategie trotz der Entwicklung im Handel zu immer größeren Flächen über Jahrzehnte hinweg quasi unverändert blieb.

\section{Vertrieb: Filialgestaltung}

Das lieblose Erscheinungsbild der Schlecker-Filialen mit ihren dunklen, engen Gängen und dem sterilen Neonlicht wurde ex-post zu einem Synonym für das Scheitern des Geschäftsmodells der Anton Schlecker e.K.

"Und das ist die gute Nachricht: das Ende von Schlecker zeigt, dass Marktwirtschaft funktioniert. Man muss nur in einer einzigen dieser düsteren, engen Drogeriefilialen gewesen sein, um zu wissen, warum Schlecker nicht überlebensfähig war: weil niemand dort gern einkaufen geht."

(Die FAZ zum Erscheinungsbild der Filialen) ${ }^{642}$

636 Vgl. S030102LZ. Wo rechtlich zulässig, teils auch in die Wohngebiete.

637 Vgl. S010823LZ. Teilweise lagen die Mietkosten unter $5 €$ je $\mathrm{m}^{2}$, vgl. S041105HB.

638 In manchen kleinen Ortschaften existierten bereits zwei Filialen oder in Städten zwei Filialen in einer Straße.

639 Vgl. S031023LZ. Schlecker übernahm auch Märkte, die die Konkurrenz öffentlich als unrentabel abgelehnt hatte; vgl. S021114LZ. Als ein Indiz für die geringe ökonomische Attraktivität der Märkte kann die Tatsache angesehen werden, dass ein Großteil der Filialen auch noch zwei Jahre nach der Insolvenz leer stehen. Rossmann prüfte 3.200 Filialen. Infrage kamen 50-80; vgl. S130821LZ.

640 Vgl. S040908MM. So verkündete Schlecker, dass nun `offensiv Standorte ausgetauscht würden und Filialen unter $200 \mathrm{~m}^{2}$ oder zwei Filialen in einem Dorf der Vergangenheit angehören sollten.

641 Vgl. S080619LZ. Es wurden im Vergleich zum Altbestand jedoch nur relativ wenige XLFilialen eröffnet.

642 Vgl. S120601FA. 
Die Filialgestaltung bei Schlecker war dabei sowohl bewusste Entscheidung an sich, als auch Konsequenz vorgelagerter Entscheidungen zur Sortiments-, Preisund Standortpolitik. So litt das Erscheinungsbild der Schlecker-Filialen auf der einen Seite unter der Zielstellung, möglichst viele Artikel auf möglichst geringer Fläche anzubieten und unter der sehr kostenbewussten Einrichtung. Auf der anderen Seite sollte das »billige« Erscheinungsbild der Filialen niedrige Preise suggerieren.

Als die Wettbewerber ab Mitte der 1990er-Jahre im Zuge ihres Trading-Up begannen, das Sortiment zu günstigen Preisen in schön gestalteten Filialen attraktiv zu präsentieren wurde diese Filialgestaltung zunehmend zum Problem. ${ }^{643}$ Es entstand ein leicht erkennbarer und immer schärfer akzentuierter Kontrast, der schlussendlich das Image des Unternehmens erheblich beeinträchtigte. ${ }^{644}$

Nichtsdestotrotz unterblieben Investitionen in die Modernisierung alter Flächen und auch die Gestaltung neuer Filialen blieb weitgehend unverändert. Erst 2008 wurde verkündet, die Filialen geräumiger und heller gestalten und somit aufwerten zu wollen. Dieser Vorsatz bezog sich jedoch vor allem auf das neue Betriebsformat Schlecker XL. ${ }^{645}$ Für die Vielzahl der tausenden kleinen Filialen wurde eine grundlegende Änderung der Filialgestaltung, erst kurz vor der Insolvenz im Rahmen des Programms »Fit for Future» erwogen, aber letztlich nicht mehr vollzogen. ${ }^{646}$

\section{Vertrieb: Vertriebskonzepte}

Die skizzierten strategischen Parameter formten in der Summe ein spezifisches Vertriebskonzept, welches trotz einer eindeutig gegenläufigen Umweltentwicklung mehr als dreißig Jahre lang beinahe unverändert verfolgt wurde.

Die Vielzahl der im Verlauf der organisationalen Entwicklung Schleckers übernommenen Unternehmen und Filialen trugen nicht zu einer Differenzierung des Marktauftritts bei. Die Akquisitionen wurden vielmehr rasch in das bestehende Konzept eingefügt und homogenisiert. Selbst die Versuche einer Neupositionierung mit den Typen Schlecker XL oder Schlecker XXL müssen eher als Versuche gewertet werden den bestehenden Betriebstyp auf einer grö-

643 Vgl. Werner (2013: 166ff.). Beispielsweise wurden Regale schräg gestellt, was die Raumnutzung mindert, aber zu mehr Übersichtlichkeit führt. Besonderen Wert wurde auf Lichtdesign gelegt.

644 Vgl. S930426SP. Das schwierige Manövrieren mit einem Kinderwagen in den engen Gängen der Filialen geriet regelrecht zu einem »Running Gag», auf den die Medien, aber auch beteiligte Akteure in ihren Darstellungen immer wieder zurückgriffen; vgl. S080325MM.

$645 \mathrm{Vgl}$. S080325MM. In den XL-Filialen wurden bis zu 13.000 Artikel auf ca. $700 \mathrm{~m}^{2}$ präsentiert.

646 Vgl. S110309LZ zur Testfiliale in Allmendingen, die deutlich breitere Gänge, niedrigere Regale und mehr Licht aufwies und als bunter und freundlicher wahrgenommen wurde. Durch entsprechende Maßnahmen konnten in der Testfiliale nach der Modernisierung aber $10 \%$ weniger Artikel angeboten werden als vor der Modernisierung. 
ßeren Fläche zu platzieren. Eine klare Differenzierung oder ein signifikantes Trading-Up fanden hier nicht statt.

„Das waren alles Etiketten. Ich würde sagen, das war Etikettenschwindel. Dahinter
stand kein einheitliches Konzept. Also da war er schon eher getrieben wie früher ALDI
auch gewachsen ist. Alles gleich. Jede Veränderung in der Organisation kostet Geld und
das stimmt ja auch. So dass man sagt >Ihr müsst versuchen, ein optimales Modell zu
finden und das muss man multiplizieren und dann hast Du natürlich die größten
Kostenvorteile.` Zumindest theoretisch. Das ging dann auch bis zum Punkt X ging das
auch. Aber wenn dann der Wettbewerb da ist, dann differenziert der Wettbewerb.»
(Ehemalige Führungskraft)

Eine Zwei-Marken-Strategie wurde im Zuge der Übernahme der Ihr Platz-Filialen erwogen, die für Schlecker das Premiumsegment abdecken sollten. Ihr Platz hatte ein doppelt so großes Sortiment wie Schlecker und sollte auf die Bereiche Wellness, Schönheit und Gesundheit ausgerichtet werden. ${ }^{647}$ Das strategische Vorhaben scheiterte jedoch, da Investitionen in ein Premiumkonzept ausblieben und viele der Ihr Platz-Filialen nach kurzer Zeit einfach in Schlecker XL-Märkte »umgeflaggt « wurden. ${ }^{648}$

\section{Einkauf und Finanzierung}

Die zweite Säule jedes Handelsbetriebs ist der Einkauf. Bei der Anton Schlecker e.K. nahm dieser Funktionsbereich und dessen Perspektive auf die betrieblichen Aktivitäten jedoch eine besonders prominente Stellung ein. So orientierte sich beispielsweise die Sortimentspolitik weniger an aktuellen Kundenbedürfnissen, denn an den Möglichkeiten zu Rabatten auf bestimmte Produkte, wie sie sich im Einkauf gerade ergaben. ${ }^{649}$ Strategischen Problemen wie niedriger Produktivität und dem zunehmenden Preiskampf wurde ebenfalls stets mit dem Versuch begegnet, im Einkauf noch bessere Konditionen zu erzielen. ${ }^{650}$

Diese Dominanz des Einkaufs blieb über die Jahrzehnte ebenso unverändert wie die Beziehung zur Herstellerindustrie und die Parameter der mit dieser vereinbarten Konditionenpolitik. Die Bedeutung dieser Politik für die Wachstumsstrategie und vor allem ihr selbstverstärkender Charakter sollen im folgenden Abschnitt zur Pfaddiagnose ausführlich analysiert werden.

647 Vgl. S071004FA; S071296LZ.

648 Vgl. S090820LZ, S120313WW: "Ihr Platz wurde in kürzester Zeit auf das Niveau von Schlecker gebracht."

649 Hierzu ein Interviewpartner: »der Einkäufer, der hat dann Zuteilungen gemacht [...] alles rein in das Lager«.

650 Vgl. S061027LZ: »Mit massiven Forderungen gegenüber der Industrie will Schlecker offenbar die anstehende Mehrwertsteuererhöhung kompensieren." 


\section{Fazit zur strategischen Persistenz}

An dieser Stelle kann jedoch festgehalten werden, dass die grundlegenden strategischen Parameter des Geschäftsmodells der Anton Schlecker e.K. in den entscheidenden Bereichen Vertrieb und Einkauf trotz einer sich wandelnden Umwelt über viele Jahrzehnte beinahe unverändert blieben. Während die Wettbewerber wie dm und Rossmann spätestens ab Anfang der 1990er-Jahre auf den Wandel der Konsumentenbedürfnisse mit einem Trading-Up reagierten, blieb die strategische Grundausrichtung der Anton Schlecker e.K. annähernd unverändert und entsprach ungefähr jener, mit der Schlecker 1975 in den Markt eingetreten war.

"'Schleckers Konzept hat sich in dreißig Jahren eigentlich so gut wie gar nicht verändert , kommentiert ein Branchenkenner die Politik des Marktführers. Was zumindest mehr als zwei Jahrzehnte Erfolgsgarant war, wird nun zunehmend zum Problem. \Jetzt bekommt er Druck von oben und von unten.` Oben sind die deutlich höherwertig positionierten $\mathrm{dm}$ und Rossmann, unten greifen insbesondere Discounter wie Lidl nach den Umsätzen von Schlecker."

(Die Lebensmittel Zeitung zum ausbleibenden Wandel bei der Anton Schlecker e.K.) ${ }^{651}$

Relevante strategische Alternativen wurden nicht entwickelt. Stattdessen wurde der Monobetriebstyp im Rahmen des etablierten Geschäftsmodells kontinuierlich multipliziert. ${ }^{652}$ Die Wettbewerber dm und Rossmann entwickelten im Verlauf der Zeit ihre Drogeriemärkte immer weiter fort und differenzierten ihr Produkt. Im Gegensatz hierzu versuchte Schlecker über Jahrzehnte hinweg bestehende Märkte mit dem Mitte der 1970er-Jahre etablierten Betriebstyp zu durchdringen. ${ }^{65}$

\begin{tabular}{|l|c|c|}
\hline \multicolumn{1}{|r|}{ Produkt } & bestehende & neue \\
\cline { 1 - 2 } Markt & Marktdurchdringung & Produktentwicklung \\
\hline Neue & Marktentwicklung & Diversifikation \\
\hline
\end{tabular}

Abbildung 11: Einordnung der Wachstumsstrategie der Anton Schlecker e.K. in die Produkt/ Markt-Matrix

(Quelle: Eigene Darstellung nach Welge \& Al-Laham [2012: 588]).

651 Vgl. S060407LZ.

652 Auch in der Handelsbetriebslehre wird Schlecker als ein Beispiel für ein »rein multiplizierendes Unternehmen« angeführt Vgl. Mattmüller \& Tunder (2004: 312).

653 Vgl. zur Wachstumsstrategie der Marktdurchdringung Ansoff (1988: 109), Welge \& AlLaham (2012: 591). Zu den Geschäftsfeldern des Unternehmens muss festgehalten werden, dass der Schlecker-Konzern bis zum Jahr 2012 auch noch die Fleischwarenfabrik, die SBWarenhäuser, sowie weitere Handelsaktivitäten wie ein Möbelhaus und eine Tankstelle führte. Diese Aktivitäten waren strategisch jedoch bedeutungslos. 
Signifikante Innovationen im Geschäftsmodell waren erst im Zuge des Turnarounds erkennbar. ${ }^{654}$

»Wir haben nur kleinkarierte Sachen ausprobiert. Heute wissen wir, dass wir unser gesamtes Geschäftsmodell umwälzen müssen.«

(Anton Schlecker in einem Interview mit dem Manager Magazin im Jahr 2010) ) $^{655}$

Angesichts dieses Festhaltens an einer Wachstumsstrategie mit einem offensichtlich unterlegenen Betriebstyp kann eine Form strategischer Persistenz attestiert werden. Diese kann zugleich als die maßgebliche Ursache für das Entstehen der Unternehmenskrise und die Insolvenz des Unternehmens ausgemacht werden. ${ }^{656}$

Im folgenden Abschnitt soll geprüft werden, inwiefern die strategische Persistenz auf die Existenz selbstverstärkender Mechanismen und auf einen hieraus resultierenden Lock-in der strategischen Entwicklung zurückzuführen ist.

\subsubsection{Pfaddiagnose}

Im Rahmen der Fallanalyse konnten zwei selbstverstärkende Mechanismen identifiziert werden: ein auf Skalenerträgen im Einkauf beruhender Mechanismus sowie positive Rückkopplungen durch Komplementaritätseffekte.

Im Folgenden wird zuerst das »critical juncture« skizziert, das diese beiden Mechanismen auslöste. Anschließend werden die Wirkweisen der Mechanismen dargestellt, welche die Anton Schlecker e.K. in den Lock-in und letztlich in die Unternehmenskrise führten.

\subsection{Selbstverstärkende Mechanismen}

Für die skizziert strategische Persistenz der Anton Schlecker e.K. lassen sich eine Reihe von Erklärungsansätzen ins Feld führen.

Auf psychologischer Ebene, mit der sich die vorliegende Arbeit nicht befasst, wären wohl individuelle Rigiditäten und Wahrnehmungsverzerrungen identifizierbar. ${ }^{657}$ Allein die Managementforschung vermag hier mit dem Konzept des

654 Nach den wenig erfolgreichen Versuchen auf größeren Flächen versuchte Schlecker ab 2011 seine Stärken der Marktpräsenz auszuspielen und auf den bestehenden Flächen ein modernes Nahversorgungskonzept zu entwerfen. Zuvor war auch der Einstieg ins E-Commerce gewagt worden, dessen Volumen aber klein blieb.

655 Vgl. S100121MM.

656 Es bestanden auch große Defizite im operativen Bereich, die im Turnaround und der Insolvenzverwaltung offen zutage traten, zum Beispiel hinsichtlich des fehlenden Warenwirtschaftssystems oder eines mangelhaften Rechnungswesens. Diese Defizite waren für die Krisenentstehung aber von nachgeordneter Bedeutung und meist Symptome tieferliegender organisationaler Probleme.

657 Diese Ebene liegt außerhalb des theoretischen und empirischen Fokus dieser sozialwissenschaftlichen Arbeit. 
»emotional attachment« der Entscheidungsträger zur etablierten Strategie einen Erklärungsansatz der strategischen Persistenz anzubieten. ${ }^{658}$

Zudem dürften das kognitive Potenzial der Gruppe der Entscheidungsträger und die Form ihrer Interaktion von großer Bedeutung für die strategische Entwicklung des Unternehmens gewesen sein. Im folgenden Teil D sollen die vom familiären Einfluss bedingten Ausprägungen dieser Variablen daher ausführlich und fallübergreifend erörtert werden.

Des Weiteren trugen zahlreiche Imprints zur Entwicklung und Stabilisierung des strategischen Musters bei der Anton Schlecker e.K. bei. So hatte die kulturelle Prägung der im Viehhandel aktiven Fleischwarenfabrik vermutlich einen Anteil daran, dass sich aus dem Gründungsunternehmen ein einkaufsorientierter Drogeriediscounter entwickelte und keine serviceorientierte Parfümeriekette. Daher sollen diese Imprints in ihrem Wirkungszusammenhang mit dem Einfluss des Faktors Familie ebenfalls fallübergreifend in Teil D dargestellt werden.

Zunächst widmet sich vorliegender Teil C den selbstverstärkenden Mechanismen und dem Lock-in und somit den bedeutsamsten Ursachen der strategischen Persistenz und Krisenentstehung. Denn die Analyse der strategischen Entwicklung und der strategischen Entscheidungsprozesse der Anton Schlecker e.K. zeigte deutlich, dass eben nicht individuelle Rigiditäten oder organisational-historische Vorprägungen für die strategische Persistenz entscheidend waren, sondern in erster Linie zwei selbstverstärkende Mechanismen. Die Suche nach Antworten auf die Frage, warum das oben skizzierte strategische Muster über Jahrzehnte beständig reproduziert wurde, muss daher bei den Wirkkräften dieser selbstverstärkenden Mechanismen beginnen.

\section{»Critical juncture«}

Die beiden bei der Anton Schlecker e.K. identifizierten selbstverstärkenden Mechanismen entwickelten sich im Gefolge einer in den Nachkriegsjahrzehnten stetig steigenden Nachfrage nach Drogeriewaren. ${ }^{659}$ Diese wachsende Nachfrage nach Artikeln der Körperpflege und Kosmetik erforderte eine kontinuierlich steigende Distributionsleistung, welche zunächst vor allem durch die Fachdrogerien und den LEH erbracht wurde. Doch nach dem Ende der Preisbindung im Jahr 1974 stand die gesamte Branche vor einem Umbruch: Zum einen nahm die Zahl der inhabergeführten Drogerien beständig ab. Zum anderen eröffneten sich für die Herstellerindustrie durch das Aufkommen der Droge-

658 Vgl. Burgelman (1994) und Kapitel D.1.2 zur emotionalen Verbundenheit zur etablierten Strategie.

659 Diese Nachfrage nach Drogeriewaren nahm im Vergleich zu vielen anderen Segmenten des Handels bis zum Jahr 2016 stetig zu; vgl. R150415HB: „Drogeriemärkte legen zu: Rossmanns wunderbare Welt des Wachstums«. 
riemärkte wie Rossmann, dm und Schlecker neue Vertriebskanäle. ${ }^{660}$ Somit war in dieser Phase des Umbruchs weitgehend offen, wer in Zukunft den rasch steigenden Bedarf der Herstellerunternehmen nach einer flächendeckenden Distribution ihrer Waren erfüllen würde.

In diesem gegebenen Rahmen offerierte die Anton Schlecker e.K. Herstellern wie Beiersdorf oder Henkel ein teils explizites, teils implizites "Angebot«: Auf der einen Seite stellte das Unternehmen in Aussicht, die erforderte Distributionsleistung durch eine rasche Expansion und fortwährende Erhöhung der Filialzahlen zur Verfügung zu stellen. Im Gegenzug für das versprochene schnelle und kontinuierliche Wachstum wurden jedoch entsprechend günstige Einkaufskonditionen gefordert.

Nicht zuletzt dank ausgeprägter organisationaler Kompetenzen im Einkauf, die auch aus den historischen Wurzeln des Unternehmens im Viehhandel erwuchsen, wurden der Anton Schlecker e.K. dann tatsächlich bereits kurz nach Markteintritt sehr günstige Konditionen gewährt. So konnte das Unternehmen bereits in den 1970er-Jahren sehr günstige Einkaufspeise erzielen und zahlreiche Rabatte und Zuschüsse aushandeln. ${ }^{661}$

Am bedeutsamsten waren allerdings die langen Zahlungsziele von ein bis drei Monaten, die der Anton Schlecker e.K. schon kurz nach dem Einstieg in den Drogeriemarkt gewährt wurden ${ }^{662} \mathrm{Da}$ die Ware üblicherweise bereits nach wenigen Tagen verkauft war, stellten diese gestreckten Zahlungsfristen eine Art zinslosen Kredit der Industrie dar, sodass Mittel für anderweitige Aktivitäten zur Verfügung standen. Die über den Cashflow erzielten Finanzmittel konnten zum Beispiel kurzfristig angelegt werden. ${ }^{663}$ Doch vor allem konnten mit den ungebundenen Finanzmitteln umgehend Investitionen in Infrastruktur und Warenbestand zur Eröffnung neuer Filialen getätigt werden. In bewusster Anlehnung an das Expansionsmodell der Discounter des LEH konnte so die weitere Expansion in der Folge weitestgehend über den Cashflow finanziert werden. ${ }^{664}$

660 Vgl. Werner (2013: 22): »Der gesamte Einzelhandel war im Umbruch«. Von 1972 sinkt die Anzahl der Fachdrogerien von 16.658 auf 8.949 (1980); 7.700 (1990) 6.050 (2000); 2.745 (2008). Vgl. Bundesverband Drogerien (2014); S090101.

661 Vgl. S810522ZE; Eine damalige Führungskraft: „Und wir kriegen dann Warenkostenzuschuss und wir kriegen Regalmeterzuschuss und wir kriegen eine Rückvergütung, ne, wenn die Jahresgespräche kamen. Und dann werden das fünf Millionen und dann werden das 10 Millionen und dann wird die Liquidität immer noch größer.»

662 Vgl. S041105HB. Zu dieser Einschätzung kommt auch Werner (2013: 232).

663 Im Jahr 2002 überstieg der Bestand der Wertpapiere sogar den kumulierten Wert aller Immobilien.

664 Vgl. S041105HB. Auf das direkte Vorbild Aldi verwiesen Interviewpartner hinsichtlich mehrerer Aspekte, zum Beispiel in Bezug auf den Mono-Betriebstyp. Vgl. hierzu auch Brandes (2001). 
"Der Blick auf den Wert des Warenbestandes (460 Millionen Mark) und die Höhe der Lieferantenverbindlichkeiten (550 Millionen Mark) förderte es zutage. Bei uns selbst lautete das Verhältnis 120 Millionen zu 50 Millionen Mark. Sprich: Die Lieferanten gaben Schlecker durch Zahlungsziele von bis zu einem Jahr kostenlose Kredite in Höhe von insgesamt 360 Millionen Mark. Davon träumen viele: Heute geliefert, in einem Jahr bezahlen«. Diese Konditionen haben Schlecker etwa 30 Millionen Mark Zinsen im Jahr erspart. Damit kann man schon die ein oder andere Filiale eröffnen."

(Götz Werner zur Einsicht aus der 1994 erstmals veröffentlichten Bilanz der Anton Schlecker e.K. $)^{665}$

Diese Konditionenvereinbarungen mit den Herstellern beförderten somit maßgeblich die rasche Expansion der Anton Schlecker e.K. in den ersten Jahren nach ihrem Markteintritt. Werner (2013) spricht entsprechend von der »Geburtshilfe der Industrie« für Schlecker. ${ }^{666}$

Rückblickend kann die Summe dieser Entscheidungen zur Konditionenpolitik zwischen der Anton Schlecker e.K. und den Herstellerunternehmen im Zeitraum 1977 bis 1984 gar als das »critical juncture« der weiteren strategischen Entwicklung verstanden werden. ${ }^{667}$

Die Bedeutung dieses »critical junctures« kann anhand der früh begonnenen, aber insgesamt misslungenen Auslandsexpansion des Unternehmens verdeutlicht werden. ${ }^{668}$ Diese scheiterte zum Teil aufgrund vertriebsseitiger Probleme wie der mangelnden Adaptionsfähigkeit an national unterschiedliche Konsumentenbedürfnisse. In erster Linie war das Scheitern der Auslandsexpansion jedoch dem Umstand geschuldet, dass Schlecker es wegen zu geringer Größe und mangelnder Marktmacht in den Auslandsmärkten nie vermochte, die günstigen Konditionen aus dem Deutschlandgeschäft in andere Länder zu »exportieren «. ${ }^{669}$ So blieb dem Unternehmen im Rahmen des etablierten strategischen Musters nur die Möglichkeit, die Ware zu den günstigen inländischen Konditionen in Deutschland zu kaufen und sie unter hohen Logistikkosten ins Ausland zu liefern.

Das »critical juncture«, als Ergebnis eines langjährigen Verhandlungsprozesses zwischen einer Vielzahl von Teilnehmern, war weder ein rein zufallsba-

665 Vgl. Werner (2013: 230).

666 Ihre Bedeutung lässt sich auch an der weitgehend misslungenen Auslandsexpansion des Unternehmens ablesen: »Dem Unternehmen ist es im Wesentlichen nicht gelungen, seine deutschen Konditionen zu exportieren. International bedeutende Lieferanten aus dem Kosmetik- und Körperpflegebereich haben bisher erfolgreich dem Druck widerstanden, im Ausland sozusagen den finanziellen Steigbügelhalter für Schlecker zu spielen und seine Marktdurchdringung über Konditionen entsprechend zu beschleunigen.» Vgl. hierzu S081204LZ.

667 Vgl. Collier \& Collier (1991); Werner (2013: 231).

668 Schlecker hatte sich früh in viele westeuropäische Märkte gewagt, war dort jedoch nur selten profitabel.

669 Vgl. S040220MM. 
siertes »small event" im Sinne der klassischen Pfadtheorie noch ein »big event" im Sinne einer einmaligen, bewussten Entscheidung. ${ }^{670}$ Zwar wurde die cashflowfinanzierte Expansionsstrategie von der Anton Schlecker e.K. intentional gewählt. Doch aus den komplexen Aushandlungsprozessen zwischen Schlecker, den Herstellern und den zahlreichen Wettbewerbern wie dm oder Rossmann hätten lange Zeit auch andere Ergebnisse resultieren können. Denn die Herstellerindustrie wäre wohl bereit gewesen, auch andere Drogeriemarktunternehmen in ähnlicher Weise indirekt zu "unterstützen«. Die spezifische Kompetenz des Unternehmens im Einkauf trug aber dazu bei, dass es schlussendlich die Anton Schlecker e.K. war, die diese Einkaufskonditionen aushandeln konnte.

»[Z]um Ärger der Wettbewerber hätschelte die Markenartikelindustrie den Emporkömmling. Schleckers Expansionsstrategie wurde mit den niedrigsten Einkaufspreisen der Branche belohnt."

(Das Manager Magazin zur Verbindung von Einkaufskonditionen und Expansion der Anton Schlecker e.K. $)^{671}$

Auf dieser Grundlage bildete sich im gemeinsamen Streben nach Wachstum in den folgenden Jahrzehnten eine symbiotische und lange Zeit interdependente Beziehung zwischen Schlecker und der Herstellerindustrie heraus, die im Verlauf der Zeit dazu führte, dass zeitweise $76 \%$ aller Drogeriemärkte in Deutschland von der Anton Schlecker e.K. betrieben wurden. ${ }^{672}$

\section{Selbstverstärkender Mechanismus aufgrund von Skalenerträgen}

Dieses zwischenzeitliche Marktergebnis ist vor allem auf einen selbstverstärkenden Mechanismus zurückzuführen, der auf Skalenerträgen im Einkauf basierte, welcher wiederum durch das »critical juncture» in Form der »Geburtshilfe« der Herstellerindustrie ausgelöst worden war.

Mit der fortschreitenden Expansion des Unternehmens stiegen sowohl die Distributionsleistung, welche die Anton Schlecker e.K. für die Herstellerindustrie übernahm, als auch die Marktmacht des Unternehmens. ${ }^{673}$ In der direkten Folge dieser höheren Distributionsleistung und der gestiegenen Marktmacht konnte Schlecker im Verlauf der Zeit jedoch auch immer bessere Konditionen aushandeln. Die so erzielten günstigeren Einkaufspreise, höheren Rabatte und Zuschüsse, sowie vor allem die stetig verlängerten Zahlungsziele befeuerten

670 Vgl. Sydow et al. (2009) zur Diskussion um »small events« und die Zufallsabhängigkeit der "trigger" von selbstverstärkenden Mechanismen. Die Autoren vermuten, dass im organisationalen Kontext solche Auslöser weniger zufallsabhängig sind als im Kontext von Marktprozessen.

671 Vgl. S110118MM.

672 Vgl. Werner (2013: 228). Diese Zahl bezieht sich auf das Jahr 2007.

673 Vgl. S050523LZ: »Wer eine Produkteinführung macht, muss einfach bei Schlecker gelistet sein, bringt es ein Lieferant auf den Punkt.» 
wiederum maßgeblich die fortschreitende Expansion des Unternehmens, durch welche wieder Distributionsleistung und Marktmacht stiegen, und so weiter. Das »critical juncture" hatte somit eine sich selbstverstärkende Wachstumsspirale ausgelöst, welche der Anton Schlecker e.K. zu einer beispiellosen Expansion verhalf.

Hinsichtlich der Skaleneffekte im Einkauf, die diesem selbstverstärkenden Mechanismus zugrunde lagen, gingen Marktteilnehmer davon aus, dass die Anton Schlecker e.K. nach und nach allein beim Einkaufspreis einen Konditionenvorteil von drei bis zehn Prozent entwickelte.

"Ziel der ungewöhnlichen Aktion: Die Markenartikler wachrütteln und ihnen mögliche Fehlentwicklungen bei der Konditionsgestaltung vor Augen führen. Mit einem sständigen Schlecker-Konditionsvorteil von mehr als fünf Prozent ‘ förderten sie geradezu das Wachstum dieses >unproduktiven Unternehmensı."

(Der Handel zu einem Rundschreiben Götz Werners an die Industrie im Jahr 1994) ${ }^{674}$

Für die fortschreitende Expansion noch bedeutsamer waren die immer zahlreicheren Rabatte und Zuschüsse, welche die Anton Schlecker e.K. gegenüber den Herstellern durchsetzen konnte. Da mit der Zeit kaum ein Hersteller mehr auf den Vertriebskanal Schlecker verzichten konnte, entwickelte das Unternehmen sukzessive ein verzweigtes Konditionensystem aus Artikelgruppenrabatten und Skonti, Werbekostenzuschüssen, Neueinlistungsgeldern, Bezugsmengenstaffeln oder Jubiläumsprämien und sogar kompletten Filialeröffnungserstausstattungen:

"Es war ein offenes Geheimnis, dass die meisten Schlecker-Lieferanten die Warenerstausstattung zu Neueröffnungen ohne Berechnung lieferten. Im Klartext: Wenn Schlecker einen neuen Laden aufmachte, bekam er die Ware dafür von der Industrie geschenkt. [...] Kein anderes Unternehmen der Branche konnte jemals solche Konditionen aushandeln [...] Ich schrieb im August 1994 deshalb empört einen offenen Brief an die Industrie, in dem ich diese Wettbewerbsverzerrung anprangerte." (Götz Werner zu den Einkaufskonditionen der Anton Schlecker e.K.) ${ }^{675}$

Infolge der stetig steigenden Marktmacht gelang es gar, Forderungen durchzusetzen, die weit über die eigentliche Warenaustauschbeziehung zwischen der Anton Schlecker e.K. und der Herstellerindustrie hinausreichten. So ließ sich

674 Vgl. S940101DH; auch Werner (2013: 231) und S040220MM. Nach der Übernahme von Ihr Platz durch Schlecker zeigte sich, dass die Konditionen von Ihr Platz um mehrere Prozentpunkte schlechter waren.

675 Vgl. Werner (2013: 231); auch S050523LZ: »Die Erstausstattung der neuen Märkte sei quasi zum Nulltarif geliefert worden und außerdem habe man darüber hinaus lange Zahlungsziele gewährt. Vgl. auch Brandes (2001: 209) für eine allgemeine Auflistung solcher Rabatte und Zuschüsse im Handel. Branchenkenner schätzten, dass bei Schlecker der Anteil der Werbekostenzuschläge, Boni und sonstigen Vergütungen bezogen auf den Umsatz kaum geringer ausfiel als der Rohertrag. Vgl. S031023LZ. 
Schlecker sowohl die Einführung von EC-Karten-Terminals als auch die Errichtung der meisten Logistikzentren von den Lieferanten bezahlen. ${ }^{676}$

Den verbleibenden Anteil an solchen Investitionen konnte das Unternehmen aus dem laufenden Cashflow begleichen und musste mithin nicht einmal für den teuren Neubau der Unternehmenszentrale in Ehingen einen Bankkredit aufnehmen.

Für diese Finanzierung über den Cashflow war von größter Bedeutung, dass es dem Unternehmen mit wachsender Marktmacht gelang die ohnehin langen Zahlungsziele immer weiter zu verlängern. Schlussendlich wurden gar Zahlungsfristen von bis $\mathrm{zu}$ sechs Monaten eingeräumt. ${ }^{677}$

»[...] dann wird die Liquidität immer noch größer [...] und ich kaufe für eine Million heute ein und zahle in vier Wochen. Dann ist das, was ich in den vier Wochen verkauft habe, natürlich schon mein Geld und jetzt verhandele ich mit fünf Wochen und mit sechs Wochen und mit sieben Wochen und mit acht."

(Ehemalige Führungskraft der Anton Schlecker e.K. in der Zeit der Expansion)

In der Folge dieser Konditionenpolitik addierten sich die Lieferantenverbindlichkeiten zum Beispiel in der Bilanz 2001 auf 1,3 Mrd. DM, denen unverkaufte Vorräte von lediglich einer Milliarde Mark gegenüberstanden. Die Barreserven hingegen beliefen sich auf 284 Millionen Mark. ${ }^{678}$

Diese Konditionen basierten zum Teil auf Marktmacht und einer sehr harten Verhandlungsführung. ${ }^{679}$ Sie waren aber auch dem Umstand geschuldet, dass die Hersteller maßgeblich von der dynamischen Expansion der Anton Schlecker e.K. und deren steigenden Umsätzen im Zuge der dynamischen Expansion profitierten. In der symbiotischen Beziehung zwischen der Anton Schlecker e.K. und der Industrie wurde wachsende Distributionsleistung gegen günstige Konditionen eingetauscht. ${ }^{680}$

So stiegen die Skalenerträge im Zeitverlauf immer weiter an, teilweise sogar

676 Vgl. Werner (2013: 232), der einen ihm vorliegenden Brief an einen Lieferanten sinngemäß mit den folgenden Worten zitiert: "Sehr geehrte Damen und Herren, wir haben jetzt in Schneverdingen unser Lager eröffnet. Dort haben wir 1 Million Euro investiert. Gemäß ihrem Umsatzanteil bei uns entfällt auf Sie ein Beitrag von 3.650 Euro. Wir bitten Sie, das auf eines der unten stehenden Konten zu überweisen.»

677 Vgl. S040220MM; S041105HB.

678 Vgl. Schlecker (2001: 3); auch S040220MM.

679 Vgl. S020725LZ. Auch deshalb versucht Schlecker bei den Lieferanten noch über Centbeträge zu verhandeln. Markenartikler berichten, dass sie mitunter sieben- bis achtmal in der Ehinger Zentrale vorsprechen müssten, bevor sie einen Liefervertrag erhielten. S050512LZ: "Sich schlappe 10.000 Multifunktionsterminals gratis zu besorgen [...] das hat Klasse, die nicht allein mit der Marktmacht des Drogeriemarktprimus zu erklären ist.»

680 Vgl. S110616LZ: „Schlecker kreierte über Jahre ein Konditionsgefüge mit bemerkenswerter Vielfalt an Einzelposten. Ein Ansatz für zusätzliche Forderungen ließ sich da immer finden. Wenngleich die Hersteller ob der Belastung ächzten, unterm Strich konnten sie lange von der starken Dynamik profitieren.» 
überproportional zum Wachstum. ${ }^{681}$ Innerhalb dieses Kreislaufs finanzierte die Aussicht auf Expansion die Expansion.

»Wir konnten öffnen, wo wir wollten, der Wettbewerb war ja noch ein anderer. Also wir müssen eigentlich nur Filialen besorgen, dann fließt Liquidität. Auch unterstützt durch die Industrie. Wenn ihr überall expandiert, machen wir Umsätze, wir werden uns dran beteiligen, da kam Geld rein. Und dann haben wir... klar, dann kommen die berühmten, die ganzen Kosten-Degenerationselemente."

(Ehemalige Führungskraft der Anton Schlecker e.K.)

Diese Strategie wurde rückblickend als »Schneeballsystem» bezeichnet:

"Auch die sbesondere Art der Unternehmensfinanzierung، kritisieren die Staatsanwälte: Schlecker hatte außergewöhnlich lange Zahlungsfristen, der Erlös aus bereits verkaufter, aber noch nicht bezahlter Ware diente zur Unternehmensfinanzierung, dies sei eine Art >Schneeballsystem`.»

(Der Spiegel zu den Ermittlungen der Staatsanwaltschaft hinsichtlich einer vermeintlichen Insolvenzverschleppung $)^{682}$

Für die strategische Ausrichtung des Unternehmens von großer Bedeutung war jedoch, dass die Dynamik dieser Expansionsstrategie nur mit einem bestimmten komplementären Betriebstyp erzielt werden konnte.

\section{Komplementaritätseffekte}

Die Expansionsstrategie der Anton Schlecker e.K., die auf den steigenden Skalenerträgen im Einkauf basierte und eine Finanzierung des Wachstums über den Cashflow ermöglichte, war eng mit dem oben skizzierten Betriebstyp verbunden.

So band das kleine und schnelldrehende Sortiment wenig Umlaufvermögen und die ungebundenen Finanzmittel konnten umgehend wieder in die fortschreitende Expansion investiert werden. ${ }^{63}$ Eine höhere Anzahl von Artikeln durch ein breiteres oder tieferes Sortiment hätte zwar ebenfalls die Umsätze erhöhen können, aber auch eine Verringerung der Umschlaggeschwindigkeit und somit des Nettozuflusses liquider Mittel zur Folge gehabt. Das Fehlen dieser Finanzmittel hätte die Wachstumsdynamik allerdings stark gebremst.

681 Vgl. S050523LZ: „Niemand hat die Lieferanten dazu gezwungen«, kommentiert ein Branchenkenner die Klagen.

682 Vgl. S120813SP; auch Werner (2013): Banken hätten in Schleckers »Riesenreich Probleme gehabt, Sicherheiten für Kredite zu finden. Mussten sie auch nicht, weil Schlecker sich weitgehend über Lieferantenkredite finanzierte. Das einem Schneeballsystem vergleichbare Konzept [...].»

683 Vgl. zur Bedeutung des kleinen Sortiments Mattmüller \& Tunder (2004:38); auch Brandes (2001: 20), der den Aldi-Gründer Theo Albrecht mit den Worten zitiert: »Zu unserem Warensortiment möchte ich weiter ausführen, dass es ca. 250 bis 280 Artikel umfasst [...] Grund [...]: 1. die Umsatzgeschwindigkeit, 2. die Verkaufsgeschwindigkeit.» 
Im Lichte der Expansionsstrategie besitzt auch der im Vergleich zu den Wettbewerbern geringere Anteil von Eigenmarken im Sortiment einen funktionalen Aspekt. So hätte Schlecker durch den Aufbau stärkerer Eigenmarken im Sortiment zwar auf der einen Seite zweifelsohne weiteren Druck auf die Herstellerindustrie ausüben können. Auf der anderen Seite hätte das Unternehmen bei einem steigenden Anteil an Eigenmarken aber auf einen größeren Teil der Finanzierungsleistungen und Zuschüsse verzichten müssen, die von der Herstellerindustrie erbracht wurden.

Besonders deutlich wird der Zusammenhang zwischen der Expansionsstrategie und den Parametern des Betriebstyps allerdings in Bezug auf die Standortpolitik: Die rasche Expansion war nicht zuletzt deshalb möglich geworden, weil die gewählte Standortpolitik eine rasche Akquise von Flächen ermöglichte. Während sich für die Wettbewerber die Suche nach hoch frequentierten Innenstadtlagen wegen des begrenzten Angebots sehr zeit- und kostenintensiv gestaltete, hatte Schlecker bei seiner Expansion in der Provinz oder städtischen Randlagen eine deutlich größere Auswahl. In Verbindung mit der konstanten strategischen Ausrichtung auf ein kleines Sortiment konnte das Unternehmen dort wenig nachgefragte, oftmals auch leerstehende Objekte akquirieren.

»[...] für die Expansion war das ein sehr, sehr geschickter Weg. Weil dadurch brauchten wir auch diese Umsätze nicht. Hatten durch die günstige Miete immer noch eine günstige Relation. Das Geschäft lief, wir waren ja noch konkurrenzfrei.»

(Ehemalige Führungskraft Schleckers)

Dank der sich aus der Standortpolitik häufig ergebenden lokalen Monopolstellung ging das Unternehmen zugleich mancherorts dem harten Preiswettbewerb aus dem Weg. So konnte es im Vergleich zur Konkurrenz deutlich höhere Preise durchzusetzen, gerade wenn diese Konkurrenz vor Ort gar nicht vertreten war. $^{684}$

Ein Trading-Up des Leistungsprogramms durch die Anmietung besser gelegener Standorte oder die Erweiterung des Sortiments, welches eben auch mehr Fläche benötigt, hätte demnach unweigerlich zu einer Verlangsamung der Expansion geführt. ${ }^{685}$

Dies gilt auch für eine höherwertige Filialausstattung. Die im Rahmen der Preispolitik der Preisführerschaft erfolgte »billige« Ladenausstattung erfor-

684 Vgl. S991227LZ; S070504LZ. Eine Ausweitung des Sortiments hätte eine komplette Änderung der Standortpolitik erforderlich gemacht. Die Verbindung von Sortiments-, Preis- und Standortpolitik wird hier deutlich.

685 Die schwierige Suche nach geeigneten Lagen erschwerte die Expansion der beiden Wettbewerber dm und Rossmann. Vgl. R150327LZ: "Drogisten kämpfen um Standorte", oder R101112LZ: »Zweikampf um Top-Standorte«. 
derte nur geringe Investitionen und band nur wenige Finanzmittel. ${ }^{686}$ Das Unternehmen hätte nicht so rasch expandieren können, hätte es auch nur einen Teil jener Mittel in die Modernisierung der eigenen Filialen gesteckt, die dm und Rossmann in das jahrzehntelange Trading-Up ihrer Filialen investierten.

Gleiches gilt für die Möglichkeit der Entwicklung verschiedener Betriebstypen. ${ }^{67}$ Diese wäre strategisch aufwendig und sehr investitionsintensiv gewesen. Sie hätte Ressourcen gebunden, die der Anton Schlecker e.K. nicht mehr für die Expansion zur Verfügung gestanden hätten.

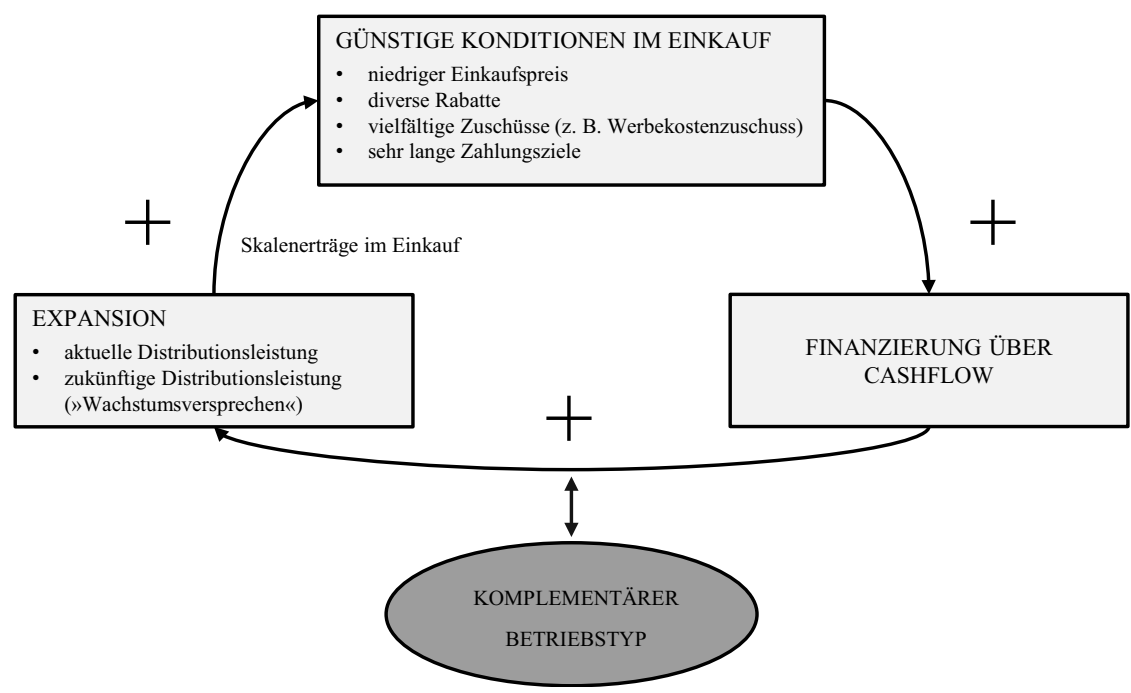

Abbildung 12: Sich selbst verstärkende Wachstumsdynamik der Anton Schlecker e.K. und komplementärer Betriebstyp

(Quelle: Eigene Darstellung).

Die auf Skalenerträgen im Einkauf beruhende Expansionsstrategie Schleckers führte zu sich selbst verstärkenden Komplementaritätseffekten im Vertrieb: Je schneller das Unternehmen wuchs und somit immer lukrativere Konditionen erzielen konnte, desto stärker tendierte es zur Reproduktion des bestehenden

686 Hierzu eine ehemalige Führungskraft: »[D]ann wird die Liquidität immer noch größer. Heißt also, ich mach ganz billige Filialen, wo eigene Leute, wir haben die Handwerker ja alle selber organisiert, die Platten neu gelegt haben, so primitiv, wie es gegangen ist, und billige Regale und billige Lampenstränge und [...].»

687 Die durchgängige Monostruktur war auch beim Vorbild Aldi eines der wichtigsten Geschäftsprinzipien. Vgl. hierzu: Brandes (2001: 181). Die Konkurrenten Schleckers, dm und Rossmann, operierten hingegen zwar auch nur über eine Marke, variierten aber deutlich stärker in der Ausgestaltung der Filialen. Vgl. hierzu Werner (2013: 166ff.), der die eigene »Illusion der Gleichheit beschreibt«. 
Betriebstyps im Vertrieb. Die Attraktivität der cashflowgestützten Wachstumsspirale erhöhte die Attraktivität des komplementären Vertriebskonzepts.

Daher verfestigte sich das strategische Muster aus Expansionsstrategie und dem komplementären Betriebstyp nach und nach; ein Abweichen von diesem etablierten Muster wurde immer schwieriger.

\subsection{Lock-in}

Viele Jahrzehnte wurde das strategische Muster der fortwährenden Expansion mit einem komplementären Betriebstyp vor allem deshalb nicht verändert, weil die beschriebene Wachstumsspirale für die Anton Schlecker e.K. äußerst lukrativ war.

Über die Reproduktion des etablierten strategischen Musters erhöhte sich nicht nur die Anzahl der Filialen, sondern insbesondere auch der Gewinn des Unternehmens. So stieg das Eigenkapital der Anton Schlecker e.K. noch in den Jahren 1996 bis 1999 um rund 270 Mio. DM bzw. 65 \%. 1999 betrug der Gewinn 300 Mio. DM bei einem Umsatz von knapp 9 Mrd. DM. ${ }^{688}$

»Sie müssen sich vorstellen, dass ja bis zum Jahr 2000 noch sehr gutes Geld verdient worden ist. Die Handelsrendite, die liegt so zwischen ein und drei Prozent. Und wir lagen dazwischen. Das ist also für einen Discounter ein guter Wert [...] Das heißt, wir haben 100 Millionen im Jahr verdient, die konnten wir gar nicht investieren. Wir hatten zu der Zeit Bankverbindlichkeit nur... also das ist kein Witz aus der Presse, sondern da standen vielleicht zwei, drei Millionen Schulden und Hunderte von Millionen Guthaben. Wir wussten ja gar nicht, was wir mit dem Geld machen sollten. Wir hatten zwei Mitarbeiter, die haben nichts anderes gemacht als zu überlegen >Was machen wir mit dem vielen Geld?«.»

(Ehemalige Führungskraft der Anton Schlecker e.K.)

Doch je länger die selbstverstärkenden Mechanismen wirkten, desto stärker wurde die weitere strategische Entwicklung von deren Regime bestimmt.

\section{Geringe Produktivität des Filialnetzes}

Von hoher Relevanz für die zunehmende Determination der strategischen Entwicklung durch die selbstverstärkenden Mechanismen waren die Auswirkungen des etablierten strategischen Musters auf die Produktivität des Filialnetzes.

So hatte die skizzierte Wachstumsspirale der Anton Schlecker e.K. auf der einen Seite zwar zu einer flächendeckenden Expansion und einem lange Zeit auch hohen Gewinn des Gesamtunternehmens geführt. Doch auf der anderen Seite stagnierten in direkter Konsequenz der eingeschlagenen Expansionsstra-

688 Vgl. Schlecker (1996), Schlecker (1997), Schlecker (1998), Schlecker (1999); auch S010823LZ. 


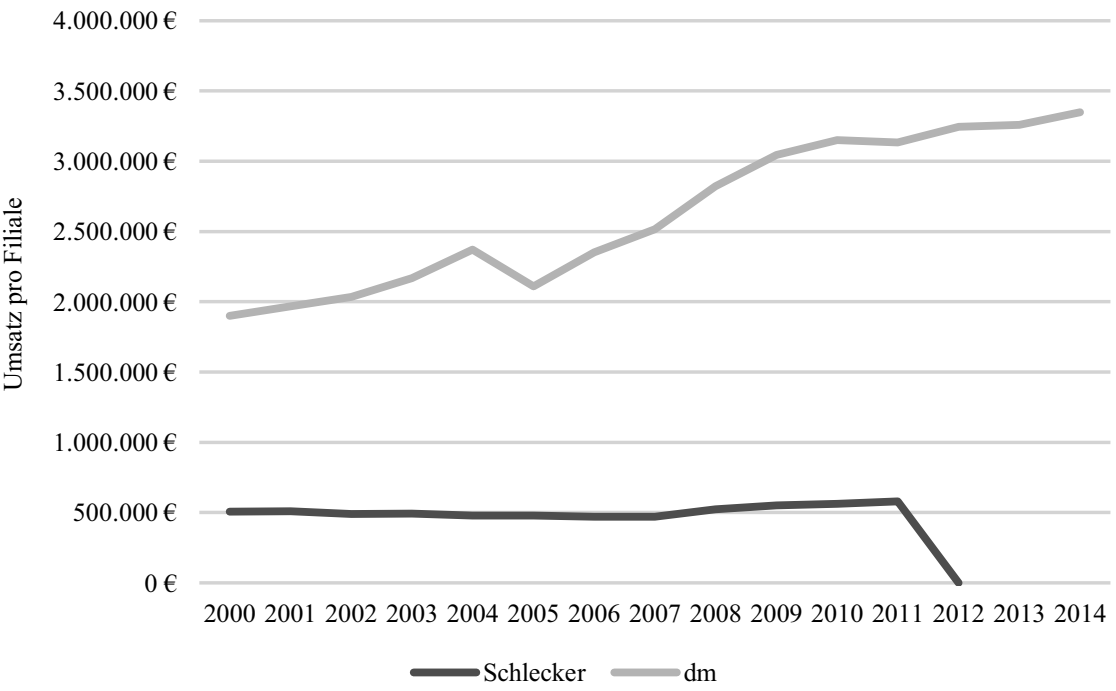

Abbildung 13: Entwicklung des Umsatzes pro Filiale Anton Schlecker e.K. und dm-Drogeriemarkt

(Quelle: Eigene Darstellung. Daten basierend auf Angaben von EHI).

tegie mit dem komplementären Vertriebstyp die Durchschnittsumsätze vieler Filialen auf einem im Branchenvergleich sehr niedrigen Niveau. ${ }^{69}$

Die Wachstumsspirale trieb das Unternehmen auf der Suche nach Expansionsmöglichkeiten immer weiter in städtische Randgebiete und kleine, entlegene Ortschaften. Nur hier ließ sich im etablierten strategischen Rahmen noch versuchen, jene Umsatzsteigerungen zu erzielen, an die viele der günstigen Konditionen im Einkauf geknüpft waren.

Allerdings waren in diesen Randlagen sowohl die Kundenfrequenz viel zu niedrig, als auch die Kaufkraft des Umfelds zu gering, um die branchenüblichen Umsätze pro Verkaufsstelle zu generieren. Hinzu kam, dass sich in der Spätphase der Expansion auch die Filialumsätze der einzelnen Filialen zu kannibalisieren begannen, weil sich die Verkaufsstellen nicht selten in zu großer Nähe zueinander befanden. ${ }^{690}$

Diese Umstände trugen maßgeblich dazu bei, dass im Jahr 2000 eine Schlecker-Filiale einen jährlichen Durchschnittsumsatz von lediglich ca. 900.000 DM erzielte. ${ }^{691}$ Dies war ungefähr ein Drittel dessen, was andere Filialsysteme in der

689 Vgl. S110304LZ zur Flächenproduktivität der vier führenden Drogeriemärkte im Jahr 2010; auch Statista (2016).

690 Vgl. S040220MM: „Statistisch gesehen gibt es hier zulande heute alle drei Kilometer eine Filiale, in manchen Einkaufsstraßen drängeln sich gleich zwei Märkte auf engstem Raum. Die Folgen dieser Kannibalisierung sind bereits spürbar."

691 Vgl. S010823LZ. 
Branche erreichten. 2004 erreichte eine dm-Filiale im Durchschnitt bereits gar das Sechsfache des Schlecker-Umsatzes pro Verkaufsstelle. Selbst in Anbetracht der deutlich größeren Standorte der Konkurrenz war die Flächenproduktivität der Anton Schlecker e.K. den Wettbewerbern somit zunehmend unterlegen. ${ }^{62}$

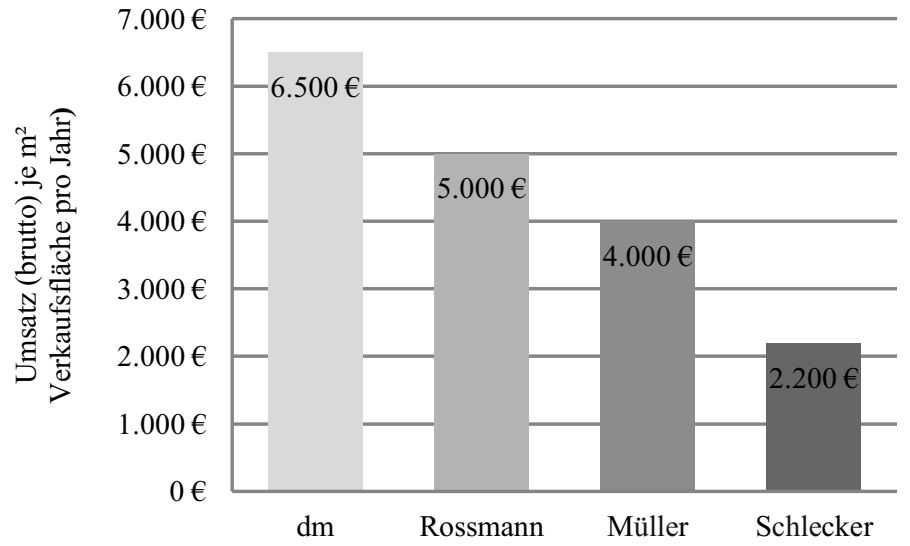

Abbildung 14: Vergleich der Flächenproduktivität der führenden Drogeriemarktunternehmen Deutschlands im Jahr 2010

(Quelle: Eigene Darstellung, Daten nach EHI).

Das etablierte strategische Muster führte jedoch nicht nur zu Tausenden von Filialen mit geringen Durchschnittsumsätzen und einer schwachen Produktivität, sondern auch zu sehr hohen Kosten des Gesamtfilialnetzes, die mit fortschreitender Expansion in einer zunehmend ungünstigen Relation zu besagten niedrigen Durchschnittsumsätzen standen.

So konnten durch die gezielte Einstellung von geringqualifizierten Frauen in ländlichen Gegenden mit einer schwachen Nachfrage am Arbeitsmarkt zwar die absoluten Personalkosten relativ gering gehalten werden. ${ }^{693}$ Doch in Bezug zu den geringen Umsätzen vieler Filialen führten die fixen Personalkosten zu relativen Personalkosten, die insgesamt weit über dem Branchendurchschnitt lagen. ${ }^{694}$

"Die Personalkosten laufen dem Branchenprimus davon, weil Schlecker sechs Filialen betreiben muss, um den vergleichbaren Umsatz einer einzigen DM-Verkaufsstelle zu erreichen. Oder anders ausgedrückt: Von jedem Euro Umsatz reicht er mehr als 21 Cent

692 Die Flächenproduktivität ist zu verstehen als das Verhältnis zwischen Erträgen bzw. Umsätzen und den eingesetzten Flächen; vgl. hierzu auch Statista (2016).

693 Ein Aspekt, aufgrund dessen die Anton Schlecker e.K. schon früh in die Kritik der Öffentlichkeit geriet.

694 Vgl. S041105HB. 
an die Mitarbeiter weiter, bei Rossmann sind es 14 Cent, bei DM elf und bei Aldi nicht einmal fünf.»

(Das Handelsblatt im Jahr 2004 zu den Kostenproblemen der Anton Schlecker e.K.) ${ }^{695}$

Zugleich verursachte das weitverzweigte Filialnetz hohe Logistikkosten. Jede der kleinen umsatzschwachen Verkaufsstellen in abgelegenen Ortschaften musste ganz genauso separat beliefert werden wie die umsatzstarke $1.000 \mathrm{~m}^{2}$ große Filiale eines Wettbewerbers in der Innenstadt. Daher musste die Anton Schlecker e.K. eine teure logistische Infrastruktur betreiben, die schlussendlich 17 große Regionallager allein in Deutschland umfasste. Zum Vergleich: Bei dm ist die Logistik bis zum heutigen Tage im Wesentlichen auf zwei große Verteilzentren konzentriert. ${ }^{696}$ Zudem resultierten aus den kleineren Liefermengen und den weiten Wegen höhere logistische Stückkosten. Diese waren teils gar so hoch, dass manche kleine Filiale nur zweimal pro Woche beliefert wurde. ${ }^{697}$

Viele Filialen konnten somit die aus der flächendeckenden Expansion resultierenden hohen Kosten hinsichtlich Personal oder Logistik nicht durch entsprechende Umsätze decken. Dies bedeutet, dass ein signifikanter und zunehmender Teil des Filialnetzes für die Anton Schlecker e.K. nicht rentabel war.

Mit dieser geringen Produktivität und fehlenden Rentabilität vieler Filialen hatte das Unternehmen nicht erst seit der Manifestation der Krise Ende der 2000er-Jahre zu kämpfen. ${ }^{698}$ Vielmehr wies schon zu Beginn der 1990er-Jahre eine Reihe an Filialen im Branchenvergleich niedrige Umsätze und eine geringe Produktivität auf. Angesichts dessen bezeichnete Götz Werner die Anton Schlecker e.K. bereits im Jahr 1994 in einem offenen Brief an die Herstellerindustrie als das »unproduktivste Unternehmen« der Branche:

695 Vgl. S041105HB; auch Werner (2013: 230); S070503LZ: IIm Vergleich der Personalkosten allerdings bleibt Schlecker abgeschlagen. Die Bilanz nennt 1,17 Mrd. Euro, das sind fast 19 Prozent vom Nettoumsatz." S092109LZ: »Aufgrund der vergleichsweise niedrigen Umsätze pro Filiale ist die Personalquote der Ehinger zuletzt auf über 20 Prozent gestiegen - und liegt damit rund doppelt so hoch wie bei den Wettbewerbern dm und Rossmann" [...] auch in den Schlecker-Kernfilialen versuchen die Ehinger nach Verdi-Angaben, die Tarifbindung zu unterlaufen Es werden zum Teil Gehälter von 6,5 Euro pro Stunde gezahlt, sagt Neumann. Das liege klar unter den Tarifen, die sich je nach Region zwischen knapp 7 Euro und 9,5 Euro bewegten."

696 Vgl. auch S041105LZ; S091119LZ.

697 Vgl. S120602HB: „Die Logistikkosten sind außer Kontrolle geraten. Schleckers Reich ist riesig, aber seine Filialen sind klein. Die weiten Wege für kleine Stückzahlen lassen jedes Shampoo, jede Zahnbürste teurer werden.« Vgl. S040220MM: "Kleinere Filialen werden zweimal pro Woche beliefert, die Kosten für die aufwändige Logistik würden sonst ausufern. Da kann es schon mal sein, dass Windeln über Tage ausverkauft sind.»

698 Bereits die Fleischwarenfabrik hatte eine verhältnismäßig hohe Anzahl von unrentablen Filialen. Eine damalige Führungskraft hierzu: »Also Filialabrechnungen und sämtliche Gehaltsrechnungen gemacht. Und dann hab ich schnell festgestellt, wenn ich acht Filialen oder neun hab, haben drei nicht schwarze Zahlen. Und das ist auch wieder etwas, was in der Schluss vielleicht mit meinen Ausführungen reinspiegelt, wo wir 5.000 Filiale hatten." 
»Werner rechnete nach und ab. Seine Reaktion: ein Rundschreiben an die führenden Lieferanten. Ihnen rechnete er die Zahlen des Wettbewerbers noch einmal genau vor. Seine Bilanz: ১Schlecker ist das unproduktivste Unternehmen der Branche überhaupt. Mit einem Umsatz von 4.800 Mark pro Quadratmeter Verkaufsfläche liege der Discounter weit unterhalb des Branchendurchschnitts. Mit einem >ständigen SchleckerKonditionsvorteil von mehr als fünf Prozent` förderten sie geradezu das Wachstum dieses >unproduktiven Unternehmensı."

(Die Zeitschrift »Der Handel« zum offenen Brief Götz Werners an die Lieferanten im Jahr 1994) $)^{699}$

\section{Steigende Abhängigkeit von den Konditionen im Einkauf}

Das etablierte strategische Muster führte somit auf der einen Seite zu einem größtenteils direkt aus der Expansionsstrategie resultierenden Produktivitätsund Kostennachteil. Auf der anderen Seite resultierten aus dem steten Wachstum aber so hohe Skalenerträge im Einkauf, dass die Kostennachteile über viele Jahrzehnte mehr als kompensiert werden konnten.

Die Wachstumsstrategie der Anton Schlecker e.K. zielte im Kern nie auf hohe Durchschnittsumsätze der einzelnen Filialen. Das Ziel war stets ein hoher und wachsender Gesamtumsatz des Unternehmens, der durch die Multiplikation der Verkaufsstellen gesteigert werden sollte. ${ }^{700}$

Die lange Zeit hohen Gewinne der Anton Schlecker e.K. resultierten zum einen aus der großen Summe der niedrigen Gewinne der vielen Filialen, zum anderen aber auch in nicht unerheblichem Maße aus betriebsfremden Erträgen aus Wertpapiergeschäften, die über den Cashflow finanziert wurden, als auch aus den von den Herstellern gewährten Boni und Zuschüssen. ${ }^{701}$

Da diese für das finanzielle Gesamtergebnis des Unternehmens maßgeblichen Konditionen in weiten Teilen ebenso an die gegenwärtige, wie an die zukünftige Höhe der Distributionsleistung gekoppelt waren, musste die Anton Schlecker e.K. allerdings auch stetig wachsen.

»'Erstklassige Einkaufskonditionen gibt es von der Industrie vor allem für Wachstum und nicht für rückläufige Umsätze`, verweist ein Industrie-Manager auf das Problem für Schlecker.»

(Die Lebensmittel Zeitung zu den an das Wachstum gekoppelten Einkaufskonditionen $)^{702}$

699 Vgl. den offenen Brief von Götz Werner 1994 an die Herstellerindustrie; auch S942106DH.

700 Vgl. S120401BE: "Die Masse sollte es machen - und dabei die Summe vieler kleiner Einheiten sein."

701 Vgl. S010823LZ; S041105HB.

702 Vgl. S040858LZ: Erstklassige Einkaufskonditionen gibt es von der Industrie vor allem für Wachstum und nicht für rückläufige Umsätze«, verweist ein Industriemanager auf das Problem für Schlecker.» Vgl. auch S050523LZ. 
Ohne die fortschreitende Expansion wäre nicht nur das gesamte Konditionengefüge, sondern das gesamte Geschäftsmodell mitsamt der Finanzierung ins Wanken geraten. ${ }^{703}$

»Verflucht zum Wachstum. Immer mehr, immer bessere Konditionen von Lieferanten und damit noch irgendwie versuchen, einen Ertrag zu erwirtschaften. Genau." (Der Insolvenzverwalter Arndt Geiwitz) ${ }^{704}$

Innerhalb dieses Systems war es der Anton Schlecker e.K. beinahe unmöglich, unmittelbar und in größerem Stil unrentable Filialen zu schließen, denn mit der Aufgabe unrentabler Filialen hätte sie nicht nur auf deren Umsätze verzichten müssen, sondern auch auf die mit ihnen verbundenen Konditionenvorteile.

"Sie haben sehr viele Konditionen im Handel, die von der Umsatzgröße abhängen. Es gibt Konditionen, die im Wesentlichen auch durch positive Umsatzveränderungen erst gezahlt werden, und wenn Sie so eine Restrukturierung machen, das geht ja über Jahre, dann buchen Sie natürlich auch Umsätze bei ganz starken Herstellern aus. Und die werden das nicht honorieren [...] da verlieren Sie."

(Ehemalige Führungskraft der Anton Schlecker e.K.) ${ }^{705}$

Auf diese Konditionenvorteile war die Anton Schlecker e.K. aufgrund der mit der Expansionsstrategie einhergehenden niedrigen Produktivität in zunehmendem Maße angewiesen. Daher setzte das Unternehmen die Expansion auch dann noch fort, als es für sie mit dem bestehenden Betriebstyp kaum noch rentable Investitionsmöglichkeiten gab.

Es hatte somit einen Entwicklungsweg eingeschlagen, den es immer schwerer verlassen konnte. Je weiter das Unternehmen im etablierten strategischen Muster expandierte, desto schwieriger wurde es, vom eingeschlagenen strategischen Entwicklungsweg abzuweichen. Im Sinne eines graduellen Verständnisses von Pfadabhängigkeit verlief die strategische Entwicklung des Unternehmens somit bereits ab Mitte der 1990er-Jahre immer "pfadabhängiger«.

Den Zustand des Lock-in erreichte das Unternehmen nach der Jahrtausendwende. In diesen Jahren stieß erstens der eingeschlagene Wachstumspfad an seine Sättigungsgrenzen:

»Und das Geschäftsmodell ist hoch fragil - trotz hoher Eigenkapitalbasis und nur weniger Bankschulden. Denn Schlecker stößt allmählich an Sättigungsgrenzen. 10550

703 Einige wenige Marktbeobachter meinten daher schon früh ein riskantes Schneeballsystem erkannt zu haben; vgl. zu dieser Einschätzung Werner (2013); auch S940621DH.

$704 \mathrm{Vgl}$. S041105HB: „Schlecker ist zum Wachstum verdammt«, formuliert es ein Berater; auch S041005LZ: "Das System Schlecker, zum Wachstum verdammt."

705 Vgl. S090129LZ: „Denn bezogen auf die gesamte Distributionsleistung für die Markenartikelindustrie der Branche ist Schlecker nach wie vor führend [...]. Nur deshalb schleppt er den vermeintlichen Ballast von tausenden Kleinfilialen mit sich, die gerade mal 70 Euro pro Artikel pro Jahr umsetzen. Da fressen alleine die Personalkosten die Spanne auf.» 
Filialen betreibt er allein in Deutschland; vor acht Jahren noch sah Schlecker die ökonomisch sinnvolle Obergrenze bei 8000 Läden. Statistisch gesehen, gibt es hier zu Lande heute alle drei Kilometer eine Filiale, in manchen Einkaufsstraßen drängeln sich gleich zwei Märkte auf engstem Raum. Die Folgen dieser Kannibalisierung sind bereits spürbar. Im Geschäftsjahr 2002 sank der durchschnittliche Umsatz pro Filiale laut Marktforschungsinstitut M+M Eurodata von 531000 auf 504000 Euro. Das Betriebsergebnis soll Insidern zufolge um zwei Drittel eingebrochen sein. Mit neuen Filialen, so ein Branchenkenner, setze Schlecker nurmehr halb so viel um.«

(Die Lebensmittelzeitung im Jahr 2006) 706 $^{70}$

Zweitens verschärfte sich der Wettbewerb durch die zunehmende Expansion der qualitativ überlegenen und Ende der 1990er-Jahre schnell gewachsenen Konkurrenten dm und Rossmann, auf welche die Industrie seit 2004 als alternative bundesweite Distributionskanäle zurückgreifen konnte.

»Ich mein, das hast du ja gemerkt, wenn, wenn hier ein Schlecker war, gegenüber hat ein DM aufgemacht, dann haben wir einfach 50 Prozent vom Umsatz verloren, wenn es reicht. Und so ging's halt an jedem Standort, und nachdem der DM und Rossmann halt auch Hunderte von Filialen aufgemacht haben, hat es jedes Mal den Schlecker getroffen. Der hatte das schlechtere Konzept [...] wenn einer so schwach ist und du setzt nur dein Konzept für die Masse. Er hatte dem nichts entgegenzusetzen.«

(Ehemalige Führungskraft der Anton Schlecker e.K.)

Das Unternehmen hatte Anfang des Jahrtausends kaum mehr strategischen Handlungsspielraum:

"Schlecker ist zum Handeln gezwungen, doch die Möglichkeiten scheinen begrenzt. Bei rund 10550 Filialen werden die Standorte in Deutschland rar. Unmittelbar neben dm-, Rossmann- oder Müller-Filialen tun sich die Ehinger ausgesprochen schwer. Der Gang in kleine Ortschaften bringt aber auch Probleme mit sich und treibt angesichts fehlender Umsätze die Personalkosten in die Höhe: `Schlecker kann eigentlich nur noch bei den Sortimenten und den Öffnungszeiten reagieren`, schlussfolgert ein Handelsmanager."

(Die Lebensmittel Zeitung zur »Zwickmühle« der Anton Schlecker e.K.) ${ }^{707}$

\section{Umkehrung der Wachstumsspirale}

Der noch verbleibende Handlungsspielraum schrumpfte Ende 2003 nochmals dramatisch. Denn als aufgrund des Erreichens der Sättigungsgrenze und des sich verschärfenden Wettbewerbs in diesem Zeitraum zum ersten Mal die Gesamtumsätze der Anton Schlecker e.K. einbrachen, verkehrte sich die einstmalige Wachstumsspirale in ihr Gegenteil. ${ }^{708}$

706 Vgl. S060819LZ.

707 Vgl. S040520LZ.

708 Vgl. S041105HB: "[J]etzt stößt das zügellos wuchernde Imperium offenbar erstmals an seine Grenzen, der Drogeriemarkt ist weitgehend gesättigt.« 
Aufgrund der schwächeren Distributionsleistung begannen die Lieferanten die Konditionen dem sinkenden Marktanteil anzupassen: Sie erhöhten die Einkaufspreise, kürzten Rabatte und Zuschüsse und nahmen die langen Zahlungsfristen wieder zurück ${ }^{709}$

")Ohne Wachstum keine Rabatte`, formuliert es ein Lieferant. DM und Rossmann legen zweistellig zu, erzielen die besseren Konditionen und treiben Schlecker im Preiskampf vor sich her."

(Die Lebensmittel Zeitung zu den Einkaufskonditionen von Schlecker) $)^{710}$

Die ungünstigeren Konditionen schlugen sich umgehend und immer stärker im Ergebnis des Unternehmens wieder.

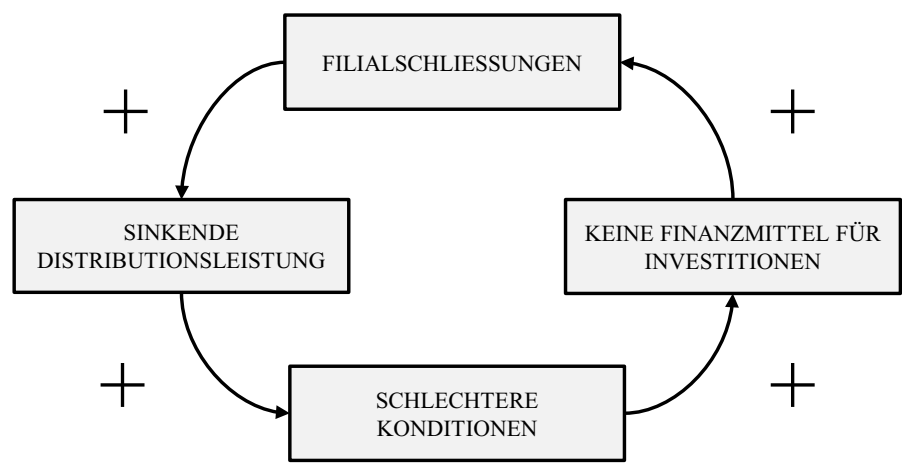

Abbildung 15: Umkehrung der Wachstumsspirale der Anton Schlecker e.K. (Quelle: Eigene Darstellung).

Ebenso wie die Konditionen Schleckers sich mit zunehmender Distributionsleistung überproportional verbessert hatten, verschlechterten sie sich nun bei rückläufigen Umsätzen.

»Ab jetzt verkürzten die Hersteller ihre Zahlungsziele, erhöhten die Preise. Damit geriet Schleckers ganzes System ins Wanken. Seine Lieferanten waren seine Bank. Jetzt reduzierten sich praktisch seine Kreditlinien. Bei Schlecker wurde es finanziell eng. Ein

709 Vgl. S100924LZ »Die Industrie kündigt an, sie wolle Schlecker die rückläufige Entwicklung in den Jahresgesprächen spüren lassen. Schlecker fordert hingegen eine Konditionenverbesserung um mindestens weitere 5 Prozent."

710 Vgl. S080325LZ; S100924LZ: „Die Industrie will bei den Jahresgesprächen Widerstand gegen Schlecker leisten. Die Verschiebungen im Markt müssten sich auch in den Konditionen widerspiegeln, heißt es zur Begründung. Die Stärke der Konkurrenz bei gleichzeitiger Schwäche des Marktführers sorgt für neues Selbstbewusstsein auf Seiten der Industrie. "Wir sind nicht mehr in dem Maße abhängig von Schlecker, wie das noch vor Jahren der Fall warı, verdeutlicht der Manager eines Markenartiklers.» 
Direktor erzählt: > Ab 2004 haben wir nur noch Preise erhöht, um uns halten zu können`.» (Das Handelsblatt zu den Wendejahren 2003 und 2004) $)^{711}$

Die Anton Schlecker e.K. reagierte auf diese Umweltdynamik innerhalb des etablierten strategischen Musters. Sie versuchte auf der einen Seite noch härter $\mathrm{zu}$ verhandeln und auf der anderen Seite wieder $\mathrm{zu}$ wachsen. So wurden nun auch in Ortschaften mit 1.500 Bewohnern Filialen mit $150 \mathrm{~m}^{2}$ eröffnet. Auf diesem Wege akquirierte das Unternehmen allein 2004 nochmals 400 Standorte. ${ }^{712}$ Zudem wurde verstärkt versucht über Zukäufe wie die Übernahme von Ihr Platz Wachstum zu erreichen. ${ }^{713}$

" Das Thema Masse ist in Deutschland fast ausgereizt. Jetzt kann er nur über eine bessere Qualität gehen oder sein System im Ausland multiplizieren`, schätzt ein Industriemanager."

(Die Lebensmittel Zeitung zu den verbliebenen Handlungsspielräumen der Anton Schlecker e.K. im Jahr 2004) $)^{714}$

Parallel wird die Expansion ins Ausland forciert. Wie jedoch oben bereits umrissen wurde, fehlten hier im Rahmen des etablierten Geschäftsmodells die günstigen Einkaufskonditionen für ein dynamisches Wachstum und einen erfolgreichen Betrieb. ${ }^{715}$

Die strategischen Reaktionen zeigen, dass der Anton Schlecker e.K. nur noch wenige Handlungsoptionen verblieben waren. Da die mit Zukäufen und Auslandsexpansion erzielten Umsatzsteigerungen durch die Einbrüche auf der Altfläche in kürzester Zeit wieder verloren gingen, verschlechterten sich die Konditionen weiter und die relativen Kosten des Filialnetzes erhöhten sich. ${ }^{716}$ Die Rentabilität nahm dramatisch ab.

So fehlten der Anton Schlecker e.K. irgendwann die finanziellen Mittel für einen wirklichen strategischen Wandel, wie er 2010 mit dem Programm »Fit for Future« skizziert wurde. Für einen solchen Wandel hätte es nicht nur des Aus-

711 Vgl. S120602HB.

712 Vgl. S050331LZ: "Die Absicht, an neuen Standorten Filialen mit mehr als $200 \mathrm{qm} \mathrm{zu}$ eröffnen, ließ sich bislang nur begrenzt verwirklichen. Gut zwei Drittel der Neueröffnungen von 2004 sind kleiner als $200 \mathrm{qm}$ «.

713 Obwohl diese Akquisition in der Öffentlichkeit als Beginn einer Zwei-Marken-Strategie kommuniziert wurde, diente sie in erster Linie dazu, das Einkaufsvolumen nach langer Zeit wieder substanziell zu steigern und somit die verbliebenen Konditionen legitimieren zu können. Vgl. S071004LZ; S071004FZ.

714 Vgl. S040819LZ.

715 Vgl. S040819LZ.

716 Vgl. S110616LZ: „Nun aber wendet sich das Blatt. Vor allem die für Schlecker typischen Fixbeträge bei den Werbekostenzuschüssen werden zum Stein des Anstoßes [...]. Posten wie der `Regalsicherungs-^ oder `Listungszuschuss` sind letztlich an der Platzierung der Produkte in vormals 10.000 Schlecker-Filialen orientiert - heute sind es noch 7.200. Vgl. auch S120126LZ; S120601LZ. 


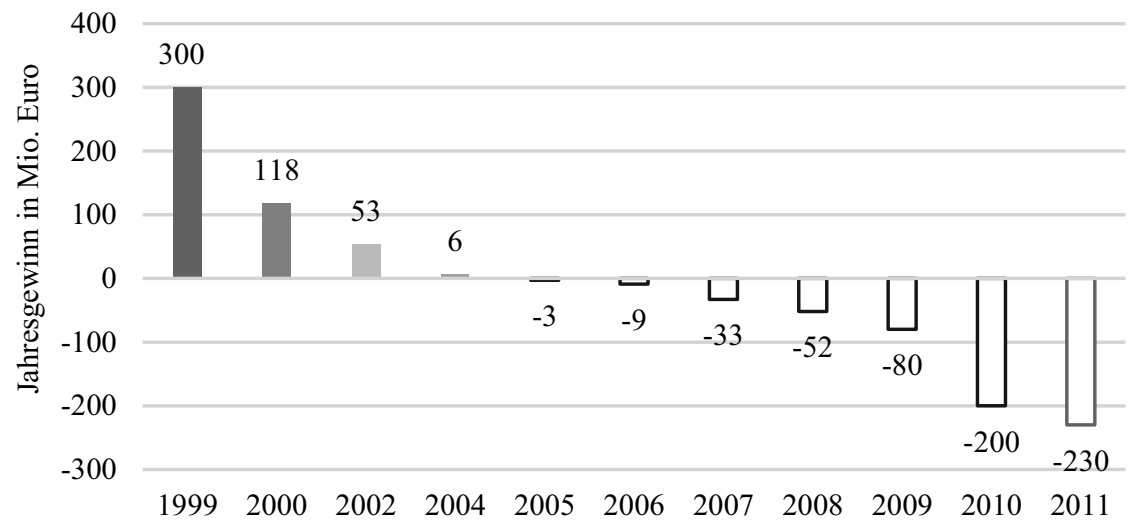

Abbildung 16: Gewinnentwicklung der Anton Schlecker e.K. in den Jahren 1999-2011 (Quelle: Eigene Darstellung auf Grundlage von Daten aus Geschäftsberichten der Anton Schlecker e.K.).

tauschs einiger Hundert Filialen bedurft. Vielmehr hätten bei einem Bestand von über 10.000 Filialen allein in Deutschland Tausende Altfilialen geschlossen werden müssen. Dies war im Rahmen eines Geschäftsmodells, das auf ebendieser Größe und Expansion beruhte, ab einem gewissen Zeitpunkt kaum noch möglich. ${ }^{717}$ Die Anton Schlecker e.K. wurde hier schlussendlich Opfer ihres auf den selbstverstärkenden Mechanismen basierenden Erfolgs. ${ }^{718}$

Als dann 2008 mit Schlecker XL eine Modifikation des bisherigen Vertriebskonzeptes eingeführt wurde, fehlten bereits die Ressourcen um diese Betriebstypenvariante am Markt zu etablieren.

"')etzt weiß ich nicht, ob das damals schon ein Investitionsproblem war, ne. nicht hergehen konnte, um zu sagen: ১Jetzt dürfen wir keine Filiale mehr eröffnen, die nicht 500 Quadratmeter groß ist, die hell ist, die gespiegelt ist, die personell entsprechend ausgestattet ist. I Ich glaub, da war es schon zu spät. Wir sind da schon einbetoniert gewesen."

(Ehemalige Führungskraft)

Der Anton Schlecker e.K. verblieb also nur noch die Möglichkeit den eingeschlagenen strategischen Entwicklungsweg zu Ende zu gehen. Sie war zur Reproduktion des strategischen Musters gezwungen und auf dem strategischen Pfad gefangen.

717 Vgl. S081030LZ: "Deshalb wird es dabei bleiben: Schlecker schließt hier ein paar hundert Märkte, tauscht dort einige Dutzend Kleinflächen gegen größere Standorte aus. Und hofft, dass die Industrie die flächendeckende Distribution weiterhin ordentlich honoriert.«

718 Vgl. S120126LZ: Schlecker »[...] wurde zum Opfer des Erfolgs seines ausschließlich und unumkehrbar auf Multiplikation und Wachstum getrimmten Modells«. 
»Zwang zum Weitermachen: Anton Schlecker ist - und das ist für den ambitionierten Unternehmer wohl das schmerzlichste [sic!] an der aktuellen Lage - buchstäblich in der Zwickmühle. Denn ihm bleibt trotz der unerquicklichen Zustandsbeschreibung dennoch nichts anderes übrig als weiterzumachen. Generell in ein attraktiveres Geschäftsmodell mit größeren Flächen zu wechseln, ist kaum praktikabel, wenn das Gros der Märkte unter 200 qm liegt. 7700 Märkte kann man nicht schließen. Schlecker weiß das und akquiriert weiter Märkte in Ortschaften ab 1500 Einwohner. Und hofft, dass der Wettbewerb ihm dort genug Spielraum lässt und die Industrie die flächendeckende Distribution inklusive Neueinführungen weiter ordentlich honoriert.»

(Die Lebensmittel Zeitung im Jahr 2005 zum strategischen Lock-in des Unternehmens)

Der empirische Verlauf der Pfadgenese und Krisenentstehung zeigt jedoch, dass die Anton Schlecker e.K. diesen strategischen Wandel trotz der wirkmächtigen selbstverstärkenden Mechanismen bis zu einem gewissen Punkt hätte vollziehen können.

$»[\ldots]$ in so einem Filialsystem haben Sie mal zehn, fünfzehn Prozent Filialen, wo man nicht richtiges Geld verdient. Und wenn Sie diese Grenze mal erreicht haben, dann müssen Sie handeln. Das geht normalerweise mit einer ganz normalen Fluktuation. Hinten abschneiden und vorne wieder aufmachen. Aber bitteschön nach einem neuen Konzept. So, wie man es ganz am Schluss, dann 2011 auch gemacht hat.[...] zehn Jahre zu spät, aber wenn es damals schon so gewesen wäre, bin ich überzeugt, dass es den Schlecker auch noch in 30 Jahren gegeben hätte. Denn wir hatten auch ganz enorme strategische Vorteile. Aber, dass dieser Wettbewerb gekippt ist, das war in $2000 \mathrm{ab}-$ sehbar. Weil einfach dieser Fundus an schwachen Filialen, der wurde jetzt so erdrückend, dass das eben Geld gekostet hat."

(Ehemalige Führungskraft der Anton Schlecker e.K. zum ausbleibenden strategischen Wandel $)^{719}$

Der empirische Verlauf der Pfadgenese wirft somit die Frage auf, warum eine solche strategische Reaktion in der Phase der Pfadformation unterblieb. Dieser Frage wird in Teil D unter Berücksichtigung des Faktors Familie nachgegangen.

Zuvor rückt mit der Berentzen-Gruppe AG jedoch noch der empirische Repräsentant der zweiten Fallgruppe in das Zentrum des Interesses.

\subsection{Fallstudie zur Fallgruppe II: Berentzen-Gruppe AG}

Die Berentzen-Gruppe AG ist ein mittelständisches Unternehmen der Getränkeindustrie, dessen geschäftlicher Schwerpunkt auf der Herstellung und dem

719 Vgl. hierzu auch die Einschätzung eines ehemaligen Beraters der Anton Schlecker e.K.: »Und ich sag mal ab '95, im Grunde ab '95 hätte man anfangen können mal drüber zu reflektieren, ist das eigentlich noch das Zukunftsmodell, das wir haben. Und da wurde, also da wurde, einfach fleißig weiter multipliziert.« 
Vertrieb von Spirituosen, sowie alkoholfreien Getränken liegt. Neben den traditionellen Produkten des Unternehmens wie dem Berentzen-Apfelkorn werden heute Marken wie Puschkin, Bommerlunder, Licor 43 und Sinalco vertrieben. Während in den 1990er-Jahren zeitweise ein Umsatz von 350 Mio. Euro erzielt werden konnte, erwirtschafteten im Jahr 2014491 Mitarbeiter einen Umsatz von 158 Mio. Euro. ${ }^{720}$ Nach mehr als 250 Jahren in der Hand der Familie Berentzen befindet sich die Berentzen-Gruppe seit 2008 unter der Kontrolle des Finanzinvestors Aurelius AG.

\subsubsection{Historische Entwicklung des Unternehmens und dessen Branchenumwelt}

\section{Vorspann}

Die Entwicklung der späteren Berentzen-Gruppe beginnt mit der Gründung einer kleinen Schmiedewerkstatt, in der zugleich auch destilliert wird, im Jahre $1758 .^{721}$ Von da an wird die Familie Berentzen 250 Jahre lang über viele Generationen hinweg im emsländischen Haselünne Korn brennen. ${ }^{722}$ Der Betrieb ist stets lokal orientiert, baut sich im Laufe vieler Jahrzehnte aber in der ganzen Region einen guten Ruf auf. Diesen lässt er sich bereits 1898 auch markenrechtlich schützen. Neben ihrer ökonomischen Aktivität ist die Familie Berentzen in Haselünne stets politisch und gesellschaftlich engagiert und stellt immer wieder Ratsherren und Bürgermeister. ${ }^{723}$

In der Nachkriegszeit beginnt der Betrieb dann unter der kaufmännischen Führung von Johannes (»Hans«) Berentzen und der technischen Leitung von Friedrich Berentzen nach und nach zu wachsen. Die beiden Brüder hatten 1954 nach dem Tod des Vaters den Betrieb übernommen, der in diesem Geschäftsjahr einen Umsatz von 4 Mio. DM verzeichnete. ${ }^{724}$

1958 gelingt der Einstieg in den Vertrieb alkoholfreier Getränke und 1960 kann gar eine regionale Pepsi-Konzession erworben werden. ${ }^{725} 1969$ beträgt der Umsatz J. B. Berentzen dann bereits 26,7 Mio. DM; 1975 sogar 49,2 Mio. DM. Der

720 Vgl. Berentzen (1998) und Berentezen (2015). Angaben wie Umsatz-, Gewinn- oder Mitarbeiterentwicklung sind größtenteils den veröffentlichten Geschäftsberichten der Berentzen-Gruppe AG entnommen und werden in der Regel nur bei abweichenden Quellen separat belegt.

721 Vgl. Berentzen (1981: 1).

722 In Haselünne, der "Stadt der Kornbrenner«, gibt es zahlreiche weitere Brennereien. Vgl. Berentzen (1981: 5).

723 Vgl. Berentzen (1981: 11f.); auch zur markenrechtlichen Sicherung des »Berentzen vom alten $\mathrm{Fa} ß$.

724 Vgl. Berentzen (1981: 32).

725 Vgl: Berentzen (1981: 32) zur Gründung der Emsland-Getränke GmbH. 
Großteil des Umsatzes wird dabei mit dem Traditionsprodukt Berentzen-Korn erwirtschaftet.

Der 1857 gegründete Spirituosenhersteller Eckes setzt in diesen Jahren ebenfalls zum großen Sprung an. So wird 1953 der Weinbrand Eckes Chantré eingeführt, welcher den Umsatz innerhalb eines Jahres auf 9 Mio. DM verdreifacht. ${ }^{726}$ Durch diesen Erfolg wird das Unternehmen unter der kaufmännischen Leitung von Ludwig Eckes und dessen Vetter Peter Eckes, der den technischen Bereich betreut, zum Marktführer in diesem Segment und verzeichnet 1957 bereits einen Umsatz von 100 Mio. DM. ${ }^{727}$

Ein Jahr später erweitert die Eckes AG das 1931 begründete Geschäft mit Fruchtsäften um den Orangensaft hohes C. Das Produkt hat sofort Erfolg am Markt und avanciert zum Marktführer bei den Orangensäften. Dennoch wächst das Unternehmen in den folgenden Jahrzehnten vor allem dank umsatz- wie margenstarker Spirituosen wie dem 1961 eingeführten Weinbrand Mariacron oder dem Kirschlikör Eckes Edelkirsch. ${ }^{728}$

Das Florieren der beiden Spirituosenhersteller Berentzen und Eckes wird in den Nachkriegsjahrzehnten von einem wachsenden Markt getragen. Mit dem wachsenden Einkommen der Bevölkerung steigt auch der Pro-Kopf-Verbrauch an Spirituosen: von 1960 bis 1976 von 4,2 l pro Kopf um rund $95 \%$ auf 8,2 1 pro Kopf. $^{729}$

Mit diesem Wert ist allerdings der Höhepunkt erreicht und in den folgenden Jahren geht der Konsum an Spirituosen kontinuierlich zurück. Innerhalb weniger Jahre reduziert er sich bis 1982 um beinahe $20 \%$ auf 6,8 1 pro Kopf. Ausschlaggebend für diesen Rückgang sind zunächst die mehrfachen Erhöhungen der Branntweinsteuer, welche die Verschiebung gesellschaftlicher Normvorstellungen in Richtung einer gesünderen Lebensweise und eines bewussteren Umgangs mit Alkohol reflektieren. ${ }^{730}$

Dennoch beginnt für die J. B. Berentzen nun erst die Zeit des ganz großen Wachstums: Mit dem »Apfelkorn« gelingt dem Unternehmen 1976 die erfolg-

726 Vgl. Eckes (2007: 21).

727 Neben Ludwig und Peter Eckes ist in der vierten Generation der Unternehmensfamilie noch ein weiterer Vetter eines dritten Stammes im Unternehmen aktiv. Dieser Stamm scheidet nach »langen unerfreulichen Auseinandersetzungen« jedoch 1955 aus dem Unternehmen aus und wird ausbezahlt. Vgl. Eckes (2007: 21).

728 Vgl. Eckes (2007: 25ff.). Der Mariacron ist 1974-1990 Marktführer bei den Weinbränden.

729 Vgl. die Übersicht B130621 des BDS zum jährlichen Verbrauch von Spirituosen im Zeitraum 1960-2013.

730 Vgl. Brendel \& Oberender (1981: 114), denen zufolge die Steuer 1966-1981 um 87,5 \% stieg. Die Abkehr vom Alkohol zeigt sich nicht nur im Bereich der Spirituosen, sondern spiegelt sich auch im Anteil alkoholischer Getränke am Gesamtgetränkekonsum: Dieser Anteil lag 1970 nach Brendel \& Oberender (1981: 125) noch bei $32 \%$, 2014 nur noch bei $17 \%$. Vgl. Statista (2016). 
reichste Markteinführung einer Spirituose nach dem Zweiten Weltkrieg und innerhalb von nur vier Jahren kann der Absatz von 2,5 Mio. Flaschen 1976 auf 25,2 Mio. Flaschen verzehnfacht werden. Damit ist der Apfelkorn 1979 die zweitmeist verkaufte Spirituose in Deutschland und wird bald auch ins Ausland exportiert. $^{731}$

In den folgenden Jahren wächst das Unternehmen dank des Erfolgs dieses Produkts und trotzt so dem Marktumfeld. ${ }^{732}$ Dieses wird immer schwieriger, da zum einen der Spirituosenkonsum rückläufig bleibt, zum anderen vor allem die traditionellen deutschen Hersteller unter übermächtiger internationaler Konkurrenz leiden. Seit der EG-Liberalisierung 1973 drängen um ein Vielfaches größere Unternehmen wie Pernod Ricard, Campari oder Bacardi mit starken Marken internationaler Strahlkraft auf den deutschen Markt, der auf Herstellerseite noch stark fragmentiert und mittelständisch geprägt ist. In der Folge konsumieren die deutschen Verbraucher immer häufiger internationale Markenprodukte und greifen nur noch in den unteren Preislagen zu klassischen deutschen Erzeugnissen wie Korn. Dieser Entwicklung können die vielen kleinen, traditionsreichen Hersteller mit ihren meist nur regional bekannten Marken wenig entgegensetzen. ${ }^{733}$ So fällt die deutsche Spirituosenbranche hinsichtlich Internationalisierung und Export immer weiter hinter die internationalen Konkurrenten zurück: Während im Zuge der Liberalisierung des EGBinnenmarktes der Import von Spirituosen um 73 \% auf 1,155 Mio. hl im Jahr 1976 ansteigt, können lediglich 0,059 Mio. hl, also 5 \% der importierten Menge, in andere Länder exportiert werden. ${ }^{734}$ Insbesondere der deutschen "Nationalspirituose», dem Kornbrand, gelingt der Durchbruch im Ausland nicht.

Zugleich führt die immer höhere Konzentration im LEH durch die wachsende Marktmacht der Handelsunternehmen zu einem zunehmenden Preisdruck, welcher durch das immer stärkere Aufkommen der Handelsmarken weiter verstärkt wird. ${ }^{735}$

Diesen Entwicklungen kann sich auch der Marktführer Eckes nicht entziehen. Obwohl ein Jahr zuvor zum ersten Mal die Umsatzgrenze von 1 Mrd. DM überschritten werden konnte, werden 1982 aufgrund von Umsatzrückgängen und der insgesamt "unbefriedigenden Geschäftsentwicklung» gar die Feier-

731 Vgl. Brendel \& Oberender (1981: 129); B801110SP.

732 Der Pro-Kopf-Konsum von Spirituosen sinkt nach B130621WS von 8,0 11980 um 22,5\% auf $6,211987$.

733 Vgl. Berekoven (1981: 31f.); Brendel \& Oberender (1981: 112) zu den Ursachen der Fragmentierung.

734 Vgl. Brendel \& Oberender (1981: 122). Zu den Daten: Berekoven (1981: $31 \mathrm{f}$ )); Brendel \& Oberender (1981: 127).

735 Im Zeitraum 1960-1975 geht die Anzahl der Lebensmitteleinzelhandelsgeschäfte um mehr als die Hälfte zurück, vgl. Berentzen (1981: 43). 
lichkeiten zum 125jährigen Firmenjubiläum auf das Jahr 1983 verschoben. ${ }^{736}$ Dieses Jubiläum wird von der mittlerweile fünften Generation der Unternehmensfamilie begangen, die nach organisationalen Veränderungen wie der Etablierung einer Familienstiftung, der Gründung eines Beirats und der Etablierung eines familienfremden Managements 1974 in die Geschäftsführung eingetreten war. $^{737}$

Bei der J. B. Berentzen stehen ebenfalls Veränderungen an: Im Rahmen des schwierigen Marktumfeldes fusioniert Berentzen 1988 mit dem auf Handelsmarken spezialisierten Hersteller Pabst \& Richarz, der sich nach mehreren Zusammenschlüssen und Übernahmen im Eigentum der Familien Pabst, Richarz und Wolf befindet. ${ }^{738}$ Durch diese Fusion entsteht die Berentzen-Gruppe, die mit einem kumulierten Umsatz von ca. 500 Mio. DM nun auf einen Schlag eines der größten Spirituosenunternehmen Deutschlands ist. ${ }^{739} \mathrm{Zu}$ einem Zeitpunkt, zu dem der kleinste der zehn führenden internationalen Konzerne immer noch drei- bis viermal so groß wie der größte deutsche Hersteller ist, soll die Gruppe eine »deutsche Antwort auf die internationale Herausforderung sein «. ${ }^{740}$

»Die neue J. B. Berentzen GmbH \& Co.« mit Sitz in Haselünne gehört nach vollzogener Fusion am 1. Januar 1988 mit einem Absatz von fast 60 Millionen Flaschen und einem Umsatz von knapp 500 Millionen DM [...] zu der von Eckes angeführten Spitzengruppe der Branche."

(Das Handelsblatt im Jahr 1987 zu der Fusion von Berentzen und Pabst \& Richarz) ${ }^{741}$

Das Eigentum an der neuen Gesellschaft wird in einem Verhältnis von 56:46 für Berentzen zwischen den Eigentümergruppen der beiden Unternehmen aufgeteilt und die Organe im Unternehmen allesamt paritätisch besetzt. ${ }^{742}$ Der Vorsitz der Geschäftsführung wird von Dr. Hans Berentzen ausgeübt, aber mit Jan B. Berentzen und Jürgen Pabst rücken zugleich auch Vertreter der jungen Gene-

736 Vgl. Eckes (2007: 32). Die Umsatzschwelle von 1 Mrd. DM wird erst 1987 wieder erreicht. 737 Harald Eckes-Chantré ist der Sohn von Ludwig Eckes; Peter Eugen und Michael sind die Söhne von Peter Eckes. Vgl. Eckes (2007: 71) und die Übersicht im Anhang. Ludwig Eckes scheidet 1973 aus, Peter Eckes 1977.

738 Vgl. B870909NZ; auch ein ehemaliger Vorstand: "noch ein weiteres Familienunternehmen, und die, die hatten auch wieder drei Stämme, Pabst, Richarz und Wolff«.

739 Vgl. B870808NZ; B970908HB; B870908NZ.

740 Vgl. B870808NZ; B970908HB; B870908NZ.

741 Vgl. B80909HB.

742 Vgl. B870909NZ; auch B870908NZ: »Stellvertretender Vorsitzender ist Carl Maria Richarz, der Rechnungs- und Personalwesen verantwortet. Als weiterem stellvertretendem Vorsitzenden obliegen Friedrich Berentzen die Bereiche Produktion, Forschung und Entwicklung. Hans Wolff führt den Gesamtbereich Handelsmarken und lose Ware. Dr. Jan-Bernd Berentzen ist zuständig für den Markenvertrieb In- und Ausland und Jürgen Pabst für nationales und internationales Marketing." 
rationen in den Vorstand auf. Hinsichtlich des Unternehmenssitzes wird sich darauf verständigt, dass dieser im traditionsreichen Haselünne verbleibt.

Nach der Fusion wird das Wachstum der Berentzen-Gruppe stark forciert. So erfolgt 1988 die Übernahme des regional tätigen Herstellers Ammerländer. 1989 dann ein Mehrheitserwerb an der König \& Schlichte GmbH samt deren Traditionsmarke Schinkenhäger und der Wodka-Marke Puschkin. Die erweiterte Gruppe rückt damit neben Eckes und Underberg in die Spitzengruppe der größten deutschen Spirituosenhersteller auf. ${ }^{743} 1990$ übernimmt die BerentzenGruppe dann die Mehrheit der Dornkaat AG, dem Marktführer im Bereich Korn, welcher 1992 komplett übernommen wird. Im Zuge all dieser Übernahmen steigt der konsolidierte Gruppenumsatz innerhalb von fünf Jahren von unter 500 Mio. um 80 \% auf rund 900 Mio. DM im Jahr $1992 .{ }^{744}$ Dies wird nicht zuletzt befeuert vom Boom der Wiedervereinigung.

Diese öffnet auch für Eckes die Möglichkeit zu Expansion und Wachstum im Osten Deutschlands. 1990 wird mit der Nordbrand Nordhäuser der größte Produktionsbetrieb der ehemaligen DDR übernommen. ${ }^{75}$ Zugleich wird das Unternehmen in eine AG umgewandelt und in zwei strategisch eigenständig agierende Geschäftsbereiche aufgeteilt, die danach beide florieren: Während in der Alkoholsparte 1993 zum Beispiel mit dem Weinbrand Chantré im Gefolge der Wiedervereinigung ein neuer Absatzrekord aufgestellt werden kann, übernimmt die Fruchtsaftsparte 1994 die Mehrheit an der Firma Granini, die im Gegensatz zu den deutschen Spirituosen- und Fruchtsaftmarken bereits international vertreten ist ${ }^{746}$ In den folgenden Jahren wird die Fruchtsaftsparte durch weitere Übernahmen und Beteiligungen kontinuierlich ausgebaut. ${ }^{747}$

Parallel zu diesen Veränderungen im strategischen Portfolio von Eckes finden organisationale Veränderungen statt. So scheiden 1993 im Zuge einer Neuausrichtung der Familienrepräsentanz im Unternehmen Harald EckesChantré und Peter Eugen Eckes aus dem Vorstand aus und wechseln in den Aufsichtsrat. ${ }^{748}$ Das Topmanagement des Unternehmens ist nun vollkommen familienfremd.

743 Vgl. B891218HB. Der Umsatz steigt durch diese Übernahme um $24 \%$ auf rund 900 Mio. DM.

744 Vgl. B930327NZ. Es wird von regelrechten »Umsatzsprüngen« gesprochen.

745 Vgl. Eckes (2007: 36).

746 Vgl. Eckes (2007: 36f.). Die Aktien verbleiben jedoch in den Händen der Familie.

747 Vgl. Eckes (2007: $37 \mathrm{ff}$.): »Die erfolgreiche Internationalisierung«. Die Sparte Fruchtsäfte war bereits in den 80er-Jahren durch signifikante Investitionen in neue Produktionskapazitäten erweitert worden. Von 1982 bis 1986 wurden 26 Mio. DM in das Werk in Bröl investiert, 1989 für 70 Mio. DM ein neues Werk in Fallinbostel errichtet.

748 Vgl. Eckes (2007: 37). Im Aufsichtsrat sind daneben noch zwei weitere Familienmitglieder aktiv. 
Zur gleichen Zeit finden auch bei der Berentzen-Gruppe personelle Veränderungen statt: 1992 wechselt der langjährige Vorsitzende der Geschäftsführung, Dr. Hans Berentzen, in den Aufsichtsrat. ${ }^{749} \mathrm{Ihm}$ folgt zunächst sein Stellvertreter Carl-Maria Richarz und somit ein Vertreter der älteren Generation der Eigentümerfamilien nach.

1994 wagt die Berentzen-Gruppe dann den großen Sprung nach vorne und geht als erster deutscher Spirituosenhersteller an die Börse - die BerentzenGruppe AG entsteht. ${ }^{750}$ In den freien Handel geht allerdings nur die Hälfte des Eigenkapitals in Form von stimmrechtslosen Vorzugsaktion. Die stimmberechtigten Stammaktien verbleiben in den Händen der bisherigen Eigentümerfamilien. Zum Sprecher des Vorstands wird Jan B. Berentzen bestimmt. Neben ihm fungieren drei Manager als Vorstand, unter ihnen Jürgen Pabst als Vertreter des Stammes Pabst \& Richarz.

In Bezug auf das Produktsortiment wird Anfang der 1990er-Jahre versucht, durch diverse Markteinführungen das Kernprodukt Berentzen-Apfelkorn zu variieren und in dessen Lebenszyklus zu verlängern. ${ }^{751}$ Zugleich finden weitere Akquisitionen statt. So wird 1996 wird die Strothmann Brennerei in Minden übernommen. ${ }^{752}$

\section{Strategiekrise}

An der Börse beginnt nach dem Allzeithoch 1996, als der Kurs 34 Euro pro Aktie beträgt, jedoch ein kontinuierlicher Sinkflug, der bis 2003 anhält und in dessen Zuge das Unternehmen zeitweise gar bis zu $90 \%$ an Wert verliert. ${ }^{753}$

Im sinkenden Börsenwert schlagen sich die nun trotz der vielen Übernahmen sinkenden Umsätze des Unternehmens und der mit 7,1 Mio. Euro deutliche Verlust des Jahres 1997 nieder. Darüber hinaus macht sich in der Öffentlichkeit zunehmend Skepsis angesichts der Kosten der Integration der vielen übernommenen Unternehmen und Marken breit. ${ }^{754}$

Sorgen bereiten den Anlegern zudem die allgemeinen Probleme der Spirituosenbranche: Während die Pro-Kopf-Verbräuche bei alkoholfreien Getränken Jahr für Jahr leicht zulegen, gehen die Verbräuche bei alkoholischen Getränken und insbesondere Spirituosen weiter kontinuierlich zurück. ${ }^{755}$ Zugleich belastet

749 Vgl. B920620NZ:»Berentzen wechselt in den Aufsichtsrat«; B080725NZ.

750 Vgl. zum Börsengang B940330NZ; B940715NZ. Der Kurs steigt am Einführungstag von 345 DM auf 352 DM.

751 Der Saure Apfel kommt 1993 auf den Markt, der Winterapfel 1995. Vgl. B960620NZ.

752 Vgl. B960515NZ: »Mit Strothmann haben wir einen richtig dicken Fisch geangelt.»

753 Vgl. die Daten von boerse.de (2015) und B971224.

754 Es folgt ein erstes Rationalisierungsprogramm. Zum Beispiel wird die im Zuge der Übernahmen gestiegene Anzahl an Abfüllanlagen für Spirituosen von 27 an sechs Standorten auf 18 an drei Standorten reduziert.

755 Der Aufschwung zur Wendezeit Anfang der 1990er-Jahre bleibt nur ein kurzes Strohfeuer, 
im Jahr 1997 die misslungene Markteinführung von sog. Alkopops das Ergebnis der Berentzen-Gruppe.

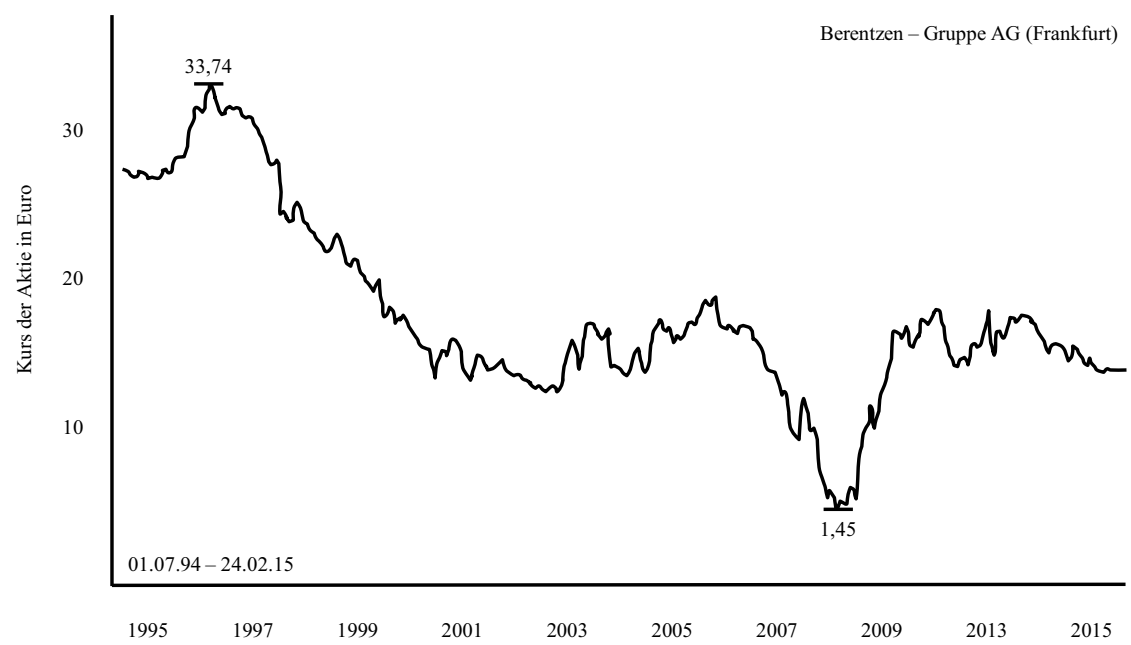

Abbildung 17: Entwicklung der Aktie der Berentzen-Gruppe AG im Zeitraum 1995-2015 (Quelle: Eigene Darstellung auf der Datengrundlage von boerse.de [2015]).

Zum Jahreswechsel 1998/99 wird trotz dieser negativen Entwicklung die Dethleffsen Spirituosen KG in Flensburg mit ihrer bekannten Marke Bommerlunder übernommen. ${ }^{756}$ Mit dieser Übernahme, der größten in der Geschichte von Berentzen, wird das Unternehmen neben der Eckes AG zum größten Spirituosenanbieter im deutschen Markt. ${ }^{757}$

\section{Erfolgskrise}

In den 2000er-Jahren setzt sich der Umsatzrückgang ungebrochen fort und auch der Ertrag nimmt kontinuierlich ab.

Zugleich gibt es in der Berentzen-Gruppe AG einige personelle Veränderungen: zunächst zieht sich im Jahr 2000 Hans Berentzen aus Altersgründen als Aufsichtsratsvorsitzender zurück. Seine Nachfolge tritt ein familienfremder

da sich der mehr als doppelt so hohe Pro-Kopf-Konsum im Osten rasch dem niedrigeren Westniveau annähert. Hingegen steigt 1997 der Verbrauch von alkoholfreien Getränken pro Kopf von 230,6 1 auf 238,8 l. Vgl. Schwedler (2013: 77).

756 Berentzen übernahm damit die 1979 fünft-, acht- und dreizehntgrößten Markenspirituosenhersteller in Deutschland. Vgl. Brendel \& Oberender (1981: 137).

757 Das Dethleffsen-Spirituosensortiment ergänzt das Berentzen-Sortiment primär um Markenspirituosen in Segmenten wie Rum und Aquavit. In diesen war Berentzen bislang nicht vertreten, ist nun aber mit Marken wie Hansen Rum oder Bommerlunder Marktführer. Vgl. Berentzen (1998: 5); Berentzen (1999: 7). 
Spitzenmanager aus der Lebensmittelindustrie an. Ein Jahr darauf tritt der Sohn von Friedrich Berentzen, Christian Berentzen, in den erweiterten Führungskreis des Unternehmens ein. ${ }^{758} 2001$ verlässt auch Jürgen Pabst den Vorstand. Nach seinem Rückzug ist die Eigentümergruppierung Pabst \& Richarz nicht mehr im Vorstand vertreten. Ein Jahr später erfolgt eine Neubesetzung des Aufsichtsrates und neben drei weiteren Aufsichtsräten zieht sich Friedrich Berentzen aus dem Aufsichtsrat zurück. Das letzte Familienmitglied aus der Familie Berentzen in leitender Funktion ist somit Jan B. Berentzen.

Probleme bereitet dem Unternehmen nun auch der steigende Anteil von Handelsmarken am Spirituosenmarkt, der 2001 um weitere 3,4 \% auf 22,7\% steigt. ${ }^{759}$ Unter dieser Entwicklung leiden insbesondere die unzähligen unbekannten und schwachen Marken der Berentzen-Gruppe. Diese verlieren im Wettbewerb Jahr um Jahr deutlich Marktanteile.

Im Jahr 2003 soll dann mit der Übernahme der Alkoholsparte der Eckes AG der Sprung aus der Krise gewagt werden. ${ }^{760}$ Wenige Monate zuvor hatte die Eckes AG bekanntgegeben, dass das Unternehmen sich aufgrund des rückläufigen Marktes vom traditionsreichen ursprünglichen Kern des Spirituosengeschäfts trennen möchte, obgleich dieser Geschäftsbereich zu diesem Zeitpunkt noch immer für einen Großteil des Umsatzes und vor allem des Gewinns verantwortlich ist. ${ }^{761}$ Das Unternehmen möchte sich in Zukunft jedoch vor allem auf die wachsende Sparte der Fruchtsäfte konzentrieren.

»Abschied vom Alkohol - der Familienkonzern will sich von Edelkirsch \& Co. trennen. Der Verkaufserlös soll in die Fruchtsaftsparte fließen [...] `Eckes ohne Alkoholsparte, das ist wie Volkswagen ohne Autos`, sagt der Betriebsratsvorsitzende Uwe Michalski.« (Das Manager Magazin zur Bekanntgabe der Eckes AG, die Alkoholsparte veräußern zu wollen $)^{762}$

Doch scheitern die Verhandlungen zwischen Eckes und Berentzen. Offiziell werden unterschiedliche Preisvorstellungen als Ursache genannt. In der Presse wird allerdings auch über Widerstände aus dem Aufsichtsrat der BerentzenGruppe AG berichtet. ${ }^{763}$ Im Zuge der Berichterstattung zur geplatzten Über-

758 Vgl. zu den Personalwechseln B010504NZ; B020612LZ. Friedrich Berentzen bekleidet die neu geschaffene Position des "Generalbevollmächtigten« für Supply-Chain-Management.

759 Vgl. Berentzen (2001: 36).

760 Vgl. B030326NZ. Zur Konzentration der Eckes AG auf die Fruchtsäfte Eckes (2007: 42 ff.).

761 Vgl. E021008MM: Die Sparte Fruchtgetränke erreicht 2001 einen Umsatz von 574 Mio. Euro bei einem Gewinn von 23,7 Mio. Euro, die Alkoholsparte einen Gewinn von 23,8 Mio. Euro bei einem Umsatz von 678 Mio. Euro.

762 E021008MM.

763 Vgl. B030513HB, B030513NZ. Der Aufsichtsrat soll Bedenken hinsichtlich der Absicherung der Finanzierung und der Höhe des Preises gehabt haben. 
nahme wird in den Medien gar offen über einen erzwungenen Abschied von Jan B. Berentzen spekuliert. ${ }^{764}$ Dieser bleibt jedoch vorerst auf seinem Posten.

Wenige Monate später sagt die Eckes AG den Verkauf der Alkoholsparte dann mangels preislich akzeptabler Angebote vorläufig ganz ab. In der Folge wird 2004 die Spirituosenproduktion am traditionsreichen Standort Nieder-Olm geschlossen und in Nordhausen konzentriert. ${ }^{765}$

Die Berentzen-Gruppe AG hingegen versucht Ende 2003 mit der Aufnahme diverser Lizenzprodukte den Problemen im Kerngeschäft zu begegnen. ${ }^{766}$ So werden nun die US-Biermarke Bud, die australische Weinmarke Yellow Tail, aber auch Spirituosen wie Linie Licor 43 vertrieben. Berentzen möchte nun verstärkt die »world of fine drinks« anstatt deutschen Schnaps anbieten.

Allerdings können die teilweise recht erfolgreichen, aber volumenschwachen Marken die Rückgänge im Kerngeschäft mit volumenstarken Traditionsprodukten bei Weitem nicht kompensieren: 2004 sind ein weiterer massiver Umsatzrückgang um 7 \% auf 180,1 Mio. Euro und ein neuer Rekordverlust in Höhe von -13,8 Mio. Euro zu verzeichnen. Daraufhin wird ein Restrukturierungsprogramm für den Bereich Markenspirituosen eingeleitet, welches zunächst aber auch erst einmal das Ergebnis belastet. ${ }^{767}$ Aufgrund der angespannten finanziellen Lage muss im Laufe des Jahres 2005 dann die sehr profitable Beteiligung an Linie Aquavit verkauft werden. ${ }^{768}$

Zugleich finden einige personelle Veränderungen im Aufsichtsrat und Topmanagement statt. ${ }^{769}$ So verkündet das Unternehmen im Juni 2005, dass der Vorstandssprecher Jan B. Berentzen ab 2007 in den Aufsichtsrat wechseln wird. Das Topmanagement der Berentzen-Gruppe AG solle ab diesem Zeitpunkt an rein familienfremd geführt werden. Ein Jahr später wird bekannt, dass sich Jan B. Berentzen bereits zum 01. Juli 2006 und somit ein Jahr früher als geplant in den Aufsichtsrat zurückziehen wird. ${ }^{770}$ Vorausgegangen waren fortgesetzte, auch öffentliche Auseinandersetzungen im Gesellschafterkreis. Im Dezember 2006 tritt Axel Dahm als neuer Vorstandsvorsitzender an. ${ }^{771}$ Die Leitung des Unternehmens liegt nun zum ersten Mal nicht mehr in den Händen der Eigentümerfamilien.

764 Vgl. zum Beispiel B030522MM: »Der Chefdestillator verflüchtigt sich.»

765 Vgl. Eckes (2007).

766 Vgl. Berentzen (2003: 2); Berentzen (2005: 38).

767 Vgl. Berentzen (2005: 8). Im Februar 2005 wird ein Sanierungsberater engagiert; hierzu B050524NZ.

768 Vgl. Berentzen (2005: 9). Für diese Beteiligung können 29 Mio. Euro erlöst werden.

769 Vgl. B050608NZ; B050626NW: »Berentzen-Chef räumt Sessel vorzeitig«.

770 Vgl. B060619HB; B061218FT.

771 Vgl. Berentzen (2006: 13). Der neue Vorstandsvorsitzende war bereits 1993-1997 für das Markengeschäft bei Berentzen verantwortlich gewesen. 
Der neue Vorstandsvorsitzende mahnt große Veränderungen für den Konzern an und in der Folge wird die Marktbearbeitung deutlich intensiviert.

»Bei Berentzen geht es nicht darum, ob das neue Management etwas ändern möchte wir müssen vieles ändern, und zwar jetzt. Veränderungen beginnen mit einem neutralen Blick auf die Situation. [...] das Kerngeschäft von Berentzen heißt Spirituose und dort wurden 133 Millionen Euro Umsatz erreicht, im Hauptmarkt Deutschland sind letztes Jahr noch 104 Millionen Euro erwirtschaftet worden. Geht man fünf Jahre zurück, lag der Spirituosenumsatz im Inland bei annähernd 140 Millionen Euro, vor zehn Jahren waren es über 200 Millionen Euro - ohne die zwischenzeitlich erworbenen Marken und Produkte wie Hansen oder Bommerlunder und ohne Lizenzmarken. Um noch einmal den Gruppenumsatz inklusive Branntweinsteuer anzusprechen: 1996 und in D-Mark gerechnet war Berentzen ein Milliardenunternehmen und die Nummer 1 im deutschen Spirituosenmarkt!

Es soll hier keine glorreiche Vergangenheit heraufbeschworen werden, denn seither haben sich wirtschaftliches Umfeld, Spirituosenmarkt und Konsumgewohnheiten geändert. Es führt aber kein Weg an der Erkenntnis vorbei, dass Berentzen deutlich an Boden verloren hat. [...] die Entwicklung der Geschäftszahlen sagt: Es reicht nicht und wir können nicht so weitermachen. Der Umsatz ist nicht nur geschrumpft, sondern sinkende Markenumsätze in Verbindung mit steigendem Handelsmarkengeschäft und durchgängig fallenden Margen untergraben die Ertragskraft.«

(Der neue Vorstandsvorsitzende Axel Dahm im Vorwort des Geschäftsberichts 2006) ${ }^{772}$

Im Ergebnis macht sich allerdings der wachsende Anteil des Handelsmarkengeschäfts am Umsatz bemerkbar. Berentzen setzt im Jahr 2007 mit 67,7 Mio. Flaschen ein Viertel mehr Handelsmarken-Spirituosen ab als 2006. Dieser Anstieg steigert zwar den Umsatz, bedroht aber massiv die Ertragskraft der gesamten Gruppe.

Währenddessen gibt es auch bei der Eckes AG personelle Veränderungen. 2005 verlassen alle verbliebenen Familienmitglieder den Aufsichtsrat und werden in der Folge von ihren Bevollmächtigen vertreten. ${ }^{773} 2006$ wird dann der große Schritt gewagt. Zunächst werden die restlichen $26 \%$ Prozent Anteile an Granini gekauft. Anschließend verkauft das Unternehmen die Spirituosensparte an Rotkäppchen-Mumm und veräußert das nicht mehr benötigte Betriebsgelände in Nieder-Olm. ${ }^{774}$ Damit endet das 150 Jahre andauernde Engagement von Eckes in der Spirituosenbranche.

772 Vgl. Berentzen (2006: 8); auch B070725BH, B080326HB. Groß angelegte Marketingkampagnen laufen an, der Marketingetat wird deutlich von 27 Mio. Euro auf 33 Mio. erhöht.

773 Vgl. zur Schlussphase der Abspaltung der Spirituosensparte bei der Eckes AG Eckes (2007: 44).

774 Vgl. Eckes (2007: 44). 


\section{Liquiditätskrise, Verkauf und Turnaround}

Bei der Berentzen-Gruppe steigen derweil die Verluste. So muss für das Jahr 2007 erneut ein Verlust von -11,40 Mio. Euro verzeichnet werden und für das Geschäftsjahr 2008 zeichnen sich noch höhere Verluste ab, die sich bei einem Umsatz von knapp 200 Mio. Euro schlussendlich auf -22,50 Mio. Euro belaufen.

Diese Entwicklung führt dazu, dass die die gesamte Berentzen-Gruppe im Sommer 2008 zum Verkauf steht. Nachdem kein strategischer Käufer gefunden werden konnte und der Verkaufsprozess zeitweilig auch gestoppt werden musste, verkaufen die Eigentümerfamilien letztlich im Juli 75,1 \% der Anteile zu einem nicht öffentlich gemachten Preis an den Finanzinvestor Aurelius. ${ }^{775}$

Mit dem neuen Mehrheitsaktionär soll eine "neue Sichtweise« kommen, "Denkverbote« sollen »entlarvt und zur Seite gelegt«, etablierte Strukturen" überprüft werden. ${ }^{776}$ Hierzu wird ein neues Management bestellt und der Aufsichtsrat neu besetzt. Die Priorität im Rahmen des Turnarounds liegt zunächst auf der Kostenreduktion: Durch Reorganisationen im Vertrieb, das vorläufige Zurückfahren des zuvor erhöhten Marketingetats, die Konzentration der Spirituosenproduktion in Minden und die damit verbundene Stilllegung des traditionellen Standortes in Haselünne sowie das Outsourcing von Verwaltungsabteilungen werden bei einem Nettoumsatz von rund 200 Mio. Euro im Jahr 2009 dann tatsächlich Gemeinkosteneinsparungen von insgesamt 17,4 Mio. Euro realisiert. ${ }^{777}$ Hierauf aufbauend, versucht der neue Gesellschafter die Ertragskraft des Unternehmens durch eine strategische Konzentration auf die zu verjüngenden und revitalisierenden Kernmarken zu stärken. ${ }^{778}$

Der finanzielle Turnaround gelingt. Nach einem Verlust von 11,4 Mio. Euro im Jahr 2007 und 22,5 Mio. Euro im Jahr 2008 wird 2009 wieder die Gewinnschwelle überschritten und 2010 ein Gewinn von 8,6 Mio. Euro erzielt.

Nach der finanziellen Sanierung wird in der dritten Phase des Turnarounds ab dem Jahr 2010 die internationale Expansion in Märkte wie die Türkei, China und Indien forciert. ${ }^{779}$ Insbesondere die wichtigen Kernmarken Berentzen und

775 Vgl. Berentzen (2008: 4ff.); B080627MM. Zum Stopp des Verkaufsprozesses auch B080716HB. Nur die Familien Berentzen und Wolf halten kurze Zeit noch 24,9\% der Aktien, die aber später auch von Aurelius übernommen werden.

776 Vgl. Berentzen (2008: 4): »Mit dem neuen Mehrheitsaktionär kam eine neue Sichtweise: ,Denkverbote` werden entlarvt und zur Seite gelegt, etablierte Strukturen überprüft und neuen Anforderungen angepasst, das Produktivvermögen soll mehr leisten. Dass diese Sichtweise seit Herbst 2008 mit Priorität auf die Kosten zielt, wird angesichts des Potenzials nicht überraschen."

777 Vgl. Berentzen (2009: 4), auch B081216HB. Somit kann das EBIT gegenüber dem Vorjahr um 12,5 Mio. Euro auf + 7,3 Mio. Euro verbessert werden.

778 Die traditionellen Marken sollen als bodenständige Lifestyle-Produkte am Markt positioniert werden und zum Beispiel mit TV-Spots wieder intensiver beworben werden.

779 Vgl. Berentzen (2008: 9); auch B080722MM. Zur Finanzierung dieser Strategie gibt das 
Puschkin legen im Absatz 2014 wieder deutlich zu. Darüber hinaus gelingt mit dem Kauf eines Anbieters von Frischsaftsystemen der Einstieg in ein neues und wachstumsträchtiges Marktsegment, das den veränderten Kundenbedürfnissen nach gesundheitsbewussterer Ernährung Rechnung trägt. ${ }^{780}$ Gleich im ersten Jahr trägt die neue Sparte ungefähr ein Drittel zum Jahresüberschuss von 4,2 Mio. Euro bei und kann von 2014 bis 2015 den Umsatz deutlich von 3,4 auf 17,3 Mio. Euro steigern. 250 Jahre nach ihren Anfängen brennt die BerentzenGruppe somit kaum mehr Schnaps in ihrer Heimat Haselünne, sondern lässt zunehmend Orangensaft pressen.

\subsubsection{Pfadanalyse}

Analog zum Fall der Anton Schlecker e.K. soll zur Pfadanalyse zunächst die Persistenz der strategischen Entwicklung nachgewiesen werden. In einem zweiten Schritt wird anschließend die Bedeutung der selbstverstärkenden Mechanismen und des Lock-in für die Entstehung dieser strategischen Persistenz aufgezeigt.

\subsubsection{Analyse der strategischen Persistenz}

Zur Untersuchung der strategischen Persistenz der Berentzen-Gruppe AG wird im Folgenden zum einen deren strategische Entwicklungsrichtung im Kontext ihrer Umweltdynamik betrachtet. Zum anderen wird die strategische Dynamik der einzelnen Geschäftsbereiche im Rahmen der strategischen Gesamtausrichtung des Unternehmens untersucht. ${ }^{781}$

\subsection{Organisationale Entwicklungsrichtung: »Wachsen oder weichen«}

Im obigen Abschnitt wurde dargestellt, wie sich ab Mitte der 1970er-Jahre das Marktumfeld für die klassischen deutschen Markenspirituosenhersteller beständig zu verschlechtern begann. Zum einen sank der Pro-Kopf-Konsum an Spirituosen infolge sich verändernder gesellschaftlicher Normvorstellungen. Zum anderen wurden die deutschen Traditionsunternehmen in diesem schrumpfenden Markt "von oben « durch die großen internationalen Spirituosenkonzerne mit ihren starken Premiummarken angegriffen, während "von unten« die preisgünstigen Handelsmarken zunehmenden Preisdruck ausübten.

Unternehmen 2012 eine Unternehmensanleihe heraus, die mit einem Emissionsvolumen in Höhe von 50 Mio. Euro erfolgreich am Markt platziert werden kann.

780 Vgl. Berentzen (2014: 6); auch B160324HB: »Orangensaft statt Apfelkorn«.

781 Die Untersuchung erfolgt somit entlang dem gängigen Verständnis von Gesamtunternehmensstrategien, welche die Frage nach der allgemeinen organisationalen Entwicklungsrichtung beantworten und anschließend festlegen sollen, in welchen Geschäftsfeldern die Unternehmung tätig ist und wie die dem Unternehmen zur Verfügung stehenden Ressourcen entsprechend verteilt werden. Vgl. Steinmann \& Schreyögg (2005: 171). 
Dieser Druck wurde durch die zunehmende Konzentration im LEH noch verstärkt.

In diesem schwierigen Marktumfeld entschloss sich das Management der J. B. Berentzen - beflügelt vom Erfolg des Berentzen Apfelkorns - Mitte der 1970er-Jahre zu einer Gesamtunternehmensstrategie des »Wachsens oder Weichens«. Das Unternehmen sollte wachsen, um der steigenden Macht des Handels und der Expansion der internationalen Konzerne adäquat begegnen zu können. $^{782}$

»Der Doktor Hans Berentzen [...], der hat mir mal ein Buch geschenkt, >Wachsen oder Weichen «."

(Eine ehemalige Führungskraft der Berentzen-Gruppe AG zur strategischen Ausrichtung des Unternehmens)

In der Folge konnte der Absatz tatsächlich innerhalb von nur vier Jahren von 2,5 Mio. Flaschen im Jahr 1976 auf 25,2 Mio. Flaschen im Jahr 1979 verzehnfacht werden. Doch bereits nach wenigen Jahren erreichte der Lebenszyklus des Erfolgsprodukt Apfelkorn sein Reifestadium und die Absatzzahlen begannen zu sinken: von 25,2 Mio. Flaschen im Jahr 1979 auf ca. 16 Mio. 1986, und 9,2 Mio. Flaschen im Jahr $1990 .^{783}$

Daraufhin wurde versucht, durch vom Apfelkorn ausgehende konzentrische Produktmodifikationen ein Sortiment der fruchtigen Spirituosen aufzubauen. ${ }^{784}$ Allerdings konnten diese Produktvariationen die Umsatzrückgänge des Apfelkorns nicht kompensieren.

Das Unternehmen Berentzen stand nun vor einer grundlegenden strategischen Wahl: Zum einen konnte versucht werden das Rentabilitätsniveau in einem schrumpfenden Markt durch eine erhöhte Effizienz aller Unternehmensaktivitäten zu konsolidieren. Zum anderen konnte die Wachstumsstrategie forciert werden. ${ }^{785}$ Das Management von Berentzen wählte die offensive Variante und suchte das Heil des Unternehmens in einer "offensiven und wachstumsorientierten Strategie«.

"An seiner offensiven und nach vorn gerichteten Strategie wird Berentzen auch in Zukunft festhalten."

(Pressemitteilung von Berentzen aus dem Jahr 1989) ${ }^{786}$

782 Die Strategie wurde von mehreren Interviewpartnern so charakterisiert. Vgl. aber auch Angermann (1970); B000622NZ. Auf die Notwendigkeit der Kooperation innerhalb der Branche verweist bereits Berentzen (1981: 39).

783 Vgl. Berentzen (1981: 25); Brendel \& Oberender (1981: 129); B910214NZ.

784 Es folgen Variationen des Apfelkorns mit Erdbeer, Moosbeere oder Grapefruit. Vgl. B790929NZ.

785 Vgl. zu den Möglichkeiten von Konsolidierungsstrategien Welge \& Al-Laham (2012: 588).

786 B891231PM. 
Da aber auch der Export des Apfelkorns ab dem Jahr 1979 nicht ausreichte, um die nachlassende Nachfrage im Inland zu kompensieren, ging das Unternehmen im Sinne der Vision des "Wachsens oder Weichens einer Strategie des organischen Wachstums zu einer Strategie des externen Wachstums über. ${ }^{787}$

Diese neue strategische Ausrichtung führte im Jahr 1988 zur Fusion mit dem auf Handelsmarken spezialisierten Hersteller Pabst \& Richarz. ${ }^{788}$ Die maßgeblichen Akteure einte dabei eine gemeinsame Vision von der Entwicklung der Branche und der notwendigen strategischen Reaktion:

»Es ist unsere Absicht [...] auf die internationale Herausforderung und deren Wirkungen im deutschen Markt eine offensive und eine deutsche Antwort zu geben."

(Der damalige Vorsitzende der Geschäftsführung Dr. Hans Berentzen) ${ }^{789}$

Das erklärte Ziel der Fusion war der Aufbau einer »Alternative zu Großkonzernen«. Zudem sollte durch die Fusion von Marktanteilen dem immer mächtiger werdenden Handel ausreichend Stärke entgegensetzt werden. Vor allem sollte mit der Fusion jedoch auch die Grundlage für weiteres externes Wachstum gelegt und das Tempo der Akquisitionen beschleunigt werden. ${ }^{790}$

"Dann kam also die Fusion und wir haben auch erst schöne Synergien gezogen, und hatten auch eine sehr schöne Wachstumsphase [...]. Es boten sich eine Reihe von Unternehmen und Marken an, zum Kauf, im Laufe der Jahre.»

(Ehemaliger Vorstand der Berentzen-Gruppe AG)

Im Rahmen dieser Strategie des externen Wachstums wurden in der Folge zahlreiche klassische deutsche Spirituosenhersteller und deren traditionsreiche Marken übernommen: 1989 erfolgte der Mehrheitserwerb an der König \& Schlichte GmbH (Umsatz 1988: 158 Mio. DM) mit ihrem Traditionsprodukt Schinkenhäger und der starken Wodka-Marke Puschkin. ${ }^{791} 1991$ folgte die Übernahme der Mehrheit bei der Doornkaat AG 1991 (Umsatz 1991: 354 Mio. DM) ${ }^{792}$ Unmittelbar nach diesen großen Akquisitionen wurde verlautbart, dass der Kauf weiterer Unternehmen beabsichtigt sei.

787 Hierzu eine ehemalige Führungskraft der Berentzen-Gruppe: »Also es war so die Zeit 'fressen und gefressen werden . Und man hat sich dann eher fürs Fressen entschieden.»

788 Vgl. B830630NZ. Durch die Fusion steigt Berentzen nun in das wachsende Handelsmarkengeschäft ein, das zu einer Auslastung der Produktionskapazitäten beiträgt, aber naturgemäß mit geringer Ertragskraft einhergeht.

789 B870909NZ:»Eine deutsche Antwort am Spirituosenmarkt«.

790 Vgl. B870909HB: "Ausdrücklich wird denn auch von Dr. Hans Berentzen und von Carl Maria Richarz darauf hingewiesen, dass man in Zukunft jederzeit für weitergehende $\mathrm{Zu}$ sammenschlüsse offen ist."

791 Vgl. B631030SP. Puschkin ist zu diesem Zeitpunkt die am dritthäufigsten verkaufte WodkaMarke in Deutschland.

792 Vgl. B910720NZ; B910830NZ. 
»Im Zusammenhang mit der Fusion der Unternehmen Berentzen und Pabst \& Richarz haben sich die beteiligten Unternehmerfamilien das Ziel gesetzt, eine deutsche Antwort auf die internationale Herausforderung zu formulieren. Das heißt, für ein typisch deutsches Spirituosensortiment Ansprechpartner des Handels zu sein. Ein solches Spirituosensortiment besteht nun einmal in allen Segmenten aus traditionsreichen Produkten beziehungsweise Marken."

(Jan B. Berentzen 1992 in einem Interview mit dem Magazin Absatzwirtschaft) ${ }^{793}$

Zur besseren Handhabung der gestiegenen Komplexität und der Vorbereitung der weiteren Wachstumsschritte wurde daraufhin die rechtliche Umwandlung der bislang als J. B. Berentzen $\mathrm{GmbH} \&$ Co. KG firmierenden Gruppe in eine Holding-Gesellschaft begonnen. Diese Entwicklung mündete im Jahr 1994 im nächsten großen Wachstumsschritt des Börsengangs. Durch den Gang an den Kapitalmarkt sollte vor allem eine belastbare Kapitalgrundlage für weiteres externes Wachstum geschaffen werden:

»Wie das Haselünner Unternehmen am Dienstag mitteilte, soll damit die Kapitalbasis zur Fortsetzung eines ertragsorientierten Wachstums gestärkt werden."

(Die Neue Osnabrücker Zeitung zum Börsengang) ${ }^{794}$

Mit den Erlösen aus der Emission wurden dann 1996 die Strothmann Brennerei in Minden und 1999 die Dethleffsen Spirituosen KG in Flensburg übernommen. Die erklärten Ziele dieser Akquisitionen waren die Steigerung von Marktanteil und Markmacht sowie die Verbreiterung des Sortiments. ${ }^{795}$

Auf diesem Wege versuchte die Berentzen-Gruppe in einem schrumpfenden Markt zu wachsen, denn nach dem kurzen Strohfeuer der Wiedervereinigung ging der Konsum von Spirituosen weiter stetig zurück. ${ }^{796}$

Diese Entwicklung schlug sich ab Anfang der 1990er-Jahre in sinkenden konsolidierten Gesamtumsätzen und ab Ende der 1990er-Jahre auch im Ergebnis und dem Börsenwert der Berentzen-Gruppe AG nieder.

793 B920101AW; vgl. auch B911204HB: Die Akquisition weiterer "geeigneter« Produktlinien oder Unternehmen sei beabsichtigt.

794 Vgl. B940704NZ. Der Vorstandssprecher der Berentzen-Gruppe, Carl-Maria Richarz, benannte demnach die Finanzierung des weiteren Wachstums als vorrangiges Ziel des Börsengangs.

795 Vgl. Berentzen (1999: 7); B981228NZ. Berentzen übernahm damit die laut Brendel \& Oberender (1981: 137) 1979 fünft-, acht- und dreizehntgrößten Markenspirituosenhersteller in Deutschland. Durch die Übernahme von Strohtmann stieg der Umsatz (inkl. Branntweinsteuer) von 472 auf 562 Mio. Euro; vgl. B960515NZ. Als zweitgrößter deutscher Hersteller setzt Berentzen 1999 nun konsolidiert 128,1 Mio. Flaschen ab (1998: 98 Mio.).

796 Vgl. BSI (2012: 10), Brendel \& Oberender (1981: 125). Betrug der Anteil von alkoholischen Getränken am Gesamtgetränkeverbrauch 1970 noch $32 \%$ und $197928 \%$, so hatte er im Jahr 2011 nur noch einen Anteil von 18,5\%. 


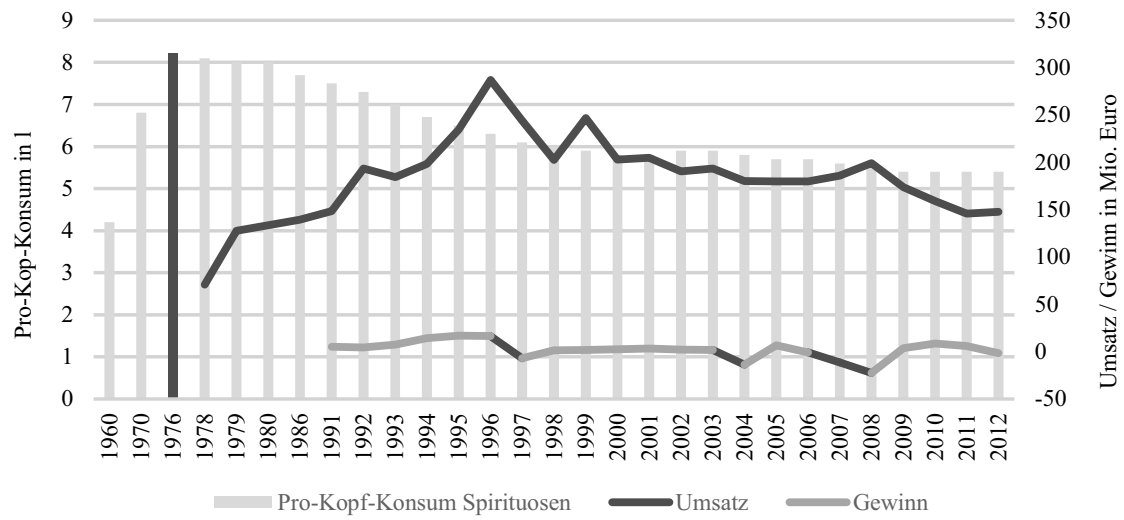

Abbildung 18: Entwicklung Pro-Kopf-Konsum Spirituosen in D 1960-2012 / Umsatz- und Gewinn der Berentzen-Gruppe AG

(Quelle: Eigene Darstellung auf Grundlage von Daten der Berentzen-Gruppe AG und Daten des Ifo-Instituts).

"In diesem Jahr [1997] herrscht bei den Aktionären einiger Spirituosenfirmen schon vor der Silvesterfeier Katerstimmung. Besonders schlimm hat es Berentzen erwischt: Deren Vorzugsaktie befindet sich seit Jahresbeginn im Sinkflug, der Börsenwert des Unternehmens schmolz auf ein Drittel."

(Das Handelsblatt 1997 zur Kursentwicklung der Berentzen-Gruppe AG) ${ }^{797}$

Der seit Jahrzehnten schrumpfende Markt hatte spätestens Mitte der 1990erJahre die anderen verbliebenen großen deutschen Markenspirituosenhersteller zu einer Anpassung ihrer strategischen Ausrichtung veranlasst. So hatte die zu diesem Zeitpunkt bereits diversifizierte Dethleffsen-Holding gezielt an die Berentzen-Gruppe veräußert. ${ }^{798}$ Die Racke-Gruppe strich weitergehende Investitionen in das Spirituosengeschäft und schaltete auf eine Strategie der Konsolidierung um:

"Auf eine reine >Erhaltungsstrategie` bei Spirituosen beschränkt sich künftig die Racke-Gruppe [...] Moller-Racke begründet die verordnete Zurückhaltung mit der fortschreitenden Negativentwicklung von Spirituosen: `Deutsche Spirituose leidet weiterı."

(Die Lebensmittel Zeitung im Jahr 2002) (999 $^{9}$

Doch insbesondere die 2002 verkündete Entscheidung der Eckes AG sich vollkommen aus dem Spirituosengeschäft zurückzuziehen, signalisierte eine zur

\footnotetext{
797 B971224HB.
}

798 Vgl. B130614NZ. Die Dethleffsen-Holding hatte einen Gebäudereiniger übernommen, sich an einem Medienhaus beteiligt und ein Immobilienunternehmen akquiriert.

799 Vgl. E021008LZ. 
Wachstumsstrategie von Berentzen konträre Umweltdynamik. ${ }^{800}$ Dabei sorgte die Ankündigung von Eckes nicht nur deshalb für großes Aufsehen in der Branche, weil die Alkoholsparte von Eckes für sich genommen zu diesem Zeitpunkt das viertgrößte europäische Spirituosenunternehmen darstellte, sondern auch weil die Sparte ungefähr die Hälfte des Umsatzes wie des Gewinns der Eckes AG beisteuerte. Trotz dieser vorhandenen Profitabilität waren die Entscheidungsträger bei der Eckes AG zur Ansicht gekommen, dass es für die langfristige Entwicklung des Gesamtunternehmens vorteilhafter wäre sich aus dem schrumpfenden Markt der Spirituosen zurückzuziehen und alle verfügbaren Ressourcen auf den Wachstumsmarkt der Fruchtgetränke zu verwenden.

»Das Familienunternehmen Eckes verdient seit 1857 mit hochprozentigen Getränken Geld. In den vergangenen Jahren baute das Unternehmen [...] jedoch seine Position bei Fruchtsäften zum Marktführer in Europa aus. Mit den Einnahmen aus dem Verkauf möchte die Gruppe weitere Safthersteller im europäischen Ausland kaufen. Fruchtgetränke lägen im gesamtgesellschaftlichen Trend zu mehr Gesundheit, Sport und Wellness und stellten einen Wachstumsmarkt dar.»

(Die Lebensmittel Zeitung im Jahr 2002 zum verkündeten Rückzug des Eckes AG aus dem Spirituosengeschäft) ${ }^{801}$

Ungeachtet dieser Entwicklungen in der Umwelt des Unternehmens verfolgte die Berentzen-Gruppe AG jedoch ihre Strategie des externen Wachstums unbeirrt weiter. Trotz des schrumpfenden Marktes und der eigenen rückläufigen Umsätze wurde sogar versucht die deutlich größere Spirituosensparte der Eckes AG zu übernehmen.

"Mit der Übernahme wäre die deutlich kleinere Berentzen-Gruppe zum größten Spirituosenhersteller Deutschlands aufgestiegen.«

(Das Handelsblatt im Jahr 2003 zum Übernahmeversuch der Spirituosensparte der Eckes AG durch die Berentzen-Gruppe AG) $)^{802}$

Auch nach dem gescheiterten Übernahmeversuch hielt die Berentzen-Gruppe an der Strategie des externen Wachstums fest.

"Berentzen machte auch deutlich, dass sein Unternehmen nach einem vergeblichen, 1,8 Millionen teuren Akquisitionsversuch "weiter aktiv auf der Suche« nach Firmen sei, deren Übernahme die Marktposition der Emsländer stärken könne."

(Die Neue Osnabrücker Zeitung zur Hauptversammlung im Jahr 2004) (03 $^{803}$

800 Vgl. Eckes (2007: 42).

801 E021008LZ; vgl. auch E021017LZ: »Großes Echo auf Pläne von Eckes«; E021017LZ2: »Zu Richtungsentscheidungen sehen sich immer mehr Unternehmen wegen negativer Rahmenbedingungen gezwungen $«$.

802 Vgl. B030513HB.

803 Zur Hauptversammlung im Jahr 2004 vgl. B040625NZ. 
Erst im Laufe des Jahres 2004 wurde teilweise und Schritt für Schritt von der Strategie des externen Wachstums abgewichen. Intentionale und signifikante Umsatzreduktionen zur Konsolidierung des Unternehmens wurden allerdings erst ab 2007 unter dem neuen Vorstandsvorsitzenden und dann ab 2008 unter dem neuen Eigentümer vorgenommen. ${ }^{804}$

Somit lässt sich hinsichtlich der organisationalen Entwicklungsrichtung ein hohes Maß an strategischer Kontinuität feststellen, die angesichts der divergenten Entwicklungsdynamik der Umwelt auf eine Form von strategischer Persistenz verweist. Zur näheren Analyse dieser Persistenz sollen im folgenden Abschnitt zunächst die Entwicklungen der einzelnen Geschäftsfelder in der strategischen Gesamtkonfiguration der Berentzen-Gruppe dargestellt werden.

\subsection{Entwicklung der Geschäftsfelder in strategischer Gesamtkonfiguration}

Die Berentzen-Gruppe verfügte über zwei Sparten. Zum einen die Sparte Spirituosen, die einen Umsatzanteil von 85 bis $90 \%$ besaß, zum anderen die Sparte alkoholfreie Getränke. ${ }^{805}$

Die Sparte der Spirituosen lässt sich in vier Geschäftsbereiche unterteilen: den Bereich der Kernmarken Berentzen und Puschkin, die akquirierten deutschen Traditionsmarken wie Doornkaat oder Bommerlunder, die durch die Fusion mit Pabst \& Richarz zum Unternehmen hinzugekommenen Handelsmarken, sowie die internationalen Lizenzmarken wie Linie Aquavit oder Licor 43, die seit Ende der 1990er-Jahre von der Berentzen-Gruppe vertrieben wurden.

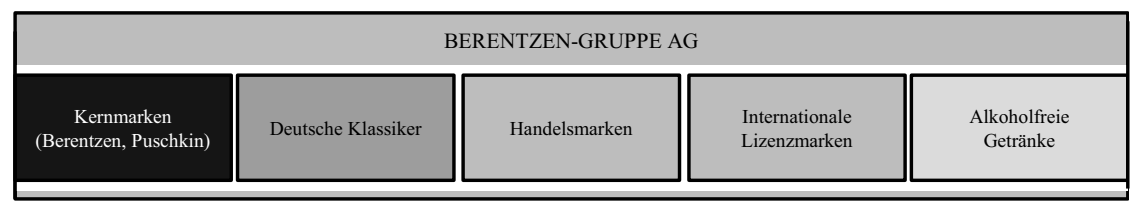

Abbildung 19: Sparten und Geschäftsbereiche der Berentzen-Gruppe AG (Quelle: Eigene Darstellung).

Im Folgenden wird aufgezeigt, wie sich diese Geschäftsfelder im Rahmen der oben skizzierten kontinuierlich verfolgten Wachstumsstrategie einzeln und in ihrem Verhältnis zueinander entwickelten. So soll die Persistenz des strategischen Musters als Summe dieser Teile näher geprüft werden.

804 Zugleich wurde ein Restrukturierungsprogramm eingeleitet, welches auf massive Kostensenkungen durch tiefgreifende Veränderungen in Produktion, Vertrieb und Verwaltung abzielte.

805 Vgl. Berentzen (1998: 9); Berentzen (1999: 11). 


\section{Alkoholfreie Getränke}

Im Gegensatz zu den alkoholischen Getränken ist der Markt für alkoholfreie Getränke seit der Nachkriegszeit ein Wachstumsmarkt: Allein von 1980 bis 1994 stieg der Pro-Kopf Konsum von 398,2 1 um 27,2 \% auf 506,7 1. ${ }^{806}$

In diesem stark wachsenden, aber hartumkämpften Markt verfolgte die Berentzen-Gruppe seit den 1970er-Jahren eine "Strategie der maßvollen Expansion «. ${ }^{807}$ Durch kleinere Übernahmen von Getränkefachgroßhändlern, den regionalen Vertrieb von Mineralwässern und Erfrischungsgetränken unter der eigenen Marke "Emsland « und vor allem durch den regionalen Vertrieb von Pepsi wuchs die Sparte moderat, blieb aber hinter den Wachstumsraten des Markts zurück. ${ }^{808}$

Im Gegensatz zur Eckes AG wurde diese Sparte im Verlauf der Unternehmensentwicklung weder durch größere Investitionen ausgebaut, noch durch bedeutsame Akquisitionen strategisch erweitert. Während die Eckes AG bereits in den 1980er-Jahren durch hohe Investitionen die Produktionskapazitäten erweiterte und zum Beispiel 1989 für 70 Mio. DM ein neues Werk errichtete, hielt die Berentzen-Gruppe an einer Strategie des maßvollen Wachstums fest. ${ }^{809}$ Diese strategische Ausrichtung war aber weniger mangelnden Ressourcen geschuldet, sondern vielmehr bewusste Entscheidung für eine Wachstumsstrategie, deren Fokus auf dem Ausbau des Spirituosengeschäfts lag.

»Der Firmenchef unterstrich, dass das Management inzwischen von dem ursprünglichen Plan abgerückt sei, langfristig ein Drittel des Geschäfts mit alkoholfreien Getränken zu bestreiten. Dem weiteren externen Wachstum des Spirituosengeschäfts sollten keine Fesseln angelegt werden."

(Die Neue Osnabrücker Zeitung zur Jahrespressekonferenz der Berentzen-Gruppe AG) $)^{810}$

An dieser Strategie des maßvollen Wachstums wurde im Rahmen der oben skizzierten Wachstumsstrategie bis zum Verkauf an den Investor Aurelius festgehalten.

Erst unter dem neuen Eigentümer gelang 2014 mit dem Einstieg in den Frischfruchtsaftbereich eine weitergehende horizontale Diversifikation innerhalb der Getränkebranche. ${ }^{811}$

»Orangensaft statt Apfelkorn. Der Spirituosenhersteller Berentzen ist bekannt für seinen Apfelkorn. Doch der Konzern schwört dem Alkohol zunehmend ab und ver-

806 Vgl. zu diesen Daten Meffert et al. (2009: 242).

807 Vgl. Berentzen (1981: 44), Schwedler (2013: 77).

808 Vgl. Berentzen (1981: 42 ff.).

809 Vgl. Eckes (2007). Darüberhinaus wurden zum Beispiel 1982 bis 198626 Mio. DM in das Werk in Bröl investiert.

810 Vgl. B000428NZ.

811 Vgl. B140902HA; B160324HB. 
ordnet sich stattdessen eine Saftkur. [...] der Blick in die Sparten zeigt: Die Gruppe besteht aus einem schwierigen Altgeschäft und einer dynamischen, viel kleineren zugekauften Tochter, die im Alleingang Umsatzplus und wohl auch den Gewinn sichert [...] `Citrocasa ist das Flaggschiff der Neuorientierung unseres Unternehmens`, sagte Schübel.»

(Das Handelsblatt zum Strategieschwenk der Berentzen-Gruppe AG) ${ }^{812}$

Obgleich die Berentzen-Gruppe die Kündigung des Vertriebsvertrags seitens des langjährigen Partners Pepsi verkraften musste, stieg der Umsatz der alkoholfreien Getränke durch das Wachstum des akquirierten Geschäftsfelds Frischfruchtsysteme somit stark an. ${ }^{813}$

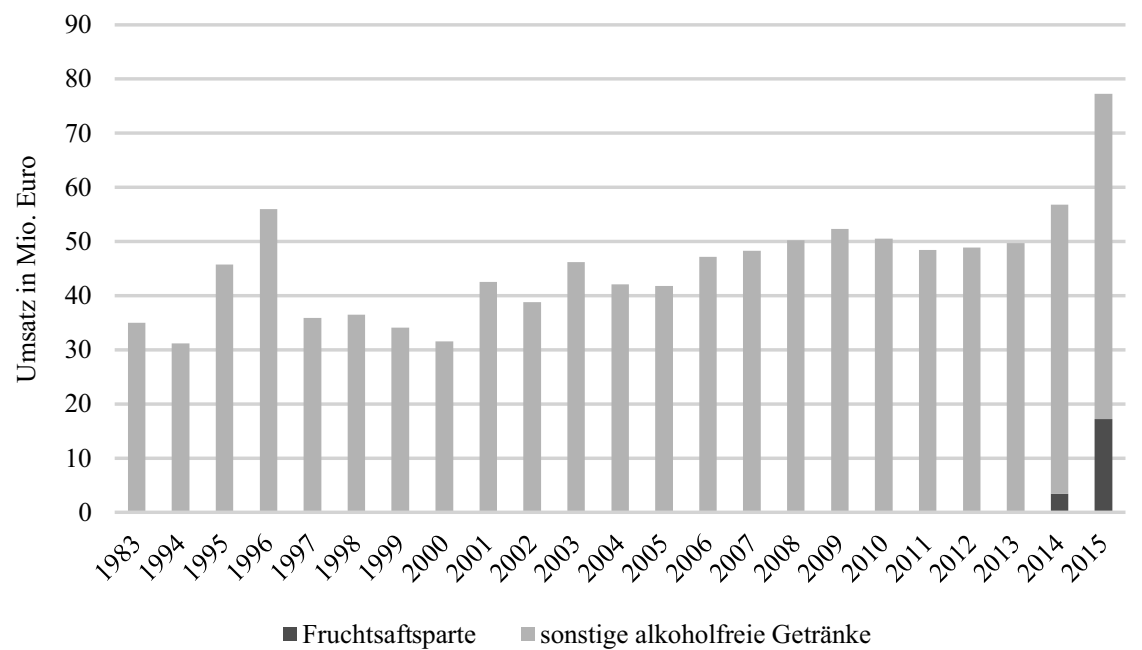

Abbildung 20: Berentzen-Gruppe AG: Entwicklung des Umsatzes der Sparte alkoholfreie Getränke 1983-2015

(Quelle: Eigene Darstellung auf Grundlage von Daten der Berentzen-Gruppe AG).

Insbesondere für den langen Zeitraum vor dem Eigentümerwechsel kann für das Geschäftsfeld der alkoholfreien Getränke jedoch ein hohes Maß an Kontinuität der strategischen Entwicklung festgestellt werden.

\section{Spirituosen: Handelsmarken}

Seit der Fusion mit dem auf Handelsmarken spezialisierten Hersteller Pabst \& Richarz im Jahr 1988 wurde das Handelsmarkengeschäft immer bedeutsamer.

812 Vgl. B160324HB.

813 Vgl. B160324HB. Zum Wechsel von Pepsi zu Sinalco B140121HB: „Seit 1960 produziert der Schnapshersteller Berentzen Cola im Auftrag von Pepsi. Damit ist bald Schluss. Ein anderer Brause-Hersteller springt in die Bresche." 
So stieg der Anteil der Handelsmarken am Absatz von $50 \%$ im Jahr 1988 auf rund $66 \%$ im Jahr 2008. ${ }^{814}$

Obwohl die primäre Ursache für die zunehmende Ertragsschwäche der Berentzen-Gruppe AG im sinkenden Markenspirituosenabsatz der Kernmarken und deutschen Klassiker lag, trug der wachsende Anteil des Geschäftsbereichs der ertragsschwachen Handelsmarken seinen Teil zu dieser Entwicklung bei. ${ }^{815}$

»Es reicht nicht und wir können nicht so weitermachen. Der Umsatz ist nicht nur geschrumpft, sondern sinkende Markenumsätze in Verbindung mit steigendem Handelsmarkengeschäft und durchgängig fallenden Margen untergraben die Ertragskraft.« (Aus dem Geschäftsbericht der Berentzen-Gruppe AG des Jahres 2006) (16 $^{816}$

Dennoch wurde diese strategische Gesamtkonfiguration beständig reproduziert. Erst im Jahr 2009 sank der Anteil der Handels- und Zweitmarken wieder, dann aber deutlich um $28 \%$. In diesem Rückgang spiegelt sich eine bewusste strategische Neuausrichtung unter dem neuen Eigentümer Aurelius, der die mittelfristige Ertragskraft der Berentzen-Gruppe stärken wollte. ${ }^{817}$

\section{Deutsche Klassiker}

Die oben skizzierte Wachstumsstrategie der Berentzen-Gruppe beruhte über einen Zeitraum von ungefähr fünfzehn Jahren vor allem auf der Akquisition typisch deutscher Spirituosenmarken von Korn und Weinbränden. Bei der Konsolidierung der Spirituosenbranche wollte die Berentzen-Gruppe federführend sein und »eine offensive und eine deutsche Antwort auf die internationale Herausforderung « schmieden. ${ }^{818}$ Durch die Vielzahl von Akquisitionen sollte nicht nur das Sortiment verbreitert werden, sondern durch den Aufkauf von Marktanteilen vor allem auch Marktmacht aufgebaut werden.

»Und damals folgt man einer Idee, die heißt, deutsche Traditionsmarken. Ja? Da kommen dann auch so sagenhafte, tolle Weltmarken wie Ammerländer Löffeltrunk oder was dann dazu."

(Ehemaliger Aufsichtsrat der Berentzen-Gruppe AG)

Allerdings hatten die erworbenen Unternehmen und Beteiligungen zum Zeitpunkt der Akquisitionen allesamt bereits seit geraumer Zeit mit großen wirt-

814 Vgl. Berentzen (2005: 18). Obwohl immer wieder in moderatem Umfang kaum profitable Geschäfte im Handelsmarkenbereich aufgegeben werden.

815 So korreliert der Rekordverlust des Jahres 2008 von -22,5 Mio. Euro im Jahr 2008 bspw. mit einem Anstieg des Anteils der Handelsmarken am Absatz von $56 \%$ im Jahr 2005 auf $66 \%$ im Jahr 2008, da der Absatz von Handelsmarken von 52 Mio. Flaschen auf 71,1 Mio. Flaschen emporschnellt.

816 Berentzen (2006: 8).

817 Berentzen (2009: 10).

818 Vgl. B870908HB; B870909NZ. 
schaftlichen Problemen zu kämpfen. So waren die 1988 und 1992 übernommenen Unternehmen Doornkaat und König \& Schlichte Mitte der 1960er-Jahre mit Umsätzen von 200 und 250 Mio. DM noch die beiden größten Spirituosenhersteller Deutschlands gewesen, hatten aber den Zenit ihrer Unternehmensentwicklung damit schon seit langer Zeit überschritten. ${ }^{819}$ König \& Schlichte hatte mit seiner Traditionsmarke Schinkenhäger schon vor der Übernahme durch Berentzen Jahr für Jahr Marktanteile verloren und allein 1988 einen Umsatzrückgang um $10 \%$ von 175 Mio. DM auf 158 Mio. DM verzeichnen müssen. Dieser Rückgang setzte sich nach der Übernahme durch die Berentzen-Gruppe fort: Zum konsolidierten Umsatz 1990 trug König \& Schlichte nur noch 70 Mio. DM bei. ${ }^{820}$ Während Doornkaat 1976 noch 19,7 0,7-Liter-Flaschen verkauft hatte, waren es im Jahr der Übernahme 1991 nur noch 3,7. ${ }^{821}$

"[...] also da sind wir auch aber dann richtig teuer schlauer geworden. Wir haben also zwei Jahre lang auch eine, auch prämierte Fernsehkampagne mit so 'nem DoornkaatSong gefahren [...], um also den Trend zu drehen, positiv für die Marke Doornkaat. Das hat nicht funktioniert. Also es war damals tatsächlich so, dass der DoppelkornMarkt, der rasselte einfach nach unten, und da war nichts mehr zu halten, eine Zeitlang. Ja, also da wurden alle Marken mitgerissen."

(Ehemalige Führungskraft der Berentzen-Gruppe AG)

All diese traditionsreichen Marken verloren somit nicht zuletzt aufgrund einer ungünstigen strategischen Positionierung in einem ohnehin rückläufigen Markt beständig an Marktanteilen. ${ }^{822}$

Als Markenanbieter hatten sie Unternehmen wie Doornkaat seit jeher Tradition und ein entsprechendes Marketing, nicht den Parameter Preis, in den Vordergrund ihrer Verkaufsbemühungen gestellt. ${ }^{823}$ Dennoch waren sie hinsichtlich des Markenimages bereits in den 1970er-Jahren von den internationalen Marken wie Bacardi oder Campari weit überholt worden. Letztere besaßen nicht nur eine exotischere Strahlkraft als die nur regional bekannten ländlichen norddeutschen Spirituosen, sondern vor allem auch ein um ein Vielfaches höheres Marketingbudget. ${ }^{824}$ Somit war das Segment der Premiumspirituosen für die deutschen Traditionsmarken bereits zu einem frühen Zeitpunkt versperrt.

819 Vgl. B631030SP.

820 Vgl. B891218NZ. Betrug der kumulierte Umsatz 1988 noch 648 Mio. DM, so waren es konsolidiert im Jahre 1990 nur noch 583 Mio. DM, wozu König \& Schlichte 70 Mio. DM beisteuerten.

821 Vgl. Brendel \& Oberender (1981: 132); B920201HA.

822 Vgl. Berekoven (1981: 29).

823 Brendel \& Oberender (1981: 130) konstatiert hier eine Inflexibilität des Verhaltens der mehrheitlich konservativen Unternehmer »aus Gründen der Pflege des Images ihres Unternehmens und ihrer Produkte«.

824 Vgl. Berekoven (1981: 29); Brendel \& Oberender (1981: 130). 
Stattdessen haftete Korn bereits ab Anfang der 1980er-Jahre ein zunehmend negatives Image an und wurde mit »Billigschnaps« assoziiert. ${ }^{825}$ In der Folge tranken jüngere Konsumenten nicht nur allgemein weniger Spirituosen, sondern griffen vor allem auch immer seltener zu den klassischen deutschen Spirituosen wie Korn. Diese Entwicklung führte bald zu einer Überalterung der Konsumentenstruktur der deutschen Spirituosenhersteller. ${ }^{826}$

Da klare Spirituosen darüber hinaus nur wenige Möglichkeiten zur geschmacklichen Differenzierung bieten, griffen die Verbraucher immer stärker gleich auf die deutlich günstigeren Handelsmarken zurück, denn diese waren im Gegensatz zum traditionsreichen »Billigschnaps" auch tatsächlich preiswert, weil sie in erster Linie über den Preis konkurrierten und hierauf ihre gesamte Wertschöpfungskette ausgerichtet hatten.

Trotz dieser früh einsetzenden und sich über einen langen Zeitraum stetig vollziehenden negativen Umweltdynamik hielt die Berentzen-Gruppe im Rahmen ihrer "klar definierten, überzeugenden Strategie» viele Jahre an der Akquisition deutscher Traditionsmarken fest. ${ }^{827}$ Nach dem Börsengang 1994 wurde der Kauf traditioneller deutscher Spirituosenhersteller mit der Übernahme von Strothmann (1996) und Dethleffsen (1998) trotz der negativen Marktentwicklung sogar noch einmal forciert. ${ }^{828}$

"[...] dass sie eben durch diese, durch den Börsengang entsprechende Mittel zur Verfügung hatten, die sie jetzt wiederum nicht in die alte Marke investiert haben, um die abzusichern, sondern in neue Firmen, die sie gekauft haben. [...] diese Firmen, die sie gekauft haben, das waren natürlich Firmen, die waren schon im Abschwung." (Ehemaliges Mitglied des Aufsichtsrates der Berentzen-Gruppe AG) ${ }^{829}$

Infolge dieser Übernahmen stiegen zwar Umsatz und Marktanteile der Berentzen-Gruppe, doch bald zeigten sich auch die hohen Kosten der Übernahmen. So stiegen die Kosten im Marketing von 66 Mio. DM im Jahr 1994 auf knapp 118 Mio. DM im Jahr 1997, da die vielen erworbenen Marken jeweils separat

825 Berekoven (1981) spricht hier in einer Beurteilung der Lage der deutschen Spirituosenindustrie im Auftrag der Doornkaat AG früh von einer »Image-Tragik» für die deutschen klaren Spirituosen.

826 BSI (2012: 49).

827 Vgl. B000622NZ; B920101AW.

828 Vgl. B981229NZ; B920101AW: "Jan B. Berentzen: ‘[...] weil erfolgreiche Innovationen in dieser traditionsgeprägten Genusswelt so selten sind, scheint es uns in der Regel sinnvoller und auch preiswerter zu sein, selbst auf den ersten Blick teure Unternehmen und damit Marken zu kaufen oder Beteiligungen daran zu erwerben."

829 Vgl. B061218FT: »Berentzen hat Marken zusammengerafft, die einfach zu haben waren, weil ihre Hersteller bereits angeschlagen waren<, sagt der Chef eines Konkurrenten. `Im Verbund werden die Marken aber nicht automatisch wertvoller.« 
gepflegt und beworben werden mussten. ${ }^{830}$ Die Pflege der kontinuierlich zugekauften Traditionsmarken stand hinsichtlich der Ressourcenverteilung somit in zunehmender Konkurrenz zu alternativen strategischen Ansätzen wie der Internationalisierung der Kernmarken. Vor allem belasteten zunehmend hohe Abschreibungen auf übernommene und bilanzierte materielle und immaterielle Vermögenswerte das Ergebnis der Berentzen-Gruppe. ${ }^{831}$ Sie stiegen innerhalb weniger Jahre von 5,5 Mio. Euro auf 19 Mio. Euro im Jahr 1999. Insbesondere die abnehmende Werthaltigkeit der Marken belastete hier die Bilanz und später auch das Eigenkapital.

»Eine Mehrheitsbeteiligung an Doornkaat, ein Mehrheitserwerb von König \& Schlichte oder auch der Totalerwerb eines regional tätigen Unternehmens wie Ammerländer bedeuten Investitionen von jeweils deutlich zweistelligen Millionenbeträgen. Insoweit ist die Frage, ob man sich als mittelständisches Unternehmen daran verschlucken kann, berechtigt."

(Der Sprecher des Vorstands Jan B. Berentzen in einem Interview mit der Zeitschrift Absatzwirtschaft im Jahr 1991) $)^{832}$

Neben der fortschreitenden Polarisierung des Verbraucherverhaltens zwischen billigen Handelsmarken einerseits und exklusiven, internationale Markenprodukten andererseits, in deren Folge der Absatz bei rückläufigen Preisen immer weiter sank, zeichneten sich zunehmend auch Kannibalisierungseffekte zwischen den verschiedenen Marken innerhalb derselben Segmente ab.

"Sie haben auch nie geschafft in den Jahren, bei den sogenannten, die haben das dann Klassiker genannt, die klassische Marken, so wie Strothmann, Hansen Rum, Doornkaat [...] die wieder nach oben, nach oben zu bringen. Ja. Also haben die das, das- Da haben sie sich eigentlich auch gar nicht drum gekümmert, das, das ging einfach nach unten, ne. Und hat den, hat den, sozusagen den Deckungsbeitrag oder den Ertrag weggefressen. So dass sie immer mehr in Schwierigkeiten kamen."

(Ehemaliges Vorstandsmitglied der Berentzen-Gruppe AG)

Trotz dieser negativen Entwicklungen wurde im Jahr 2003 sogar noch versucht die deutlich größere Spirituosensparte der Eckes AG mit ihren Traditionsprodukten wie Eckes Edelkirsch, Mariacron oder Nordhäuser Doppelkorn zu übernehmen. Diese Marken verzeichneten zu diesem Zeitpunkt jedoch ebenfalls seit längerer Zeit sinkende Absatzzahlen. ${ }^{833}$

830 Erst nach dem ersten Verlust des Jahres 1997 wurden die Marketing- und Vertriebsausgaben dann wieder auf 70 Mio. DM reduziert.

831 Vgl. die Geschäftsberichte der Berentzen-Gruppe AG. 1994: 10,8 Mio. DM; 1995: 16,9 Mio. DM; 1996: 20,3 Mio. DM; 1997: 26,1 Mio. DM; 1998: 24,4 Mio. DM; 1999: 36,9 Mio. DM.

832 Vgl. B920101AW.

833 Vgl. B030513LZ. 
"Was wollen wir denn mit noch einem Eckes Edelkirsch um Gottes Willen. Dass die Eckes das verkaufen wollen, verstehe ich total. Dass die sich lieber auf hohes C, oder was sie sonst haben, konzentrieren, wunderbar. Aber ich kann jetzt nicht so richtig nachvollziehen, warum wir unter den veränderten Bedingungen, die ich eben genannt hab, unser Glück jetzt darin suchen sollen, Zinnaer, Klostertaler, oder Ammerländer Löffelschluck und so weiter zu kaufen. Eckes Edelkirsch, zu dem Zeitpunkt schon, war eine alte Tante, die in den Stift gehört. Jetzt letzter Lebensabschnitt, Heim für betreutes Wohnen, das war damals unsere Vorstellung jedenfalls von dieser Marke.«

(Ehemaliges Aufsichtsratsmitglied der Berentzen-Gruppe AG)

Erst nach diesem gescheiterten Akquisitionsversuch verkündete die Berentzen-Gruppe eine Abkehr vom etablierten strategischen Muster in diesem Geschäftsfeld. Doch obgleich man sich nun von einem »eher deutsch geprägten Spirituosenhersteller zu einem Vermarkter mit internationalem Zuschnitt« wandeln wollte, erfolgte keine klare strategische Abkehr von diesen deutschen Traditionsmarken. ${ }^{834}$

Daher kann die strategische Entwicklung dieses Geschäftsfelds der Berentzen-Gruppe AG insbesondere im Zeitraum 1988 bis 2008 ebenfalls als weitgehend konstant betrachtet werden.

\section{Internationale Lizenzmarken}

Im Zuge der eben dargestellten Fokussierung auf deutsche Traditionsmarken hatte sich die Berentzen-Gruppe AG Anfang der 1990er-Jahre von den im Portfolio befindlichen Importmarken getrennt.

"Das zweite, das zu streichen ist, sind Artikel, die gegen die Zielrichtung stehen, dem Handel gegenüber Ansprechpartner Nummer $1 \mathrm{zu}$ sein für ein deutsches Sortiment. Wir haben sämtliche Importmarken abgegeben.«

(Der Sprecher des Vorstands Jan B. Berentzen in einem Interview mit der Zeitschrift Absatzwirtschaft im Jahr 1992) $)^{835}$

Erst nachdem die akquirierten Traditionsmarken über viele Jahre konstant rückläufige Ergebnisse erzielt hatten, wurde sich ab dem Jahr 2004 unter dem Motto der "world of fine drinks« wieder verstärkt diesen internationalen Lizenzmarken zugewandt.

"Die Berentzen-Gruppe beabsichtigt daher sich von einem eher deutsch geprägten Spirituosenanbieter zu einem Vermarkter mit internationalem Zuschnitt zu wandeln,

834 Vgl. Berentzen (2004: 4): "Große deutsche Traditionsmarken, wie Doornkaat oder Strothmann, sollen im Sortiment durchaus noch sihren Stellenwert behalten`.» Allerdings liefen diese mit sinkenden Absätzen und Umsätzen größtenteils einfach weiter mit. Hierzu ein ehemaliger Vorstand: "Aber, aber die Klassiker, die haben sie einfach vernachlässigt. Die haben sich nicht drum gekümmert.« Vgl. auch Berentzen (2005: 18): »Die `Klassiker entwickelten sich überwiegend parallel zur Tendenz in ihren Marktsegmenten.»

835 B920101AW. 
um so die rückläufige Entwicklung des Massenmarktes in deutschen Traditionssegmenten zu kompensieren."

(Die Berentzen-Gruppe AG im Konzernlagebericht 2003 zur zukünftigen strategischen Ausrichtung) $)^{836}$

So wurden die Marken Linie Aquavit und Licor 43 in das Portfolio aufgenommen, ebenso die Biermarke Bud und die Weinmarke Yellow Tail. Die Vermarktung dieser Marken wurde dabei durch die Übernahme der Ende 2003 akquirierten Columbus Getränke $\mathrm{GmbH}$ gestützt. ${ }^{837}$

Diese Versuche wurden jedoch mit einem relativ geringen Ressourceneinsatz durchgeführt und wiesen trotz einiger sehr profitabler Beteiligungen wie jener an Linie Aquavit nie ein signifikantes Umsatzniveau auf, welches die Rückgänge im Kerngeschäft der Spirituosen hätte kompensieren können. ${ }^{838}$ Dieser vom etablierten strategischen Muster abweichende strategische Versuch blieb somit in den Anfängen stecken.

\section{Kernmarken}

Das Geschäftsfeld der Kernmarken umfasste die Gruppe der fruchtigen Spirituosen um die Kernmarke Berentzen und die Wodka-Marke Puschkin, die 1989 akquiriert worden war.

Hinsichtlich dieses Geschäftsfelds konzentrierte sich die Marktbearbeitung vor allem auf die Marke Berentzen. Nach dem Abflauen der ersten Erfolgswelle Mitte der 1980er-Jahre wurde hier über Jahrzehnte in zyklischen Abständen die Marktbearbeitung in Marketing und Vertrieb intensiviert, um den relativen Marktanteil wieder zu erhöhen, und wieder zurückgefahren, um Kosten zu sparen. ${ }^{839}$

Zudem wurden vom Sauren Apfel bis zum Winterapfel verschiedenste Variationen des etablierten Sortiments auf den Markt gebracht, um das Kernsegment der fruchtigen Spirituosen zu beleben. Diese Geschmacksvariationen sorgten jedoch meist nur für eine kurzfristige Belebung und konnten sich ohne die anfangs stets hohe marketingtechnische Unterstützung in den seltensten Fällen dauerhaft am Markt etablieren.

836 Berentzen (2003: 8f.).

837 Vgl. Berentzen (2003:9ff.); Berentzen (2005: 18).

838 Hierzu ein ehemaliger Vorstand: "Aber dieses Yellow Tail, das waren so Versuche, die man nebenbei her... ich hab es ja als Hobby gesehen, ganz ehrlich, aber die hätten das Unternehmen aus meiner Sicht nicht weitergebracht, weil die Kernkompetenz war und ist Spirituose [...], wenn sie Weine machen, oder Sekt, oder Champagner, brauchen sie ein ganz anderes Kompetenzumfeld, eine ganz andere Zielgruppe, ganz andere Absatzkanäle [...], hat aber auch nur verantwortbare Ressourcen beansprucht, dieser Versuch."

839 Vgl. Berentzen (2000: 5); Berentzen (2007: 17). 
»[...] ein Fehler nach dem anderen. Dann, was machen wir denn jetzt, äh Saurer Apfel war letztes Jahr, jetzt bringen wir mal die Saure Johannisbeere. Und dann fangen Sie an, in >Diversifikation` zu ticken und zu denken, setzen Ihre gesamte Forschung und Entwicklung darauf, diversifizierte Produkte aus dem Kernprodukt Berentzen Apfelkorn abzuleiten. Und am Ende ist hier der Marketingtopf, das Marketinggeschehen, das zu überproportional großen Teilen für diese Neueinführung, und die, die nennt man dann Innovationen. Ja? Also Sie nehmen immer eine andere Fruchtidee, und setzen die mit Berentzen in Zusammenhang [...] Ja, dann gibt es mal kleine Impulse, die gehen hoch, aber Sie konnten sehen, in dem Moment, wo die Werbung ausgelaufen ist, oder auch nur halb ausgelaufen, buff, fällt es runter.«

(Ehemaliger Aufsichtsrat der Berentzen-Gruppe AG)

Hinter diesen Maßnahmen der Marktdurchdringung blieb die Entwicklung wirklich neuartiger Produkte zurück. Zwar wurden vereinzelt neue Produkte entwickelt, doch spätestens nach dem Misserfolg mit den Alkopops 1997 war die Berentzen-Gruppe hier deutlich zurückhaltender. ${ }^{840}$

»Und dann hat man in der Folge sich ja weniger auf neue Produkte konzentriert sondern mehr darauf noch mal, sage ich mal, Wettbewerb fahren [...] Portfolio zu übernehmen, wie dann Ende der Neunziger Dethlefsen, die auch eher so Dinge wie Bommerlunder und, und Co. an Bord hatten. Und wenn man dann, ich sag mal, sich so grob jetzt mal unabhängig vom Unternehmen, sondern eher so die Landkarte anschaut, wohin entwickeln sich Trends und wie sind auch Trends so langfristig zu sehen, muss man heute sagen, die, die deutsche Spirituose zählt sicherlich seit Jahren nicht mehr zu den Trendprodukten, und man hat sich dann damit eher, ich sag mal, ein Stückweit Ballast an Bord geholt."

(Ehemaliges Mitglied der Geschäftsführung)

Hinter den Bemühungen um die Marke Berentzen und das Sortiment der fruchtigen Spirituosen trat die zweite Kernmarke Puschkin stets etwas zurück. Die Erfolge eines kompletten Marken-Relaunchs ab 1994 samt der Einführung von Softspirituosen auf Wodka-Basis ebbten bald wieder ab und insgesamt konnte die ehemals führende Wodka-Marke Deutschlands nur unterdurchschnittlich vom Wodka-Boom profitieren. ${ }^{841}$

Hinsichtlich der Internationalisierung dieser beiden Kernmarken wurde insbesondere mit der Kernmarke Berentzen seit Ende der 1970er-Jahre kontinuierlich und in moderaten Tempo auch die Entwicklung neuer Märkte im Ausland verfolgt. Im Vergleich zu den Investitionen in die Strategie der

840 Hierzu ein ehemaliges Mitglied des Vorstands: «[E]s wurde die Linie verfolgt, die Marken weiter werbetechnisch extrem nach vorne zu bringen. Das hat aber nicht zu dem Erfolg geführt. [...]. Die Marke hat einen hohen Bekanntheitsgrad gehabt, da war eigentlich nicht mehr was rauszuholen. Man hätte eigentlich schon sich überlegen müssen, welche artverwandten Produkte kann man in neue Märkte etablieren. Das [...] ist nicht gemacht worden.«

841 Vgl. B940708LZ zum Marken-Relaunch 1994 mit Marken wie Puschkin Black Sun. 
Marktdurchdringung wurden diese strategischen Vorstöße jedoch mit relativ geringen Ressourcen unterstützt. So stieg in der Folge der Exportanteil am Absatz zwar moderat, aber stetig an und betrug $199810 \%$, 2003 gar 17,8 \%. ${ }^{842}$ Diese steigende Exportquote war jedoch nicht nur auf die Erfolge der Internationalisierungsstrategie zurückzuführen, sondern eben auch auf den stetig sinkenden Inlandsumsatz. In absoluten Zahlen betrachtet relativierte sich der Erfolg der Internationalisierung: Während der Auslandsabsatz im Zeitraum 1999 bis 2006 von 13,8 Mio. Flaschen um nicht einmal 6 Mio. Flaschen auf 19,3 Mio. Flaschen stieg, sank der Inlandsabsatz im gleichen Zeitraum dramatisch um 55 Mio. Flaschen von 128,1 Mio. auf 73,1 Mio. Flaschen. ${ }^{843}$

Erst unter dem neuen Eigentümer wurden gezielt Auslandsmärkte wie die Türkei oder China erschlossen, um die Abhängigkeit von Inlandsmarkt zu reduzieren. ${ }^{844}$

\section{Fazit zur strategischen Persistenz}

Ordnet man die strategischen Aktivitäten der verschiedenen Geschäftsfelder der Berentzen-Gruppe AG in die Produkt/Markt-Matrix der Wachstumsstrategien ein und gewichtet den zugrunde liegenden Ressourceneinsatz, so ergibt sich folgendes Bild. ${ }^{845}$

\begin{tabular}{|c|c|c|c|c|}
\hline Produkt & \multirow{2}{*}{\multicolumn{2}{|c|}{ bestehende }} & \multirow{2}{*}{\multicolumn{2}{|c|}{ neue }} \\
\hline Markt & & & & \\
\hline \multirow[t]{2}{*}{ bestehende } & $\begin{array}{c}\text { Marktdurchdringung } \\
\text { Erwerb deutscher Traditionsmarken }\end{array}$ & +++ & \multirow[t]{2}{*}{$\begin{array}{l}\text { Produktentwicklung } \\
\text { Alkopops (1996/97) }\end{array}$} & \multirow[t]{2}{*}{+} \\
\hline & Konzentration auf Kernmarken & ++ & & \\
\hline neue & $\begin{array}{c}\text { Marktentwicklung } \\
\text { Internationale Lizenzmarken } \\
\text { Expansion ins Ausland }\end{array}$ & $\begin{array}{l}+ \\
+\end{array}$ & $\begin{array}{c}\text { Diversifikation } \\
\text { alkoholfreie Getränke }\end{array}$ & o \\
\hline
\end{tabular}

Abbildung 21: Die Geschäftsfelder der Berentzen-Gruppe AG im Rahmen der Produkt/MarktMatrix

(Quelle: Eigene Darstellung).

Zusammenfassend lässt sich somit festhalten, dass die Berentzen-Gruppe seit Mitte der 1970er-Jahre bis Mitte der 2000er-Jahre kontinuierlich ein strategi-

842 Vgl. B790929NZ; B851026NZ zu den Anfängen der Internationalisierung. Bis 1985 steigt der Exportanteil - maßgeblich bedingt durch den Erfolg des Apfelkorns - auf 8 \%. Vgl. Berentzen (2003: 9).

843 Vgl. die Angaben in den Geschäftsberichten Berentzen (1999) bis Berentzen (2006).

844 Allerdings mit gemischten Ergebnissen; vgl. B140325HB; B121221MA.

845 Vgl. Ansoff (1988: 109); Welge \& Al-Laham (2012: 591). 
sches Muster verfolgte, welches grundlegend auf der Idee des »Wachsens oder Weichens« beruhte.

Vor dem Hintergrund dieser Idee wurde eine entsprechend offensive Gesamtunternehmensstrategie verfolgt, die konsequent und beharrlich vor allem auf der Durchdringung bestehender Märkte mit bestehenden Produkten beruhte. Diese Strategie der Marktdurchdringung basierte zunächst auf der Kernmarke Berentzen und dem Sortiment der fruchtigen Spirituosen, ab Ende der 1980er-Jahre jedoch vor allem auf der Akquisition anderer traditioneller deutscher Unternehmen der Spirituosenindustrie und deren Marken. Auf die Akquisitionen wurde ein großer Teil der verfügbaren Ressourcen verwandt. Hinter diesen strategischen Aktivitäten blieben Versuche der Internationalisierung mittels Vertrieb von internationalen Lizenzmarken oder die Entwicklung neuer Märkte mit den eigenen Kernmarken ebenso deutlich zurück wie die Ansätze zur Diversifikation mit der Sparte der alkoholfreien Getränke. Diese wurde bewusst nicht entscheidend gestärkt.

An dieser strategischen Konfiguration und Entwicklungsrichtung wurde bis zum Verkauf an den neuen Eigentümer Aurelius beinahe unverändert festgehalten. Da diese strategische Kontinuität jedoch in einem immer stärkeren Kontrast sowohl zur allgemeinen Dynamik der Branchenumwelt, als auch der negativen finanziellen Entwicklung des eigenen Unternehmens stand, kann die festgestellte Kontinuität als eine Form von strategischer Persistenz interpretiert werden.

»Erstaunlich ist, dass das sich dann über Jahre entwickelt hat, fünf, sechs, sieben Jahre, und keiner hat was getan. [...] Keiner hat etwas getan. Man konnte das sehen. Ich hab mir die Zahlen als ich kam mal angeguckt, der letzten Jahre, und es ging wirklich so nach unten. Bumm! Da muss man sich fragen, warum hat da keiner gegengehalten? Warum hat in den Jahren davor keiner was getan? Und, und wenn du dich nicht wehren kannst, alles wiederholst, dann musst du das abschneiden, ne. Also dann musst du es cutten. Aber du kannst es doch nicht einfach zugucken.»

(Ehemaliges Vorstandsmitglied der Berentzen-Gruppe AG)

Im folgenden Abschnitt wird aufgezeigt, dass diese strategische Persistenz vor allem auf die Wirkkräfte eines selbstverstärkenden Mechanismus und den daraus resultierenden Lock-in der strategischen Entwicklung zurückzuführen war.

\subsubsection{Pfaddiagnose}

Im Zuge der Fallanalyse der Berentzen-Gruppe AG zeigte sich, dass für deren dargestellte strategische Persistenz vor allem ein sich verschärfender Konflikt im Gesellschafterkreis ausschlaggebend war. Denn dieser Konflikt begann als sich selbst verstärkender sozio-emotionaler Mechanismus zunehmend die weitere 
strategische Entwicklung des Unternehmens zu bestimmen und trug maßgeblich zur Aufrechterhaltung und Reproduktion des etablierten strategischen Musters bei.

Im Folgenden wird daher zunächst das »critical juncture» skizziert, welches diesen Mechanismus auslöste, bevor anschließend die selbstverstärkende Wirkkraft des Konflikts aufgezeigt wird, die das Unternehmen zunächst in den strategischen Lock-in und schlussendlich in die Unternehmenskrise führte.

\subsubsection{1 Der Konflikt als sich selbst verstärkender sozio-emotionaler Mechanismus} Ebenso wie bei der Anton Schlecker e.K. lassen sich für die in den obigen Abschnitten dargestellte strategische Persistenz auch bei der Berentzen-Gruppe AG verschiedene Erklärungsansätze ins Feld führen.

So wurde der strategische Entscheidungsraum früh und nachhaltig durch verschiedene Aspekte des sozio-emotionalen Vermögens der Eigentümerfamilien bedingt. Beispielsweise spielten hinsichtlich der strategischen Frage, ob die relativ unproduktive Produktion am traditionellen Standort Haselünne geschlossen wird oder nicht, auch die Einbettung der Unternehmensfamilie in lokal-soziale Zusammenhänge und das Streben nach einer Bewahrung des transgenerationalen familiären Erbes eine bedeutende Rolle. Diese Imprints, die in engem Zusammenhang mit dem familiären Einfluss auf das Unternehmen standen, werden daher fallübergreifend in Teil D behandelt.

Die Analyse der strategischen Entwicklung und der strategischen Entscheidungsprozesse bei der Berentzen-Gruppe AG zeigt jedoch deutlich, dass es grundlegend ein selbstverstärkender Mechanismus war, der dazu führte, dass es das Unternehmen unter den Eigentümerfamilien nicht mehr vermochte, den eingeschlagenen Entwicklungsweg zu verlassen und sich strategisch zu wandeln.

Im Gegensatz zu dem auf Skalenerträgen beruhenden Mechanismus bei der Anton Schlecker e.K. beruhte dieser Mechanismus jedoch nicht auf ökonomischen Rückkopplungen, sondern auf der sozio-emotionalen Dynamik eines eskalierenden Konflikts innerhalb der dominanten Koalition.

Hinsichtlich solcher Dynamiken wiesen Schreyögg et al. (2003) im Rahmen der konzeptionellen Übertragung des klassisch-ökonomischen Pfadkonzepts auf den organisationalen Kontext bereits früh darauf hin, dass sich selbst verstärkende sozio-emotionale Prozesse zu einem Lock-in organisationaler Entwicklungen führen könnten. ${ }^{846}$ Allerdings wurden solche sozialen Dynamiken im Gegensatz zu den ausführlich erörterten nutzenorientierten Dynamiken von der organisationalen Pfadforschung in der Folge kaum näher untersucht oder thematisiert. Daher soll im Zuge der Darstellung der Konfliktdynamik bei der

846 Vgl. Schreyögg et al. (2003: 270f.): Die »Dynamik von Emotionen« und »mikropolitische Prozesse« könnten zu Pfadabhängigkeiten führen. 
Berentzen-Gruppe AG der Konflikt in Anknüpfung an die bestehende Forschung zu Konflikten ausführlicher als ein spezifischer sich selbst verstärkender sozioemotionaler Mechanismus in den theoretischen Bezugsrahmen der organisationalen Pfadtheorie eingeführt werden. Dabei knüpft die Darstellung insbesondere an das Modell der stufenförmigen Konflikteskalation nach Glasl (2013) an, welches die selbstverstärkende Wirkkraft der sozio-emotionalen Dynamik deutlich herausarbeitet. ${ }^{847}$

Der selbstverstärkende Charakter von Konflikten kommt jedoch bereits im systemtheoretischen Konfliktverständnis zum Ausdruck.

»Es ist ebenfalls ganz normal, dass es [...] zu Äußerungen kommt, die vorausgesetzten oder (und das wird schon heikler) geäußerten Erwartungen widersprechen. Zur Störung der Kommunikation wachsen sich solche Bagatellvorwürfe aus, wenn auf ein Nein mit einem Gegennein geantwortet wird; denn das bringt die Versuchung mit sich, beim Nein zu bleiben und das Nein auf beiden Seiten durch weitere Kommunikation zu verstärken. In einem solchen Falle wollen wir von Konflikt sprechen.«

(Luhmann, 1993) ${ }^{848}$

Konfliktäre Prozesse sind somit dadurch charakterisiert, dass in ihrem Verlauf eine bestimmte Position, eine Meinung oder eine Handlungsanweisung verneint wird und diese Negation wiederum verneint wird. Die Oszillation zwischen zwei Positionen, die sich gegenseitig negieren, erhöht durch das beständige Aufeinander-Bezug-Nehmen der Negationen und der Negationen der Negationen die Wahrscheinlichkeit der Fortsetzung der Kommunikation. ${ }^{849}$ Jede Partei möchte nun das »letzte Wort« haben. Somit bieten Konflikte ein stabiles Fundament für die Fortsetzung des Konflikts, solange es zu keiner Entscheidung kommt. Der Konflikt bindet die Konfliktparteien durch Kommunikation und treibt sich beständig selbst an. Die systemtheoretisch orientierte Konflikttheorie spricht hier auch von der Autopoiesis des Konflikts.

"Als soziale Systeme sind Konflikte autopoietische, sich selbst reproduzierende Einheiten. Einmal etabliert, ist ihre Fortsetzung zu erwarten und nicht ihre Beendigung.» (Luhmann, 1984) $)^{850}$

In diesem Zuge erhalten sich viele Konflikte nicht nur, sondern verstärken sich auch selbst. Dieser Konflikten inhärente Charakter der Reproduktion und Selbstverstärkung kommt insbesondere im weitrezipierten Stufenmodell der

847 Auf diesem Wege wird auch der Forderung der Pfadtheorie entsprochen, der zufolge bei der Referenz sozio-emotionaler Prozesse stets geprüft werden müssen, ob denn eine selbstverstärkende Wirkkraft im Sinne der Pfadtheorie vorliege. Vgl. Schreyögg et al. (2003: 271).

848 Luhmann (1993: 565); vgl. auch Simon (2012: 13).

849 Vgl. Luhmann (1984: 530); auch Simon (2012: 11).

850 Luhmann (1984: 537). Konflikte können durch diese Form der Selbsterhaltung über sehr lange Zeiträume, gar über Generationen andauern. 
Konflikteskalation nach Glasl (2013) zum Ausdruck. ${ }^{851}$ Durch dieses Modell wird beschrieben, wie eine dem Konflikt inhärente Dynamik dazu führen kann, dass der Konflikt sich über neun Phasen immer weiter beschleunigt und schlussendlich in der totalen Eskalation mündet. ${ }^{852}$

Anhand dieser Stufen soll nun im Folgenden die selbstverstärkende Dynamik des Konflikts innerhalb der dominanten Koalition der Berentzen-Gruppe AG nachgezeichnet werden.

\section{»Critical juncture«}

Seinen Ausgangspunkt nahm der selbstverstärkende Mechanismus des Konflikts im Nachfolgeprozess in den Jahren nach der Fusion 1988 zwischen der J. B. Berentzen und Pabst \& Richarz zur Berentzen-Gruppe. ${ }^{853}$

Nach einigen Jahren professioneller Zusammenarbeit kam es in dieser Zeit zu ersten Auseinandersetzungen hinsichtlich der Frage, wer dem langjährigen Vorstandsvorsitzenden Hans Berentzen in dessen Position nachfolgen sollte. Die Familien Pabst und Richarz hielten den von den Familienstämmen Hans und Friedrich Berentzen vorgeschlagenen Sohn von Hans Berentzen, Jan B. Berentzen, für nicht ausreichend qualifiziert und lehnten ihn als neuen Vorstandsvorsitzenden ab. Dessen ungeachtet setzten die beiden Berentzen-Stämme Jan B. Berentzen schlussendlich als neuen Vorstandsvorsitzenden durch. ${ }^{854}$

"Können wir ihm das mittlerweile ja doch ziemlich groß gewordene Unternehmen anvertrauen? Das geht nicht, da muss ein anderer her. Und da zieht, passierte es immer, oder häufig dasselbe, dass dann die Emotion anfängt zu wirken. Dann geht man von der rationalen Ebene runter. Und dann geht es darum, wie können wir das durchsetzen? Und da plötzlich kommt dann die andere Seite und erklärt, Verwaltungsrat, papperlapapp, es gibt kein imperatives Mandat. Und wir haben hier eine Stimmenmehrheit

851 Vgl. Glasl (2013: 235 ff.). Dieses Modell der Konfliktforschung findet auch Eingang in die Lehrbücher der Managementtheorie; vgl. Steinmann \& Schreyögg (2005: 636).

852 Es ist dabei wichtig zu betonen, dass nicht jeder Konflikt alle diese Abwärtsstufen durchläuft und im Abgrund endet. Das Modell dient eher dazu, aufzuzeigen, wohin Konflikte führen können, wenn nicht eingegriffen wird. Vgl. Steinmann \& Schreyögg (2005: 636). Für eine Darstellung solcher eskalierenden Dynamiken im Kontext von Familienunternehmen vgl. auch Großmann (2014: 192ff.).

853 Von der Literatur zu Familienunternehmen wird der Nachfolgeprozess als eine der bedeutendsten Ursachen für Konflikte erachtet. Vgl. Levinson (1971); Harvey \& Evans (1994). von Schlippe \& Kellermanns (2008: 48): "Wohl keine Situation ist so kritisch wie die Nachfolge in Familienunternehmen." Großmann (2014: 193 ff.)Großmann (2014)Großmann (2014)Großmann (2014)Großmann (2014)Großmann (2014)Großmann (2014) verweist in seiner Studie auf die Bedeutung solch »einschneidender Ereignisse« für die Entwicklung von Konflikten.

854 Vgl. hierzu die Aussage eines ehemaligen Aufsichtsrates: „In der Vergangenheit waren die beiden Berentzen-Stämme verbündet und die hatten dann den Doktor Jan-Bernd Berentzen in den Vorstandsvorsitz gebracht. Pabst \& Richarz war das immer ein Dorn im Auge.» 


\begin{tabular}{|c|c|c|c|}
\hline Stufe & Bezeichnung & Charakteristika & Wichtige Schwellen \\
\hline 1 & Verhärtung & $\begin{array}{c}\text { Einander konträr } \\
\text { gegenüberstehende Standpunkte, } \\
\text { die sich zunehmend verhärten. } \\
\text { Noch keine starren Lager und } \\
\text { Meinungen. }\end{array}$ & $\begin{array}{c}\text { Ebene der rein } \\
\text { sachlichen } \\
\text { Auseinandersetzung }\end{array}$ \\
\hline 2 & $\begin{array}{l}\text { Debatte und } \\
\text { Polemik }\end{array}$ & $\begin{array}{l}\text { Macht und Selbstbehauptung } \\
\text { werden bedeutsamer. Erste } \\
\text { taktische Vorstöße. Aktionen } \\
\text { führen zu Reaktionen. Es bilden } \\
\text { sich Subgruppen; Standpunkte } \\
\text { verhärten sich weiter. }\end{array}$ & Einseitige Handlungen \\
\hline 3 & $\begin{array}{l}\text { Taten statt } \\
\text { Worte }\end{array}$ & $\begin{array}{l}\text { Gefühl: Reden hilft nicht mehr - } \\
\text { jetzt müssen Taten folgen. Keine } \\
\text { Partei möchte nachgeben; } \\
\text { Kontrahenten sollen einsichtig } \\
\text { werden. }\end{array}$ & \\
\hline 4 & $\begin{array}{l}\text { Gewinnen } \\
\text { oder verlieren }\end{array}$ & \begin{tabular}{|c|} 
Es bilden sich Klischees, der \\
»Gegner« wird zum »Feind«. Es \\
wird zunehmend um Anhänger und \\
Mitstreiter geworben. Koalitionen \\
verfestigen sich.
\end{tabular} & \\
\hline 5 & Gesichtsverlust & $\begin{array}{c}\text { Öffentliche Bloßstellung. } \\
\text { Diffamierung des Gegenübers. }\end{array}$ & »Entscheidungsschlacht» \\
\hline 6 & Drohstrategien & $\begin{array}{l}\text { Drohungen und Gegendrohungen } \\
\text { eskalieren. }\end{array}$ & \\
\hline 7 & Scharmützel & $\begin{array}{l}\text { Begrenzte Attacken. Der »Feind» } \\
\text { wird immer mehr zur »Sache«. }\end{array}$ & \\
\hline 8 & Krieg & $\begin{array}{l}\text { Der »Feind« muss vernichtet } \\
\text { werden; das feindliche System soll } \\
\text { zugrunde gehen. }\end{array}$ & $\begin{array}{l}\text { Aufgabe der eigenen } \\
\text { materiellen und sozialen } \\
\text { Existenz }\end{array}$ \\
\hline 9 & $\begin{array}{l}\text { Gemeinsam in } \\
\text { den Abgrund }\end{array}$ & $\begin{array}{c}\text { Totaler Krieg. Der Feind soll auch } \\
\text { um den Preis der Selbstvernichtung } \\
\text { zerstört werden. }\end{array}$ & \\
\hline
\end{tabular}

Tabelle 9: Stufen der Konflikteskalation nach Glasl (2013)

(Quelle: Glasl (2013: 235ff.) und Steinmann \& Schreyögg (2005)).

und damit setzen wir jetzt unseren Mann durch [...] Ich will nur sagen, dass so etwas zu einer ersten großen Friktion führt, ist ja klar.»

(Ehemaliger Aufsichtsrat der Berentzen-Gruppe AG)

Trotz dieses ersten Konflikts fand der um Jan B. Berentzen und Jürgen Pabst formierte neue Vorstand eine konstruktive Ebene der Zusammenarbeit und verfolget mit der Unterstützung des Aufsichtsrats die Vision des »Wachsens oder Weichens«. 


\section{Stufe 1: Verhärtung}

Allerdings entwickelten sich innerhalb des Vorstands mit der Zeit in mehreren Sachfragen zunehmend konträre Standpunkte, die zum Teil persönlichen Überzeugungen und Ansichten entsprangen, aber auch aus den unterschiedlichen kulturellen Hintergründen der Familien und Unternehmen resultierten.

Diese unterschiedlichen persönlichen Ansichten und strukturell divergenten Perspektiven trugen dazu bei, dass im Laufe der Jahre immer häufiger entlang der gleichen Positionen Reibungen auftraten und die Akteure Widerspruch zunehmend als Resistenz erfuhren. ${ }^{855}$ Schritt für Schritt nahm so die anfängliche Offenheit und Unbefangenheit im gegenseitigen Umgang ab.

Da es oftmals nicht gelang, diese auftretenden Differenzen allein durch sachliche Auseinandersetzung und Diskussion zu überwinden, begann sich nach und nach das soziale Klima im Unternehmen zu wandeln.

\section{Stufe 2: Debatte und Polemik}

Im Laufe der Zeit reiften im Schatten sachlicher Differenzen Fragen von Prestige und Macht, Selbstbehauptung und Status zu bedeutsamen Punkten heran und erschwerten so früh das Nachgeben auf sachlicher Ebene.

In dieser angespannten Situation kam es immer häufiger zu intensiven verbalen Auseinandersetzungen. Obgleich die Beteiligten durchaus noch an einer Kooperation interessiert waren, führten die Streitigkeiten dazu, dass insbesondere im Topmanagement-Team die bestehenden Unterschiede zwischen den Akteuren immer stärker wahrgenommen wurden und die Beziehungen zunehmend als Konkurrenzsituation interpretiert wurden.

In diesem zunehmend kompetitiven Rahmen versuchten die Beteiligten nun mithilfe diverser Taktiken der eigenen Position und Partei einen Vorteil oder ein Übergewicht zu erarbeiten. Allerdings veranlassten solche »Vorstöße« die andere Seite meist nur dazu, das leicht gekippte Gleichgewicht durch »Gegenstöße» umgehend wiederherstellen zu wollen. Somit begann sich Schritt für Schritt ein zirkulärer Prozess zu etablieren, in welchem Aktion stets zu Reaktion führte. ${ }^{856}$

"Also letztlich haben wir alle Fehler bei dem einfachen Grundsatz gemacht, also wer die Balance verletzt, muss auch damit leben, dass andere reagieren. Also Aktion führt zu Reaktion, wie auf dem Schachbrett, und die Reaktion ist umso stärker, je stärker der eine oder die eine Seite empfindet, dass eben zu seinen Lasten die Balance, ja, das Gleichgewicht, mit dem alle leben können, verletzt wurde. Das ist eigentlich so, für mich so die Kernerfahrung, ja.«

(Ehemaliger Vorstand der Berentzen-Gruppe AG)

$855 \mathrm{Zu}$ generellen Merkmalen dieser Konfliktstufe vgl. Glasl (2013: 236ff.).

856 Diese Dynamik wird in der Konfliktliteratur bereits auf unterer Ebene als »Teufelskreis» beschrieben. Vgl. Glasl (2013: 249). 
Dabei hatte die Subtilität der Beeinflussungsversuche zur Folge, dass die Kommunikation in der Wahrnehmung der Akteure zunehmend als vieldeutig wahrgenommen wurde. ${ }^{857}$

Glasl (2013) grenzt diese Phase der Konflikteskalation dadurch ab, dass sich die Konflikte noch rein auf der Ebene der verbalen Kommunikation bewegen. ${ }^{858}$ Doch bei der Berentzen-Gruppe AG führte nicht zuletzt der steigende Druck auf die Akteure, der aus der Ende der 1990er-Jahre sich verschärfenden problematischen Unternehmensentwicklung resultierte, dazu, dass das Heil in Konflikten immer häufiger auch in der Handlung gesucht wurde.

\section{Stufe 3: Taten statt Worte!}

Nach und nach entstand bei den in den Konflikt involvierten Akteuren des Topmanagements das Gefühl, dass weitere Gespräche und Diskussionen weder zur Lösung der zwischenmenschlichen Differenzen, noch zur Lösung der Probleme des Unternehmens beitragen könnten. So nahmen die Akteure des Managements insbesondere im Licht der immer deutlicher zu Tage tretenden Krisensymptome verstärkt eine wechselseitige Blockade wahr.

"[...] und dann ging das ein halbes Jahr gut und dann gingen die Zwistigkeit wieder los. Ja, also wir blockierten uns dann wieder gegenseitig, Also wenn er sagte `Nein`, konnte der ganze Vorstand nichts machen. Und wenn wir sagten `Ja<, dann hat er eben auch `Nein, nein` gesagt oder umgekehrt. Wenn er `Ja`sagte, haben wir denn auch mal `Nein` gesagt. Also es war auf jeden Fall für das Unternehmen nicht, nicht besonders produktiv.«

(Ehemaliges Vorstandsmitglied der Berentzen-Gruppe AG)

Ab einem gewissen Punkt versuchten die Akteure dann, diese wahrgenommene Blockade nicht mehr allein durch Argumentation zu durchbrechen, sondern auch durch Handlungen.

Die Attraktivität von Handlungen nimmt Glasl (2013) zufolge mit zunehmender Dauer der Blockade im Vergleich zur Diskussion zu, können sie doch einseitig und autonom erfolgen und den Konfliktparteien so zumindest für den Moment das Gefühl verschaffen, die wechselseitige Abhängigkeit überwinden zu können. ${ }^{859}$

Vor dem Hintergrund einer solchen Motivlage versuchten die Familien-

857 Glasl (2013: 248f.) verweist darauf, dass im Zuge der so ansteigenden Komplexität die Akteure nun häufig beginnen im Verhalten der anderen Partei vermeintlich typische Denkweisen und Handlungen zu erkennen, um die Komplexität wieder zu reduzieren.

858 Vgl. Glasl (2013: 251): „Für die ersten beiden Eskalationsstufen war das Wort das wichtigste Mittel der Auseinandersetzung."

859 Vgl. Glasl (2013: 252), gemäß dem das Gefühl der wechselseitigen Blockade nun das Klima der Interaktion bestimmt. Vgl. auch Schelling (1958), der die Vorteile aktiven Handelns für das Autonomiegefühl der handelnden Partei aufzeigt. 
stämme Berentzen um die Jahrtausendwende dann Jürgen Pabst zum Rücktritt aus dem Vorstand zu bewegen.

»Der Streit währt bereits viele Jahre. Spätestens als Gesellschafter und Vorstand Jürgen Pabst Ende 2001 aus seinem Amt gedrängt worden war, sind die Fronten verhärtet.« (Die Lebensmittel Zeitung im Jahr 2005 zum Konflikt bei der Berentzen-Gruppe AG) ${ }^{860}$

Dieser Versuch der Autonomisierung zog nach sich, dass die verbale Kommunikation zwischen den Konfliktparteien und den Eigentümerfamilien drastisch abnahm. Da von den Eigentümerfamilien nur noch ein Stamm operativ im Unternehmen tätig war, entfiel die tägliche Interaktion deren Vertreter über die operative Arbeit im Unternehmen. In der Folge verlagerte sich der Schwerpunkt der Auseinandersetzung quasi zwangsläufig von der Arena des Topmanagements in die Sphären des Gesellschafterkreises und zunehmend auch in den Aufsichtsrat.

Eine solche Abnahme der Kommunikation führt gemäß Glasl (2013) in der Regel auch zu einer verstärkten kognitiven Kanalisierung der Akteure und des Konflikts, denn je stärker der direkte Austausch zwischen den Konfliktparteien abnimmt, desto mehr gewinnen die individuellen und kollektiven Interpretationen der noch verfügbaren Kommunikationsakte an Gewicht. ${ }^{861}$ Die noch verfügbare Kommunikation wird verstärkt auf »Untertöne« geprüft und entsprechend interpretiert. Die Richtigkeit und Aufrichtigkeit der Aussagen der Akteure der anderen Konfliktpartei wird grundsätzlich immer stärker bezweifelt. Der in diesem Zuge ansteigenden Komplexität der Kommunikation versuchen die Akteure daher mit einer kognitiven Komplexitätsreduktion zu begegnen: Einzelne Wahrnehmungen werden zu einem in sich stimmigen Gesamtbild verbunden und es beginnt sich ein stark kontrastiertes Stereotyp der anderen Partei zu entwickeln. Nach Eisenhardt \& Bourgeois (1988) nimmt in der Konsequenz dieser Stereotypisierungen bereits recht früh die Fähigkeit zur differenzierten Wahrnehmung und Empathie für die Anliegen und Gefühle der anderen Konfliktpartei ab. ${ }^{862}$

Dabei werden das Entstehen und die Wirkkraft solcher Stereotype durch die im Zuge des Konflikts zunehmende Kohäsion innerhalb der eigenen Gruppe maßgeblich befördert. ${ }^{863}$ Die Kohäsion nahm auch bei den Konfliktparteien der Berentzen-Gruppe AG immer mehr zu. Denn je mehr sich die Auseinandersetzungen um Personalien oder die strategische Entwicklung des Unternehmens intensivierten, desto mehr verstärkte sich innerhalb der Konfliktparteien ein

860 B050526LZ.

861 Vgl. Glasl (2013: $253 \mathrm{ff}$.$) .$

862 Vgl. Eisenhardt \& Bourgeois (1988: 763).

863 Vgl. Steinmann \& Schreyögg (2005: 658); ausführlich zu Sterotypen Tajfel \& Stroebe (1982). 
Gefühl der Zusammengehörigkeit. ${ }^{864}$ Zudem versprach ein Bezug auf die größere Gruppe auch eine Erhöhung der eigenen Handlungsmacht. In jedem Fall rückten die Gruppenmitglieder zunächst enger zusammen. ${ }^{865}$

Eine solche zunehmende Kohäsion führt in der Regel dazu, dass kritische Meinungen zunehmend nicht mehr geäußert werden und sich kollektiv dominante Interpretationen der Umwelt entwickeln. Diese können in diesem Stadium der Konflikteskalation an schwach ausgeprägte Formen von "group think« erinnern. ${ }^{866}$

Nicht zuletzt solche Effekte der zunehmenden Kohäsion innerhalb der Konfliktparteien trugen bei der Berentzen-Gruppe dazu bei, dass die Eigentümerfamilien immer weiter auseinander drifteten und ein immer stärker voneinander getrenntes Eigenleben zu führen begannen.

\section{Stufe 4: Gewinnen und Verlieren}

Durch diese zunehmende Separierung und die Herausbildung spezifischer Umweltinterpretationen und Dynamiken wurde das Verhältnis zwischen den Eigentümerfamilien kompetitiver. Die verbliebene kollaborative Orientierung an gemeinsamen ökonomischen Zielen und die sachliche Problemlösung traten zugunsten eines Denkens im Schema »Sieg und Niederlage« in den Hintergrund. Die eigenen Haltungen wurden nun aggressiver vertreten.

Gemäß Glasl (2013) beeinflusst die zunehmend feindselige Einstellung gegenüber der anderen Partei das bereits entwickelte stereotype Bild von der anderen Konfliktpartei. ${ }^{867}$ Das Stereotyp wird von bestimmten Verhaltensweisen auf generelle Eigenschaften wie die fachliche Kompetenz oder die Persönlichkeit ausgedehnt. Zugleich ist das Bild im Verlauf dieser Phase der Konflikteskalation bald so stark verankert, dass es nur noch sehr schwer durch andere Erfahrungen revidiert werden kann.

In der Folge überlagern solche Stereotype immer stärker die Interaktion zwischen den Konfliktparteien. In der Interaktion werden die der anderen Partei attribuierten Eigenschaften nicht selten erst durch das eigene Verhalten bewusst oder unbewusst evoziert und somit in der eigenen Wahrnehmung wiederum bestätigt. $^{868}$

864 Vgl. hierzu eine Aussage eines ehemaligen Aufsichtsrates: "Jetzt passiert dann immer das gleiche. Zunächst mal, Blut ist dicker als Wasser, pflocken die, ja, das heißt, die setzen sich zusammen, und verbünden sich.«

865 Vgl. Eisenhardt \& Bourgeois (1988); Pettigrew (1973).

866 Vgl. Glasl (2013: 252f.); grundlegend zum Phänomen des "groupthink« Janis (1972).

867 Der moralische Wert ist ausdrücklich noch nicht eingeschlossen. Es geht noch nicht um "gut« und »böse«. Vgl. Glasl (2013: 259): »Der Gegner ist nicht kompetent! Er hat Defizite im Wissen und Können [...].«

868 Glasl (2013: 263) verweist hier auf das Konzept des »double-bind« nach Watzlawick et al. (2007) und das Beispiel der dominanten Ehefrau und des introvertierten Ehemanns. In 
In Kontrast zu diesem zunehmend negativen und stereotyp gezeichneten Bild der anderen Partei verbessert sich das Selbstbild der Konfliktparteien im Verlauf des Konflikts meist beständig. ${ }^{869}$ So stilisierten sich bei der BerentzenGruppe AG die Akteure zunehmend zu Vertretern der "richtigen" Sache, die allein über die notwendige Erfahrung und Kompetenz verfügten, um die Krise des Unternehmens zu bewältigen. Im Zuge dieser Selbststilisierungen entwickelte sich auf Seiten der Familien Berentzen das Bild der Bewahrer der Tradition, während sich die Vertreter der Seite Pabst und Richarz immer stärker als Vertreter eines modernen Professionalismus begriffen.

Zugleich breitete sich der Konflikt mit zunehmender Dauer immer weiter im sozialen Raum aus. Was als Auseinandersetzung zwischen einzelnen Mitgliedern des Topmanagement-Teams begonnen hatte und sich im Verlauf der Zeit im Gesellschafterkreis etabliert hatte, beschränkte sich nun nicht mehr nur auf die Mitglieder der Eigentümerfamilien, sondern ergriff in zunehmenden Maße auch die Kontroll- und Aufsichtsorgane des Unternehmens.

Je mehr Akteure in den Konflikt verwickelt wurden, desto mehr Themen gingen in ihn ein. Neben einer Vielzahl von sachlichen Streitpunkten und Statusfragen wurden so auch immer mehr der Konflikt an sich, dessen Ursachen und mögliche Lösungswege zu einem konfliktären Thema und in das Konfliktnetz eingewoben. Auch die Definition der Krise und mögliche Ursachen wurden zum Thema des Konflikts.

»Ich hab alle Termine wahrgenommen, zehn Prozent Aufsichtsratstätigkeit gemacht, aber 90 Prozent Familiengespräche."

(Ehemaliger familienfremder Aufsichtsrat der Berentzen-Gruppe AG)

Mit dieser zunehmenden Verflechtung der Themen und der Ausweitung im sozialen Raum nahm die Politisierung des Konflikts und der Entscheidungsprozesse $\mathrm{zu}$, denn mit der steigenden Komplexität des Konfliktgeschehens wurde der Einsatz von Macht zur Überwindung der gegenseitigen Abhängigkeit im Entscheidungsprozess attraktiver. ${ }^{870}$ Macht wurde mithin als attraktives Instrument der Konfliktlösung erachtet und rückte immer stärker in den Mittelpunkt der Auseinandersetzung. Daher zielte das sichtbare wie verdeckte

diesem Beispiel sei für die Beteiligten ihr Verhalten jeweils nur Reaktion auf das Verhalten des anderen. Die Frau müsse führen, weil der Mann so apathisch sei. Dieser erklärt, er ziehe sich zurück, weil die Frau so dominant und herrisch sei.

869 Vgl. Glasl (2013: 260).

870 Zur klassischen Annahme, dass Konflikte in Organisationen zu politischen Prozessen führen vgl. March (1962); Eisenhardt \& Bourgeois (1988); Pfeffer (1981). Unter Politik sollen mit Eisenhardt \& Zbaracki (1992) all jene beobachtbaren, aber häufig versteckten Handlungen verstanden werden, durch welche die Akteure oder Gruppen versuchen ihre Macht zur Beeinflussung einer Entscheidung oder eines Prozesses zu vergrößern. Vgl. auch Eisenhardt \& Bourgeois (1988: 738). 
"politische" Handeln der Akteure immer mehr auf die Macht im Unternehmen und die Möglichkeiten zur Machterweiterung. Diese voranschreitende Politisierung war von großer Bedeutung für die Fortschreibung und zunehmende Eskalation eines Prozesses, der immer mehr zu einer Aushandlung der Machtordung avancierte.

"Also rückbetrachtet eben war also nichts mehr zu machen, weil es ging dann auch beiden Seiten immer weniger um die Sache und immer mehr um die Macht [...] auch wenn der Ausgangspunkt verletzte Balance war oder verletzt empfundene Balance, das Ziel war später nicht mehr die Balance, sondern erkennbar die ganze Macht im Unternehmen."

(Ehemaliger Aufsichtsrat der Berentzen-Gruppe AG)

Zur Vergrößerung und Erlangung dieser Macht wurde auf verschiedenste politische Taktiken und Mittel zurückgegriffen. ${ }^{871}$ So wurden zur Vergrößerung der Machtbasis insbesondere im Aufsichtsrat Unterstützer gesucht und es wurde versucht Koalitionen zu bilden. ${ }^{872}$

»Einflussnahmen wurden in vielfältiger Art ausgeübt. Jeder einzelne Familienstamm versuchte, mich in eine Richtung zu manipulieren, und es war wirklich Manipulation, um sicher zu stellen, dass ich im Sinne des jeweiligen Familienstammes im Aufsichtsrat entscheide. Das wäre nicht nur ein Verstoß gegen das Aktiengesetz und gegen Corporate Governance gewesen. Sondern das wäre für das Unternehmen auch schädlich gewesen. Ich hatte von Anfang an darauf hingewiesen, dass ich stets im Sinne des Unternehmens, und nicht im Sinne der, nicht unbedingt immer im Sinne der Familienstämme, Einzelinteressen, entscheiden würde. Woraufhin alles jubelnd sagte, ja selbstverständlich, dafür haben wir Sie ja auch ausgewählt. [...] Im richtigen Leben hat sich das dann hinterher natürlich etwas anders herausgestellt. Dass das eher ein Lippenbekenntnis war, und man dann doch versuchte, die Partikularinteressen durchzusetzen."

(Ehemaliger Aufsichtsrat der Berentzen-Gruppe AG)

Positionen im Management wurden mit »eigenen Leuten« besetzt, deren Loyalität teils wichtiger war als ihre Sachkenntnisse. ${ }^{873}$

871 Hierzu eine ehemalige Führungskraft: „Es ist dieses - wie sagt man so schön - diese Hidden Agenda Geschichte. Also, sie haben- im Vordergrund haben sie eine offizielle Agenda, die muss gespielt werden, die wird auch kommuniziert und dieses und jenes. Aber im Hintergrund, in Anführungszeichen im Hintergrund, laufen Dinge, die konnte ich zum damaligen Zeitpunkt nicht fassen. [...] Und da kam dann für mich das erste Mal der Punkt, wo ich sagte, `Hoppla, der- wir haben doch nicht alle dieselbe Zielsetzungı."

872 Vgl. Eisenhardt \& Bourgeois (1988: 759), denen zufolge Koalitionen eine hohe Persistenz aufweisen, wenn sich diese erst einmal gebildet haben; vgl. auch Glasl (2013: 264f.).

873 Vgl. folgende Aussagen ehemaliger Führungskräfte: »[W]enn man diese Politik weiter getrieben hat, haben dann die jeweiligen Vorstände die, die Projektgruppen natürlich auch eher so nach Loyalitätsaspekten zusammengestellt. Und ich würde auch aus heutiger Sicht sagen, dass in den Projektgruppen nicht, nicht zwingend, praktisch M\&A-Experten ge- 
»Wir hatten also richtige Frontenbildung, auch quer durchs Unternehmen, was dem Unternehmen nicht gut getan hat auch, weil wir haben dann Manager danach beurteilt [...] ob sie mehr auf unserer Seite stehen oder auf der Seite der anderen, und nicht nur nach ihrer Leistung.[...] Und da haben wir also schon mal Entscheidungen getroffen, die nicht nur, sage ich jetzt mal, rein sachlich aus Unternehmenssicht bedingt waren, sondern die aus Gesellschaftersicht erfolgten, ja. Wer hält mit wem zu. Ja, also Freund oder Feind war wichtiger, als gutes Unternehmen oder schlechtes Unternehmen." (Ehemaliges Vorstandsmitglied der Berentzen-Gruppe AG)

Dabei nahm die Tendenz zu Bildung von Koalitionen mit dem Druck auf die Akteure zu, der aus dem Konflikt und der Unternehmenskrise resultierte. ${ }^{874}$

Über diese Koalitionsbildung hinaus, zeigten sich im Konfliktverlauf der Berentzen-Gruppe AG eine Vielzahl weiterer politischer Mittel. So versuchten die Konfliktparteien immer wieder die Kontrolle über die organisationale Agenda zu gewinnen, um durch Überraschungen oder Täuschungsmanöver bestimmte Positionen durchzusetzen. ${ }^{875}$ Solche Maßnahmen reichten von der plötzlichen Abänderung der Tagesordnung der Aufsichtsratssitzung bis hin zu geheimen Absprachen mit anderen Unternehmen, die erst später allgemein publik gemacht wurden.

Diese Beispiele verweisen zusätzlich auf die Bedeutung der Steuerung des Informationsflusses, die bei der Berentzen-Gruppe als taktisches Instrument eingesetzt wurde. ${ }^{876} \mathrm{Je}$ mehr der Konflikt eskalierte, desto häufiger wurden wichtige Informationen zurückgehalten oder gar bewusst manipuliert, um der eigenen Konfliktpartei einen Vorteil im Machtkampf zu verschaffen. Zum Teil wurden bewusst Gerüchte in Umlauf gebracht.

Durch den Rückgriff auf dieses Repertoire an politischen Mitteln wurde versucht den sich verschärfenden Konflikt durch den Einsatz von Macht und Druck auf die andere Partei zu lösen. Dabei zeigten sich die Akteure aber nicht als kühl kalkulierend und rational abwägend, sondern eher als Getriebene ihrer eigenen Handlungen und stark emotional gesteuert. ${ }^{877}$

Zugleich fiel es den Akteuren im Zuge der zunehmenden Politisierung des Entscheidungsprozesses immer schwerer noch eine kollektive Handlungsbasis

sessen haben.«. «Das heißt, gewisse Managementpositionen dann verändert wurden, um dann sozusagen da irgendwie seine Schäfchen da in die, in die richtigen Bahnen zu lenken."

874 Vgl. Staw et al. (1981) zur entsprechenden Hypothese der »threat-rigidity«, der zufolge immer stärker auf habituelle Handlungen wie zum Beispiel das Agieren in festen Koalitionen zurückgegriffen wird, je höher der zeitliche Druck für die Akteure wird. Dieses Verhalten wurde u. a. auch von Eisenhardt \& Bourgeois (1988) beobachtet.

875 Vgl. hierzu allgemein Pfeffer (1981); Pfeffer (1992).

876 Vgl. Eisenhardt \& Bourgeois (1988); Pettigrew (1973).

877 Vgl. Eisenhardt \& Bourgeois (1988). Die »politischen« Beiträge der Managementforschung porträtierten die Akteure häufig als kühl kalkulierende »Strippenzieher«. Die empirische Untersuchung verwies allerdings eher darauf, wie begrenzte Rationalität, Emotionalität und politische Prozesse im Konflikt zusammenwirken. 
zu finden. In diesem Zusammenhang verweisen Pfeffer (1981) auf das »law of political entropy«. Demzufolge reproduziert sich der politische Charakter von Aktivitäten, wenn sich Politik erst einmal in der Organisation etabliert hat und erschwert so die Findung gemeinsamer Perspektiven und Entscheidungen immer mehr. ${ }^{878}$

Auch bei der Berentzen-Gruppe AG gelangt es den Akteuren nach der Politisierung des Entscheidungsprozesses nicht mehr, eine gemeinsame Handlungsbasis herzustellen. Im sich verschärfenden Machtkampf wurde hingegen versucht, die Glaubwürdigkeit der anderen Konfliktpartei durch direkte und persönliche Angriffe auf deren Repräsentanten zu erschüttern. Auf diesem Wege sollte der anderen Konfliktpartei die Legitimität ihrer Anliegen und mit fortschreitendem Konfliktverlauf prinzipiell die Legitimität ihrer Präsenz im Unternehmen entzogen werden. In diesem Kampf um »Rechtmäßigkeit» wurden beispielsweise traditionelle Symbole referenziert und Narrationen konstruiert, um die eigenen Positionen zu legitimitieren und die Ansprüche der Konkurrenten zu delegitimieren. Politik wurde so mithin auch zum »Management of Meaning « ${ }^{879}$.

Wenngleich auf der vierten Stufe des Konflikts bereits ein offener Machtkampf ausgetragen wird, achten Glasl (2013) zufolge die Beteiligten in solchen Konfliktsituationen meist sorgsam darauf, dass ein wirklicher »Gesichtsverlust « einer Konfliktpartei unterbleibt. ${ }^{880}$ Geschieht dies absichtsvoll oder versehentlich doch, kann für einen gewissen Zeitraum noch auf einige kulturell etablierter Korrekturrituale wie die öffentliche Entschuldigung zurückgegriffen werden. ${ }^{881}$ Erfolgen solche korrigierenden Maßnahmen aber nicht, oder zeigen sie nicht die gewünschte Wirkung, überschreitet der Konflikt eine weitere Schwelle und erreicht die nächste Stufe.

Laut Konflikttheorie ist diese Schwelle hoch - und deutlich höher als die später folgenden. Bei der Berentzen-Gruppe AG trieben sowohl die steigende Komplexität des Konflikts, der sich im sozialen Raum immer weiter ausgebreitet und immer mehr Themen vereinnahmt hatte, als auch die negative Dynamik der sich beständig verschärfenden Unternehmenskrise die Akteure über diese Schwelle. Der Konflikt eskalierte bis zum »Gesichtsverlust« einer Konfliktpartei. $^{882}$

878 Vgl. Pfeffer (1981: 32).

879 Vgl. Pettigrew (1987: 659); der die Legitimität näher thematisiert.

880 Vgl. Glasl (2013: 266ff.). Zum Konzept des »Gesichts« vgl. Goffman (1955); zum klassischen Gesichtsverlust im Rahmen von Degradierungszeremonien Garfinkel (1956).

881 Vgl. Simon (2012).

882 Hier ließ sich die auch von Großmann (2014: 204ff.) und Rüsen (2009) beobachtete negativ dynamisierende Wechselwirkung zwischen dem Konflikt und der sich verschärfenden Unternehmenskrise beobachten. Der weiter eskalierende Konflikt verschärfte die Unter- 


\section{Stufe 5: Gesichtsverlust}

Für Goffman (1955) ist das "Gesicht» der soziale Wert, den eine Person für sich in Anspruch nimmt. Dieser beanspruchte Wert wird ihr vom sozialen Umfeld zugestanden, kann auf verschiedene Weise aber auch wieder entzogen werden. Mit einem direkten Angriff auf das "Gesicht» des Gegenübers wird dessen soziale Identität grundsätzlich infrage gestellt. ${ }^{883}$ Garfinkel (1956) spricht hier von »Degradierungszeremonien«, in deren Zuge auf die öffentliche Anklage eine Form der rituellen Destruktion einer Person folgt. ${ }^{84}$

Ein solcher Angriff auf das »Gesicht« eines Vertreters einer Konfliktpartei erfolgte bei der Berentzen-Gruppe AG, als mit fortschreitender Konflikteskalation versucht wurde, den Sprecher des Vorstands, Jan B. Berentzen, durch mehrere Angriffe in der Öffentlichkeit zu diskreditieren. Es konnten mehrere Presseartikel veröffentlicht werden, in denen nicht nur vehemente Zweifel an der Möglichkeit des Verbleibs des Vorstandssprechers im Amt geäußert wurden, sondern auch öffentlich dessen professionelle Kompetenz und gar Eigenschaften als Person infrage gestellt wurden.

»Jan Berentzen hat aller Voraussicht nach Vertrauen und Stellung verspielt. Der Chef der gleichnamigen emsländischen Schnapsbrennerei ging beim geplanten Kauf des Schnapsproduzenten Eckes allzu selbstherrlich vor.»

(Das Manager Magazin im Jahr 2003 zur Zukunft von Jan B. Berentzen in der BerentzenGruppe AG) ${ }^{885}$

Laut Glasl (2013) bietet sich durch solche direkten, persönlichen Angriffe nicht nur die Möglichkeit zur Reduktion der angestauten emotional-kognitiven Spannungen, sondern auch eine Erklärung des Konflikts. ${ }^{886}$ Denn das Verhalten der anderen Partei lässt sich nach dem Überschreiten dieser Schwelle nun nicht mehr nur durch deren Inkompetenz erklären, sondern auch mit mangelnder moralischer Glaubwürdigkeit und Integrität. So wird nun häufig auch das vergangene Geschehen vor dem Hintergrund der veränderten Interpretationsschablone betrachtet. In der Folge erscheint die andere Konfliktpartei nun auch rückwirkend in einem "ganz anderen Licht «. ${ }^{887}$

In diesem Zusammenhang beginnt sich Simon (2012) zufolge meist auch das eigene Selbstbild weiter zu wandeln: je »dunkler« die andere Seite nun erscheint, desto heller, ehrenhafter und anständiger leuchtet das komplementäre eigene

nehmenskrise, während die sich verschärfende Krise durch den zunehmenden Handlungsdruck oder gegenseitige Schuldzuweisungen zur fortschreitenden Eskalation des Konflikts beitrug.

883 Vgl. Goffman (1955); auch Glasl (2013: 268f.).

884 Vgl. Garfinkel (1956); auch Glasl (2013: 275).

885 B030522MM; vor allem auch B060619HB mit dem Titel »Der Realität entrückt«.

886 Vgl. Glasl (2013: 270).

887 Vgl. Glasl (2013: 269), der auf die Beobachtungen von Garfinkel (1967) verweist. 
Selbst. So wird der Antagonismus der beiden Konfliktparteien bald nicht mehr nur als Auseinandersetzung zwischen "richtig" und »falsch" erlebt, sondern verstärkt als Kampf zwischen "gut« und »böse«. Die andere Partei wird zum »Feindbild «. ${ }^{888}$

Die Stärke solcher Bilder verhindert, dass die eigene Vorstellung von der anderen Partei oder sich selbst noch entscheidend erschüttert wird. Vielmehr wird der Konflikt meist immer ideologischer und berüht immer stärker fundamentale Werte. Bei der Berentzen-Gruppe drehte sich der Konflikt so längst nicht mehr nur um den ökonomischen Sinn einer Verlagerung der Produktion vom traditionsreichen Stammsitz in Haselünne zum modernen Standort in Minden. Vielmehr standen neben dem reinen Machtkampf vor allem der Wert der Tradition und die eigene Identität als Unternehmensfamilie im Zentrum der Auseinandersetzung. Ungeachtet der Legitimität solcher Werte erschwerten solche Überhöhungen aber natürlich immer weiter die Kompromissfindung. ${ }^{889}$ In Verbindung damit, dass der anderen Konfliktpartei auch die moralische Integrität abgesprochen wurde, wurden in der Folge die meisten Vorschläge der anderen Partei von vornherein rundheraus abgelehnt, da $\mathrm{zu}$ einem solchen Gegner kein Vertrauen mehr bestehen konnte. Hingegen musste stets das Schlimmste befürchtet werden.

"Also, wir hatten uns auch als Typen gegeneinander festgefahren. Ich war ihm gegenüber voller Misstrauen, und das merkte er natürlich. Wenn er von irgendeinem Thema anfing, und sei es, sei es nur Fußball oder Familie, hatte ich sofort irgendwo, irgendwie einen falschen Braten gerochen, so schlimm war das dann zum Schluss.« (Ehemalige Führungskraft der Berentzen-Gruppe AG)

In solchen Situationen werden eigene Zugeständnisse meist vom Entgegenkommen der anderen Konfliktpartei abhängig gemacht. ${ }^{890}$ Da einseitige Zugeständnisse im konfliktären sozialen Rahmen aber in der in der Regel ausbleiben, führt diese reziproke Erwartungshaltung zu einer immer tieferen Verankerung der gegenseitigen Blockade.

An diesem Punkt gerät die Konfliktdynamik im Allgemeinen an eine Wegscheide: Zum einen besteht die Möglichkeit, dass das Konfliktgeschehen in einem scheinbar ausweglosen Patt und »Stellungskrieg« erstarrt. Zum anderen können die Akteure auch versuchen, die Flucht nach vorne anzutreten und den Konflikt einer Form der »Entscheidungsschlacht« zuzuführen, in welcher er durch Sieg und Niederlage endgültig entschieden werden soll. Bei der Berentzen-

888 Vgl. Simon (2012: 66); ausführlich Omer et al. (2007).

889 Vgl. Glasl (2013: 274), welcher die Feststellung von Mack \& Snyder (1957) hervorhebt, demnach bei Wert- oder Ideologiekonflikten Kompromisse als Lösungsweg beinahe ausgeschlossen erschienen.

890 Vgl. Watzlawick et al. (1974). 
Gruppe steuerte der Konflikt Mitte der 2000er-Jahre auf eine solche Entscheidungsschlacht zu.

\section{Stufe 6: Drohstrategien}

Zur Herbeiführung einer endgültigen Entscheidung des Konflikts bei der Berentzen-Gruppe AG wurde nun verstärkt auch auf "unlautere« Mittel wie substanzielle Drohungen und Drohmanöver zurückgegriffen. ${ }^{891}$ Gemäß Glasl (2013) gehen Konfliktparteien in diesem Stadium meist davon aus, dass jedes Zeichen der Schwäche vom Gegner sofort ausgenutzt würde und so daher nur Unbeugsamkeit und Demonstration der eigenen Stärke zum Erfolg verhelfen. ${ }^{892}$

Bei der Berentzen-Gruppe AG führten entsprechende Drohungen lediglich dazu, dass der Konflikt weiter akzelerierte. Zur Glaubhaftmachung der Drohungen musste bereits entsprechendes Drohpotenzial aufgebaut und zur Schau gestellt werden. Diese Aufrüstung wirkte für die Gegenseite wiederum so bedrohlich, dass entsprechend nachgerüstet wurde. Somit kam eine Spirale der Aufrüstung an kommuniziertem Macht- und Drohpotenzial in Gang, die schlussendlich zum Überschreiten der nächsten Schwelle der Konflikteskalation führte, denn mit fortschreitender Konflikteskalation und sich erhöhendem Stress fühlten sich die Parteien zunehmend in die Enge getrieben.

Die aus solchem Stress resultierende kognitive Verengung schränkt im Allgemeinen die von den Akteuren wahrgenommenen Handlungsalternativen mindestens ebenso stark ein wie das faktische Handeln der Parteien. ${ }^{893}$ Auf diesem Wege treiben sich die Konfliktparteien immer stärker in einen sich verengenden Graben hinein: der wiederum wachsende Stress führt dann jedoch dazu, dass irgendwann Drohungen auch zu Aktionen werden.

Bei der Berentzen-Gruppe AG war für diese fortschreitende konfliktdynamisierende Verengung des Konfliktgeschehens auch die zunehmende Publizität des Konflikts von großer Bedeutung. Bereits um die Jahrtausendwende war in den Medien von Unstimmigkeiten innerhalb der Berentzen-Gruppe AG berichtet worden, doch spätestens im Laufe des Jahres 2005 wurde der Konflikt unter den Augen der Öffentlichkeit geführt und medial entsprechend begleitet. $^{894}$

891 Dabei wird häufig ein bewusstes Spiel mit den Ängsten der anderen Partei getrieben. Großmann (2014: 182) beschreibt im Kontext eines Konflikts zwischen zwei Brüdern die glaubhaft vorgebrachte Drohung, das eigene Vermögen mehr oder weniger wahllos an eigene Freunde zu vererben.

892 Vgl. Glasl (2013: 284); auch Kahn (1965).

893 Vgl. Glasl (2013: 281), der diese Situation mit den in Kapitel B.1 thematisierten Krisen vergleicht, in denen Entscheidungsträger unter einem besonderen Zeitdruck und Stress stehen. Vgl. Hermann (1972); Schreyögg \& Ostermann (2014).

894 Aufgrund der Vielzahl von Medienberichten sah sich der Vorstandssprecher Jan B. Be- 


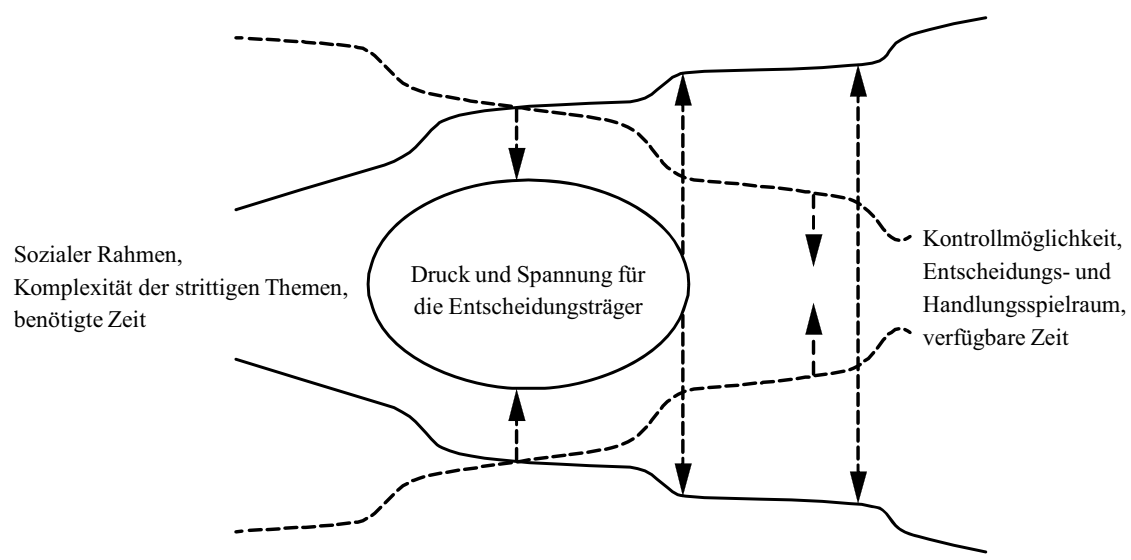

Abbildung 22: Dualität von Handlungsdruck und Handlungsspielraum im Krisenprozess (Quelle: Eigene Darstellung in Anlehnung an Glasl [2013: 283]).

"Krach in der Berentzen-Gruppe. [...] Bei Berentzen geht der schwelende Familienzwist in eine neue Runde. Nach Verlusten von 13,8 Mio. Euro 2004 wollen einige Gesellschafter trotz erkennbarer Erholungstendenzen offenbar einen umfassenden Personalwechsel [...] Der Streit eskaliert an der Frage der strategischen Ausrichtung und dem weiteren Weg der Sanierung und Umstrukturierung [...] Wechselnde Fronten im Aufsichtsrat [...]Wie zu hören ist, sind sich die beiden Berentzen-Stämme Dr. Hans Berentzen und Friedrich Berentzen nicht mehr einig. Christian Berentzen, Sohn von Friedrich Berentzen, sei als Hauptaktionär `inzwischen der Kragen geplatztı und soll sich auf die Seite von P\&R geschlagen haben."

(Die Lebensmittel Zeitung im Jahr 2005 zu den Konflikten bei der Berentzen-Gruppe AG)

Im Zuge der zunehmenden Publizität hatten sich die Akteure nun auch öffentlich auf bestimmte Positionen festgelegt, von denen sie nun nicht mehr ohne Weiteres abweichen konnten ohne ihren Ruf zu verlieren. Hinsichtlich solcher Situationen sprechen Deutsch \& Lewicki (1970) von einem »locking-in« der Akteure, welches die Denk- und Handlungsalternativen beider Parteien stark einschränke. ${ }^{895}$ Nicht nur der Bedrohte, sondern auch der öffentlich Drohende, der sich in seinem zukünftigen Verhalten mit der Drohung bereits konditioniert hat, hänge nun von der Reaktion der anderen Partei ab.

rentzen gar zu der Aussage veranlasst, dass bei Berentzen »keine fernsehreife Familiensaga« abliefe. Vgl. B050524MM, B050524NZ.

895 Vgl. Deutsch \& Lewicki (1970) im Rahmen des spieltheoretischen »chicken game«. Diese Bindung vollzieht sich meist unbewusst, wird in einigen Fällen aber auch als bewusste Strategie der Selbstbindung gewählt. Vgl. Glasl (2013: 279ff.). 


\section{Stufen 7-9: Von begrenzten Vernichtungsschlägen bis zum Rand des Abgrunds}

Nach einer Vielzahl von Drohungen und eskalierten Auseinandersetzungen konnte sich spätestens ab Mitte der 2000er-Jahre keine der Konfliktparteien eine gemeinsame Zukunft in der Berentzen-Gruppe AG mehr vorstellen. Die andere Konfliktpartei wurde zunehmend als ein ganzheitliches Hindernis gesehen, welches die eigene Identität und materielle Existenz gefährdete. Daher wurden nun versucht, durch gezielte juristische Schritte und Attacken im sozio-emotionalen Bereich den Gegner aus dem Unternehmen zu treiben. ${ }^{896} \mathrm{Da}$ das affektive Moment in Relation zur ökonomischen Vernunft immer mehr an Gewicht gewann, waren die Akteure dabei auch immer stärker bereit, zur Erreichung dieses Ziels auch eigene Verluste in Kauf zu nehmen. ${ }^{897}$

In dieser Phase erfolgen nun vermehrt gegenseitige persönliche Angriffe auf die Repräsentanten der Konfliktparteien, um latent vorhandene Spannungen innerhalb der Konfliktparteien zu verschärfen und so deren Einflusspotenzial zu schwächen. Nicht zuletzt aufgrund dieser Angriffe kam es bei der BerentzenGruppe AG tatsächlich vermehrt zu Zersplitterungen und Fraktionsbildungen innerhalb der lange hoch kohäsiven Gruppen. ${ }^{898}$

In Reaktion auf solche aufbrechenden internen Verwerfungen versuchten die Protagonisten innerhalb der jeweiligen Konfliktparteien umso stärker und entschlossener aufzutreten. Dessen ungeachtet entwickelte sich jedoch insbesondere bei der Konfliktpartei Berentzen erheblicher Widerstand gegen die eigene Führung. Letztlich kam es gar zum Bruch zwischen den beiden Stämmen der Unternehmensfamilie Berentzen; der Stamm um Christian Berentzen wechselte in das Lager von Pabst \& Richarz.

»Derweil eskaliert der Streit im Eigentümerkreis. Vorstandschef Jan Berentzen hat nun offenbar nicht nur die Aktionärsgruppe Pabst \& Richarz (34,5 Prozent der stimmberechtigten Stammaktien) gegen sich, sondern auch den Stamm seines Onkels Friedrich Berentzen (34,3 Prozent).«

(Das Manager Magazin zu den eskalierenden Konflikten im Gesellschafterkreis) ${ }^{899}$

Im Zuge dieser Auseinandersetzungen im Gesellschafterkreis verließen nun die ersten Aufsichtsrats- und Vorstandsmitglieder das Unternehmen. Mitte Mai 2005 legte der stellvertretende Vorsitzende des Aufsichtsrats sein Mandat nieder. Kurz danach verließ auch der Marketingvorstand das Unternehmen. Im Oktober verkündete der Aufsichtsratsvorsitzende, dass er sein Amt zum Ende

896 Vgl. B080717KR: »Kleinkrieg in der Brennerei-Idylle«; auch B061218FT.

897 Vgl. Glasl (2013: 297), der von einer auf dieser Stufe der Konflikteskalation typischen Grundeinstellung des »lose-lose« spricht.

898 Vgl. B061218FT; allgemein Glasl (2013: 301); auch Kahn (1965: 300), der von Angriffen auf die zzentrale Nervensystem des Feindes« spricht.

899 B050524MM. 
des Jahres niederlege. Zuvor, im Juni 2005, hatte das Unternehmen zwar bekanntgegeben, dass der Vorstandssprecher Jan B. Berentzen ab 2007 in den Aufsichtsrat wechseln würde. Zum ersten Mal in der Historie der BerentzenGruppe sollte das Management also vollkommen familienfremd sein..$^{900}$ Doch rissen auch nach dieser Bekanntmachung die Auseinandersetzungen im Gesellschafterkreis nicht ab. Im Juni 2006 gab das Unternehmen bekannt, dass sich der Vorstandssprecher Jan B. Berentzen nun bereits kurzfristig aus der Leitung des Unternehmens zurückziehen würde. Da aufgrund der öffentlichen Auseinandersetzungen monatelang kein Nachfolger gefunden werden konnte, besaß die Berentzen-Gruppe AG bis Ende 2006 keinen Vorstandsvorsitzenden. ${ }^{901}$

Doch die Berentzen-Gruppe kam auch nach dem Rückzug der Eigentümerfamilien aus der Führung des Unternehmens nicht zur Ruhe. Gegen Ende des Jahres verließen der Sanierungsberater und der erst seit Kurzem im Unternehmen tätige Marketingvorstand das Unternehmen. ${ }^{902}$ Im Mai 2007 trat dann der amtierende Vorsitzende des Aufsichtsrates nach nicht einmal zwei Jahren im Amt zurück. Es wurden Differenzen hinsichtlich der zukünftigen strategischen Ausrichtung des Unternehmens vermutet.

"Schwindelerregende Rotation [...] Seit einem halben Jahr dreht sich das Personalkarussell beim Schnapsbrenner Berentzen: Aufsichtsrat, Vorstands- und Vertriebschef - kaum ein Manager blieb, wo er war.«

(Das Manager Magazin zu den vielen personellen Veränderungen bei der BerentzenGruppe AG)

Im Juni 2008 wurde dann bekannt gegeben, dass die Berentzen-Gruppe zum Verkauf stehe und nach einen strategischen Investor oder einem Käufer gesucht werde. ${ }^{903}$ Doch aufgrund einer erneuten Eskalation des Konflikts musste der Verkaufsprozess drei Wochen nach Bekanntgabe der Verkaufsabsichten Mitte Juli gestoppt werden. Nach einer vorläufigen Einigung hatten die beiden Familienstämme Berentzen, die sich zuvor einander wieder angenähert und auf ein gemeinsames Vorgehen verständigt hatten, wegen unterschiedlicher Vorstellungen über die Form des Verkaufs und den Preis ihre Zustimmung zum Verkauf zurückgezogen. ${ }^{904}$

Nach diesem Eklat legten der neue Aufsichtsratsvorsitzende, erst seit einem Jahr im Amt, dessen Stellvertreter und ein weiteres Mitglied des Aufsichtsrates ihre Ämter nieder. Kurz darauf trat auch der Vorstandsvorsitzende Axel Dahm

900 B050607MM, B050608NW.

901 Vgl. B060629HB: „Die Berentzen-Gruppe AG [...] muss zunächst ohne neuen Vorstandssprecher auskommen. Nach wie vor stehe kein Nachfolger für den Ende Juni ausschei-

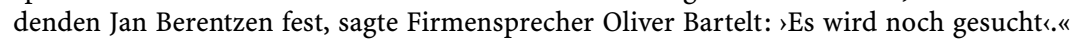

902 Vgl. Berentzen (2016: 13); B061002FM.

903 Vgl. B080627HB.

904 Vgl. B080716HB, B08717MM, B080720MM, B080721HB. 
zurück, drohte durch den geplatzten Verkauf doch eine existenzgefährdende Liquiditätskrise. ${ }^{905}$

Zur Aufgabe der eigenen materiellen und sozialen Existenz war schlussendlich allerdings keine der beteiligten Parteien bereit. ${ }^{906}$ So konnten sich die Konfliktparteien im letzten Moment noch auf einen Verkauf an den Finanzinvestor Aurelius einigen.

\section{Submechanismen der Konflikteskalation}

$\mathrm{Zu}$ dieser eskalierenden sozio-emotionale Dynamik des Konflikts trugen drei Submechanismen bei, die als "Eskalationstreiber « den Konflikt beförderten und die Parteien mithin immer weiter in den Konflikt führten: die Ausweitung der strittigen Themen, die Ausweitung der sozialen Arena und die Beschleunigung durch Drohungen..$^{907}$

So wurden von den Konfliktparteien der Berentzen-Gruppe vom Alkoholgehalt der Produkte über Personalentscheidungen bis hin zur Frage des Verkaufs des Unternehmens immer mehr Thematiken vom Konflikt erfasst. ${ }^{908}$ Die strittigen Punkte hatten natürlich stets auch einen sachlichen Ursprung, dienten aber im Zuge des eskalierenden Konflikts vor allem auch der Stärkung der eigenen Argumentation und Position, sowie der Schwächung der anderen Partei. Daher wurde bewusst immer mehr argumentatives Material in den Konflikt hineingetragen.

Zugleich wurden neben dieser bewussten Ausweitung der Streitpunkte unbewusst viele Aspekte in Unternehmen und Familie erfasst, die bislang nicht von größerer Bedeutung gewesen waren. Zudem besaß der Konflikt in seinem Verlauf eine Tendenz zur Generalisierung, die der Beschreibung von Luhmann (1975) nahe kommt, der konstatiert, dass

905 Vgl. B080717KR; auch B08717MM:»Der Aufsichtsratschef [...] und zwei weitere Mitglieder des Kontrollgremiums sind zurückgetreten. Der Verkauf des Unternehmens ist laut Medienberichten gescheitert."

906 Glasl (2013)Vgl. B080721HB. Die letzte der von Glasl (2013) skizzierten Schwellen wurde somit nicht überschritten. Derartige selbstzerstörerische Dynamiken wurden aber in der explorativen Vorstudie beschrieben: » ja, klar. Das kommt in allen möglichen Spielarten vor, dass, also dass mit dieser Verhärtung, das so weit geht, dass in Kauf genommen wird, dass das Unternehmen dabei scheitern kann an dieser Obstruktion. Und das den Leuten egal ist sogar, nicht, ich gehe hier mit fliegenden Fahnen unter, und opfere sogar mein Eigentum, Hauptsache, die andere Seite kommt nicht zu ihrem Ziel. Das gibt es.« Vgl. Glasl (2013: 301f.), auch Großmann (2014: 206); Rüsen (2009: 322).

907 Glasl (2013: 209f.) nennt fünf Basismechanismen: (1.) die »zunehmende Projektion bei wachsender Selbstfrustration", (2.) die "Issue-Lawine und Simplifizierung", (3.) die "wechselseitige Kausalitätsumkehrung bei gleichzeitiger Simplifizierung der Kausalitätsbeziehungen«, (4.) die "Ausweitung der sozialen Arena bei gleichzeitiger Tendenz zum Personifizieren «, (5.) die "Beschleunigung durch pessimistische Antizipation".

908 Glasl (2013: 106ff.) spricht hier von »Konflikt-Issues«. 
»[...] Konflikte an sich zur Generalisierung tendieren, zur Ausdehnung auf alle Eigenschaften, Lagen und Beziehungen und Mittel der Gegner. In dem Maße, als Dissens und wechselseitige Behinderung bewusst werden, ergreifen sie mehr und mehr Themen, und zugleich ziehen die Gegner mehr und mehr soziale Beziehungen in den Konflikt, die an sich miteinander verträglich waren."

(Luhmann, 1975) $)^{909}$

Je mehr strittige Punkte in den Konflikt einbezogen wurden, desto stärker nahm die Komplexität des Konflikts zu. Zur Handhabung dieser ansteigenden Komplexität griffen die Konfliktparteien nun häufiger auf Simplifizierungen zurück. ${ }^{910}$ Organisationsstrukturelle und strategische Probleme wurden personifiziert und ihre Ursachen der anderen Partei zugeschrieben. Zum Beleg dieser Thesen mussten jedoch wiederum neue Indizien ins Feld geführt werden. Auf diesem Wege eskalierte der Konflikt immer weiter.

Doch nicht nur der Umfang des Katalogs an Streitgegenständen vergrößerte sich im Verlauf des Konflikts und trieb diesen beständig weiter an, auch die fortschreitende Ausweitung der sozialen Arena trug maßgeblich zur Eskalation des Konflikts bei.

So versuchten alle Konfliktparteien Unterstützung für die eigene Position außerhalb der bisherigen Konfliktarena zu finden - nicht immer nur, weil die Unterstützung bereits benötigt worden wäre, sondern manchmal auch, um der anderen Partei zuvorzukommen, von der angenommen wurde, dass sie im Konfliktgeschehen bislang neutrale Akteure für ihre Seite gewinnen wollte. Auf diesem Wege weitete sich der vom Topmanagement ausgehende Konflikt so lange über den Kreis der Gesellschafter bis hin zu den extern bestellten Führungskräften und Arbeitnehmervertretern im Aufsichtsrat aus, dass letztlich gar in der Öffentlichkeit, über die Medien, für die eigene Sache gestritten und Unterstützung gesucht wurde. Der Konflikt wurde somit auf einer immer größeren Bühne re-inszeniert. ${ }^{911}$ Analog zur Ausweitung der Themen führte die Ausweitung der sozialen Arena dazu, dass sich die Komplexität des Konflikts und der Entscheidungsprozesse erhöhte, denn jeder hinzugezogene Akteur brachte neue Wahrnehmungen, Einstellungen und Handlungsweisen mit in den Konflikt. Die Wahrscheinlichkeit von Missverständnissen und Fehlinterpretationen, welche wiederum den Konflikt weiter befeuerten, stieg.

Ein weiterer Submechanismus, der bei der Berentzen-Gruppe die selbstverstärkende Konfliktdynamik antrieb, war die wechselseitige Zuschreibung der

909 Luhmann (1975: $101 \mathrm{ff}$.); vgl. auch Glasl (2013: 217).

910 Vgl. Glasl (2013: 209) der von einer »Ausweitung der strittigen Themen bei gleichzeitiger kognitiver Komplexitätsreduktion« spricht.

911 Vgl. Wimmer et al. (2005: 8). 
Schuld an der Entstehung des Konflikts und an dessen Fortsetzung. ${ }^{912}$ Jede Konfliktpartei hatte zunehmend stärker akzentuierte Ansichten darüber, was den Konflikt verursacht und verschärft hatte. Aufgrund dieser unterschiedlichen Perspektiven zu den Ursache- und Wirkungszusammenhängen kam es zum Konflikt über den Konflikt. Für die eine Partei war Ursache, was der anderen Partei als Wirkung erschien. So führte die Kommunikation über den Konflikt und dessen Lösung meist nur zu neuen Konflikten. ${ }^{913}$

Der Umstand, dass den Konfliktparteien die eigenen Handlungen in wachsendem Maße als Reaktionen auf Entscheidungen oder Handlungen der anderen Partei erschienen, führte beide Seiten jedoch in eine Pattsituation. ${ }^{914}$ Eine signifikante Verbesserung der Situation wurde gemäß dieser Logik vor allem durch veränderte Handlungen der Gegenseite erwartet.

Wie bereits an mehreren Stellen thematisiert, führten diese Subdynamiken zu einer immer höheren Komplexität des Konflikts und einem stetig steigenden Druck auf die Entscheidungsträger, der durch die sich verschärfende Unternehmenskrise noch gesteigert wurde. Dies führte dazu, dass die Akteure eine Flucht nach vorne als die einzige Lösung des Konflikts ansahen und entsprechend versuchten sich im Machtkampf einen Vorteil und Vorsprung zu verschaffen. Auf diesem Wege bildeten sich nun aber selbstverstärkende Erwartungshaltungen aus, die den in der Pfadtheorie thematisierten adaptiven Erwartungen ähneln. Sie erhöhten den Handlungsdruck und beschleunigten den Konflikt. ${ }^{915}$ Jede Konfliktpartei ging davon aus, dass die jeweils andere bereits Maßnahmen zur Durchsetzung der eigenen Interessen planen könnte, und traf daher selbst entsprechende vorbeugende Maßnahmen. Da solche Maßnahmen jedoch nicht lange unentdeckt blieben, zogen sie wiederum entsprechende Gegenmaßnahmen der anderen Konfliktpartei nach sich. Auf diesem Wege etablierte sich ein selbstverstärkender Zirkel der Konflikteskalation. ${ }^{916}$

Diese sich selbst verstärkende sozio-emotionale Dynamik des Konflikts trieb nicht nur die Akteure immer weiter in die Konfrontation, sondern führte auch

912 Vgl. Glasl (2013: 218), der von einer »wechselseitigen Kausalitätsumkehrung bei gleichzeitiger Simplifizierung der Kausalitätsbeziehungen« spricht.

913 Wohl nicht zuletzt deshalb, weil allgemein im Allgemeinen im Zuge der zunehmenden Konflikteskalation die Fähigkeit zur Wahrnehmung der Interessen, aber auch Ansichten der anderen Parteien immer stärker leidet. Vgl. Eisenhardt \& Bourgeois (1988: 763).

914 Vgl. allgemein Glasl (2013: 221).

915 Vgl. Glasl (2013: 226ff.); auch Simon (2012: 104). In der Pfadtheorie vgl. North (1990: 95ff.); Pierson (2000: $251 \mathrm{ff}$.); Schreyögg et al. (2003: 263).

916 Vgl. Glasl (2013: 227), der auf zahlreiche andere Beiträge verweist, die den zugrunde liegenden Charakter dieser sozio-emotionalen Dynamik als »circuli vitiosi« beschreiben. Glasl macht explizit auf die Nähe zu sich selbst zurückkoppelnden »Feedback-Schlaufen« aufmerksam und führt als Beispiel einer mathematischen Modellierung den Beitrag von Richardson (1960) an. 
das Unternehmen immer weiter in die Krise. ${ }^{917}$ Denn je mehr der Konflikt eskalierte, desto schwieriger wurde es im kollektiven Entscheidungsprozess vom etablierten strategischen Muster und dem eingeschlagenen strategischen Entwicklungsweg abzuweichen. Der selbstverstärkende Mechanismus hielt das strategische Muster somit aufrecht bis die Berentzen-Gruppe AG nicht mehr vom eingeschlagenen Entwicklungsweg abweichen konnte und in einem $\mathrm{Zu}$ stand des strategischen Lock-in gefangen war.

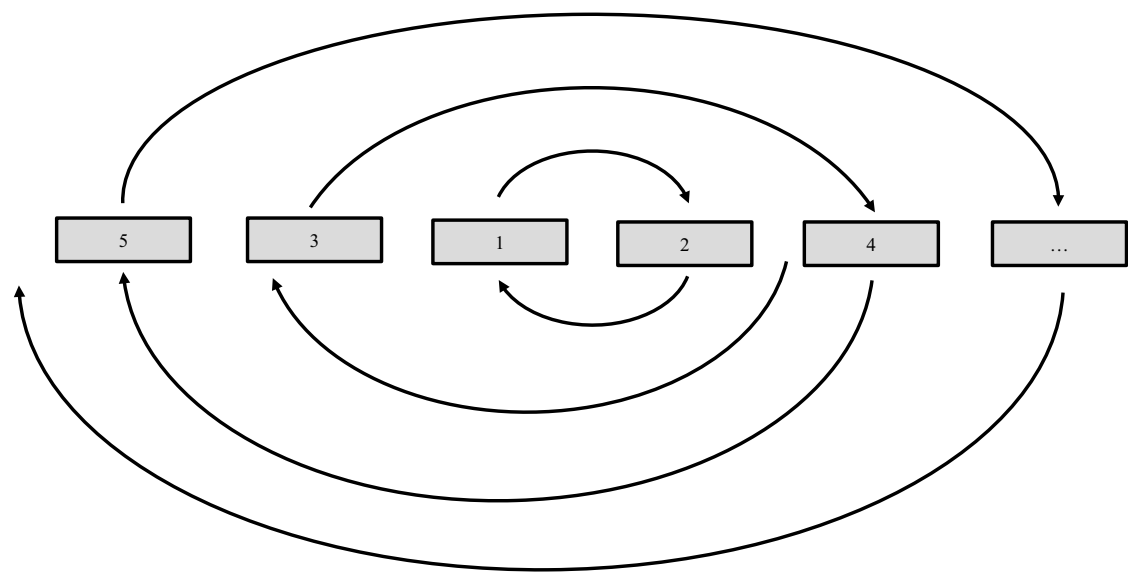

Abbildung 23: Zirkulärer Prozess der Konflikteskalation (Quelle: Glasl [2013: 228]).

\subsection{Lock-in}

Mit zunehmender Dauer und Eskalation des Konflikts war dieser bei der Berentzen-Gruppe AG zu einem allgegenwärtigen Phänomen geworden. Der Konflikt nahm Denken und Handeln der Akteure immer stärker in Besitz, erforderte immer mehr Zeit und absorbierte Kraft und Ressourcen des Unternehmens. Er nistete sich als "parasitäres System « im strategischen Kommunikations- und Entscheidungsraum ein und überlagerte dort mehr und mehr das strategische Management. Dies senkte unmittelbar die Produktivität der Unternehmensführung.

»Zum Schluss war ich also mindestens die Hälfte des Tages befasst, mit Gesellschafterund Aufsichtsratsgesprächen. Ja, also das heißt, dann wurde man dem Unternehmen auch gar nicht mehr gerecht."

(Ehemaliges Vorstandsmitglied der Berentzen-Gruppe AG)

917 Vgl. allgemein zur krisendynamisierenden Wirkung von Konflikten und die Parallelität der sich wechselseitig bedingenden Familienkrisen und Unternehmenskrisen Großmann (2014: 223); Rüsen (2009: 178ff.). 
Das stetig ansteigende Misstrauen ließ die Agenturkosten beständig ansteigen, denn die Konfliktparteien entwickelten zunehmend das Gefühl, jede Aktivität der anderen Seite überwachen zu müssen, um unliebsame Überraschungen und politische Manöver zu vermeiden.

Des Weiteren waren die Informationsflüsse in höchstem Maße gestört. Nicht zuletzt, weil die Kontrolle über Informationen und deren gezielte Lenkung eine wichtige Machtressource darstellte. All dies beeinträchtigte die Güte der den Entscheidungsträgern zur Verfügung stehenden Informationen sowie die Kapazitäten der Informationsaufnahme und -verarbeitung innerhalb der dominanten Koalition. Die Kommunikationsbarrieren begannen früh den strategischen Entscheidungsprozess zu verlangsamen und mündeten zu teils dysfunktionalen Kompromissen zwischen den verschiedenen sachlichen Positionen und Interessen. ${ }^{918}$

»Und da machte sich aber schon bemerkbar, dass also wir Mühe hatten [...] auf einen strategischen gemeinsamen Nenner zu kommen. Das wurde dann im Laufe der Jahre immer schlimmer. [...] Also bitte nicht die Grädigkeit auf, auf 15 absenken, und dann kommt so ein fauler Kompromiss dabei raus, die, die Hälfte senkt man auf 15 Prozent Alkohol ab, die andere Hälfte auf 18, ja. Da haben Sie die Strategiekrise.[...] Wir haben dem Markt keine klaren Vorgaben mehr gemacht."

(Ehemaliges Vorstandsmitglied der Berentzen-Gruppe AG)

$\mathrm{Ab}$ einem gewissen Punkt kam die strategische Kommunikation beinahe vollständig zum Erliegen und war gänzlich vom Konflikt überlagert. ${ }^{919}$

Allerdings behinderte der Konflikt den notwendigen strategischen Wandel nicht nur, weil im Zuge seiner zunehmenden Eskalation die produktive Auseinandersetzung um die zukünftige strategische Richtung auf der Strecke blieb. Vor allem führte der eskalierende Konflikt zur strategischen Persistenz, weil sich die Parteien im Entscheidungsprozess gegenseitig blockierten. Ungeachtet der sachlichen Sinnhaftigkeit wurden strategische Initiativen der anderen Partei meist schon allein aus politischen Gründen zurückgewiesen, nicht zuletzt, weil mit der Bewahrung der eingeschlagenen Strategie und deren Wandel auch eine Stabilisierung und Re-Allokation von Macht assoziiert wurde. ${ }^{920}$

918 Vgl. allgemein Eisenhardt \& Bourgeois (1988: 765); Pettigrew (1973).

919 Hierzu ein ehemaliger Aufsichtsrat: "Man hat sich glaube ich zum Schluss viel zu sehr mit sich selbst beschäftigt. Eine Sitzung jagte die andere, die eigentlich nie die Themen hatte, wo wird das Unternehmen zukünftig stehen, wie stellt es sich neu auf?»

$920 »[\ldots]$ haben dann schnell da jemanden reingesetzt, der nie auch nur einen Fuß, einen, einen Zentimeter und eine Sekunde lang bei den Aufsichtsräten der anderen den Fuß auf die Erde kriegte. Also sobald er irgendwas vortrug, wurde er unterbrochen, und es wurden ihm Aufträge erteilt, und wenn er beim nächsten Mal, bei der nächsten Aufsichtsratssitzung dazu präsentieren wollte, wurde gesagt: > Dafür haben wir jetzt keine Zeit`, und es wurde von der Tagesordnung runtergenommen [...].» 
Auf diesem Wege führte die Konfliktkommunikation dazu, dass mit Eskalation des Konflikts überhaupt keine strategischen Entscheidungen mehr getroffen wurden, sodass natürlich auch keine signifikanten strategischen Veränderungen mehr vorgenommen werden konnten. ${ }^{921}$ Vom selbstverstärkenden Mechanismus des Konflikts aufrechterhalten, reproduzierte sich das etablierte strategische Muster einfach weiter.

"Das Problem ist aber, dass das Innenverhältnis der Familie nicht mehr stabil war und dadurch waren eigentlich keine klaren Entscheidungen, ob jetzt Personalabbau oder Konzentration oder so, getroffen worden."

(Ehemaliger Aufsichtsrat der Berentzen-Gruppe AG)

Ungeachtet der gegenseitigen Blockaden und des daraus resultierenden Stillstands des strategischen Entscheidungsprozesses setzte sich die negative Umweltdynamik fort, verschärfte die Unternehmenskrise und verringerte den Handlungsspielraum weiter. Ein radikaler strategischer Kurswechsel wurde zwar immer notwendiger, doch der eskalierende Konflikt und die sich aufzehrenden finanziellen Ressourcen schränkten den Handlungsspielraum immer weiter ein.

»Noch komplizierter als die Marktlage stellt sich das Verhältnis der Gesellschafter dar [...] Beide Gesellschafter liegen seit Jahren im Clinch. In diesen Konflikt habe sich der langjährige Chef Jan Bernd Berentzen zuletzt so verstrickt, dass er für einen radikalen Kurswechsel des Unternehmens im Aufsichtsrat nicht mehr die notwendige Mehrheit fand, berichten unternehmensnahe Kreise."

(Die Lebensmittelzeitung im Jahr 2005) (22 $^{922}$

Der strategische Entscheidungsprozess war somit geprägt von der Dualität eines zunehmenden Handlungsdrucks und eines abnehmenden Handlungsspielraums. ${ }^{923} \mathrm{Ab}$ einem gewissen Zeitpunkt konnten die Entscheidungsträger im etablierten organisationalen Rahmen nicht mehr vom eingeschlagenen strategischen Entwicklungsweg abweichen. Die Berentzen-Gruppe AG war auf einem strategischen Pfad gefangen, der das Unternehmen in eine existenzbedrohende Krise führte.

921 Ein ehemaliger Aufsichtsrat: $[$ W] $]$ enn dann die Familiengeschichten nicht sauber laufen, dann ist es schwierig [...] harte Entscheidungen, die konnten dann nicht mehr getroffen werden. So war der Krise nicht beizukommen.«

922 B061023HB.

923 Diese Dualität von Handlungsdruck und Handlungsspielraum wird in der Unternehmenskrisenforschung häufig beschrieben. Siehe auch Abbildung 3, die diese Dualität aufgreift. 


\section{$3 \quad$ Zwischenfazit zur empirischen Untersuchung}

Im Rahmen der beiden Fallanalysen wurden in den obigen Abschnitten zunächst die jeweiligen strategischen Entwicklungen der Anton Schlecker e.K. und der Berentzen-Gruppe AG im Kontrast zu ihrer Umweltentwicklung skizziert und auf diesem Wege die strategische Persistenz der beiden Unternehmen dargestellt. Anschließend wurde im Zuge der jeweiligen Pfaddiagnosen aufgezeigt, dass diese strategischen Persistenzen maßgeblich auf die Wirkkräfte von selbstverstärkenden Mechanismen zurückzuführen sind.

Im ökonomischen Kontext des Marktes erklären solche selbstverstärkenden Mechanismen die Entstehung pfadabhängiger Entwicklungsverläufe beinahe restlos, denn die Wirkkräfte der ökonomischen Mechanismen sind recht früh so stark, dass keiner der atomisierten Marktteilnehmer auch nur annähernd einflussreich genug wäre, um sich diesen Wirkkräften entgegenzustellen und sich gegen die Genese des Pfades zu stemmen.

Im Gegensatz hierzu war im organisationalen Kontext der untersuchten Unternehmen auch in der Phase der Pfadformation zweifelsohne noch ein hohes Maß an Macht vorhanden, mit welcher der Genese der strategischen Pfade potenziell hätte begegnet werden können. ${ }^{924}$ So hätte die dominante Koalition bei der Anton Schlecker e.K. lange Zeit mehr als ausreichend Machtmittel besessen, um den auf steigenden Skalenerträgen basierenden Mechanismus noch zu brechen. Die Wirkkraft des Mechanismus hatte keineswegs innerhalb kürzester Zeit in den Lock-in geführt, sondern allmählich, im Verlauf mehrerer Jahrzehnte. Auch bei der Berentzen-Gruppe AG besaß die dominante Koalition lange Zeit ausreichend Einfluss, um vom etablierten strategischen Muster der Wachstumsstrategie durch Marktdurchdringung abzuweichen.

Aus diesem Sachverhalt ergibt sich für den organisationalen Kontext des theoretischen Bezugsrahmens somit eine Erklärungslücke. Ob der Existenz dieser verbliebenen Macht können die selbstverstärkenden Mechanismen allein die Pfadgenese nicht vollends ausleuchten. Hierzu müssen die Wirkweise und Richtung machtvoller Kontextfaktoren wie der Familie in die Analyse einbezogen werden. Der familiäre Einfluss auf den strategischen Entscheidungsprozess und dessen Antezedenten müssen in der Erklärung der Pfadgenese neben die Komponenten der Pfadtheorie treten, um nachvollziehen zu können, warum den selbstverstärkenden Mechanismen in der Phase der Pfadformation nicht gegengesteuert wurde, als dies noch möglich war.

924 Die Betonung liegt auf dem »noch«. Denn es ist ja gerade ein theoretisches Charakteristikum der Pfadtheorie, dass dieser Einfluss im Verlauf der Pfadgenese immer weiter schwindet - so lange, bis in der dritten Phase der Pfadabhängigkeit weitestgehend jeglicher strategischer Handlungsspielraum und Einfluss auf die strategische Entwicklung abhanden gekommen ist. 
Daher werden im Folgenden die in der Pfadanalyse als besonders relevant identifizierten Struktur- und Interaktionsvariablen Macht, Diversität und Konflikt analysiert und es wird aufgezeigt, welchen Einfluss der Faktor Familie auf diese beobachteten Ausprägungen und somit die Genese und Dynamik der strategischen Pfade hatte. 
Open-Access-Publikation im Sinne der CC-Lizenz BY 4.0

(C) 2018, VeR unipress GmbH, Göttingen 


\section{Der Einfluss des Kontextfaktors Familie im Prozess der Pfadgenese}

Im vorigen Teil C wurde im Zuge der ersten beiden Schritte der Pfadanalyse aufgezeigt, wie bei der Anton Schlecker e.K. und der Berentzen-Gruppe AG aus der prozessualen Dynamik selbstverstärkender Mechanismen pfadabhängige strategische Entwicklungsverläufe erwuchsen und wie diese Pfadabhängigkeit die beiden Unternehmen in die Krise führte. In Teil D soll sich nun der Frage gewidmet werden, welche Bedeutung der familiäre Einfluss für die Genese und Dynamik dieser strategischen Pfade besaß.

Im Zuge dieses Unterfangens werden im ersten Kapitel zunächst jene Einflüsse und Ereignisse skizziert, die als Imprints in der ersten Phase der Pfadgenese den strategischen Handlungs- und Entscheidungskorridor prägten und ausformten. ${ }^{925}$ Denn schon lange bevor das Regime der selbstverstärkenden Mechanismen zunehmend die strategische Entwicklung bestimmte, wirkten Imprints wie die lokal-soziale Einbettung der Unternehmen oder organisationskulturelle Aspekte beschränkend auf die strategischen Entwicklungsmöglichkeiten der Unternehmen. Dabei entsprangen diese Imprints entweder direkt dem Einfluss des Faktors Familie oder wurden maßgeblich aufgrund dessen "tradierender Kraft « innerhalb der Organisation fortgeschrieben. ${ }^{926}$

Doch insbesondere hinsichtlich der zweiten Phase der Pfadgenese, der Phase der Pfadformation, bedarf es einer Integration des Kontextfaktors Familie in die Analyse. Denn die oben skizzierte graduelle Pfadgenese bei der Anton Schlecker e.K. und der Berentzen-Gruppe verweist darauf, dass vor allem aufgrund der machtvollen Position der Unternehmensfamilien lange Zeit noch ausreichend "managerial discretion" vorhanden war, um der zunehmenden Wirkkraft der selbstverstärkenden Mechanismen durch einen machtvollen Eingriff noch entscheidend zu begegnen, gewann das Regime der selbstverstärkenden Mechanismen doch erst nach und nach so viel Zugkraft, dass es schlussendlich die

925 Vgl. Sydow et al. (2009: 692f.), die von der "pre-formation phase« sprechen, und Kapitel B.1.3.2.1.

$926 \mathrm{Zu}$ tradierenden Kräften im Allgemeinen vgl. Stinchcombe (1965: 169). 
strategische Entwicklung übernehmen konnte. ${ }^{927}$ Während im klassischen, machtfreien ökonomischen Kontext der Pfadgenese die selbstverstärkenden Mechanismen die Durchsetzung einer bestimmten Lösung fast vollständig erklären, verbleibt hier somit im organisationalen Rahmen noch Erklärungsbedarf. Denn angesichts des noch vorhandenen Machtpotenzials stellt sich die Frage, warum die dominanten Koalitionen nicht rechtzeitig gegensteuerten, als sie noch hätten gegensteuern können.

Zur Annäherung an Antworten auf diese Frage wird sich daher im zweiten Kapitel den vom Faktor Familie geprägten Struktur- und Interaktionsvariablen des strategischen Entscheidungsprozesses gewidmet.

\section{Die Phase der historischen Vorprägung: Die Historizität des strategischen Entscheidungsraumes und die prägende Wirkung organisationaler Imprints}

$\mathrm{Zu}$ Beginn der untersuchten strategischen Entwicklungen erschienen die strategische Handlungsräume der Anton Schlecker e.K. und der Berentzen-Gruppe AG noch weit aufgespannt. Es waren zahlreiche alternative ergebnisoffene Entwicklungsverläufe denkbar.

Grenzenlos unbeschränkt waren diese Entscheidungsräume und strategischen Entwicklungen allerdings weder bei der Anton Schlecker e.K. und der Berentzen-Gruppe AG noch bei den komparativen Vergleichsfällen der Dirk Rossmann $\mathrm{GmbH}$ und der Eckes AG. ${ }^{928}$ Vielmehr war der strategische Entscheidungsraum in allen Unternehmen durch Einflüsse aus der historischen Umwelt der Unternehmen, deren lokale Einbettung in gewachsene soziale Räume oder die zur organisationalen Kultur geronnenen Werte und Einstellungen der Gründerzeit historisch stark vorgeprägt.

Diese Imprints bildeten das historische Fundament der Unternehmen und ihrer strategische Entwicklung. ${ }^{929}$ Vor allem aber wirkten sie auch linear aus der Vergangenheit auf gegenwärtige Entscheidungsprozesse ein.

927 Vgl. Hambrick \& Finkelstein (1987), aber vor allem auch das Modell der Pfadgenese nach Sydow et al. (2009), in welchen die verbleibende "managerial discretion « im Zentrum des Interesses steht.

928 Vgl. Schreyögg et al. (2003: 261); Teece et al. (1997: 522); auch Sydow et al. (2009: 692f.): "Phase I should build on a historically framed or imprinted contingency and, therefore, neither on the assumption of determinacy nor on that of completely unrestricted choice."

929 Vgl. grundlegend zum Ansatz des Stinchcombe (1965); auch Boeker (1989); die Literaturübersicht von Marquis \& Tilcsik (2013); zusammenfassend und mit der Pfadtheorie kontrastierend Sydow et al. (2009). 
PHASE I

(»Historische Vorprägung«)

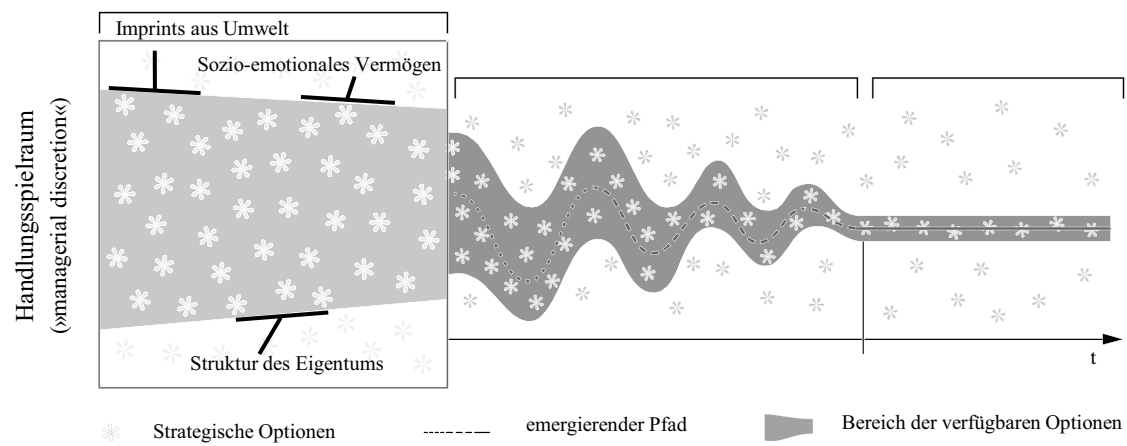

Abbildung 24: Ausbildung eines Entwicklungskorridors durch Imprints in der Phase der historischen Vorprägung

(Quelle: Eigene Darstellung in Anlehnung an Sydow et al. [2009]).

"Just as for a child, the conditions under which an organization is born and the course of its development in infancy have important consequences for its later life.«

(Kimberly, 1979) ${ }^{930}$

Sie führten zwar noch keineswegs zu einer determinierten Entwicklungstrajektorie, doch durch die historische Prägung der Organisation schlossen sie zu späteren Zeitpunkten manche strategischen Handlungsoptionen beinahe gänzlich aus oder ließen andere Optionen wiederum als besonders attraktiv oder notwendig erscheinen. Somit rahmten die historischen Imprints den strategischen Entscheidungsraum der Gegenwart, schränkten im Sinne des »history matters « durch etablierte kollektive Orientierungsmuster und organisationale Strukturen die Veränderungsvarianz der strategischen Entwicklung ein und formten auf diesem Wege früh einen strategischen Entwicklungskorridor.

Dabei zeigte die empirische Untersuchung, wie dauerhaft solche Imprints in die Organisation eingebrannt sind und dass ihre prägende Kraft aufgrund des Prinzips der historischen Sequenzialität meist nachhaltig und kaum reversibel ist. $^{931}$

930 Kimberly (1979: 438); vgl. auch Marquis \& Tilcsik (2013); Mahoney (2000: 511); Baron et al. (1999: 528): »Organizations founding conditions [...] exerting an indelible and enduring influence an how enterprises evolve."

$931 \mathrm{Vgl}$. Pierson (2000: 263): »[E]arlier parts of a sequence matter much more than later parts, an event that happens stoo later may have no effect, although it might have been of great consequence if the timing had been different." 
»Imprinting refers to a process by which events occurring at certain key developmental stages have persisting, if not lifelong, consequences."

(Hannan et al., 1996) ${ }^{932}$

Imprints wie das dynastische Vermächtnis vorheriger Generationen und die Reputation der Familie im Rahmen des sozio-emotionalen Vermögens oder die Prägung der Eigentümerstruktur entsprangen direkt der strukturellen Kopplung des Unternehmens mit der Familie und der Ko-Evolution dieser beiden Systeme.

Auf andere Imprints nahm die Familie einen anders gearteten, aber nicht minder bedeutsamen Einfluss. So erwuchsen weder die prägenden Kräfte der Gründerpersönlichkeiten noch historische ökonomische oder technologische Einflüsse in besonders umweltsensitiven Phasen der organisationalen Entwicklung direkt der strukturellen Kopplung des Unternehmens mit der Familie. Auch in anderen ökonomischen Kontexten, in denen keine Familie im nahen Umfeld des Unternehmens operiert, prägen Gründer das entstehende Unternehmen und Einflüsse des institutionellen und technologischen Kontext zum Zeitpunkt der Gründung formen die Organisation aus. Doch zeigte die empirische Studie, dass in familiär beeinflussten Unternehmen die häufig beobachtbare besondere Permanenz und fortgesetzte Wirkkraft solcher historischer Imprints eben nicht zuletzt auf die »tradierende Kraft« der Familie zurückzuführen sind. Denn die Familie absorbiert als kultureller Träger solche historischen Einflüsse und reproduziert sie mittels verschiedener Mechanismen teilweise über Generationen. ${ }^{933}$

Im Folgenden werden die wichtigsten in der empirischen Untersuchung identifizierten historischen Imprints in ihrer Wirkung auf die Organisation und den strategischen Entscheidungsraum skizziert. Zugleich soll auch reflektiert werden, welche Bedeutung der Faktor Familie für die Ausprägung und Permanenz dieser Imprints besaß. ${ }^{934}$

932 Hannan et al. (1996: 507).

933 Stinchcombe (1965) zufolge erklärt sich die Permanenz bestimmter organisationaler Charakteristika als Resultat zweier separater Kräfte: den prägenden Kräften, die die ursprünglichen Charakteristika in einer kurzen Phase der organisationalen Sensitivität wie der Gründungszeit in die Organisation einprägen und den »tradierenden Kräften«, welche in der Folgezeit diese Charakteristika aufrechterhalten.

934 Aufgrund des für die Darstellung begrenzten Raumes können nicht zu jedem Imprint die empirischen Ausprägungen jedes Falls skizziert werden. Daher werden die meist in mehreren Fällen nachgewiesenen Imprints meist an einem Beispiel illustriert, bei dem das Imprint besonders ausgeprägt war. 


\subsection{Imprints aus der externen Umwelt der Organisation}

Hinsichtlich der individuellen Persönlichkeitsentwicklung gibt es Phasen wie die Kindheit oder die Pubertät, in denen Menschen für Einflüsse aus ihrer Umwelt besonders sensitiv sind. In vergleichbarer Weise gibt es aber auch in der Evolution von Organisationen besonders umweltsensitive Phasen. So macht nicht zuletzt der grundlegende Beitrag des Imprinting-Ansatzes von Stinchcombe (1965) darauf aufmerksam, wie Organisationen in diesen besonders umweltsensitiven Stadien ihrer organisationalen Entwicklung Elemente der Umwelt übernehmen und in ihre formellen und informellen Strukturen integrieren. ${ }^{935}$ Die Literatur zum Imprinting verweist hier insbesondere auf gesamtökonomische und institutionelle Bedingungen oder in der Branche vorherrschende technologische und organisatorische Lösungen. ${ }^{936}$ Diese Imprints formen die sich gerade ausbildenden organisationalen Strukturen und Praktiken, schleifen sich im Zuge eines Institutionalisierungsprozesses ein und werden dann später eben auch unter veränderten Umweltbedingungen weiter praktiziert. Auf diesem Wege können die zu einem bestimmten Zeitpunkt aus der Umwelt absorbierten Elemente die organisationalen Strukturen prägen, langfristig in der Organisation wirken und so deren Evolution beeinflussen.

Solche als Imprints wirkenden historischen Umwelteinflüsse aus der Zeit der Gründung oder anderen wichtigen Unternehmensphasen fanden sich auch in den in der empirischen Studie untersuchten Unternehmen.

So trug zum Beispiel der von Wachstum und Aufbruchsstimmung geprägte gesellschaftliche und ökonomische zeitgeschichtliche Rahmen maßgeblich zur Herausbildung der familiären Vision und organisationalen Identität der Anton Schlecker e.K. als wachsendes, expandierendes Unternehmen bei.

In den Jahren eines bis dahin nicht bekannten gesamtökonomischen Wachstums galt es das Alte hinter sich zu lassen und sich mit vollem Einsatz und Fleiß dem Neuen zuzuwenden. Die hohen Wachstumsraten und der steigende materielle Wohlstand hinterließen in der Nachkriegsgesellschaft kein Gefühl der Sättigung, sondern weckten vielmehr ein Streben nach Höherem. ${ }^{937}$

935 Vgl. Stinchcombe (1965); auch Kimberly (1979: $441 \mathrm{ff}$.).

936 Vgl. Stinchcombe (1965: 153): »[T]he organizational inventions that can be made at a particular time in history depend on the social technology available at that time.« Für einen Überblick über solche Umwelteinflüsse vgl. Marquis \& Tilcsik (2013: $205 \mathrm{ff}$.). Prägende Rahmenbedingungen können auch dazu führen, dass innerhalb einer Branche und Population über verschiedene Organisationen hinweg lange Zeit ähnliche Merkmalsausprägungen beobachtet werden können. Stinchcombe (1965) untersucht zum Beispiel branchenübergreifend identifizierbare Arbeitsstrukturen bestimmter historischer Epochen. Raynard et al. (2013) zeigen auf, wie das maoistische Konzept der Selbstversorgung große Teile der Schwerindustrie in China prägt.

937 Zur Rolle von Familienunternehmen in diesem historischen Kontext vgl. Klein (2010: 20ff.). 
Bereits viele Jahre vor der Übernahme der Geschäftsleitung durch Anton Schlecker jun. und dem Einstieg in die Drogeriemarktbranche prägten Aufbauwille und unbedingte Wachstumsorientierung auch die Zielstellungen im Unternehmen Schlecker. Innerhalb weniger Jahre war aus einer einzelnen Metzgerei eine Fleischwarenfabrik entstanden und durch die Eröffnung der ersten SB-Verbrauchermärkte hatte das Unternehmen Schlecker weiter erfolgreich expandiert. Eine »Goldgräberstimmung" prägte das Unternehmen und dessen junge Mitarbeiter, die später unter Anton Schlecker jun. die beispiellose Expansion als Drogeriemarktunternehmen verantworten sollten. ${ }^{938}$

"Auf jeden Fall, das war die Geburtsstunde von unserm Betriebsglück [...]. Gebrummt! Und ich bin ja erst später dazu gekommen. Das heißt, ich war beim Bauen dabei, ich hab da auch mit Geräten geschafft, die ich noch gar nicht in der Hand gehabt hatte. Dazu, das war so eine Goldgräberstimmung [...] Du willst größer werden. Die Expansion ist entscheidend. Und das ist auch immer, ich komm später noch drauf, wo wir dann unsere Drogeriemärkte eröffnen, immer die Frage zur richtigen Zeit. Und wir haben, wir hatten die richtige Zeit, aber die Zeiten ändern sich.»

(Ehemalige Schlecker-Führungskraft zum Aufbau des Unternehmens in den 1960erJahren)

Noch bedeutsamer für die Konstitution und Permanenz des auf Expansion getrimmten Geschäftsmodells der Anton Schlecker e.K. sollte sich jedoch der von Aldi forcierte Aufstieg der Betriebsform Discount ab Anfang der 1960er-Jahre erweisen.

Kennzeichen dieses neuen Discount-Konzepts waren das in Deutschland bis weit in die 1950er-Jahre hinein unbekannte Konzept der Selbstbedienung, ein relativ schmales und flaches Warensortiment sowie eine schlichte Präsentation der Waren. Diese wurden nicht mehr in Läden mit teurer Ladeneinrichtung und Dekoration, sondern in schlichten Räumlichkeiten von Paletten auf selbstgefertigten Holzregalen verkauft. Zudem wurde zugunsten dauerhaft niedriger Preise auf Kreditverkäufe und Rabatte verzichtet und auf eine konsequente, alle Funktionsbereiche umfassende Kostensenkungsstrategie gesetzt. $^{939}$

Der Aufstieg dieser Betriebsform übte in der gesamten Handelswelt einen nachhaltigen Einfluss auf das Verständnis der Geschäftsmodelle aus. So auch in der Drogeriemarktbranche.

938 Eine ehemalige Führungskraft: »Lieblingsvergleiche von ihm waren immer McDonalds, Microsoft. So nach dem Motto weltweite Firmen. >Wir können die Drogeriemärkte auch in USA, in Australien, 100.000 Märkte können wir machen`. Aber das war jetzt so abgehoben [...]."

939 Vgl. Brandes (2001: 19ff.). 
»Die Idee lag in der Luft [...] der gesamte Einzelhandel war im Umbruch. [...] Es war naheliegend, das Discounter-Prinzip auch in anderen Handelsbereichen anzuwenden.« (Götz Werner) $)^{940}$

Zunächst übernahm Dirk Roßmann die Prinzipien des Discounts aus dem Lebensmitteleinzelhandel. Kurze Zeit später folgte Götz Werner, der von der Sortimentspolitik über die Warenpräsentation bis hin zur Mitarbeiterführung ebenfalls dem Vorbild Aldi folge. ${ }^{941}$

Am nachhaltigsten schlug sich der Aufstieg des von Aldi vorangetriebenen Discount-Konzeptes aber bei der Anton Schlecker e.K. nieder. So kann sogar der Einstieg in die Drogeriemarktbranche zum Teil auf das Aufkommen des Discount-Prinzips zurückgeführt werden. Denn dessen Ausbreitung ließ die Margen der bis dahin erfolgreichen SB-Warenhäuser dramatisch sinken und zwang die Führungskräfte Schleckers strategische Alternativen zu prüfen. Vor diesem Hintergrund wurde durch eine Art »Spin-off« des margenstarken Drogeriesegments aus den SB-Verbrauchermärkten der Einstieg in die Drogeriemarktbranche gewagt.

"Handel ist Wandel, kam dann diese Discountgeschichte. Nämlich das weiße Sortiment, Butter, Zucker, Mehl, alles musste so verkauft werden quasi zum Einkaufspreis. Und jetzt war das große Fest vorbei. [...] und ich hab natürlich Abrechnungen gemacht und ich hab schon gesehen, wie viel da gibt's und Zahnpasta, Kosmetik, die haben die, eine ganz starke Kalkulation gehabt, für uns starke Kalkulation. 33 Prozent minus, nach dem noch mal minus 10 Prozent und vielleicht auch noch drei Prozent Skonto. Und das waren diese Artikel, die wir dann hauptsächlich so da reingestopft haben. Und ach, können wir doch probieren. Ja, eigentlich gar keine schlechte Idee, ganz klar. Kann man ja machen.«

(Ehemalige Schlecker-Führungskraft zum Einstieg in die Drogeriemarktbranche)

Als der Einstieg in die Drogeriemarktbranche dann tatsächlich vollzogen wurde, orientierte man sich, wie die Konkurrenten Rossmann und dm, auch bei der Anton Schlecker e.K. hinsichtlich der Begrenzung des Sortiments und der spärlichen, bewusst billig wirkenden Ausstattung der Filialen am großen Vorbild Aldi. Der Aufstieg dieses Discounters diente der Anton Schlecker e.K. und deren Entscheidungsträgern jedoch vor allem als Vorlage für die eigene Expansion. So wurde wie bei Aldi ein vereinheitlichter Betriebstyp konzipiert und dann

940 Vgl. Werner (2013: 197); auch den Artikel R140723HB, in dem Dirk Roßmann mit den Worten zitiert wird: "Mir war klar, jetzt tut sich was im Handel.»

941 Vgl. Werner (2013: 52): »Durch Pepis [Mitgeschäftsführer Götz Werners] Aldi-Erfahrungen gab es keine langen Diskussionen. $>$ Das machen wir so, wie ich das gewohnt bin! [...] Wir würden das Harzburger Modell einführen. Wir bildeten Filialbezirke [...] Auch für die Mitarbeitersuche übernahmen wir den Prozess von Aldi.« Selbst der Text für die Stelleninserate wurde von Aldi übernommen. 
mit möglichst geringen Veränderungen und Anpassungskosten in der Fläche ausgerollt. $^{942}$

"Also, da war er schon eher getrieben wie früher Aldi auch gewachsen ist. Alles gleich. Jede Veränderung in der Organisation kostet Geld und das stimmt ja auch. So dass man sagt >Ihr müsst versuchen, ein optimales Modell zu finden und das muss man multiplizieren`, und dann hast Du natürlich die größten Kostenvorteile.»

(Langjährige Führungskraft der Anton Schlecker e.K. zur Bedeutung des homogenen Betriebstyps für die Expansion)

Vor allem diente die Expansion Aldis der Anton Schlecker e.K. aber als ein Beispiel für eine spezifische Form der Finanzierung der Expansion über den Cashflow, war doch eine der maßgeblichen Ursachen für die rasche Ausbreitung Aldis, dass das Unternehmen das für die Expansion notwendige Kapital selbst erwirtschaftete. ${ }^{943}$ Der rasche Warenumschlag des schnelldrehenden Sortiments innerhalb von zehn Tagen generierte in Verbindung mit der sofortigen Barzahlung der Kunden und dem üblichen Zahlungsziel der Lieferanten von 30 Tagen eine so hohe Liquidität, dass die Eröffnung neuer Filialen nicht über teure Kredite von Banken, sondern über den eigenen Cashflow finanziert werden konnte. Der Erfolg dieses Finanzmodells aus der ökonomischen Umwelt prägte die strategischen Entscheidungsträger bei Schlecker nachweislich stark und trug durch die Übertragung auf das eigene Unternehmen maßgeblich zur Herausbildung des oben skizzierten Geschäftsmodells von Schlecker bei.

Doch nicht nur in der Drogeriemarktbranche, sondern auch in der Spirituosenindustrie prägte der Aufstieg des Discount-Modells zahlreiche Unternehmen. So führte die zunehmende Bedeutung des Discounts und dessen Handelsmarken bei der später mit der J. B. Berentzen fusionierten Pabst \& Richarz GmbH zur Entwicklung eines im Vergleich zum traditionellen Markengeschäft gänzlich anders gearteten Geschäftsmodell. Dieses war in einem von äußerst niedrigen Margen geprägten Umfeld nicht mehr auf die Pflege der Marke und die Betonung der eigenen Tradition fokussiert, sondern auf die Generierung möglichst hoher Umsätze, bei möglichst niedrigen Kosten. Die in dieser Zeit bei der Pabst \& Richarz GmbH bestehende organisationale Prägung und die Herausbildung kollektiver Orientierungsmuster sollte später zu zahlreichen

942 Selbst das Sortiment in den Auslandsmärkten war trotz der unterschiedlichen Konsumpräferenzen der Kunden und vieler negativer Marktreaktionen lange Zeit vereinheitlicht; vgl. S040220MM: "Was der Schwabe bei seiner zentral organisierten Brachialstrategie überdies außer Acht lässt, sind länderspezifische Eigenheiten. Das deutsche Einheitssortiment der Ehinger funktioniert selbst in Österreich nicht." S. auch Kapitel C.2.1.2.1.2.

943 Eine ehemalige Führungskraft: »Finanzieren mussten wir nichts [...] Also das war eher so cashflowgetrieben wie früher Aldi auch gewachsen ist." Führungskräfte aus der Gründungszeit bestätigten diese Orientierung am Vorbild Aldi auch hinsichtlich dieses Aspekts." 
Spannungen im fusionierten Gesamtunternehmen führen, da diese historische Prägung sehr stark von jener des traditionellen Markenherstellers Berentzen abwich und zu divergierenden Perspektiven und Positionen in den strategischen Entscheidungsprozessen beitrug. ${ }^{944}$

Die Nachhaltigkeit und Permanenz solcher Umwelteinflüsse in »key developmental stages« werden von neoinstitutionalistischen Autoren mit der von der Umwelt gewünschten institutionellen Verlässlichkeit erklärt. Ähnlich argumentieren Vertreter der evolutionstheoretischen Perspektive mit ihrer Betonung der existenziellen Notwendigkeit organisationaler Trägheit zur Aufrechterhaltung von Stabilität. ${ }^{945}$ Wenngleich solche isomorphen Kräfte natürlich auch innerhalb der untersuchten Branchen eine gewisse Wirkkraft besaßen und sicherlich die Notwendigkeit organisationaler Stabilität zur Bewahrung von Strukturen und Praktiken beitrug, erklären diese theoretischen Perspektiven doch nicht die Differenzierung, die Diversität und den Wandel der Imprints innerhalb der jeweiligen Branche. ${ }^{946}$

In Bezug auf diese dynamische Differenzierung im Verlauf der Zeit zeigte sich in der empirischen Untersuchung deutlich der Einfluss der Familie als tradierende Kraft solcher Imprints. So lässt sich die im Vergleich zu ihren Konkurrenten Rossmann oder dm deutlich stärker ausgeprägte Permanenz der historischen Prägungen bei der Anton Schlecker e.K. zum Großteil auf die tradierende Kraft des familiären Einflusses zurückführen.

In diesem Zuge erweist sich neben allgemeinen Faktoren wie der Dauer der erfolgreichen Praktizierung bestimmter organisationaler Imprints vor allem die aus dem familiären Einfluss und dem Strukturmerkmal der Personenorientierung hervorgehende personale Kontinuität der organisationalen Entscheidungsträger als bedeutsam. ${ }^{947}$ Diese trug einen großen Teil zur Präservation einstmals adoptierter organisationaler Charakteristika bei. Dies geht zum einen darauf zurück, dass die Eigentümer in der Phase der Prägung das Unternehmen führten und selbst eine zentrale Rolle bei der Adaption von Umwelteinflüssen und der Formulierung von Strategien einnahmen. Ihre fortgesetzte aktive Rolle erschwerte eine spätere Abkehr von den frühen organisationalen Prägungen, weil die Eigentümer diese Imprints organisational über

944 S. zu dieser Dimension der Diversität Kapitel D.2.2.2.1.

945 Vgl. DiMaggio \& Powell (1983); Meyer \& Rowan (1977); evolutionstheoretisch Hannan \& Freeman (1984).

946 Vgl. zu dieser Einschätzung auch Baron et al. (1999: 542). Wenngleich jüngere Beiträge des Neo-Institutionalismus nun stärker die institutionelle Anpassung und organisationale Evolution in veränderten gesellschaftlichen Rahmenbedingungen betonen. Diese Beiträge erklären dann allerdings nicht, warum sich in Unternehmen wie der Anton Schlecker e.K. die Imprints dennoch präservieren.

947 Vgl. Boeker (1989: 497), gemäß dem die Imprints im Unternehmen sich umso nachhaltiger verankern und wirken, desto erfolgreicher das Unternehmen am Markt agiert. 
lange Zeit einbetten konnten. ${ }^{948}$ Zum anderen war jedoch insbesondere bei der Anton Schlecker e.K. ein Großteil der Führungskräfte über lange Zeit im Management aktiv. Viele Führungskräfte aus der Gründungsphase des Unternehmens als Fleischwarenfabrik und der Phase des Einstiegs als Discounter in die Drogeriebranche waren viele Jahrzehnte im Unternehmen tätig.

»Es gilt das Motto `Einmal Schlecker, immer Schlecker [...]. ^Ich habe noch nie jemanden im Management verloren`, sagt Anton Schlecker.»

(Das Manager Magazin im Jahr 2008) ${ }^{949}$

Die langjährigen Führungskräfte trugen zur Präservation von Umwelteinflüssen aus den entscheidenden Phasen der organisationalen Entwicklung bei, indem sie entsprechende Charakteristika in die organisationale Kultur einbetteten und über praktizierte kollektive Orientierungsmuster über viele Jahrzehnte in der Organisation weitervermittelten. ${ }^{950}$ So wurde innerhalb dieses stabilen personellen Rahmens im Zuge der Auslandsexpansion noch Jahrzehnte später die aus dem Discount-Prinzip abgeleitete Idealvorstellung einer möglichst weit gehenden Homogenität von Betriebstyp und Sortiment nicht wirklich hinterfragt. Gleiches galt lange für das von Aldi übernommene und jahrzehntelang unverändert praktizierte Finanzierungsmodell der Expansion über den Cashflow.

Wie in Kapitel B als allgemeines strukturelles Merkmal des familiären Einflusses herausgearbeitet, trug die Unternehmensfamilie Schlecker maßgeblich zur personalen Kontinuität der Führungskräfte bei. In einem an einzelnen Personen orientierten System partikularistischer Beziehungen wurde an Führungskräften, die der Familie als besonders loyal, vertrauenswürdig und fleißig erschienen, unter Umständen viel länger festgehalten als sachlich vielleicht geboten erschien. ${ }^{951}$

"Das waren jetzt schon Leute, die konnten sie gar nicht mehr ersetzen, weil, das waren dann auch die Leute der ersten Stunde. Die waren also noch zehn Jahre länger da als ich. Die haben noch die ersten Filialen mit eingeräumt. Das sind so die mit Herzblut, ne? [...] Und da war ja nun doch ein kräftiger Bedarf an qualifizierten Leuten, an auslandserfahrenen Leuten. Das wurde dann immer irgendwie verschoben, runtergeredet, und da würde ich also schon sagen, das war das Handicap `Familie`.»

(Ehemalige Führungskraft der Anton Schlecker e.K.)

948 Vgl. Boeker (1989: 495f.), der davon ausgeht, dass die Imprints eine höhere Permanenz aufweisen, wenn die Eigentümer im Unternehmen tätig sind.

949 S080325MM.

950 S. allgemein zur Bedeutung der Zusammensetzung der dominanten Koalition Kapitel D.2.2.

951 Vgl. McConaughy (2000) zur personalen Kontinuität; Daily \& Dollinger (1992); GomezMejia et al. (2001); Shleifer \& Vishny (1997) zum »entrenchment« altgedienter Führungskräfte. 
Das Strukturmerkmal der Personenorientierung äußerte sich am deutlichsten im fortgesetzten Wirken von Reinhold Freudenreich. Er trug als »rechte Hand « des Gründers Anton Schlecker sen. maßgeblich zum Aufbau des Unternehmens in den Nachkriegsjahrzehnten bei und war anschließend auch unter dem Nachfolger Anton Schlecker jun. noch jahrzehntelang in entscheidender Position im Unternehmen tätig. So führte Reinhold Freudenreich noch 2007 für Anton Schlecker die Verhandlungen zum Kauf von Ihr Platz mit Goldman Sachs. ${ }^{952}$

»Aber er war natürlich der langjährige Generalstabsoffizier von Anton Schlecker [...] Den hat man ja dann zurückgeholt, dann ging der wieder, dann hat man ihn wieder zurückgeholt, obwohl der Mann schon lange in Rente wollte. Man hat ihn immer über Geld geködert, hat ihm dann einfach das Gehalt verdoppelt und noch mal verdoppelt und dann hat er noch ein Jahr gemacht und noch ein Jahr gemacht. An der Stelle einfach, das ist ein ganz klares Zeichen dafür, dass man nicht bereit war, in den wirklichen Wechsel zu gehen."

(Ehemalige Führungskraft der Anton Schlecker e.K.)

Bei der Anton Schlecker e.K. waren das Strukturmerkmal der Personenorientierung und die damit einhergehende personale Kontinuität wegen der ausgesprochen engen strukturellen Kopplung zwischen dem Unternehmen und der Familie besonders stark ausgeprägt. Doch auch in den anderen Unternehmen trug die personale Kontinuität maßgeblich zur tradierenden Kraft der Familie und somit zur Permanenz prägender Umwelteinflüsse bei.

\subsection{Sozio-emotionales Vermögen}

Als wichtige historische Imprints zeigten sich neben den historischen Umwelteinflüssen auch verschiedene Facetten des sozio-emotionalen Vermögens der Unternehmensfamilien. ${ }^{953} \mathrm{Zu}$ nennen sind hier zum Beispiel die im Laufe

952 Vgl. S080325MM: „Der Kauf von Ihr Platz illustriert die eigenwillige schleckersche Hauskultur, jene Mischung aus Professionalität und Provinz. Die Verhandlungen mit Goldman Sachs führte nicht Anton Schlecker, sondern Reinhold Freudenreich, ein knapp 81-jähriger Schlecker-Pensionär. Er ist seit Urzeiten dabei, er arbeitete schon unter Vater Schlecker und gilt im Konzern als die graue Eminenz, mit der sich Anton Schlecker auch heute noch jede Woche einmal trifft. Der alte Mann von der Schwäbischen Alb verblüffte die ausgebufften Investmentbanker aus London mit seiner Bauernschläue und seiner Fitness. Auch in der letzten entscheidenden Verhandlungsrunde, die bis in die frühen Morgenstunden dauerte, war der Veteran hellwach und spitzfindig. Freudenreich ist der Prototyp des SchleckerManagers: arbeitseifrig, detailbesessen, trickreich - und vor allem loyal. Für ihn wie für fast alle der rund 400 Beschäftigten in der Ehinger Konzernzentrale gilt das Motto `Einmal Schlecker, immer Schlecker «."

953 Vgl. Gómez-Mejía et al. (2007), die unter sozio-emotionalem Vermögen die »non-financial 
der Jahrzehnte erworbene familiäre Reputation, die nicht durch strategische Kurswechsel gefährdet werden sollte, oder das dynastische familiäre Erbe, welches gewahrt werden sollte. Hinsichtlich dieser Aspekte ihres sozio-emotionalen Vermögens wirkte die Familie somit nicht nur als tradierende Kraft von anderer Stelle ausgehender Imprints, sondern direkt auf den strategischen Handlungsraum.

\section{Einbettung der Familie in den lokalen sozialen Raum und die Bewahrung der familiären Reputation}

So prägten bei der Berentzen-Gruppe AG der Wunsch nach einer Aufrechterhaltung der im Laufe vieler Jahrzehnte verankerten lokal-sozialen Einbettung sowie der über Generationen erworbenen Reputation der Unternehmensfamilie stark den strategischen Entscheidungsraum des Unternehmens.

Hinsichtlich der lokal-sozialen Einbettung hatte der Einfluss der Familie im Zuge der Gestaltung der allgemeinen Umweltbeziehungen der Organisation bei der Berentzen-Gruppe zu einem besonderen lokalen Engagement des Unternehmens und der Familie geführt. ${ }^{954}$ So war die Familie Berentzen neben ihrer ökonomischen Aktivität in Haselünne stets auch politisch und gesellschaftlich in der Gemeinde engagiert gewesen und hatte über die Jahrhunderte auch immer wieder Ratsherren und Bürgermeister gestellt. ${ }^{955}$ Dieses Engagement führte zu einer starken regionalen Verwurzelung von Familie und Unternehmen.

»Berentzen war immer für die Region da, die Familie. Und als, als Berentzen verkauft wurde, war das Unternehmen 250 Jahre alt [...] und in 250 Jahren prägt man eine Region."

(Interview mit einem ehemaligen Aufsichtsrat der Berentzen-Gruppe AG)

Auch die letzten beiden im Unternehmen tätigen Generationen waren in hohem Maße politisch und gesellschaftlich engagiert. Hans Berentzen war in der Kommunalpolitik tätig, mehrere Jahre Honorarkonsul der Niederlande und Präsident der regionalen Industrie- und Handelskammer. Sein Bruder Friedrich, mit dem er das Unternehmen nach dem Zweiten Weltkrieg in neue Höhen geführt hatte, machte sich durch die Pflege und Restaurierung des historischen Stadtkerns um die Heimatstadt verdient. Beide waren in dort hochgeachtete Persönlichkeiten, die höchste Wertschätzung erfuhren.

aspects of the firm that meet the family's affective needs such as identitiy, the abilty to exercise family influence, and the perpetuation of the family dynasty « verstehen. Vgl. auch Felden \& Hack (2014: $47 \mathrm{ff}$.).

954 Allgemein zu dieser regionalen Verankerung von Familienunternehmen: Gallo \& Pont (1996: 46); vgl. auch Schüßler (2008: 137) in der Pfadforschung.

955 Vgl. Berentzen (1981: 11 ff.). 
"Am Firmensitz aber, ihrem Wohn- und Geburtsort Haselünne, werden die zwei Brüder [...] als Förderer, ja als Retter der mehr als 1000 Jahre alten 13 000-EinwohnerGemeinde am Flüsschen Hase verehrt. >Die Berentzens schaffen ihr Geld nicht in die Schweiz, sondern stecken es hier rein`, sagt der scheidende Stadtdirektor Klaus Schütte. >Das tut der Stadt richtig gutı."

(Die Wirtschaftswoche im Jahr 2004) ${ }^{956}$

Der letzte Vorstandsvorsitzende aus den Reihen der Familie, Jan B. Berentzen, war ebenfalls politisch und gesellschaftlich in der Region präsent und übernahm, wie sein Vater vor ihm, beispielsweise Orgeldienste in der Gemeinde. ${ }^{957}$ Seine Frau war ebenfalls in der Kommunalpolitik aktiv und erhielt aufgrund ihres außerordentlichen Engagements für die Region das Bundesverdienstkreuz. Im Rahmen der entsprechenden Preisverleihung wurde sie als »Engel von Haselünne« gepriesen. ${ }^{958}$

Diese starke soziale Einbettung der Familie und des Unternehmens - welche vom sozialen Umfeld, aber auch von Familienmitgliedern nicht unbedingt als getrennte Einheiten wahrgenommen wurden - in lokale Strukturen wurde selbst für neue Führungskräfte des Unternehmens rasch offensichtlich.

"[...] als neuer, neuer Vorstand und nach, keine Ahnung, drei, vier Tagen, nachdem du mal rausgehst aus dem Büro und [...] da gehst du einmal durch die Stadt und plötzlich grüßen dich wildfremde Menschen, die du noch nie gesehen hast. Die auch nicht bei Berentzen arbeiten. Aber [...] das ist ein Ort, da heißt es, das ist der neue Vorstand bei Berentzen. Und automatisch wirst du gegrüßt. Das ist jetzt - das, das zeugt positiv kommen oder positiv zu beschreiben, die Situation, Berentzen ist da oben immer eine Institution gewesen."

(Ehemaliger Vorstand der Berentzen-Gruppe AG)

Eine solche regionale Verwurzelung gehört aus gesellschaftspolitischer Perspektive sicherlich zu den positiv zu bewertenden Charakteristika von Familienunternehmen. Aus rein ökonomischer Perspektive betrachtet kann die aus der aktiven Rolle der Familie erwachsende Einbettung in lokale Zusammenhänge ein zweischneidiges Schwert darstellen. Ungeachtet aller möglicherweise vorhandenen altruistischen Motive oder ethisch-moralischer Verantwortungsgefühle der Unternehmensfamilie können aus dieser Einbettung in spezifische lokale und soziale Zusammenhänge auf der einen Seite handfeste ökonomische Vorteile erwachsen. So kann in ländlicheren Regionen aus einer teilweise quasi-

956 B040818WW.

957 Vgl. B030514: „Der Vater von vier Kindern ist fest in seiner Heimatstadt Haselünne verwurzelt, wo seine Vorfahren das Unternehmen als Kornbrennerei im Jahre 1758 gründeten. In seiner Freizeit setzt sich der etwa 1,80 Meter große, schlanke Mann schon mal an die Orgel in einem örtlichen Altersheim. Außerdem ist er Landesvorsitzender und stellvertretender Bundesvorsitzender des Wirtschaftsrats der CDU.«

958 Vgl. B131218. 
monopolistischen Stellung heraus der lokale Arbeitsmarkt genutzt werden. Grundsätzlich kann über viele Jahre aufgebautes Vertrauen im lokalen oder regionalen Stakeholder-Netzwerk die Transaktionskosten deutlich mindern. ${ }^{959}$ Auf der anderen Seite kann eine solche historisch gewachsene regionale Verwurzelung aber auch maßgeblich den strategischen Handlungsspielraum einschränken. ${ }^{960}$

Im Fall der Berentzen-Gruppe AG wurde über viele Jahre die Konzentration der gesamten Produktion am modernen Standort in Minden diskutiert, um strukturell und nachhaltig das Kostenniveau zu senken. ${ }^{961}$ Diese Maßnahme hätte jedoch auch die Schließung der Produktion am traditionellen Standort des Unternehmens in Haselünne mit sich gebracht, an dem die Familie Berentzen seit fast 250 Jahren Schnaps gebrannt hatte.

Aufgrund der emotionalen Verbundenheit mit der Region und der dargestellten Einbettung in lokale politische und wirtschaftliche Zusammenhänge war dieser ökonomisch zweifelsohne sinnvolle Schritt für die meisten Familienmitglieder unvorstellbar. Zu groß erschienen die empfundene Verantwortung für die Region und die an die Familie gerichteten gesellschaftlichen Erwartungen. Nicht zuletzt, weil die Berentzen-Gruppe einer der wenigen verbliebenen Kornbrenner in Haselünne - der "Stadt der Kornbrenner« - war. ${ }^{962}$

$\mathrm{Zu}$ hoch muteten zudem die sozio-emotionalen Kosten an, denn versteht man Reputation als symbolisches Kapital, das auf kollektiver Anerkennung des ökonomischen, kulturellen und sozialen Kapitals des Reputationsträgers basiert und diesen mit gesellschaftlicher Legitimität ausstattet, dann wäre mit der Schließung der Standorts Haselünne zweifelsohne viel davon verloren gegan-

959 Vgl. für eine Übersicht zur Diskussion um Cluster und regionale Produktionsnetzwerke wie das »Dritte Italien« und deren Einbettung in familiäre Strukturen Bathelt \& Glückler (2012: 248ff.).

960 Vgl. Schüßler (2008: 143):»Bekannte Fabrikanten wie Desch hatten außerdem einen Ruf zu verlieren.« Sie zitiert einen Interviewpartner: "Die haben das Licht angemacht, um vorzutäuschen, sie hätten Arbeit. Stand alles still, da ist nichts gelaufen [...] die hätten eher trocken Brot gegessen, als Leute zu entlassen."

961 Ein ehemaliger Aufsichtsrat: „Es hätte eine Zusammenführung der Produktionsstätten stattfinden müssen [...] wäre besser gewesen, das alles in Minden zu konzentrieren. Da gab es also intern einige Möglichkeiten, die Kosten zu senken.»

962 Eine ehemalige Führungskraft: »[A]lso die, die Stadt Haselünne hatte, sag ich mal, vor 200 Jahren noch, ich glaub, mehrere Dutzend Kornbrennereien am Standort gehabt [...]. Das heißt, man kennt ja dann die Begriffe, wie Fuseltown-City, Schnapshauptstadt und so weiter." Zur Bedeutung dieser Tradition für den strategischen Entscheidungsraum ein ehemaliger Aufsichtsrat: [...] noch drei Schnapsbrenner und aus der Historie gab's irgendwann mal, ich hätte fast gesagt, fast 50 , so dass also diese Region immer in diesem Bereich stark geprägt war. Und das führt natürlich dazu, dass, dass es einer Familie dann schwer fällt, auch unangenehme Entscheidungen zu treffen, die Arbeitsplätze abzubauen oder Produktionslinien zu konzentrieren.» 
gen. ${ }^{963}$ Dieses Motiv trat in der explorativen Vorstudie immer wieder zum Vorschein:

"Eben aus dem Aspekt, dass man sich nicht vorstellen konnte, zum Beispiel die Produktion in Nordhorn aufzugeben, praktisch in Konkurs gegangen [...]. Eben diesen Aspekt vernachlässigt hat, auch unter dem irrationalen Phänomen, wir sind hier so verwurzelt, und so angesehen und so weiter, das können wir gar nicht. Übrigens auch ein allgemeines Merkmal, wenn es darum geht, aus wichtigen Aspekten, sei es der Technologie, sei es der Strategie, erhebliche Einschnitte vorzunehmen, auch Sozialpläne aufzustellen, Entlassungen vorzunehmen. Da besteht in Familienunternehmen große Scheu, da. So nach dem Motto, das können wir uns nicht leisten. Wobei der Aspekt der sozialen Verantwortung häufig nur vorgeschoben wird, im Kern der Befindlichkeiten ist es häufig die Reputation. Nicht, wie stehen wir da? Wir können uns doch in unserem Dorf, in unserem Städtchen nicht mehr sehen lassen, wenn da was passieren würde. So, das sind natürlich Dinge, die dann einschränkend wirken.»

(Ehemaliger Aufsichtsrat in verschiedenen Familienunternehmen im Rahmen der explorativen Vorstudie)

Solche Erwartungen oder Erwartungserwartungen können sich wohl auch im Zuge des Wirkens eines einzelnen Gründers herausbilden, dürften aber sicherlich deutlich stärker ausgeprägt und tiefer im lokalen Raum sowie der Familie verankert sein, wenn sie sich auf ein Unternehmen in der fünften Generation der gleichen Unternehmensfamilie beziehen. Hierbei mag hinsichtlich der Höhe des potenziell zu verlierenden symbolischen Kapitals auch der Umstand eine Rolle spielen, ob das Unternehmen den Namen der Familie trägt oder nicht. ${ }^{964}$

"Aber wahr ist, Berentzen war da, also hat man damals zugestimmt, ja gut, dann lass uns doch den berühmteren Namen halten. [...] Berentzen ist Berentzen, steht ja auch fett dran. So. Das hätte man nicht machen sollen. Man hätte den Namen aufteilen sollen, was weiß ich, Pabst, Richarz, Berentzen oder Berentzen, Pabst, Richarz, irgend sowas machen sollen. Oder was ganz Neues ... Das hätte, weil das am Ende aus der dynastisch

963 Vgl. Bourdieu (2012). Ein ehemaliger Aufsichtsrat: „Also da ist sicherlich auch in vielen Bereichen weniger jetzt nach sachlichen Kriterien bewertet worden [...], sondern mehr nach Loyalität und Familie und, und nach, ich sag mal, ja den, den, den Beziehungen innerhalb von so 'nem kleinen Ort wie Haselünne.« Vgl. allgemein auch Gimeno et al. (1997), die zeigen, dass die Wahrscheinlichkeit einer Firmenschließung negativ mit dem Grad des "psychic income from entrepreneurship» korreliert.

964 Ein ehemaliger Aufsichtsrat: »Wenn ich aber hergehe in einem familiengeführten Unternehmen, und nehme meinen Familiennamen auf ein Produkt, dann baue ich da schon strategisch eine riesen Gefahr ein, weil das ist ja wie ein Kind, da steht mein Name auf der Flasche. Und natürlich werde ich alles tun, um dieses Kind zu fördern [...] und Werbung, die gemacht wird, hey da wird mein Name ins Fernsehen gehauen, oder auf Großflächenplakate. Das ist toll, das schmückt, das macht stolz [...] Nur bei der Frage, stecke ich das Geld hinter Puschkin oder Doornkaat oder Malteser oder Berentzen, raten Sie mal, was denn die Berentzens fühlen, und denken und tun?» 
emotionalen Ecke eine große Bedeutung bei Debatten hatte. Wenn einer sagt, wie stehen wir denn da, wenn wir das machen. Dann sagen die natürlich das immer stark bezogen auf den Prestigeverlust, den man befürchtet für den eigenen Familiennamen.» (Ehemaliger Aufsichtsrat der Berentzen-Gruppe AG)

Da die weiteren am Unternehmen beteiligten Familien, die ihre Anteile an der Berentzen-Gruppe im Zuge der Fusion mit der Weinbrennerei Pabst \& Richarz erworben hatten, weder in der Region verankert waren noch auf die Reputation des Firmennamens Rücksicht nehmen mussten, nahmen sie im Entscheidungsprozess zur Schließung des Standorts Haselünne auch eine gänzlich andere Perspektive und Position ein. Im Vergleich zu den von ihnen gehaltenen ökonomischen Werten waren ihre sozio-emotionalen Vermögenspositionen viel niedriger und so votierten sie folgerichtig für die Schließung des Standortes.

\section{Bewahrung des transgenerationalen familiären Erbes}

$\mathrm{Zu}$ diesen Sorgen um die familiäre Reputation und die lokal-soziale Einbettung, die stärker auf negative Umweltreaktionen bezogen waren, trat als weiterer Imprint das stärker nach innen gerichtete dynastische Streben der Familie Berentzen nach der Bewahrung des transgenerationalen Erbes.

Dieses Streben nach transgenerationaler Kontinuität wurde in der theoretischen Einleitung als ein spezifisches Strukturmerkmal familiär beeinflusster Unternehmen herausgearbeitet, welches auf einem häufig stark ausgeprägten Verständnis eines familiären Vermächtnisses beruht, das gewahrt und an kommende Generationen weitergereicht werden soll. ${ }^{965}$

Bei der Berentzen-Gruppe AG zeigte sich dieses Streben nach transgenerationaler Kontinuität nicht nur im familiären Wunsch, das Familienunternehmen an sich zu bewahren. Vielmehr bezog sich dieser dynastische Anspruch zumindest bei manchen Mitgliedern der Unternehmensfamilie auch stark auf die Bewahrung spezifischer Aspekte des Unternehmens wie des traditionellen Standorts Haselünne oder des Geschäftsbereichs der Spirituosen. Das Unternehmen sollte im Grunde in seiner ursprünglichen Gesamtkonfiguration erhalten werden.

»Diese Generation, die jetzt vielleicht noch mal eine andere Bindung an das Unternehmen hatte, an familiäre Tradition, an Historie, an das Weiterreichen des Vererbten, das auf der einen Seite sicherlich, ja, zusammengehalten hat ein Stückweit, auf der anderen Seite aber auch, was Entwicklung und Moderne betrifft, ein Stückweit stark auf der Bremse gestanden hat und das Thema Haselünne, Tradition, Portfolio und so

965 An dieser Stelle sei nochmals an die Definition von Familienunternehmen nach Chua et al. (1999) erinnert: »[A] business governed and/or managed with the intention to shape and pursue the vision of a dominant coalition $[\ldots]$ in a manner that is potentially sustainable across generations." Siehe ausführlich zum Merkmal der "Continuity« Kapitel B.2.3.2. 
weiter, eher versucht hat, alles so zu bewahren, wie es war.»

(Ehemalige Führungskraft der Berentzen-Gruppe AG)

Die Forschung zu Familienunternehmen führt solche familiären Bestrebungen auch darauf zurück, dass der Verkauf der Keimzelle des Unternehmens oder die Schließung eines traditionellen Standortes sowohl vom Umfeld als auch von den handelnden Akteuren selbst als persönliches Scheitern und Nicht-Erfüllung des transgenerationalen Auftrages wahrgenommen werden kann. ${ }^{966}$

Letztlich können aus solch gefühlten Verpflichtungen gegenüber vorherigen Generationen und den Erwartungen des familiären Umfeldes wie bei der Berentzen-Gruppe AG signifikante Beschränkungen des aktuellen strategischen Entscheidungsraumes erwachsen, die in der Konsequenz die strategische Wandlungsfähigkeit des ganzen Unternehmens bedrohen. Simon et al. (2005) sprechen daher wohl nicht zu Unrecht von der Paradoxie von Tradition und Innovation, mit der sich viele Entscheidungsträger in Familienunternehmen konfrontiert sehen. ${ }^{967}$

\section{Emotionale Verbundenheit}

Als eine weitere Facette des familiären Strebens nach der Bewahrung des sozioemotionalen Vermögens zeigte sich in der empirischen Studie das theoretisch eher auf der Ebene des Individuums angesiedelte »emotional attachment" im Sinne einer besonderen persönlichen und emotionalen Verbundenheit zu einem Produkt, einem Standort oder einer spezifischen Strategie. ${ }^{968}$

Eine solche individuelle Verbundenheit erwies sich in der empirischen Studie an vielen Stellen als ein bedeutsames Imprint, welches den strategischen Handlungsspielraum signifikant prägte. Sie zeigte sich beispielsweise bei der Eckes AG hinsichtlich des Verkaufs der Spirituosensparte:

»Emotional war das natürlich für die Familienmitglieder, für einige war das natürlich ein großer, ein großer Schritt, weil das war, wie wenn man ein Kind veräußert, hat mir einer der Gesellschafter gesagt, `Ich komm mir vor, wie wenn ich heut mein Kind verkauft hätte.ı Na ja, aber die, die Ratio und die strategische Weitsicht war schon richtig, das so zu tun. Ansonsten wird es die Firma wahrscheinlich nicht mehr in der Form geben, wie sie's heute gibt."

(Ehemaliges Vorstandsmitglied der Eckes AG)

966 Vgl. Sharma \& Manikutty (2005: 294ff.).

967 Vgl. Simon et al. (2005); auch Sharma \& Manikutty (2005: 296).

968 Vgl. Burgelman (1994). Für viele Unternehmer wird das Unternehmen zu einem Hauptbestandteil ihrer Identität und für manche stellt das Unternehmen gar den Versuch dar die eigene Sterblichkeit zu überwinden; vgl. Kets de Vries (1993: 68); Kets de Vries (1996:34ff.) auch Pierce et al. (2001) zur psychological ownership«; Zellweger \& Astrachan (2008) zum "emotional value of owning a firm». 
Noch deutlicher zeigte sie sich bei der Anton Schlecker e.K., in deren Konfiguration Unternehmen und Familie allerdings auch deutlich enger strukturell gekoppelt waren als bei der Eckes AG. So erwuchs bei Schlecker aus der emotionalen Verbundenheit zur Expansionsstrategie und dem Lebenswerk an sich eine individuelle emotionale Herausforderung, auch nur einzelne der vielen Tausend Filialen zu schließen.

»Du musst von deinen 10.000 Filialen runter auf 4.000. Und jede Filiale ist ein Baby. Das will er nicht, das willst du nicht schließen [...] Emotionalität in der Sache, es geht nicht nur drum, Kasse zu machen. Das ging bei Anton Schlecker gar nicht. Das ist sein Lebenswerk. Das ist er, das ist meine Identität und das sind meine Filialen. Dann geb ich lieber einen einzelnen Finger bevor ich einen Filiale aufgebe, ja, das ist eine, da ist ein ganz anderes- Das ist ein Familienunternehmer, das ist ein Unternehmertum, wo das sein eigenes Ich ist [...] das ist das psychologische Moment. Ein Unternehmer so vom Schlage Anton Schlecker, dessen Leben zu 95 Prozent aus dem Unternehmen besteht. Da ist jeder Schritt, jede Entscheidung, die er trifft und macht ist eine ganz persönliche und individuelle. Jeder Standort, der aufgemacht wurde, hat er mit entschieden und unterschrieben. Und das ist vom, und da ist auch alles, was du dann wieder zurückbauen musst und wieder aufgibst, ist eine, ich sag mal, wie eine persönliche Niederlage. Du hast es mal aufgebaut, jetzt musst du es wieder abgeben.» (Langjährige Führungskraft der Anton Schlecker e.K.)

Entscheidend für die fortgesetzte Expansion war der oben skizzierte wirkungsmächtige selbstverstärkende Mechanismus. Doch die persönliche Verbundenheit Anton Schleckers zur Wachstumsstrategie beschränkte ebenfalls in nicht unerheblichem Maße den strategischen Handlungsspielraum des Managements.

"Aber schließen und/ das war nie sein Thema. Ich kann mich gut dran erinnern, als wir nach Holland gegangen sind, das war auch Ende der 80er-Jahre, da lief das in Österreich sehr gut. In Holland überhaupt nicht. Fünf Jahre Verluste, zehn Jahre Verluste. Da bin ich dann auch in sein Büro und hab den Ordner genommen und hab den mal gegen die Wand geschmissen. Mit Holland bräuchte er nicht mehr zu kommen. Also wenn wir da jetzt nicht schließen, ist der größte Schwachsinn aller Zeiten. Der hat das, glaub ich, 23 Jahre gehalten. Wohl wissentlich, dass Millionen jedes Jahr darüber flossen. Das muss man sich mal vorstellen. Immer in der Hoffnung, irgendwann haben die Holländer es kapiert.

Die kommen wieder. Die kommen wieder! Dann bringt der Ihnen eine Filiale mit. Eine. Wir hatten 14.000. Und die war vorher zwei Jahre im Minus. Und im dritten Jahr hat sie plus acht Prozent gemacht. Ja, und so machen das die anderen bald auch! [...] Und da hat sein Herz dran gehangen und Schließen gab's gar nicht. Also, schließen war für ihn ... wenn Sie das Wort `Schließung ‘ in den Mund genommen haben, konnten Sie eigentlich schon nach Hause gehen, an dem Tag. Mochte er nicht hören. >Wir müssen da ein bisschen gucken, wir müssen auch mal aufräumen, wir müssen mal wieder renovieren.» Sowas kam dann."

(Langjährige Führungskraft der Anton Schlecker e.K.) 
Selbst kleinste Veränderungen am Filialnetz und gar die Aufgabe der Vision einer endlosen Expansion fielen Anton Schlecker persönlich sehr schwer. ${ }^{969}$ Dies zeigt sich bei der Anton Schlecker e.K. auch an der aus ökonomischer Perspektive irrational, weil strategisch und finanziell vollkommen aussichtslos anmutenden Aufrechterhaltung der fünf SB-Verbrauchermärkte und der traditionellen Fleischwarenfabrik. Diese erwirtschafteten keinerlei Profite und wurden allein aus emotionaler Verbundenheit über Jahrzehnte weitergeführt. ${ }^{970}$

»Aber selbst solche einfachen Dinge, also die Großfläche wieder abzugeben, denn die hat ja auch Arbeit gemacht, da waren ja auch 400-500 Leute, die damit beschäftigt waren, so ein Nullsummenspiel zu machen. Es gab keine strategische Option. Der Zug war weg. [...] Und der war nicht in der Lage, diese Flächen zu schließen. Ganz am Schluss meiner Zeit haben wir dann die Fleischproduktion eingestellt und haben nur noch Fleisch verpackt. Aber diese Großflächen sind bis auf den Tag, wo ich da war, geblieben. Ein völlig sinnloses Unterfangen für eine solche Firma, die so aufgestellt war wie wir. Und das kennzeichnet ihn wohl auch. Er gibt nichts ab. Vielleicht können wir es nochmal gebrauchen."

(Langjährige Führungskraft der Anton Schlecker e.K.)

All diese Facetten des sozio-emotionalen Vermögens der Familie trugen somit dazu, dass sich in den untersuchten Unternehmen bereits früh strategische Handlungskorridore etablierten, die die weitere strategische Entwicklung zunächst in relativ feste Bahnen lenkte.

\subsection{Kultur der Organisation}

Prägenden Einfluss auf die strategischen Entscheidungsräume der untersuchten Unternehmen hatten zudem die spezifischen Sets an gemeinsamen Überzeugungen, Werten und Symbolen im Rahmen der jeweiligen Organisationskulturen. ${ }^{971}$

Diese kulturellen Muster reflektierten in vielerlei Hinsicht den historischen Einfluss der Unternehmensfamilien und einzelner Protagonisten aus vergan-

969 Vgl. allgemein Gimeno et al. (1997).

970 Vgl. S120316SW zu den Schließungen dieser Geschäfte im Jahr 2012 : »Wann nun der Ehinger Schlecker-Baumarkt, das Restaurant, die Tankstelle, das Fleischwerk und das Kinderland endgültig schließen, steht noch nicht fest [...] Ebenfalls dicht machen sollen die rund 20 Fleischtheken in den Schlecker-Märkten [...]. Davon betroffen ist übrigens auch die Fleischtheke, die sich in der Schlecker-Filiale in der Ehinger Hauptstraße befindet. Die Filiale, in der der Vater einst den Grundstein für das Unternehmen legte.«

971 Vgl. allgemein zur Organisationskultur (Schreyögg, 2006: 448ff.); zu deren Wirkung als Imprint vgl. Marquis \& Tilcsik (2013: 216). Die Autoren verweisen zum Beispiel darauf, dass starke Kulturen als prägende Imprints großen Einfluss auf die Gestaltung von Karrierewegen in Unternehmen nehmen. 
genen Perioden der organisationalen Entwicklung, der über die tradierende Kraft der Familie erhalten wurde. Dieser Zusammenhang kann wiederum besonders deutlich am Beispiel der Anton Schlecker e.K. aufgezeigt werden, in deren Sphäre Unternehmen und Familie ausgesprochen eng gekoppelt waren.

Eines der auffälligen Merkmale der organisationalen Kultur der Anton Schlecker e.K. war der ausgeprägte und das ganze Unternehmen durchziehende Drang zur Sparsamkeit. ${ }^{972}$ So wurde aus Kostengründen nicht nur an der Ladenausstattung, sondern lange Zeit auch an den Telefonanschlüssen der Filialen gespart. In wichtigen Fällen war das Personal angehalten, eine Telefonzelle oder den Anschluss eines benachbarten Geschäfts zu nutzen. Doch auch in der Verwaltung wurde nach Kräften gespart. Da hier kaum »organizational slack« existierte, blieben die Versuche von Unternehmensberatungen und Insolvenzverwaltung, hier Einsparpotenziale zu realisieren, weitgehend erfolglos. ${ }^{973}$

»Und insofern Führungsstrukturen extrem flach. Wir haben noch nie ein Unternehmen erlebt, wo wir überhaupt keine Kosteneinsparungspotenziale gesehen haben, überhaupt nicht. Im Gegenteil, wir mussten, hätten investieren müssen. Sehr, sehr schwäbisch geführt. Kostensensibel geführt."

(Ehemaliger Berater der Anton Schlecker e.K.)

Die tief in der Organisation verankerte Sparsamkeit äußerte sich in unterlassenen Investitionen und Modernisierungen auf verschiedenen organisationalen Ebenen. $^{974}$

»Was allerdings tatsächlich extrem schwierig war bei Schlecker, ist, dass der aus lauter Sparsamkeit nicht mal ein Warenwirtschaftssystem hatte. Die Bestandslisten haben die Verkäuferinnen händisch ausgefüllt und per Fax verschickt [...]. Schlecker hat immer auf Menge und den billigsten Einkaufspreis geschaut, aber nicht modernisiert. Wir wussten nicht mal, was in den 6.000 Filialen sich so wo genau befindet. Und das ist schon ein extremer Aufwand gewesen, uns hier einen Überblick zu verschaffen." (Insolvenzverwalter der Anton Schlecker e.K.)

972 Vgl. S930426.

973 Zum »organizational slack« vgl. Cyert \& March (1963).

974 Auf tieferer organisationaler Ebene wurde zum Beispiel die Anschaffung von Einweghandhandschuhen für Lagermitarbeiter immer wieder aufgeschoben. Die völlig veralteten Kassensysteme wurden erst ausgetauscht, als kein Anbieter mehr gefunden werden konnte, der noch eine kompatible Software hätte programmieren können. Vgl. S040212. Der Insolvenzverwalter zu dieser Sparsamkeit: "Sie müssen sich vorstellen, die haben kein EDVgeschlüsseltes Warenwirtschaftssystem in den Läden gehabt. Das heißt, die Kassiererin hat abends auf einer Excel-Liste, auf einem Blatt Papier, nicht auf einer Excel-Liste, das ist falsch, auf einem Blatt Papier, auf einem Vordruck, ausgefüllt, was sie für Artikel haben wollte.» 
Ein zweites hervorstechendes kulturelles Merkmal war das ausgeprägte Misstrauen, das sich vor allem gegenüber den Angestellten des Unternehmens zeigte. ${ }^{975}$ Auf allen organisationalen Ebenen wurden Listen missliebiger Mitarbeiter geführt, die durch Betriebsratstätigkeit oder sonstige unerwünschte Betätigungen aufgefallen waren. Des Weiteren waren die Bezirksleiter und Filialleiter angewiesen regelmäßig die Taschen und Spinde der Mitarbeiter zu kontrollieren. Damit von den Mitarbeitern mitgebrachte Zigaretten oder Damenbinden bei solchen Kontrollen nicht als gestohlen gewertet wurden, galt es diese von Kolleginnen bei Dienstantritt gegenzeichnen zu lassen

»Dieses konsequente Druckmachen, dieses permanente Kontrollieren, dieses tagtägliche Anschieben, dieses immer wieder sich wiederholende Ermahnen und Hinweisen gehört gewiss nicht zu den angenehmsten Tätigkeiten einer Führungskraft, mit Sicherheit aber zu den erfolgsentscheidenden."

(Aus einer internen Schleckerbroschüre)

Das in der Organisationskultur der Anton Schlecker e.K. verankerte Menschenbild und die aus diesem Bild resultierenden formalen und informellen Kontrollsysteme ähnelten den von McGregor (1960) in seiner "Theorie X" beschriebenen Einstellungen und deren organisationalen Konsequenzen. ${ }^{976}$ Der Kontrolle dienten letztlich auch die über Jahrzehnte fortwährenden wöchentlichen Filialbesuche von Anton und Christa Schlecker.

"Es gibt Menschen, ja und du glaubst an das Gute im Menschen und an das Positive. Die mit dem X, die mit dem Y. Und klar war Schlecker eine Kultur, wo die Theorie, jetzt weiß ich nicht, was gut und böse war. Auf jeden Fall, die das den Menschen erst mal: Mensch ist faul, Mensch ist böse, Mensch ist schlecht, will sich nicht bewegen und nicht arbeiten und darauf stelle ich mich ein."

(Langjährige Führungskraft der Anton Schlecker e.K.)

975 Vgl. S110502LZ; S031204MM; S040220MM. Mitarbeitern, die sich mehrfach für längere Zeit krank gemeldet hatten, wurden Auflistungen mit ihren Fehlzeiten zugeschickt. Begleitet vom Hinweis, dass man in Bezug auf die "gesetzliche Fürsorgepflicht « werde prüfen müssen, ob der Gesundheitszustand eine Weiterbeschäftigung zulasse. Erst spät wurden diese Entwicklungen von der dominanten Koalition reflektiert. Vgl. S110118MM: »Bei Schlecker hatte sich langsam eine Angstkultur eingeschlichen «, teilte Lars Schlecker dem Handelsblatt mit. »Ich habe klargestellt, dass Abmahnungen kein Mittel zur Personalentwicklung sind."

976 Vgl. McGregor (1960), Schreyögg (2006: 193). Hierzu eine ehemalige Führungskraft: „Es gehört zu der Facette, dass es ums Betrügen, ich sag's mal grob, Bescheißen und, und um ein Bewertungsraster geht, Arbeit, Arbeitsergebnis, Arbeitsleistung so Person 10:1. Du macht das, wenn du nicht, dann findet man andere, ne. Du bist eigentlich gar nichts wert. Und wirklich in der Firma ist man mehr als über Leichen gegangen. Ich hab das oft genug festgestellt. Furchtbar. Mensch, keinen Wert.» 
Diese kulturellen Ausprägungen waren jedoch weniger auf Anton Schlecker zurückzuführen, als vielmehr auf den Gründer Anton Schlecker sen. und dessen erste Führungskräfte.

"Anton ist nie laut geworden, der war zurückhaltend, also der war zurückhaltend, vernünftig eher schon. Der hat halt seine Vorstellung gehabt, die war, hat er geprägt durch die Vergangenheit.«

(Ehemalige Führungskraft der Anton Schlecker e.K.)

Durch die prägende Wirkkraft der Protagonisten der Gründungsphase hatten sich die kulturellen Ausprägungen wie die Sparsamkeit und das allgemeine Misstrauen schon viele Jahre vor dem Einstieg des Sohnes in das Unternehmen und dessen Expansion in die Drogeriemarktbranche herausgebildet. Diese Persönlichkeiten waren nicht nur die Architekten der organisationalen Struktur, sondern prägten auch entscheidend die entstehende organisationale Kultur. ${ }^{977}$ Wie in vielen Organisationen dienten diese Protagonisten aufgrund ihrer hierarchischen Autorität, ihres Expertenstatus und der Gründungsleistung als organisationaler Kristallisationspunkt der Anton Schlecker e.K. und definierten auf diesem Fundament in weiten Teilen die Mission, Struktur und Kultur der Organisation. Durch frühe Entscheidungen und Handlungen setzten sie den organisationalen Rahmen, der anschließend nachhaltig auf die Organisation wirkte. ${ }^{978}$ Der Einfluss des Gründers zeigte sich hier insbesondere in Bezug auf die Gestaltung der internen sozialen Kontrolle, die Strukturierung von Aufgaben und Prozessen und das Verhältnis der organisationalen Beziehungen zur Umwelt. ${ }^{979}$ So lässt sich das organisationskulturelle Merkmale der Sparsamkeit mit der Persönlichkeit des Gründers Anton Schlecker sen. in Verbindung setzen, denn der "alte Schlecker« war auch noch als etablierter Unternehmer in seiner Heimatstadt Ehingen für seinen Geiz bekannt. So soll er an zu Fußballspielen häufig erst zur zweiten Halbzeit erschienen sein, um den Eintritt zu sparen. ${ }^{980}$

Diese Sparsamkeit lebte Anton Schlecker sen. auch im jungen Unternehmen vor. Den Preisen im Einkauf und möglichen Rabatten widmete er höchste

977 Harvey \& Evans (1994) sprechen hier von einem »initial cultural imprint«, der kulturelle, aber auch strategische Veränderungsprozesse erschwere; vgl. allgemein zur prägenden Kraft des Gründers Nelson (2003: $707 \mathrm{ff}$ ); vor allem Schein (2010:219): „Cultures basically spring from three sources: (1) the beliefs, values, and assumptions of founders of organizations; (2) the learning experiences of group members as their organization evolves; and (3) new beliefs, values, and assumptions brought in by new members and new leaders. Though each of these mechanisms plays a crucial role, by far the most important for cultural beginnings is the impact of founders."

978 Vgl. Baron et al. (1999: 532); Gimeno et al. (1997); Nelson (2003: 710): »Once formulated and articulated, a founders's organizational blueprint alikely >locks in the adoption of particular structures, as well as certain premises that guide decision-making."

979 Vgl. Kimberly (1979: 447).

980 Vgl. anekdotisch zum Geiz des Vaters: S071005HB; S120602HB. 
Aufmerksamkeit und kommunizierte gegenüber Mitarbeitern, dass er Sparsamkeit für eine Tugend und Verschwendung für verachtenswert hielt. Die Fleischereifilialen und die SB-Verbrauchermärkte sollten möglichst billig und in Eigenregie eingerichtet werden. In dieser ausgeprägten Sparsamkeit im Vertrieb lag sicherlich auch ein Teil der Wurzeln jener billigen Filialgestaltung, die später zu einem Synonym für das Schlecker'sche Geschäftsmodell und dessen Scheitern werden sollte. ${ }^{981}$ Doch vor allem im für den Erfolg der Fleischwarenfabrik entscheidenden Einkauf von Schlachtvieh zeigte sich der Gründer als unnachgiebiger Verhandlungspartner, der für einen guten Preis unter Umständen nicht davor zurückschreckte, auf unlautere Mittel zurückzugreifen. Auf dem Wege der alläglich vorgelebten Praxis beeinflusste dieser »Geist« des Gründers in hohem Maße die sich formierenden Einstellungen, Werte und Normen im Unternehmen. ${ }^{982}$

»[...] haben wir abgeliefert. Hat der, in der Küche hat das gar nicht gemerkt, statt 875 Kilo bestellter und bezahlter Ware, dass er bloß 87,5 bekommen hat. Also hast du schon mitgekriegt, um was es eigentlich alles nur geht."

(Langjährige Führungskraft der Anton Schlecker e.K.)

Nach und nach begannen sich so die organisationale Identität des Unternehmens und dessen Kultur auszuprägen. Diese wurde durch eine Vielzahl informeller Mechanismen geteilt, verfestigte sich im Laufe der Zeit jedoch vor allem durch eine entsprechende Auswahl neuer Organisationsmitglieder. Diese wurden entweder gemäß ihrem bereits ausgebildeten und komplementären Wertegerüst selektiert, oder waren wegen ihrer Jugend noch »formbar «. ${ }^{983}$ Viele der späteren Führungskräfte von Anton Schlecker jun. fanden in dieser Zeit den Weg ins Unternehmen und machten im preissensitiven Milieu des Viehhandels ihre ersten beruflichen Erfahrungen. ${ }^{984}$ Auf diesem Wege bildeten sie indivi-

981 Eine damalige Führungskraft: »Wir haben Mühe gehabt, diese Eröffnung nahezubringen mit primitivsten Mitteln. Und darunter war es ganz schlecht. Gar nichts geht, keine Zwischendecke drin, furchtbar! Regale, sonst nichts."

982 Vgl. allgemein Schein (2010: 235ff.) zu den Mechanismen der kulturellen Einbettung persönlicher Werte und Einstellungen in die emergierende Kultur. Ein "Wert» soll hier mit Rokeach (1973) verstanden werden als "an enduring belief that a specific mode of conduct or end-state is personally or socially preferable to an opposite or converse mode of conduct or end-state."

983 Vgl. Schein (1983: 21 f.); Schein (2010: 219).

984 Eine langjährige Führungskraft: »Immer eigentlich zusammen mit der, mit dem Alten, also Freudenreich [...], mit dem alten Schlecker. [...] Jetzt ist der Betrieb so abgelaufen, man hat Schweine aufgekauft in der Gegend und hat Vieh aufgekauft in der Regel in Memmingen, ja, auf dem Großmarkt. Erst später hat man dann auch in Stuttgart gekauft. Ich als Lehrling hab alles gemacht. Ich bin mitgefahren nach Stuttgart, wo wir die Schweine haben zuerst ins Wasser hineinschmeißen müssen, weil sie zu viel verdreckt, ne. Und ich hab Betäubung hier gemacht mit dem Elektroschocker.» 
duelle Fähigkeiten aus, die später als organisationale Kompetenzen Eingang in die Organisation fanden und maßgeblich zur strategischen Ausrichtung des Unternehmens auf den Einkauf und $\mathrm{zu}$ dem in Teil C skizzierten »critical juncture« des selbstverstärkenden Mechanismus beitrugen.

Zugleich übernahmen sie unter der persönlichen Führung des Gründers und dessen erster Führungsriege nach und nach - auch gegen persönliche Zweifel einen Großteil deren Einstellungen, oder zeigen zumindest ein kulturkonformes Verhalten: ${ }^{985}$

"Und man kommt auch sehr schnell in ein Korsett, das man eigentlich zuerst gar nicht anziehen wollte, aber dann viele Zwänge kommen. Und plötzlich ist man der Helfer vom Henker, ja. Und man meint noch, man macht alles gut, man kriegt wieder mehr und wird Prokurist und wird was weiß ich was. [...] wir hatten zu hohe Personalkosten. Also wir müssen statt 40 Stunden 44 Stunden schaffen. Und von den 250 Überstunden lass ich nur 100 stehen.« Etwas Gemeines! Gemeineres gibt's gar nicht. Aber Ich, ich, ich hab's gesagt, der Henkers Gehilfe.

(Langjährige Führungskraft der Anton Schlecker e.K.)

Auch das zweite oben skizzierte kulturelle Merkmal der späteren Anton Schlecker e.K., das auffällige Misstrauen gegenüber der Umwelt, war bereits lange vor dem Einstieg Anton Schleckers jun. in der organisationalen Kultur verankert. Es zeigte sich früh im Verhältnis zu den eigenen Mitarbeitern und dem daraus resultierenden Bedürfnis nach deren Kontrolle.

"Also der alte Schlecker hat gesagt zu seinem Lehrling: »du musst immer das schlechteste vom Menschen denken, und dann kommst du grade so hin."

(Ehemalige Führungskraft der Anton Schlecker e.K.)

In Übereinstimmung mit der Imprinting-Literatur ist das von diesem Menschenbild des Gründers ausgehende Arbeitsregime mit seinen vielen Mechanismen der Kontrolle der Mitarbeiter als besonders nachhaltiges Imprint $\mathrm{zu}$ charakterisieren. ${ }^{986}$ Das Misstrauen gegenüber der Umwelt manifestierte sich aber auch im Verhältnis zu den Kunden:

985 Ehemalige Führungskraft: „Frau Ebbensteiner war Chefsekretärin, aber in an so einem kleinen Tisch und der Herr Freudenreich war vis-a-vis von ihr und von mir. Ich bin am Küchentisch, neben der Ebbensteiner, platziert worden. Herr Freudenreich durch die Scheibe von mir getrennt und der Schlecker, der alte Schlecker ein bisschen weiter hinten. [...]. Und die hat mir dann gesagt, was ich zu tun hab, nämlich die Kuverts fertig zu machen mit den Viehzetteln. [...] Die hat gesagt, wenn du fertig bist, bloß nicht aufgucken. Jetzt war ich nach anderthalb Stunden fertig an dem Tag, und ich durft nicht aufgucken. Ich hab das gemacht...«.

986 Vgl. Baron et al. (1999), die die Permanenz des organisationalen Beschäftigungsmodells des Gründers in Bezug auf die Dimension der Verbindung der Mitglieder zur Organisation, die Koordination und Kontrolle der Angestellten und die Selektion der Mitglieder nachweisen. 
»Man hat ja so blöd gebaut damals, das hat alles mit der Hysterie vom alten Schlecker noch zu tun gehabt. [...] Alle klauen! Und deshalb musste die Ausgangs- und Eingangstür klein sein, in so einem großen Riesenhaus, zur Überwachung ist das gewesen. Und er hat mit seinen Kunden gestritten [...]. Da hat er gestanden oft, der alte Schlecker. ২Packen Sie mal bitte die Taschen aus, ne.« - Ja - er hat es sich leisten können."

(Langjährige Führungskraft der Anton Schlecker e.K.)

Im Zuge der familieninternen Nachfolge wurde nun ein großer Teil der fest im Unternehmen verankerten kulturellen Muster, für die beispielhaft die Sparsamkeit und das Misstrauen skizziert wurden, vom Nachfolger Anton Schlecker übernommen.

"Also das hat er da sicherlich auch übernommen und daraus hat sich auch ein gewisses Misstrauen entwickelt. Aber auch ein Misstrauen gegenüber den eigenen Leuten. Und ich fürchte sogar auch zum Teil gegenüber der Kundschaft.«

(Langjährige Führungskraft der Anton Schlecker e.K.)

Wie von Schein (2010) allgemein beschrieben, hatte die Entscheidung zur familieninternen Nachfolge im Unternehmen Schlecker somit bedeutsame Konsequenzen für die Stabilität der Organisationskultur. ${ }^{987}$ Mit der Entscheidung für eine Übergabe der Kontrolle über das Unternehmen innerhalb der Familie geht häufig eine fortwährende Reproduktion der kulturellen Muster einher - ist der kulturelle Bruch meist doch geringer, als wenn zunehmend familienfremde Führungskräfte mit der Leitung des Unternehmens betraut werden, die eine vollkommen andere Sozialisation, unterschiedliche Erfahrungen und sehr verschiedene Annahmen in die Organisation einbringen und einen inkrementellen kulturellen Wandel befördern. So weist Schein (1983) darauf hin, dass Werte wie das Streben nach Unabhängigkeit in einem signifikant stärkeren Maße in der Organisation reproduziert werden, wenn Familienmitglieder im Unternehmen in leitender Tätigkeit tätig bleiben. ${ }^{988}$

In diesem Zusammenhang zeigt sich bei der Anton Schlecker e.K. wiederum die kulturvermittelnde Funktion der Familie als System, welches seit Jahrtausenden verlässlich Werte und Normen von Generation zu Generation weiterreicht. ${ }^{989} \mathrm{Da}$ familiäre Normen, sofern sie nicht grundsätzlich infrage gestellt werden, meist unbewusst von der nachfolgenden Generation im Zuge ihrer Sozialisation übernommen werden, trug die familieninterne Nachfolge und die

987 Vgl. Schein (2010: 259): »The decision whether or not to retain private ownership [...] may appear to be a financial decision, but it has enormous cultural consequences."

988 Vgl. Schein (1983: 27); auch Grundström et al. (2012) gehen davon aus, dass in der Nachfolge des Familienunternehmens durch Familienangehörige ein Großteil der für Familienunternehmen spezifischen Werte tendenziell erhalten bleibt. Vgl. auch Garcia-Alvarez et al. (2002: 189ff.); Sharma \& Salvato (2013: 43).

989 Vgl. Gersick et al. (1997: 149); Nave-Herz (2014: 3ff.); Stamm (2013: 33). 
Übertragung von historisch gewachsenen Überzeugungen, Werten und Zielen innerhalb der Familie und durch die Familie im Unternehmen bei der Anton Schlecker e.K. zur Stabilität der bereits stark akzentuierten kulturellen Muster bei. ${ }^{990}$ Nicht zuletzt, weil sich im Unternehmen keine kurzfristige Betriebsübergabe vollzog, sondern Anton Schlecker als sehr junger designierter Nachfolger in ein Unternehmen mit einer bereits sehr stark akzentuierten Organisationskultur eintrat und hier fast zehn Jahre unter seinem Vater arbeitete.

Wenngleich die meisten Beiträge der allgemeinen Organisations- und Managementforschung in Bezug auf die Bedeutung der kulturellen historischen Vorprägung für spätere Entscheidungsprozesse vor allem die Gründungsphase der Organisation fokussieren, erweist sich bei der Anton Schlecker e.K. der Übergang von einer Generation zur nächsten im Zuge der familieninternen Unternehmensnachfolge als beinahe ebenso bedeutsam. ${ }^{991}$ Hervorzuheben ist in diesem Zusammenhang, dass sich im Zuge des Generationenübergangs im Unternehmen Schlecker wiederum das Strukturmerkmal des familiären Einflusses der ausgeprägten Personenorientierung zeigte. Im Rahmen der etablierten und stark familiär und partikularistisch geprägten Organisationskultur übernahm Anton Schlecker jun. das gesamte vom Vater bestellte Topmanagement-Team. Diese transgenerationale Kontinuität der Führungskräfte stabilisierte durch selbst praktizierte Reproduktion der Muster und Sozialisation nachrückender Generationen in hohem Maße die im Unternehmen etablierten Werte und Normen.

Im Laufe der sich über Jahrzehnte erstreckenden Zusammenarbeit bildet sich in einem Unternehmen eine »dominant logic« aus, die sich als sehr beharrlich erweist und häufig auch dann die Kommunikationen und Entscheidungen der Manager leitet, wenn die Logik von diesen nicht geteilt wird. ${ }^{992}$ Aufgrund dieser nachhaltigen Prägkraft wird die ursprüngliche Zusammensetzung der Entscheidungsträger von der allgemeinen Imprinting-Forschung gar als ein wichtiges originäres Imprint erachtet. ${ }^{993}$

990 Vgl. Dyer (1988: 46); Hall et al. (2001: 195); Simon et al. (2012: 135).

991 Vgl. Kimberly (1979); Marquis \& Tilcsik (2013); Stinchcombe (1965).

992 Vgl. Prahalad \& Bettis (1986); Nelson (2003: 711). Es kann davon ausgegangen werden, dass insbesondere die beruflich weitgehend unerfahrene Frau von Anton Schlecker, die mehr und mehr mit der Leitung des Konzerns betraut war, von dieser etablierten Kultur stark geprägt wurde. Vgl. S100517ZE, wo Christa Schlecker mit den Worten »Das Personal ist wie ein Möbelstück: Man kann es jederzeit verrücken oder abschaffen« zitiert wird. Eine Führungskraft zu den Auseinandersetzungen der Anton Schlecker e.K. mit der Gewerkschaft: »[D]iesen Personalkampf, den, glaub ich, aber eher Frau Schlecker zu vertreten hatte, weil sie dafür verantwortlich war, für das Personal und da einen persönlichen Krieg geführt hat, mit der Gewerkschaft.»

993 Vgl. Baron et al. (1999: 543):»[A]n important founding condition bearing on organizationbuilding may be the social composition of an organization's initial work force." 
Bei der Anton Schlecker e.K. zeigte sich die Wirkkraft dieses Imprints vor allem am Beispiel von Reinhold Freudenreich, der wichtigste Vertrauensperson von Anton Schlecker sen. Dieser trug durch seine konsequente Orientierung am Menschenbild des Gründers, seine eigene Persönlichkeit und sein eigenes Führungsverständnis in erheblichem Maße zur Ausprägung der sich formierenden Organisationskultur in der Gründungszeit bei. ${ }^{994}$

"Und Freudenreich war menschenverachtend. [...] wenn er gesehen hat, dass der alte, der alte Schlecker kommt, hat er den Lehrling fertiggemacht bis zum Gehtnichtmehr, nur damit der nachher sieht, der Schlecker, was er, mein lieber Mann, das ist ein Ochsentreiber, ne [...] er hat die Menschen ganz klein machen können. Da ist fast kein Tag vergangen, wo der nicht nur geschrien hat, wo er getobt hat, wo er Menschen klein gemacht hat, ganz im Sinne vom alten Schlecker. Ein Beispiel, [...] hat er geheißen [...].. Ich war im Büro, so schräg vis-à-vis. Immer mittags ist der Freudenreich da rauf und hat geschrien, dass es auf 200 Meter Entfernung zu hören, so quasi wie wenn ich da stünde."

(Langjährige Führungskraft zur Rolle von Reinhold Freudenreich zu Zeiten der Fleischwarenfabrik)

Vor allem aber trug Freudenreichs über Jahrzehnte fortwährendes Wirken in erheblichem Maße zur Reproduktion der strategischen Muster und der Stabilisierung der formierten Kultur in der späteren Phase des Unternehmens in der Drogeriemarktbranche bei.

»Aber er war natürlich der langjährige Generalstabsoffizier von Anton Schlecker, der alles, so jetzt aus meiner, wie ich ihn interpretiere dann einfach, dieser Stil hat das Unternehmen geprägt auch. Er war der Umsetzer, der harte Einpeitscher. Wenn du nicht schnell genug über den Hof gelaufen bist, dann hat er hoch, hochgepfiffen und so. Es ist einfach ein Managertyp 80er-Jahre [...]."

(Ehemalige Führungskraft zur Rolle Reinhold Freudenreichs in der späteren Anton Schlecker e.K.)

Wie von Dyer (1988) für Familienunternehmen allgemein postuliert, begann sich die Organisationskultur nicht zuletzt aufgrund des direkt wie indirekt konservierend wirkenden Einflusses der Familie erst im Zuge der schlussendlichen Manifestation der Unternehmenskrise zu wandeln. ${ }^{995}$

Solche organisationskulturellen Prägkräfte determinierten natürlich keineswegs die späteren organisationalen Entwicklungen der untersuchten Unternehmen. Allerdings rahmten sie ebenso wie die Umwelteinflüsse aus der Gründungszeit des Unternehmens und die Facetten des sozio-emotionalen Vermögens

994 Eine ehemalige Führungskraft: „Freudenreich hat die Menschen ganz klein machen können. Da ist fast kein Tag vergangen, wo der nicht nur geschrien hat, wo er getobt hat, wo er Menschen klein gemacht hat, ganz im Sinne vom alten Schlecker." Vgl. auch S120602HB. 995 Vgl. Dyer (1988: 46). 
den strategischen Entscheidungsraum der Prozesse und schränkten durch etablierte kollektive Orientierungsmuster oder die Selektion kulturkomplementärer Organisationsmitglieder die Veränderungsvarianz der strategischen Entwicklung ein. Sie trugen somit ebenfalls zur Formation eines strategischen Entwicklungskorridors bei.

So ließen die scharf akzentuierten kulturellen Merkmale der Sparsamkeit und des Misstrauens gegenüber der Umwelt bei der Anton Schlecker e.K. die Entwicklung eines harten Discount-Konzepts deutlich wahrscheinlicher erscheinen als zum Beispiel die Diversifikation und Expansion über eine serviceorientierte Parfümeriekette. Vielmehr zeichneten sich die Umrisse des späteren Geschäftsmodells - starke Fokussierung auf die Konditionen im Einkauf und weitgehende Vernachlässigung vertrieblicher Aspekte - bereits lange vor dem Eintritt des Unternehmens in den Drogeriemarkt ab.

"Die wichtigste Schlecker-Tugend ist Sparsamkeit, fast das gesamte Management entstammt interner Aufzucht - wer sollte da einen schönen Laden konzipieren können?»

(Das Handelsblatt im Jahr 2011) $)^{996}$

Zugleich hätte es eines grundlegenden kulturellen Wandels und des frühen Austauschs eines großen Teils der Führungskräfte bedurft, um in den 1990erJahren ein Geschäftsmodell zu entwickeln, in dem die sich verändernden Bedürfnisse des Kunden und nicht die im Einkauf erzielten Konditionen im Mittelpunkt stehen.

\subsection{Image der Organisation und deren Marken}

Ein weiteres Imprint, welches den grundsätzlichen strategischen Handlungsraum in den untersuchten Fällen absteckte und später auch beschränkte, waren das Image der Unternehmen und deren Marken. Unter einem solchen Image soll hier das mehrdimensionale Einstellungskonstrukt verstanden werden, welches das in der Psyche relevanter externer Stakeholder fest verankerte, ganzheitlich verdichtete und wertende Vorstellungsbild von einem Unternehmen wiedergibt. ${ }^{997}$

Jedes der in der empirischen Studie untersuchten Unternehmen wies nicht nur einen überaus hohen Bekanntheitsgrad bei den Konsumenten und in der

996 S110118HB.

997 Vgl. zur Definition Meffert et al. (2015: 118). Unter einer Marke kann dabei als eine in der Psyche des Konsumenten und sonstiger Bezugsgruppen fest verankertes, unverwechselbares Vorstellungsbild von einem Produkt oder einer Dienstleistung verstanden werden. 
allgemeinen Öffentlichkeit auf, sondern eben auch ein stark ausgeprägtes Image mit klar definierten Attributen.

So war der Name Berentzen nicht nur eng verbunden mit dem traditionellen Produkt Apfelkorn, sondern auch mit der Vorstellung der ländlichen Lebenswelt eines norddeutschen Gutshofs. Dieses Image entsprang der natürlichen Umwelt des Produktionsstandorts Haselünne im Emsland und wurde über viele Jahrzehnte bewusst und sorgsam gepflegt. In Rahmen dieser Image- und Markenpflege wurde der real existierende Berentzen-Hof in Haselünne auch immer wieder explizit in Marketingmaßnahmen eingebunden. ${ }^{998}$

Durch diese bewusst gepflegte Verbindung zur Tradition entstand auf der Seite der Stakeholder ein klar umrissenes Bild, das sicherlich verkaufsfördernd wirkte. Doch auf der anderen Seite schränkte das eindeutige Image den strategischen Handlungsspielraum weit über den Funktionsbereich des Marketings hinaus ein. So begrenzte dieses traditionelle, ländliche Image massiv die Möglichkeiten der Diversifikation des Produktportfolios. Die mehrmaligen Versuche der Berentzen-Gruppe, sich zum Beispiel mit exotischen Fruchtmischgetränken vom Traditionsprodukt des Apfelkorn zu emanzipieren und sich als szenige Life-Style-Marke am Markt zu präsentieren, waren aufgrund dieser historischen Vorprägung fast vom Vornherein zum Scheitern verurteilt. ${ }^{999}$

Darüber hinaus schränkte die Verbundenheit des Images mit dem traditionellen Standort Haselünne den Spielraum für strategische Richtungsentscheidungen wie die Schließung des Produktionsstandorts Haselünne ein.

"[...] so, wie die Marke Berentzen als Markenwelt aufgebaut wurde, ich sag mal, auch, also ländlich traditionell an dem Berentzen-Hof, den es ja dann auch tatsächlich real in Haselünne gibt, sehr stark verankert ist. [...] Dass es eben nicht so das Produkt ist, wo man sagt, also das ist eigentlich egal, wo es hergestellt wird, ob's auf der grünen Wiese in Minden oder sonst wo. Da spielt natürlich genau diese emotionale Komponente rein."

(Ehemaliger Vorstand der Berentzen-Gruppe AG)

998 Vgl. B950817WS.

999 Vgl. die vollkommen konträren TV-Spots: Während 1996 der Spot mit den Worten »komm raus zu uns, kaum raus aufs Land» beginnt und eine Großmutter ihrem Besuch »Großmutter's Schoko-Geheimnis" präsentiert (B160212YT) trinken nur wenige Jahre später junge, gutgelaunte Menschen Berentzen-Limone auf einem Surfbrett am Strand (B061125YT). B020812YT zeigt Berentzen dann wiederum völlig anders als Szene-Getränk "on the rocks« in einer Cocktailbar, während neueste Spots unter dem neuen Eigentümer wieder das beim Konsumenten verankerte Bild des "authentischen « Spaßes auf dem Land ausrufen. Eine ehemalige Führungskraft: »[W]ir haben uns auf unseren Kernmarkt zu besinnen, ja, und zu beschränken. Und, klar, das wird natürlich auch auf dem Schützenfest oder so, ,ja, die Berentzens machen jetzt einen auf international, da trinken wir lieber was Authentischesı.» 
Die Eckes AG war in ähnlicher Weise historisch geprägt. Ihre Produkte wie die Weinbrände Mariacron und Chantré oder der Likör Eckes Edelkirsch waren jahrzehntelang die Marktführer ihrer Kategorien gewesen, als Marken äußerst bekannt und im Sortiment fest etabliert. ${ }^{1000}$ Doch auch hier war das Image dieser Marken auf Seiten der Verbraucher so stark vorgeprägt, dass es nicht nur das Image des gesamten Unternehmens gefärbt hatte, sondern auch zumindest die Alkoholsparte in ihrer strategischen Entwicklung beschränkte. So fand sich nach dem Entschluss, sich auf den Fruchtsaftbereich zu konzentrieren, nicht zuletzt deshalb lange Zeit kein Käufer für die Alkoholsparte, weil sich der Großteil der Marken nicht mehr glaubhaft einer Verjüngung zuführen ließ. ${ }^{1001}$

Doch in Unternehmen, die sehr eng mit einer Familie gekoppelt sind und sich ko-evolutionär mit dieser entwickeln, kann im Laufe der Zeit nicht nur das Image der Produkte auf das Unternehmen abfärben, sondern auch das Image der Familie oder einzelner Persönlichkeiten. ${ }^{1002}$ Dass aus dieser familiären Prägung des Unternehmensimages gar eine Beschränkung der strategischen Entwicklungsmöglichkeiten erwachsen kann, lässt sich besonders eindrücklich am Beispiel der Anton Schlecker e.K. verdeutlichen.

In diesem Unternehmen war die Omnipräsenz des eigenen Familiennamens ein wichtiger Bestandteil des sozio-emotionalen Vermögens und wurde beständig gepflegt. ${ }^{1003}$ So wurde die Eigenmarke AS nach dem Inhaber Anton Schlecker benannt und die Mitarbeiterzeitschrift nach dessen Frau Christa. Zugleich war das immer gleiche Portrait der Inhaber nicht nur in den Verwaltungsgebäuden des Unternehmens allgegenwärtig, sondern auch in den Filialen des Unternehmens. Darüber hinaus wurde der Name Schlecker in den Publikationen des Unternehmens nicht nur auffallend häufig verwandt, sondern auch immer in Großbuchstaben gesetzt.

Diese enge Verbindung des Unternehmens mit der Familie in der Außendarstellung der Anton Schlecker e.K. nahm im Zuge der organisationalen Entwicklung allerdings auch einen zunehmenden Einfluss auf die strategischen Entwicklungsmöglichkeiten des Unternehmens. Im Laufe der Jahre hatte sich das Unternehmen durch eine Vielzahl verschiedenster Skandale in der Öffentlichkeit ein negatives Image erworben. Verunreinigte Babynahrung, der Miss-

1000 Vgl. Eckes (2007: 30f.).

1001 Vgl. E021008LZ; E030210LZ.

1002 Vgl. Simon et al. (2005: 77f.): »Als Familie heißt man eben nicht ungestraft wie eine Marke [...] die familiäre Identität und die ihrer Mitglieder sind kaum vom Unternehmen zu trennen."

1003 Vgl. S131009WS. Zur Verwendung der Familienbilder im Unternehmen S120313WW; S080325MM. Auf die Frage, was denn für ihn das Schlimmste an der Pleite des Unternehmens sei, antwortete Anton Schlecker gegenüber einem der Interviewpartner im Privaten, dass "nun der Familienname nicht mehr überall zu lesen sei«. 
brauch von Leiharbeitsregelungen, der schließlich zur »lex Schlecker « durch Bundesarbeitsministerin von der Leyen führte, die Verurteilung von Anton und Christa Schlecker wegen Lohnbetrugs, der von der Gewerkschaft immer wieder angeklagte Druck auf die Angestellten und die Verhinderung der Bildung von Betriebsräten - in Reaktion auf all diese Vorfälle formte sich in der Öffentlichkeit ein scharf konturiertes Bild des Unternehmen.

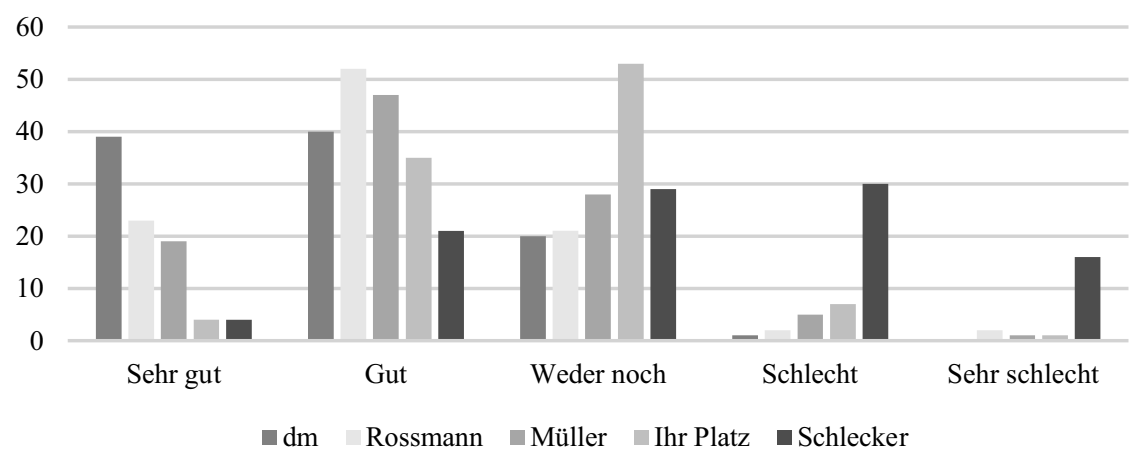

Abbildung 25: Unternehmensimage der fünf führenden Drogeriemärkte Deutschlands im Jahr 2009

(Quelle: Eigene Darstellung auf Grundlage von Daten von handelsdaten.de / Grass Roots).

Infolge der engen Verbindung des Unternehmens mit der Familie im Zuge der Außendarstellung begannen die Konsumenten zunehmend dieses negative, mit einer starken affektiven Komponente verbundene Image zu personifizieren. ${ }^{1004}$ Durch die wenigen existierenden Bilder der Familie bekam das Unternehmen ein Gesicht und diese Personifizierung trug dazu bei, dass sich die Vorstellung vom Unternehmen nachhaltig in den Köpfen der Konsumenten verankerte. Für viele Menschen wurde insbesondere Anton Schlecker - nach den Daten der empirischen Untersuchung teils sicherlich zu Unrecht - gar zum Idealtyp des skrupellosen und gierigen Unternehmers.

»Nach einer Umfrage des `Handelsblatts` gilt Anton Schlecker als der mit Abstand unbeliebteste Unternehmer in Deutschland [...]. ^Ideale Manager sind in den Augen der Menschen dagegen Bosch-Chef Franz Fehrenbach und dm-Gründer Götz Werner mit 88 und 87 Prozent Zustimmung."

(Das Handelsblatt im Jahr 2012) ${ }^{1005}$

Eine wichtige Rolle für die Permanenz des Negativimages spielte hier die starke Umweltabschottung des Unternehmens, die in der theoretischen Einleitung als ein strukturelles Merkmal eines steigenden familiären Einfluss herausgearbeitet

1004 Vgl. Meffert et al. (2015: 116) zu den Komponenten des Images.

1005 S120316HB. 
wurde und gerade bei der Anton Schlecker e.K. direkt auf den Einfluss der selbst vollkommen abgeschieden lebenden Familie Schlecker zurückzuführen ist. Diese Umweltabschottung war bei der Anton Schlecker e.K. derart stark ausgeprägt, dass das Unternehmen sogar weitgehend auf Öffentlichkeitsarbeit oder andere Maßnahmen zur Verbesserung des Images verzichtete. ${ }^{1006}$ So wurde der Öffentlichkeit kaum die Möglichkeit gegeben, ihr Negativbild zu revidieren.

Erst kurz vor der Insolvenz begann das Unternehmen professionell und nachhaltig an seinem Image zu arbeiten. Über ein neues Logo und erstmals gesendete TV-Spots wurde versucht den Neuanfang als »sympathischer Nachbar Schlecker « zu kommunizieren, für den nun die Kinder Lars und Meike Schlecker als die neuen Gesichter des Unternehmens in die Öffentlichkeit traten. ${ }^{1007}$

Doch das langfristig erworbene und fest in den Köpfen der Konsumenten verankerte Negativimage des Unternehmens und der Familie behinderte als historischer Imprint die Ansätze zum strategischen Wandel im Zuge des Turnarounds massiv. Die Modernisierung einiger Hundert Filialen erwies sich als einfacher als der Imagewandel. Viele Konsumenten boykottierten Schlecker aus sozialen Beweggründen bis zur Insolvenz das Unternehmens. ${ }^{1008}$

"Zwar sei Schlecker eines der bekanntesten Handelsunternehmen Deutschlands, sagte der Geschäftsführer des Kölner Instituts für Handelsforschung (IFH), Manfred Hunkemöller [...]. Der Ruf der Kette sei aber schwer belastet. ১Die Diskussionen um Dumpinglöhne und Mitarbeiterschikanen bleiben beim Kunden in Erinnerung. Ähnlich sieht es der Art Directors Club Deutschland: `Als Marke hat Schlecker seine letzte Chance gehabt und verspielt. Der alte Name und Markenauftritt [...] steht für den Unterschicht-Billig-Drogeriemarkt $\_$«

(Ausschnitt aus einem Bericht von Spiegel-Online B120128)

Der Unternehmens- und Familienname Schlecker wurde von Seiten der hinzugezogenen Berater gar als so große Belastung für den strategischen Wandel erachtet, dass im Zuge des Turnaround-Versuchs 2010 gar auf eine vollständige Umbenennung des gesamten Unternehmens gedrängt wurde. ${ }^{1009}$

1006 S. zu kulturellen und strukturellen Mustern der Abschottung ausführlich Kapitel D.2.1.2. 1007 Vgl. S110523LZ. Lars Schlecker 2011 vor Mitarbeitern: »Wir wollen keine Negativschlagzeilen mehr. Wir wollen nicht mehr der Buhmann für Deutschland sein [...] um unseren Verkauf nachhaltig zu befeuern, müssen wir auch unser Arbeitgeber-Image verbessern.«; vgl. S110624.

1008 Vgl. S110613: „FAZ: „Sie werben im Fernsehen mit lustigen Spots. Sollten Sie nicht eher betonen, dass Sie Ihre Leute gut behandeln? Viele boykottieren Schlecker aus sozialen Gründen. Lars Schlecker: »In der Tat denken wir darüber nach, unsere Mitarbeiter mehr in die Werbung einzubeziehen. Wir haben sicher Fehler gemacht, können das Ruder aber nicht über Nacht herumreißen."

1009 Vgl. S120602HB. 


\subsection{Eigentumsstruktur und familial-organisationale Konfiguration}

So bedeutsam die historischen Umwelteinflüsse aus der Gründungszeit der untersuchten Unternehmen für deren spätere Entwicklung waren und so sehr spätere strategische Entwicklungen von tradierten kulturellen Mustern der Organisation geprägt wurden - als das für die spätere strategische Entwicklung mit Abstand am bedeutsamste Imprint erweisen sich die Strukturen des Eigentums. ${ }^{1010}$ Die Eigentumsstrukturen und die daraus erwachsenden formalen organisationalen Strukturen an der Spitze der Organisationen prägten unmittelbar die strategischen Entscheidungsräume der untersuchten Unternehmen und die sie durchlaufenden Entscheidungsprozesse. In jedem der untersuchten Unternehmen spielte die Eigentumsstruktur eine maßgebliche Rolle für den Verlauf der Entscheidungsprozesse und das Entstehen der strategischen Pfade. Dies gilt sowohl für die Anton Schlecker e.K., in der die Unternehmensfamilie innerhalb der dominanten Koalition weitgehend unbeschränkt und unkontrolliert entscheiden konnte, als auch für die Berentzen-Gruppe AG, bei der die strategischen Entscheidungsprozesse von einer komplexen Eigentumsstruktur mit mehreren Zentren innerhalb der dominanten Koalition geprägt waren.

"Ich hab aus der Berentzen-Sache eines auch gelernt, diese Begriffe wie familienfremdes Management, ich sage ihnen ganz ehrlich, das, das interessiert mich heute nicht mehr. Das ist für mich teilweise Grundvoraussetzung. Ich schaue mir aber heute, wenn ich nochmal in so eine Situation käme, die, die Mehrheitsverhältnisse, die Besitzverhältnisse, die Aufsichtsratskonstellation hundert Mal an, hundert Mal. Weil das passiert mir persönlich, und jetzt spreche ich persönlich, das passiert mir persönlich nicht mehr, weil ich dafür viel zu viel Energie in solche Themen investieren musste [...], da war ein großes U, stand ein, ein Tisch mit, keine Ahnung, zwanzig, dreißig Plätzen. Waren alle besetzt. Und das waren alles irgendwie Familienangehörige. Und da müssen sie wissen, wer hat bei dem Ehepaar dahinten das Sagen und wie verstehen die sich und wie - was sagt die zu der ihrer Tasche. Und, und solche, solche Sachen spielen da leider eine Rolle. Jetzt nicht Berentzen-spezifisch, sondern generell. Und das muss man wissen. Leider."

(Ehemaliges Vorstandsmitglied der Berentzen-Gruppe AG)

Im Allgemeinen wird im Zuge der organisationalen Strukturierung von Einfluss, Verantwortlichkeit und Kontrolle Entscheidungsträgern auf der einen Seite Handlungsautonomie im formal definierten Rahmen verliehen; auf der anderen Seite geht der Aufbau von Strukturen immer mit Einschränkungen der Handlungsmöglichkeiten der Akteure und der im System zugelassenen Hand-

1010 Vgl. allgemein zur Bedeutung historisch geprägter organisationaler Strukturen für den strategischen Handlungsraum Kimberly (1987: 234); Koch (2011); auch Gutenberg (1976: 488). 
lungsverknüpfungen einher. ${ }^{1011}$ Nach erfolgter Strukturierung wird von den Entscheidungsträgern erwartet, dass sie sich innerhalb des von den formalen Strukturen der Organisation vorbestimmten Handlungsspielraums bewegen und ihre formal definierte Entscheidungsautonomie nicht überschreiten. ${ }^{1012}$

Diese handlungsbeschränkende organisationale Strukturierung von Entscheidungskompetenz und Kontrolle wird insbesondere in Bezug auf den strategischen Entscheidungsraum an der Spitze der organisationalen Hierarchie maßgeblich vom Faktor Eigentum geprägt, denn zum einen sind es die Eigentümer, die innerhalb des allgemeinen rechtlichen Rahmens und der Besonderheiten der jeweiligen Rechtsformen schlussendlich die Ausgestaltung der organisationalen Entscheidungs- und Kontrollstrukturen bestimmen. Zum anderen ist es die Struktur des Eigentums, die maßgeblich die Verteilung von Macht innerhalb dieser Strukturen und die Möglichkeiten deren Veränderung bedingt.

Im Vergleich zu den oben skizzierten Imprints wie dem Image des Unternehmens oder den Facetten des sozio-emotionalen Vermögens führt die Eigentumsstruktur somit auch weniger zu einer direkten Beschränkung auf einen spezifischen, inhaltlich determinierten strategischen Korridor. ${ }^{1013}$ Vielmehr setzt die Eigentumsstruktur maßgeblich den organisationalen Rahmen, in dem die späteren strategischen Entscheidungsprozesse verlaufen und nimmt auf diesem Wege entscheidenden Einfluss auf deren Verlauf und Ergebnis, wie im Fall Berentzen:

»[...] die Stämme Pabst und Richards [haben]damals eine, eine, eine, ja, eine Minorität gehabt. Allerdings, [...] da- da bin ich schon davon überzeugt, das war vielleicht einer der Fehler, dass man gesagt [hat], ,Ihr habt zwar eine Minorität, aber Ihr habt eine [Sperrminorität], Ihr könnt mit dieser Minorität sämtliche Entscheidungen verhindern. ( Damals hat man gesagt, ja, man mag sich ja eh und ist gut miteinander, geben wir ihnen das Recht, wir haben ja schon die höheren Anteile. Im Nachhinein muss man sagen, ist das natürlich eine Katastrophe gewesen. Weil, sonst hätte man ja später gar nicht die Grundlage gehabt, jede betriebswirtschaftliche Entscheidung zu behindern. [...] Eine klassische Pattsituation. Insofern, das war sicherlich ein Fehler, dass man da zu viel Vertrauen reingelegt hat, im Rahmen der Übernahme und dadurch sich ei-

1011 Vgl. Schreyögg (2006: 12).

1012 Vgl. Schreyögg (2006: 109). Systemtheoretisch gesehen, ergibt sich gemäß Luhmann (1984:397) die Strukturierung des strategischen Aufgabenvollzugs durch den Aufbau von Erwartung, und Erwartungen entstehen durch Einschränkungen.

1013 Wenngleich viele Beiträge den Einfluss auf allgemeinere strategische Ausprägungen zurückführen. So weisen zum Beispiel Baysinger et al. (1991) nach, dass mit dem Grad der Konzentration des Eigentums auch der Anteil der F\&E-Ausgaben steigt. Bethel \& Liebeskind (1993) zeigen, wie die Struktur des Eigentums die Wahrscheinlichkeit einer organisationalen Restrukturierung beeinflusst. 
gentlich die eigene Handlungsweise in die Zukunft projiziert, eingeschränkt.»

(Ehemalige Führungskraft der Berentzen-Gruppe AG)

Wie in vielen Unternehmen waren die Eigentumsstrukturen der in der empirischen Studie untersuchten Unternehmen stark historisch geprägt und lassen sich in weiten Teilen aus der evolutionären Entwicklung der Unternehmen ableiten. So sind die mehrstämmigen Eigentumsstrukturen der Berentzen-Gruppe AG und der Eckes AG ebenso auf teils Jahrzehnte zurückliegende Entscheidungen und evolutionäre Prozesse zurückzuführen wie die dominante Stellung der Unternehmensfamilie Schlecker in der Anton Schlecker e.K.

»The structural properties of an organization are perhaps the most evident source of how history is reflected in the development of an organization."

$(\text { Koch, 2011) })^{1014}$

Im Zuge dieser evolutionären Prozesse erwies sich vor allem der Einfluss des Kontextfaktors Familie im Zuge der Ko-Evolution der beiden Systeme für die Entwicklung der Eigentums- und Organisationsstrukturen als bedeutsam. Aufgrund der besonders engen Kopplung der Systeme im Bereich des Eigentums beeinflussten die Irritationen der Familien nicht nur die Ausübung der Eigentümerrechte innerhalb der gegebenen organisationalen Strukturen, sondern prägten vor allem auch deren historische Evolution. ${ }^{1015}$ Hier zeigte sich, dass vorhergehende Generationen nicht nur, wie oben beschrieben, die kulturellen Muster der Organisation prägen, sondern zum Ende ihrer Wirkungszeit im Unternehmen mit der Entscheidung für eine bestimmte Form des generationalen Transfers von Leitung und Eigentum vor allem auch unmittelbar das Fundament für die zukünftige organisationale Struktur des Unternehmens legen.

Während durch den Einfluss der Familie bei der Anton Schlecker e.K. so die monozentrische Konfiguration reproduziert wurde, welche auch den Kontrastfall der Dirk Rossmann GmbH kennzeichnete, hatte sich durch die Prägung der Familie bei der Berentzen-Gruppe AG ebenso wie bei der Eckes AG im Laufe der Zeit unter dem familiären Einfluss eine polyzentrische familial-organisationale Konfiguration herausgebildet. ${ }^{1016}$

1014 Koch (2011: 338); auch Kimberly (1987: 234); Baron et al. (1999: 528): »[D]ifferences in how administrative structures evolve might be [...] 'programmed « in an organizations' infancy."

1015 Vgl. Klein (2010: 152 ff.); Wimmer et al. (2005: 101). Dieser Umstand verweist wiederum auf die Bedeutung der Nachfolge als Schlüsselperiode für die historische Prägung der Organisation.

1016 Vgl. zu diesem anhand von Konfigurationen strukturierten Analyse in der strategischen Prozessforschung Mintzberg (1984); (Pettigrew 2001: 702). 


\section{Monozentrische Konfiguration}

Die Fleischwarenfabrik Schlecker war ein in hohem Maße auf die Person des Gründers und einige wenige um ihn gruppierte Führungskräfte zugeschnittenes Unternehmen. Diese monozentrische dominante Koalition vereinigte alle wichtige Entscheidungskompetenz auf sich und war keiner weiter gehenden Kontrolle der Entscheidungen durch organisationale Gremien oder formalisierte Entscheidungsprozesse unterworfen.

Diese monozentrische Struktur wurde im Zuge der Nachfolge von Anton Schlecker sen. zu Anton Schlecker jun. organisational reproduziert. Grundlegend für dieser Reproduktion war die Entscheidung des Gründers Anton Schlecker, Eigentum und Kontrolle am Unternehmen in Analogie zum bäuerlichen Anerbenrecht oder der feudalen Primogenitur an lediglich einen einzelnen Nachfolger zu übertragen. ${ }^{1017}$

An dieser Nachfolge lassen sich in Bezug auf die Strukturen der Organisation vor allem jene tradierenden Kräfte der Familie zeigen, die auch hinsichtlich der anderen Imprints zutage traten. Bereits im Allgemeinen erweisen sich analog zur Reproduktion kultureller Muster - spezifische Formen der organisationalen Strukturierung als meist umso nachhaltiger, je länger der Gründer oder dessen Familie im Unternehmen präsent sind. ${ }^{1018}$ Doch insbesondere im Zuge von Nachfolgen nach dem »Thronfolgermodell« übernimmt die nachfolgende Generation häufig die Aufgabenstrukturierungen, Kommunikationsformen und Führungsverständnisse der vorhergehenden Generation. ${ }^{1019}$ Denn insbesondere in solchen einpoligen Strukturen kann die nachfolgende Generation in vielfältiger Weise den großen Einfluss des Übergebers im Unternehmen wahrnehmen und entwickelt in der Folge häufig ein von diesem erlebten Unternehmerbild geprägtes Rollenverständnis. Zugleich wird die Übernahme dieses an den Werten von Autonomie und Kontrolle orientierten organisationalen Modells vom Übergeber aber auch explizit nahegelegt.

Im Fall der Anton Schlecker e.K. dürften sowohl implizite wie explizite Einflüsse zur Reproduktion der organisationsstrukturellen Komponenten der monozentrischen Konfiguration beigetragen haben. Als Anton Schlecker jun.

1017 Vgl. Simon (2012: 74ff.); Wiechers (2006: 191). Die Verbreitung dieser Erbpraktik zeigt sich an den Zahlen zum Alleineigentum. Gemäß Klein (2010: 161) sind $52 \%$ aller Unternehmen in der ersten Generation im Alleinbesitz, in der zweiten Generation $29 \%$ und in der dritten Generation $14 \%$.

1018 Nach Hannan \& Freeman (1984) gehört die Form der organisationalen Strukturierung allgemein zu den Aspekten der Organisation mit der größten Trägheit. Vgl. auch Baron et al. (1999: 531); Nelson (2003: 722).

1019 Vgl. Klein (2010: 165); Simon et al. (2005: 78):»Im Rückblick gesehen war nach dem Krieg die Rolle von Rudolf-August Oetker zentral. Er wirkte als Integrationsfigur in der Familie wie im Unternehmen. Eine Erwartung, die heute auf die persönlich haftenden Gesellschafter übergegangen ist." 
die Nachfolge im Unternehmen antrat, hatte er bereits zehn Jahre gemeinsam mit dem willensstarken Vater in der von diesem etablierten und dominierten Kultur und Struktur zusammengearbeitet. In dieser langen Phase dürfte er durch den sozialisierenden Einfluss der Familie und die kulturelle Prägung im Unternehmen nach und nach eine Vorstellung einer »idealen« Organisationsform persönlich entwickelt haben.

"Ja. Also wenn Sie es so sehen, glaub ich, dass, wenn einem klar ist, dass die nächste Familiengeneration weitermacht, dann wird der Stil des Vaters und der Mutter erst einmal weitergelebt. Länger, als wenn etwas anders/ wenn etwas übergeben wird, zumindest mit Abstand. Weil wiederum ja auch Personifizierungen erfolgen.»

(Ehemalige Führungskraft der Anton Schlecker e.K.)

In gewisser Weise wurde die dominante organisationale Rolle, die Anton Schlecker sen. in seinem Unternehmen ausübte, aber auch explizit von ihm an seinen Sohn Anton Schlecker jun. vermacht.

"Schleckers Schwestern überging der Vater, auch wenn er die beiden finanziell bestens versorgte. Er wollte die Firma in einer Hand wissen. Einer entscheidet, sonst gibt's Chaos, sagte er immer."

(Das Handelsblatt im Jahr 2012) $)^{1020}$

In jedem Falle mussten die organisationalen Strukturen der monozentrischen Konfiguration durch die praktizierte Übergabe der Rolle von Anton Schlecker sen. an Anton Schlecker jun. nicht angepasst werden. ${ }^{1021}$ Hingegen wurde lediglich die Position an der Spitze der monozentrischen familial-organisationalen Konfiguration neu besetzt.

»Bisher war ich der Schlecker und das der Junior. Jetzt drehen wir es um. Ab sofort ist er der Schlecker, und ich mach nichts mehr."

(Anton Schlecker sen. 1974 vor seinen Mitarbeitern) ${ }^{1022}$

Im Rahmen dieser reproduzierten monozentrischen Konfiguration gruppierten sich zwar einige wenige Führungskräfte, seine Frau Christa und später die beiden Kinder um Anton Schlecker zur dominanten Koalition. Doch innerhalb dieser Gruppe lag die letztendliche Entscheidungsgewalt vollständig in den Händen von Anton Schlecker. So konnte er nicht nur die Entscheidungsprozesse dominieren, sondern eben auch die organisationale Struktur nach Belieben auf sich und seine Frau zuschneiden. ${ }^{1023}$

Als alleiniger Eigentümer traf er so zunächst die Entscheidung das Unter-

1020 S120602HB.

1021 Vgl. allgemein zu dieser Rollenübertragung Gersick et al. (1997: 67).

1022 S120602HB.

1023 Ein ehemaliger Berater hierzu:»Und die Führungsstruktur war ganz klar. Alles über Herrn Schlecker und Frau Schlecker, sag ich mal.» 
nehmen in der Rechtsform des eingetragenen Kaufmanns zu führen. Mit dieser Rechtsformwahl ging auf der einen Seite die persönliche Haftung als Unternehmer einher, auf der anderen Seite aber auch die beinahe vollständige Kontrolle über das Unternehmen.

»Und die Gesellschaftsform, das war eine Diskussion bis zum Gehtnichtmehr. Weil jeder Steuerberater, jeder Wirtschaftsprüfer, jeder Notar, alle haben gesagt: । Ändere das. Wenn Du mal in die Haftung musst...২. - >Meiner Firma passiert nichts.» So hat er gesagt. Wollte die Kontrolle nicht abgeben."

(Ehemalige Führungskraft der Anton Schlecker e.K.)

Der Umstand, dass die Unternehmensfamilie auf die Einrichtung eines Kontrollund Aufsichtsorgans verzichtete, trug ebenfalls zur aus der Konzentration des Machtpotenzials resultierenden asymmetrischen Machtverteilung innerhalb der dominanten Koalition bei.

Diese monozentrische Konfiguration wurde in der Folge unverändert über mehrere Jahrzehnte beibehalten. Erst im Zuge des Turnaround-Versuchs 2010 wurde die Organisationsstruktur grundlegend geändert. Mit der Position des COO und des CFO wurde eine neue organisationale Hierarchieebene eingezogen und das operative Geschäft größtenteils an familienfremde Manager übertragen. ${ }^{1024}$ Nach dieser Änderung der Organisationsstruktur berichteten die für die Funktionsbereiche zuständigen Direktoren nun nicht mehr direkt an die Familie Schlecker, sondern an den COO und CFO.

"Seit etwa einem Jahr berichten bei Schlecker nahezu alle Direktoren an einen der beiden Manager, nicht mehr an die Familie. Anfang 2011, nach Jahrzehnten der Alleinherrschaft, beschloss Anton Schlecker, ein externes Duo in die oberste Führungsebene zu lassen. Eine Doppelspitze direkt unterhalb der Eigentümerfamilie, in der bis dato nur einer entschied: der Patriarch.»

(Das Handelsblatt im Jahr 2012) ${ }^{1025}$

$\mathrm{Zu}$ dieser Kontinuität der organisationalen Struktur im Rahmen der monozentrischen Konfiguration trugen wiederum familiäre Einflüsse bei. Die fortwährende Fortschreibung dieser Konfiguration beruhte vor allem auch auf der Aussicht, Eigentum und Kontrolle am Unternehmen sowie die selbst ererbte Rolle in der Organisation nochmals ohne strukturelle Änderungen innerhalb der Familie an die beiden Kinder übergeben zu können. Ohne diese Perspektive der innerfamiliären transgenerationalen Kontinuität wäre Anton Schlecker bereits deutlich früher dazu gezwungen gewesen, zur Übergabe des Unterneh-

1024 Vgl. S110120HB: "Der COO, ein Mann aus dem internen Nachwuchs, übernahm neben dem Einkauf auch den Vertrieb und das Marketing. Der CFO, der extern rekrutiert wurde, war neben den Bereichen Finanzen und Controlling auch für Logistik- und Verwaltungsentscheidungen verantwortlich."

1025 S120214HB. 
mens an familienfremde Manager professionellere und personenunabhängigere Strukturen aufzubauen - hätte die Integration familienfremder strategischer Entscheidungsträger wie eines $\mathrm{CEO}$ doch die Etablierung gänzlich anders gearteter organisationaler Strukturen bedurft. Da jedoch die Aussicht auf eine familieninterne transgenerationale Kontinuität bestand, übte der familiäre Einfluss eine konservierende Wirkung auf die organisationale Struktur aus.

"[...] dass das ein bisschen, diese organisationale Struktur auch gestützt hat. Wenn man zum Beispiel sieht bei LIDL, da haben die Kinder ganz klar gesagt, `Nee, machen wir auf keinen Fall.ı Da war Herr Schwarz gezwungen, auf jeden Fall Professionalisierungsschritte einzuleiten. So was machte Anton Schlecker nicht, weil er dachte, dass seine Kinder den Laden irgendwann einfach weiterführen."

(Ehemalige Führungskraft der Anton Schlecker e.K.)

\section{Polyzentrische Konfiguration}

In vielen Unternehmensfamilien wird das Eigentum am Unternehmen nicht nach dem Thronfolgermodell vererbt, sondern gleichverteilt an alle Kinder. Diese Erbpraktik der Gleichverteilung ist teilweise dem Umstand geschuldet, dass schlicht die Mittel zur Kompensation nicht berücksichtigter Kinder fehlen. In den meisten Fällen ist sie jedoch auf die unserer Gesellschaft vorherrschende Gerechtigkeitslogik von Gleichheit und Gleichbehandlung der Kinder in Familien zurückzuführen. ${ }^{1026}$ Selbst wenn hinsichtlich potenziell negativer Auswirkungen auf die zukünftige Entscheidungsfindung im Unternehmen Bedenken bestehen sollten, können sich doch nur die wenigsten Übergeber dieser in Gesellschaft und systemischer Logik des Systems Familie verankerten Norm schlussendlich entziehen. Entsprechend stellt die gleichmäßige Vererbung an alle existierenden Nachkommen die dominante familiäre Erbpraktik der letzten Jahrzehnte dar, nicht zuletzt deshalb, weil ein derart gestalteter Erbvorgang im normativen und rechtlichen Bezugsrahmen der Gesellschaft auch eine Regel etabliert, die häufig richtungsweisenden und institutionellen Charakter für alle folgenden Generationen besitzt. ${ }^{1027}$ Während nämlich im Falle des Thronfolgermodells dessen Legitimität im Zuge des nächsten Erbgangs mit guten Erfolgsaussichten wieder infrage gestellt werden kann, ist die legitimatorische Kraft der Erbnorm der Gleichverteilung so hoch, dass davon in späteren Generationenübergängen nur schwerlich wieder abgerückt werden kann. Einer der Gründe dafür ist, dass im Falle einer angestrebten stärkeren Bündelung des

1026 Vgl. Simon (2002).

1027 Vgl. Simon (2012: 70f.); Wiechers (2006: 190). Die nachhaltige Wirkkraft und Unumkehrbarkeit dieser Lösung wurde auch in der explorativen Vorstudie immer wieder hervorgehoben: »Also, ja, es macht, die zweite Generation macht es, und ist dann nicht mehr aufhebbar [...], wenn es einmal eingeführt war, können Sie es nicht mehr auflösen, weil keiner das Geld dazu hat, weil es häufig auch nur einstimmig geht [...].» 
Eigentums zu den hohen emotionalen Kosten auch eine hohe finanzielle Belastung tritt, müssten doch die auf ihren Eigentumsanteil verzichtenden Familienmitglieder entsprechende Kompensation erhalten.

Da viele Übergeber diese Kosten scheuen, erhöht sich im Zuge der generationalen Transfers unter dem Einfluss der familiären Logik der Kreis der Eigentümer durch das Bruchteilseigentum in vielen Fällen von Erbgang zu Erbgang immer weiter. ${ }^{1028}$ Zusammengehalten von der Zentripetalkraft des gemeinsamen Eigentums wächst dabei auch das System Familie von Generation zu Generation immer weiter an. Letztlich können in diesem Rahmen gar soziale Systeme erwachsen, die hinsichtlich Größe und unpersönlicher Beziehungen mehr den Verwandtschaftsnetzwerken eines Clans oder einer Sippe ähneln als einer bürgerlichen Kleinfamilie. ${ }^{1029}$ Somit werden mit der Wahl der familiären Erbpraktik der Gleichverteilung in vorhergehenden Generationen bereits die Weichen für die spätere Entwicklung und Gestalt der familial-organisationalen Konfiguration gestellt.

Bei der Berentzen-Gruppe AG resultierte die Eigentumsstruktur zunächst aus dem familiären Transfer von Eigentum und Leitung von Johann B. an die Brüder Hans und Friedrich Berentzen. Zu diesen beiden Familienstämmen traten im Zuge der Fusion mit der Weinbrennerei Pabst \& Richarz drei weitere Familien (Pabst, Richarz, Wolff).

Nach der Fusion existierten innerhalb der dominanten Koalition zwei Machtzentren: auf der einen Seite das Zentrum der Familien Berentzen, um die seit Jahrzehnten eng zusammenarbeitenden Brüder Hans und Friedrich Berentzen. Auf der anderen Seite gab es das zweite Zentrum um die Familien Pabst und Richarz. Zusammengenommen besaßen die Familien Berentzen zwar eine knappe Mehrheit des Eigenkapitals, aber eine vertragliche Regelung sollte verhindern, dass eine Seite die andere »beherrschen« könnte.

"Am Kapital der neuen J.B. Berentzen GmbH \& Co [...] wird Berentzen zwar die Mehrheit besitzen, doch ist, wie betont wurde, vertraglich festgelegt, dass keine Seite beherrscht werden kann."

(B87088)

Diese vertragliche Regelung ist zum einen darauf zurückzuführen, dass im Zuge der recht rasch zwischen den Familien vereinbarten und durchgeführten Fusion die genaue Bewertungsgrundlage der Unternehmen zunächst offen blieb, zum

1028 Vgl. Gersick et al. (1997); Klein (2010: 153).

1029 Vgl. Simon (2012: 72); James (2006). Traditionsreiche dynastische Unternehmensfamilien wie Haniel, in denen auf diese Art und Weise schon viele Erbgänge vollzogen wurden, zählen so mittlerweile mehr als 600 familiäre Gesellschafter. Rechnet man noch die Angehörigen wie Ehepartner, Kinder bislang ohne Anteile etc. hinzu, so kommt man in diesen Familien auf eine Anzahl von bis zu 1.000 »Familienmitgliedern«. 
anderen aber auch darauf, dass zwischen den Familien ein hohes Vertrauen herrschte. Schlussendlich führte diese vertragliche Vereinbarung in der Organisation zu einer paritätischen Besetzung der entscheidenden Gremien.

»Ja, und schwupp wurde die Fusion vorbereitet, und 1988 durchgeführt. Und da gab es dann Schwierigkeiten am Ende in der Bewertung der beiden Firmen. Wie soll man abgrenzen, wie kommt man zu einer ausgewogenen Wertdarstellung? Und dann haben die gesagt, papperlapapp, wir einigen uns mal zunächst rein numerisch auf $56 \mathrm{Be}-$ rentzen, 44 Pabst \& Richarz. Aber das ist eigentlich eine vage Veranstaltung. Das liegt jetzt an der Anzahl der Flaschen, die wir produzieren, und weiß der Teufel was. Wertmäßig war das gar nicht so klar. Da haben die gesagt, gut, wir machen Folgendes, in allen Gremien behandeln wir uns pari, fifty-fifty. Damals gab es einen Verwaltungsrat, das Unternehmen war ja noch nicht an der Börse. Und dann hat man gesagt, damit diese Wertschätzung zum Ausdruck kommt und wir uns nicht hier nur auf eine noch nicht abgeschlossene Wertbeurteilung verständigen können, wir aber loslegen wollen, besetzen wir alle Gremien paritätisch.«

(Ehemaliger Aufsichtsrat der Berentzen-Gruppe AG)

Im Zuge des Börsengangs 1994 wurde dann zwar die Hälfte des Eigenkapitals am Kapitalmarkt als Vorzugsaktien verkauft. Das Kapital der stimmberechtigten Stammaktien verblieb aber komplett in den Händen der bisherigen Eigentümerfamilien und ergab sich die auf der folgenden Seite dargestellte Eigentumsstruktur: ${ }^{1030}$

Die nominellen Relationen der Eigenkapitalanteile hatten sich somit zwar leicht verschoben, doch die vertraglich vereinbarte Nichtbeherrschung und festgelegte paritätische Besetzung der Gremien wurde in die neue Rechtsform transformiert. So wurden die sechs neben den drei Arbeitnehmervertretern zu besetzenden Plätze im Aufsichtsrat dem Prinzip der Parität entsprechend mit drei von den Familien Berentzen bestellten Mitgliedern und drei vom Stamm Pabst \& Richarz bestellten Mitgliedern besetzt. Die Geschäftsführung wurde, wie seit einigen Jahren, weiterhin von Jan B. Berentzen, dem Sohn von Hans Berentzen, und Jürgen Pabst, dem Vertreter des Stammes Pabst und Richarz, gebildet. Die familial-organisationale Konfiguration der Berentzen-Gruppe AG war somit dadurch gekennzeichnet, dass sich das Einflusspotenzial innerhalb der dominanten Koalition nicht wie bei der Anton Schlecker e.K. asymmetrisch in einem Machtzentrum konzentrierte, sondern sich innerhalb der dominanten Koalition symmetrisch auf mehrere Zentren verteilte. ${ }^{1031}$

1030 Vgl. B940330NZ, B970405NZ.

1031 Symmetrie des Einflusspotenzials muss nicht zwangsläufig bedeuten, dass das Eigenkapital oder die Stimmrechte exakt 50:50 aufgeteilt sind. Eine Symmetrie im hier verstandenen Sinne kann vielmehr bereits dann vorliegen, wenn die Handlungen und Entscheidungen der einen Seite nicht ohne das Einverständnis der anderen Seite getroffen werden können. 
Mit der Skizzierung der monozentrischen bzw. polyzentrischen Eigentumsstruktur als letzter Aspekt sind nun die wichtigsten historischen Einflüsse skizziert, die in der empirischen Studie identifiziert wurden und die entweder direkt auf den Faktor Familie zurückzuführen sind oder maßgeblich aufgrund dessen tradierender Kraft innerhalb der Organisation fortgeschrieben wurden.

Diese historischen Einflüsse übten zwar keine determinierende Wirkung auf die späteren Entscheidungsprozesse aus, aber sie formten bereits in der ersten Phase der Pfadgenese, der Phase der »historischen Vorprägung«, in den untersuchten Unternehmen einen strategischen Entscheidungskorridor und wirkten beschränkend auf die strategische Entwicklung der Unternehmen.

Im nächsten Kapitel gilt es zu analysieren, welche vom Faktor Familie beeinflussten Variablen des strategischen Entscheidungsprozesses in der Phase der Pfadformation zur Genese und Dynamik der strategischen Pfade beitrugen.

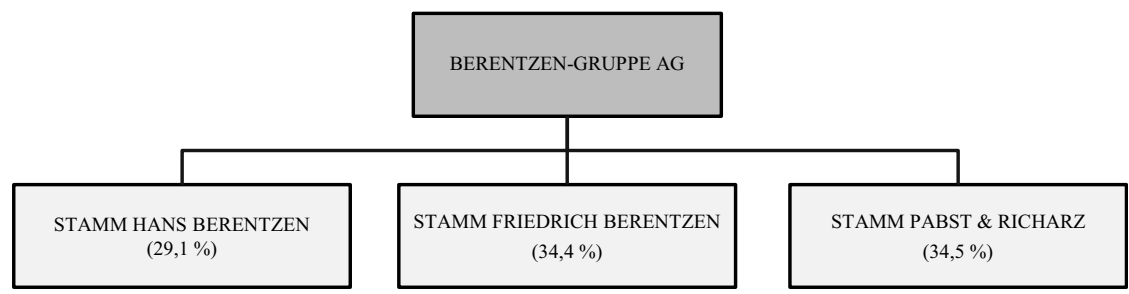

Abbildung 26: Struktur des Eigentums der Berentzen-Gruppe AG (Quelle: Eigene Darstellung; vgl. B050524).

\section{Der strategische Entscheidungsprozess in der Phase der Pfadformation}

Im vorigen Kapitel wurde die historische Prägung des strategischen Entscheidungsraums der untersuchten Unternehmen skizziert, die auf familiär bedingte oder tradierte Imprints zurückgeht. Diese historischen Imprints bildeten früh einen Evolutionskorridor für die weitere strategische Entwicklung der Unternehmen. So war es beispielswiese bei der Anton Schlecker e.K. vor dem Hintergrund der historischen Prägung als Fleischwarenfabrik unwahrscheinlich, dass sich im Zuge des Einstiegs in die Drogeriebranche ein auf Kundenorientierung und Service basierendes Vertriebskonzept entwickeln würde. Die historischen Wurzeln strebten eher zu dem von günstigen Einkaufskonditionen getriebenem Geschäftsmodell.

Doch so bedeutsam diese historische Vorprägung für die strategische Entwicklung der Unternehmen gewesen sein mag - sie war nicht primäre Ursache für die spätere Genese der strategischen Persistenzen und der daraus resultie- 
renden Unternehmenskrisen. Die historische Vorprägung führte zwar zu einer beachtlichen Einschränkung der strategischen Entwicklungsmöglichkeiten, aber keineswegs zu deren Determination.

Dieser Umstand wird deutlich, wenn man sich vergegenwärtigt, dass nicht nur die später strategisch pfadabhängigen Unternehmen stark historisch geprägt waren, sondern auch die komparativen Vergleichsfälle. So beeinflusste beispielsweise der historische Imprint des Aufstiegs des Discount-Modells die Dirk Rossmann GmbH kaum weniger als die Anton Schlecker e.K., während die aus dem historischen Einfluss der Familie resultierende polyzentrische Eigentümerstruktur den strategischen Entscheidungsraum bei der Eckes AG ebenso strukturierte wie bei der Berentzen-Gruppe AG. Ungeachtet ähnlicher Imprints und historischer Parallelen entwickelte sich in diesen komparativen Vergleichsfällen aus der historischen Vorprägung keine strategische Pfadabhängigkeit. Stattdessen gelang diesen Unternehmen im Rahmen des ihnen noch zur Verfügung stehenden Entwicklungsspielraums im Verlauf der Zeit ein bemerkenswerter strategischer Wandel.

Dies verweist auf die herausragende Bedeutung der in Teil $\mathrm{C}$ dargestellten Regime der selbstverstärkenden Mechanismen für die Entwicklung der pfadbedingten strategischen Persistenzen bei der Anton Schlecker e.K. und der Berentzen-Gruppe AG. Die selbstverstärkenden Mechanismen erklären den größten Teil der strategischen Persistenz. Die empirische Studie zeigt somit auf, dass Pfadabhängigkeit »mehr ist « als »history matters«. Allerdings: Während die selbstverstärkenden Mechanismen im klassischen, machtfreien ökonomischen Kontext der Pfadtheorie die Durchsetzung einer bestimmten Lösung umfassend erklären, verbleibt im organisationalen Rahmen der untersuchten Unternehmen ein Erklärungsdefizit.

Die in Teil C skizzierte graduelle Genese der Pfadabhängigkeit bei der Anton Schlecker e.K. und der Berentzen-Gruppe AG illustriert, dass in der Phase der Pfadformation nicht nur Zeit, sondern über einen längeren Zeitraum auch ausreichend "managerial discretion" vorhanden war, um der zunehmenden Verengung der strategischen Entwicklungsmöglichkeiten mit einem machtvollen Eingriff in den selbstverstärkenden Mechanismus noch zu begegnen. Zum einen gewann das Regime der selbstverstärkenden Mechanismen erst nach und nach so viel Kraft, dass es schlussendlich das Ruder der strategischen Entwicklung übernehmen konnte, zum anderen besaßen die dominanten Koalitionen insbesondere aufgrund der Eigentümerfunktionen der Unternehmensfamilien eine ausgesprochen machtvolle Position.

Vor diesem Hintergrund stellt sich die Frage, warum die dominanten Koalitionen der Anton Schlecker e.K. und der Berentzen-Gruppe AG in der frühen Phase der Pfadformation, als diese Dynamiken noch nicht ihre volle Wirkkraft 
entfaltet hatten, den selbstverstärkenden Mechanismen nicht durch einen machtvollen Eingriff Einhalt geboten.

PHASE II

(»Pfadformation«)

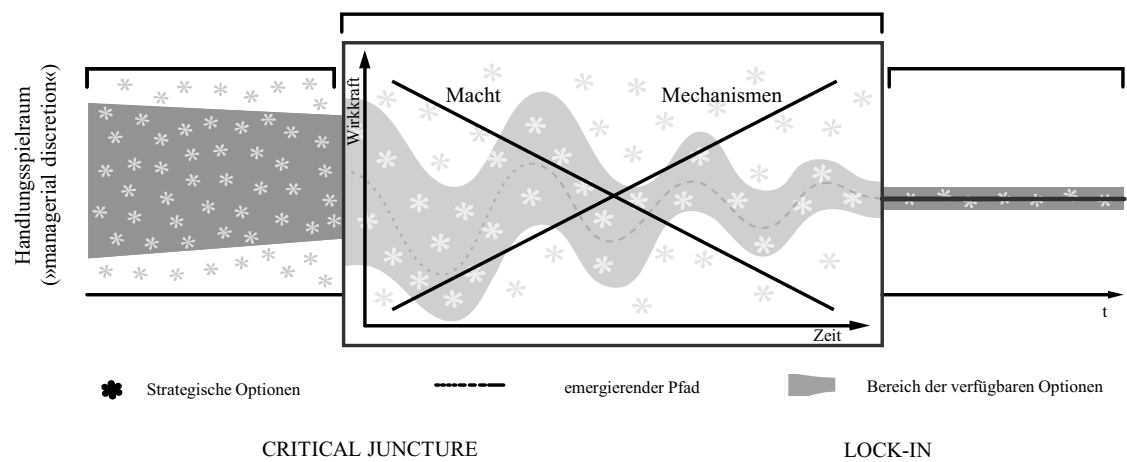

Abbildung 27: Die Phase der Pfadformation im Prozess der Pfadgenese (Quelle: Eigene Darstellung in Anlehnung an Sydow et al. [2009]).

Zur Klärung dieser Frage wurden die strategischen Entscheidungsprozesse der Anton Schlecker e.K. und der Berentzen-Gruppe AG im Zuge der Pfadanalyse mit jenen der Dirk Rossmann GmbH und der Eckes AG verglichen. Im Zuge dieser kontrastierenden Analyse kristallisierten sich die Ausprägungen und Wirkungszusammenhänge dreier Struktur- und Interaktionsvariablen als besonders bedeutsam für die strategischen Entscheidungsprozesse und die Pfadgenese bei der Anton Schlecker e.K. und der Berentzen-Gruppe AG heraus: Macht, Diversität und Konflikt. Die empirischen Ausformungen dieser drei organisationalen Variablen und ihre Wirkungszusammenhänge prägten in der Phase der Pfadformation die kollektiven strategischen Entscheidungsprozesse der dominanten Koalitionen in Verlauf und Ergebnis entscheidend und erklären größtenteils das Ausbleiben der strategischen Reaktion. ${ }^{1032}$

Die große Machtfülle der dominanten Koalitionen sowohl innerhalb der jeweiligen Organisation als auch gegenüber deren Umwelt spannte einen Rah-

1032 Zur Annahme einer allgemeinen Kollektivität von Entscheidungsprozessen, in welchen die letzte Entscheidung vielleicht von einem einzelnen Akteur getroffen wird, aber vorgeschaltet stets kollektiv wahrgenommen, interpretiert und kommuniziert wird, vgl. Hambrick (2007: 334); Schreyögg (2006: 436) auch Fredrickson (1986: 281): »Moreover, numerous authors agree that only in the most simple of organizations are all of the activities controlled by one individual.« Vgl. auch Mintzberg (1979); Thompson (1967). Zudem hat sich in zahlreichen empirischen Studien mittlerweile erwiesen, dass die Untersuchung von Gruppen von Entscheidungsträgern meist die höhere Erklärungskraft besitzt. Vgl. Finkelstein et al. (2009: 11). 
men auf, der die Entscheidungsprozesse der dominanten Koalition in weiten Teilen gegen exogene Einflüsse immunisierte. Diese Immunität wurde durch bestimmte Merkmale der Struktur und Kultur zusätzlich verstärkt. In der Steuerung und Kontrolle ihrer Entscheidungsprozesse waren die dominanten Koalitionen der Anton Schlecker e.K. und der Berentzen-Gruppe AG daher weitestgehend auf sich und ihre endogenen Potenziale und Dynamiken zurückgeworfen. So kam in der Bearbeitung der Komplexität der Umwelt und deren Irritationen dem Grad an Diversität und dem Niveau an Sach- und Beziehungskonflikt in den strategischen Entscheidungsprozessen ein besonders hoher Stellenwert zu. ${ }^{1033}$

In den folgenden Abschnitten sollen jeweils die in der Phase der Pfadformation empirisch beobachteten Ausprägungen und Auswirkungen dieser Variablen skizziert werden. Zugleich soll herausgearbeitet werden, inwieweit und wie der Kontextfaktor Familie diese Ausprägungen und Auswirkungen beeinflusste.

\subsection{Macht und Immunität der dominanten Koalitionen}

Die Analyse des Einflusses des Faktors Familie in der Phase der Pfadformation muss ihren Ausgangspunkt bei der Variable Macht nehmen. Gemeint ist die Macht der um die Unternehmensfamilien gruppierten dominanten Koalitionen über die untersuchten Unternehmen, mit der sie im Zuge der strategischen Entscheidungsprozesse auf die zunehmende Wirkkraft der selbstverstärkenden Mechanismen hätten reagieren können. ${ }^{1034}$

Im folgenden ersten Abschnitt werden die Machtfülle der dominanten Koalitionen der Anton Schlecker e.K. und der Berentzen-Gruppe AG sowie die daraus resultierenden Probleme der Selbstkontrolle sowie der Immunität der dominanten Koalition gegenüber exogenen Einflüssen skizziert.

Im zweiten Abschnitt werden dann jene Ausprägungen der Struktur und Kultur dargestellt, welche die Immunität zusätzlich erhöhte.

1033 Siehe Kapitel B.1.2.1 zum Verhältnis von Umwelt und Organisation. Zur allgemeinen Bedeutung von Entscheidungsträgern für die Ergebnisse von Entscheidungsträgern vgl. Carpenter et al. (2004: 750); auch Finkelstein et al. (2009: 2) die nach Jahrzehnten der Forschung zum Einfluss der Entscheidungsträger auf die strategischen Wahlentscheidungen und die Performance von Unternehmen festhalten, dass »top executives can greatly influence what happens in organizations - for good and for ill."

1034 Wie in der Heranführung an den theoretischen Bezugsrahmen skizziert, soll Macht im Sinne von Weber (1972: 28) verstanden werden. Vgl. ähnlich Pfeffer (1981: 2f.) oder Finkelstein (1992: 506): »capacity of individual actors to exert their will.» 

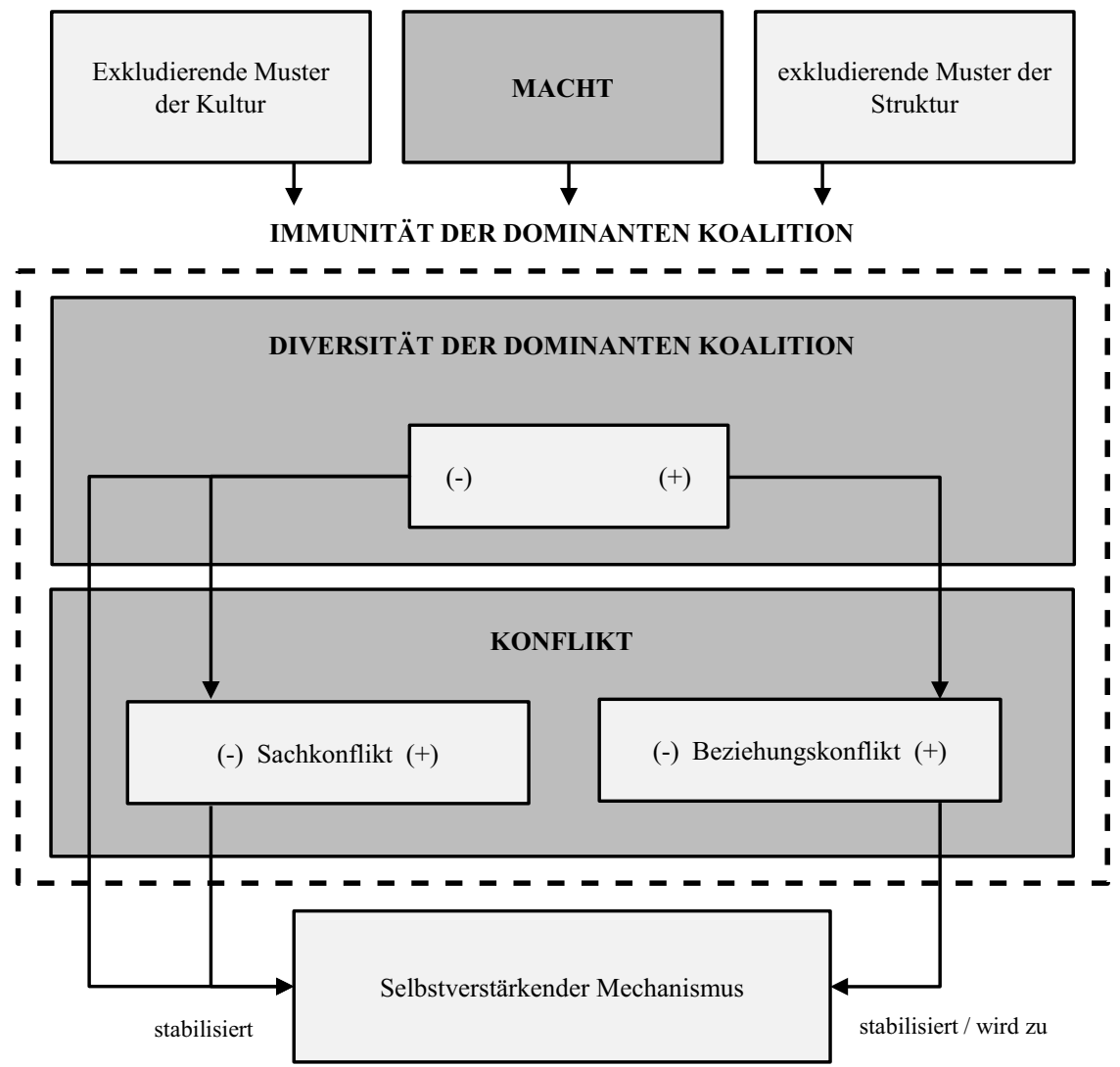

Abbildung 28: Wirkungszusammenhang organisationaler Variablen in den Entscheidungsprozessen der Phase der Pfadformation

(Quelle: Eigene Darstellung).

\subsubsection{Macht, Entscheidungsautonomie und Selbstkontrolle der dominanten Koalition}

Organisationale Macht beruht im Kern auf dem Vermögen der Akteure, die externen und internen Unsicherheiten der Umwelt zu handhaben. Für diese Bewältigung der Umwelt können die Akteure auf die Machtmittel des Eigentums, der Autorität der organisationalen Hierarchie oder der Expertise und des Prestiges zurückgreifen. ${ }^{1035}$

1035 Vgl. Finkelstein (1992: 508); Hambrick (1981); Pfeffer (1981: 97ff.); Thompson (1967). So besitzt ein Akteur oder eine Gruppe Macht, wenn sie durch ihr Eigentum am Unternehmen dessen fortlaufende Finanzierung sicherstellt. In Bezug auf die internen Quellen von Unsicherheit besitzt ein organisationaler Akteur Macht, wenn er zum Beispiel durch die Kontrolle 
In den untersuchten Unternehmen waren all diese Machtmittel in vergleichsweise hohem Maße in den dominanten Koalitionen der Unternehmen konzentriert. Diese Bündelung - insbesondere das konzentrierte Eigentum der Unternehmensfamilien - verhalf den dominanten Koalitionen aber nicht nur zu einer hohen Machtfülle, sondern verschaffte ihnen auch ein hohes Maß an Entscheidungsautonomie. ${ }^{1036}$ Die dominanten Koalitionen verfügten über einen sehr hohen Grad an Selbstbestimmung und Entscheidungsfreiheit sowohl innerhalb der Organisationen als auch gegenüber deren Umwelt.

\section{Entscheidungsautonomie der dominanten Koalitionen gegenüber der organisationalen Umwelt}

Die Entscheidungsautonomie gegenüber der Umwelt beruhte in beiden Unternehmen fundamental auf der Konzentration des Eigentums innerhalb der dominanten Koalitionen. So befand sich bei der Anton Schlecker e.K. das gesamte Eigenkapital in den Händen der Unternehmensfamilie. Die Berentzen-Gruppe AG war zwar börsennotiert, aber die stimmberechtigten Stammaktien befanden sich vollständig in Besitz der Unternehmensfamilien.

Durch die kontinuierliche und stabile Kapitalisierungsfunktion der Unternehmensfamilien standen die Unternehmen und ihre Protagonisten unter einem geringeren Druck externer, marktbasierter Governance-Mechanismen. ${ }^{1037}$ Negative Unternehmensentwicklungen erforderten keine kurzfristigen Gegenmaßnahmen zur Marktberuhigung oder der Prävention feindlicher Übernahmen. Der fehlende Druck des Kapitalmarkts und des Marktes für Unternehmenskontrolle erhöhte somit die strategische Unabhängigkeit der Entscheidungsträger gegenüber der organisationalen Umwelt und eröffnete den dominanten Koalitionen einen größeren Handlungsspielraum.

Dieser Umstand kann am Beispiel der Anton Schlecker e.K. besonders deutlich illustriert werden. Am jahrzehntelangen Marktführer der Drogerie-

der Informationsflüsse oder das Setzen der Agenda die nie vollkommen deckungsgleichen Ansichten über eine bestimmte strategische Entscheidung integrieren kann.

1036 Dem Alleinbestimmungsprinzip nach Gutenberg (1976) zufolge steigt mit zunehmender Macht auch die Entscheidungsautonomie; vgl. Gutenberg (1976: 460ff.); Hambrick \& Finkelstein (1987: 371). Entsprechend wurde in der Heranführung an den theoretischen Bezugsrahmen in Kapitel B.2.3.1. festgehalten, dass mit einem steigenden Einfluss der Familie über den Kanal »Macht« auch die Autonomie der dominanten Koalition zunimmt. Die hohe Entscheidungsautonomie kann somit als eine Facette des familiären Einflusses verstanden werden.

1037 Zum fehlenden Druck des Kapitalmarkts vgl. Anderson \& Reeb (2004: 213); Hambrick \& D'Aveni (1988: 17). Die Berentzen-Gruppe AG war zwar am Kapitalmarkt notiert, die Kapitalgeber hatten durch die Trennung der Aktien in Stamm- und stimmrechtslose Vorzugsaktien rechtlich und faktisch jedoch nur wenige Mitbestimmungsmöglichkeiten. Zugleich war das Unternehmen dem Markt für Unternehmenskontrolle entzogen. Vgl. Morck et al. (1989). 
marktbranche waren neben Anton Schlecker keine weiteren Gesellschafter beteiligt. Daher konnte das Unternehmen in der Rechtsform des »eingetragenen Kaufmanns" geführt werden, in welcher das Alleinbestimmungsprinzip der Eigentümer in seiner reinsten Form verwirklicht wird. In diesem rechtlichen Rahmen konnte die um Anton Schlecker und dessen Familie gruppierte dominante Koalition weitgehend unbeschränkt über das Unternehmen verfügen. Die Entscheidungsträger waren in ihren Entscheidungsprozessen weder auf die Zustimmung weiterer Eigenkapitalgeber angewiesen, noch unterlagen sie gegenüber Stakeholdern weiter gehenden Rechenschaftspflichten zur Lage und Entwicklung des Unternehmens. Rechtlich war Anton Schlecker als Einzelunternehmer im Kern nur sich selbst Rechenschaft schuldig.

Die auf diesem Wege gesetzlich verankerte Entscheidungsautonomie der dominanten Koalition konnte durch eine unternehmerische Strategie der Reduktion von Umweltabhängigkeiten weiter erhöht werden. ${ }^{1038}$ So kämpfte das Unternehmen lange Zeit erfolgreich gegen die Einführung von Tarifverträgen und sämtliche Formen der Mitarbeitermitbestimmung. Diese Bemühungen galten zu einem gewissen Teil der Reduktion von Personalkosten, doch mindestens ebenso bedeutsam war das Streben nach Bewahrung der hohen Entscheidungsautonomie der dominanten Koalition. ${ }^{1039}$

Seinen deutlichsten Ausdruck fand dieses Streben nach Unabhängigkeit aber in der Finanzierung des Unternehmens. Da mit der Aufnahme von Fremdkapital Abhängigkeiten und Rechenschaftsplichten - hauptsächlich gegenüber Banken - verbunden gewesen wären, wurde die Finanzierung über Fremdkapital strikt vermieden. Stattdessen wurde die Anton Schlecker e.K. beinahe ausschließlich über den Cashflow finanziert. Im Jahr 1999 beliefen sich die Kredite des Unternehmens - bei einer Bilanzsumme von 2 Mrd. DM und einem Wertpapier- und Geldvermögen von knapp 800 Mio. DM - auf nur rund 3,7 Mio. DM. ${ }^{1040}$ In diesem Rahmen konnten weder die Banken noch andere potenzielle Fremdkapitalgeber Einfluss auf den strategischen Entscheidungsprozess der dominanten Koalition ausüben.

1038 Vgl. S110118MM zu diesem selbst kurz vor der Insolvenz geschätzten Wert: „Es wird also keinen Verkauf oder Teilverkauf geben?« »Auf gar keinen Fall. Wir sind froh über unsere Eigenständigkeit ohne irgendeine Mitsprache von Investoren oder Anteilseignern. Diese Selbstständigkeit ist ein Gut, das wir uns nicht nehmen lassen.»

1039 Dieses Streben nach einer Bewahrung der unternehmerischen Unabhängigkeit äußerte sich u. a. in den sehr kurzfristigen Mietverträgen, die die Anton Schlecker e.K. abschloss. Während der Wettbewerber dm zur Erzielung günstiger Konditionen zum Beispiel Verträge von zehn bis 25 Jahren Laufzeit abschloss, wurden bei der Anton Schlecker e.K. trotz kaum nennenswerter Filialschließungen meist nur Mietverträge von einem Jahr abgeschlossen. Vgl. Werner (2013: 125); S091008LZ.

1040 Vgl. Schlecker (1999); S010823LZ. 
Das Streben nach Unabhängigkeit prägte sogar die externe Kommunikation der dominanten Koalition. So lautete der erste von vier knappen Sätzen der Lageberichte der Jahresabschlüsse aus den Jahren 2005 bis 2010 stets:

»Wie bisher konnte auch im Geschäftsjahr 2010 die Geschäftstätigkeit ohne mittel- und langfristige Bankkredite ausgeübt werden.«

(Der erste Satz der Lageberichte aus den Jahren 2005 bis 2010) ${ }^{1041}$

Noch ein Jahr vor der Insolvenz teilte Lars Schlecker in einem Zeitungsinterviews mit:

»Wir sind gottfroh, dass wir nicht auf Banken angewiesen sind.»

(Lars Schlecker im Jahr vor der Insolvenz) $)^{1042}$

Die dominante Koalition der Anton Schlecker e.K. war zur Wahrung der hohen Autonomie selbst dann noch nicht zur Aufnahme größerer Summen Fremdkapital bereit, als die Liquiditätskrise im Jahr 2010 innerhalb des Unternehmens bereits offen zutage getreten war. ${ }^{1043}$

In etwas schwächer ausgeprägter Form lassen sich die Dynamiken und Resultate eines solchen Autonomiestrebens auch bei der Berentzen-Gruppe AG feststellen. Dort existierten mit den Streubesitzaktionären zwar noch weitere Eigenkapitalgeber, denen gegenüber diverse Informations- und Rechenschaftspflichten bestanden. Aufgrund der bewussten Trennung des Grundkapitals in die emittierten Vorzugsaktien, mit denen kein Stimmrecht verbunden war, einerseits und die in den Händen der Eigentümerfamilien verbliebenen Stammaktien, die mit Stimmrecht einhergingen, andererseits waren diese Stakeholder jedoch vom strategischen Entscheidungsprozess vollkommen ausgeschlossen. ${ }^{1044}$ Trotz einer im Vergleich zur Anton Schlecker e.K. höheren Fremdkapitalquote waren die Entscheidungsträger der dominanten Koalition in der Steuerung und Kontrolle der strategischen Entwicklung somit ebenfalls in hohem Maße autonom gegenüber der Umwelt.

1041 Schlecker (2005); vgl. auch Schlecker (2006), Schlecker (2008; Schlecker (2010).

1042 S110118MM.

1043 Bereits im Jahr 2010 hatte der 2008 ins Unternehmen eingetretene Finanzvorstand die Notwendigkeit expliziert, sich von einem Insolvenzexperten beraten zu lassen. Nachdem die Familie Schlecker dies ablehnte, verließ der Finanzvorstand das Unternehmen; vgl. S120602. Diese Vorgänge illustrieren die Erkenntnisse von Gómez-Mejía et al. (2007), die in ihrer Studie feststellten, dass viele Unternehmensfamilien der Bewahrung des familiären Einflusses über das Unternehmen selbst dann höchste Priorität zukommen lassen, wenn damit ein erhebliches Risiko für das Überleben des Unternehmens einhergeht. Vgl. auch von Schlippe (2009: 191); Wimmer et al. (2005: 158).

1044 Vgl. B940330NZ. Dieser Ausschluss vom strategischen Entscheidungsprozess wurde von Seiten der Aktionäre auch immer wieder beklagt; vgl. hierzu B000621MM. 


\section{Entscheidungsautonomie der dominanten Koalitionen innerhalb der Organisation} Die Macht der Unternehmensfamilien führte in den untersuchten Unternehmen aber nicht nur zu einer erhöhten Unabhängigkeit gegenüber der Umwelt, sondern auch $\mathrm{zu}$ einer ausgeprägten Entscheidungsautonomie der dominanten Koalitionen innerhalb der Organisation.

Diese Autonomie beruhte vor allem auf der weitgehenden Einheit von Eigentum und Leitung in den Unternehmen und deren Konzentration innerhalb der dominanten Koalitionen. Aufgrund der Machtakkumulation bildeten sich weder im Topmanagement noch bei einem Kontrollorgan ein zweites "Zentrum der betrieblichen Willensbildung « im strategischen Entscheidungsprozess. ${ }^{1045}$

"In Familienunternehmen kann ich nicht der Sprecher der Geschäftsleitung werden, kann ich nicht die Nummer eins sein - das ist der Unternehmer. Ende der Durchsage. Das muss ich wissen. [...] egal ob der Unternehmer eine, eine, ein ganz toller Typ ist oder ein Vollidiot ist, er wird immer die Nummer eins sein. Das ist gottgegeben. Das muss ich hinnehmen. [...] zwischen dem externen Geschäftsführer und dem Unternehmer ist alles klar; weil ich kann nie seine Position erreichen, das Unternehmen gehört mir halt nicht. Er hat schlussendlich das letzte Wort [...] wir hatten oft den, den Streit darüber, dass er sagte: 'Ich möchte, dass du jetzt endlich sagst: Das ist unser Unternehmen und nicht sagst, das ist dein Unternehmen.` Also wenn ich, also sag ich: ,Weißt du, nehmen wir mal an, ich komm dir richtig dumm. Was machst du dann? \& Sagt er: `Dann schmeiß ich dich raus.`Sag ich: ১Eben.〈 Sag ich: `Nehmen wir mal an, du kommst mir richtig dumm, was ist dann? ‘ Sagt er : >Das musst du hinnehmen. 'Sag ich: ‘Eben, deshalb ist es dein Unternehmen. Deswegen liegt die letzte Entscheidung bei dir. [...]."

(Langjährige Führungskraft bei der Dirk Rossmann GmbH) $)^{1046}$

Anstatt einen unabhängigen Pol der Entscheidungsfindung mit eigenem finalen Entscheidungsanspruch zu bilden, gruppierten sich die familienfremden Führungskräfte innerhalb der dominanten Koalitionen um die Protagonisten der Eigentümerfamilien zu einem einzigen Zentrum der Willensbildung.

Zur Herausbildung der einpoligen Struktur trugen allerdings nicht nur das Machtpotenzial des Eigentums und die Wirkkraft der familiären Akteure in ihren Doppelrollen als Eigentümer und Führungskräfte bei. Vielmehr war sie auch auf die Schwäche des familienfremden Topmanagements zurückzuführen. Insbesondere bei der Anton Schlecker e.K. setzte sich das Topmanagement zum

1045 Vgl. Gutenberg (1976: 473).

1046 Vgl. hierzu die Aussage einer ehemaligen Führungskraft der Anton Schlecker e.K.: »Sie können ja nicht ... ich kann ja nicht entscheiden. Ich kann ja nicht sagen `Okay, Sie haben das alles verstanden, ich gehe also morgen zum Amtsgericht und mach eine neue Firmengründung, dann gründen wir eine Aktiengesellschaft. Ist ja alles ausgerechnet . Hat er nicht gemacht ... und somit haben wir das nicht gemacht. So nach dem Motto >Geben Sie mal her, machen Sie mal eine Ausarbeitung`, ging dann in die Schublade, `machen wir später`. Und das hat sich eigentlich nie geändert.« 
Teil aus langjährigen Mitarbeitern zusammen, die nicht zuletzt eben aufgrund ihrer Seniorität und Loyalität zur Unternehmensfamilie in diese Position gelangt waren. Zur Wahrung der Autonomie der Eigentümerfamilie wurden teilweise auch bewusst geringer qualifizierte oder persönlich weniger starke Führungskräfte in das Topmanagement berufen. ${ }^{1047}$

"[W]eil meine Eltern eben aus ihren früheren Jahren kennengelernt hatten sozusagen, es ist gefährlich, wenn man sich gute Leute heranzüchtet, weil die können sich selbstständig machen [...] deshalb war Strategie meiner Eltern immer, lieber mit, sagen wir mal, mittelmäßigeren Leuten arbeiten, deren Horizont beschränkt ist und wo diese Gefahr nicht auftritt. Und dann lieber selber mehr machen müssen." (Ehemaliger Familienunternehmer im Rahmen der explorativen Vorstudie)

Zur Autonomie der dominanten Koalitionen trug wiederum auch die aus der familiensystemischen Orientierung an Personen hervorgehende personale Kontinuität der Entscheidungsträger bei. ${ }^{1048}$ Aufgrund vergangener Erfolge oder einfach ob ihrer langjährigen Zugehörigkeit zum Unternehmen genossen viele Entscheidungsträger eine von außerhalb der dominanten Koalition beinahe unangreifbare Position. Diese Sicherheit erhöhte deren strategische Entscheidungsfreiheit. ${ }^{1049}$

»Wenn Sie ein angewachsener Nichtfamiliengeschäftsführer sind [...] die eben so lange dabei sind, dass sie sich auf die Loyalität ihrer Gesellschafter verlassen können, die sind im Übrigen häufig dann diejenigen, die eben schon als Junge ins Unternehmen reinkamen und mit hochgewachsen sind mit den Eigentümern und halt schon immer da waren, und zu denen hat man dann schon Loyalität, und die haben dann eine größere, also eine viel größere Freiheit."

(Ehemaliger Fremdgeschäftsführer eines börsennotierten Familienunternehmens)

Die Entscheidungsfreiheit der dominanten Koalitionen wurde zudem dadurch gestärkt, dass die Kontroll- und Aufsichtsorgane nur eine schwach ausgeprägte Autonomie aufwiesen. ${ }^{1050}$

So erhöhte bei der Berentzen-Gruppe AG eine besondere Form von »CEO duality« die Entscheidungsautonomie der dominanten Koalition. Lange Zeit

1047 Dieses Motiv wurde auch in der explorativen Vorstudie wiederholt genannt: «Also da, aber das ist auch wieder normal, ein starker Führer, ein starker Vorsitzender des Vorstandes holt sich starke Leute rein. Ein schwacher holt sich willfährige Leute rein, die ihn nicht zu sehr bedrängen."

1048 Wie oben bereits dargestellt, baute bei der Anton Schlecker e.K. Reinhold Freudenreich mit Anton Schlecker sen. in den 1950er-Jahren die Fleischwarenfabrik auf und führte Jahrzehnte später 2007 für Anton Schlecker jun. noch die Verhandlungen mit Goldman Sachs zum Kauf von Ihr Platz. Siehe zum Strukturmerkmal des familiären Einflusses der personalen Kontinuität Kapitel B.2.3.1.

1049 Vgl. Gomez-Mejia et al. (2001: 83); Schein (1983: 24) zu solchen »secure positions«.

1050 Vgl. zur Bedeutung von Kontrollorganen für den Grad an Entscheidungsfreiheit der strategischen Entscheidungsträger insb. die Studie von Crossland \& Hambrick (2007). 
kontrollierte hier der Vater als Aufsichtsratsvorsitzender den als Vorstandssprecher tätigen Sohn. ${ }^{1051} \mathrm{Da}$ die Positionen im Aufsichtsrat von den einzelnen Stämmen doch gezielt mit Interessenvertretern besetzt wurden, kann aufgrund Loyalitätsverpflichtungen oder gar familiärer Bindungen auch die Unabhängigkeit weiterer Aufsichtsratsmitglieder als zumindest eingeschränkt betrachtet werden. ${ }^{1052}$ Der Aufsichtsrat der Berentzen-Gruppe AG war somit kein unabhängiges Kontroll- und Aufsichtsorgan in einem System von "checks and balances«, sondern aufgrund des komplexen Beziehungsgeflechts seiner Mitglieder eher eine weitere Arena derselben dominanten Koalition innerhalb des einen Zentrums der Willensbildung. ${ }^{1053}$

Dies bedeutet keineswegs, dass der Aufsichtsrat sich vollständig der Kontrolle des Managements entzogen hätte. Im Verlauf der Konflikteskalation wurde er gar zu einer Art Opposition zum Vorstand. Aufgrund der Rollenüberlagerungen und -verschränkungen und der mangelnden Unabhängigkeit konnte der Aufsichtsrat aber nicht als exogenes Regulativ des strategischen Entscheidungsprozesses fungieren. Da er in diesen Prozessen stets endogene Kraft war, mussten sich die Akteure der dominanten Koalition in der Konsequenz letztlich selbst kontrollieren.

Gleiches gilt verstärkt für die Entscheidungsträger der dominanten Koalition der Anton Schlecker e.K. zu. Aufgrund der gewählten Rechtsform war das Unternehmen nicht zur Einrichtung eines Kontrollorgans verpflichtet. Zugunsten der Wahrung der höchstmöglichen Autonomie wurde tatsächlich auch kein Gremium eingerichtet, welches die Entscheidungsprozesse der dominanten Koalition hätte beaufsichtigen und kontrollieren können.

Zur Autonomie der dominanten Koalitionen trug zudem die Macht des Prestiges und die hohe Legitimität bei, welche die Unternehmensfamilien in der Leitung der Unternehmen genossen. ${ }^{1054}$ Sowohl bei der Anton Schlecker e.K. als auch bei der Berentzen-Gruppe AG wurde die Führung des Unternehmens durch

1051 Solche Konstellation begünstigen in der Regel auch Formen des »entrenchment«. Vgl. Finkelstein \& D'aveni (1994); auch Finkelstein et al. (2009: 31); Krause et al. (2014: 256ff.).

1052 Solche Maßnahmen zur Wahrung des eigenen, partikularen Einflusspotenzials sind nicht nur, aber insbesondere in Familienunternehmen gängige Praxis; vgl. Anderson \& Reeb (2004: 232).

1053 Hierzu ein ehemaliger Aufsichtsrat der Berentzen-Gruppe AG: »[W]enn man sich so das ganze Konstrukt anschaut, dass da, ich sag mal, ja 'ne starke Verwebung aus Gesellschaftern, Aufsichtsratsämtern, Vorstandsposten bis hin dann oberste Managementebene.«Bei der Eckes AG ließ sich zu Beginn der untersuchten strategischen Entwicklung eine ähnliche Konstellation feststellen. In diesem Unternehmen wurde das Beziehungsgeflecht im Verlauf der Zeit aber entflochten und die Unabhängigkeit des Kontrollorgans erhöht.

1054 Vgl. zur Macht aus Prestige Finkelstein (1992: 510) und zur Legitimation von Führung in der Familienunternehmensforschung Klein (2010: 205ff.). 
die Familie und die um sie gruppierte dominante Koalition innerhalb der Organisation als rechtmäßig empfunden und nicht infrage gestellt. ${ }^{1055}$

Diese Legitimität beruhte auf der Leistung der Unternehmensgründung und der Macht der Idee: Unter der Leitung Anton Schleckers hatte sich aus der regional tätigen Fleischwarenfabrik eines der größten Einzelhandelsunternehmen Deutschlands entwickelt. Die Familie Berentzen führte beinahe 250 Jahre lang das Unternehmen erfolgreich. Diese Tradition und die vergangenen Erfolge ließen die Leitung des Unternehmens durch die um die Unternehmensfamilien gruppierten dominanten Koalitionen legitim und trotz sachlicher Differenzen über alle Zweifel erhaben erscheinen.

"Ich denke mal, das liegt an dem, an diesem Gesellschafter, Gesellschafter-Vorstand [...] Jan Berentzen. Nicht unbedingt in seiner Person begründet, aber daran, dass er Gesellschafter ist und dass er dem Gründer folgte, der die Firma mal gegründet hat, mehr oder weniger [...]. Als Repräsentant dieser Familie hat keiner Kritik an ihm geübt. No-, Nobody. Er war wie Gott.«

(Ehemaliger Aufsichtsrat der Berentzen-Gruppe AG)

Somit erwuchs aus der Legitimität der Unternehmensleitung Autorität, die als Herrschaftsmechanismus neben die Macht der dominanten Koalitionen trat und maßgeblich zu deren Autonomie innerhalb der Organisation beitrug. ${ }^{1056}$

\section{Hoher Grad an Zentralisierung}

In der Macht der dominanten Koalitionen gründete aber nicht nur eine hohe Entscheidungsautonomie, sondern auch die Möglichkeit, die »internal rules of the game« zu bestimmen und wichtige organisationsstrukturelle Dimensionen zu prägen. ${ }^{1057}$ In beiden Unternehmen ließ sich der hohe Grad an Zentralisierung nachweisen, der in Teil B als strukturelles Merkmal des familiären Einflusses herausgearbeitet wurde. ${ }^{1058}$

1055 Diese Feststellung bezieht sich auf die zugesprochene Legitimität der Führung von der restlichen Organisation außerhalb der dominanten Koalition. Innerhalb der dominanten Koalition wurde diese Legitimation gerade bei der Berentzen-Gruppe AG stark infrage gestellt.

1056 Unabhängig davon, wie in den untersuchten Unternehmen schlussendlich entschieden wurde: Selbst bei großer sachlicher Differenz billigten die Organisationsmitglieder außerhalb der dominanten Koalition den Entscheidungsträgern meist zu, dass sie zumindest das Recht hatten, so zu entscheiden. Vgl. zum Verhältnis von Macht und Autorität Pfeffer (1981: 4ff.).

1057 Vgl. Fama \& Jensen (1983: 302). Hier liegt ein bedeutender Unterschied zwischen Eigentümern und dem angestellten Topmanagement.

1058 Vgl. die Kategorie »command« in Kapitel B.2.3.1. Zur Bedeutung solcher Strukturen für den strategischen Entscheidungsprozess und Wandel vgl. Bower (1970:67):»[S]tructure may motivate or impede strategic activity." Vgl. auch Pettigrew (1987); Van de Ven \& Poole (1995). 
Sowohl bei der Anton Schlecker e.K. als auch bei der Berentzen-Gruppe AG liefen die strategischen Entscheidungsprozesse in kleinem Kreise ab, bestehend aus den Protagonisten der Eigentümerfamilien und einigen wenigen vertrauten Mitarbeitern. Innerhalb dieser Zirkel waren die Entscheidungskompetenzen und Verantwortlichkeiten, aber auch die Möglichkeiten der Initiierung strategischer Aktivitäten konzentriert. ${ }^{1059}$ Bei der Berentzen-Gruppe AG war dieser Kreis in einer diffusen Sphäre zwischen Vorstand, Aufsichtsrat und Gesellschafterkreis angesiedelt. Trotz der komplexen Gesellschaftsstruktur wurden dort die maßgeblichen strategischen Entscheidungen getroffen.

Noch ausgeprägter war die Zentralisierung bei der Anton Schlecker e.K. Hier lief selbst der größte Teil der operativen Informationsflüsse in der formalen Hierarchie erst bei Anton und Christa Schlecker zusammen, die dann den entsprechenden Reaktionsbedarf koordinierten.

»Überhaupt kommunizieren wir intern viel professioneller. Früher haben 40 Führungskräfte direkt an die Familie berichtet, das war ineffizient.«

(Lars Schlecker in einem Interview mit der FAZ im Jahr 2011) ${ }^{1060}$

In diesen vertikal strukturierten Bahnen verlief die Kommunikation mehrere Jahrzehnte unverändert vor allem über Berichte: Die Funktionsbereiche berichteten strikt vertikal in der Hierarchie von unten nach oben und empfingen in entgegengesetzter Richtung ihre Anweisungen. Stand beispielsweise im Einkauf die Entscheidung zur Aufnahme eines neuen Produkts in das Sortiment an, so wurde diese Frage nicht selten in der Hierarchie vom Einkäufer über den »Category Manager« zum zuständigen Direktor und schlussendlich zu Anton und Christa Schlecker übermittelt. Dort wurde eine Entscheidung getroffen, die schlussendlich wieder über viele Hierarchiestufen nach unten kommuniziert wurde. ${ }^{1061}$ Horizontale oder laterale Kommunikation außerhalb dieser auf ein Zentrum zulaufenden Bahnen fand kaum statt.

Nicht nur die Entscheidungsprozesse und Informationsflüsse waren zentralisiert, sondern auch die Machtressourcen zur Belohnung und Bestrafung. ${ }^{1062}$ Der Aufstieg in der Hierarchie war sowohl bei der Anton Schlecker e.K. als auch bei der Berentzen-Gruppe AG an das persönliche Wohlwollen der über mehrere Jahrzehnte gleichen Entscheidungsträger gebunden.

1059 Vgl. zu dieser Konzeption von Zentralisierung Fredrickson (1986: 285).

1060 S110613FA. Vgl. Bower (1970: 287): »[W]hen management chooses a particular organization form, it is providing not only a framework for current operations but also the channels along which strategic information will flow.»

1061 Vgl. S120602HB.

1062 Vgl. zu dieser Machtressource French et al. (1959: 253f.). 


\section{Geringe Formalisierung}

In beiden Unternehmen lässt sich zudem jene geringe Formalisierung der strategischen Entscheidungsprozesse ausmachen, die in Teil B als weitere Manifestation eines hohen familiären Einflusses identifiziert wurde. ${ }^{1063}$

In beiden Unternehmen wurden die strategischen Entscheidungsprozesse nur von wenigen, im Vergleich zu kapitalmarktorientierten Unternehmen nur relativ schwach formalisierten Managementsystemen und Kommunikationsstrukturen gerahmt. Der Rahmen des strategischen Entscheidungsprozesses wurde vor allem von Personen gesetzt, weniger durch Strukturen oder Programme. So verliefen die strategischen Entscheidungsprozesse nicht so sehr in formal determinierten Bahnen, sondern basierten eher auf proaktiven Initiativen wichtiger Protagonisten und besaßen häufig Ad-hoc-Charakter. ${ }^{1064}$ Entsprechend erwuchsen die strategischen Ziele meist aus persönlichen Bestrebungen und positiven Visionen, weniger aus permanenten und routinisierten Kontrollen der Umweltdynamik und daraus abgeleiteten Handlungsbedarfen.

Bei der Anton Schlecker e.K. fanden jahrzehntelang überhaupt keine formalisierten Sitzungen zur strategischen Unternehmensentwicklung statt. Solche strukturierten Kommunikationsanlässe zu strategischen Thematiken wurden vielmehr als bürokratisch und unnötige Zeitverschwendung erachtet.

"Und auch in dieser Zeit ist er dann mal von dem Herrn Schwarz, das ist der Inhaber von Lidl, dort in den Aufsichtsrat berufen worden. Da saß er jetzt auf einmal in ganz erlauchtem Kreis. Sehr kompetente Leute, in dem Gremium [...] hat [es] ihm nicht gefallen. Da wird ja geschwätzt und da wird ja erzählt [...].«

(Ehemalige Führungskraft der Anton Schlecker e.K.)

Die Berentzen-Gruppe AG wies aufgrund ihrer Rechtsform einen höheren Formalisierungsgrad der Monitoring- und Kontrollmechanismen auf. Dennoch zeigte sich auch hier in den Entscheidungsprozessen eine Tendenz zur Vermeidung oder Umgehung struktureller Reglementierung. ${ }^{1065}$

1063 Es wird mit der Forschung zu Familienunternehmen davon ausgegangen, dass aufgrund der langfristigen strategischen Ausrichtung der Eigentümerfamilien, der aus den besonderen Governance-Strukturen erwachsenden Kontrollmöglichkeiten der Agenten durch die Prinzipale sowie des Strebens von Familienunternehmen nach kultureller »community« mit steigendem Einfluss der Familie der Grad an Formalisierung des Umweltscreenings, der Entscheidungsfindung und der strategischen Kontrolle abnimmt. Vgl. König et al. (2013: 424).

1064 Vgl. hierzu für Familienunternehmen im Allgemeinen Schulze et al. (2001: 104); Ward (1987: 37); Wimmer et al. (2005: 111).

1065 Eine ehemalige Führungskraft: »[U]nd da hab ich mir doch vorm Kamin mit dem schon die Hand drauf geschüttelt, so machen wir das. Und auf die Frage, ja, musst Du nicht erst mal ein paar Leute fragen zu Hause, wurde zur Antwort gegeben, ach phh, das, lass das nur meine Sorge sein." 
»Regeln wurden nicht eingehalten. Die haben alleine Verhandlungen geführt, unterschrieben und das war's denn."

(Ehemaliger Aufsichtsrat der Berentzen-Gruppe AG)

In diesem schwach formalisierten Rahmen des strategischen Entscheidungsprozesses gab es für die mittlere Managementebene somit vergleichsweise wenige vorstrukturierte Anlässe und Kanäle, um am strategischen Entscheidungsprozess zu partizipieren. Dies galt nicht nur für die schlussendliche Entscheidungsfindung, sondern vor allem auch bezüglich der vorgeschalteten Analyse der Umweltdynamik und der generellen Unternehmensentwicklung. Dies mündete nicht zuletzt darin, dass die strategischen Entscheidungsträger einer sehr schwach ausgeprägten unmittelbaren prozessbegleitenden Kontrolle des strategischen Entscheidungsprozesses durch Akteure außerhalb der dominanten Koalition unterworfen waren.

\section{Problem der Selbstkontrolle und Immunität der dominanten Koalitionen}

Letztlich führten die hohe Entscheidungsautonomie der dominanten Koalition, der hohe Grad der Zentralisierung und die geringe Formalisierung der strategischen Entscheidungsprozesse dazu, dass die um die Unternehmensfamilien gruppierten dominanten Koalitionen der Anton Schlecker e.K. und der Berentzen-Gruppe AG sich in einer außergewöhnlich machtvollen Lage befanden, um Einfluss und Kontrolle über die strategischen Entscheidungsprozesse ihrer Unternehmen auszuüben. ${ }^{1066}$ Sie konnten die strategische Entwicklung der von ihnen geführten Unternehmen weitestgehend unbeschränkt und selbstbestimmt steuern und genossen somit einen außergewöhnlich hohen Grad an »managerial discretion ${ }^{1067}$

Die Entscheidungsfreiheit der dominanten Koalitionen war so hoch, dass selbst weitreichendste Entscheidungen zu Akquisitionen oder anderen strategischen Richtungsentscheidungen ohne langwierige Abstimmungsprogramme innerhalb kürzester Zeit getroffen werden konnten. Dies illustriert anschaulich der Einstieg der Anton Schlecker e.K. in das eCommerce-Geschäft, der innerhalb weniger Wochen beschlossen und dann über hohe Investitionen rasch implementiert wurde. ${ }^{1068}$

1066 Für eine allgemeine Einschätzung der machtvollen Position von Eigentümerfamilien vgl. Anderson \& Reeb (2003: 1304); Anderson \& Reeb (2004: 213).

1067 Vgl. Hambrick \& Finkelstein (1987); Finkelstein \& Hambrick (1990); Sydow et al. (2009: 701).

1068 Vgl. S001207LZ: Es ist der unbestreitbare Vorzug des Unternehmers, in Grenzsituationen wie der jetzigen eindeutige Zeichen zu setzen. Während in anders verfassten Organisationen Gremien tagen, qualvolle Entscheidungs- bzw. Nicht-Entscheidungsprozesse ihren Lauf nehmen und allenthalben Bedenkenträger den Finger heben, kann der Unternehmer 
»Ende der 90er-Jahre war ja hier der E-Commerce-Boom weltweit. Und da möchte ich Ihnen jetzt mal in zwei Minuten erzählen, wie da ein Entscheidungsprozess ablief. An irgendeinem/ es war immer der Dienstag, lag so ein Taschenbuch >E-CommerceHandel in Deutschland unverzichtbar`. So ungefähr hieß das. Sagt er: `Ich habe hier ein neues Buch. Lesen Sie mal.^ Okay. Nächste Woche. \Und? \Ja wie und?^〉Haben Sie das Buch gelesen? ‘ Nein`, sag ich, 'hatte ich eigentlich Weihnachten vor. ` $\mathrm{Ne}$, lesen Sie mal.< Da hab ich gesagt: ^Ich kann doch ein Buch jetzt nicht lesen. ২Doch gehen Sie ins Büro und lesen Sie. $[$ [...] Und habe dann gelesen `E-Commerce wird kommen`, so wie er eben heute auch da ist weltweit. Und dann die Meinung abgefragt bei mir: >Und wie war's? > Brillant. Finde ich Wahnsinn. Aber ich sag: ‘Das werden wir ja wohl kaum machen können oder?^>Warum nicht?^ Da habe ich dann auch gestutzt. E-Commerce, das wäre jetzt mal was Neues. Da ist ja noch keiner dran von den Drogeriemärkten. ১Ja, dann kümmern Sie sich mal um das Thema. Wie geht sowas?^ Ich sag: ১Wissen Sie was, ich fahre nach Düsseldorf. Da wohnt der, der das Buch geschrieben hat.« Und dann sind wir da runter [...] \Ja bei Schlecker, da könnte man das auch machen. Ihr habt den Riesenvorteil, Ihr seid bekannter als der Bundeskanzler.^ Da kamen gerade die Statistiken raus. 99 Prozent aller Deutschen kennen Schlecker. [...] Super. Ja. Sie glauben es nicht. Zwei Wochen später war die Entscheidung gefallen, wir machen E-Commerce. Ohne eine einzige Berechnung. Ohne Dritte zu fragen.»

(Ehemalige Führungskraft der Anton Schlecker e.K.)

Derart verlaufende Entscheidungsprozesse waren nur möglich, da spiegelbildlich zur hohen Machtfülle der dominanten Koalitionen bei der Anton Schlecker e.K. und der Berentzen-Gruppe AG weder organisationsextern noch organisationsintern ausreichend machtvolle Regulative außerhalb der autonomen dominanten Koalitionen existierten, die diese in ihren Entscheidungsprozessen entscheidend hätten irritieren oder kontrollieren können. ${ }^{1069}$

"Es gibt zwei, die Sie genannt haben, das sind beides keine sehr positiven Beispiele, aber die haben natürlich auch damit was zu tun, dass das Regulativ gefehlt hat, das Regulativ bei Schlecker waren seine Frau und seine zwei Kinder, das hat nicht ausgereicht."

(Ehemaliges Vorstandsmitglied der Eckes AG)

Da die hohe Machtkonzentration die strategischen Entscheidungsträger weitgehend von externen und internen Governance-Mechanismen isolierte, waren die dominanten Koalitionen dieser Unternehmen in der Steuerung und Kontrolle ihrer Entscheidungsprozesse weitestgehend auf sich und ihre Eigengesetzlichkeit zurückgeworfen. ${ }^{1070}$ Sie unterlagen als Gruppe somit dem von der neueren Agenturtheorie thematisierten Problem der Selbstkontrolle. ${ }^{1071}$

frei nach Luther eindeutige Signale geben: `Hier stehe ich. Ich kann nicht anders. Gott helfe mir.«

1069 In den komparativen Vergleichsfällen der Dirk Rossmann GmbH und Eckes AG entwickelten sich im Laufe der organisationalen Entwicklung solche Regulative.

1070 Vgl. Schulze et al. (2003: 181): »[P]rivate ownership isolates them from the discipline 
Dieses aus den Strukturmerkmalen des familiären Einflusses resultierende Problem der Selbstkontrolle zeigt hinsichtlich der Frage, warum die dominanten Koalitionen nicht auf die emergierenden Pfade reagierten, die Richtung der Suche nach Antworten auf: Die in den beiden empirischen Fällen beobachtete hohe Autonomie verweist genau auf jene in Teil B skizzierte Problematik schwacher exogener Kräfte zur Korrektur von Fehlentwicklungen, die die institutionelle Pfadforschung als ursächlich für die im Vergleich zu Marktprozessen höhere Anfälligkeit von Institutionen und Organisationen für das Entstehen von Pfadabhängigkeit ansieht.

"The characteristics of politics change the picture considerably: the absence or weakness of efficiency-enhancing mechanisms of competition [...] makes increasing returns processes in politics particularly intense. They increase the difficulty of reversing the course down which actors have started.«

(Pierson, 2000) $)^{1072}$

Da Akteure in Organisationen im Vergleich zum Marktgeschehen in einer weit weniger transparenten und zugänglichen Umgebung operieren, seien sie auch nicht im gleichen Maße den für Fehlentwicklungen sensiblen und korrigierenden Kräften des Marktes ausgesetzt. Ohne die unmittelbare und volle Wirkkraft des Marktdrucks können sich Stabilitäts- und Kontinuitätstendenzen in Organisationen leichter entfalten und erhalten.

"In this view organizations (in particular, formal political institutions) are more prone to bring about path-building forces than markets, because they act in sa far, far murkier environment $[$ [...] with weaker forces to correct inefficient courses of action over time."

(Sydow et al., 2009) $)^{1073}$

external markets provide." Zugleich deckt sich die empirische Beobachtung mit der etymologischen Herkunft der Autonomie. Das griechische "autonomia « zielt einerseits im Außenverhältnis auf den Umstand der Selbstständigkeit, darüber hinaus im Innenverhältnis aber auch auf die Eigengesetzlichkeit. Vgl. dict (2016).

1071 Vgl. Schulze et al. (2001: 99): »Private ownership and owner management not only reduce the effectiveness of external control mechanisms, they also expose firms to a sself-control problem created by incentives that cause owners to take actions which harm themselves as well as those around them ‘." Jensen (1994: 12): "Constrained at the time by our economists' view of rationality, Meckling and I discussed only one source of agency costs, that which emanates from the conflicts of interest between people. There is clearly a second major source of agency costs, the costs incurred as a result of the self control problems discusses above - that is the actions that people take that harm themselves as well as those around them - what Richard Thaler years ago characterized as agency problems with one's self.« Vgl. hierzu Thaler \& Shefrin (1981). Von diesen Beiträgen wird das Problem der Selbstkontrolle auf der Ebene des Individuums angesiedelt.

1072 Pierson (2000: 257). Pierson spricht hier von Politik, diese Argumentation wird von der Forschung aber auch auf Organisationen übertragen; siehe hierzu Kapitel B.1.3.5.

1073 Sydow et al. (2009: 701); vgl. auch Pierson (2000: 260). 
Insbesondere die Macht und deren strukturelle Verteilung in der Organisation können einen entstehenden Pfad vor korrigierenden Kräften schützen und somit in der Genese stabilisieren. ${ }^{1074}$

Genau dies zeigt die Pfadgenese der Anton Schlecker e.K. und der BerentzenGruppe AG. Sie offenbart, wie die Macht der dominanten Koalitionen die strategischen Entscheidungsprozesse in der Phase der Pfadformation dergestalt ummantelte, dass aus der hohen Autonomie der dominanten Koalitionen gar eine Form der Immunität der Entscheidungsträger und der Entscheidungsprozesse gegenüber exogenen Einflüssen hervorging. ${ }^{1075}$ Diese Immunität der Entscheidungsträger trug einen Teil zur ausbleibenden strategischen Reaktion bei, da mit ihr das Fehlen exogener Kräfte einherging, welche die dominanten Koalitionen machtvoll zu einem Gegensteuern hätten bewegen können. ${ }^{1076}$

Als hinreichende Erklärung der ausbleibenden strategischen Reaktion kann diese machtbasierte Immunität aber nicht dienen, da das Fehlen eines machtvollen Regulativs nicht zwangsläufig ausschließt, dass die dominanten Koalitionen von sich aus auf die Dynamik der Umwelt und Fehlentwicklungen im Unternehmen reagieren. Auf diesen Umstand verweist auch der Kontrast zu den Vergleichsfällen der Dirk Rossmann GmbH und Eckes AG. In diesen beiden Unternehmen ließen sich nämlich ebenfalls eine starke Machtkonzentration und eine daraus abgeleitete hohe Entscheidungsautonomie der dominanten Koalitionen feststellen. So war in beiden Vergleichsfällen der Großteil des Eigenkapitals in den Händen der Eigentümerfamilien konzentriert. ${ }^{1077}$ Diese hohe Eigentumskonzentration ermöglichte eine ausgeprägte Unabhängigkeit der strategischen Entscheidungsträger gegenüber der Umwelt der Organisationen.

Innerhalb der Organisationen wurde die Autonomie der Entscheidungsträger der dominanten Koalition zwar durch unabhängigere Kontroll- und Aufsichts-

1074 Vgl. Ackermann (2003: 232f.); Beyer (2010: 4); Pierson (2000: 257): »[F]our prominent and interconnected aspects of politics that make this realm of social life conducive to increasing returns processes: (1) the central role of collective action; (2) the high density of institutions; (3) the possibilities for using political authority to enhance asymmetries of power; and (4) its intrinsic complexity and opacity.«

1075 Diese Immunität ähnelte der Unantastbarkeit der politischen Immunität hoher Mandatsträger. Vgl. Gomez-Mejia et al. (2001: 83): »[I]mplicit immunity top-level executives gain from their family status. «Zudem Schein (1983: 26): »Have secure position by virtue of ownership «; Miller (1991: 34): »influence, autonomy and desire to resist external pressures for change." Die organisationale Pfadtheorie spricht hinsichtlich der dritten Phase von einer Immunität der strategischen Entscheidungsprozesse gegenüber äußeren Einflüssen. Vgl. Holtmann (2008: 67).

1076 Zur Bedeutung exogenen Drucks zur Auslösung strategischer Reaktionen vgl. Hambrick \& D’Aveni (1988: 17).

1077 Bei der Eckes AG befanden sich die Unternehmensanteile vollständig in den Händen der Familie. Bei der Dirk Rossmann $\mathrm{GmbH}$ war neben der Eigentümerfamilie noch die Hannover Finanz am Unternehmen beteiligt. 
organe sowie eine stärkere Trennung von Eigentum und Verfügungsgewalt eingeschränkt. Doch auch hier waren die Entscheidungsprozesse relativ stark zentralisiert und die Entscheidungsträger genossen aufgrund ihres Eigentümerstatus und ihrer langjährigen Unternehmenszugehörigkeit ein hohes Maß an Legitimität und Autorität in der Führung des Unternehmens. ${ }^{1078}$ In diesem organisationalen Rahmen konnte die dominante Koalition der Dirk Rossmann $\mathrm{GmbH}$ die strategischen Entscheidungsprozesse weitgehend autonom steuern und kontrollieren. ${ }^{1079}$ Auch die Entscheidungsträger der dominanten Koalition bei der Eckes AG genossen in ihren Entscheidungsprozessen einen sehr hohen Grad an Unabhängigkeit.

"Ja also ich, ich behaupte, dass ich ein, ja also mit meinem, mit meinem Management ein Höchstmaß an Freiheit hatte. Ein Höchstmaß an Freiheit [...] also einem extrem hohen Freiheitsgrad."

(Ehemaliges Vorstandsmitglied der Eckes AG)

Demnach waren, obwohl die Kräfte zur Korrektur von Fehlentwicklungen in den beiden Vergleichsfällen etwas stärker ausgeprägt waren als bei der Anton Schlecker e.K. und der Berentzen-Gruppe AG, auch bei der Dirk Rossmann GmbH und der Eckes AG die dominanten Koalitionen in der Steuerung und der Kontrolle der strategischen Entscheidungsprozesse weitgehend auf sich selbst zurückgeworfen. ${ }^{1080}$ Nichtsdestotrotz konnten diese beiden Unternehmen frühzeitig auf die in Teil C skizzierte sich verändernde Umweltdynamik in der Branche reagieren und einen bemerkenswerten strategischen Wandel vollziehen.

Die schwach ausgeprägten exogenen Regulative allein können die ausbleibende Reaktionen auf die Pfadgenese bei der Anton Schlecker e.K. und der BerentzenGruppe AG somit nicht erklären. Der Kontrast mit den in Verlauf und Ergebnis so divergenten strategischen Entscheidungsprozessen der Vergleichsfälle verweist vielmehr auf die Bedeutung von Struktur- und Interaktionsvariablen des strategischen Entscheidungsprozess innerhalb der dominanten Koalitionen. Die Ausprägungen dieser Variablen und die entsprechenden Wirkungszusammenhänge werden in den folgenden Unterkapiteln näher thematisiert. Zuvor werden im folgenden Abschnitt jedoch jene Aspekte der organisationalen Kultur und

1078 Die strategischen Entscheidungsprozesse bei der Dirk Rossmann GmbH wurden innerhalb der dominanten Koalition fast zwei Jahrzehnte lang von drei bis vier Personen geprägt.

1079 Mindestens bis zum Verkauf der Eigenkapitalanteile der Hannover Finanz an den Konzern Hutchison Whampoa. Vgl. Michler (2009: 104): »So gehören heute 40 Prozent an dem deutschen Familienunternehmen den Chinesen. Mehr allerdings sollen sie nicht bekommen. \Ich verkaufe nichts«, sagt Roßmann. Satte 60 Prozent der Anteile blieben in der Familie. So könne ihm niemand reinreden. >Ich bin und bleibe Herr im Haus.«"

1080 Diese schwächere Ausprägung des Strukturmerkmals der Entscheidungsautonomie könnte als ein Indiz für die graduelle Wirkkraft des Einflusses der Familien interpretiert werden. Siehe hierzu Kapitel B.2.3.1. 
Struktur dargelegt, die bei der Anton Schlecker e.K. und der Berentzen-Gruppe die machtbasierte Immunität der dominanten Koalition zusätzlich verstärkten und somit das Problem der Selbstkontrolle nochmals erhöhten.

\subsubsection{Die immunisierende Wirkung organisationaler Kulturen und Strukturen}

Neben der machtbasierten Immunität, die einen direkten Zugriff exogener Regulative auf die dominanten Koalitionen verhinderte, zeigt sich in den Entscheidungsprozessen der Anton Schlecker e.K. und der Berentzen-Gruppe AG eine zweite Form von Immunität: Exkludierende kulturelle Muster und formelle Strukturen zogen um die dominanten Koalitionen Grenzen, die von den Irritationen der Umwelt nur schwer durchdrungen wurden. ${ }^{1081}$ Während die machtbasierte Immunität dazu beitrug, dass die dominanten Koalitionen sich nicht irritieren lassen mussten, trugen Abschottung und Selbstimmunisierung dazu bei, dass sie von den Perturbationen der Umwelt tatsächlich kaum irritiert wurden und irritiert werden konnten.

\section{Exkludierende kulturelle Muster gegenüber der Umwelt und der restlichen Organisation}

Die Konstruktion der Umwelt gehört in jeder Organisation zu den grundlegenden Orientierungs- und Vorstellungsmustern. ${ }^{1082}$ Eine zentrale Rolle im Konstruktionsprozess nimmt vor allem die dominante Koalition ein: Über formelle und informelle Mechanismen prägt sie, ob die Umwelt in der Organisation grundsätzlich als feindlich und bedrohlich oder als Ort voller Möglichkeiten wahrgenommen wird. Im Zuge der Gestaltung des Grenzmanagements bestimmt sie letztlich, ob sich die Organisation den Möglichkeiten der Umwelt öffnet oder sich vor deren Gefahren abschottet und nach innen orientiert.

Vor dem Hintergrund der bisherigen Forschung zu Familienunternehmen und den Indizien der explorativen Vorstudie wurde in Teil B herausgearbeitet, dass die Ko-Evolution von Familie und Unternehmen häufig zu Manifestationen von Umweltabgewandtheit und sozialer Innenorientierung führt. ${ }^{1083}$

Solche kulturellen Muster der Abschottung konnten in der empirischen

1081 Siehe Kapitel B.1.2.1 zur Bedeutung dieser Irritationen für den Wandel der Organisation. 1082 Vgl. Schein (2010: 27 ff.); Schreyögg (2006: 452).

1083 Vgl. Litz (1997: 60); Miller \& LeBreton-Miller (2005: 279); Ward (1987: 173). Allerdings hat bei der Anton Schlecker e.K. wahrscheinlich auch die Persönlichkeit der Protagonisten zu diesen exkludierenden Mustern beigetragen. Hierzu eine ehemalige Führungskraft: »Also man hat uns da auch eingeladen, mal hinzukommen. Aber das hätte natürlich immer einen anderen Auftritt verlangt und ich glaube, dem wollte er sich auch nicht stellen [...] aber ich glaube, er hat sich das nicht zugetraut." 
Studie insbesondere bei der Anton Schlecker e.K., zum Teil auch bei der Berentzen-Gruppe AG ausgemacht werden.

»Dann kam die Auslandsexpansion. Und da war ja nun doch ein kräftiger Bedarf an qualifizierten Leuten, an auslandserfahrenen Leuten. Das wurde dann immer irgendwie verschoben, runtergeredet, und da würde ich also schon sagen, das war das Handicap >Familie`. Die wollten für sich bleiben, das sollte >familiär ` bleiben.«

(Langjährige Führungskraft der Anton Schlecker e.K.)

Bei der Anton Schlecker e.K. wurde die Umwelt von vielen Mitgliedern der um die Familie gruppierten dominanten Koalition als widrig und feindlich wahrgenommen. Es herrschte eine Mentalität des »Wir gegen den Rest der Welt« vor, die sich im Zuge der zahlreichen Auseinandersetzungen mit den Stakeholdern des Unternehmens immer weiter vertiefte. ${ }^{1084}$

Mit einer möglichst weit gehenden Abschottung versuchte sich die dominante Koalition gegenüber der als feindselig wahrgenommenen Umwelt zu immunisieren. So führte die Familie Schlecker nicht nur ein vollkommen zurückgezogenes Privatleben. ${ }^{1085}$ Sie schottete vielmehr das gesamte Unternehmen möglichst weitgehend von der Öffentlichkeit ab. Während die Protagonisten der bedeutendsten Wettbewerber, Götz Werner und Dirk Roßmann, ihre Unternehmen medial inszenierten, traten weder die Familie Schlecker noch andere Mitglieder der dominanten Koalition je öffentlich in Erscheinung: Interviewanfragen der Presse wurden konsequent abgelehnt, Auftritte in der Öffentlichkeit fanden nicht statt und selbst Gespräche mit führenden Politikern oder Wirtschaftsvertretern wurden verweigert. ${ }^{1086}$ Sogar in kommunikativen Krisensituationen wie den Skandalen um Lohn-Dumping oder verunreinigte Babynahrung verzichtete das Unternehmen weitgehend auf eine mediale Eigendarstellung. Die erste Pressekonferenz des Unternehmens, das durch seine zeitweise mehr als 14.000 Filialen im öffentlichen Raum lange Zeit so präsent

1084 Hierzu eine langjährige Führungskraft: »[...] dann war natürlich auch die Öffentlichkeit nicht gut ihm, sag ich mal, ja. Also er hat Teile, nichts Positives zurückerhalten und dann sagt man immer mal, da sagt dann so'n Schwabe >Ihr könnt mich alle am Arsch lecke. Das interessiert mich nicht, was ihr über mich sagt, ja. Ich hab hier mein Unternehmen, das ist erfolgreich, ich hab meine Familie. Und das, das ist, was mir wichtig ist.«

1085 Vgl. S940415ZE; S140125SW: „Es gibt Interesse, aber wenig Bedauern oder gar Mitleid für Anton Schlecker. Der Firmenchef hat als Person kaum eine Rolle gespielt in der Stadt. Er nahm nicht am öffentlichen Leben teil, viele in der Stadt kennen ihn nur von jahrzehntealten Fotos.« Dieser Rückzug ins Private lässt sich nicht allein mit der Entführung der beiden Kinder Meike und Lars im Jahre 1987 erklären, da diese Muster der Abschottung sich schon zwanzig Jahre vorher im Unternehmen etabliert hatten.

1086 Vgl. S940415ZE; S120324TA. Lediglich zur Veröffentlichung der verkürzten Bilanzen wurden eine Handvoll ausgewählter Journalisten eingeladen, die aber keine kritischen Fragen stellen durften. Vgl. S041026MM: "Selbst heimische Politiker und Wirtschaftsvertreter betonen: `Persönliche Kontakte zu Anton Schlecker gibt es nichtı.» 
gewesen war, fand im Jahr 2012 im Zuge der Eröffnung des Insolvenzverfahrens statt. Doch auch hier setzten die Protagonisten der dominanten Koalition, Anton und Christa Schlecker, ihre Abschottung gegenüber der Umwelt fort und wurden von der Tochter vertreten. ${ }^{1087}$

»[B]in ich auch mal angesprochen worden/ ein Staatssekretär war das manchmal >Von Schlecker hört man ja/ kommen Sie uns doch mal besuchen. Uns interessiert das was der da macht. Prima. Und dann kriegten wir Wochen später nochmal einen Brief von dem Herrn Teufel, damals Ministerpräsident, also: Er sliest das ja gerne in der Zeitung. Schlecker wächst und tolles Image für das Land. Er möchte sich mit ihm treffen. $\$ Jetzt dürfen Sie raten, was passiert ist [...]. Nichts. Den hat man einfach liegenlassen, den Brief dann.«

(Langjährige Führungskraft der Anton Schlecker e.K.)

Ausgehend von der Unternehmensfamilie wurde sowohl durch implizite Praktik als auch explizite Anweisungen ein kultureller Rahmen geprägt, in dem die Abschottung gegenüber der vermeintlich feindlichen Umwelt zu einem klaren handlungsleitenden Orientierungsmuster wurde. ${ }^{1088}$ Die von der Unternehmensfamilie Schlecker etablierten Normen und Symbolsysteme waren dabei so eindeutig, dass den weiteren Mitgliedern der dominanten Koalition unzweifelhaft klar war, dass Diskretion erwünscht war. ${ }^{1089}$

"Auch eine Darstellung nach außen nicht. Ich selbst bin also immer wieder angesprochen worden, hier auch Vorträge zu halten, hier auch im Wirtschaftsforum, oder an der Universität hier in Ulm, oder so, weil das eben so rasant lief, ob man da auch mal was erzählen könnte. Das hat er mir, ich würd mal sagen, fast untersagt. [...] Ich möchte nicht, dass Sie da über die Firma sprechen.«

(Langjährige Führungskraft der Anton Schlecker e.K.)

Das kulturelle Muster der Abschottung gegenüber der Umwelt manifestierte sich auch in der Beibehaltung der Rechtsform des eingetragenen Kaufmanns, die nicht nur Entscheidungsautonomie, sondern aufgrund der geringen Rechenschaftspflichten auch maximale Diskretion gewährte. Im Rahmen dieser Rechtsform war die Anton Schlecker e.K. nicht einmal zur Veröffentlichung eines Geschäftsberichts verpflichtet. In der Tat publizierte das Unternehmen erst seit 1994 verkürzte Jahresabschlüsse, die so knapp wie möglich gehalten wurden: Außer der verkürzten Bilanz und Gewinn- und Verlustrechnung enthielten sie einen Lagebericht, der grundsätzlich aus lediglich vier knappen Sätzen bestand: ${ }^{1090}$

1087 Vgl. S120204SP:»[I]n der Pleite agiert die Unternehmerfamilie genau so, wie sie mehr als 30 Jahre lang die Geschäfte führte: als Phantom."

1088 Vgl. zur Prägnanz von Kulturen Schreyögg (2006: 464).

1089 Wobei das kulturkonforme Verhalten eher Resultat einer kalkulierten Anpassung denn Ausdruck einer wirklichen Internalisierung dieser Norm war.

1090 Vgl. S040220MM; auch Werner (2013: 230f.). Hierzu S070503LZ: »Während sich in anderen Geschäftsberichten des Handels die Lageberichte und Risikobewertungen umfas- 
»Zum Bilanzstichtag betrug die Eigenkapitalquote rund $32 \%$. Wie bisher konnte auch im Geschäftsjahr 2005 die Geschäftstätigkeit ohne mittel- und langfristige Bankkredite ausgeübt werden. Die für die gesamte Geschäftstätigkeit in 2005 notwendige Liquidität war stets in sicherem und ausreichendem Umfang vorhanden. Der Markt ist nach wie vor stark belegt und birgt weiterhin eine angespannte Wettbewerbssituation."

(Der Schlecker-Konzernabschluss im Jahr 2005 zur Lage des Unternehmens)

Über diese spärlichen Informationen hinaus veröffentlichte das Unternehmen keine Zahlen zur aktuellen Geschäftsentwicklung.

Die Interaktion mit anderen Stakeholdern war von einer starken Trennung in »innen« und »außen« und dem kulturellen Muster der Abschottung geprägt. Neben dem hohen Autonomiestreben trug dies zur strikten Vermeidung der Aufnahme von Fremdkapital bei. In der Spätphase der Liquiditätskrise war die dominante Koalition zwar schlussendlich zur Aufnahme von Fremdkapital in Höhe von 300 bis 400 Mio. Euro und der damit verbundenen Aufgabe der absoluten Autonomie über das Unternehmen bereit. Allerdings scheiterten die Gespräche mit den kontaktierten Banken daran, dass die Unternehmensfamilie weder den Banken noch den hinzugezogenen Unternehmensberatern einen Einblick in die tatsächliche finanzielle Lage des Unternehmens oder gar in die familiären Vermögensverhältnisse gewähren wollte. ${ }^{1091}$ Der zentrale Protagonist des Unternehmens, Anton Schlecker, weigerte sich grundsätzlich, persönlich mit den als nicht vertrauenswürdig erachteten Banken zu sprechen. ${ }^{1092}$

"Die Frage, die nie diskutiert werden durfte, wo ich auch im Nachhinein, das war eigentlich das, deshalb ist es gescheitert, behaupte ich, zum Schluss, das war die Kapitalfrage. $\mathrm{Da}$ ist letztendlich nun, hat er das die eigenen Leute machen lassen, glaube ich, die mit Krisensituationen keine Erfahrung hatten, wir hatten mit Externen teilweise gesprochen, die zum Teil auch hergebracht, aber sobald man jetzt konkret wurde, hat Anton Schlecker verweigert, mit den Leuten zu sprechen [...] jeder Versuch dahin, den wir da in die, in die Hinsicht unternommen hatten, haben die, hat uns die Familie nicht gelassen."

(Ehemaliger Berater der Anton Schlecker e.K.)

Ungeachtet des Stellenwerts der Finanzierungsfrage bei der Implementierung des Turnaround-Konzepts und der erfolgreichen Bewältigung der Krise wurde

send mit zahlreichen kritischen Details des Gesamtmarktes befassen, kommt Schlecker mit wenigen erklärenden Kernsätzen aus. « Zudem wurden diese Jahresabschlüsse stets mit knapp zwei Jahren Verzögerung veröffentlicht. Im Januar 2000 wurde die Bilanz des Jahres 1998 publiziert, im Januar 2001 wurde Einblick in den Geschäftsverlauf des Jahres 1999 gegeben etc.

1091 Vgl. S120602HB.

1092 Hierzu eine langjährige, in der Krise noch aktive Führungskraft: »Aber so, dann im Grunde brauchst du das Geld, aber dann hat aber Anton Schlecker mit denen nicht geredet. Ja, er hat immer noch nicht mit denen geredet, sondern hat er seinen 35-jährigen Sohn vorgeschoben." 
von der Unternehmensfamilie um den Bereich der Finanzierung eine Grenze gezogen, die nur jahrzehntelang im Unternehmen beschäftigte und somit als vertrauenswürdig erachtete Mitarbeiter überschreiten durften.

Innerhalb der Branche schotteten sich die Entscheidungsträger der dominanten Koalition der Anton Schlecker e.K. - auf explizites Drängen der Familie ebenfalls möglichst ab.

"Man hat also das gemieden. Es war auch gar nicht gewünscht, mit anderen Firmen in Kontakt zu treten. Auch die Möglichkeit hat's ja gegeben, bei Kongressen oder so. Das wurde nicht gewünscht. Dass man dort, wenn man jetzt sich so mit wenigen Leuten ... natürlich kann man nicht die Unterlagen austauschen ... Einfach mal: `Wie habt ihr den Einkauf organisiert?» >Ach so, ihr seid schon da dran ..... Und: `Läuft, oder läuft nicht?, dass man da sagt >Also das können wir eigentlich auch machen, da sind die schon weiter und so. Das war eigentlich ... nicht eigentlich, sondern das war auch kein Thema. Das wurde nicht gewünscht und nicht gerne gesehen."

(Langjährige Führungskraft der Anton Schlecker e.K.)

Darüber hinaus prägte das kulturelle Muster der Abschottung die Auswahl der Führungskräfte. Führungspositionen bei der Anton Schlecker e.K. wurden nur in sehr seltenen Fällen mit extern rekrutierten Managern besetzt. Vielmehr wurden vakante Stellen über viele Jahrzehnte nahezu ausschließlich an unternehmensinterne »Eigengewächse« vergeben.

"Und sie haben's mit Eigenbau versucht, die Akademikerquote war extrem gering bei Schlecker, da gab es kaum Leute, die Konzepterfahrung hatten bei Schlecker. Das waren halt alles dann, die waren 20 Jahre dabei, die wussten, wie man den Laden führt, aber die wussten nicht, wie man ein neues Konzept entwickelt. [...] Also es waren sogenannte Abiturslehrlinge oder was, die, die kamen nach dem Abitur und dann haben sie ein Programm, im Grunde war es eine Art Trainee-Programm, bei Schlecker gemacht, und es war alles in den 80er-Jahren, in der Zeit, wo die gewachsen sind, und da sind dann die, die Fleißigen, sag ich mal, die sind dann sehr schnell in Führungspositionen hereingewachsen, ob Sie da den Vertriebschef nehmen, den Marktleiter, den Personalchef, das waren alle die Leute, die da aus dem eigenen Saft kamen und die haben da Karriere gemacht, und die haben dann plötzlich Milliarden verantwortet, würde ich grade mal, ja. Aber nicht hier über ERP-Systeme und Controlling.... Genau das hat dann halt gefehlt da."

(Ehemaliger Berater der Anton Schlecker e.K.) ${ }^{1093}$

Selbst vollkommen neuartige Projekte außerhalb des etablierten operativen Geschäfts, wie die Einführung neuer IT oder eine grundlegende strategische

1093 Dabei waren für den Aufstieg im Unternehmen vor allem die Dauer der Unternehmenszugehörigkeit und die Loyalität zu Unternehmen wichtig. Hierzu eine langjährige Führungskraft des Unternehmens: „Für mich ging das ja nur nach oben. Ne, alle ein, zwei Jahre wurde man ja da befördert. Man brauchte sich da nicht groß drängeln, das ging automatisch.» 
Neuausrichtung des gesamten Unternehmens, wurden lieber vertrauten Mitarbeitern oder Familienmitgliedern übertragen, als extern qualifiziertes Personal zu rekrutieren. ${ }^{1094}$

"Als der Drogerie-Gigant 2008 eine neue Strategie brauchte, wurden kurzerhand die Führungskräfte zusammengetrommelt. Am länglichen Konferenztisch hatte Christa Schlecker das Kommando - und ließ nicht locker, bis das Konzept fertig war. [...] `Federführend war meine Frau<, bestätigt Selfmademan Schlecker : `Wir brauchen keine Strategieberaterı."

(Die Süddeutsche Zeitung im Jahr 2010) ${ }^{1095}$

Abschottung der dominanten Koalition gegenüber der Umwelt kann in bestimmten Bereichen - wie bei den mit dem Börsengang einhergehenden Transparenzpflichten - auch bei der Berentzen-Gruppe AG nachgewiesen werden. ${ }^{1096}$ Allerdings war sie bei Weitem nicht so stark ausgeprägt wie bei der Anton Schlecker e.K. Hinsichtlich der meisten Angelegenheiten, die eine größere Distanz zur definierten Privatsphäre aufwiesen, war die Berentzen-Gruppe AG von der oben skizzierten Einbettung der dominanten Koalition in lokal-soziale Zusammenhänge geprägt.

Allerdings tendierte die dominante Koalition der Berentzen-Gruppe AG zu einer Form der Abschottung gegenüber dem Rest der Organisation:

»Der Vorstand hat von seinem Führungsstil her patriarchisch geherrscht. Er hat mit seinem Vorstandkollegen und dem Syndikus in einem separaten Gebäude residiert, und wenn der Marketingchef, oder der Vertriebschef, oder der Finanzchef mal was mit ihm zu besprechen hatte, musste um einen Termin gebeten werden, der dann drei oder vier Wochen später stattfand. Also er hatte den Kontakt zur Basis meiner Einschätzung nach völlig verloren. Und das hat mir auch, das haben mir auch die Vertreter im

1094 »In dieser Zeit sind mir dann im Laufe der Jahre dann auch verschiedene Projekte angetragen worden. Klar, auch die interne Konkurrenzsituation war nicht so groß. Ja, das können Sie sich vorstellen. Das ist eine Handvoll Leute gewesen, die sich überhaupt mit solchen, ich sag mal, übergeordneten Aufgaben, auseinandersetzen wollten." Eine ehemalige Führungskraft: »[...] weil ich gehöre zu der Generation, die am neuen IT-System nicht mehr dabei war und ich mich selber so wie der Schlecker abgeschottet habe, das heißt ich hab dann einen Geschäftsführer geschickt. Der soll sich das alles anhören und so weiter [...] und später sollte ich mich dann aber in meinem Bereich um die IT kümmern, obwohl ich selber kein großes Licht war ...«.

1095 S100517SÜ.

1096 So gab in den Reihen der dominanten Koalition schwere Bedenken hinsichtlich der mit der Notierung am Kapitalmarkt verbundenen Transparenz- und Offenlegungspflichten und es wurde beispielsweise längere Zeit verhindert, die Gehälter der Geschäftsführung zu veröffentlichen. Ein ehemaliger Aufsichtsrat:»[...] dann muss ich veröffentlichen... Ich muss als erstes mal veröffentlichen, wie viel Gehälter die Vorstände haben. Nee, das wollen wir nicht, das wollen wir gar nicht. [...] Das wollen wir gar nicht. Dabei war das alles bescheiden, das ist alles bescheidene Masse, hätte man ruhig veröffentlichen können. Aber das ist das erste, was sie gemacht haben, auf der Aufsichtsratssitzung zu, zu verhindern, dass das veröffentlicht wird, ne." 
Aufsichtsrat des, der Belegschaft, also die Arbeitnehmervertreter mitgeteilt. Die sich bitterlich darüber beschwert haben, dass die Kommunikation aufgrund dieser Abgehobenheit überhaupt nicht funktionierte."

(Ehemaliger Aufsichtsrat der Berentzen-Gruppe AG)

Doch gilt wieder: Solche Manifestationen der Abschottung der dominanten Koalition gegenüber dem Rest der Organisation waren bei der Anton Schlecker e.K. deutlich stärker ausgeprägt. Anton Schlecker und seine Frau Christa arbeiteten vollkommen abgeschottet von den Mitarbeitern in dem für sie allein reservierten sechsten Stockwerk der Konzernzentrale. In diese private Etage gelangten die Ehepartner über einen exklusiven Eingang in der Tiefgarage, der sie zu einem nur ihnen zur Verfügung stehenden privaten Aufzug führte, welcher ohne Halt direkt in den sechsten Stock fuhr. Obgleich die Familie durch die überall im Unternehmen vorzufindenden Portraits der Protagonisten und der Schriftzüge des Familiennamens beinahe omnipräsent war, geriet insbesondere Anton Schlecker zu einer beinahe amorphen Figur. ${ }^{1097}$

»Von Angesicht zu Angesicht habe ich ihn das letzte Mal vielleicht vor zehn Jahren gesehen."

(Der Portier der Schlecker-Zentrale im Jahr 2012 gegenüber dem Spiegel) ${ }^{1098}$

Das exkludierende kulturelle Muster äußerte sich auch in den Interaktions- und Kommunikationsstrukturen der Führungskräfte, die von zahlreichen Grenzen zwischen den einzelnen Führungskräften und Funktionsbereichen geprägt waren. Eine Kommunikation in Gruppen fand in der Anton Schlecker e.K. auf der Ebene des höheren Managements kaum statt und die einzelnen Funktionsbereiche kommunizierten sehr selten horizontal. Vielmehr führten Anton und Christa Schlecker mit den jeweils für einen Bereich zuständigen Direktoren bilaterale Gespräche, die den Charakter von verbalen Berichten hatten. In diesen vertikalen Bahnen vollzog sich über viele Jahrzehnte unverändert das Gros der strategisch relevanten Kommunikation.

"Ja, ganz, ganz, das ist ein ganz wichtiger Punkt. Ich sag ja, ich war mal hier, ich muss mal sehen in den Sechzigern, ja, unsere hat der Schlecker noch die Begrüßung gemacht, Mikrofon und so weiter. Als ich dann unter die, die Deutschlandschönheit da hatte und Presse sich angesagt hat, und ich mich schon vorbereitet hatte, dann ist er alte Schlecker noch gekommen: >Anton, das musst doch du machen!, und so weiter, ne [...]. Und da ist bei ihm, wo er dann bei der Beerdigung auch noch was gesagt hat, aber

1097 Vgl. S120313WW zu den ersten Veränderungen in der Ihr-Platz-Zentrale nach der Übernahme durch Schlecker: »Eine ihrer ersten Amtshandlungen war, gerahmte Fotos von Anton und Christa Schlecker an den Wänden aufzuhängen und auf den Schreibtischen aufzustellen.« Auch in jedem Sozialraum der Filialen der Anton Schlecker e.K. war ein Portrait der Protagonisten vorzufinden.

1098 Vgl. S120602HB; S120204SP. 
dann ging das kontinuierlich zurück [...]. Ja und so hat er sich zurückgezogen, dieses Harzburger Modell ist sehr schnell versandet. Besprechen wir mit jedem Direktor separat, ne, ja, ich nur mit ihm separat, und: `Hast du deine Berichte abgegeben?, und dies und jenes. Das war's dann schon."

(Langjährige Führungskraft der Anton Schlecker e.K.)

Die Interaktions- und Kommunikationsmuster verweisen auf die »Zwanghaftigkeit« der organisationalen Kultur. ${ }^{1099}$ Ausgehend von der Basisannahme einer bedrohlichen und chaotischen Umwelt, versuchte die dominante Koalition der Anton Schlecker e.K. die Komplexität der Welt durch eine perfektionierte Ordnung zu zähmen, in der beinahe sämtliche operativen Aufgaben und Verantwortlichkeiten über Jahrzehnte hinweg in der beständig gleichen Form vorbedacht und geregelt waren.

")Er konnte einem schon im März sagen, welche Termine er im Juni wahrnehmen würde`, erzählt ein Schlecker-Vorstand.»

(Aus einem Artikel des Handelsblatts im Jahr 2012) $)^{1100}$

Anton und Christa Schlecker folgten jede Woche dem stets gleichen Arbeitsprogramm, das neben der Kenntnisnahme der Berichte der Direktoren vor allem aus den von Donnerstag bis Samstag stattfindenden Kontrollfahrten zu Filialen bestand. Auf diesen Inspektionen wurde überprüft, ob die Regale korrekt eingeräumt waren und der Boden geputzt war. ${ }^{101}$ Durch eine solche Detailbesessenheit sollten Überraschungen vermieden und nichts dem Zufall überlassen werden. Die Programme und Routinen sollten helfen, die vermeintlich absolute Kontrolle über die zunehmende Komplexität des wachsenden Unternehmens zu wahren. $^{1102}$

Diese Grundannahmen und Einstellungen spiegelten sich in den Strukturen der Organisationen wider, denn die eigene Machtfülle verlieh den dominanten Koalitionen die Möglichkeit zur Gestaltung der formalen Strukturen entlang ihrer kulturellen Leitlinien.

1099 Vgl. Kets De Vries \& Miller (1986: 461); für eine zusammenfassende Übersicht über diesen und andere Kulturtypen siehe Schreyögg (2006: $459 \mathrm{ff}$.).

1100 S120602HB.

1101 Die Protagonisten der dominanten Koalition, Anton und Christa Schlecker, erreichten jeden Morgen um 06.45 über ihren privaten Aufzug die Zentrale. Es folgten die Berichte der Direktoren, die stets von gleicher Länge waren. Mittags um 12.30 Uhr Pause bis $14.00 \mathrm{Uhr}$, danach wieder Berichte bis zum Feierabend um 19.00 Uhr. Dieser Takt prägte den Arbeitsalltag von Montag bis Mittwoch. Donnerstagmittag begannen dann die Kontrollfahrten, die bis Samstagmittag dauerten. Vgl. S120602HB.

1102 Auch Kets De Vries \& Miller (1986) vermuten, dass solchen kulturellen Mustern häufig das unbewusste Leitmotiv der Unternehmensspitze nach einer Wahrung der Autonomie und Kontrolle zugrunde liegt. Vgl. Kets De Vries \& Miller (1986: 266). 


\section{Abschottende organisationale Strukturen}

Hinsichtlich der Abschottung durch organisationale Strukturen erweisen sich wieder die familiären Strukturmerkmale des hohen Zentralisierungsgrads und der geringen Formalisierung als bedeutsam. Diese Merkmalsausprägungen führten innerhalb der Organisation zu strukturellen Grenzen um die dominanten Koalitionen, die von den Irritationen der Umwelt und der restlichen Organisation nur noch schwer durchdrungen werden konnten.

Mit der hohen Zentralisierung waren vielstufige Kommunikationsprozesse verbunden. ${ }^{1103}$ Aufgrund dieser Vielstufigkeit wiederum gingen auf dem Weg zur organisationalen Spitze zwangsläufig viele wichtige Informationen verloren, bevor sie die dominanten Koalitionen erreichten. Dies liegt zum einen darin begründet, dass relevante Informationen nicht unverändert als "Pakete« über viele Stufen hinweg transportiert werden können, sondern vor dem Hintergrund individueller Perspektiven und Präferenzen in jedem kommunikativen Vorgang neu wahrgenommen und interpretiert werden. Mit jedem Zwischenschritt gehen Umdeutungen, aber auch Verluste der ursprünglichen Information einher. Zum anderen werden gerade in stark zentralisierten Strukturen mit konzentrierter Belohnungs- und Sanktionierungsmacht Informationen vom mittleren Management häufig bewusst zurückgehalten. Es werden dann nur Informationen weitergegeben, die den antizipierten Erwartungen der Entscheidungsträger entsprechen und dem eigenen Aufstieg in der Hierarchie nützlich erscheinen. ${ }^{1104}$ Wahrgenommene Warnsignale werden nicht oder nur äußerst diplomatisch übermittelt. Am Ende des Instanzenwegs erhalten die Entscheidungsträger an der Spitze der Organisation letztlich nur noch einen Bruchteil der in der Organisation vorhandenen Informationen.

Zugleich wird die Informationsübermittlung in zentralisierten Organisation wie der Anton Schlecker e.K. und Berentzen-Gruppe AG durch ressourcenbedingte und kognitive Grenzen erschwert. Einerseits wollten insbesondere die Protagonisten der Unternehmensfamilie über möglichst alle Vorgänge informiert sein und diese kontrollieren. Da die Entscheidungsträger aber andererseits jeweils nur eine begrenzte Menge der transportierten Informationen aufnehmen und verarbeiten können, bleiben weitere Informationen auf der Strecke. ${ }^{105}$

Aus diesen Gründen überwanden viele Irritationen der Umwelt die struktu-

1103 Vgl. Fredrickson (1986: 286); Mintzberg (1979).

1104 Vgl. Schreyögg (2006: 109ff.) zur Dualität von Differenzierung und Integration im Zuge der Strukturierung von Aufgaben sowie den mit den verschiedenen Lösungsansätzen verbundenen Vor- und Nachteilen.

1105 Vgl. zu dieser Grundannahme der verhaltenswissenschaftlich orientierten Managementtheorie Simon (1957). 
rellen Grenzen der dominanten Koalitionen auch dann nicht, wenn sie von anderen Subsystemen der Organisationen wahrgenommen worden waren. ${ }^{1106}$

In diesem Zusammenhang ist die geringe Formalisierung des strategischen Entscheidungsprozesses von Bedeutung. Aufgrund der geringen Formalisierung der strategischen Analyse der Umwelt und der Unternehmensentwicklung gab es sowohl bei der Anton Schlecker e.K. als auch bei der Berentzen-Gruppe AG nur vergleichsweise wenige vorstrukturierte kommunikative Plattformen und Kanäle, über die irritierende Informationen aus dem mittleren Management nach oben an die dominanten Koalitionen hätten übermittelt werden können. ${ }^{1107}$

\section{Immunität der dominanten Koalitionen}

Im Teil B wurde aus systemtheoretischer Perspektive erörtert, dass durch den Aufbau formaler und informaler Ordnung die Komplexität der Umwelt maßgeblich reduziert und für die Organisation überhaupt erst bearbeitbar gemacht wird. Allerdings wurde auch darauf aufmerksam gemacht, dass die Umstellung von der Komplexität der Welt auf den Modus der Systemkomplexität lediglich eine Problemverschiebung ist, denn Reduktion von Komplexität bedeutet zwangsläufig Vereinfachung. ${ }^{1108}$ Es werden nur noch bestimmte Teile der Welt wahrgenommen, nur noch bestimmte Fragen an die Welt gestellt, evtl. nur noch bestimmte Antworten zugelassen. Diese Selektion ist zwingend notwendig, sie erzeugt durch die Schaffung virtueller Sicherheiten aber zwangsläufig auch neue Unsicherheit. Dies gilt insbesondere für Kulturen und Strukturen, wie sie bei der Anton Schlecker e.K. und teilweise auch bei der Berentzen-Gruppe AG beobachtet werden konnten. Hier führten die formalen und informalen Ordnungen infolge der Abschottung und Selbstimmunisierung gegen die Komplexität und Irritationen der Umwelt zu übermäßig starken Selektionen und Vereinfachungen. ${ }^{1109}$

"Ein geschlossenes System. Was heißt das? Du nimmst, wenn du jetzt hier aus dem Fenster schaust, eine Million Eindrücke auf in deinem Gehirn, sag ich mal, macht 100 daraus oder zehn. Das heißt, die Umwelt ist viel, viel, viel komplexer und die wirkt auch

1106 Vgl. Bower (1970: 67); Fredrickson (1986: 285).

1107 Vgl. allgemein zu dieser Beobachtung Fredrickson (1986: 286). Auf der anderen Seite machen Cyert \& March (1963: $101 \mathrm{ff}$.) darauf aufmerksam, dass ein sehr hoher Grad an Formalisierung und »standard operating procedures« des Umweltscreenings dazu führt, dass verstärkt in bereits bekannten Gebieten nach Umweltveränderungen gesucht wird und vor allem auf Ansätze zurückgegriffen wird, die bereits erfolgreich verwendet wurden.

1108 Vgl. Steinmann \& Schreyögg (2005: 140ff.); Luhmann (1984: 141); Martens \& Ortmann (2006: 427).

1109 Vgl. Kets De Vries \& Miller (1986: 275f.); Schreyögg (2006: 477). Die Titulierung von Systemen als "geschlossen« ist dabei als Beschreibung von Phänomenen zu verstehen und keineswegs mit der theoretisierten Funktionsweise autopoietischer sozialer Systeme bei Luhmann (1984) gleichzusetzen. 
so. Und mit zunehmendem Alter sind immer noch eine Million da, aber aus den 100 werden 50, 30, 20, eins, zwei gemacht. Das heißt, du wirst ignorant, auf Deutsch. Du wirst zu einem geschlossenen System. Und jetzt mal dieses System Schlecker war die ganze Umwelt von Schlecker.»

(Ehemaliger Berater der Anton Schlecker e.K.)

Die exkludierenden kulturellen und strukturellen Muster trugen so zur Immunität der dominanten Koalitionen bei und verringerten maßgeblich die Irritationsfähigkeit deren Entscheidungsprozesse. In der Konsequenz dieser exkludierenden kulturellen und strukturellen Muster wurden relevante Umweltveränderungen und »weak signals« von den Entscheidungsträgern nicht mehr wahrgenommen. ${ }^{110}$

"Die Wahrnehmung aber war bei Schlecker gestört, und dies nicht nur beim Chef. ^Ich fand das immer erstaunlich, wie sehr die Leute von Schlecker in ihrer Scheinwelt gefangen waren`, sagt ein dm-Manager. `Wenn eine Führungskraft von Schlecker zu uns wechselte, fragten wir ihn immer, was ihn denn hier am meisten überrascht hat. Und alle antworteten: Dass ihr so billig seid!«

(Ausschnitt aus einem Artikel des Handelsblatts im Jahr 2012) ${ }^{1111}$

Bei der Anton Schlecker e.K. wurden noch im Endstadium der Erfolgskrise, als das Scheitern des Geschäftsmodells längst offen zutage getreten war, kaum bestreitbare Tatsachen geleugnet. ${ }^{1112}$

"Das Image von Schlecker sei nicht im Geringsten beschädigt, sagte Anton Schlecker 2010 in einem seiner seltenen Interviews. Da war Anton Schlecker längst Deutschlands meistgehasster Unternehmer."

(Der Spiegel im Jahr 2012) ${ }^{1113}$

Die Möglichkeiten exogener Kräfte, Druck auszuüben, war somit nicht nur geringer; zusätzlich wurde vorhandener Druck aufgrund der exkludierenden kulturellen und strukturellen Muster gar nicht in seiner vollen Stärke wahrgenommen. Diese beiden Formen der Immunität können somit als erste Antworten auf die Frage dienen, warum die dominanten Koalitionen in der Phase

1110 Vgl. Klein (2010: 280), die die Bedeutung der Offenheit für von innen wie außen angestoßene Veränderungen betont. Die Auswirkungen der exkludierenden Tendenzen entsprechen den Beobachtungen von Kets de Vries (1993: 67). Die Forschung zu Unternehmenskrisen beschreibt, dass eine solche Abwendung von der Umwelt in vielen Unternehmen in der Strategiekrise vorzufinden ist. Vgl. Weitzel \& Jonsson (1989: 98).

1111 S120602HB.

1112 Ehemalige Führungskraft: "Als der Wettbewerb aufkam, wurde der lange Zeit einfach nicht wahrgenommen. Wurde auch nicht wahrgenommen, dass die Kunden eben - es geht nicht mehr nur um Produktpreis, sondern sich brauch - Es geht auch um Marke, es geht um ein Einkaufsgefühl und es geht darum, dass, ja, wie soll ich sagen, dass man mit viel Sympathie auf den Kunden zugeht ...«.

1113 S120718SP. 
der Pfadformation nicht entscheidend auf die Genese der Pfade reagierten obwohl sie noch die Macht dazu gehabt hätten. Ist es doch eben genau der in den beiden Unternehmen schwach ausgeprägt oder nicht in seiner ganzen Dimension wahrgenommen Druck der Umwelt, der in Unternehmen häufig zum strategischen Wandel führt. ${ }^{114}$

Dennoch waren die dominanten Koalitionen natürlich nicht vollkommen isoliert von ihrer Umwelt. Trotz der beschriebenen ausgeprägten Immunität drangen immer noch zahlreiche Irritationen zu ihnen durch. In der Bearbeitung dieser Irritationen waren die dominanten Koalitionen der Anton Schlecker e.K. und der Berentzen-Gruppe AG aufgrund ihrer hohen Autonomie und Immunität allerdings weitgehend auf ihre endogenen Potenziale und Dynamiken zurückgeworfen.

\subsection{Die Bedeutung von Diversität und Konflikt für die ausbleibende strategische Reaktion}

Im obigen Unterkapitel wurde dargestellt, wie die Machtkonzentration die strategischen Entscheidungsträger der dominanten Koalitionen gegen exogene Einflüsse immunisierte und wie kulturelle und strukturelle Muster diese Immunität erhöhten. Die Immunität verringerte direkt die Rezeptivität der dominanten Koalitionen für Irritationen der Umwelt und erklärt die ausbleibende strategische Reaktion in der Phase der Pfadformation zum Teil. Vor allem führte die Immunität der Entscheidungsträger aber dazu, dass die dominanten Koalitionen der Unternehmen in der Steuerung und Kontrolle ihrer Entscheidungsprozesse weitestgehend auf sich selbst zurückgeworfen waren. Es lag in sehr ausgeprägter Form an ihnen, die Dynamiken der Umwelt und Gefahren der Unternehmensentwicklung $\mathrm{zu}$ identifizieren und entsprechende strategische Reaktionen einzuleiten. ${ }^{1115}$

$\mathrm{Ob}$ ihrer Immunität waren die dominanten Koalitionen in der Bearbeitung dieser komplexen und mehrdeutigen strategischen Aufgaben jedoch stark von der Leistungsfähigkeit der eigenen Entscheidungsprozesse abhängig. ${ }^{116}$ In diesem Zusammenhang zeigt insbesondere die kontrastierende Analyse mit den

1114 Vgl. Hambrick \& D’Aveni (1988: 17); Tushman \& Romanelli (1985: 214).

1115 Zur Immunität der dominanten Koalition bei der Anton Schlecker e.K. sagt eine ehemalige Führungskraft: »Ich meine, man kann sich nicht verändern, wenn man selber nicht die Bereitschaft zu hat. [...] Es gibt niemanden, der da ansetzen könnte.« Vgl. zu diesen Aufgaben des strategischen Entscheidungsprozesses Dutton \& Duncan (1987); Mintzberg (1979) und zur Bedeutung der dominanten Koalition Carpenter et al. (2004: 750).

1116 Vgl. Amason (1996: 123); Wiersema \& Bantel (1992: 92) zur Komplexität der Aufgaben der Entscheidungsträger im strategischen Entscheidungsprozess. 
sich strategisch wandelnden Vergleichsfällen, dass die Qualität der Entscheidungsprozesse in der Phase der Pfadformation von zwei fundamentalen Einflussgrößen abhängig war, und zwar zum einen von den kognitiven Potenzialen der dominanten Koalitionen:

"Und in der Zeit ist mir das eigentlich im Nachhinein doppelt klar geworden, dass alles hat uns geführt. Das haben wir damals geahnt und vermutet, dass es so, so nicht weitergeht, weil die Komplexität ist einfach zu groß. Auch wir waren überfordert. Ja [...] sie müssen ein ganz anderes Management-Potenzial mitbringen. Und das war alles nicht vorhanden."

(Ehemalige Führungskraft der Anton Schlecker e.K.) ${ }^{1117}$

Zum anderen waren die Interaktions- und Kommunikationsprozesse der dominanten Koalitionen eine wichtige Einflussgröße, denn die Interaktionen und Kommunikationen bestimmen, inwieweit die vorhandenen kognitiven Potenziale für die strategischen Entscheidungsprozesse auch fruchtbar gemacht werden können. ${ }^{1118}$

"Und wir haben dann mehr in den Aufsichtsratssitzungen und Gesprächen haben die sich mehr um sich selbst gedreht, möchte ich mal sagen. Sie haben sich gegenseitig beschuldigt, die haben sich gegenseitig Vorwürfe gemacht. Dann haben sie sich wieder verteidigt. Die haben sich belauert, nicht, ich weiß nicht, wie ich das sonst ausdrücken soll, die, die, die haben auf einem Feld miteinander gerungen, was nicht den Kopf frei machte, für strategische Überlegungen.[...] das ist natürlich eine Atmosphäre, die nicht unbedingt geeignet ist, sagen wir mal eine, eine vernünftige strategische Diskussion zuzulassen, mal in Ruhe in die Vergangenheit zu schauen, wo kommen wir her und mal in Ruhe in die Zukunft zu schauen, wo gehen wir hin, mit welchen Maßnahmen steuern wir in die richtige Richtung? [...] So, so wurde da nicht mehr gesprochen [...] Man hatte das Gefühl, die haben sich mit der Sache überhaupt nicht beschäftigt, rein geschäftlich. Es ging nur um Macht, Einfluss [...] und das ist dann der Boden, wo so was dann schief geht, ne. Ganz eindeutig."

(Ehemaliges Vorstandsmitglied der Berentzen-Gruppe AG)

1117 Eine ehemalige Schlecker-Führungskraft: »Also diese Modelle waren da. Denen waren sie auch aufgeschlossen. Aber es wurde nie umgesetzt [...], vielleicht, weil es zu kompliziert war.» Vgl. auch die Aussage des Insolvenzverwalters in S120604LZ: Das alte SchleckerManagement sei »überfordert gewesen [...], wir haben hier Defizite gehabt in der Führungsqualität."

1118 Diese beiden Antezedenzien der Entscheidungsqualität sind in der Forschung zu strategischen Entscheidungsprozessen fest etabliert. Die Forschung zu strategischen Entscheidungsprozessen versteht die Ergebnisse strategischer Entscheidungsprozesse schon lange primär als Funktion des Zusammenwirkens der in den Prozess beteiligten Akteure. Vgl. Amason (1996: 124): "One stream of this research, focusing on the quality of the decisions themselves, has identified two principal antecedents of decision quality: the cognitive capabilities of a top management team and the interaction process through which the team produces its decisions.« Vgl. auch Eisenhardt et al. (1997); Pelled et al. (1999); Simons et al. (1999). 
In den folgenden Abschnitten sollen die beobachteten empirischen Ausprägungen dieser beiden Einflussgrößen skizziert werden, bevor die Auswirkungen auf den strategischen Entscheidungsprozess dargestellt werden. Anschließend wird geprüft, inwiefern familiäre Einflüsse für diese Ausprägungen und Wirkungszusammenhänge von Bedeutung waren.

Hinsichtlich der ersten Einflussgröße des strategischen Entscheidungsprozesses, des kognitiven Potenzials der dominanten Koalitionen, sind allerdings zunächst noch einige kurze theoretische Vorüberlegungen anzustellen.

Gruppen sind in der Bearbeitung der Umwelt von der jeweiligen kognitiven Basis der einzelnen Gruppenmitglieder abhängig, da Gruppen als solche nicht wahrnehmen oder interpretieren können. ${ }^{1119}$ Somit werden die kollektiven Entscheidungsprozesse in mehrerlei Hinsicht von den individuellen Werten und insbesondere der jeweiligen kognitiven Basis der Entscheidungsträger beeinflusst. Zunächst limitiert die kognitive Basis das Blickfeld der einzelnen Entscheidungsträger und somit auch die Bereiche der Umwelt, in denen die Gruppe nach Möglichkeiten und Risiken für die Entwicklung des Unternehmens sucht. Des Weiteren bedingt sie im Zuge der stets selektiven Wahrnehmung, welchen Stimuli und Irritationen innerhalb dieses eingeschränkten Blickfelds Aufmerksamkeit geschenkt wird. Des Weiteren prägen die kognitiven Filter, wie die wahrgenommenen Irritationen der Umwelt interpretiert werden.

Auf diesem Wege bestimmt die jeweilige kognitive Basis der einzelnen Gruppenmitglieder das gesamte kognitive Potenzial der Gruppe und somit den strategischen Entscheidungsprozess, denn was individuell nicht wahrgenommen und prozessiert wird, kann auch in der Gruppe nicht dem kollektiven Entscheidungsprozess zugeführt werden. ${ }^{120}$

Eine nähere Einschätzung der kognitiven Potenziale der untersuchten dominanten Koalitionen entzog sich natürlich dem Zugang dieser rekonstruierenden sozialwissenschaftlichen Arbeit. Da sie zur Erklärung der ausbleibenden strategischen Reaktion aber von Bedeutung sind, wurden für die Analyse der strategischen Entscheidungsprozesse die Annahmen des Upper-EchelonAnsatzes nach Hambrick \& Mason (1984) herangezogen. ${ }^{1121}$ Gemäß diesem Ansatz der Managementforschung wird dem schwierigen Zugang zu den kognitiven Potenzialen der Entscheidungsträger begegnet, indem beobachtbare demografische Charakteristika wie das Alter der Entscheidungsträger oder

1119 Auf diesen Umstand macht gerade die neuere Systemtheorie mit ihrer Unterscheidung in psychische und soziale Systeme aufmerksam; vgl. Luhmann (1984: 16f.). Unter der kognitiven Basis können mit Hambrick \& Mason (1984: 195) die Annahmen über zukünftige Ereignisse, die Kenntnis von Alternativen und das Wissen um die damit verbundenen Konsequenzen verstanden werden. Vgl. auch Wiersema \& Bantel (1992: 94).

1120 Vgl. Amason (1996: 124); Hambrick \& Mason (1984: 202); Wiersema \& Bantel (1992: 94).

1121 Vgl. Carpenter et al. (2004: 750); Hambrick \& Mason (1984); auch Bresser (2010: 36ff.). 
deren funktionale Spezialisierung als Indikatoren der dahinterliegenden kognitiven Basis einbezogen werden. Der Upper-Echelon-Ansatz knüpft hinsichtlich dieses Schlusses an die Erkenntnisse der verhaltenswissenschaftlichen Entscheidungstheorie an. Es wird davon ausgegangen, dass die kognitive Basis der Entscheidungsträger maßgeblich von deren persönlichen Werdegängen und Erfahrungen geprägt wird, ${ }^{1122}$ denn aus diesen Erfahrungen resultieren im Verlauf der Zeit bestimmte Perspektiven auf die Umwelt oder Einstellungen, die die Wahrnehmung und Interpretation beeinflussen. Da sich diese Erfahrungen in den beobachtbaren Merkmalen des Alters oder der Ausbildung widerspiegeln, sei es zulässig, von einem bestimmten beobachtbaren Merkmal der Entscheidungsträger auf eine bestimmte Umweltperspektive und entsprechende strategische Wahlentscheidungen zu schließen. ${ }^{1123}$

Auf der Grundlage dieser theoretischen Annahmen wurden in der Analyse der Entscheidungsprozesse in der Phase der Pfadformation demografische Charakteristika der Entscheidungsträger als Indikatoren einer bestimmten Perspektive auf den strategischen Wandel genutzt. ${ }^{124}$ Im Zuge der Analyse trat jedoch weniger die Bedeutung einzelner Merkmalsausprägungen hervor. Vielmehr erwies sich vor allem von Belang, inwiefern sich die Mitglieder der dominanten Koalitionen hinsichtlich dieser demografischen Attribute unterscheiden. Dieser Grad an Abweichung, welcher das Maß an Heterogenität beziehungsweise Homogenität der dominanten Koalitionen adressiert, soll im Folgenden unter dem Begriff der Diversität behandelt werden. ${ }^{1125}$

In Bezug auf die zweite bedeutsame Einflussgröße im strategischen Entscheidungsprozess, den Prozess der Interaktion und Kommunikation der dominanten Koalition, erwies sich vor allem das Niveau an Konflikt als bedeutsam für die Qualität der Bearbeitung der strategischen Aufgaben und das Ausbleiben

1122 Vgl. zur verhaltenswissenschaftlichen Entscheidungstheorie Cyert \& March (1963); March \& Simon (1958). So gehen Hambrick \& Mason (1984: 124) beispielsweise davon aus, dass eine langfristige Tätigkeit in einem bestimmten Funktionsbereich dazu führt, dass Probleme vor dem Hintergrund mentaler Schablonen, die man sich angeeignet hat, definiert werden und in dieser Richtung auch Lösungen gesucht werden.

1123 Zum Beispiel zwischen der Dauer der Unternehmenszugehörigkeit und dem Streben nach der Bewahrung des Status quo, vgl. Miller (1991). Die UE-Perspektive ist damit sowohl Theorie wie auch Methode. Obwohl solche Zusammenhänge von der Forschung quantitativ in großen Stichproben nachgeprüft wurden, können sie auch deduktiv zur Stützung von Einzelbeobachtungen herangezogen werden, wenn in den Daten selbst entsprechende Indizien vorliegen. Vgl. Bresser (2010: 38); Carpenter et al. (2004) zur Validität dieser Charakteristika als Indikatoren strategischer Handlungen.

1124 Der verstärkte Einsatz von qualitativen Methoden wird in der entsprechenden Forschung seit Längerem gefordert, aber noch selten praktiziert. Vgl. Priem et al. (1999: 939); Nielsen (2010: 308f.).

1125 Vgl. zu diesem definitorischen Verständnis Pelled et al. (1999: 1); Wiersema \& Bantel (1992: 91). 
der strategischen Reaktion. Der Grad an Sachkonflikt und Beziehungskonflikt bestimmte maßgeblich, inwieweit die kognitiven Potenziale der dominanten Koalition für den Entscheidungsprozess fruchtbar gemacht werden konnten. ${ }^{1126}$

Im Folgenden sollen die Ausprägungen und Wirkungszusammenhänge der beiden Variablen Diversität und Konflikt näher erörtert werden. Dies erfolgt getrennt für die beiden Fallgruppen der monozentrischen und polyzentrischen Konfiguration, denn die empirische Studie zeigt, dass die Verteilung der Macht innerhalb der dominanten Koalitionen, welche vor allem aus dem Imprint der Eigentumsstruktur erwuchs, den Wirkungszusammenhang der Variablen Diversität und Konflikt in den untersuchten Fällen maßgeblich beeinflusste. Den Ausprägungen von Diversität und Konflikt kam ihre Bedeutung für das Ausbleiben der Reaktion auf die Genese der strategischen Pfade vor allem im gegebenen Rahmen der jeweiligen Machtverteilungen zu.

\subsubsection{Diversität und Konflikt in der monozentrischen Konfiguration der Macht}

Sowohl bei der Anton Schlecker e.K. als auch bei der Dirk Rossmann GmbH war die Macht innerhalb der weitgehend autonomen dominanten Koalitionen stark asymmetrisch verteilt. Mit Anton Schlecker und Dirk Roßmann vereinigten zwei Akteure einen Großteil der Machtmittel auf sich und konnten so die Entscheidungsprozesse innerhalb der dominanten Koalitionen potenziell dominieren.

Da aufgrund dieser Machtasymmetrie von einer gewissen Kongruenz zwischen Elementen der Persönlichkeiten und den Strukturen und Strategien der Unternehmen ausgegangen werden kann, ließe sich das Festhalten an der sich selbst verstärkenden Wachstumsspirale in der Phase der Pfadformation auch durch Persönlichkeitsmerkmale des dominanten Akteurs erklären. ${ }^{127}$ Formen

1126 Vgl. zu dieser etablierten Unterscheidung von Sachkonflikt und Beziehungskonflikt Jehn \& Bendersky (2003); Pelled et al. (1999: 2). Dieses Begriffspaar findet sich auch auch unter "cognitive« und "affective conflict« oder »substantive« und »interpersonal conflict». Vgl. Amason (1996); Eisenhardt et al. (1997). Nach Ansicht der meisten Autoren sind die Definitionen jedoch weitgehend deckungsgleich. Unter Sachkonflikt werden jene Konflikte subsumiert, die auf unterschiedlichen Ansichten zu Sachaufgaben, Zielen, Prozeduren oder auch strategischen Alternativen beruhen. In Beziehungskonflikten geht es um interpersonale Auseinandersetzungen, die Fragen von Status oder auch Sympathie betreffen.

1127 So zeigen beispielsweise Miller et al. (1982) in Anknüpfung an Rotter (1966), dass die Kontrollüberzeugung in einem direkten Zusammenhang mit der praktizierten Corporate Strategy steht. So würden eher extern kontrollbewusste Menschen tendenziell konservativere Strategien verfolgen, monolithische und abgeschlossene Strukturen aufbauen und häufiger in sehr stabilen Umwelten operieren. Die Richtung der Kausalwirkung ist dabei häufig allerdings nicht vollends klar. Vgl. ähnlich auch Kets De Vries \& Miller (1986), die die Bedeutung der Machtkonzentration für ihre Ergebnisse betonen. 
individueller Rigidität dürften ebenfalls zur Genese und Verfestigung des strategischen Pfades in der Phase der Pfadformation beigetragen haben.

Doch greift die Analyse, schränkt man sie auf die Persönlichkeitsmerkmale und Einstellungen des Entscheidungsträgers Anton Schlecker ein, für das Verständnis der Pfadformation zu kurz. ${ }^{1128}$ Selbst wenn die letztgültige Entscheidungsgewalt bei der Anton Schlecker e.K. juristisch gesehen meist bei einem einzelnen Akteur lag, entsprangen die Entscheidungen nicht vollständig autarken und individuellen Prozessen. Am vorgeschalteten Entscheidungsprozess waren auch bei der Anton Schlecker e.K. mehrere Akteure beteiligt, deren Wahrnehmungen, Interpretationen und Kommunikationen in den strategischen Entscheidungsprozess einflossen und diesen mitprägten.

Gerade im Rahmen der monozentrischen Konfigurationen der Anton Schlecker e.K. und der Dirk Rossmann GmbH kam den der schlussendlichen Entscheidung vorgeschalteten Aspekten eine besondere Bedeutung zu. Wie alle Organisationsmitglieder waren auch die dominanten Akteure geprägt von ihren persönlichen Erfahrungen und den sich hieraus entwickelten Einstellungen und Interpretationsmustern. ${ }^{1129}$ Im Gegensatz zu allen anderen Entscheidungsträgern konnten sie wegen ihrer Machtfülle im Wettbewerb alternativer Entscheidungsmöglichkeiten aber den eigenen Vorstellungen potenziell auch stets zur Durchsetzung verhelfen. Um dem Gefährdungspotenzial zu begegnen, das langfristig aus solchen autarken Einzelentscheidungen für die strategische Entwicklung der Unternehmen erwachsen kann, galt es daher insbesondere in diesen monozentrischen Konfigurationen die strategischen Entscheidungsprozesse ausreichend zu irritieren. ${ }^{1130}$

Für diese Irritation erweisen sich in der kontrastierenden Analyse zwischen der Anton Schlecker e.K. und der Dirk Rossmann GmbH zunächst die Zusammensetzungen der Gruppen bedeutsam, die sich um den machtvollen Protagonisten zur dominanten Koalition gruppierten.

\subsubsection{Homogenität der dominanten Koalition}

Die Managementforschung macht bereits seit einigen Jahren auf die Bedeutung der demografischen Zusammensetzung von Topmanagement-Teams für

1128 Vgl. Miller et al. (1982: 267). Die Ergebnisse zum Zusammenhang von Zusammensetzungen von Gruppen und strategischen Ergebnissen haben auch eine deutlich höhere Validität.

1129 Vgl. Hambrick \& Fukutomi (1991); auch Finkelstein et al. (2009: 85); Miller (1991). Dies führt nicht zuletzt dazu, dass solch dominante Akteure vor allem auf jene Praktiken vertrauen, die ihnen in der Vergangenheit zum Erfolg verholfen haben.

1130 Vgl. Simon (1957); Cyert \& March (1963). Für eine Übersicht zu empirischen Studien zu Rationalität und deren Begrenzungen im Kontext strategischer Entscheidungsprozesse vgl. Eisenhardt \& Zbaracki (1992). Zu den Nachteilen von Einzelentscheidungen vgl. Schweiger et al. (1986). 
die Qualität der Entscheidungsprozesse und die strategische Wandlungsfähigkeit aufmerksam. Hierbei verweist sie auf die Bedeutung des Alters der Entscheidungsträger, deren funktionale Spezialisierung und das Ausbildungsniveau. Des Weiteren fokussiert sie die Dauer der Unternehmenszugehörigkeit der Entscheidungsträger und die Kontinuität der Gruppenzusammensetzung. ${ }^{1131}$

\section{Empirische Ausprägungen}

Hinsichtlich der meisten dieser Dimensionen lassen sich bei der Anton Schlecker e.K. eindeutige Merkmalsausprägungen und somit eine in vielerlei Hinsicht sehr hohe Homogenität der dominanten Koalition feststellen.

»Und das war zum Beispiel auch nicht vermittelbar, dass in diese Länder, in dieser Länderführung die jeweiligen Ausländer reingehören. Und nicht Deutsche. Es waren immer nur Deutsche. Die hatten da das Sagen. Aber keiner konnte die Sprache noch nicht mal.»

(Ehemalige Führungskraft der Anton Schlecker e.K.)

Die Entscheidungsträger der dominanten Koalition gehörten über viele Jahrzehnte zumeist der gleichen Alterskohorte an. Hieraus resultierte nicht nur eine sehr homogene Altersstruktur, sondern im Verlauf der Zeit auch ein recht hohes Durchschnittsalter. Der 81-jährige Generaldirektor Reinhold Freudenreich kann hier wieder als Beleg dienen. ${ }^{1132}$

Dieses Beispiel verweist aber zugleich auch auf die sehr lange Unternehmenszugehörigkeit der meisten Entscheidungsträger der dominanten Koalition. In der kritischen Phase der Pfadformation Ende der 1990er-Jahre, als der Wirkkraft der selbstverstärkenden Mechanismen noch hätte begegnet werden können, war Anton Schlecker bereits 35 Jahre im Unternehmen tätig, viele seiner engsten Mitarbeiter kaum weniger lang oder wie Reinhold Freudenreich gar länger.

"Das waren jetzt schon Leute, die konnten sie gar nicht mehr setzen, weil, das waren dann auch die Leute der ersten Stunde. Die waren also noch zehn Jahre länger da als ich. Die haben noch die ersten Filialen mit eingeräumt."

(Ehemalige Führungskraft der Anton Schlecker e.K.)

Hinsichtlich der funktionalen Spezialisierung der Mitglieder der dominanten Koalition zeigt sich wiederum die historisch bedingte starke Fokussierung des Unternehmens auf den Funktionsbereich des Einkaufs. Selbstverständlich waren

1131 Vgl. für eine Übersicht zu solchen Studien Finkelstein et al. (2009); Jehn \& Bendersky (2003); Wiersema \& Bantel (1992).

1132 S120602HB: "Die Verhandlungen mit Goldman Sachs führte nicht Anton Schlecker, sondern Reinhold Freudenreich, ein knapp 81-jähriger Schlecker-Pensionär. Er ist seit Urzeiten dabei, er arbeitete schon unter Vater Schlecker und gilt im Konzern als die graue Eminenz.» 
im Topmanagement alle Funktionsbereiche vertreten, doch die einflussreichsten Akteure wie Anton Schlecker, der Generaldirektor Freudenreich oder der spätere CEO Rusch waren alle eher vom Bereich des Einkaufs geprägt. Dieser Funktionsbereich war auch personell in quantitativer wie qualitativer Hinsicht am besten ausgestattet. ${ }^{1133}$

Eine deutliche Merkmalsausprägung ist in Bezug auf das formale Qualifikationsniveau der Ausbildung zu konstatieren: Es lässt sich innerhalb der dominanten Koalition ein sehr niedriger Anteil an Akademikern registrieren. ${ }^{1134}$ Auch die Protagonisten der dominanten Koalition hatten nicht studiert, sondern eine Ausbildung absolviert: Anton Schlecker zum Metzgermeister, seine Frau Christa zur Fremdsprachensekretärin.

"Also Sie müssen sich vorstellen, wenn Sie da als ich sag mal als Akademiker da bei uns starten, dann waren Sie ein Exot. Ich glaub, als ich damals anfing, waren zwei oder drei Volkswirte oder so da. Das war's dann."

(Ehemalige Führungskraft der Anton Schlecker e.K.)

Aufgrund dieser recht einseitigen Ausprägungen bedeutender demografischer Charakteristika lässt sich für die dominante Koalition der Anton Schlecker e.K. ein sehr niedriger Grad an Diversität konstatieren.

Im Vergleich hierzu zeichnete sich die dominante Koalition der Dirk Rossmann GmbH durch eine höhere Diversität aus. So besaß der Funktionsbereich des Vertriebs in der dominanten Koalition einen ebenso hohen Stellenwert wie der Einkauf und war entsprechend organisational verankert. ${ }^{135}$ Das Niveau der formalen Qualifikation war ebenfalls recht unterschiedlich. Dirk Roßmann hatte nicht studiert, sondern eine Lehre zum Drogisten absolviert. Neben ihm waren in der dominanten Koalition des Unternehmens aber auch zahlreiche Akademiker tätig. Hinsichtlich der Dimensionen des Alters, der Dauer der Unternehmenszugehörigkeit und der Zusammenarbeit innerhalb der dominanten Koalition sind auch bei der Dirk Rossmann GmbH relativ eindeutige Merkmalsausprägungen feststellen, denn auch in diesem Unternehmen hatte in den Jahren des Wachstums fast zwei Jahrzehnte eine dominante Koalition in der gleichen personellen Konstellation zusammengearbeitet.

Zusammengenommen lässt sich für die Dirk Rossmann GmbH aber dennoch

1133 Eine ehemalige Führungskraft: »Die haben zum Beispiel auch über viele Jahre den Einkauf zum Beispiel personell sehr verstärkt. Mit wirklich qualifizierten Leuten.« Im Gegensatz hierzu ein ehemaliger Berater zu den Funktionsbereichen Vertrieb und Marketing: »[...] und wir haben intern das Unternehmen angeschaut, alle Funktionsbereiche: Vertrieb, Marketing. Und das war, mal salopp gesagt, das Unternehmen war marode bis zum Anschlag. Also, alles, was dort an Professionalität - die gab's nicht."

1134 Vgl. S080325MM: »Er fördert seine Leute, die ganz selten Akademiker sind.«

1135 Hierzu ein ehemaliges Mitglied der dominanten Koalition bei Rossmann: "Also wir, wir sind, hier haben wir die, immer die Balance gehabt zwischen Einkauf und Vertrieb." 
ein im Vergleich zur Anton Schlecker e.K. deutlich höherer Grad an Diversität konstatieren.

\section{Auswirkungen der geringen Diversität bei der Anton Schlecker e.K.}

Die eindeutigen Merkmalsausprägungen und die vergleichsweise geringe Diversität der dominanten Koalition der Anton Schlecker e.K. blieben nicht ohne Auswirkungen auf deren strategische Entscheidungsprozesse und die Fähigkeit zur Reaktion auf die sich verändernde Umwelt. ${ }^{136}$

So dürfte das zunehmende Alter der dominanten Koalition zur abnehmenden Bereitschaft für eine entschiedene Reaktion auf den emergierenden Pfad beigetragen haben. ${ }^{1137}$ Denn zum einen gehen mit zunehmendem Alter im Allgemeinen nicht nur abnehmende kognitive Fähigkeiten, sondern auch ein größeres Commitment zum Status quo einher. Darüber hinaus wird davon ausgegangen, dass Entscheidungsträger mit zunehmendem Alter in steigendem Maße sowohl materielle als auch sozio-emotionale Sicherheit wertschätzen. Sie sind daher in der Regel riskanten Entscheidungen wie einem grundlegenden strategischen Wandel mehr und mehr abgeneigt. ${ }^{1138}$

Parallel zum steigenden Alter der dominanten Koalition dürfte die zunehmende Verweildauer der Entscheidungsträger im Unternehmen dazu beigetragen haben, dass in der Phase der Pfadformation keine Abkehr vom etablierten strategischen Muster erfolgte.

»Also das sind seine strategischen Innovationen gewesen. Unstrittig. Da gibt's auch nicht, wo andere sagen `Das war meine Idee` oder so. Das ist seine Sache und dann hat das mit der Zeit aber aufgehört. Dann hat er solche Sachen, meines Erachtens, nicht mehr gebracht. Je länger das lief, wirklich nur noch gedacht `Multipliziere und der Erfolg ist gigantisch`.»

(Ehemalige Führungskraft der Anton Schlecker e.K.)

Diese empirischen Indizien werden von der entsprechenden Managementforschung gestützt. So merken Hambrick \& Fukutomi (1991) an, dass bereits nach

1136 Diese Auswirkungen wurden unmittelbar aus den empirischen Daten abgeleitet, werden im Folgenden aber zur Stützung der Zusammenhänge zusätzlich durch Beiträge der bisherigen Forschung zur Zusammensetzung von TMT untermauert. Vgl. Finkelstein et al. (2009); Wiersema \& Bantel (1992).

1137 Vgl. Hambrick \& Mason (1984: 198). So beobachtet Child (1974), dass organisationales Wachstum mit der Jugend des Managements verbunden ist; vgl. auch Hitt \& Tyler (1991). Für die Forschung zu Familienunternehmen beobachteten Hall et al. (2001), wie ehemals hochinnovative Unternehmer im Verlauf der Unternehmensentwicklung ihre »entrepreneurial capacity « verloren.

1138 Vgl. Hambrick \& Mason (1984: 198). Zu den kognitiven Fähigkeiten vgl. Child (1974: 181f.); zum Sicherheitsbedürfnis Carlsson \& Karlsson (1970); Vroom \& Pahl (1971) und spezifisch zum Eingehen von unternehmerischen Risiken in Familienunternehmen Zahra (2005). 
nur fünf Jahren der Unternehmenszugehörigkeit das Verhalten der meisten Entscheidungsträger immer stärker von etablierten Paradigmen bestimmt wird, die auf impliziten mentalen Modellen beruhen, ${ }^{1139}$ insbesondere dann, wenn sich dieses unbewusste Paradigma bislang als erfolgreich erwiesen hat. ${ }^{1140}$

Zudem nehmen gemäß vielen Beiträgen mit zunehmender Verweildauer im Unternehmen sowohl die Menge als auch die Qualität an Irritationen ab. ${ }^{141} \mathrm{Je}$ länger die Entscheidungsträger im Unternehmen seien, desto stärker orientierten sie sich an bewährten und »verlässlichen« Quellen. Zudem würden die hinzugezogenen Informationen immer stärker durch die zunehmend akzentuierte kognitive Basis gefiltert. Dies trage, ebenso wie das erarbeitete große operative Sachwissen im bestehenden Gefüge, dazu bei, dass die Entscheidungsträger mit zunehmender Verweildauer im Unternehmen immer seltener vom etablierten strategischen Muster abweichen. ${ }^{1142}$

Hinsichtlich der funktionalen Spezialisierung wirkte sich vor allem die Einkaufsorientierung der dominanten Koalition auf den strategischen Entscheidungsprozess aus. Die jahrzehntelangen beruflichen Erfahrungen ihrer Protagonisten in diesem Bereich hatten zu entsprechend geprägten Perspektiven auf die Umwelt und die Entwicklung des Unternehmens geführt und so wurde strategischen Herausforderungen wie dem zunehmenden Preiskampf in der Branche meist über taktische Maßnahmen im Einkauf begegnet. ${ }^{1143}$ Diese Einkaufsorientierung hemmte die Abkehr vom lange Zeit erfolgreichen, über die Einkaufskonditionen getriebenen selbstverstärkenden Mechanismus. Auf der anderen Seite erschwerte sie aber auch die Entwicklung eines neuen strategischen Musters. ${ }^{1144}$

1139 Vgl. Hambrick \& Fukutomi (1991) zu einem idealtypischen Verlauf der Schaffenskraft von Entscheidungsträgern. Vgl. auch Finkelstein et al. (2009: 85): »Research and theory on executive tenure has clustered generally around one major idea: long-tenured executives tend not to make major changes in their organizations. In fact, there is considerable evidence of this important phenomenon."

1140 Vgl. Miller (1991) zum Verhältnis von Unternehmenszugehörigkeit und zunehmendem Selbstbewusstsein.

1141 Vgl. Finkelstein et al. (2009: 87); Miller (1991).

1142 Hambrick \& Fukutomi (1991). Miller (1991) spricht vom Phänomen, dass die Entscheidungsträger mit zunehmender Verweildauer im Unternehmen »stale in the saddle« würden.

1143 Vgl. grundlegend Dearborn \& Simon (1958); auch Eisenhardt et al. (1997: 48); Finkelstein et al. (2009: 97). Als Mechanismen für eine solche Prägung führen Finkelstein \& Hambrick (1990: 487) zum Beispiel die Sozialisation im Funktionsbereich oder vergangene Lernerfahrungen an.

1144 Vgl. Chaganti \& Sambharya (1987) und Thomas et al. (1991), denen zufolge Unternehmen strategisch eher als »defender« agieren, wenn der Anteil von Entscheidungsträgern aus durchsatzorientierten Funktionen wie Produktion, Einkauf oder Rechnungswesen hoch ist. Zum Begriff »defender« vgl. Miles et al. (1978). 
»Fast das gesamte Management entstammt interner Aufzucht - wer sollte da einen schönen Laden konzipieren können?«

(Das Manager Magazin im Jahr 2011) $)^{1145}$

Hinsichtlich der Schwierigkeiten, eine neue, zukunftsweisende Strategie zu entwickeln, dürfte auch die einseitige Merkmalsausprägung der beruflichen Qualifikation von Bedeutung gewesen sein. Die meisten Führungskräfte der Anton Schlecker e.K. hatten sich zwar über viele Jahrzehnte ein großes Funktionswissen im bestehenden Gefüge angeeignet. Sie waren aber wohl weder von ihrer formalen Ausbildung noch von ihren beruflichen Erfahrungen her in der Lage, im Zuge der sich verändernden Umwelt aktiv eine neue Strategie und zum Beispiel einen neuen Vertriebstyp zu entwickeln. ${ }^{1146}$

»Und sie haben's mit Baumitteln, das, die Akademikerquote war extrem gering bei Schlecker, da gab es kaum Leute, die Konzepterfahrung hatten bei Schlecker. Das waren halt alles dann, die waren 20 Jahre dabei, die wussten, wie man den Laden führt, aber die wussten nicht, wie man ein neues Konzept entwickelt [...] , sie hatten alle nur diesen Schlecker-Horizont, das muss man halt auch sagen, und dann dieses analytische Vorgehen, das gab es nicht."

(Ehemaliger Berater der Anton Schlecker e.K.)

Die eindeutigen Ausprägungen dieser einzelnen Merkmale dürften somit direkt das etablierte strategische Muster gestützt und die Rezeptivität für den strategischen Wandel gemindert haben. Vor allem dürfte sich jedoch die Homogenität der dominanten Koalition negativ auf die inhaltliche und prozessuale Qualität der strategischen Entscheidungsprozesse und die Fähigkeit zur Reaktion auf den emergierenden strategischen Pfad ausgewirkt haben. Verweist die Managementforschung doch darauf, dass Gruppen mit einem sehr niedrigen Grad an Diversität in der Regel deutlich schlechtere Ergebnisse erzielen als heterogene Gruppen. ${ }^{1147}$

Dies gilt insbesondere, wenn die Gruppen mit solch komplexen und unstrukturierten Aufgaben wie der strategischen Steuerung eines Unternehmens betraut sind. ${ }^{148}$ Bei einer niedrigen Diversität fehlt es hier an unterschiedlichen

1145 S110118MM.

$1146 \mathrm{Zu}$ Auswirkungen von formaler Qualifikation auf strategische Entscheidungsprozesse vgl. Finkelstein et al. (2009: 106ff.); Wiersema \& Bantel (1992: 92).

1147 Vgl. allgemein Steinmann \& Schreyögg (2005: 627f.); Wanous \& Youtz (1986). Für eine ausführliche Darstellung der Vor- und Nachteile von Diversität vgl. Milliken \& Martins (1996); Williams \& O’Reilly III (1998). Zur Bedeutung von Diversität in Familienunternehmen Klein (2010: 237).

1148 Vgl. Amason (1996: 124); Bantel \& Jackson (1989); Bresser (2010: 39); Milliken \& Martins (1996: 416); Wiersema \& Bantel (1992). (Wenngleich später gezeigt werden wird, dass Diversität ein zweischneidiges Schwert ist und ein zu hoher Grad an Diversität auch sehr negative Konsequenzen haben kann.) 
Wahrnehmungen und Perspektiven, um Probleme möglichst frühzeitig und ganzheitlich $\mathrm{zu}$ beleuchten. Zum anderen mangelt es an unterschiedlichen Ideen, um Probleme einer kreativen Lösung zuzuführen und aktiv Innovation zu generieren. Diese Nachteile im strategischen Entscheidungsprozess erweisen sich vor allem in dynamischen Phasen der Umweltentwicklung als sehr nachteilig. ${ }^{1149}$

Dies zeigt sich auch bei der Anton Schlecker e.K. Hier wurden die Wahrnehmung und Interpretation der sich verändernden Umwelt durch die geringe kognitive Diversität der dominanten Koalition beeinträchtigt. Die geringe Diversität verstärkte hier die Effekte der exkludierenden kulturellen Muster und organisationalen Strukturen.

»Herbst 2004. Selten hatte sich Anton Schlecker, Gründer und Inhaber der gleichnamigen Drogeriekette, zuvor gegenüber Journalisten geäußert. Doch an jenem Tag wollte er Recherchen der WirtschaftsWoche entgegentreten, sein Konzern geriete immer stärker unter den Druck der Rivalen - und gewährte Einlass in sein Reich. >Die Konkurrenz wird auch nicht mehr so stark wachsen`, gab sich Schlecker damals überzeugt. Sein Unternehmen? >Das Konzept stimmt, es muss nur weiter verfeinert werden`, befand der Drogeriefürst und deutete voller Stolz auf eine Karikatur an der holzgetäfelten Wand des Besprechungsraums: Anton Schlecker als strahlender Gondoliere, seine Frau als Galionsfigur am Bug der schönsten und größten Gondel. Dahinter paddeln Schleckers Rivalen, dm-Gründer Götz Werner und Dirk Roßmann. >Bis die anderen Schlecker überholt haben, gibt es mich nicht mehr, sagte der Drogeriekönig."

(Die Wirtschaftswoche im Jahr 2012) (1150 $^{15}$

Diese verzerrte Wahrnehmung und unzureichende Interpretation der Umwelt war keineswegs auf die Protagonisten der Unternehmensfamilie beschränkt. Sie kennzeichneten einen großen Teil der dominanten Koalition.

»Bei Schlecker war die Wahrnehmung der Selbsteinschätzung untergeordnet. >Wir sind die Größten, also sind wir auch die Billigsten`, so das Credo der Schlecker-Manager. An dieser Überzeugung hielten sie auch fest, als Schleckers Konkurrenten durch ihren Erfolg Schlecker längst im Preis unterbieten konnten.«

(Das Handelsblatt im Jahr 2012) $)^{1151}$

Letztlich trugen diese Auswirkungen des geringen Grads der Diversität der dominanten Koalition somit vor allem dazu bei, dass ein Großteil der domi-

1149 Vgl. Carpenter et al. (2004: 765).

1150 S120128WW. Die verzerrte Wahrnehmung war insbesondere deshalb gefährlich für die weitere strategische Entwicklung, weil sich wie in vielen Krisenunternehmen auch bei der Anton Schlecker e.K. die tatsächliche strategische Lage des Unternehmens lange Zeit nicht in der Bilanz niederschlug. Vgl. Hambrick \& D'Aveni (1988: 17).

1151 S120602HB. Vgl. auch S120604SP und die Aussage des Insolvenzverwalters: »Die Realität war in vielen Bereichen eine ganz andere als das Management selbst geglaubt hat.» 
nanten Koalition lange Zeit nicht die Gefahr erkannte, die sich aus der Wachstumsspirale des selbstverstärkenden Mechanismus entwickelte.

\section{Einfluss des Faktors Familie}

Die homogene Zusammensetzung der dominanten Koalition der Anton Schlecker e.K. lässt sich zum Teil auf den Einfluss des Faktors Familie zurückführen. ${ }^{1152}$

So zog das familiär geprägte Streben nach personaler Kontinuität fast zwangsläufig die lange Unternehmenszugehörigkeit der Entscheidungsträger und das ebenso hohe wie homogene Durchschnittsalter der dominanten Koalition nach sich. Innerhalb eines partikularistischen und an einzelnen Personen orientierten Beziehungsgefüges wurde auch dann noch an vertrauten Entscheidungsträgern festgehalten, wenn deren fachliche Qualifikation nicht mehr vollkommen mit den Anforderungen ihrer Position korrespondierte. ${ }^{1153}$

Zudem trugen jene Manifestationen des familiären Einflusses, welche allgemein die hohe Immunität der dominanten Koalition begründeten, auch spezifisch zu deren niedrigen Diversität bei. So führten die abschottenden kulturellen Muster dazu, dass Führungsposten meist ausschließlich mit intern rekrutierten Mitarbeitern besetzt wurden. ${ }^{1154}$ Zugleich erschwerten die exkludierenden Muster den Rückgriff auf externes Wissen und Potenziale, die die eigene geringe Diversität zumindest partiell hätten kompensieren können.

Diese strukturellen Merkmale des familiären Einfluss trugen somit zum geringen Grad an Diversität bei, dessen dysfunktionale Auswirkungen auf den strategischen Entscheidungsprozess wiederum die ausbleibende Reaktion auf die Genese der strategischen Pfade zum Teil erklären.

Allerdings weist der Vergleich mit der Dirk Rossmann GmbH darauf hin, dass auch nach der Berücksichtigung der dysfunktionalen Auswirkungen der geringen Diversität noch ein Erklärungsdefizit besteht. Denn obgleich die Diver-

1152 Diese Frage lässt sich einordnen in die Fokusverschiebung der Upper-Echelon-Forschung zu den Ursachen der spezifischen Zusammensetzungen von TMT. Vgl. Carpenter et al. (2004: 759f.); Nielsen (2010:309). Zudem Hambrick (2007: 338): »There is a need to turn upper echelons theory on its head by considering executive characteristics as consequences rather than as causes [...]. Why do top management teams look the way they do?» Manche Autoren schreiben Familienunternehmen generell eine hohe Homogenität von TMT zu. Vgl. Gersick et al. (1997: 15); Hatum et al. (2010: 262f.).

1153 Über die Tendenz zum Partikularismus erklären auch Singal \& Gerde (2015) die von ihnen beobachtete geringere Ausprägung von Diversity-Maßnahmen in Familienunternehmen.

1154 Vgl. S031207LZ: „Nachfolger des langjährigen und verdienten Einkaufschefs wird Helmut Glaser, 45, der dem Unternehmen ebenfalls bereits seit über 10 Jahren angehört.« Von einem »internen Wechsel bei der Vertriebsführung« berichtet eine weitere Quelle. Vgl. S040520LZ. Ouchi \& Jaeger (1978) zeigen, dass japanische Unternehmen eine solche interne Rekrutierung praktizieren, um die gewünschte Homogenität im Management zu erreichen. 
sität der dominanten Koalition der Dirk Rossmann GmbH höher war als jene der Anton Schlecker e.K., war die Differenz nicht so groß, dass sie die gesamte beobachtete Varianz in Verlauf und Ergebnis der strategischen Entscheidungsprozesse plausibel machen könnte.

Vielmehr zeigen sich im kontrastierenden Vergleich der Entscheidungsprozesse über die Diversität hinaus vor allem die unterschiedlichen Kommunikationsmuster als höchst bedeutsam für Verlauf und Ergebnis der strategischen Entscheidungsprozesse in der Phase der Pfadformation. Denn nur jene unterschiedlichen Wahrnehmungen und Perspektiven konnten die strategischen Entscheidungsprozesse der beiden Unternehmen bereichern, die - auch gegen Widerstände - in die Kommunikationsprozesse einflossen und so soziale Bedeutung erlangen. Auf der Grundlage nicht kommunizierter individueller Perzeptionen und Annahmen konnten die dominanten Koalitionen keinen Wandel anstoßen. ${ }^{1155}$

\subsubsection{Mangelnder Sachkonflikt in monozentrischen Konfigurationen}

Im Rahmen der monozentrischen Konfigurationen der Anton Schlecker e.K. und der Dirk Rossmann GmbH kam den Interaktions- und Kommunikationsprozessen der dominanten Koalitionen eine besondere Bedeutung zu. Galt es doch das vorhandene kognitive Potenzial der dominanten Koalitionen in den strategischen Entscheidungsprozess einfließen zu lassen, um die dominanten Akteure im Entscheidungsprozess ausreichend $\mathrm{zu}$ irritieren und autarke Einzelentscheidungen $\mathrm{zu}$ vermeiden. ${ }^{1156}$

"[...] da ist die Frage, wer ist der Diskussionspartner, der Counterpart für den Unternehmer? Führt der ein Selbstgespräch? Hat der eine Umgebung, die so emanzipiert ist?"

(Auf Familienunternehmen spezialisierter Unternehmensberater im Rahmen der explorativen Vorstudie)

1155 Vgl. Hambrick \& Mason (1984: 230); auch Simon (2011: 38f.). Mittlerweile gehen die meisten Beiträge davon aus, dass die Beziehung zwischen der Zusammensetzung der dominanten Koalition und deren Performance im Entscheidungsprozess durch Gruppenprozesse moderiert wird. Vgl. Carpenter et al. (2004: $763 \mathrm{ff}$.) für eine Übersicht intervenierender Variablen. Vgl. Smith et al. (1994); Lawrence (1997) zu ersten Forderungen nach deren verstärkter Berücksichtigung, um den teils inkonsistenten Ergebnissen der Forschung zur Zusammensetzung von TMT zu begegnen. Vgl. auch Bantel \& Jackson (1989) Eisenhardt \& Schoonhoven (1990) oder Murray (1989). Beiträge wie Amason (1996) und Pelled et al. (1999) versuchen entsprechend die Validität der Ergebnisse durch Integration des Konfliktniveaus als intervenierende Prozessvariable zu erhöhen.

$1156 \mathrm{Zu}$ den wahrscheinlichen Defiziten solcher Einzelentscheidungen vgl. Amason (1996: 127): "[T] he synthesis that emerges from the contesting of the diverse perspectives is generally superior to the individual perspectives themselves. "Vgl. auch Schweiger et al. (1986); Schweiger et al. (1989). 


\section{Empirische Ausprägungen}

Wie im obigen Abschnitt festgestellt wurde, waren die kognitiven Potenziale zur Irritation des strategischen Entscheidungsprozesses bei der Anton Schlecker e.K. aufgrund der geringen Diversität der dominanten Koalition vergleichsweise schwach ausgeprägt. Doch auch hinsichtlich der Nutzbarmachung dieser Potenziale zeigten sich im Unternehmen sehr ausgeprägte Interaktions- und Kommunikationsmuster.

"[...] da sehr sehr viele Leute waren, die nicht nachgedacht haben. Die machen alles. Da müssen Sie sich Leute vorstellen, die haben Funktionen später innegehabt, die hätten die nie bekleiden dürfen. Da sind einfachste Leute zum Teil befördert worden, weil sie lange genug dabei waren, weil sie gebuckelt haben. Ja, da gibt's also sehr sehr viel unterwürfige Mitarbeiter, die alles gemacht haben, nur um diesen Job zu bekommen. Und die haben oft auch einen Job gehabt, der war vielleicht 2.500 Euro wert, die haben den für 6.000 Euro gekriegt. Mit denen konnten Sie machen, was Sie wollten. Und da kam nie Kritik. Nie.»

(Langjährige Führungskraft der Anton Schlecker e.K.)

Bis hin zu den strategischen Entscheidungsträgern der dominanten Koalition war die Interaktion und Kommunikation von der Norm weitgehender Konfliktvermeidung geprägt. ${ }^{1157}$ Die offene Aussprache wurde von den meisten Entscheidungsträgern grundsätzlich gemieden und kontroverse Diskussionen erst gar nicht geführt.

»Einmal im Jahr bestellte Schlecker seine fünf engsten Angestellten samt Gattinnen zum Weihnachtsessen [...]. Schlecker, am Tischende thronend, stellte Fragen, seine Untergebenen antworteten. Nacheinander. Keiner wagte es, zu unterbrechen oder ihm ins Wort zu fallen, erzählt ein Teilnehmer dieser Runden. Schlecker schaffte es, ein Essen in großer Runde in eine Serie von Vier-Augen-Gesprächen zu verwandeln.»

(Das Handelsblatt im Jahr 2012) $)^{1158}$

In den Entscheidungsprozessen wurde sowohl bezüglich operativer als auch strategischer Thematiken Wert auf eine rasche Entscheidungsfindung und eine zügige Abarbeitung der anstehenden Aufgaben gelegt. Auf die Erörterung von Problemen und die Diskussion möglicher Lösungswege wurde hingegen weniger Zeit und Aufmerksamkeit verwendet. ${ }^{1159}$ In der Gruppe gab es kaum Auseinandersetzungen um die Ziele des Unternehmens oder offene, gar kontroverse

1157 Vgl. zu solchen Gruppennormen Jehn (1995: 262f.); Tjosvold (1991). Vgl. auch Morrison \& Milliken (2000) zum Phänomen der »organizational silence« und deren Ursachen.

1158 S120602HB.

1159 Vgl. die Aussage eines Turnaround-Beraters in S111125LZ: »Durch das Projekt ist Schlecker kein Debattierklub geworden. Im Gegenteil: Die Familie ist der klare Machtpromotor im Hintergrund. Wer nicht mitzieht, ist im falschen Film. « Ein weiterer Berater zu den Formaten der Meetings: »[...] also die Meetings sehr häufig waren immer bilateral. Also er hat, ja, so nicht bei großen Runden, das hat er nicht geliebt.« 
Diskussion hinsichtlich der Wege und strategischen Maßnahmen, um diese Ziele zu erreichen. ${ }^{1160}$ Ebenso wenig existierten Formate, in denen die strategischen Entscheidungsträger der dominanten Koalition regelmäßig in Gruppengröße zusammengekommen wären, um die Chancen und Bedrohungen der Umweltdynamik zu diskutieren oder sich intensiv mit den Risiken der Unternehmensentwicklung auseinanderzusetzen. Somit fehlte jedoch eine Plattform, auf der die verschiedenen Vertreter der Funktionsbereiche ihre Perspektiven, Zweifel oder Meinungen zur strategischen Entwicklung des Unternehmens hätten einbringen können. Die latent vorhandenen Meinungsunterschiede zur strategischen Entwicklung des Unternehmens und zu adäquaten Reaktionen auf die sich verändernde Umwelt wurden nur selten zum Ausdruck gebracht. Eine kritische Erörterung der gegenwärtigen und zukünftigen strategischen Ausrichtung des Unternehmens fand innerhalb der dominanten Koalition bei der Anton Schlecker e.K. demnach im Grunde kaum statt.

Lediglich mit einigen wenigen vertrauten Führungskräften führte der dominante Akteur Anton Schlecker Gespräche zu Themen, die über die Abarbeitung operativer Aufgaben hinausgingen. Doch auch diese - meist bilateralen Gespräche mündeten selten in Diskussionen und ein Ringen um Positionen. Vielmehr nahmen sie meist eher die Form von Berichten oder Vorträgen der Führungskräfte zu bestimmten Aspekten der Unternehmensentwicklung an, in deren Zuge offener Widerspruch vor allem vom dominanten Akteur Anton Schlecker für gewöhnlich vermieden wurde. Einzelne Führungskräfte konnten in diesen spezifischen Kommunikationssituationen so zwar teilweise relativ offen ihre Vorschläge präsentieren. Da diese Vorschläge aber selten negiert wurden, erwuchsen aus diesen Gesprächen keine konstruktiven Auseinandersetzungen oder sachlichen Konflikte, die sich selbst getragen hätten und über längere Zeit fortgeführt worden wären. ${ }^{1161}$

"[...] hat nicht dagegen argumentiert. Das war ja nicht so. Das ist immer eine sehr einseitige Diskussion gewesen [...], da kam dann nichts. Da wusste man jetzt immer nicht, nimmt er es jetzt mit? Denkt er nochmal drüber nach? Und ich glaube auch nicht, dass er das dann mit anderen groß diskutiert hat. Und der Rest hat diese Diskussionen nicht geführt.«

(Ehemalige Führungskraft der Anton Schlecker e.K.)

1160 Vgl. zur Definition und Beschreibung von Sachkonflikt Amason (1996: 127); Pelled et al. (1999: 2).

1161 Vgl. das in Teil C dargestellte Konfliktverständnis nach Luhmann (1993: 565), nach dem die Negation der Negation zur Fortsetzung der Kommunikation führt. 
In Bezug auf die familienfremden Mitglieder der dominanten Koalition wurden Diskussionen und sachlicher Konflikt somit weniger explizit unterdrückt als vielmehr strukturell und kulturell gestützt vermieden. ${ }^{1162}$

Auf der mittleren Managementebene hingegen ist eine Konfliktunterdrückung zu verzeichnen. ${ }^{163}$ Im Gegensatz zu den familienfremden Angehörigen der dominanten Koalition wurden strategische Themen zwischen den Mitgliedern der Unternehmensfamilie teilweise auch kontrovers diskutiert. Allerdings konnten und sollten kritische Meinungen nicht so intensiv und nachhaltig vertreten werden, dass sich hier innerhalb der Familie länger anhaltende sachliche Konflikte zur strategischen Entwicklungsrichtung des Unternehmens entwickelt hätten. ${ }^{1164}$

"Jetzt stellen Sie sich vor, der hätte noch zwei starke Brüder. Ne, der eine macht das Europageschäft. Der Schlecker macht hier das Geschäft und der Dritte, das ist meinetwegen der Logistiker [...] und ich hätte jetzt mit zwei seinen Brüdern diese Themen besprochen, ich würde mit Ihnen um eine Millionen wetten, da hätte sich was geändert. Aber diese Unternehmensfamilie war nicht da. Die bestand ja jetzt nur in der Frau oder in den Kindern. Das ging nicht. Die Kinder waren viel zu jung und mit der Frau ging das auch nicht. Da ging es auch intellektuell nicht. Das war nicht ihr Thema. Und dadurch gab es keinen Gesprächspartner, soweit das Auge reicht, wo man sagt: >Rede Du doch mal mit ihm. Mach ihm das mal kları."

(Ehemalige Führungskraft der Anton Schlecker e.K.)

Zusammenfassend lässt sich für die dominante Koalition der Anton Schlecker e.K. somit ein insgesamt sehr niedriges Niveau an Sachkonflikt konstatieren.

Die Interaktions- und Kommunikationsmuster der dominanten Koalition der Anton Schlecker e.K. standen in klarem Gegensatz zu jenen der Dirk Rossmann GmbH. Wo bei der Anton Schlecker e.K. eine hohe kommunikative Distanz herrschte, versuchte der dominante Akteur Dirk Roßmann grundsätzlich eine kommunikative Nähe im Unternehmen und insbesondere in der dominanten Koalition aufzubauen. ${ }^{1165}$ Weit über eine hohe Interaktions- und Kommunika-

1162 Vgl. Simon (2011: 94) zur konfliktunterdrückenden Wirkung von Macht.

1163 Diesbezüglich eine ehemalige Führungskraft der Anton Schlecker e.K.: »Aber hier ist schon auch ein bisschen mit der Angst gespielt worden. Die Leute hatten Angst, ihren Arbeitsplatz zu verlieren. Und haben dann fast auch alles mitgemacht. [...] /Wenn Du hier nicht parierst, kannst Du gehen. Da sind auch viele Exempel statuiert worden. Wenn dann noch einer was gesagt hat, der war dann auch ruckzuck weg."

1164 Vgl. S110118MM: „Oft hört man in anderen Familienunternehmen: Jetzt redet der Alte immer noch hinein. Für mich ist das kein Hineinreden, sondern ein Ratgeben.» S110613FA: "In unserer Familie setzt sich keiner durch, alle Beschlüsse fallen einstimmig. Eher wird eine Entscheidung verschoben, als dass sie gegen Widerstand durchgeboxt wird."

1165 Eine ehemalige Führungskraft der Dirk Rossmann GmbH: »Das zeigt sich dann in der Unternehmenskultur [...], dass die Geschäftsführer, die dann da waren und die leitenden Angestellten, sich immer mit ihm duzten. Ne. Das wollte er so, [...], das hat er so ent- 
tionsdichte hinausgehend, wurde in diesem offenen kommunikativen Rahmen vor allem der an der Sache orientierte Konflikt nicht nur gestattet, sondern auch ausdrücklich erwünscht und angeregt.

»Man kann ja auch mal strittig sein, ohne dem anderen böse zu sein. Ich glaube, das ist das Wichtigste, was ein Chef als Kultur in einer Gemeinschaft, in einem Unternehmen entwickeln muss - wenn man's kann: eine Streitkultur."

(Dirk Roßmann im Jahr 2014 in einem Interview mit dem H1) ${ }^{1166}$

Explizit wurde bei der Dirk Rossmann GmbH versucht, das gewünschte hohe $\mathrm{Maß}$ an sachorientiertem Konflikt in der organisationalen Kultur zu verankern. So wurden beispielsweise Führungskräfte des mittleren und oberen Managements zu spezifischen Konflikttrainings entsandt. ${ }^{1167}$ Von der mittleren Managementebene bis hinauf zu den Entscheidungsträgern der dominanten Koalition wurden die Führungskräfte vom Protagonisten Dirk Roßmann zu Widerspruch ermutigt.

"[...] eine Einladung zur Auseinandersetzung. Ich sag mal weil, ich glaube wie jeder, jeder gute oder starke Unternehmer, er will zwar keinen Widerspruch, aber er braucht ihn und das weiß er auch. Und er will Leute, die ihm widersprechen [...] wir haben dann einen Pakt geschlossen, der hieß letztendlich, wir dürfen uns in allen Punkten streiten, haben wir auch getan. Reibung, Streiten um den richtigen Weg. das war ein entscheidender Durchbruch, weil wir hatten, es war dann so, dass wir offen Kritik auch an ihm ausgeübt haben, das war, das fand er nicht gut. Muss er auch nicht, wer findet das schon gut? Aber es hat funktioniert."

(Ehemalige Führungskraft der Dirk Rossmann GmbH)

Auch zwischen den im Unternehmen tätigen Mitgliedern der Unternehmensfamilie Roßmann existierte ein hohes Konfliktniveau.

»Wir streiten manchmal ganz schön heftig und kritisieren uns, und da wird es auch mal laut. Ich bin ja manchmal etwas emotional. Wir bellen uns an, tun das aber in herzlicher Verbundenheit.«

(Dirk Roßmann in einem Interview mit der Welt im Jahr 2012) ${ }^{1168}$

wickelt. Aber es hat, es war halt so nie, es war kein Schmusekurs über die Jahre, aber über die Jahre so zusammenwachsen, also ein gegenseitiges Respektieren auch." Diese kommunikative Nähe bei der Dirk Rossmann GmbH stand diametral zur Anton Schlecker e.K., in der der dominante Akteur gegenüber langjährigen Mitarbeitern nach einiger Zeit vom "Du« wieder zum »Sie« überging; vgl. auch R100218HB.

1166 R140203H1.

1167 Vgl. R110418SZ. Roßmann: »Wir haben in den vergangenen Jahren mehr als 500 Mitarbeiter des mittleren und oberen Managements an sogenannten Rossmann-Jahresgruppen teilnehmen lassen. [...]. Man lernt [...] mit Kritik konstruktiv umzugehen, zu streiten und sich und andere sensibler wahrzunehmen."

1168 R120414WE; auch R150416HB. »Bei Rossmann ist es so, dass die Kinder gelernt haben, von ihrem Vater schon, nämlich genau diese Kultur. Du musst kritisieren. Also die, die Kinder kritisieren ihn auch halb öffentlich. [...] vor allen Dingen der eine Sohn, der hat 
Auswirkungen des geringen Grads an Sachkonflikt bei der Anton Schlecker e.K. Bei der Dirk Rossmann GmbH manifestierte sich der hohe Grad an sachorientiertem Konflikt unmittelbar im Verlauf und Ergebnis der Entscheidungsprozesse, denn durch die Reibungen und Auseinandersetzungen ließ sich der dominante Akteur in seinen persönlichen Ansichten und Meinungen irritieren und unter Umständen auch in seiner Entscheidungsfindung beeinflussen. ${ }^{1169}$

"[...] aber wenn's um strategische Entscheidungen im Unternehmen ging, dann war es immer eine Entscheidung, die im Gremium fallen musste, wobei er auch mal überstimmt wurde. Also das war nicht so, dass nur gemacht wurde, was er will. Das war nicht gottgegeben. Es gab auch Phasen in diesem Unternehmen, und die waren nicht so selten, dass man nicht miteinander gesprochen hat, weil man sich uneins war, ne. [...] Aber daraus zog er dann letztlich eine Entscheidung, die auch ein Kompromiss sein konnte."

(Ehemalige Führungskraft der Dirk Rossmann GmbH)

Die letztlich getroffene Entscheidung beruhte bei der Dirk Rossmann GmbH somit meist auf einem vorausgegangenen Spiel verschiedener Annahmen und Alternativen zur strategischen Entwicklung des Unternehmens, die zum Teil erst über den Sachkonflikt in den Entscheidungsprozess eingeflossen waren. Diese irritierende und teilweise auch korrigierende Wirkung der intensiven Auseinandersetzungen entsprach der vom dominanten Akteur auch explizierten Erwartung an diese Kommunikationsmuster. ${ }^{1170}$

Während die traditionelle Organisationstheorie Konflikte lange vor allem als ein die organisationalen Abläufe störendes Element betrachtete, verweisen mittlerweile viele Beiträge der Managementforschung auf diese funktionalen Auswirkungen sachorientierter Konflikte. ${ }^{171}$ Auseinandersetzungen in der Sache fördern demnach den Austausch vorhandener Perspektiven, Meinungen,

seinen Vater massiv kritisiert. Und auch da dasselbe, er liebt seinen Sohn, ne, keine Frage, aber auch die sprechen manchmal zwei Wochen nicht mehr miteinander."

1169 Vgl. Eisenhardt \& Zbaracki (1992: 21): »[...] research indicates how decision makers can move along the rationality vs. bounded rationality continuum, typically by increasing conflict."

1170 Eine ehemalige Führungskraft der Dirk Rossmann GmbH: ")Ihr hättet mich davon abhalten müssen. Dafür werdet ihr bezahlt.` Also so klar war er dann auch. Sagt er: >Ich erwarte von euch, dass ihr euch dann auch durchsetzt, ne`."Vgl. auch R120602HB: »[...] es kann passieren, dass mich einer davon auch mal temperamentvoll zur Brust nimmt. Es ist fast immer so: Die Mehrheit ist schlauer als der Einzelne."

1171 Vgl. Jehn \& Bendersky (2003: 188): »Before 1990, much of the organizational literature presumed that conflict was counterproductive." Zur klassisch negativen Sicht auf Konflikte beispielsweise Blake \& Mouton (1984); Pondy (1967). Vgl. Jehn \& Bendersky (2003: 189ff.) für eine Übersicht über ähnliche Perspektiven. Neuere Beiträge wie Amason (1996); Jehn (1995); Jehn (1997); Schweiger et al. (1989) betonen hingegen die positiven Auswirkungen. In der Familienunternehmensforschung vgl. Simon (2012: 113); von Schlippe \& Kellermanns (2008: 41). 
Annahmen oder Ideen. ${ }^{1172}$ Zudem führe der Sachkonflikt dazu, dass mehr Informationen in die Gruppe und deren Entscheidungsprozesse einfließen, denn die Auseinandersetzung mit anderen Meinungen und Perspektiven sei meist damit verbunden, dass die Akteure sich auf die Suche nach neuen Informationen begeben, um die eigene Position zu stützen oder im Lichte der Gegenposition kritisch zu hinterfragen. Sie arbeiten sich so tiefer in die Materie hinein und entwickeln dabei ein multi-perspektivisches Verständnis des Problems und möglicher Lösungsansätze. Auf diesem Wege verhindern sachorientierte Konflikte eine vorschnelle Entscheidungsfindung und fördern eine intensivere Auseinandersetzung mit den strategischen Aufgaben. Das ringende Wechselspiel der Positionen, Perspektiven und Alternativen trage somit dazu bei, synthetische, innovative und tiefer durchdachte Ideen und Lösungen zu generieren. ${ }^{1173}$ Letztlich erhöhen die funktionalen Auswirkungen sachorientierter Konflikte insgesamt die Qualität der Bearbeitung von Aufgaben und die Leistung der Gruppe im strategischen Entscheidungsprozess. ${ }^{1174}$

Genau diese funktionale, irritierende und kontrollierende Wirkung sachorientierter Konflikte entfiel in der Steuerung der strategischen Entscheidungsprozesse der Anton Schlecker e.K. weitgehend. Da sich aufgrund der ausgeprägten Konfliktvermeidung die unterschiedlichen Wahrnehmungen, Interpretationen und Perspektiven nicht aneinander rieben, konnte auch das kognitive Potenzial der Gruppe nicht ausgeschöpft werden. So flossen zu wenige Annahmen, Meinungen und Ideen in den Entscheidungsprozess ein. Ob der ungenügenden Irritation fehlte es an jener Informations- und Perspektivenvielfalt, die es bedarf, um der begrenzten individuellen und kollektiven Rationalität zu begegnen. ${ }^{1175}$ Ein höheres Maß an Sachkonflikt hätte die etablierten Wahrnehmungs- und Interpretationsmuster erschüttern und somit den individuellen und kollektiven Wahrnehmungshindernissen entgegenwirken können. ${ }^{1176}$ Mehr Konfliktkommunikation hätte auch das Ignorieren der strategischen Probleme und der entstehenden Unternehmenskrise erschwert. ${ }^{1177} \mathrm{Da}$

1172 Vgl. zu den funktionalen Auswirkungen des Konflikts Eisenhardt et al. (1997: 43); Pelled et al. (1999: 9).

1173 Vgl. Amason (1996: 127); Jehn (1995: 275); auch Schweiger et al. (1986: 66): »[...] programmed conflict is useful in improving the quality of strategic decisions."

1174 Vgl. Eisenhardt et al. (1997), die beobachten, dass TMT in überdurchschnittlich erfolgreichen Unternehmen mehr Aufgabenkonflikte hatten als unterdurchschnittlich erfolgreiche Unternehmen. Ähnlich: Amason (1996: 123ff.); De Dreu \& Weingart (2003: 747); Jehn (1994); spezifisch für Familienunternehmen Cosier \& Harvey (1998: 75).

1175 Vgl. Cosier \& Schwenk (1990); Janis (1972); Schweiger et al. (1986); Schweiger et al. (1989).

1176 Vgl. zu diesen Wahrnehmungshindernissen Schreyögg \& Ostermann (2014: 124).

1177 Vgl. S120602HB: »Zwischen 2005 und 2010 hat Schlecker 400 Millionen Minus geschrieben. Aus eigener Kraft kann das Familienunternehmen Anton Schlecker nicht mehr ge- 
diese Auseinandersetzungen von großen Teilen der dominanten Koalition aber unterlassen wurden, konnte in der Kommunikation der dominanten Koalition lange Zeit die Erklärung einer temporären Umweltentwicklung aufrechterhalten werden. ${ }^{1178}$ Die praktizierte Konfliktvermeidung führte so zwar zu einem oberflächlichen Konsens, vor allem aber zu einer großen Apathie und »organizational silence $\lll .{ }^{1179}$ Diese Apathie trug maßgeblich dazu bei, dass sich gerade die Protagonisten der dominanten Koalition, Anton und Christa Schlecker, zu spät eingestanden, dass die Wachstumsspirale zu weit gedreht worden war und sich das Unternehmen auf dem Weg in eine existenzbedrohende Krise befand. ${ }^{1180}$ Ein frühzeitiges Erkennen, Verstehen und Eingeständnis der Krisenentstehung in der Phase der Pfadformation wäre aber von zentraler Bedeutung für die Einleitung strategischer Gegenmaßnahmen gewesen. ${ }^{1181} \mathrm{Ab}$ einem gewissen Zeitpunkt war die Wirkkraft der selbstverstärkenden Mechanismen zu hoch, als dass noch hätte reagiert werden können.

Ähnliche Zusammenhänge wurden auch im Rahmen der explorativen Vorstudie thematisiert:

"Das ist so ein Thema, das viel zu wenig diskutiert wird bei der Erforschung der Familienunternehmen, weil es hier immer nur um die Streitvermeidung geht, und den Streit vermeide ich natürlich, wenn ich Alleingeschäftsführer bin, bloß, der führt das halt regelmäßig an die Wand.»

(Auf Familienunternehmen spezialisierter Rechtsanwalt im Rahmen der explorativen Vorstudie)

\section{Einfluss der Familie}

Dass bei der Anton Schlecker e.K. und der Dirk Rossmann GmbH die Variable Konflikt unterschiedlich ausgeprägt war, lässt sich, wie oben deutlich wurde, auch auf die unterschiedlichen Persönlichkeiten Anton Schlecker und Dirk Roßmann zurückführen. Während Dirk Roßmann Konflikte offen befürwortete,

sunden. ১Schreiben Sie das nicht auf`, sagt Schlecker zu Beck. >Wenn es auf dem Papier steht, dann wird es auch wahr.` Vgl. zu solchen Verdrängungsmechanismen in Krisenunternehmen Hambrick \& D’Aveni (1988: 16); Nystrom \& Starbuck (1984: 55f.); Argenti (1976: $93 \mathrm{ff}$.) zu bilanzpolitischen Maßnahmen, um den Ernst der Lage zu beschönigen.

1178 Vgl. Albach \& Freund (1989: 255); Trahms et al. (2013: 1290). Barker \& Patterson (1996) weisen darauf hin, dass TMT mit steigender Verweildauer Krisen eher externen Ursachen zuschreiben.

1179 Vgl. Eisenhardt et al. (1997: 77): »The absence of conflict is not harmony, it’s apathy.« Vgl. auch Morrison \& Milliken (2000).

1180 Vgl. Whetten (1980: 580). Zudem Starbuck et al. (1978: 118), die für dieses fehlende Eingeständnis auch die Bedrohung der persönlichen Emotionen und der Reputation verantwortlich machen.

1181 Vgl. zur Bedeutung der Krisenerkennung Schreyögg \& Ostermann (2014: 122ff.); Slatter \& Lovett (1999: 61ff.). Ob, wann und wie eine Krise wahrgenommen wird, gilt in der Unternehmenskrisenforschung als eine entscheidende Determinante des Krisenmanagements. Vgl. auch Penrose (2000: 156). 
ging Anton Schlecker vielleicht auch aus persönlicher Unsicherheit und der Angst vor Kontrollverlust Konflikten weitestgehend aus dem Weg. ${ }^{1182}$ Doch darüber hinaus kann der geringe Grad an Konflikt auch wieder auf einige jener Merkmale zurückgeführt werden, die in Teil B als Indikatoren des familiären Einfluss identifiziert wurden. ${ }^{183}$

Bereits die aus dem Streben nach Autonomie und exkludierender Abschottung resultierende Beibehaltung der Rechtsform des eingetragenen Kaufmanns schützte vor unliebsamen Konflikten in den Entscheidungsprozessen der Anton Schlecker e.K. ${ }^{1184}$ Zudem förderte die ausgeprägte Zentralisierung den niedrigen Grad an Konflikten im strategischen Entscheidungsprozess. Da ein großer Teil wichtiger operativer und beinahe ausschließlich alle strategischen Fragen von Anton oder Christa Schlecker entschieden wurden, blieb in den dyadischen Gesprächen kaum Raum für intensive Diskussionen und Auseinandersetzungen. Doch schützte auch die Reduktion auf bilaterale Kommunikationssituationen an sich vor Konflikten. So wurde Gruppenkommunikation von vornherein vermieden, in deren Zuge schwerer kontrollierbare Gruppendynamiken in Konflikte hätten münden können.

$\mathrm{Zu}$ der weitgehenden Konfliktfreiheit im Entscheidungsprozess trug des Weiteren die nur schwach ausgeprägte Formalisierung der strategischen Analyse der Umweltdynamik und der eigenen Unternehmensentwicklung bei, da es deswegen an institutionalisierten Plattformen zum Austausch gegenteiliger Perspektiven und Meinungen fehlte. Auf der anderen Seite dämmt die aus dem Kontrollstreben resultierende Routinisierung der operativen Aufgaben das Konfliktpotenzial ein, ${ }^{185}$ denn die routinisierte Erledigung der operativen Aufgaben bot nur wenig Raum für kritische Auseinandersetzung.

Vor allem aber dürften die aus der Orientierung an Personen und die aus dem partikularistischen Beziehungsgefüge erwachsende personale Kontinuität zum niedrigen Grad an Sachkonflikt beigetragen haben. Die lange Zusammenarbeit in der dominanten Koalition reduzierte das Konfliktniveau erheblich, erstens,

1182 Vgl. R120602HB. Eine ehemalige Führungskraft: »[...] aber das hätte natürlich immer einen anderen Auftritt verlangt und ich glaube, dem wollte er sich auch nicht stellen [...], aber ich glaube, das hat er sich nicht zugetraut.« Vgl. theoretisch zum Zusammenhang zwischen Persönlichkeitsmerkmalen des CEO und Dynamiken im TMT Peterson et al. (2003).

$1183 \mathrm{Vgl}$. Zattoni et al. (2015), die allgemein einen negativen Zusammenhang zwischen familiärem Einfluss und dem Niveau an Konflikt in der dominanten Koalition feststellen.

1184 Vgl. Simon (2012: 75); auch S140603WI: »Zu den Vorteilen des eingetragenen Kaufmanns zählt eine schnelle Entscheidungsfindung. Es sind keine aufwendigen Abstimmungsprozesse erforderlich, da der eingetragene Kaufmann allein verantwortlich und entscheidungsberechtigt ist. Hierdurch gibt es auch keine Konflikte über die Art der Unternehmensführung."

1185 Vgl. Jehn (1995: 275f.); Pelled et al. (1999: 7). 
weil die Führungskräfte um die rigiden Perspektiven der Protagonisten wussten und mit der Zeit nur noch solche Themen ansprachen und Maßnahmen vorschlugen, von denen sie ausgingen, dass sie angehört und befürwortet würden: ${ }^{1186}$

")Natürlich haben unsere Manager gesehen, dass sich da etwas verändertı, sagt ein Direktor von Schlecker. >Das Problem war, dass Probleme nicht akzeptiert wurden. Das fing ganz oben an: bei Anton Schlecker. $>$ Er brauchte ja nicht mal mehr Ja oder Nein zu sagen`, erzählt ein Altdirektor. Wir haben ihm natürlich keine Sachen vorgeschlagen, von denen wir wussten, dass er sie ablehnen würde."

(Ausschnitt aus einem Artikel des Handelsblatts im Jahr 2012) ${ }^{1187}$

Zweitens hatte sich im Zuge der herausgebildeten Interaktions- und Kommunikationsmuster in der dominanten Koalition Resignation ausgebreitet:

»Weil ich weiß ja von meinen Kollegen, dass die gesagt haben >Naja mit dem spreche ich da nicht mehr drüber. Also die hatten keine Lust mehr. Da gab es eine gewisse Resignation [...]. Das ist schon lange so gewesen.«

(Ehemalige Führungskraft der Anton Schlecker e.K.)

$\mathrm{Zu}$ dem geringen Ausmaß an Sachkonflikt trug des Weiteren der Transfer der familiären "Konsensfiktion« auf das Unternehmen bei, aufgrund derer die sachliche Verständigung als Nebenprodukt der langjährigen sozialen Beziehungen erwartet wurde. ${ }^{1188}$

Über die Auswirkungen dieser in Teil B herausgearbeiteten Strukturmerkmale hinaus spiegeln sich in den Daten Aspekte eines familiären Harmoniestrebens wider, welches zur Reduktion von Konflikten innerhalb der Unternehmensfamilie beigetragen haben mag. ${ }^{189}$ Allerdings finden sich bei der Anton Schlecker e.K. keine Indizien dafür, dass dieses Harmoniestreben für die Kommunikation innerhalb des strategischen Entscheidungsraumes der dominanten Koalition übernommen wurde.

Eher trug die persönliche Identifikation und Verflechtung der Unterneh-

1186 Vgl. hierzu Katz (1982), nach dem Mitglieder in lange bestehenden Arbeitsgruppen sich in ihren Meinungsäußerungen entweder den antizipierten Erwartungen und Reaktionen der anderen Mitglieder anpassen oder Kritik ganz vermeiden; auch Ouchi \& Jaeger (1978), die diesen gewünschten Zustand der Konfliktfreiheit in japanischen Unternehmen aufzeigen.

1187 S120602HB.

1188 Vgl. zu dieser Konsensfiktion Wimmer et al. (2005: $110 \mathrm{f}$.).

1189 Vgl. S120324TA: "Anfang Februar in Wien hat Lars Schlecker erklärt: ১Wir halten als Familie zusammen wie Pech und Schwefel.« Vgl. allgemein Simon (2012: 110ff.) und Ward (1987), die ein Streben nach Harmonie und die Vermeidung von Konflikt als ein charakteristisches Merkmal von Familienunternehmen ansehen. Dies resultiere nicht zuletzt daraus, dass die Akteure um die potenziell destruktive Kraft von eskalierenden Konflikten wüssten. Ein solches Streben nach Bewahrung emotionaler Beziehungen wird auch von der allgemeinen Forschung zu Konflikten in Entscheidungsprozessen als ein Grund für die Vermeidung von Sachkonflikten genannt; vgl. Jehn (1997). 
mensfamilie mit dem Unternehmen, der eingeschlagenen Strategie und selbst dem gewählten Betriebstyp des Drogeriediscounters dazu bei, dass Anzeichen von Misserfolg nicht auch konfliktär adressiert wurden. ${ }^{1190}$

Für den geringen Grad an sachorientiertem Konflikt dürfte jedoch vor allem auch die im letzten Abschnitt skizzierte geringe Diversität der dominanten Koalition verantwortlich sein. Dies legt insbesondere die kontrastierende Analyse mit den strategischen Entscheidungsprozessen der Dirk Rossmann GmbH nahe. Denn hier entstand ein Großteil der Sachkonflikte direkt aus der Reibung zwischen den verschiedenen Funktionsbereichen und den divergenten Perspektiven.

"[...] ein Handelsunternehmen, das nur einkaufsbetrieben ist, ist Scheiße. Man muss das Zeug auch verkaufen können. Und eins, was nur vertriebsgetrieben ist, geht auch nicht. Ein schöner Laden ist auch nicht alles, ne, [...]. Also man braucht die Reibung zwischen diesen beiden Bereichen. Da hat natürlich jeder Funktionsbereich hat seine eigene Perspektive, seine eigenen Probleme, die muss man irgendwie überkreuz legen. Und wenn, wenn die sich sachlich reiben, ist das gut."

(Ehemalige Führungskraft der Anton Schlecker e.K.)

Die Homogenität der dominanten Koalition dürfte hier das Gegenteil bewirkt haben, denn in homogenen und kohäsiven Managementteams kann meist nicht zuletzt deshalb schneller entschieden werden, weil die Homogenität auch das Niveau an Sachkonflikt reduziert. ${ }^{191}$

Mit diesem beobachteten Zusammenhang zwischen dem Grad an Diversität und dem Niveau an Konflikt schließt sich der Kreis. Diese beiden so unterschiedlichen Ausprägungen der Variablen bei der Anton Schlecker e.K. und der Dirk Rossmann GmbH leisten einen Erklärungsbeitrag zu Verlauf und Ergebnissen der strategischen Entscheidungsprozesse der beiden untersuchten Unternehmen. Die Ausprägungen von Diversität und Konflikt sind von großer Bedeutung für das Verständnis, warum die Dirk Rossmann GmbH es vermochte, sich über die Jahre kontinuierlich strategisch zu wandeln, während die Anton Schlecker e.K. strategisch nicht adäquat auf die Dynamik der Umwelt und die Gefahr des selbstverstärkenden Mechanismus reagierte.

Der Zusammenhang zwischen Diversität und Konflikt kann jedoch auch eine

1190 Vgl. S130620WS: So stand noch 2013 auf der Unternehmenswebsite: »Dabei beschränkt sich SCHLECKER konsequent auf den SCHLECKER Drogeriemarkt, der im Durchschnitt auf 200 Quadratmetern Verkaufsfläche rund 4.000 Artikel führt.« Vgl. Wimmer et al. (2005: 124); Voigt (1990: 76).

1191 Vgl. Eisenhardt et al. (1997: 46): »Teams that have members who differ across demographic characteristics [...] are more likely to have confict. [...] In contrast, many low conflict teams have little diversity.« Vgl. auch Pelled et al. (1999: 20f.); Milliken \& Martins (1996); Morrison \& Milliken (2000: 711). 
andere, eine dysfunktionale Seite annehmen. Darauf verweisen die strategischen Entscheidungsprozesse der Unternehmen der zweiten Fallgruppe.

\subsubsection{Diversität und Konflikt in der polyzentrischen Konfiguration der Macht}

Im obigen Abschnitt wurden im Rahmen der beiden monozentrischen Konfigurationen die empirischen Ausprägungen der Variablen Diversität und Konflikt dargestellt, deren Auswirkungen auf den strategischen Entscheidungsprozess maßgeblich zum Ausbleiben der Reaktion auf die Genese der strategischen Pfade in der Phase der Pfadformation beitrugen. In diesem Zuge wurde aufgezeigt, wie die geringe Diversität und das niedrige Niveau an Sachkonflikt innerhalb der weitgehend immunen dominanten Koalition der Anton Schlecker e.K. zu einer ungenügenden Irritation des strategischen Entscheidungsprozesses führten. Darüber hinaus wurde erläutert, inwieweit und wie der Einfluss des Faktors Familie diese Manifestationen und Wirkungszusammenhänge beeinflusste.

Analog zu diesem Vorgehen werden im folgenden Abschnitt die Ausprägungen und Auswirkungen der Variablen Diversität und Konflikt im Rahmen der polyzentrischen Machtkonfiguration der Berentzen-Gruppe AG und der Eckes AG dargestellt. ${ }^{1192}$

\subsubsection{Heterogenität der dominanten Koalition}

Zur Beurteilung der Diversität und der dahinter liegenden Kognitionen und Werte wird zum einen wiederum auf die klassischen demografischen Charakteristika der Managementforschung zurückgegriffen, zum anderen aber auch auf jene Dimensionen, die sich darüber hinaus in der empirischen Untersuchung als bedeutsam für die Ausprägung der Diversität identifizieren ließen. ${ }^{1193}$

\section{Empirische Ausprägungen}

Hinsichtlich der demografischen Charakteristika ist bei der Berentzen-Gruppe AG eine grundsätzlich hohe Variation an Merkmalsausprägungen festzustellen.

Das demografische Charakteristikum des Alters betreffend ist vor allem in der frühen Phase der Pfadformation eine große Streuung innerhalb der dominanten Koalition ersichtlich. ${ }^{1194}$ Von Seiten der Unternehmensfamilien waren

1192 Mit dem Terminus »Koalition« wird häufig eine Einigkeit der Entscheidungsträger assoziiert, auf die der Begriff aber grundsätzlich nicht abstellt. Hambrick (1994) schlagen entsprechend vor, weniger von Topmanagement-Teams als von "top management groups « zu sprechen, um mögliche Fragmentierungen in der Gruppe begrifflich nicht zu verdecken. Vgl. auch Carpenter et al. (2004: $768 \mathrm{ff}$.).

1193 Vgl. Finkelstein et al. (2009: 83ff.); Wiersema \& Bantel (1992: $97 \mathrm{ff}$.$) .$

1194 Hierzu eine ehemalige Führungskraft: »Diese Rollenverteilung im Unternehmen, die 
lange Zeit verschiedene Generationen im Unternehmen aktiv und auch innerhalb der Generationen war die Altersspanne groß. Zudem unterlagen die familienfremden Mitglieder des Topmanagements und des Aufsichtsrats einer natürlichen Fluktuation, die ebenfalls zu Altersunterschieden der Entscheidungsträger führte. Mit dieser Fluktuation gingen sehr unterschiedliche Zeitspannen der Unternehmenszugehörigkeit einher. Auf der einen Seite waren in der dominanten Koalition Mitglieder wie Hans und Friedrich Berentzen vertreten, welche das Unternehmen über viele Jahrzehnte geprägt hatten. Auf der anderen Seite umfasste die dominante Koalition zahlreiche Führungskräfte, die neu zur Berentzen-Gruppe AG gestoßen waren und vorher in anderen Unternehmen tätig gewesen waren. ${ }^{1195}$

Obwohl der Bereich des Vertriebs und Marketings eine hervorgehobene Stellung einnahm und die meisten der Protagonisten ihre beruflichen Erfahrungen in diesem Funktionsbereich gesammelt hatten, ist auch in Bezug auf die funktionale Spezialisierung der Entscheidungsträger keine derart ausgeprägte Ungleichgewichtung zu sehen, wie sie bei der Anton Schlecker e.K. hinsichtlich des Einkaufs vorzufinden war. ${ }^{1196}$

Insgesamt lässt sich hinsichtlich der Merkmale der demografischen Zusammensetzung für die dominante Koalition der Berentzen-Gruppe AG somit eine recht hohe Diversität konstatieren. Über diese demografischen Charakteristika hinaus wies die dominante Koalition aber auch noch hinsichtlich weiterer Merkmale eine hohe Diversität auf. Deren Heterogenität war grundlegend davon geprägt, dass die Mitglieder der dominanten Koalition unterschiedlichen Familien angehörten oder in besonderer Weise mit einem der drei Familienstämme verbunden waren, die sich infolge des Imprints der polyzentrischen Konfiguration formiert hatten. Die meisten der familienfremden Führungskräfte und Aufsichtsräte wiesen zu einem der Subzentren eine besondere emotionale oder professionelle Nähe auf. ${ }^{1197}$

Die Grenzen zwischen diesen verschiedenen Subzentren der dominanten

hatte, glaube ich, einfach was mit den Altersunterschieden zu tun. Der eine war Vertriebschef, da hatte der andere gerade erst mit dem Studium begonnen." Vgl. auch B061218FT.

1195 Vgl. B050929WE:»[...] wechselt ein ehemaliger Spitzenmanager des von Barilla übernommenen Bäckereikonzerns Kamps in den Vorstand der Berentzen-Gruppe.»

1196 Eine ehemalige Führungskraft der Berentzen-Gruppe AG: »Und es gab den Marketingvorstand Jürgen Pabst von Pabst \& Richards. Und es hat sich so ergeben, dass, ja, die nun, wie soll ich sagen, die Marketingabteilung unter der Leitung vom Herrn Pabst sehr stark angewachsen ist und irgendwann nicht mehr so ganz dem entsprach, wie man sich das für einen mittelständischen Spirituosenhersteller vorstellt."

1197 »Wir hatten also richtige Frontenbildung, auch quer durchs Unternehmen, was dem Unternehmen nicht gut getan hat, auch, weil wir haben dann Manager danach beurteilt [...], ob sie mehr auf unserer Seite stehen oder auf der Seite der anderen." 
Koalition waren klar gezogen und relativ stabil. ${ }^{1198}$ Die Abgrenzung wurde grundsätzlich durch die Permanenz des Merkmals der Familienzugehörigkeit befördert. ${ }^{1199}$ Des Weiteren trugen formelle und informelle Mechanismen wie die separate Bestellung der Aufsichtsratsmitglieder nach dem Proporzprinzip oder reziproke Erwartungshaltungen der Solidarität und Loyalität zwischen den Mitgliedern der Subzentren zur Stabilisierung der Grenzen bei. Von erheblicher Bedeutung war, dass die Grenzziehung hinsichtlich bestimmter Aspekte wie der paritätischen Besetzung der Gremien auch rechtlich festgeschrieben worden war. ${ }^{1200}$

Zwei weitere mit der Familienzugehörigkeit verbundene Merkmale verstärkten die Heterogenität der dominanten Koalition in der Phase der Pfadformation: die Trennung der Subzentren in aktiv in die Leitung des Unternehmens eingebundene Gesellschafter einerseits und passive Eigentümer andererseits sowie die unterschiedlichen kollektiven Identitäten der Unternehmensfamilien.

Die Trennung in aktive und passive Gesellschaftergruppen hatte sich nach dem Rückzug von Jürgen Pabst aus dem Vorstand im Jahr 2001 ergeben, nach dem Jan B. Berentzen das letzte in der operativen Leitung des Unternehmens verbliebene Mitglied einer der Unternehmensfamilien war. Somit war nur noch ein Subzentrum im obersten Management des Unternehmens vertreten, während die beiden anderen Stämme das Unternehmen als "passive« Gesellschaftergruppen über die Gremien des Gesellschafterkreises und über den Aufsichtsrat leiteten. ${ }^{1201}$ Die Rollenverteilung im Unternehmen brachte einen sozialen Statusunterschied zwischen den Subzentren und deren Mitgliedern mit sich. Der aktive Stamm verfügte aufgrund der direkten Beteiligung an der Unternehmensführung nicht nur über größere Machtressourcen. Er konnte innerhalb und außerhalb der Familie im Verhältnis zur Eigenkapitalbeteiligung auch einen überproportional großen Anteil des sozio-emotionalen Vermögens am Unternehmen genießen, ${ }^{1202}$ zum Beispiel hinsichtlich des Prestiges des Unternehmerdaseins oder der Befriedigung in der Ausübung von Kontrolle. Es ist

1198 Die Subzentren unterschieden sich hier von den in der Managementforschung häufig als fluide und vom jeweiligen Sachgegenstand abhängig beschriebenen Koalitionen; vgl. Pfeffer (1981); Eisenhardt \& Bourgeois (1988).

1199 Die Familienzugehörigkeit kann ähnlich wie die Rasse oder das Geschlecht als ein unveränderbares Merkmal verstanden werden und somit zu vergleichbar differenzierenden Effekten führen. Vgl. Pelled et al. (1999: 5).

1200 Zur Bedeutung der Institutionen und Mechanismen der Governance für die Intensität der Gruppen- und Stammesbildung vgl. Gersick et al. (1997: 50); Simon et al. (2005: 68).

1201 Der Terminus des "passiven« Gesellschafters wird aufgrund des Machtpotenzials und der aktiven Beteiligung im strategischen Entscheidungsprozess häufig nicht der tatsächlichen Bedeutung solcher Gesellschafter gerecht, aber hier wegen seiner häufigen Verwendung in der Familienunternehmensforschung dennoch verwendet.

1202 Vgl. Berrone et al. (2012); Gómez-Mejía et al. (2007). 
davon auszugehen, dass diese Elemente des sozio-emotionalen Vermögens aber nicht nur den tatsächlich aktiven Protagonisten des Subzentrums zugutekamen, sondern auch den mit ihnen eng verbundenen Mitgliedern des Subzentrums.

Allerdings gab es auch innerhalb der Subzentren große Unterschiede in den Gesellschafterrollen. Während manche Mitglieder Posten im mittleren Management bekleideten, waren andere im Aufsichtsrat aktiv. Wiederum andere hatten über die Wahrnehmung der Pflichten als Eigentümer hinaus überhaupt keinen professionellen Bezug zum Unternehmen. Diese unterschiedlichen Rollen im Unternehmen führten nicht nur zu einem sozialen Statusunterschied zwischen den aktiven und den passiven Familienmitgliedern, sondern erhöhten auch die Differenzen hinsichtlich des sozio-ökonomischen Status der Gesellschafter. Dieser basierte grundsätzlich darauf, dass die Gesellschafter unterschiedlichste Ausbildungswege absolviert hatten und an teils weit voneinander entfernten Wohnorten in diversen Berufsfeldern arbeiteten. Da sie zudem über unterschiedlich hohe Einkommen und finanzielle Ressourcen verfügten, befanden sie sich insgesamt in sehr heterogenen Lebenslagen.

"Weil der eine Stamm ist eben tätig, der andere ist nichttätig, die anderen haben studiert, die anderen haben nicht studiert. Völlig unterschiedlich, divergent."

(Ehemaliger Aufsichtsrat der Berentzen-Gruppe AG)

Die Mitglieder der dominanten Koalition gehörten außerdem familiären Subzentren an, die sehr unterschiedlich ausgeprägte kollektive Identitäten aufwiesen. ${ }^{1203}$

»[...] diese Familienstammstruktur ist auch geprägt durch - Berentzen ist Marke und Pabst und Richards war Handelsmarke. Da kommt das auch her, dieses Thema." (Ehemaliger Vorstand der Berentzen-Gruppe AG)

Im Verlauf divergenter Ko-Evolutionen der Familien mit ihren strategisch unterschiedlich positionierten Unternehmen hatten sich bereits lange vor der Fusion zur Berentzen-Gruppe eigene Identitäten entwickelt: Die Subzentren der Großfamilie Berentzen verstanden sich als Eigentümerfamilien eines 250 Jahre alten traditionellen Familienunternehmens und vor allem - ungeachtet der Börsennotierung und der Verteilung der Stammaktien - als die eigentlichen, angestammten Eigentümer der Berentzen-Gruppe. Im Gegensatz hierzu begriff sich das Subzentrum Pabst \& Richarz eher als professionell orientierte Gesellschaftergruppe eines Getränkekonzerns. Diesem unterschiedlichen Selbstverständnis lag nicht nur zugrunde, dass die Gesamtgruppe den Namen der Familie Berentzen trug, sondern wohl auch der Umstand, dass sich

1203 Vgl. zu Identitäten von Unternehmensfamilien Lubinski (2010); Stamm (2013). Zur Bedeutung von sozialen Identitäten in Gruppenbeziehungen vgl. auch Abrams \& Hogg (1990) und vor allem Tajfel (2010). 
das Unternehmen Pabst \& Richarz im Verlauf seiner Evolution zu einem Handelsmarkenhersteller entwickelt hatte, in dessen volatilem Umfeld mit niedrigen Gewinnmargen operiert wurde und in dem strategisch weniger das Marketing der Familientradition als vielmehr eine größtmögliche Produktivität und Effizienz im Vordergrund stand. ${ }^{1204}$

Aufgrund dieser abweichenden Merkmale der Gesellschafter und der unterschiedlichen Merkmalsausprägungen der klassischen demografischen Charakteristika kann somit zusammenfassend eine sehr hohe Diversität der dominanten Koalition der Berentzen-Gruppe konstatiert werden.

Gleichermaßen von einer hohen Diversität gekennzeichnet war aber auch die dominante Koalition des Vergleichsfalls der Eckes AG. ${ }^{1205}$ In diesem Unternehmen waren nicht nur das familienfremde Topmanagement und der Aufsichtsrat heterogen zusammengesetzt, sondern auch der Gesellschafterkreis.

Diese Diversität war vor allem darauf zurückzuführen, dass bereits mit der vierten Generation in den 1930er-Jahren eine Stammesbildung eingesetzt hatte, die sich in späteren Generationenübergängen reproduziert und weiter verzweigt hatte. ${ }^{1206}$ Aus dieser Aufsplittung resultierte eine Gesellschafterstruktur, in der manche Familienmitglieder noch aktiv in die strategischen Entscheidungsprozesse involviert waren, während andere sich eher passiv auf ihre Gesellschafterrolle zurückzogen und an den Entscheidungsprozessen in deutlich geringerem Maße partizipierten. ${ }^{1207}$

"Das Traditionsunternehmen und die gebundenen Vermögenswerte sind in den vergangenen Jahren zusehends einer hausgemachten Erosion ausgesetzt. Mit jeder weiteren Generation wächst die Zahl der Anspruchssteller auf Erbe oder Ausschüttung, gleichzeitig aber lässt deren unmittelbares Engagement für die Firma nach.»

(Die Lebensmittel Zeitung im Jahr 2012) ) $^{1208}$

1204 Siehe zu dieser Dimension auch die Skizzierung des Imprints des sozio-emotionalen Vermögens in Kapitel D.1. Eine ehemalige Führungskraft zum Kontrast: "Das war 'ne schwierige Geschichte [...] Wer gewohnt ist, wie soll ich das mal sagen, so'n Dampfschiff da zu fahren, wo die Erträge stimmen, hatte plötzlich mit'm Rennboot zu tun."

1205 Vgl. Eckes (2007: 15): »1931: Ludwig Eckes (vierte Generation, Zweig Jakob), zwei Jahre später Peter Eckes (vierte Generation, Zweig Heinrich) und Herbert Eckes (vierte Generation, Zweig Richard) drei Urenkel von Firmengründer Peter Eckes I., beginnen [...] ihre Tätigkeit im Unternehmen. Sie werden über mehr als 40 Jahre den Ausbau des Unternehmens bestimmen."

1206 Vgl. Eckes (2007: 70ff.) zu einem Stammbaum über sechs Generationen.

1207 Vgl. E100825HB.

1208 E100825LZ. Weiter:»Die eigenen Kinder und Verwandten vermochten es kaum, es Ludwig Eckes recht zu machen. Von seinem Selbstverständnis her forderte er größeren Einfluss und mehr Anteile am Unternehmen. Sein Vetter Peter Eckes, selber als eher solider Arbeiter im Hintergrund beschrieben, willigte schließlich ein. Der Polarisierung innerhalb des Clans gab dies zusätzliche Nahrung, so meinen jedenfalls Zeitzeugen [...]. Die Kinder von Peter Eckes, Peter-Eugen (71) und Michael (66), hatten es leichter. In Reichweite ihres 
Diese Rollenverteilung brachte auch bei der Eckes AG Unterschiede im sozialen wie im sozio-ökonomischen Status mit sich. Doch im Gegensatz zur dominanten Koalition der Berentzen-Gruppe AG gehörten alle Subzentren noch demselben Verwandtschaftsnetzwerk an und referenzierten die gleiche Unternehmens- und Familienhistorie. Sie besaßen daher noch eine ausgeprägte gemeinsame kollektive Identität. Summa summarum war die dominante Koalition der Eckes AG somit zwar durchaus divers, wies allerdings einen geringeren Diversitätsgrad auf als die dominante Koalition der Berentzen-Gruppe AG.

\section{Auswirkungen der hohen Diversität bei der Berentzen-Gruppe AG}

Dieser hohe Grad an Diversität innerhalb der dominanten Koalition blieb in der Berentzen-Gruppe AG nicht ohne Auswirkungen auf den Kontext des strategischen Entscheidungsprozesses.

Einerseits dürfte die Heterogenität - entsprechend der oben skizzierten theoretischen Annahmen - das kognitive Potenzial der Gruppe der Entscheidungsträger maßgeblich erhöht haben. Aufgrund der heterogenen Altersstruktur sowie der Mischung aus langjährigen Mitarbeitern und extern rekrutierten Führungskräften kann vermutet werden, dass sehr verschiedene Annahmen über die zukünftige Entwicklung der Umwelt und divergente Perspektiven auf das eigene Unternehmen in den Entscheidungsprozess einflossen. Dies erhöhte das Potenzial einer multiperspektivischen Analyse der strategischen Probleme und einer auf dieser Perspektiven- und Informationsvielfalt beruhenden synthetischen und kreativen Lösungsfindung. ${ }^{1209}$

Andererseits führten insbesondere die Zugehörigkeit zu verschiedenen Unternehmensfamilien, die Trennung in aktive und passive Gesellschafter sowie die voneinander abweichenden kollektiven Identitäten auch zu völlig verschiedenen Perspektiven und divergenten Interessen im strategischen Entscheidungsprozess.

"Pabst und Richards war ja interessiert, eine Handelsmarke zu machen. Das heißt, Marke- schwarz weiß, Marke ist mir egal, push mal meine Produkte, weil ich bin der Abfüller und da hab ich eventuell mehr davon. Und Berentzen sagt, ich bin 300 Jahre Edelobst oder was auch immer, versuche mal, mich zu forcieren. Und vielleicht liegt in dieser Struktur schon, schon [...] das Spannungsfeld und eines der Grundübel, warum das alles so ist."

(Ehemaliger Vorstand der Berentzen-Gruppe AG)

Onkels Ludwig begnügen sie sich mit Funktionen in der Rohwaren-Beschaffung und Technik. Später konzentrieren sie sich auf die Rolle als Gesellschafter.»

1209 Vgl. Finkelstein et al. (2009: 153): »The quality of strategic decisions (as defined by the generation of multiple feasible alternatives and the comprehensive evaluation of those alternatives) is positively associated with TMT heterogeneity [...].«Vgl. auch Milliken \& Martins (1996: 416); Steinmann \& Schreyögg (2005: 627f.). 
Aus der Trennung in aktive und passive Gesellschafter resultierten Informationsasymmetrien, die das Entstehen von abweichenden Betrachtungsweisen auf die strategische Entwicklung des Unternehmens beförderten. Was vom aktiven Subzentrum - dem mehr und bessere Informationen zur Verfügung standen als kurzfristiger Gewinneinbruch aufgrund von Einmaleffekten wahrgenommen wurde, erschien den passiven Gesellschaftern lediglich als Fortsetzung einer langanhaltenden Negativentwicklung.

Die unterschiedlichen sozioökonomischen Hintergründe der Gesellschafter führten zu materiellen Interessenunterschieden, zum Beispiel hinsichtlich der Höhe eines zufriedenstellenden Ertrags der Eigenkapitalanteile oder der Möglichkeiten eines Verzichts auf solche Erträge im Zuge der Unternehmenskrise. ${ }^{1210}$ Während manche Gesellschafter über höhere finanzielle Ressourcen verfügten, waren andere Mitglieder des heterogenen Gesellschafterkreises in deutlich höherem Maße auf diese Erträge angewiesen.

An der Frage der zufriedenstellenden Rendite des Eigenkapitals lässt sich die Auswirkung des unterschiedlich verteilten sozio-emotionalen Vermögens am Unternehmen illustrieren. Während das aktive Subzentrum und vor allem dessen aktive Gesellschafter durch das Sozialprestige des Unternehmerdaseins oder die Kontrollmacht über das Unternehmen in höheren Maße für niedrigere finanzielle Erträge kompensiert wurden, konnten die passiven Gesellschafter nicht von einer vergleichbaren kompensatorischen Wirkung profitieren. Sie waren entsprechend auch in geringerem Maße zu finanziellem Verzicht bereit.

Die Positionen zu solchen Thematiken wurden auch von den unterschiedlich starken emotionalen Bindungen und der jeweiligen Identifikation mit dem Unternehmen beeinflusst. Während das aktive Subzentrum der Familien Berentzen eine hohe emotionale Verbundenheit mit dem Unternehmen aufwies und sich auch das eher passive Subzentrum der Familie noch stark mit dem Unternehmen und bestimmten organisationalen Lösungen identifizierte, waren

1210 Vgl. Klein (2010: 148); allgemein auch Wöhe \& Döring (2005: 268): »[J]e größer die Zahl der Gesellschafter und je ungleicher das Privatvermögen ist, desto unterschiedlicher ist in der Regel die Interessenlage. « Zu solchen Formen der Heterogenität im Gesellschafterkreis und Möglichkeiten deren Managements vgl. auch Felden \& Hack (2014: 287ff.). Ein Gesellschafter: »[...] eine Firma ist, in der unser gesamtes Vermögen steckt. Und da, das ist auch unseres, nicht nur ihres, sie kann ja sagen, ich verzichte doch gerne mal drei oder zehn Jahre auf Einkünfte. Das soll sie ja gerne machen. Aber es ist auch unseres, wir können da gar nicht drauf verzichten. Wir dürfen nicht vergessen, es sind ja Anteilseigner nicht nur reiche Leute. Da haben Sie auch in der Verwandtschaft Leute, die dringend angewiesen sind darauf, dass es eine Dividende gibt, eine Ausschüttung.« Im Allgemeinen kommen die unterschiedlichen ökonomischen Interessen in Fragen zur Finanzierung des Unternehmens, der Höhe der Ausschüttungen oder der strategischen Ausrichtung des Unternehmens zum Ausdruck. Vgl. Felden \& Hack (2014: 287); Gersick et al. (1997: 45). 
solche emotionalen Bindungen im Subzentrum Pabst \& Richarz naturgemäß deutlich schwächer ausgeprägt.

»Und da war es, glaube ich, dass es emotional unterschiedliche Bindungen zum Unternehmen gab, was die, was die verschiedenen Familienstämme betrifft.«

(Ehemaliger Aufsichtsrat der Berentzen-Gruppe AG)

Für das Subzentrum Pabst \& Richarz rückten daher finanzielle Aspekte stärker in den Vordergrund und seine Vertreter betrachteten das Unternehmen in erster Linie als Vermögenswert und Einkommensquelle. Aus der emotionsgeladenen Perspektive der Berentzen-Subzentren musste eine solche Haltung wiederum so wirken, als agiere der Stamm Pabst \& Richarz als passiver Nutznießer des eigenen langjährigen Engagements für das Unternehmen.

Mit den unterschiedlichen Identitäten gingen zudem divergente Werthaltungen und Einstellungen einher. Da sich die Subzentren der Familie Berentzen als die angestammten Eigentümerfamilien eines 250 Jahre alten Traditionsunternehmen begriffen, legten sie entsprechend Wert auf die Bewahrung der familiären Reputation und Tradition sowie den Erhalt des transgenerationalen Erbes in seiner bisherigen Form. ${ }^{1211}$ Sie maßen daher der Autonomie des Unternehmens einen ebenso hohen Wert bei wie der familiären Kontrolle der Familie über die Berentzen-Gruppe in Form einer aktiven Beteiligung am operativen Management.

Im Subzentrum Pabst \& Richarz hatte sich im Verlauf der Ko-Evolution mit ihrem Unternehmen ein anderes Wertesystem gebildet und die Unternehmensfamilie maß zum Beispiel der Professionalität einen deutlich stärkeren Stellenwert bei als der Tradition. ${ }^{1212}$ Sie räumte auch der familiären Beteiligung an der Geschäftsführung eine geringere Bedeutung ein und erachtete die Unabhängigkeit der Berentzen-Gruppe nicht als ein derart hohes Gut, wie es die namensgebenden Eigentümerfamilien taten.

Diese Abweichungen in Interessen, emotionalen Bindungen und Werthaltungen führten schlussendlich zu unterschiedlichen Perspektiven und Präferenzen im strategischen Entscheidungsprozess.

»So, und als wir dann sagten, Haselünne, daraus machen wir doch jetzt am besten ein Museum. Weil das ist ja schön, wenn die Busse mit den Kaffeerentnern da ankommen,

1211 Vgl. hierzu die Skizzierung des Imprints des sozio-emotionalen Vermögens in Kapitel D.1. Zur Bedeutung von Werten für die Entscheidungen des TMT vgl. die theoretischen Hypothesen von Hambrick \& Brandon (1988). Die Autoren machen vor allem deutlich, dass sich die Wertabhängigkeit der Handlungen weder unterbinden noch vollständig auf ihre Interessen zurückdrängen lässt. Vgl. hierzu auch Bresser (2010: 37).

1212 Ein Gesellschafter: »[...] und unsere Leute stellten relativ schnell fest, dass dort eine nie gesehene unprofessionelle Vorgehensweise da war.« Ebenso ein ehemaliger Aufsichtsrat: "[E]s sind ja in der Regel die Probleme dann da, wenn zwei Welten aufeinander treffen. Wenn Sie eine hochprofessionelle Welt gegen eine relativ konservative Welt stellen." 
dann führen wir die durch, fahren, und der Onkel Paul kann erzählen, wie hat das alles angefangen, und wie macht man überhaupt einen Weinbrand oder einen Korn. Das ist ja schön. Hinterher auf den Hof mit den Leuten, dann können sie noch was einkaufen auf der Tenne, und kriegen vielleicht ein Würstchen oder was. Alles wunderbar, nur mit moderner Produktionsmechanik hat das hier nix zu tun. Und wir sind in der Produktion zu teuer. Wenn Sie das darstellen, oder per Frage beantwortet haben wollen, auch in der Gegenüberstellung mit anderen Produktionsstätten, haben wir uns aber gar nicht mehr darüber unterhalten. Sondern, jetzt kommt es, wenn wir das hier wegnehmen und woanders hin verlagern, dann braucht der Onkel Karl nie wieder sich um das Landratsamt zu bewerben, und Tante Ilse wird nie im Leben wieder gewählt als Bürgermeisterin von Haselünne. Das war die Qualität der Auseinandersetzung dann.« (Ehemaliger Aufsichtsrat der Berentzen-Gruppe AG)

Über die Entwicklung solcher divergenten Perspektiven hinaus führte die Diversität zwischen den Subzentren aber vor allem auch dazu, dass sich aus den eigenständigen kollektiven Identitäten der Subzentren ein immer stärkeres Gemeinschaftsgefühl (»Wir-Gefühl«) entwickelte, dessen Bezugspunkte nicht mehr nur in sich selbst lagen, sondern vor allem auch in der Abgrenzung zur anderen, als andersartig wahrgenommenen Gruppe. Dies erhöhte die Kohäsion in der eigenen Gruppe, führte aber letztlich dazu, dass die Differenz zur anderen Gruppe Bestandteil der eigenen Gruppenidentität wurde und die verschiedenen Subzentren sich immer stärker zu sozial gegeneinander abgegrenzten Einheiten entwickelten. ${ }^{1213}$ Die Identifikation mit den anderen Subzentren und der Gesamtgruppe wurde mithin erschwert. Diese Entwicklung beförderte die Entstehung von Stereotypen, vor allem gediehen in diesem Wahrnehmungsrahmen Neid und Missgunst gegenüber den anderen Subzentren.

Die Heterogenität schuf so großes Konfliktpotenzial, das von einer ähnlichen Merkmalsausprägung noch verstärkt wurde: Die beiden Repräsentanten der Stämme im Management der Berentzen-Gruppe AG wiesen ungefähr das gleiche Alter auf und über die soziale Vergleichsperspektive trug dieses gleiche Alter zu Rivalitäten zwischen der Subzentren bei. ${ }^{1214}$

1213 Vgl. Steinmann \& Schreyögg (2005: 635) zu dieser Form der Identitätsbildung oderverstärkung in Prozessen zwischen Gruppen und deren Konsequenzen; für einen grundlegenden Überblick über verschiedene Aspekte der sozialen Identitätstheorie vgl. auch Abrams \& Hogg (1990). Der Grad an Integration von Gruppen in Entscheidungsprozessen nimmt im Allgemeinen mit zunehmender Diversität ab; vgl. Milliken \& Martins (1996: 403).

1214 Vgl. Pelled et al. (1999: 21); auch Hambrick (1994: 202), der erhöhte Rivalität dann beobachtet, wenn »several group menbers were the same age and vying to be the next group leader«. Die Forschung zu Familienunternehmen beschreibt Fälle von mangelndem Selbstwertgefühl, das aus solchen Statusunterschieden resultiert und das es dann teilweise in anderer Weise oder zu einem späteren Zeitpunkt zu kompensieren gelte. Vgl. Baus (2013: $41 \mathrm{f}$.). 


\section{Einfluss des Faktors Familie}

Die Diversität der Mitglieder der dominanten Koalition der Berentzen-Gruppe AG erwuchs zum Teil direkt aus der Ko-Evolution des Unternehmens mit der Familie. Im Verlauf des Wachstums von Unternehmen und Familie war der Kreis der Gesellschafter von Generation zu Generation stetig angewachsen und mit der steigenden Anzahl der Gesellschafter ging eine zunehmende Heterogenität einher. Mit steigendem Verwandtschaftsgrad nahm die emotionale Bindung zwischen den weiter entfernten Familienmitgliedern ab, während die emotionale Bindung aufgrund der höheren Kommunikationsdichte zwischen den enger verwandten Mitgliedern der gleichen Linie gewahrt blieb. Diese familiären Dynamiken trugen zur Bildung von Subzentren entlang der Abstammungslinien der Unternehmensfamilien bei, zwischen denen dann auch die Status- und Interessenunterschiede immer deutlicher hervortraten. ${ }^{1215}$ Der größere Teil der Komplexität der sozialen Architektur der Berentzen-Gruppe AG und der Heterogenität der dominanten Koalition resultierte allerdings direkt aus der Fusion der J. B. Berentzen mit der Weinbrennerei Pabst \& Richarz. Im Zuge dieser Fusion traten gar drei weitere Unternehmensfamilien ins Unternehmen ein. ${ }^{1216}$

\subsubsection{Sach- und Beziehungskonflikte in polyzentrischen Konfigurationen}

Aufgrund der hohen Diversität verfügte die dominanten Koalition der Berentzen-Gruppe über ein grundsätzlich hohes Managementpotenzial, um im Zuge ihrer Entscheidungsprozesse auf den emergierenden strategischen Pfad $\mathrm{zu}$ reagieren und einen strategischen Wandel zu vollziehen. Allerdings zeigte sich auch bei der Berentzen-Gruppe AG, dass es entscheidend von den Interaktionsund Kommunikationsmustern innerhalb der weitgehend autonomen dominanten Koalition abhängt, inwiefern diese Potenziale tatsächlich für den strategischen Entscheidungsprozess fruchtbar gemacht werden können.

\section{Empirische Ausprägungen}

Bei der Berentzen-Gruppe AG wurden die Interaktion und die Kommunikation der dominanten Koalition in der Phase der Pfadformation maßgeblich von der skizzierten Heterogenität beeinflusst. ${ }^{1217}$ Aus der mit der Größe und den ver-

1215 Die Forschung zu Familienunternehmen betont ebenfalls die Bedeutung familiärer Dynamiken für die Entstehung von Subzentren im Unternehmen. Vgl. Gersick et al. (1997: 50); Simon et al. (2005: 82). Obgleich im Fall der Berentzen-Gruppe AG auch die Fusion mit Pabst \& Richarz von Bedeutung war, entstehen die meisten polyzentrischen Konfigurationen allein aufgrund solcher familiärer Prozesse.

1216 Vgl. allgemein zu Familienunternehmen im Besitz mehrerer Familien Klein (2010: 184ff.); zu Mechanismen deren Komplexitätsbewältigung Pieper et al. (2015).

1217 Vgl. Steinmann \& Schreyögg (2005: 634), die für Gruppen im Allgemeinen konstatieren, die »Art der Interaktion zwischen Gruppen« sei »sehr stark durch ihre Unterschiedlichkeit bestimmt «. Sie weisen darauf hin, dass das Konfliktpotenzial mit einer steigenden Anzahl 
schiedenen Dimensionen der Diversität einhergehenden Heterogenität resultierten zu Beginn der Pfadformation strukturelle Reibungen entlang der divergenten Interessen und unterschiedlichen Identitäten. An diesen entfachte sich der Konflikt innerhalb der dominanten Koalition, der sich schlussendlich zu der in Teil C dargestellten selbstverstärkenden sozio-emotionalen Dynamik auswuchs. ${ }^{1218}$

Die unterschiedlichen professionellen Erfahrungen der Akteure in Markenund Handelsmarkenunternehmen ermöglichten zunächst einen fruchtbaren Austausch unterschiedlicher Perspektiven und konstruktive Auseinandersetzungen. Mit der Zeit erreichte der Grad an sachorientiertem Konflikt aber ein sehr hohes Niveau.

»[...] als der Markt immer schwieriger wurde, und man sich auch nicht so recht auf eine gemeinsame Strategie verständigen konnten, das merkt man dann, das können Sie dann als Kern der Strategiekrise bezeichnen [...] Also ich nehm mal ein Beispiel, die von Pabst in Teilbereichen durchgesetzte weitere Grädigkeitsabsenkung auf 15 oder 16 Prozent. Haben wir gesagt, das ist keine Spirituose mehr, da sind wir ganz sicher, dass das also ein Korntrinker nicht mehr mitmacht [...] hatten selber das Empfinden, das trinken wir nicht mehr, wenn ein Apfelkorn 15 Prozent hat. und da machte sich aber schon bemerkbar, dass also wir Mühe hatten auf einen strategischen gemeinsamen Nenner zu kommen. Das wurde dann im Laufe der Jahre immer schlimmer.» (Ehemaliger Aufsichtsrat der Berentzen-Gruppe AG)

Über Konflikte zu Sachfragen hinaus führten die Interessenunterschiede im heterogenen Gesellschafterkreis zu einem Wettbewerb um die knappen Ressourcen des Unternehmens. ${ }^{1219}$ Strittig waren zum Beispiel die Höhe der Bezüge der aktiven Gesellschafter oder die Höhe der Ausschüttungen.

Die unterschiedlichen Interessen trugen des Weiteren zur Auseinandersetzung um Einfluss und Kontrolle bei. Zur Verfolgung der Partikularinteressen bedurfte es entsprechender Machtressourcen, mit denen die eigenen Ziele gegenüber den anderen Subzentren durchgesetzt werden konnten. Die Machtressourcen konnten aber, zumindest relativ gesehen, nur auf Kosten der anderen Subzentren erhöht werden. Somit entzündeten sich an den Interessenunter-

von Familienmitgliedern zunimmt. Vgl. auch Eisenhardt \& Bourgeois (1988: 759); Williams \& O'Reilly III (1998).

1218 Vgl. Amason \& Sapienza (1997) zur positiven Korrelation von TMT-Größe und Sach- und Beziehungskonflikten; auf diesen Zusammenhang weisen auch von Schlippe \& Kellermanns (2008: 48) und Simon (2012: 73) hin.

1219 Vgl. Steinmann \& Schreyögg (2005: 635). Die Höhe der Bezüge der aktiven Gesellschafter und die Höhe der Ausschüttungen sind zwei klassische Streitgegenstände in Familienunternehmen. Vgl. u. a. die empirische Studie zu Konflikten in Familienunternehmen von Großmann (2014: 184). 
schieden nicht nur Sachkonflikte, sondern auch Beziehungskonflikte um Macht und Einfluss. ${ }^{1220}$

"Der wollte ihn unbedingt ablösen und hat daran gearbeitet. Das ist das einzige, was ihn interessiert hat [...] alles andere interessierte ihn nicht. [...]. Ja, der hat überall die Schalthebel gedreht und versucht, sich 'ne Position aufzubauen, um dahin zu kommen. Er wollte in der Sonne stehen. Da oben dachte er, steht er da in der Sonne. Da war aber alles Mist drum rum, ne. Er wollte den Job von Jan Berentzen, ja. Unbedingt, unbedingt."

(Ehemalige Führungskraft der Berentzen-Gruppe AG)

Das hohe Maß an Beziehungskonflikt speiste sich aber vor allem aus den faktischen und gefühlten Statusunterschieden innerhalb der dominanten Koalition.

"[...] aber es ist tatsächlich so, mehrfach ist die Bilanz, die Balance verletzt worden, mal von der einen, mal von der andern Seite. Also so, dass am Ende des Tages die stärkste Verletzung der Bilanz, Balance war eben vom Empfinden her - Mensch, jetzt hat man die Seite Pabst und Richarz, unsere Seite sozusagen, ist rausgedrängt, aus dem Management, was sollen wir da noch, ja? Und dann wurde eben ganz stark betrieben, ja wenn die Seite Pabst und Richarz schon rausgedrängt wurde aus dem Management, dann muss eigentlich auch die Seite Berentzen rausgedrängt werden."

(Ehemaliger Aufsichtsrat der Berentzen-Gruppe AG)

Diese Beziehungskonflikte fanden in den sich gegeneinander differenzierenden Identitäten der Subzentren einen fruchtbaren Boden, nicht zuletzt, weil diese Differenzbildung entlang des unveränderlichen Merkmals der Familienzugehörigkeit erfolgte und somit den Perspektivwechsel erschwerte. ${ }^{1221}$

Angesichts der multiplen Bruchstellen innerhalb der dominanten Koalition lässt sich ein bedeutender Unterschied zur Diversität und der ausgeprägten Konfliktkultur bei der Dirk Rossmann GmbH feststellen. Dort war die Diversität der dominanten Koalition auf aufgabenbezogene Merkmale wie den funktionalen Hintergrund der Akteure begrenzt. Die Sachkonflikte entzündeten sich entsprechend an abweichenden funktionalen Perspektiven oder anderen Annahmen über die Umweltentwicklung. Im Gegensatz hierzu basierte die Heterogenität der dominanten Koalition der Berentzen-Gruppe AG auf einer Vielzahl von anders gearteten und tiefgreifenden Merkmalsausprägungen, die von divergenten strategischen und ökonomischen Interessen bis hin zu unterschiedlichen Werten reichten. Hier führten nicht nur konträre Perspektiven aufgrund einer anderen Spezialisierung zum Sachkonflikt, sondern auch eine unterschiedlich starke emotionale Verbundenheit mit dem Sachthema oder dessen Einordnung in ein anders ausgestaltetes Wertesystem. Diese Komplexität und

1220 Vgl. zur Definition von Beziehungskonflikten Jehn \& Bendersky (2003: 200).

$1221 \mathrm{Zu}$ diesem Zusammenhang zwischen permanenten Merkmalen und Beziehungskonflikt vgl. Pelled et al. (1999: 5). 
Vielschichtigkeit der entgrenzten Diversität dürfte maßgeblich zu dem hohen Grad an Sach- und Beziehungskonflikt beigetragen haben, ${ }^{1222}$ nicht zuletzt auch deshalb, weil aufgrund der Vielfalt an bedeutsamen sozio-ökonomischen Zielen die konfliktbändigenden Kräfte ökonomischer Ziele schwächer ausgeprägt waren. ${ }^{1223}$

Bei der Eckes AG basierte die Diversität ebenfalls auf einer Mischung aus divergenten Interessen, unterschiedlicher emotionaler Verbundenheit zum Unternehmen und Statusunterschieden. ${ }^{1224}$ Auch hier resultierten nicht nur zahlreiche Sachkonflikte, sondern auch substanzielle Beziehungskonflikte.

»Der Streit unter den Familiengesellschaftern der Getränkefirma hat die Fusion mit der italienischen Campari-Gruppe scheitern lassen [...] [es] hatte sich die Familie Eckes nicht auf ein gemeinsames Vorgehen einigen können [...], nun hat der Streit einen weiteren Höhepunkt erreicht. Laut Manager Magazin beantragte Harald EckesChantré, Sohn des verstorbenen Firmenpatriarchen Ludwig Eckes, in der jüngsten Hauptversammlung, dem Vorstandsvorsitzenden Herbert Verse und dem Spirituosenvortand Axel Thies die Entlastung zu verweigern. In der Abstimmung konnte sich Harald Eckes-Chantré jedoch nicht gegen seine Verwandten durchsetzen. Kurz nach dem Eklat war der Aufsichtsratsvorsitzende, der Anwalt Brun-Hagen Hennerkes, zurückgetreten."

(Das Manager Magazin im Jahr 2000) ${ }^{1225}$

Parallel zur Ausbildung von Familienstämmen traten solche Sach- und Beziehungskonflikte bei der Eckes AG über viele Jahrzehnte immer wieder auf. So war bereits 1955 nach langwierigen Konflikten ein Stamm aus dem Unternehmen ausgeschieden. ${ }^{1226} 1983$ hatte sich ein weiterer Gesellschafter aufgrund von

1222 Die Beobachtungen in dieser Studie decken sich mit den Ergebnissen zu Konflikten in strategischen Entscheidungsprozessen aus der Managementforschung. Diese geht davon aus, dass unterschiedliche Ausprägungen hinsichtlich einiger weniger Merkmale zu funktionalen Sachkonflikten führen, während ein Konglomerat unterschiedlicher Merkmalsausprägungen das Entstehen von Beziehungskonflikten fördere. Vgl. Pelled et al. (1999: 20f.).

1223 Vgl. Eisenhardt \& Bourgeois (1988) zu der konfliktbändigenden Wirkkraft ökonomischer Ziele; Pfeffer (1981) zur erhöhten Konfliktanfälligkeit von Organisationen wie Universitäten, in denen solche weitgehend geteilte Ziele wie das Gewinnstreben fehlen.

1224 Vgl. E100825LZ; auch E100825LZ: »[....] berichten Insider von widerstrebenden Interessen, bei denen es im Wesentlichen um die Frage geht, wie es mit Eckes weitergehen soll. Überliefert sind heftige Diskussionen um eine angedachte Partnerschaft mit Campari, die im Jahr 2000 angeblich an der Bewertung des Unternehmens Eckes scheiterte. Schon damals gibt es Hinweise, wonach Harald Familienmitglieder auf Basis eines Firmenwertes von 660 Millionen DM auszahlen will. Seine Schwester hält jedoch mindestens 750 Millionen DM für angemessen."

1225 E000920MM.

1226 Vgl. Eckes (2007: 21): »Nach langen unerfreulichen Auseinandersetzungen scheidet Herbert Eckes [...], der 1933 in das Unternehmen eingetreten war, nach einem Vergleich 1955 rückwirkend aus.« Vgl. auch Eckes (2007: 32). 
Konflikten über die strategische Ausrichtung des Unternehmens aus der Leitung des Unternehmens zurückgezogen.

\section{Auswirkungen des hohen Konfliktniveaus bei der Berentzen-Gruppe AG}

Der Fall der Anton Schlecker e.K. verweist darauf, dass ein zu geringes Maß an sachorientierten Auseinandersetzungen und Konflikten zu einer nicht ausreichenden Irritation des strategischen Entscheidungsprozesses durch unterschiedliche Perspektiven und Meinungen führen kann. In Verbindung mit der geringen Diversität trug das niedrige Niveau an Sachkonflikt maßgeblich zu der ausbleibenden strategischen Reaktion der Anton Schlecker e.K. auf den emergierenden Pfad bei.

Die skizzierte Dynamik des Konflikts bei der Berentzen-Gruppe AG verweist jedoch darauf, dass auch zunächst rein sachorientierte Konflikte nicht nur positive Auswirkungen haben. ${ }^{1227}$ Die Forschung $\mathrm{zu}$ Konflikten in Entscheidungsprozessen verweist darauf, dass andauernde sachliche Auseinandersetzungen Intoleranz und Ungeduld hervorrufen können, wenn Akteure das Gefühl haben, dass die ihrer Ansicht nach korrekte eigene Ansicht zur Thematik auch nach längerer Diskussion nicht zu einem Ergebnis führt. Kritik an Ideen könne dazu führen, dass Akteure nicht nur ihren Vorschlag abgewertet sehen, sondern auch ihre Kompetenz. Die Negation der eigenen Meinung könne persönlich genommen werden und zu kleinen emotionalen Verletzungen führen. Langfristig könne ein hohes Maß an Sachkonflikt auch zum Entstehen eines raueren Konfliktklimas führen, welches wiederum die Gefahr von emotionalen Verletzungen berge. Auf diesem Wege könne aus einem hohen Grad an Sachkonflikt mit der Zeit Beziehungskonflikt erwachsen. ${ }^{1228}$ Die skizzierte Konfliktentwicklung bei der Berentzen-Gruppe AG in der Phase der Pfadformation illustriert dies.

Die hier beobachtete sozio-emotionale Dynamik zeigt, dass die Beziehungsebene vor allem dann rasch erreicht ist, wenn der Sachkonflikt substanzielle Interessen, Emotionen oder Werte berührt. Die Beziehungsebene schwingt zwar in Konflikten stets mit und die Zuteilung von Rollen und Ressourcen kann von den Beteiligten immer als soziale Positionierung und Bewertung des eigenen Status verstanden werden. Doch ist die Gefahr einer Konflikteskalation vor allem dann hoch, wenn die Identifikation mit dem Streitgegenstand sehr stark ist - so

1227 Vgl. die beiden Meta-Analysen der Konfliktforschung von de Wit et al. (2012) und De Dreu \& Weingart (2003). Diese Beiträge zeigen, dass nicht nur Beziehungskonflikte, sondern auch Sachkonflikte einen negativen Einfluss auf Gruppen und deren Dynamiken haben können. Sachkonflikt führt zwar häufig zu einer erhöhten Produktivität und Innovation, verringert aber nicht selten auch den Grad an Vertrauen und die Kohäsion innerhalb der Gruppe. Vgl. de Wit et al. (2012: $361 \mathrm{f}$.).

1228 Vgl. Amason (1996: 141f.); auch Tjosvold (1991). 
stark wie beispielsweise in der Frage der Schließung des traditionellen Standorts. $^{1229}$

Bei der Berentzen-Gruppe AG resultierten aus den sachlichen Auseinandersetzungen Spannungen, die sich im Verlauf der Zeit immer stärker auf der Beziehungsebene niederschlugen und von dort wieder auf die Sachebene zurückwirkten. ${ }^{1230}$ Infolge der Vermengung der beiden Konfliktarten und den dysfunktionalen Auswirkungen nahm in der Phase der Pfadformation die Qualität der Entscheidungsprozesse stark ab. ${ }^{1231}$ Wertvolle Ressourcen der Entscheidungsträger und des Unternehmens wurden vom Konflikt beansprucht, der von den strategischen Aufgaben ablenkte. Zudem senkte der Konflikt die Bereitschaft zum Austausch von Informationen und Ideen, nicht zuletzt weil der Beziehungskonflikt massiv das Vertrauen zwischen den Subzentren minderte und die Identifikation mit der Gesamtgruppe der dominanten Koalition verringerte. Die dysfunktionalen Auswirkungen der Konflikte verdrängten am Ende auch die funktionalen Effekte des Sachkonflikts.

Letztlich lähmte der Konflikt als "parasitäres System« die kollektiven Entscheidungsprozesse, verhinderte zugleich eine effektive Kontrolle der strategischen Entwicklung und mündete im in Teil $\mathrm{C}$ beschriebenen Lock-in der Berentzen-Gruppe AG.

Wie eingangs dieses Abschnitts dargestellt, war auch bei der Eckes AG die Interaktion und Kommunikation innerhalb der dominanten Koalition von Sachund Beziehungskonflikten negativ beeinträchtigt. Dieses Unternehmen konnte jedoch trotz des hohen Konfliktniveaus einen bemerkenswerten strategischen Wandel vollziehen. Dieser Unterschied erklärt sich vor allem dadurch, dass die Konflikte bei der Eckes AG nicht im gleichen Maße wie bei der Berentzen-

1229 Vgl. de Wit et al. (2012: 362); Pelled et al. (1999: 6f.).

1230 Vgl. zu dieser Interdependenz Pelled et al. (1999: 7).

1231 Beziehungskonflikte werden von der Konfliktforschung meist als rein dysfunktional für die Performance von Gruppen und deren Entscheidungsprozesse erachtet. Vgl. de Wit et al. (2012: 362) für einen Überblick über relevante Studien, die zu diesem Ergebnis kommen. Amason (1996) zufolge leidet die Qualität strategischer Entscheidungen unter einem hohen Grad an Beziehungskonflikt. Eisenhardt et al. (1997) stellen ebenfalls fest, dass Beziehungskonflikte die Interaktionsprozesse von Gruppen behindern und die Performance des strategischen Entscheidungsprozesses mindern. Vgl. des Weiteren Jehn (1994) und Jehn (1995: 276), die keinen negativen Zusammenhang zwischen Beziehungskonflikten und der Performance feststellen kann und vermutet, dass die Konfliktparteien dem Konflikt aus dem Weg gehen. Dies ist gerade in Entscheidungsprozessen der dominanten Koalition allerdings nicht möglich. Zu negativen Auswirkungen von Beziehungskonflikten speziell in Familienunternehmen vgl. Eddleston \& Kellermanns (2007: $545 \mathrm{ff}$.), die in ihrer Studie den negativen Zusammenhang zwischen Beziehungskonflikten und der Perfomance von Familienunternehmen nachweisen; auch von Schlippe \& Kellermanns (2008). 
Gruppe AG den strategischen Entscheidungsraum erreichten und sich dort als parasitäres System etablieren konnten.

Das Übergreifen der Konflikte auf den strategischen Entscheidungsraum lässt sich weitestgehend auf die in Teil C erläuterte selbstverstärkende sozioemotionale Dynamik zurückführen, die den Konflikt immer weiter antrieb. Doch münden, obwohl Konflikten stets eine Tendenz zur Selbstverriegelung und Fortsetzung inhärent ist, nicht alle Konflikte im Unternehmen in strategischer Persistenz des gesamten Unternehmens. ${ }^{1232}$ Hinsichtlich dieses Schritts zur strategischen Persistenz verweist insbesondere die Gegenüberstellung mit der Eckes AG auf eine Reihe weiterer Merkmale, die dazu beitrugen, dass den Wirkkräften des selbstverstärkenden Mechanismen nicht begegnet wurde und folglich nicht auf den emergierenden strategischen Pfad reagiert werden konnte. Diese Merkmale sind in weiten Teilen auf den Einfluss des Faktors Familie zurückzuführen.

\section{Einfluss der Familie}

Für die dysfunktionalen Auswirkungen der Konflikte auf den strategischen Entscheidungsprozess in der Phase der Pfadformation war bei der BerentzenGruppe AG grundsätzlich die Gestaltung der familiären Repräsentanz im strategischen Entscheidungsraum von großer Bedeutung. Dies tritt insbesondere im Vergleich zur Eckes AG deutlich hervor.

Wie bei der Berentzen-Gruppe AG durchliefen auch bei der Eckes AG die strategischen Entscheidungsprozesse lange ein einziges Zentrum der betrieblichen Willensbildung. ${ }^{1233}$ Allerdings waren bei der Eckes AG im Verlauf der Entwicklung des Unternehmens durch unterschiedliche Governance-Strukturen nach und nach verschiedene "Schotten« zwischen die familiären Dynamiken und den strategischen Entscheidungsraum eingezogen worden. ${ }^{1234}$ Bereits drei Jahre vor dem Einstieg der fünften Generation im Jahr 1975 wurden Stiftungen zur Regelungen der Gesellschafterbeziehungen gegründet sowie ein Beirat etabliert und ein Fremdgeschäftsführer bestellt. Diese Governance-Strukturen halfen dabei, die Durchschlagskraft der Konflikte, die mit dem Ausstieg des

1232 Zwischen der Selbstverriegelung des Konflikts und der »eingelockten« strategischen Entwicklung des gesamten Unternehmens muss analytisch klar unterschieden werden.

1233 In diesem Zentrum bestimmten die familiären Subzentren und deren Mitglieder nicht unbedingt die operative Aufgabenerfüllung, aber doch weitgehend den strategischen Entscheidungsprozess. Das Topmanagement und der Aufsichtsrat waren zwar weniger von der Eigentümerfamilie beeinflusst als bei der Berentzen-Gruppe AG, aber sie bildeten keinesfalls separate Zentren der Willensbildung.

1234 Für einen Überblick über Formen der "Family Business Governance« vgl. Felden \& Hack (2014: $281 \mathrm{ff}$.). 
Gesellschafters Michael Eckes aus dem Management verbunden waren, auf den strategischen Entscheidungsprozess zu mindern. ${ }^{1235}$

Auch in den folgenden Jahrzehnten erfolgten bei der Eckes AG meist erst Veränderungen in der organisationalen Struktur und familiären Repräsentanz, bevor schwierige Entscheidungen zum strategischen Wandel gefällt werden konnten. Der Übernahme der Fruchtsaftsparte von Melitta ging beispielsweise die Rechtsformänderung in eine AG und die Aufteilung des Unternehmens in die beiden eigenständigen Sparten Spirituosen und alkoholfreie Getränke voraus, insbesondere aber auch der gleichzeitige Rückzug der beiden Gesellschafter Harald Eckes-Chantré und Peter Eckes aus dem Management und deren Wechsel in den Aufsichtsrat. ${ }^{1236}$ Durch diese Anpassungen der familiären Repräsentanz an interne und externe Herausforderungen blieben im wenige Jahre später folgenden Konflikt im Kontext der Fusion mit Campari zumindest die Entscheidungsprozesse im Management weitgehend unberührt.

2005 erfolgte eine weitere Neuorganisation der Familienvertretung. ${ }^{1237}$ Nachdem sich Harald Eckes-Chantré bereits 2002 aus dem Aufsichtsrat zurückgezogen hatte, verließen nun auch Michael und Peter Eckes dieses Gremium. Von nun an wurden dort alle Familienmitglieder durch Bevollmächtigte vertreten. Diese Veränderung implizierte zwar nicht den vollständigen Rückzug der familiären Gesellschafter aus dem strategischen Entscheidungsprozess, doch wurde der Einfluss der Familie nun indirekter ausgeübt, sodass konfliktäre familiäre Dynamiken nicht direkt und in ihrer vollen Intensität den strategischen Entscheidungsraum erreichen konnten. ${ }^{1238}$ Nicht zuletzt, weil sich durch diesen taktischen Teilrückzug der Familie der strategische Entscheidungsraum wieder geöffnet hatte, konnte 2006 der entscheidende Schritt des strategischen Wandlungsprozesses vollzogen und mit dem deutschen Spirituosengeschäft der traditionsreiche Ursprungskern des Unternehmens verkauft werden.

»Ich glaub, zweiter großer wichtiger Schritt war dann irgendwann die Governance zu haben, dass dieses Unternehmen nicht von einem Familienmitglied geführt wird. Das heißt, Harald und Peter waren immer Mit-Vorstände, ich glaub, Michael war auch ein Vorstand, aber sie waren immer in der zweiten Reihe, das war sicherlich ein ganz wesentlicher Schritt. Und der dritte große Schritt war sicherlich denn irgendwann auch

1235 Vgl. Eckes (2007: $30 \mathrm{ff}$.): „Nach einer strategischen Entscheidung [...] verlässt der Vorstand [...] Michael Eckes, die Firma, bleibt aber Gesellschafter."

1236 Vgl. E000920MM; E100825LZ. Eine ehemalige Führungskraft der Eckes AG: »natürlich waren da auch oftmals viele Konflikte innerhalb des Aufsichtsrates, weil im Aufsichtsrat war die Familie ja noch."

1237 Vgl. Eckes (2007: 44).

1238 Nach dem Kauf der Spirituosensparte der Eckes AG, durch die von ihm in der Wendezeit erworbene Rotkäppchen-Mumm Sektkellereien GmbH sind Harald Eckes-Chantré und seine Töchter heute wieder stärker in die strategischen Entscheidungsprozesse eingebunden. 
mal die Entscheidung, dass man gesagt hat, im Vorstand ist zukünftig kein Familienmitglied mehr, was auch im Grunde eine sehr wichtige Entscheidung war, und damit auch den Proporz auszuschließen, weil die vier Stämme sehr unterschiedlich waren. Man konnte sich nicht auf niemand verständigen, also es war einem lieber einen von außen zu haben, der inhaltlich effektiv war. So und das waren sicherlich ganz, ganz wesentliche Weichenstellungen [...], die auch, die dann auch im, im, im Ablauf, im Zeitablauf passiert sind und die haben dann auch dazu beigetragen, dass man es dann bis 2006 geschafft hat. Natürlich waren auf diesem Weg auch oftmals viele Konflikte innerhalb des Aufsichtsrates, weil im Aufsichtsrat war die Familie ja noch bis 2005 [...], aber dann hat man auch den Aufsichtsrat nur mit familienfremden Mitgliedern besetzt."

(Ehemalige Führungskraft der Eckes AG)

Wenngleich die Kommunikation innerhalb der dominanten Koalition der Eckes AG somit häufig konfliktär war, konnte aufgrund der an die Evolution des Unternehmens und der Familie angepassten Governance-Strukturen im strategischen Entscheidungsraum des Unternehmens immer noch über strategische Themen kommuniziert und diese auch Entscheidungen zugeführt werden.

Bei der Berentzen-Gruppe AG war der Einfluss der Eigentümer und deren Dynamiken auf den strategischen Entscheidungsraum viel unmittelbarer. Hier waren Unternehmen und Familie deutlich enger gekoppelt und die strategische Kommunikation von Management, Kontrollorgan und Gesellschafterkreis durch zahlreiche Doppelrollen eng miteinander verwoben. Im Rahmen dieser Strukturen schlugen sich die Konflikte zwischen den familiären Subzentren und deren Mitgliedern kaum abgeschwächt im strategischen Entscheidungsprozess nieder.

Zur Übertragung der familiären Dynamiken in den strategischen Entscheidungsraum trug aber nicht nur die enge Verzahnung der organisationalen Entscheidungs- und Kontrollgremien bei, sondern auch der familiär geprägte kulturelle Rahmen innerhalb der dominanten Koalition. Hier führte der familiäre Einfluss auf der einen Seite zu einer grundsätzlich erhöhten Emotionalität, weil Themen wie die Schließung des Standorts Haselünne für die Akteure persönlich und aufgrund familiärer Logiken stark emotional belegt waren. ${ }^{1239}$ Zum anderen trug der familiäre Einfluss zum Beispiel über Mehrfachrollen dazu bei, dass die Expression von Emotionen in hohem Maße legitim erschien. ${ }^{1240}$ Über

1239 Vgl. zur Bedeutung des Grades an zugelassener Emotionalität Schein (2010: 105).

1240 Vgl. zum verstärkenden Einfluss dieser Offenheit für Beziehungskonflikte Jehn \& Bendersky (2003: 217). Dies ist deshalb bemerkenswert, weil manche Studien der Konfliktforschung zu dem Schluss kommen, dass die positive Korrelation zwischen Aufgabenkonflikt und der Leistung von Gruppen an der Spitze der Organisation gerade deshalb besonders hoch ist, weil die Aufgabenkonflikte hier deutlich seltener mit Beziehungskonflikten verbunden seien. Die Akteure verstünden es hier im Vergleich zu Akteuren auf niedrigeren organisationalen Ebenen besser, durch geschicktes Kommunizieren und 
diese Einflüsse trug die erhöhte Emotionalität somit zum einen zum Übergreifen der Konflikte auf den strategischen Entscheidungsprozess bei, zum anderen zu den fließenden Grenzen zwischen Sachkonflikt und Beziehungskonflikt. ${ }^{1241}$

Über diese enge Kopplung zwischen Familie und Unternehmen im strategischen Entscheidungsraum hinaus trugen noch drei weitere organisationale Merkmale dazu bei, dass der sich selbst verstärkenden sozio-emotionalen Dynamik nicht begegnet wurde und die Konflikte schlussendlich den strategischen Entscheidungsraum erreichten: die symmetrische Verteilung der Macht innerhalb der polyzentrischen Konfiguration, konfliktkanalisierende Austrittsbarrieren sowie das Fehlen einer übergeordneten Instanz, die die Konflikte einer Lösung hätte zuführen können.

Die Subzentren der dominanten Koalition besaßen einen annähernd gleich hohen Eigenkapitalanteil an der Berentzen-Gruppe AG und verfügten daher über annähernd gleich viel Macht über das Unternehmen. Weitere Machtressourcen wie die Führungsbeteiligung oder die Legitimität der Tradition konnten dieses Machtgleichgewicht nicht entscheidend beeinflussen, das somit zu einer Situation der reziproken Anhängigkeit der Subzentren im strategischen Entscheidungsprozess führte. ${ }^{1242}$

"Am Kapital der neuen I. B. Berentzen GmbH \& Co [...] wird Berentzen zwar die Mehrheit besitzen, doch ist, wie betont wurde, vertraglich festgelegt, dass keine Seite beherrscht werden kann."

(Die Neue Osnabrücker Zeitung im Jahr 1977) $)^{1243}$

Die einseitige Verletzung dieser Machtsymmetrie im Zuge des Nachfolgeprozesses trug maßgeblich zur Eskalation der Konflikte bei. Im rahmensetzenden Imprint der polyzentrischen Konfiguration führte die Symmetrie der Macht jedoch vor allem auch dazu, dass der eskalierende Konflikt nicht durch Macht und machvolle Entscheidungen gelöst werden konnte. ${ }^{1244}$ Die Machtsymmetrie verstärkte somit den Konflikten im Allgemeinen inhärenten Zustand der Unentschiedenheit, der aus der fortgesetzten Negation der Negation entspringt, und überführte diesen in einen Zustand der Unentscheidbarkeit. ${ }^{1245}$ Die Sub-

politisches Agieren Aufgabenkonflikte nicht zu manifesten Beziehungskonflikten auswachsen zu lassen. Vgl. de Wit et al. (2012: 373).

1241 Vgl. zur moderierenden Rolle von Emotionalität im Allgemeinen Jehn \& Bendersky (2003: 204).

1242 Vgl. zu der Gefahr einer solchen Abhängigkeit bei Konflikten Steinmann \& Schreyögg (2005: 637f.).

1243 B870808NZ.

1244 Wenngleich die symmetrische Machtverteilung bei der Berentzen-Gruppe zum Teil aus der Fusion resultierte, ist sie insgesamt doch typisch für familiär beeinflusste Unternehmen. Vgl. Klein (2010); des Weiteren Simon et al. (2005) zu Geschwisterpartnerschaften oder Stammesunternehmen.

1245 Vgl. Luhmann (1984: 530); Simon (2012: 11ff.). 
zentren negierten wechselseitig Interessen und Ansprüche und auch hinsichtlich der strategischen Alternativen zeigte sich eine Oszillation der Positionen. Diese Unentscheidbarkeit trug entscheidend dazu bei, dass sich die Sach- und Beziehungskonflikte über viele Jahre immer weiter fortsetzten. Sie ermöglichte letztlich erst die wechselseitige Blockade des strategischen Entscheidungs- und Veränderungsprozesses.

Die Machtsymmetrie verhinderte aber nicht nur eine Entscheidung des Konflikts, sondern trug auch zu dessen weiteren Eskalation in der Phase der Pfadformation bei. Denn gerade weil das Einflusspotenzial annähernd gleichverteilt war, war die Akkumulation von Macht in politischen Prozessen für die Mitglieder der dominanten Koalition so attraktiv. Zusätzlicher Machtgewinn versprach einen Vorteil bei der Durchsetzung der eigenen Interessen. ${ }^{1246}$

Da die Machtsymmetrie den Entscheid des Konflikts zu eigenen Gunsten für die Subzentren aber beinahe unmöglich machte, wäre eine Alternative zur Fortsetzung des Konflikts der Austritt aus dem Unternehmen gewesen. Dieser Weg wurde allerdings durch eine Reihe von Austrittsbarrieren versperrt, die in weiten Teilen auf den Einfluss des Faktors Familie zurückzuführen waren.

Die erste Austrittsbarriere entsprang aber der Aufgabe des strategischen Entscheidungsprozesses und dessen Verankerung an der hierarchischen Spitze. Im Gegensatz zu vielen Alltagskonflikten, in denen die Streitgegenstände häufig nach recht kurzer Zeit ihre Relevanz verlieren, wiesen die strittigen strategischen Thematiken bei der Berentzen-Gruppe AG über lange Zeiträume eine permanent hohe Bedeutung auf. ${ }^{1247}$ Zugleich konnten sich die Akteure im strategischen Entscheidungsraum nur in sehr begrenztem Maße ausweichen. Auf lang oder kurz mussten Entscheidungen getroffen werden und konnten von den Akteuren nicht weiter delegiert werden. Somit war ein Abschwächen der Konflikte durch Ausweichen, wie es in anderen Zusammenhängen stattfinden kann, im strategischen Entscheidungsraum der Berentzen-Gruppe AG nicht möglich. ${ }^{1248}$

Neben dieser Begrenzung der Konfliktarena waren jedoch vor allem verschiedene Formen von Austrittsbarrieren, die den Austritt einzelner Gesellschafter oder Subzentren aus dem Unternehmen verhinderten, von Bedeutung

1246 Vgl. Schulze et al. (2003: 185f.):»[T] he risk of intrafirm conflict also rises if ownership is distributed somewhat equally [...] The nonlinear relationship suggests that family firms are most vulnerable to conflict, and least willing to bear added risk, when ownership is split in relatively equal proportions."Vgl. auch Eisenhardt \& Bourgeois (1988: 750), die eine »inability to accomplish objectives without politics» in Strukturen mit mehreren ungefähr gleich einflussreichen Zentren feststellen und daher von einer erhöhten Anfälligkeit für Konflikte und politische Prozesse ausgehen.

1247 Vgl. Simon (2012: 36).

1248 Vgl. Jehn (1995: 276) zu dieser Möglichkeit des Ausweichens. 
für die weitere Eskalation des Konflikts und die ausbleibende Reaktion auf den strategischen Pfad. ${ }^{1249}$

Hier ist bei der Berentzen-Gruppe AG zunächst eine Reihe von sozio-emotionalen nachweisbar, die den Austritt von Subzentren oder einzelner Gesellschafter aus dem Unternehmen erschwerten. Für die aktiv im Management des Unternehmens aktiven Gesellschafter wäre mit dem Ausstieg aus dem Unternehmen der Verlust der Rolle als Unternehmer verbunden gewesen. Somit hätte auch auf die damit einhergehende Autonomie, Gestaltungsmacht und auf gesellschaftlichen Status verzichtet werden müssen. Zugleich stellt ein solcher Ausstieg häufig eine Herausforderung für die Re-Definition der eigenen Identität als Unternehmer dar. ${ }^{1250}$ Diese Aspekte dürften dazu beigetragen haben, dass sich das aktive Subzentrum nach dem Rückzug Jürgen Pabsts nicht ebenfalls aus dem aktiven Management zurückzog.

Für das weithin passive Subzentrum der Familie Berentzen dürften die Konsequenzen eines Ausstiegs für den familiären Zusammenhalt in keinem sinnvollen Verhältnis zum Konfliktniveau zwischen den beiden Subzentren der Familie Berentzen gestanden haben. ${ }^{1251}$

Von besonderer Bedeutung war für die Subzentren der Familie Berentzen die Bewahrung des transgenerationalen Erbes. ${ }^{1252}$ Im Unternehmen war das Bewusstsein stark ausgeprägt, dass das Vermögen nicht nur auf eigener Leistung beruht, sondern auch auf der Leistung der Vorfahren. ${ }^{1253}$ Das Streben nach der Bewahrung dieses Vermögens beeinflusste Entscheidungen wie jene über die Schließung des traditionellen Standorts in Haselünne. Es dürfte ungleich stärker den Austritt der Subzentren aus dem Unternehmen erschwert haben, denn von vielen Akteuren wird ein solcher Verkauf als persönliches Scheitern und NichtErfüllung des transgenerationalen Auftrages wahrgenommen. ${ }^{1254}$ Neben dieses Streben nach Bewahrung des transgenerationalen Erbes dürfte aber insbesondere für die Akteure der namensgebenden Unternehmensfamilie die emotionale Verbundenheit der familiären Subzentren zum Unternehmen den Austritt erschwert haben, ebenso die Sorge um die Reputation als traditionsreiche Un-

1249 Vgl. für einen Überblick zu solchen Ausstiegsbarrieren Redlefsen (2004: 24ff.)

1250 Vgl. die Ausführungen von Klein (2010: 94ff.) zum Rückzug der älteren Generation im Nachfolgeprozess und den Faktoren der Bereitschaft zu diesem Rückzug. Diese dürften auch im Kontext des Austritts von Gesellschaftern aus dem Unternehmen von Bedeutung sein.

1251 Gersick et al. (1997) prognostizieren in Fällen des Ausstiegs von Familienangehörigen aus dem Gesellschafterkreis enttäuschte Erwartungen der Angehörigen und verlorene Intimität, teilweise gar das Ende der familiären Kommunikation.

1252 Siehe Kapitel D.1 zur Beschreibung des transgenerationalen Erbes bei der BerentzenGruppe AG.

1253 Vgl. Berentzen (1981: 5 ff.).

1254 Vgl. Sharma \& Manikutty (2005: 294ff.). 
ternehmensfamilie und die Aufrechterhaltung eines entsprechenden Familienkonzepts.

$\mathrm{Zu}$ solchen sozio-emotionalen Austrittsbarrieren traten eine Reihe wirtschaftlicher Hürden, die den Ausstieg von Subzentren oder einzelnen Gesellschaftern verhinderten. ${ }^{1255}$ Aufgrund der angespannten wirtschaftlichen Lage konnten die Gesellschafter mittelfristig kaum auf substanzielle Wertsteigerungen hoffen. Allerdings erhielten die passiven Gesellschafter kontinuierliche Dividendenzahlungen und die im Unternehmen tätigen Mitglieder der dominanten Koalition ein laufendes Einkommen. Eine höhere wirtschaftliche Ausstiegsbarriere als der potenzielle Verzicht auf solche Zahlungen war der Umstand, dass es für die Anteile der Berentzen-Gruppe AG praktisch keinen Markt gab. Ein Verkauf von Anteilen an die anderen Subzentren der dominanten Koalition wäre angesichts der dafür notwendigen Finanzmittel wohl nur zu signifikanten Preisabschlägen denkbar gewesen. Ein Verkauf einzelner Anteilspakete am Markt war - ungeachtet aller etwaiger rechtlichen Regelungen - jedoch beinahe unmöglich, denn für Investoren war eine Minderheitsbeteiligung in der komplexen polyzentrischen Eigentümerstruktur äußerst unattraktiv. Die internen Zerwürfnisse waren in der Öffentlichkeit bekannt und zudem hatte sich die Krise des Unternehmens und die seit Jahrzehnten negative Branchenentwicklung bereits im Erfolg des Unternehmens niedergeschlagen. Die Entscheidungsträger der dominanten Koalition waren über das gemeinsame Eigentum am Unternehmen somit - unabhängig von ihrem Willen - aneinander gebunden.

Diese sozio-emotionalen und wirtschaftlichen Barrieren kanalisierten die Konfliktdynamik und verhinderten einen Rückzug einzelner Akteure oder Subzentren aus dem Konfliktgeschehen. Die Ausstiegsbarrieren beförderten somit erheblich die Fortführung des Konflikts über viele Jahre und das Übergreifen auf den strategischen Entscheidungsraum. ${ }^{1256}$

Die Symmetrie der Macht und die Ausstiegsbarrieren spezifizieren somit "festgefahrene Eigentümerstrukturen «, auf deren Bedeutung für die Pfadgenese die eingangs referenzierte Studie von Schüßler (2008) aufmerksam macht und

1255 Vgl. Klein (2010: 166); Redlefsen (2004: 24ff.). Die genaue Ausgestaltung der rechtlichen Ausstiegsbarrieren lässt sich aus dem vorliegenden Datenmaterial nicht rekonstruieren. Die Familienunternehmensforschung verweist hier aber zum Beispiel auf familieninterne Andienungspflichten, Zustimmungsvorbehalte der Gesellschafterversammlung oder rechtliche Regelungen zur Bewertung des Unternehmens. Vgl. Redlefsen (2004: 27ff.).

1256 Vgl. Fabis (2007: 301). Von der Forschung zu Familienunternehmen werden Zeiträume von bis zu zehn oder mehr Jahren beobachtet, in denen solche Konflikte keiner Entscheidung zugeführt werden können. Vgl. Großmann (2014: 175ff.). 
die dieser Arbeit als potenzielles Indiz für die Bedeutung des Faktors Familie für die Entwicklung strategischer Pfadabhängigkeit diente. ${ }^{1257}$

Letztlich wurden die fortgesetzte Eskalation und die ausbleibende Reaktion auf die Genese des strategischen Pfads aber auch dadurch befördert, dass es an der Spitze der Organisation keine übergeordnete oder exogene Instanz mehr gab, die den Konflikt noch entscheidend hätte irritieren oder durch eine Entscheidung beenden können. ${ }^{1258}$ Die Entscheidungsträger der dominanten Koalition waren somit nicht nur bei der Steuerung und Kontrolle des strategischen Entscheidungsprozesses, sondern auch beim Umgang mit der eskalierenden sozio-emotionalen Dynamik weitgehend auf sich selbst zurückgeworfen. An dieser Stelle schließt sich der Kreis zu der zu Beginn dieses Kapitels skizzierten Immunität der dominanten Koalitionen, die den Entscheidungsprozessen in der Phase der Pfadformation den Rahmen setzten.

\section{$3 \quad$ Fazit: Der Einfluss des Faktors Familie auf die Genese der strategischen Pfade}

Die vorhandene Forschung zu Unternehmenskrisen in Familienunternehmen erachtet den Faktor Familie meist als den alles entscheidenden Faktor der Krisenentstehung.

"Betrachtet man allerdings gescheiterte Familienunternehmen, so sind diese in der Regel an der Familie und nicht am Unternehmen gescheitert."

(Klein, 2010) $)^{1259}$

Solche Einschätzungen hängen allerdings stark von der eingenommenen Perspektive auf den Untersuchungsgegenstand ab. In Familienunternehmen beeinflussen Angehörige der Unternehmensfamilie meist maßgeblich die Entscheidungsprozesse in den Unternehmen und deren strategische Entwicklung. Fehlentscheidungen oder unterlassene Veränderungsprozesse können daher in den meisten Fällen in Verbindung zu Angehörigen der Eigentümerfamilie gebracht werden. Solche Feststellungen sind zunächst im Grunde lediglich eine kontextuelle Spezifizierung der allgemeinen Erkenntnis der Krisenursachenforschung, dass Unternehmenskrisen meist nicht von exogenen Einflüssen

1257 Vgl. Schüßler (2008: 143) und Kapitel A.2 und B.1.3.5.

1258 Vgl. Simon (2011: 93); Steinmann \& Schreyögg (2005: 634) zur Lösung von Konflikten durch Hierarchie und Programme. Das Fehlen einer solchen Instanz verweist wiederum auf die Bedeutung entsprechender Governance-Strukturen und Regelsysteme. Vgl. hierzu spezifisch im Hinblick auf den Umgang mit Konflikte aus einer juristischen Perspektive Fabis (2007).

1259 Vgl. Klein (2010: 298). 
ausgelöst werden, sondern auf endogenen Problemkonstellationen beruhen und die Unternehmensleitung hier ein entscheidender Faktor ist.

Ein anderes Bild ergibt sich, wenn man nach den spezifischen Einflüssen auf die Entscheidungsprozesse fragt, die strukturell auf die Familie und deren Systemlogik zurückgeführt können. Dies gilt auch für die abschließende Beurteilung des Einflusses des Faktors Familie auf die Genese und Dynamik der strategischen Pfade in den untersuchten Unternehmen. An den Entscheidungsprozessen, die der Pfadgenese zugrunde lagen, waren Mitglieder der Eigentümerfamilien maßgeblich beteiligt und, so liegt es zunächst nahe, dem Faktor Familie ist die entscheidende Bedeutung für die Pfadgenese zuzumessen. Tritt man jedoch einen Schritt zurück und betrachtet den Einfluss der Familie in den einzelnen Phasen der Pfadgenese auf den verschiedenen Ebenen der Phänomene Unternehmenskrise, strategischer Pfad und strategischer Entscheidungsprozess, so ergibt sich ein differenzierteres und komplexeres Bild für die Bedeutung der Familie bei der Entstehung der strategischen Pfadabhängigkeiten.

In der Phase der historischen Vorprägung entwickelten sich aus dem Einfluss des Faktors Familie direkt bedeutsame Imprints, oder Imprints aus anderen Quellen wurden über dessen tradierende Kraft in der Organisation fortgeschrieben. Der Einfluss der Familie trug somit dazu bei, dass sich in den Unternehmen früh ein Evolutionskorridor der weiteren strategischen Entwicklung der Unternehmen bildete.

Es wurde jedoch gezeigt, dass diese historische Vorprägung letztlich nicht primär ursächlich für die spätere Genese der strategischen Persistenzen ist. Diese sind in erster Linie auf die Wirkkraft der selbstverstärkenden Mechanismen und den daraus resultierenden Lock-in zurückzuführen. Die selbstverstärkenden Mechanismen erklären den größten Teil der Pfadgenese. Obgleich sich die Mechanismen in familiär beeinflussten Unternehmen entwickelten, war ihre Wirkkraft im Kern allerdings zunächst unabhängig vom Faktor Familie: Die Skalenerträge, auf denen der selbstverstärkende Mechanismus bei der Anton Schlecker e.K. beruhte, sind ein fundamentales ökonomisches Prinzip, dessen selbstverstärkende Wirkung in der Literatur vielfach beschrieben wird. Die sozio-emotionale Dynamik des Konflikts bei der Berentzen-Gruppe AG ist in ihrer Wirkkraft ebenfalls zunächst einmal unabhängig vom Einfluss des Kontextfaktors Familie. Sie vollzieht sich in derselben eskalierenden Form auch in anderen Organisationsformen.

Trotz dieser Unabhängigkeit der Wirkkräfte der selbstverstärkenden $\mathrm{Me}$ chanismen war der Faktor Familie keineswegs bedeutungslos für das Entstehen der strategischen Pfade. Vielmehr wurde gezeigt, dass die Einflüsse dieses Faktors maßgeblich die Wirkrichtung der vorhandenen organisationalen Macht in der Phase der Pfadformation prägten. Sie trugen dazu bei, dass die vorhan- 
dene Macht nicht dazu verwendet wurde, dem emergierenden strategischen Pfad durch eine entschiedene Reaktion zu begegnen.

Der Einfluss des Faktors Familie setzte hier mit der hohen Immunität der dominanten Koalitionen und den monozentrischen und polyzentrischen Machtstrukturen einen Rahmen, in dem sich die selbstverstärkenden Mechanismen in besonderer Weise entfalten konnten. Erst innerhalb des so von der Macht und deren Verteilung aufgespannten Rahmens konnten die beschriebenen Ausprägungen und Wirkungszusammenhänge der Variablen Diversität und Konflikt ihre Wirkung auf den strategischen Entscheidungsprozess entfalten. Allerdings wurden auch hier Wirkungszusammenhänge beschrieben, die in strategischen Entscheidungsprozessen unterschiedlicher Kontexte von Bedeutung sind.

Was kann nach dieser kaskadenförmigen Analyse des Einflusses des Faktors Familie auf die Genese und Dynamik der strategischen Pfade in den untersuchten Unternehmen abschließend im Kern festgehalten werden? Die Entstehung der strategischen Pfadabhängigkeit kann letztlich nicht allein auf die Existenz des Faktors Familie in der Sphäre des Unternehmens zurückgeführt werden. Sein Einfluss prägte aber einen kontextuellen Rahmen der Struktur und Kultur, in dem sich die auf den selbstverstärkenden Mechanismen beruhenden Prozesse der Pfadgenese in besonders fruchtbarer Weise entfalten konnten.

Über die untersuchten Fälle hinaus kann die Familie somit als ein Faktor verstanden werden, der im Kontext von Familienunternehmen aufgrund der mit ihm meist einhergehenden Machtkonzentration die primär auf selbstverstärkenden Mechanismen beruhende Genese strategischer Pfade in der Phase der Pfadformation potenziell ebenso stabilisieren wie ihr nachhaltig begegnen kann. 


\section{E Schlussbetrachtung und Diskussion}

\section{$1 \quad$ Zusammenfassung}

Die vorliegende Forschungsarbeit nahm ihren Ursprung in der Frage, warum manche Unternehmen dauerhaft und unverändert existenzbedrohende Strategien verfolgen. Die Arbeit schlug bei der Suche nach Antworten einen Weg ein, der mit der Unternehmenskrise seinen Ausgangspunkt beim manifestierten Resultat solcher strategischer Persistenzen nahm. Von diesem Phänomen ausgehend, wurde sich Schritt für Schritt zu einem spezifischen Ursachenkomplex vorgearbeitet.

Zwei für die Analyse strategischer Phänomene unverzichtbare, von der bisherigen Managementforschung zu Unternehmenskrisen bislang jedoch weitgehend vernachlässigte Dimensionen spannten den theoretischen Bezugsrahmen der Arbeit auf: die prozessuale Entwicklungsdynamik und deren organisationaler Kontext. Zur Adressierung dieses Forschungsdefizits wurde mit strategischen Pfaden ein spezifischer Prozess der Krisenentstehung im Kontext familiär beeinflusster Unternehmen untersucht. In dem so aufgespannten theoretischen Rahmen wurde die allgemeine Fragestellung zur leitenden Forschungsfrage spezifiziert, inwieweit und wie der Faktor Familie die Genese und Dynamik strategischer Pfade in Familienunternehmen beeinflusst.

Nach der Skizzierung der Problemstellung und der daraus abgeleiteten Forschungsfrage wurde im nächsten Kapitel des einleitenden Teils A die qualitative Forschungsstrategie dargestellt, die aufgrund des explorativen Charakters der Forschungsfrage gewählt worden war.

In Teil B wurde der durch den Prozess der Pfadgenese und den Kontextfaktor Familie bestimmte theoretische Bezugsrahmen der Arbeit näher ausgeführt. Das erste Kapitel dieses Teils widmete sich mit den strategischen Pfaden zunächst dem zu untersuchenden spezifischen Prozess der Krisenentstehung. Als erstes wurden der prozessuale Charakter von Unternehmenskrisen und die Endogenität ihrer Krisenursachen herausgearbeitet. Es wurde deutlich gemacht, dass Unternehmenskrisen in den meisten Fällen nicht auf exogene Einflüsse wie 
Konjunkturkrisen zurückzuführen sind, sondern letztlich meist nur das manifestierte Resultat eines längerfristigen Prozesses sind, in dem aufgrund bestimmter innerorganisationaler Problemkonstellationen nicht mehr adäquat auf die Herausforderungen der Umwelt reagiert wird. Diese Erkenntnis der empirischen Krisenursachenforschung konnte durch die Einnahme einer systemtheoretisch orientierten Perspektive auf das Verhältnis zwischen Umwelt und Organisation theoretisch gefestigt werden. Die systemtheoretische Perspektive verdeutlichte, dass die Umwelt die Organisation unablässig und existenziell herausfordert und die Organisation sich im Zuge ihres Managementprozesses entsprechend permanent wandeln muss, um zu überleben.

Im nächsten Schritt wurde die Theorie der organisationalen Pfadabhängigkeit und damit der zentrale theoretische Erklärungsansatz dieser Arbeit vorgestellt, der zu erklären vermag, warum der notwendige strategische Wandel manchen Unternehmen nicht gelingt. Es wurde herausgestellt, dass der theoretische Mehrwert des Pfadabhängigkeitskonzepts als Theorie der Krisenentstehung in der Fokussierung des Konstrukts des selbstverstärkenden Mechanismus liegt. Während andere theoretische Beiträge zur Erklärung von organisationaler Trägheit und Persistenz in erster Linie einzelne Korrelationen zwischen Ursachen und Persistenzen benennen, gelingt es der Pfadtheorie über das Konstrukt des selbstverstärkenden Mechanismus die resultierende Persistenz durch die Verknüpfung der zugrunde liegenden Entscheidungen im Rahmen einer kausalen Prozesskette endogen und konsistent aus dem Prozess heraus zu erklären. Aus dieser prozessual verknüpften Entscheidungsdynamik der selbstverstärkenden Mechanismen gewinnt die Theorie der Pfadabhängigkeit ihre besondere Erklärungskraft. Diese Dynamik wurde durch das vorgestellte Phasenmodell der Pfadgenese nach Sydow et al. (2009) verdeutlicht. Unter Rückgriff auf dieses Modell wurde aufgezeigt, wie in einem historisch geprägten Entscheidungsraum zunächst vielleicht unbeachtete Ereignisse oder Entscheidungen selbstverstärkende Mechanismen in Gang setzen können, die sich im Zuge der Phase der Pfadformation immer weiter verstärken und direkt oder indirekt die fortwährende Reproduktion eines bestimmten strategischen Musters begünstigen. Vor allem wurde hervorgehoben, dass aufgrund der zunehmenden Wirkkraft der selbstverstärkenden Mechanismen ein Abweichen vom eingeschlagenen strategischen Entwicklungsweg mit zunehmendem Fortschreiten immer schwieriger wird. Am Ende kann der Lock-in stehen - ein Punkt, an dem das Management beinahe jede Entscheidungsfreiheit verloren hat. Das Unternehmen ist dann auf dem eingeschlagenen Entwicklungsweg "gefangen«; es ist pfadabhängig. Das pfadabhängige Unternehmen kann sich auch dann nicht mehr wandeln, wenn in der Umwelt vorteilhaftere Alternativen auftauchen oder die relevante Umwelt sich in eine vom eingeschlagenen Weg abweichende Richtung zu entwickeln beginnt. Mit der Skizzierung dieser dritten 
und letzten Phase der Pfadformation wurde die Relevanz strategischer Pfade für die Entstehung von strategischen Persistenzen und daraus resultierenden Unternehmenskrisen verdeutlicht.

Anschließend wurde aufgezeigt, dass die Theorie der organisationalen Pfadabhängigkeit trotz ihrer hohen Erklärungskraft für die Analyse strategischer Persistenzen und daraus resultierender Unternehmenskrisen noch Erklärungsdefizite birgt. Nach einer Betrachtung der bestehenden Forschungsarbeiten wurde vor allem die stärkere Integration organisationaler Kontextvariablen in die Analyse der Pfadgenese als notwendig erachtet. Es wurde dargelegt, dass insbesondere die potenziell ebenso pfadstabilisierende wie pfadbrechende Rolle der Macht von der bisherigen organisationalen Pfadforschung vernachlässigt wurde. Während die meisten Beiträge der organisationalen Pfadtheorie die Pfadgenese in Analogie zum klassischen machtfreien Rahmen des Marktes allein über die Wirkkraft der selbstverstärkenden Mechanismen erklären, machte die vorliegende Arbeit darauf aufmerksam, dass Akteure im organisationalen Kontext - im Gegensatz zu den machtlosen Marktteilnehmern - ein hohes Maß an Macht besitzen können und dass dort, wo Macht existiert, auch die Wirkrichtung des machtvollen Einflusses auf die Pfadgenese in die Analyse integriert werden muss.

Diese theoretische Überlegung führte zu der zweiten Dimension des theoretischen Bezugsrahmens: dem sowohl von der Unternehmenskrisenforschung als auch von der Pfadforschung vernachlässigten organisationalen Kontext.

Vor dem Hintergrund des aufgezeigten Forschungsdefizits wandte sich das zweite Kapitel des Teils B dem spezifischen Kontextfaktor der Familie zu. Dessen potenzielle Bedeutung für die Pfadgenese war von der bisherigen Pfadforschung zwar mehrfach indiziert, aber noch nicht näher untersucht worden.

Nach einer Gegenüberstellung der empirischen Bedeutung dieser ökonomischen Größe mit deren marginaler Behandlung in der etablierten Managementforschung wurde im nächsten Schritt begonnen, den Faktor Familie für die weitere Untersuchung zu konzeptualisieren. In Anknüpfung an die systemtheoretische Perspektive wurden Familie und Unternehmen als zwei grundsätzlich anders operierende Systeme skizziert, die sich in der Sphäre von Familienunternehmen aber derart permanent irritieren, dass von einer strukturellen Kopplung gesprochen werden kann, in deren Folge sich die Systeme koevolutionär entwickeln.

Auf diesem systemtheoretischen Verständnis aufbauend, wurde im Anschluss der Einfluss des Faktors Familie konzeptionell erörtert. Es wurde aufgezeigt, warum die teils künstlich anmutende Dichotomie zwischen Familienunternehmen und Nicht-Familienunternehmen für die Analyse des familiären Einflusses problematisch ist, und verdeutlicht, warum zur besseren Integration des Faktors Familie in die Theoriegerüste der allgemeinen Managementforschung nicht mehr die Entität des Familienunternehmens in das Zentrum der Analyse 
gestellt werden sollte, sondern der spezifische Einfluss des Systems Familie auf ein System Unternehmen sowie die Konsequenzen dieses Einflusses.

Dieser Linie folgend rückten die Macht der Familien, deren Generationalität und kulturelle Prägung ins Zentrum der Darstellung, mithin die drei Kanäle, über welche der Faktor Familie strukturell gekoppelte Unternehmen und deren Entscheidungsprozesse beeinflussen kann. Vor allem wurden auf der Grundlage der bestehenden Forschung zu Familienunternehmen jedoch jene charakteristischen Merkmalsausprägungen erörtert, die sich in Unternehmen regelmäßig aufgrund des familiären Einflusses herausbilden und in denen sich der Einfluss des Systems Familie widerspiegelt.

Als erster Indikator des familiären Einflusses wurde eine im Vergleich zu anderen Formen ökonomischer Aktivität sehr hohe Entscheidungsautonomie der dominanten Koalition herausgearbeitet, die in erster Linie aus dem in der Familie konzentrierten Eigentum am Unternehmen erwächst. Diese hohe Entscheidungsautonomie besteht sowohl gegenüber der Umwelt der Organisation als auch intern gegenüber dem Rest der Organisation. Des Weiteren legten die Erkenntnisse der bisherigen Familienunternehmensforschung nahe, dass mit einem zunehmenden Einfluss der Familie erstens die Zentralisierung der Entscheidungsprozesse zunimmt, während zweitens der Grad der Formalisierung der Entscheidungsprozesse tendenziell sinkt: Der familiäre Einfluss schlägt sich in den Entscheidungsstrukturen des Unternehmens nieder. Darüber hinaus wurde die generationale Orientierung familiär beeinflusster Unternehmen herausgestellt. Ein zum Teil retrospektiver Blick bezieht Tradition und Historie auch in Entscheidungsprozessen ein; prospektiv wird die Bewahrung des transgenerationalen Erbes zu einer wichtigen Entscheidungsprämisse. Zu guter Letzt wurden kulturelle Muster skizziert, die sich im Zuge der Ko-Evolution mit einer Familie im Unternehmen häufig entwickeln: Die Organisationskulturen in familiär beeinflussten Unternehmen zeichnen sich häufig nicht nur durch eine deutlich erhöhte Affektivität, sondern auch durch eine gewisse Diffusität der Beziehungen aus. Als ein weiteres Merkmal des familiären Einflusses wurden eine kollektive Orientierung sowie eine Tendenz zu geschlossenen Kulturen herausgearbeitet.

Mit diesen Strukturmerkmalen stand der zweite Pfeiler des theoretischen Gebäudes, in dem sich vorliegende Studie bewegt, und es konnte sich in Teil C der empirischen Untersuchung den strategischen Pfaden in Familienunternehmen zugewandt werden.

Nach einer Darstellung und Begründung des qualitativen Forschungsdesigns und der Methoden der Datenerhebung und -auswertung wurden zunächst der Fall der Anton Schlecker e.K. und anschließend der Fall der Berentzen-Gruppe AG einer mehrstufigen Pfadanalyse zugeführt. Beide Fälle dienten als empirische Repräsentanten jener monozentrischen und polyzentrischen Konfigurati- 
on, die sich in der explorativen Vorstudie als besonders wichtig für die Pfadgenese in Familienunternehmen erwiesen hatte.

Im ersten Schritt der Pfadanalyse konnte durch Kontrastierungen der strategischen Entwicklungen der Unternehmen mit den Dynamiken ihrer Umwelt insbesondere den Entwicklungen der Vergleichsfälle Dirk Rossmann GmbH und Eckes AG - zunächst eine strategische Persistenz nachgewiesen werden. Im zweiten Schritt wurde dann jeweils demonstriert, dass diese Persistenzen maßgeblich auf das Wirken der beiden pfadtheoretischen Elemente der selbstverstärkenden Mechanismen und der daraus resultierenden Lock-ins zurückzuführen waren.

Wie aufgrund der theoretischen Erörterung in Teil B vermutet, zeigte sich in der Analyse der Pfadgenese, dass die Wirkkraft der selbstverstärkenden Mechanismen im organisationalen Kontext der Unternehmen die Pfadgenese zwar größtenteils, aber nicht restlos erklären kann. In beiden Fällen hätte zu Beginn der Phase der Pfadformation über einen Zeitraum von mehreren Jahren noch ausreichend in den dominanten Koalitionen konzentrierte Macht existiert, um der Dynamik der sich selbst verstärkenden Mechanismen entgegenzuwirken. Das Ausbleiben von entsprechenden Reaktionen kann im organisationalen Kontext der Unternehmen nicht allein durch die Wirkkraft der selbstverstärkenden Mechanismen erklärt werden.

Die Analyse der strategischen Entscheidungsprozesse verweist vielmehr darauf, dass es zur Erklärung dieser ausbleibenden Reaktion auf die Genese der strategischen Pfade der Integration des organisationalen Kontexts und des Faktors Familie bedarf, der diesen Kontext und die ihn durchlaufenden Entscheidungsprozesse maßgeblich prägte. Daher wurde der Einfluss des Faktors Familie auf den Prozess der Pfadgenese in Teil D im Rahmen des Modells der Pfadgenese nach Sydow et al. (2009) erörtert.

Im ersten Schritt wurde gezeigt, wie die grundlegende historische Vorprägung des strategischen Entscheidungsraums die Etablierung der später pfadbedingt persistenten strategischen Muster begünstigte und den strategischen Entscheidungsraum bereits vor dem Einsetzen der selbstverstärkenden $\mathrm{Me}$ chanismen signifikant beschränkte. Einige der wirksamen Imprints wie das sozio-emotionale Vermögen oder die Struktur des Eigentums waren direkt auf die strukturelle Kopplung der Unternehmen mit den Familien zurückzuführen. Hinsichtlich anderer Imprints, wie der Prägung des Entscheidungsraumes durch Umwelteinflüsse aus besonders umweltsensitiven Phasen der Unternehmensentwicklung oder spezifischer kultureller Muster, zeigte sich der Einfluss des Faktors vor allem in dessen tradierender Kraft. Die Imprints aus längst vergangenen Phasen der Unternehmensentwicklung wurden erhalten und in die Gegenwart der strategischen Entscheidungsprozesse überführt.

Im Einklang mit der organisationalen Pfadtheorie ergab die Analyse der 
Pfadgenese allerdings auch, dass diese Imprints zwar früh den strategischen Entscheidungsraum prägten und einen Korridor der strategischen Entwicklung formten, sie zu der schlussendlichen strategischen Persistenz der Unternehmen aber nur begrenzt beitrugen. Die Gegenüberstellung mit den Vergleichsfällen der Dirk Rossmann GmbH und der Eckes AG, die zwar ebenfalls historisch geprägt waren, im Verlauf ihrer Entwicklung aber keine strategische Pfadabhängigkeit entwickelten, untermauerten den Befund. Darin trat einerseits deutlich die überragende Bedeutung der in Teil C dargestellten Regime der selbstverstärkenden Mechanismen für die Entwicklung der pfadbedingten strategischen Persistenzen bei der Anton Schlecker e.K. und der BerentzenGruppe AG zutage. Andererseits lenkte der Befund den Blick auf die der Pfadgenese zugrunde liegenden strategischen Entscheidungsprozesse in der Phase der Pfadformation - jener Phase, in der den sich selbst verstärkenden Mechanismen durch einen machtvollen Eingriff noch hätte begegnet werden können.

Die strategischen Entscheidungsprozesse in dieser Phase der Pfadgenese wurden vor allem von den Ausprägungen und Wirkungszusammenhängen dreier Struktur- und Interaktionsvariablen geprägt: Macht, Diversität und Konflikt. Die empirischen Ausformungen dieser drei organisationalen Variablen und ihre Wirkungszusammenhänge prägten in der Phase der Pfadformation in entscheidender Weise die kollektiven strategischen Entscheidungsprozesse der dominanten Koalitionen in Verlauf und Ergebnis und erklärten größtenteils das Ausbleiben der strategischen Reaktion bei der Anton Schlecker e.K. und der Berentzen-Gruppe AG.

Zunächst wurde hinsichtlich der Variable Macht skizziert, dass die die familiären Strukturmerkmale der hohen Entscheidungsautonomie, des hohen Grads an Zentralisierung und der geringen Formalisierung der Entscheidungsprozesse die um die Unternehmensfamilien gruppierten dominanten Koalitionen der Anton Schlecker e.K. und der Berentzen-Gruppe AG in eine außergewöhnlich machtvolle Lage versetzten, um Einfluss und Kontrolle über die strategischen Entscheidungsprozesse der Unternehmen auszuüben. Die dominanten Koalitionen konnten die strategische Entwicklung der von ihnen geführten Unternehmen weitgehend unbeschränkt und selbstbestimmt steuern, waren gleichzeitig aber auch losgelöst von internen und externen Governance-Mechanismen und in der Steuerung und Kontrolle weitestgehend auf sich und ihre Eigengesetzlichkeit zurückgeworfen. Dieses von der bisherigen Pfadforschung schon erkannte Problem der Selbstkontrolle wurde als Immunität gegenüber exogenen Regulativen zur Korrektur von Fehlentwicklungen charakterisiert und als erster Hinweis auf die Frage nach der ausbleibenden Reaktion auf die Pfadgenese konstatiert.

Der erneute Vergleich mit der Dirk Rossmann GmbH und der Eckes AG deren dominante Koalitionen ebenfalls über ein hohes Maß an Entscheidungsautonomie verfügten - zeigte aber an, dass die Erklärung der in Verlauf und 
Ergebnis so divergenten Entscheidungsprozesse noch die Integration zusätzlicher Variablen des strategischen Entscheidungsprozesses verlangte.

In einem Schritt wurde daher die immunisierende Wirkung exkludierender kultureller und struktureller Muster aufgezeigt, die vor allem bei der Anton Schlecker e.K., in Teilen aber auch bei der Berentzen-Gruppe AG die machtbasierte Immunität der dominanten Koalitionen noch erhöhten. Es wurde dargestellt, wie die kulturellen und strukturellen Grenzen, die maßgeblich aus den familiären Strukturmerkmalen der kulturellen Geschlossenheit, des partikularistischen Beziehungsgefüges und der Zentralisierung resultierten, die dominante Koalition gegenüber dem Rest der Organisation abschotteten. Diese Grenzen konnten von den Irritationen der Umwelt nur noch schwer durchdrungen werden und beeinträchtigten mithin die Irritationsfähigkeit deren Entscheidungsprozesse. Die exkludierenden kulturellen und strukturellen Muster verringerten so weiter die Rezeptivität für strategische Wandelerfordernisse und erhöhten das Problem der Selbstkontrolle der dominanten Koalitionen.

Im nächsten Schritt konnte insbesondere anhand der kontrastierenden Analyse mit den sich strategisch wandelnden Vergleichsfällen gezeigt werden, dass die Qualität der Entscheidungsprozesse und die strategische Reaktionsfähigkeit in der Phase der Pfadformation von zwei fundamentalen Einflussgrößen abhängig war: zum einen den kognitiven Potenzialen der dominanten Koalitionen, denen sich über den Grad an Diversität der dominanten Koalitionen angenähert wurde, zum anderen den Interaktions- und Kommunikationsprozessen der dominanten Koalitionen. Es wurde gezeigt, dass im Rahmen der jeweiligen monozentrischen und polyzentrischen Konfigurationen den Ausprägungen und Wirkungszusammenhängen der Variablen Diversität und Konflikt eine besondere Bedeutung für die Handhabung des Problems der Selbstkontrolle und letztlich für das Ausbleiben der Reaktion auf die Genese der strategischen Pfade zukam.

Aufgrund der niedrigen Diversität der Anton Schlecker e.K. wurde der Entscheidungsprozess nicht ausreichend durch unterschiedliche Perspektiven und Informationen irritiert. Zudem trug die geringe Diversität wohl auch dazu bei, dass das Niveau an Sachkonflikt innerhalb der dominanten Koalition der Anton Schlecker e.K. sehr niedrig war. Hier hätte es eines permanent höheren Niveaus an sachorientiertem Konflikt bedurft, um die etablierten Wahrnehmungsschemata und Entscheidungsmuster zu irritieren. Hierauf machte nicht zuletzt der Vergleich mit der Dirk Rossmann GmbH aufmerksam, für die nicht nur eine höhere Diversität, sondern vor allem auch eine ausgeprägte Konfliktkultur als wichtige Determinanten der Irritation des dominanten Akteurs im Entscheidungsprozess und des beobachteten strategischen Wandels ausgemacht werden konnten. Anhand verschiedener struktureller Merkmale wurde aufge- 
zeigt, inwiefern die geringe Diversität und das niedrige Niveau an Sachkonflikt als Antezedenzien des strategischen Entscheidungsprozesses auf den Einfluss des Faktors Familie zurückzuführen waren.

Im Anschluss hieran wurden die Ausprägungen von Diversität und Konflikt bei den polyzentrischen Konfigurationen der Berentzen-Gruppe AG und der Eckes AG dargestellt und deren Auswirkungen auf den strategischen Entscheidungsprozess skizziert. In diesen Unternehmen stellt sich das Verhältnis zwischen den beiden Variablen quasi spiegelbildlich zur Anton Schlecker e.K. dar: Die Diversität innerhalb der dominanten Koalition war hinsichtlich verschiedenster Merkmale so hoch, dass hieraus nicht nur fruchtbare Reibungen zwischen verschiedenen Perspektiven entstanden, sondern mit der Zeit auch ein sehr hohes Maß an Sach- und Beziehungskonflikt resultierte. Aus diesen Konflikten erwuchs die sich selbst verstärkende sozio-emotionale Dynamik. Für die ausbleibende Reaktion auf diese Dynamik und deren Erreichen des strategischen Entscheidungsraumes waren noch weitere Merkmale des Kontexts, und zwar die Machtsymmetrie der Subzentren, die Existenz von Subzentren sowie das Fehlen einer übergeordneten Instanz, von Bedeutung.

Abschließend ermöglichte ein Schritt zurück die Reflexion darüber, welchen Einfluss der Faktor Familie nun auf die Genese und Dynamik der strategischen Pfade in den untersuchten Unternehmen ausübte. Festzuhalten ist, dass der größte Teil der Pfadgenese sich allein aus der - vom Einfluss des Faktors Familie zunächst unabhängigen - Wirkkraft der selbstverstärkenden Mechanismen erklärt, der Faktor Familie im Kontext von Familienunternehmen aber von Bedeutung ist, um das aus der Existenz von Macht im organisationalen Kontext verbleibende Erklärungsdefizit der organisationalen Pfadtheorie zu adressieren. Der Faktor Familie kann demnach im Kontext von Familienunternehmen aufgrund der mit ihm meist einhergehenden Machtkonzentration ein bedeutender Faktor sein, um in der frühen Phase der Pfadformation die Pfadgenese zu stabilisieren - oder dieser nachhaltig zu begegnen

\section{$2 \quad$ Kritische Würdigung}

Abschließend werden zunächst der Geltungsanspruch der Ergebnisse sowie die Limitationen der Arbeit reflektiert. Derart sensibilisiert, sollen die Ergebnisse der Arbeit hinsichtlich ihres Beitrags für die verschiedenen Forschungsfelder eingeordnet und die Implikationen für die zukünftige Forschung erörtert werden. 


\subsection{Zuverlässigkeit der Ergebnisse und Limitationen der Arbeit}

Die Frage, welchen Geltungsanspruch die oben dargestellten Erkenntnisse zur Genese und Dynamik strategischer Pfade in Familienunternehmen besitzen, wird anhand der beiden klassischen Gütekriterien der Reliabilität und Validität reflektiert, welche auch in qualitativen Beiträgen regelmäßig zur Beurteilung der Zuverlässigkeit von Forschungsarbeiten herangezogen werden. ${ }^{1260}$

\section{Reliabilität}

Im klassischen quantitativen Sinn zielt das Gütekriterium der Reliabilität auf die exakte und beliebig häufige Replizierbarkeit der Messungen und Ergebnisse unter gleichen Untersuchungsbedingungen. Aufgrund der divergenten epistemologischen Grundlagen qualitativer Sozialforschung und dem komplexeren Interaktionsverhältnis zwischen dem forschenden Subjekt und dem zu erforschenden Objekt kann dieser Anspruch allerdings nicht eins zu eins auf qualitative Forschungsfragen übertragen werden. ${ }^{1261}$ Im qualitativen Forschungsrahmen stellt sich weniger die Frage nach der exakten Wiederholbarkeit der Messvorgänge, sondern vielmehr jene nach der Zuverlässigkeit der Daten und Vorgehensweisen im Forschungsprozess. Von dieser Zuverlässigkeit der Daten und Interpretationen hängt der Geltungsanspruch der Arbeit ab.

Im Rahmen der vorliegenden Forschungsarbeit wurden daher eine Reihe von Maßnahmen zur Erhöhung der Zuverlässigkeit der Daten und der Überprüfbarkeit und Vergleichbarkeit des Forschungsprozesses getroffen.

Zur Sicherstellung der Qualität und Zuverlässigkeit der Daten wurde grundlegend im Rahmen der Datenerhebung auf bewährte Methoden der qualitativen Sozialforschung wie die narrative Interviewtechnik zurückgegriffen. Prinzipien und Ablaufschema gewährleisten nicht nur eine offene und gegenstandsangemessene, sondern auch eine zuverlässige Datenerhebung. Zudem wurde beispielsweise in mehreren Interviewworkshops die erzählstimulierende offene Eingangsfrage der Experteninterviews und der narrativ-biografischen Interviews von Dritten kritisch beleuchtet und in der Folge mehrfach angepasst.

Die Reliabilität der Arbeit sollte durch eine Triangulation der Datenquellen erhöht werden. Durch den Einbezug interner und externe Dokumente, welche die Interviewdaten ergänzten, wurde eine breite Datenbasis geschaffen, die im Zuge der Datenerhebung die Möglichkeit bot, die gewonnenen Daten gegen-

1260 Vgl. zu diesen Kriterien der qualitativen Forschung als Qualitätsstandards Przyborski \& Wohlrab-Sahr (2008: 35ff.) bzw. zu»Strategien der Qualitätssicherung« Flick (2007: 508), auch Yin (2009: 33).

1261 Vgl. zum Kriterium der Reliabilität in qualitativer Forschung und Maßnahmen zur Sicherstellung dieses Qualitätsstandards Flick (2007: 489ff.); Przyborski \& Wohlrab-Sahr (2008: $38 \mathrm{ff}$.$) .$ 
einander $\mathrm{zu}$ spiegeln und in der Folge zu verwerfen oder zu verifizieren. Im Verlauf des Forschungsprozesses diente diese breite Datenbasis analog hierzu auch zur Kontrastierung der vorläufigen Ergebnisse. So konnte die Zuverlässigkeit der aus externen oder internen Dokumenten gewonnenen vorläufigen Erkenntnisse zu den ersten beiden Schritten der Pfadanalyse erhöht werden durch den Vergleich mit den zeitlich später erhobenen Interviewdaten. Die aus diesen Daten gewonnenen Erkenntnisse zum Einfluss des Faktors Familie konnten wiederum mit der breiteren Datenbasis der externen und internen Dokumente rückgespiegelt werden.

Da sich eine Wiederholung der Datenerhebung aber allein aufgrund des schwierigen Zugangs zum Feld nicht hätte durchführen lassen, wurde darüber hinaus Wert auf eine möglichst genaue und intersubjektiv nachvollziehbare Dokumentation der Forschungsarbeit gelegt. Die erhobenen Interviewdaten wurden alle vollständig transkribiert und ebenso wie die internen und externen Dokumente stets umgehend gekennzeichnet und in die entsprechenden Datenbanken eingepflegt. Somit war ein lückenloser Nachvollzug der Datenbasis im Forschungsprozess gewährleistet.

Die so hergestellte Referenzbasis stellt aber auch den intersubjektiven Nachvollzug des Forschungsprozesses und dessen Rekonstruktion durch Dritte sicher. Diesem Ziel dient auch die Veröffentlichung der Eingangsfrage und der Fallchroniken im Anhang.

\section{Validität der Ergebnisse}

Hinsichtlich der Frage der Gültigkeit der Ergebnisse stellt sich die Frage, inwieweit die Interpretationen und Konstruktionen der Forschungsarbeit in den untersuchten Prozessen begründet sind und inwiefern sie verallgemeinert werden können. ${ }^{1262}$

Die Gegenstandsangemessenheit ist das fundamentale methodologische Ziel der dieser Arbeit zugrunde liegenden qualitativ-zirkulären Forschungsstrategie. Daher wurde ein breiter Zugang zum Forschungsfeld gewählt und versucht, sich in der Verfolgung der Forschungsfrage dem Forschungsgegenstand durch die explorative Vorstudie und die offenen Erhebungsmethoden möglichst unbeschränkt anzunähern. Mit der Wahl der Pfadtheorie wurde diese Offenheit jedoch bedeutend eingeschränkt. Daher ist zu prüfen, ob sich die beobachteten strategischen Persistenzen bei der Anton Schlecker e.K. und der BerentzenGruppe AG nicht passender durch andere Erklärungsansätze begreifen ließen. Zur Klärung dieser Frage wurden im Theorieteil der Arbeit alternative Erklä-

1262 Vgl. Eisenhardt (1989); Przyborski \& Wohlrab-Sahr (2008: 36); Yin (2009: 37f.). Gemäß Flick (2007: 492f.) lässt sich die Problematik der Validität in qualitativer Forschung auch in der Frage zusammenfassen, ob der Forscher sieht, was er zu sehen meint. 
rungsansätze vorgestellt, mit den zentralen Komponenten der Pfadtheorie abgeglichen sowie die Differenzen herausgearbeitet. Vor dem Hintergrund dieser theoretischen Reflexionsfläche wurden die strategischen Entwicklungen der Fälle aus einer pfadtheoretischen Perspektive untersucht, die offen war für alternative Erklärungsansätze. Mit den vom Imprinting-Ansatz fokussierten Prägkräften historischer Einflüsse wurden gar Bestandteile dieser Erklärungsansätze in diese Arbeit integriert.

Die Analyse der Entwicklung der strategischen Persistenzen zeigte aber deutlich, dass diese historischen Imprints zwar in bedeutender Weise den strategischen Entscheidungsraum einschränkten und früh einen Entwicklungskorridor ausformten, aber nicht für die letztlich zu beobachtende beinahe vollständige Determination der strategischen Entwicklung ursächlich waren. Hierauf verweist auch der Umstand, dass die Vergleichsfälle der Dirk Rossmann GmbH und der Eckes AG ebenfalls historisch geprägt waren, sich diese Unternehmen aber sowohl innerhalb des historisch geprägten Entwicklungskorridors strategisch wandeln als auch diesen Korridor im Zeitverlauf verschieben konnten.

Die Theorie der "structural inertia« kann zur Erklärung der Wandlungswiderstände und der ausbleibenden Reaktion auf den strategischen Pfad beitragen, indem der Aufbau von standardisierten Prozessen und Routinen oder die Institutionalisierung von Regeln und Normen beleuchtet wird. Aufgrund der fehlenden Berücksichtigung der ökonomischen und sozio-emotionalen Dynamiken erklärt die Theorie der "structural intertia" jedoch weder bei der Anton Schlecker e.K. noch bei der Berentzen-Gruppe AG den Entstehungsprozess der strategischen Persistenz.

Dies gilt auch für den Ansatz des »escalating commitment «. Diese theoretische Perspektive vermag es, zur Erklärung des teilweise pathologischen Entscheidungsverhaltens in der Phase der Pfadformation beizutragen, als sich die Ineffizienz der strategischen Entwicklung abzeichnete, aber das strategische Muster, zum Teil auch aus Angst vor Gesichtsverlust, weiter reproduziert wurde. Das Erklärungsangebot des »escalating commitment« kann jedoch nicht klären, warum in den untersuchten Unternehmen nicht nur in einzelnen Entscheidungssituationen, sondern über Jahrzehnte an den etablierten strategischen Mustern festgehalten wurde.

Im Vergleich zu diesen alternativen Ansätzen zur Erklärung des ausbleibenden strategischen Wandels kann die Pfadtheorie somit als ein gegenstandsangemessener Ansatz zur Analyse der strategischen Persistenzen erachtet werden.

Es stellt sich des Weiteren die Frage der Validität der in diesem theoretischen Rahmen identifizierten und beschriebenen Kausalzusammenhänge. Mit dem etablierten Phasenmodell der organisationalen Pfadgenese nach Sydow et al. (2009) stand ein bewährtes Analyseinstrument zur Verfügung, welches in 
sich geschlossen die strategische Persistenz theoretisch erklärt. ${ }^{1263}$ Viele Autoren der empirischen Pfadforschung in Organisationen räumen allerdings die meist schwierige analytische Rekonstruktion der empirischen Kausalzusammenhänge der strategischen Pfade ein. ${ }^{1264}$

Auch in vorliegender Studie erweist sich, dass strategische Entscheidungsprozesse komplexe Prozesse sind, die eine emergente und eine intentionale Komponente besitzen, sich auf mehreren Ebenen vollziehen und in der analytischen Rekonstruktion erst als getroffene Entscheidungen zu beobachten sind. Sie stellen somit für den Beobachter ein schwer zugängliches Analyseobjekt dar.

Mit den gewählten Methoden der Datenerhebung und -auswertung konnte die für den Nachweis der strategischen Persistenz und die Pfaddiagnose notwendige Untersuchungstiefe jedoch erreicht werden. ${ }^{1265}$ Im Rahmen der auf diesem Wege ermittelten Daten traten die Pfadkomponenten der historischen Imprints, der selbstverstärkenden Mechanismen und der Lock-in klar zutage. Diese Elemente konnten identifiziert und im Rahmen des etablierten Phasenmodells der organisationalen Pfadtheorie nach Sydow et al. (2009) in einen nachvollziehbaren Kausalzusammenhang eingeordnet werden. Die Wirkungszusammenhänge ergaben eine logische Beweiskette.

Schwieriger gestaltete sich der Nachweis des Einflusses des Kontextfaktors Familie auf diese Pfadgenese, mithin der letzte Schritt zur Beantwortung der zentralen Forschungsfrage. Wenngleich manche Beiträge der Familienunternehmensforschung dies nahelegen, können keineswegs sämtliche Entscheidungen in Familienunternehmen und die sie rahmenden Aspekte des strategischen Entscheidungsprozesses tatsächlich überzeugend auf den Einfluss des Systems Familie zurückgeführt werden. Entscheidungen von Mitgliedern der Unternehmensfamilie können beispielsweise auch aufgrund rein persönlicher Dispositionen oder politischer Überzeugungen getroffen werden.

Im Rahmen der Analyse der strategischen Entscheidungsprozesse musste daher stets gefragt werden, ob und inwiefern ein anderer Typ von Eigentümer oder Entscheidungsträger in diesen Prozessen einen signifikant anderen Einfluss ausgeübt hätte. Zur Erhöhung der Validität der Erkenntnisse dieser Arbeit hätte es hier einer noch breiteren und vor allem noch tieferen Datenbasis bedurft. Für die ersten beiden Schritten der Pfadanalyse konnten großteils bereits externe und interne Dokumente herangezogen werden konnten. Für den letzten Schritt gilt dies nicht: Familiäre Einflüsse lassen sich - wenn überhaupt - nur in offenen Interviews rekonstruieren. Daher hätten noch mehr Daten, vor allem

1263 Vgl. Sydow et al. (2009).

1264 Vgl. Holtmann (2008: 252 ff.); Scholle (2013: 419ff.).

1265 An dieser Stelle sei nochmals darauf verwiesen, dass die narrative Interviewtechnik von Schütze (1978) im Rahmen der Rekonstruktion von Entscheidungsprozessen erstmals angewandt wurde. Vgl. Przyborski \& Wohlrab-Sahr (2008: 93); zudem s. Kapitel C.1.2.1. 
Interviewdaten, erhoben werden müssen. Hilfreich wäre nicht nur eine Erhöhung der Anzahl an Interviewpartnern gewesen - insbesondere Mitglieder der Unternehmensfamilien -, sondern auch die Durchführung mehrerer, mit zeitlichem Abstand geführter Interviews mit denselben Interviewpartnern im zyklischen Forschungsprozess.

Spätestens im Kontrast solcher Idealvorstellungen zeigt sich jedoch eine grundlegende Limitation dieser Arbeit: der sehr schwierige Zugang zum Feld. Seit den frühen Jahren der empirischen Unternehmenskrisenforschung macht diese darauf aufmerksam, dass sich die Forschung zu Krisenprozessen erheblich schwieriger gestalte als beispielsweise die Forschung zu den Herausforderungen organisationalen Wachstums. Entscheidungsträgern in Krisensituationen fehle meist die Zeit und Führungskräften in gescheiterten Unternehmen meist die persönliche Bereitschaft, der Managementforschung Auskunft über den Niedergang ihres Unternehmens oder gar eigene Fehler zu geben. ${ }^{1266}$ Diese allgemeine Feststellung trifft auf Familienunternehmen in besonderem Maße zu, da die biografischen Experten hier nicht nur die Bereitschaft aufbringen müssen, über funktionale Defizite und sachliche Fehler zu sprechen, sondern sich darüber hinaus meist auch der Schilderung familiärer Dynamiken öffnen. Dieser Umstand verknappte sowohl das Potenzial der dieser Forschungsarbeit zur Verfügung stehenden Fälle als auch die Zahl der potenziellen Interviewpartner aus dem Kreise der Familie innerhalb eines Falles.

Zur Stützung der vermuteten Zusammenhänge zwischen dem Einfluss der Familie und der Pfadgenese wurde daher auf die in Teil B herausgearbeiteten Strukturmerkmale zurückgegriffen, die als Indikatoren des familiären Einflusses im Unternehmen dienten. Die Frage nach der Validität dieser Charakteristika familiär beeinflusster Unternehmen wird im folgenden Abschnitt kurz diskutiert.

Zugleich wurde in der Analyse aber stets versucht zu reflektieren, inwieweit die beobachteten Ausprägungen der Variablen auch auf andere Einflussfaktoren wie die Persönlichkeiten der Entscheidungsträger zurückzuführen sein könnten. Es spricht einiges dafür, dass in manchen Punkten ein Zusammenhang zwischen der Persönlichkeit der Entscheidungsträger und den verfolgten Strategien der Unternehmen und deren Strukturen besteht, nicht zuletzt aufgrund der meist hohen Machtkonzentration der Entscheidungsträger. Diese Einflüsse standen nicht im Fokus dieser Arbeit und konnten nicht weiter verfolgt werden. Zur Einschätzung des Einflusses der Familie konnten sie jedoch nicht ignoriert werden.

1266 Vgl. Weitzel \& Jonsson (1989). 


\section{Externe Validität}

Abschließend stellt sich die Frage nach der externen Validität und Generalisierbarkeit der Erkenntnisse der Forschungsarbeit. Hinsichtlich dieses Gütekriteriums wird an qualitative Forschungsarbeiten manchmal ein Anspruch herangetragen, welcher der Logik der statistischen Generalisierbarkeit quantitativer Forschung folgt. ${ }^{1267}$ Dem Anspruch, von den beiden Fällen auf die Gesamtpopulation zu schließen, kann die vorliegende Arbeit nicht gerecht werden - sie verfolgte diesen Anspruch jedoch auch nicht. Ihr Ziel war die Exploration möglicher Einflüsse des Kontextfaktors Familie auf den Prozess der Pfadgenese anhand der untersuchten Fälle.

Dies bedeutet jedoch nicht, dass die anhand der Fälle aufgezeigten Wirkungszusammenhänge nur für sich stehen. Sie lassen sich vielmehr in Bezug zur Pfadtheorie analytisch generalisieren. Diesbezüglich besitzt vor allem die Beobachtung, dass die selbstverstärkenden Mechanismen im organisationalen Kontext aufgrund der Existenz von Macht die Genese der Pfade nicht allein erklären können, eine hohe externe Validität: Wenn sich in einem historisch geprägten Entscheidungsraum selbstverstärkende Mechanismen entwickeln, so führt deren Wirkkraft zu strategischer Persistenz und in die Unternehmenskrise - wenn die organisationale Macht in der Phase der Pfadformation nicht für eine entschiedene Reaktion auf die Pfadgenese eingesetzt wird.

Die Einflussgrößen für diese ausbleibende Reaktion wurden für den Kontext familiär beeinflusster Unternehmen näher spezifiziert. Hinsichtlich einer möglichen Übertragbarkeit der beobachteten Zusammenhänge auf andere Fälle gilt es zwei Punkte zu reflektieren:

Die Fälle der Anton Schlecker e.K. und der Berentzen-Gruppe AG wurden nicht aufgrund einer Zufallsauswahl in die Untersuchung einbezogen, sondern repräsentieren zwei typische familial-organisationale Konfigurationen von Familienunternehmen, die nach den vorangegangenen Erkenntnissen der explorativen Vorstudie als besonders pfadsensitiv gelten konnten.

Die innerhalb dieser beiden Konfigurationen wirksamen und für die ausbleibende Reaktion bedeutsamen Zusammenhänge zwischen den Variablen Macht, Diversität und Konflikt sind auf der hohen Abstraktionsebene dieser Arbeit ausführlich diskutiert und empirisch belegt. Die Einflüsse des Faktors Familie auf diese Ausprägungen und Wirkungszusammenhänge verlangen für eine Übertragung auf andere Fälle jedoch eine stärkere Validierung. Weder die erarbeiteten Strukturmerkmale des familiären Einflusses noch deren Bedeutung

1267 Die Übertragung der quantitativen Logik auf den qualitativen Rahmen findet Ausdruck in der Frage: »Was wollen Sie denn auf der Grundlage von ein paar Interviews generalisieren?«; vgl. Flick (2007: 50). 
als Antezedenzien des strategischen Entscheidungsprozesses sind hier bislang ausreichend validiert.

\subsection{Beitrag der Arbeit und weiterführende Diskussion}

Mit der Untersuchung der Genese und Dynamik strategischer Pfade im Kontext familiär beeinflusster Unternehmen leistet die vorliegende Arbeit einen Beitrag zur Unternehmenskrisenforschung, zur organisationalen Pfadtheorie sowie zur Forschung zu Familienunternehmen. Da die Beobachtungen dieser Studie aber nicht als definitive Endpunkte des Forschungsprozesses, sondern vielmehr als Teil einer fortlaufenden theoretischen Entwicklung verstanden werden sollen, wird im Folgenden nicht nur der Beitrag der Arbeit zu den verschiedenen Forschungsfeldern aufgezeigt, sondern es werden auch von dieser Studie indizierte Anknüpfungspunkte für weitere Forschungsarbeiten skizziert.

\section{Unternehmenskrisenforschung}

Die vorliegende Arbeit nahm ihren Ausgangspunkt im Phänomen der Unternehmenskrise und der entsprechenden Managementforschung. Mit strategischen Pfaden fokussierte sie einen besonderen Prozess der Krisenentstehung, den sie im spezifischen Kontext familiär beeinflusster Unternehmen untersuchte. Sie leistete somit allgemein einen Beitrag zur Erklärung derart abgegrenzter Krisenphänomene.

Mit der Vorstellung der organisationalen Pfadtheorie als theoretischer Perspektive auf Prozesse der Krisenentstehung begegnete die Arbeit dem Theoriedefizit der Unternehmenskrisenforschung. Ein solcher Rückgriff auf etablierte Theorien der Sozialwissenschaften oder der Psychologie erscheint auch für zukünftige Forschungsarbeiten erfolgversprechender als die Versuche, zur Entwicklung einer wie auch immer gearteten originären »Theorie der Unternehmenskrise« beitragen zu wollen.

Über die Fokussierung des Prozesses der Pfadgenese als Ursachenkomplex strategischer Persistenzen adressierte die vorliegende Arbeit das eingangs identifizierte prozessuale Defizit der Managementforschung zu Unternehmenskrisen. Mithilfe des Konstrukts des selbstverstärkenden Mechanismus gelang es in der Analyse der empirischen Fälle, bestimmte Ursachen über die Wirkkräfte der Mechanismen mit dem Resultat der strategischen Persistenz zu verknüpfen und somit prozessendogen zu erklären. Auf diesem Wege machte die Studie auf eine wichtige Entwicklungsdynamik von Unternehmenskrisen aufmerksam und zeigte dabei nicht nur auf, welche Ursachen unter welchen Umständen in die Krise führen, sondern vor allem auch, wie dies geschieht.

Mit der Hinwendung zum Kontextfaktor Familie begegnete die Arbeit dem 
zweiten strukturellen Defizit der Forschung zu Unternehmenskrisen: der Vernachlässigung des organisationalen Kontexts. Die Studie knüpfte an Befunde jüngerer Beiträge der Unternehmenskrisenforschung an, die nahelegen, den potenziell bedeutsamen Einfluss von Eigentümerfamilien im Krisenprozess stärker herauszuarbeiten, und zeigte eine Vielzahl direkter und indirekter Einflüsse auf, über die der Faktor Familie auf den Prozess der Krisenentstehung wirken kann. ${ }^{1268}$

Mit der geleisteten retrospektiven Beschreibung und Analyse von Krisenprozessen ist dem Anspruch der Managementlehre als handlungsorientierter Disziplin allerdings nicht vollends Genüge getan. Auf dem Fundament eines tiefen Verständnisses der Krisenursachenkomplexe sollte die Managementlehre auch zur Vermeidung und - da dies nicht immer gelingen wird - vor allem zur Bewältigung von Unternehmenskrisen beitragen. Kommende Forschungsarbeiten sollten sich daher stärker den Möglichkeiten des Bruchs strategischer Pfade widmen.

\section{Pfadforschung}

Nachdem in dieser Studie anhand der Pfadtheorie aufgezeigt wurde, warum der notwendige strategische Wandel in manchen Fällen über viele Jahre unterbleibt, wäre es für zukünftige Forschungsarbeiten beispielsweise interessant zu untersuchen, welche Möglichkeiten die resultierenden Unternehmenskrisen bieten, um den negativen Konsequenzen des ausgebliebenen inkrementellen Wandels durch einen revolutionären Wandel im Turnaround zu begegnen. ${ }^{1269}$ Die skizzierten Fälle indizieren hier, dass die strategische Pfadabhängigkeit eine besonders herausfordernde Situation für mögliche Maßnahmen der Krisenbewältigung ist. Mit der in der Theorie und vor allem in der Praxis dominanten Vorstellung eines zweiphasigen Turnaround-Verfahrens - auf der ersten Stufe wird mit Maßnahmen des »Retrenchment« das Unternehmen stabilisiert und erst im zweiten Schritt, der "Recovery«, wird sich auf der konsolidierten Grundlage dem strategischen Wandel gewidmet - dürfte in Fällen strategischer Pfadabhängigkeit nur selten Erfolg erzielt werden. ${ }^{1270}$ Sämtliche Maßnahmen der Finanzierung oder der Kostensenkung dürften mittelfristig wirkungslos bleiben, wenn nicht von Beginn des Turnaround-Managements an die eigentlichen Ursachen der Krise adressiert werden. Die Krisenursache "strategische Pfadabhängigkeit« kann langfristig nur überwunden werden, wenn die Wirkkräfte des selbstverstärkenden Mechanismus abgeschwächt und das Unternehmen

1268 Vgl. Cater \& Schwab (2008); Trahms et al. (2013).

1269 Vgl. für eine kurze Übersicht zur Kontroverse um die situative Vorteilhaftigkeit und Durchsetzbarkeit von inkrementellem und revolutionärem strategischem Wandel Bresser (2010: 160).

1270 Vgl. zu diesem klassischen zweistufigen Modell zum Beispiel Arogyaswamy et al. (1995); Robbins \& Pearce (1992). 
aus dem Lock-in befreit werden kann. Allerdings ist es ein definitorisches Charakteristikum der strategischen Pfadabhängigkeit, dass ein solcher Bruch des Pfades - wenn überhaupt - nur sehr schwer zu bewerkstelligen ist. Wäre eine Abkehr vom pfadabhängigen Weg in die Krise durch die Anwendung des gängigen Repertoires an finanztechnischen oder juristischen Turnaround-Maßnahmen möglich, so wäre das Unternehmen nicht pfadabhängig im Sinne der hier vorgestellten organisationalen Pfadtheorie.

Um der herausfordernden Situation der Pfadabhängigkeit zu begegnen und den Bruch des Pfades zu bewerkstelligen, erscheinen vielmehr tiefer gehende Ansätze notwendig, die durch Reflexion der aufgedeckten Pfadabhängigkeit und der sie verursachenden Mechanismen eine zunächst kognitive, dann strategische Öffnung bewirken. Schreyögg et al. (2003) verweisen hier auf diskursive Ansätze, verhaltensbezogene und systemische Maßnahmen der Organisationsentwicklung sowie ressourcenbezogene Ansätze. ${ }^{1271}$

Die in dieser Studie untersuchten Fälle der Anton Schlecker e.K. und der Berentzen-Gruppe AG legen nahe, dass die Frage des Zeitpunkts solcher Maßnahmen von entscheidender Bedeutung für deren Erfolg ist. Die Anton Schlecker e.K. versuchte mit externer Hilfe im Turnaround-Versuch der Jahre 2011 und 2011 den strategischen Pfad zu verlassen, allerdings setzten diese Maßnahmen mindestens fünf oder zehn Jahre zu spät an. ${ }^{1272}$

Darüber hinaus verweisen die empirischen Fälle indirekt darauf, dass die Existenz des machtvollen Faktors Familie in Kombination mit der Situation der Unternehmenskrise ein besonderes Potenzial zum Bruch strategischer Pfade darstellen könnte. In der existenzbedrohenden Unternehmenskrise manifestiert sich die Nachteilhaftigkeit der etablierten strategischen Muster, und die vorher möglicherweise fest verankerten Wahrnehmungsschemata und Entscheidungsmuster werden erschüttert. Die Krise bietet hier somit ein besonderes Momentum für Wandel. Tritt in diese Dynamik zusätzlich ein Faktor wie die Familie, die über konzentrierte Machtmittel verfügt, um notwendige Veränderungen durchzusetzen, so kann in manchen Fällen der erforderliche strategische Wandel vielleicht noch vollzogen werden. Es erscheint allerdings fraglich, ob der Austausch der Unternehmensleitung und insbesondere der familiären Entscheidungsträger hier nicht eine essenzielle Voraussetzung für einen Bruch des

1271 Vgl. Schreyögg et al. (2003: 278f.).

1272 Vgl. eine ehemalige Führungskraft der Berentzen-Gruppe AG: »Ich glaube, wir haben bis zum letzten Tag den eingeschlagenen Weg für den richtigen gehalten. Und ich sage es nochmal: Das, was heute passiert ist oder die letzten zwei Jahre passiert ist, das ist ja letzten Endes auch zu achtzig Prozent das, was wir damals schon hatten. Aber wir waren halt damals unter den gegebenen Umständen zu spät. Das ist wie, wenn ein Patient zum Arzt geht und der eben drei Jahre später als notwendig mit der, mit der Therapie anfängt.» 
strategischen Pfades ist, um die für diesen Wandel notwendige Exogenität zu erreichen. $^{1273}$

Mit der Untersuchung der Möglichkeiten des Pfadbruchs im Kontext von Familienunternehmen könnten zukünftige Forschungsarbeiten somit nicht nur die Unternehmenskrisenforschung bereichern, sondern auch die seit Langem geführte, aber bislang weitgehend ergebnislose Diskussion um Möglichkeiten und Mittel des Bruchs von Pfaden in der Pfadforschung voranbringen. ${ }^{1274}$

Quasi einen Schritt zuvor trug vorliegende Arbeit dazu bei, den organisationalen Kontext der Pfadgenese näher zu beleuchten. In diesem Zuge machte die Studie vor allem darauf aufmerksam, dass im organisationalen Rahmen die Akteure in der Phase der Pfadformation häufig lange Zeit noch ausreichend Macht besitzen, um der Wirkkraft der selbstverstärkenden Mechanismen zu begegnen, und dass die Wirkrichtung dieser Akteure daher zu einer erklärungsbedürftigen Variable wird. Zudem zeigte sie, dass der Faktor Familie von Bedeutung sein kann, um die Wirkrichtung der Macht auf die Pfadgenese zu verstehen.

Spezifischer erbrachte vorliegende Arbeit auch den von Schreyögg et al. (2003) geforderten Nachweis, dass sozio-emotionale Prozesse mit einer mikropolitischen Komponente nicht nur eine selbstverstärkende Dynamik besitzen, sondern auch tatsächlich zur Genese von strategischer Pfadabhängigkeit führen können. ${ }^{1275}$

Mit dem gewählten offenen Design und den entsprechenden Methoden der Datenerhebung und -auswertung gelang annäherungsweise die Rekonstruktion der Prozesse in "Echtzeit $« .{ }^{1276}$ Insbesondere die der Biografieforschung entlehnte methodisch innovative sequenzielle retrospektiv-prospektiv kontrastierende Analyse der organisational-strategischen Entwicklung könnte für nachfolgende Arbeiten der Pfadforschung von Interesse sein. Diese Methode fand nach Kenntnis des Autors in der Pfadforschung bislang noch keine Anwendung, könnte aber anderen Studien helfen, um sich in der Rekonstruktion der Prozesse in die historische Position der handelnden Akteure zu versetzen und aus deren damaliger Perspektive den verbliebenen Handlungsspielraum auszuloten.

\section{Forschung zum Faktor Familie}

Vorliegende Arbeit leistete aber auch einen Beitrag zur Forschung zum Faktor Familie. Indem sie sich in die Linie der Arbeiten stellte, die weniger die Entität

1273 Zum »Topmanagement Change« und dessen Notwendigkeit für die Krisenbewältigung vgl. allgemein Pearce \& Robbins (2008); Schendel et al. (1976); Slatter \& Lovett (1999).

1274 Vgl. Schreyögg et al. (2003: 273ff.); auch Petermann (2010); Tobias (2012).

1275 Vgl. Schreyögg et al. (2003: 271).

1276 Vgl. zu diesen methodischen Überlegungen der Pfadforschung Garud et al. (2010: 770); Sydow et al. (2009). 
des Familienunternehmens fokussieren als vielmehr die spezifischen Einflüsse, die das System Familie auf das System Unternehmen ausübt, gelang es ihr den Faktor Familie in ein etabliertes Theoriegebäude der Managementforschung zu integrieren. Für diese Integration von Bedeutung waren vor allem die charakteristischen Merkmale in Struktur und Kultur, die als Indikatoren des familiären Einflusses herangezogen wurden. Über einen solchen systemtheoretisch orientierten Zugang kann sich die Forschung zum Faktor Familie noch viele weitere Theoriewelten erschließen.

Die vorliegende Arbeit indizierte zum Beispiel, dass der Faktor Familie gewinnbringend in die Upper-Echelon-Forschung integriert werden könnte. Diese fragt nach mehreren Jahrzehnten fruchtbarer Forschung zum Zusammenhang zwischen demografischen Merkmalen des Topmanagements und strategischen Outputvariablen nicht nur nach der Bedeutung moderierender Variablen wie des Konflikts, sondern eben auch nach den Antezedenzien der Zusammensetzung der Topmanagement-Teams. ${ }^{1277}$ Die Integration der Einflüsse des Systems Familie - als unabhängigem Faktor - könnten hier in vielen Fällen die Ausprägungen von abhängigen Variablen wie der Diversität verständlich machen.

Allerdings ist die Managementforschung für eine solche Integration auf eine in diesem wie in anderen Forschungskontexten auf eine belastbare Grundlage angewiesen, wie genau sich der Einfluss des Faktors Familie in welchen strukturellen und kulturellen Merkmalen niederschlägt. Diesbezüglich müsste die theoretische und qualitative Forschung zum Faktor Familie im Sinne der »4 C’s« "Command «, "Continuity«, »Community" und »Connections" noch klarer und konsistenter herausarbeiten was die Merkmale des familiären Unternehmens sind. Ein stärkerer Rekurs der Managementforschung auf das System Familie und deren Funktionslogiken durch eine engere Verknüpfung mit der etablierten Familienforschung erscheint hier vorteilhaft. Hierauf aufbauend müssten die Strukturmerkmale weitr in groß angelegten quantitativen Studien getestet werden, um nicht nur theoretisch konsistente, sondern auch valide und generalisierbare Aussagen zur Wirkung des Einflusses des Faktors Familie im Unternehmen treffen zu können.

\section{Beitrag für die unternehmerische Praxis}

Letztlich kann vorliegende Studie auch der unternehmerischen Praxis dienlich sein. Zwar generierte sie aufgrund des Charakters ihrer Forschungsfrage keine derart eindeutigen Handlungsempfehlungen, wie sie die Praxis von der Mana-

1277 Vgl. zu diesem Bestreben der Upper-Echelon-Forschung Carpenter et al. (2004: 759f.); Nielsen (2010: 309), vor allem auch Hambrick (2007: 338): »There is a need to turn upper echelons theory on its head by considering executive characteristics as consequences rather than as causes [...]. Why do top management teams look the way they do?» 
gementforschung teilweise erwartet. Die Studie kann den Akteuren der unternehmerischen und beratenden Praxis aber als theoretische und empirische Reflexionsebene dienen, um prozessuale Entwicklungsdynamiken in anderen Unternehmen zu beleuchten und besser zu verstehen.

Über diesen theoretisch abgegrenzten Rahmen weit hinaus verdeutlicht die Untersuchung über die Dramatik der dargestellten empirischen Entwicklungen eindrücklich die Notwendigkeit eines permanenten strategischen Wandels als Existenzbedingung in einer dynamischen und komplexen Umwelt. Die Arbeit mahnt diesen Wandel allerdings nicht nur an, sondern verweist auch auf dessen Voraussetzungen: kulturelle und strukturelle Offenheit einer Organisation, die sich der Umwelt nicht verschließen darf, sondern sich den Chancen öffnen und den Herausforderungen frühzeitig begegnen muss. Eine Vielfalt an unterschiedlichen Wahrnehmungen und Perspektiven der Akteure, um die Komplexität der Dynamik der Umwelt annäherungsweise zu begreifen, ist gefordert. Ebenso bedarf es Kommunikationskulturen, in denen diese verschiedenen Perspektiven zum fruchtbaren Austausch in den Entscheidungsprozess einfließen und "sich reiben " können, um zu ganzheitlicheren synthetischen Lösungen zu gelangen, ohne dass die Auseinandersetzungen in der Sache zu einem Ringen auf der Beziehungsebene ausarten.

Die Anton Schlecker e.K. und die Berentzen-Gruppe AG vermochten es auf Dauer nicht, diese organisationalen Voraussetzungen des strategischen Wandels bereitzustellen und konnten daher nicht mehr adäquat auf ihre endogenen Problemkonstellationen und den Wandel ihrer Umwelt reagieren. Ihr Scheitern mag gegenwärtigen und zukünftigen Entscheidungsträgern als mahnendes Beispiel dienen. 


\section{F Anhang}

\section{$1 \quad$ Kurzchroniken der Unternehmensentwicklungen}

\subsection{Fallgruppe 1: Anton Schlecker e.K. und Dirk Rossmann GmbH}

\begin{tabular}{|c|c|c|}
\hline Jahr & Objekt & Ereignis \\
\hline \multicolumn{3}{|c|}{ Vorspann } \\
\hline 1908 & Rossmann & Großvater von Dirk Rossmann gründet Drogerie \\
\hline 1944 & Schlecker & Anton Schlecker jun. wird in Ehingen geboren \\
\hline 1950 & Schlecker & Anton Schlecker sen. eröffnet erste Metzgerei in Ehingen \\
\hline 1965 & Schlecker & $\begin{array}{l}\text { Anton Schlecker jun. steigt als Metzgermeister in das Unternehmen } \\
\text { seines Vaters ein, aus dem sich mittlerweile eine Fleischwarenfabrik } \\
\text { mit } 17 \text { angeschlossenen Filialen entwickelt hat. } \\
\text { Eröffnung des ersten SB-Verbrauchermarkts }\end{array}$ \\
\hline $1960 \mathrm{er}$ & Umwelt & Aufstieg des Discount-Prinzips \\
\hline 1972 & Rossmann & $\begin{array}{l}\text { Dirk Roßmann übernimmt das Discount-Prinzip und eröffnet in } \\
\text { Hannover den ersten Drogeriemarkt Deutschlands. Zu diesem } \\
\text { Zeitpunkt gibt es in D noch } 17.000 \text { inhabergeführte Fachdrogerien }\end{array}$ \\
\hline 1973 & Umwelt & Götz Werner eröffnet in Karlsruhe den ersten dm-Drogeriemarkt \\
\hline \multirow[t]{2}{*}{1974} & Umwelt & Preisbindung für Drogerieartikel wird abgeschafft \\
\hline & Schlecker & Anton Schlecker jun. übernimmt die Geschäftsführung vom Vater \\
\hline 1975 & Schlecker & Schlecker steigt in den Drogeriemarkt ein \\
\hline 1977 & Schlecker & Eröffnung der 100. Filiale \\
\hline 1980 & Rossmann & Einstieg der Hannover Finanz als Minderheitsgesellschafter (10\%) \\
\hline \multirow[t]{2}{*}{1981} & Schlecker & Übernahme der Marktführerschaft von dm \\
\hline & Umwelt & Anzahl Fachdrogerien auf 8.000 gesunken \\
\hline 1984 & Schlecker & Eröffnung der 1.000. Filiale \\
\hline 1987 & Schlecker & $\begin{array}{l}\text { Start der Auslandsexpansion (1987: AU; 1989: NL + LUX + ESP; } \\
\text { 1991: FR) } \\
\text { Entführung von Lars und Meike Schlecker }\end{array}$ \\
\hline 1988 & Rossmann & Liquiditätskrise \\
\hline
\end{tabular}


(Fortsetzung)

\begin{tabular}{|c|c|c|}
\hline Jahr & Objekt & Ereignis \\
\hline \multicolumn{3}{|c|}{ Strategiekrise } \\
\hline \multirow[t]{3}{*}{$1990 \mathrm{er}$} & Umwelt & $\begin{array}{l}\text { Veränderungen im Verbraucherverhalten: Günstige Preise }+ \\
\text { differenziertes Sortiment (Bio-Produkte, Handelsmarken etc.) }\end{array}$ \\
\hline & Umwelt & $\begin{array}{l}\text { Trading-Up vieler Wettbewerber, insb. dm (Slogan von dm »Große } \\
\text { Marken. Kleine Preise« wird zu »Hier bin ich Mensch, hier kauf ich } \\
\text { ein«) }\end{array}$ \\
\hline & Schlecker & $\begin{array}{l}\text { Skandale um verunreinigte Lebensmittel, häufige Überfälle der } \\
\text { Filialen, schlechte Arbeitsbedingungen }\end{array}$ \\
\hline 1990 & Rossmann & Expansion nach Osteuropa \\
\hline 1997 & Schlecker & Wachstum um 1.000 Filialen auf nun 7.500 Filialen \\
\hline \multirow[t]{2}{*}{1998} & Schlecker & Strafbefehl gegen Anton und Christa Schlecker wegen Lohnbetrugs \\
\hline & Rossmann & Beginn des Trading-Ups \\
\hline 2000 & Schlecker & $\begin{array}{l}\text { Umsatz von 5,13 Mrd. Euro (dm: } 2 \text { Mrd. Euro; Rossmann: } 763 \text { Mio. } \\
\text { Euro) }\end{array}$ \\
\hline $2000 \mathrm{er}$ & Umwelt & Konsolidierung in der Branche durch Übernahmen \\
\hline \multirow[t]{2}{*}{2003} & Schlecker & Filialnetz umfasst 13.300 Filialen (v. a. in D) \\
\hline & Rossmann & $\begin{array}{l}\text { Rossmann beendet Einkaufskooperation mit Müller und startet } \\
\text { Expansion in den Süden Deutschlands. }\end{array}$ \\
\hline 2004 & Umwelt & $\begin{array}{l}\text { dm eröffnet erste Filiale in Berlin. Der Wettbewerb zwischen den } \\
\text { führenden Drogeriemärkten wird nun bundesweit ausgetragen }\end{array}$ \\
\hline \multicolumn{3}{|c|}{ Erfolgskrise } \\
\hline \multirow[t]{2}{*}{$2000 \mathrm{er}$} & Umwelt & $\begin{array}{l}\text { Zunehmender Preiskampf zwischen dm und Rossmann, dem } \\
\text { Schlecker nicht mehr folgen kann }\end{array}$ \\
\hline & Umwelt & $\begin{array}{l}\text { Der Umsatz von dm und Rossmann wächst mehrere Jahre mit } \\
\text { zweistelligen Raten }\end{array}$ \\
\hline 2004 & Schlecker & $\begin{array}{l}\text { 14.166 Filialen, 6,6 Mrd. Euro Umsatz, } 6 \text { Mio. Euro Gewinn } \\
\text { Deutliche Umsatzrückgänge auf Altflächen }\end{array}$ \\
\hline 2007 & Schlecker & Externes Wachstum durch Übernahme der insolventen Ihr Platz \\
\hline 2008 & Schlecker & Eröffnung erster Schlecker XL-Filialen \\
\hline 2009 & Schlecker & $\begin{array}{l}\text { Skandal um Beschäftigung von ehemaligen Schlecker-Mitarbeitern } \\
\text { in XL-Filialen zu schlechteren Leiharbeitskonditionen über } \\
\text { familieneigene Gesellschaft. }\end{array}$ \\
\hline \multicolumn{3}{|c|}{ Turnaround-Versuch } \\
\hline \multirow[t]{2}{*}{2010} & Schlecker & $\begin{array}{l}\text { Ausarbeitung eines Turnaround-Konzepts durch } \\
\text { Unternehmensberatung (»Fit for Future«). Lars und Meike Schlecker } \\
\text { beginnen das Unternehmen nach außen zu repräsentieren. }\end{array}$ \\
\hline & Schlecker & Rückzug aus einigen Ländern (2009: DK; 2010: HUN + BE + NL). \\
\hline \multirow[t]{2}{*}{2011} & Schlecker & Familienfremde Geschäftsführung \\
\hline & Schlecker & $\begin{array}{l}\text { Implementierung Turnaround-Konzept (neues Ladenkonzept, } \\
\text { zeitgemäßes Marketing etc.) }\end{array}$ \\
\hline
\end{tabular}


(Fortsetzung)

\begin{tabular}{lll}
\hline Jahr & Objekt & Ereignis \\
\hline Liquiditätskrise & \\
\hline $\mathbf{2 0 1 1}$ & Schlecker & $\begin{array}{l}\text { Probleme bei Warenversorgung. Familie sucht ergebnislos } \\
\text { Investoren }\end{array}$ \\
\hline & Schlecker & Insolvenzantrag \\
\hline $\mathbf{2 0 1 2}$ & Schlecker & $\begin{array}{l}\text { Gläubigerausschuss verkündet mangels Fortführungsperspektive } \\
\text { das Ende der Anton Schlecker e.K. }\end{array}$ \\
\hline
\end{tabular}

\subsection{Fallgruppe 2: Berentzen-Gruppe AG und Eckes AG}

\begin{tabular}{lll}
\hline Jahr & Objekt & Ereignis \\
\hline Vorspann & \\
\hline $\mathbf{1 7 8 5}$ & Berentzen & Gründung der Kornbrennerei J. B. Berentzen \\
\hline $\mathbf{1 8 5 7}$ & Eckes & Gründung der Brennerei \\
\hline $\mathbf{1 8 9 8}$ & Berentzen & $\begin{array}{l}\text { markenrechtliche Eintragung des Kornbrands »Berentzen vom alten } \\
\text { Faß« }\end{array}$ \\
\hline $\mathbf{1 9 3 1}$ & Eckes & Einstieg in das Geschäft mit Fruchtsäften \\
\hline $\mathbf{1 9 5 3}$ & Eckes & $\begin{array}{l}\text { Markteinführung des Weinbrands Chantré, der den Umsatz } \\
\text { innerhalb eines Jahres auf 9 Mio. DM verdreifacht }\end{array}$ \\
\hline $\mathbf{1 9 5 4}$ & Berentzen & $\begin{array}{l}\text { Die beiden ältesten Brüder Hans und Friedrich Berentzen } \\
\text { übernehmen nach dem Tod des Vaters die Geschäftsführung }\end{array}$ \\
\hline $\mathbf{1 9 5 5}$ & Eckes & Ein Stamm scheidet nach langwierigen Auseinandersetzungen aus \\
\hline $\mathbf{1 9 5 7}$ & Eckes & Umsatz von 100 Mio. DM \\
\hline $\mathbf{1 9 5 8}$ & Berentzen & $\begin{array}{l}\text { Einstieg in den Markt für alkoholfreie Getränke durch die Gründung } \\
\text { der Emsland-Getränke GmbH }\end{array}$ \\
\hline $\mathbf{1 9 6 0}$ & Emwelt & Markteinführung des Orangensafts hohes C \\
\hline $\mathbf{1 9 6 1}$ & Eckes & $\begin{array}{l}\text { Mark-Kopf Verbrauch Spirituosen: 4,2 l/ Kopf } \\
\text { avanciert }\end{array}$ \\
\hline $\mathbf{1 9 7 0 e r}$ & Berentzen & $\begin{array}{l}\text { Konzentration im LEH sowie Aufkommen von Discount und } \\
\text { Handelsmarken erhöhen Preisdruck }\end{array}$ \\
\hline $\mathbf{1 9 7 3}$ & Umwelt & $\begin{array}{l}\text { EG-Liberalisierung der Spirituosenbranche } \\
\text { Große internationale Unternehmen drängen auf den fragmentierten } \\
\text { geprägten deutschen Markt, während die deutschen } \\
\text { mittelständischen Unternehmen kaum exportieren }\end{array}$ \\
\hline & Eckes & $\begin{array}{l}\text { Organisationale Veränderungen (1972: Gründung von Stiftungen zur } \\
\text { Verwaltung des Eigentums; 1973: Bestellung eines } \\
\text { Fremdgeschäftsführers und Einrichtung eines Beirats; 1975: Einstieg } \\
\text { der 5. Generation in Geschäftsführung) }\end{array}$ \\
\hline & & \\
\hline
\end{tabular}


(Fortsetzung)

\begin{tabular}{lll}
\hline Jahr & Objekt & Ereignis \\
\hline $\mathbf{1 9 7 6}$ & & $\begin{array}{l}\text { Pro-Kopf Verbrauch Spirituosen: 8,2 1/ Kopf } \\
\text { (In der Folge sinkt der Konsum stetig) }\end{array}$ \\
\hline & Berentzen & $\begin{array}{l}\text { Einführung des Berentzen-Apfelkorn, der erfolgreichsten } \\
\text { Markteinführung einer Spirituose nach dem Zweiten Weltkrieg } \\
\text { Strategie des »Wachsen oder Weichens« }\end{array}$ \\
\hline $\mathbf{1 9 7 9}$ & Berentzen & Der Apfelkorn ist die zweitmeist verkaufte Spirituose in D \\
\hline $\mathbf{1 9 8 3}$ & Eckes & $\begin{array}{l}\text { Gesellschafter verlässt nach Konflikt Geschäftsführung, bleibt aber } \\
\text { Eigentümer }\end{array}$ \\
\hline $\mathbf{1 9 8 8}$ & Berentzen & $\begin{array}{l}\text { Fusion mit der Weinbrennerei Pabst \& Richarz. } \\
\text { Sitz des fusionierten Unternehmens ist Haselünne, Organe werden } \\
\text { paritätisch besetzt } \\
\text { Übernahme Ammerländer }\end{array}$ \\
& & $\begin{array}{l}\text { Akquisition von König \& Schlichte (u. a. mit Wodka-Marke } \\
\text { Puschkin) } \\
\text { Berentzen-Gruppe wird neben Eckes zu einem der größten } \\
\text { Spirituosenhersteller in D }\end{array}$ \\
\hline $\mathbf{1 9 8 9}$ & Berentzen \\
& & Akquition von Nordbrand Nordhauser, dem großten \\
& &
\end{tabular}

\begin{tabular}{|c|c|c|}
\hline 1990 & Eckes & $\begin{array}{l}\text { Akquisition von Nordbrand Nordhäuser, dem größten } \\
\text { Produktionsbetrieb der DDR }\end{array}$ \\
\hline & & $\begin{array}{l}\text { Rechtsformänderung: Eckes AG entsteht und alkoholische und } \\
\text { Fruchtsäfte werden zu getrennten Sparten }\end{array}$ \\
\hline 1992 & & $\begin{array}{l}\text { Akquisition von Doornkaat } \\
\text { Der konsolidierte Gruppenumsatz ist damit innerhalb von fünf } \\
\text { Jahren von } 500 \text { Mio. DM um } 80 \% \text { auf rund } 900 \text { Mio. DM gestiegen }\end{array}$ \\
\hline 1993 & Eckes & $\begin{array}{l}\text { Nach dem Wechsel zweier Familienmitglieder in den Aufsichtsrat ist } \\
\text { das TMT vollkommen familienfremd }\end{array}$ \\
\hline 1994 & Berentzen & $\begin{array}{l}\text { Börsengang: Die Berentzen-Gruppe AG entsteht } \\
\text { (Stammaktien verbleiben bei Unternehmensfamilien) } \\
\text { Sprecher des Vorstands wird Jan B. Berentzen }\end{array}$ \\
\hline \multirow[t]{2}{*}{1992} & Berentzen & Generationswechsel in Führung des Unternehmens \\
\hline & Eckes & Übernahmen des Fruchtsaftanbieters Granini \\
\hline \multicolumn{3}{|c|}{ Strategiekrise } \\
\hline 1996 & Berentzen & $\begin{array}{l}\text { Übernahme von Strothmann } \\
\text { Allzeithoch der Aktie, die anschließend aber stetig an Wert verliert }\end{array}$ \\
\hline 1997 & Berentzen & $\begin{array}{l}\text { Hohe Abschreibungen infolge der vielen Übernahmen und } \\
\text { misslungene Markteinführung von Alkopops belasten Ergebnis. } \\
\text { Verlust von -7,1 Mio. Euro }\end{array}$ \\
\hline 1999 & Berentzen & Übernahme der Dethleffsen Spirituosen KG \\
\hline \multicolumn{3}{|c|}{ Erfolgskrise } \\
\hline \multirow[t]{2}{*}{$2000 \mathrm{er}$} & Berentzen & Umsatzrückgang setzt sich fort, Ertrag nimmt ab \\
\hline & & $\begin{array}{l}\text { Anteil von Handelsmarken am Spirituosenmarkt nimmt stetig zu. } \\
\text { Trifft insb. kleine schwache Marken }\end{array}$ \\
\hline
\end{tabular}


(Fortsetzung)

\begin{tabular}{|c|c|c|}
\hline Jahr & Objekt & Ereignis \\
\hline 2001 & Berentzen & $\begin{array}{l}\text { Der Stamm Pabst \& Richarz zieht sich aus der aktiven } \\
\text { Unternehmensleitung im Vorstand zurück }\end{array}$ \\
\hline \multirow[t]{2}{*}{2002} & Berentzen & $\begin{array}{l}\text { Umbesetzung des Aufsichtsrats (u. a. Rückzug von Friedrich } \\
\text { Berentzen, nachdem sich Hans Berentzen schon } 2000 \text { zurückgezogen } \\
\text { hatte) }\end{array}$ \\
\hline & Eckes & $\begin{array}{l}\text { Bekanntgabe der Verkaufsabsicht der traditionsreichen } \\
\text { Spirituosensparte. Mangels akzeptablem Kaufangebots wird der } \\
\text { Verkauf aber gestoppt }\end{array}$ \\
\hline \multirow[t]{3}{*}{2003} & Berentzen & $\begin{array}{l}\text { Übernahmeversuch der deutlich größeren Spirituosensparte der } \\
\text { Eckes AG, die aber aufgrund unterschiedlicher Preisvorstellungen } \\
\text { und Uneinigkeit im Gesellschafterkreis scheitert }\end{array}$ \\
\hline & & Rekordverlust von $-13,8$ Mio. $€$ \\
\hline & & $\begin{array}{l}\text { Aufnahme diverser internationaler Lizenzprodukte ins Sortiment } \\
\text { (»world of fine drinks«) }\end{array}$ \\
\hline \multirow[t]{2}{*}{2004} & Eckes & $\begin{array}{l}\text { Schließung des traditionsreichen Standorts Nieder-Olm und } \\
\text { Konzentration der Spirituosenproduktion in Nordhausen }\end{array}$ \\
\hline & Berentzen & $\begin{array}{l}\text { massiver Umsatzrückgang und Rekordverlust von -13,8 Mio. Euro. } \\
\text { Restrukturierungsprogramm }\end{array}$ \\
\hline \multirow[t]{3}{*}{2005} & Berentzen & Konflikte im Gesellschafterkreis werden publik \\
\hline & Berentzen & $\begin{array}{l}\text { Ankündigung, dass der Vorstandssprecher Jan B. Berentzen } 2007 \text { in } \\
\text { den Aufsichtsrat wechseln wird }\end{array}$ \\
\hline & Eckes & Alle Familienmitglieder verlassen Aufsichtsrat \\
\hline \multirow[t]{2}{*}{2006} & Berentzen & $\begin{array}{l}\text { Jan B. Berentzen tritt im Juni als Vorstandssprecher zurück. Ihm folgt } \\
\text { im Dezember der erste familienfremde Vorstandsvorsitzende }\end{array}$ \\
\hline & Eckes & $\begin{array}{l}\text { Verkauf der Spirituosensparte an Rotkäppchen-Mumm und } \\
\text { Akquisition der restlichen Anteile an Granini }\end{array}$ \\
\hline \multicolumn{3}{|c|}{ Liquiditätskrise, Verkauf und Turnaround } \\
\hline 2007 & Berentzen & Erneuter Verlust von -11,4 Mio. Euro \\
\hline 2008 & Berentzen & $\begin{array}{l}\text { Die Berentzen-Gruppe AG wird im Juni zum Verkauf gestellt und im } \\
\text { Juli an den Finanzinvestor Aurelius verkauft. } \\
\text { Zwischenzeitlich war der Verkaufsprozess wegen eines Zerwürfnisses } \\
\text { im Gesellschafterkreis gestoppt worden, woraufhin der } \\
\text { Vorstandsvorsitzende und einige Aufsichtsräte von ihren Ämtern } \\
\text { zurückgetreten waren und eine Liquiditätskrise drohte. } \\
\text { Dem Verkauf folgt eine Neubesetzung von Vorstand und Aufsichtsrat, } \\
\text { sowie ein umfassendes Restrukturierungsprogramm (u. a. Stilllegung } \\
\text { des Standorts Haselünne) }\end{array}$ \\
\hline 2010 & Berentzen & $\begin{array}{l}\text { Finanzielle Sanierung gelingt } \\
\text { (Verlust 2008: -22,4 Mio. Euro; Gewinn 2010: 8,6 Mio. Euro) } \\
\text { Internationale Expansion wird forciert }\end{array}$ \\
\hline
\end{tabular}


(Fortsetzung)

\begin{tabular}{lll}
\hline Jahr & Objekt & Ereignis \\
\hline 2014 & Berentzen & Akquisition eines Anbieters von Orangensaftpressen \\
& & Dieser trägt maßgeblich zum Gewinn bei und kann bis 2015 seinen \\
& & Umsatz von 3,4 Mio. Euro auf 17,9 Mio. Euro steigern \\
\hline
\end{tabular}

\section{Quellen referenzierter Daten}

Finanzielle Kennzahlen aus Jahresabschlüssen

\begin{tabular}{lll}
\hline Kürzel & Quelle & Titel \\
\hline Berentzen (1998) & Berentzen-Gruppe AG & Geschäftsbericht 1998 \\
\hline Berentzen (1999) & Berentzen-Gruppe AG & Geschäftsbericht 1999 \\
\hline Berentzen (2000) & Berentzen-Gruppe AG & Geschäftsbericht 2000 \\
\hline Berentzen (2001) & Berentzen-Gruppe AG & Geschäftsbericht 2001 \\
\hline Berentzen (2002) & Berentzen-Gruppe AG & Geschäftsbericht 2002 \\
\hline Berentzen (2003) & Berentzen-Gruppe AG & Geschäftsbericht 2003 \\
\hline Berentzen (2004) & Berentzen-Gruppe AG & Geschäftsbericht 2004 \\
\hline Berentzen (2005) & Berentzen-Gruppe AG & Geschäftsbericht 2005 \\
\hline Berentzen (2006) & Berentzen-Gruppe AG & Geschäftsbericht 2006 \\
\hline Berentzen (2007) & Berentzen-Gruppe AG & Geschäftsbericht 2007 \\
\hline Berentzen (2008) & Berentzen-Gruppe AG & Geschäftsbericht 2008 \\
\hline Berentzen (2009) & Berentzen-Gruppe AG & Geschäftsbericht 2009 \\
\hline Berentzen (2010) & Berentzen-Gruppe AG & Geschäftsbericht 2010 \\
\hline Berentzen (2011) & Berentzen-Gruppe AG & Geschäftsbericht 2011 \\
\hline Berentzen (2012) & Berentzen-Gruppe AG & Geschäftsbericht 2012 \\
\hline Berentzen (2013) & Berentzen-Gruppe AG & Geschäftsbericht 2013 \\
\hline Berentzen (2014) & Berentzen-Gruppe AG & Geschäftsbericht 2014 \\
\hline Schlecker (1998) & Anton Schlecker e.K. & Jahresabschluss 1998 \\
\hline Schlecker (1999) & Anton Schlecker e.K. & Jahresabschluss 1999 \\
\hline Schlecker (2000) & Anton Schlecker e.K. & Jahresabschluss 2000 \\
\hline Schlecker (2001) & Anton Schlecker e.K. & Jahresabschluss 2001 \\
\hline Schlecker (2002) & Anton Schlecker e.K. & Jahresabschluss 2002 \\
\hline Schlecker (2003) & Anton Schlecker e.K. & Jahresabschluss 2003 \\
\hline Schlecker (2004) & Anton Schlecker e.K. & Jahresabschluss 2004 \\
\hline Schlecker (2005) & Anton Schlecker e.K. & Jahresabschluss 2005 \\
\hline Schlecker (2006) & Anton Schlecker e.K. & Jahresabschluss 2006 \\
\hline Schlecker (2007) & Anton Schlecker e.K. & Jahresabschluss 2007 \\
\hline & & \\
\hline
\end{tabular}


(Fortsetzung)

\begin{tabular}{lll}
\hline Kürzel & Quelle & Titel \\
\hline Schlecker (2008) & Anton Schlecker e.K. & Jahresabschluss 2008 \\
\hline Schlecker (2009) & Anton Schlecker e.K. & Jahresabschluss 2009 \\
\hline Schlecker (2010) & Anton Schlecker e.K. & Jahresabschluss 2010 \\
\hline
\end{tabular}

Referenzierte Quellen aus externen Dokumenten

\begin{tabular}{|c|c|c|c|}
\hline Kürzel & Quelle & Datum & Titel \\
\hline B631030SP & Der Spiegel & $\begin{array}{l}30.10 . \\
1963\end{array}$ & »Weil er hart ist» \\
\hline B790929NZ & $\begin{array}{l}\text { Neue Osnabrücker } \\
\text { Zeitung }\end{array}$ & $\begin{array}{l}29.09 . \\
1979\end{array}$ & $\begin{array}{l}\text { "Berentzen klettert die Erfolgsleiter } \\
\text { weiter empor» }\end{array}$ \\
\hline B801110SP & Der Spiegel & $\begin{array}{l}10.11 \\
1980\end{array}$ & »Branntweinsteuer. Grausige Mixtur» \\
\hline B830630NZ & $\begin{array}{l}\text { Neue Osnabrücker } \\
\text { Zeitung }\end{array}$ & $\begin{array}{l}30.06 \\
1983\end{array}$ & $\begin{array}{l}\text { "Berentzen hat auch in den USA Fuß } \\
\text { gefasst» }\end{array}$ \\
\hline B851026NZ & $\begin{array}{l}\text { Neue Osnabrücker } \\
\text { Zeitung }\end{array}$ & $\begin{array}{l}26.10 . \\
1985\end{array}$ & $\begin{array}{l}\text { "Berentzen streckt Fühler nach China } \\
\text { und Japan aus» }\end{array}$ \\
\hline B870808NZ & $\begin{array}{l}\text { Neue Osnabrücker } \\
\text { Zeitung }\end{array}$ & $\begin{array}{l}08.09 . \\
1987\end{array}$ & »Fusion am Spirituosenmarkt» \\
\hline B870908NZ & $\begin{array}{l}\text { Neue Osnabrücker } \\
\text { Zeitung }\end{array}$ & $\begin{array}{l}08.09 . \\
1987\end{array}$ & $\begin{array}{l}\text { "Fusion zwischen Berentzen und Pabst } \\
\text { \& Richarz» }\end{array}$ \\
\hline B870909NZ & $\begin{array}{l}\text { Neue Osnabrücker } \\
\text { Zeitung }\end{array}$ & $\begin{array}{l}09.09 . \\
1987\end{array}$ & $\begin{array}{l}\text { "Eine deutsche Antwort am } \\
\text { Spirituosenmarkt» }\end{array}$ \\
\hline B891218HB & Handelsblatt & $\begin{array}{l}18.12 . \\
1989\end{array}$ & $\begin{array}{l}\text { „Berentzen übernimmt König \& } \\
\text { Schlichte» }\end{array}$ \\
\hline $\mathrm{B} 891231 \mathrm{PM}$ & Pressemitteilung & $\begin{array}{l}31.12 . \\
1989\end{array}$ & $\begin{array}{l}\text { "Erfolgreicher Geschäftsverlauf von } \\
\text { Berentzen in 1989. Optimistische } \\
\text { Perspektiven für die 90er Jahre» }\end{array}$ \\
\hline B910214NZ & $\begin{array}{l}\text { Neue Osnabrücker } \\
\text { Zeitung }\end{array}$ & $\begin{array}{l}26.02 . \\
1991\end{array}$ & $\begin{array}{l}\text { „Berentzen-Gruppe: Gesteckte Ziele } \\
1990 \text { weit übertroffen« }\end{array}$ \\
\hline B910720NZ & $\begin{array}{l}\text { Neue Osnabrücker } \\
\text { Zeitung }\end{array}$ & $\begin{array}{l}20.07 . \\
1991\end{array}$ & $\begin{array}{l}\text { "Berentzen will Mehrheit bei Doornkaat } \\
\text { übernehmen« }\end{array}$ \\
\hline B910830NZ & $\begin{array}{l}\text { Neue Osnabrücker } \\
\text { Zeitung }\end{array}$ & $\begin{array}{l}30.08 . \\
1991\end{array}$ & $\begin{array}{l}\text { "Berentzen bei Doornkaat jetzt } \\
\text { Mehrheitsaktionär« }\end{array}$ \\
\hline B911204HB & Handelsblatt & $\begin{array}{l}04.12 . \\
1991\end{array}$ & $\begin{array}{l}\text { "Die Spirituosen-Gruppe soll neu } \\
\text { formiert werden« }\end{array}$ \\
\hline B920101AW & Absatzwirtschaft & $\begin{array}{l}01.01 . \\
1992\end{array}$ & $\begin{array}{l}\text { "Berentzen GmbH \& Co./ Wie viel } \\
\text { Differenzierung braucht die } \\
\text { Dachmarke?» }\end{array}$ \\
\hline B920201HA & Der Handel & $\begin{array}{l}01.02 . \\
1992\end{array}$ & "Doornkaat-Beherrschungsvertrag" \\
\hline
\end{tabular}


(Fortsetzung)

\begin{tabular}{|c|c|c|c|}
\hline Kürzel & Quelle & Datum & Titel \\
\hline B920620NZ & $\begin{array}{l}\text { Neue Osnabrücker } \\
\text { Zeitung }\end{array}$ & $\begin{array}{l}20.06 . \\
1992 \\
\end{array}$ & »Berentzen wechselt in den Aufsichtsrat“ \\
\hline B930327NZ & $\begin{array}{l}\text { Neue Osnabrücker } \\
\text { Zeitung }\end{array}$ & $\begin{array}{l}27.03 . \\
1993\end{array}$ & $\begin{array}{l}\text { „Berentzen-Gruppe will neue Märkte } \\
\text { erschließen« }\end{array}$ \\
\hline B940330NZ & $\begin{array}{l}\text { Neue Osnabrücker } \\
\text { Zeitung }\end{array}$ & $\begin{array}{l}30.03 . \\
1994\end{array}$ & $\begin{array}{l}\text { "Berentzen-Gruppe geht im Sommer an } \\
\text { die Börse" }\end{array}$ \\
\hline B940705NZ & $\begin{array}{l}\text { Neue Osnabrücker } \\
\text { Zeitung }\end{array}$ & $\begin{array}{l}05.07 . \\
1994\end{array}$ & $\begin{array}{l}\text { "Berentzen-Vorzugsaktien zum Preis } \\
\text { von } 345 \mathrm{DM} \text { « }\end{array}$ \\
\hline B940715NZ & $\begin{array}{l}\text { Neue Osnabrücker } \\
\text { Zeitung }\end{array}$ & $\begin{array}{l}15.07 . \\
1994\end{array}$ & »Berentzen-Kurs mit $352 \mathrm{DM}$ eingeführt \\
\hline B960515NZ & $\begin{array}{l}\text { Neue Osnabrücker } \\
\text { Zeitung }\end{array}$ & $\begin{array}{l}15.05 . \\
1996\end{array}$ & "Mit Strothmann dicken Fisch geangelt» \\
\hline B960620NZ & $\begin{array}{l}\text { Neue Osnabrücker } \\
\text { Zeitung }\end{array}$ & $\begin{array}{l}20.06 . \\
1996\end{array}$ & $\begin{array}{l}\text { "Die hochgelegte Latte wurde noch } \\
\text { übersprungen" }\end{array}$ \\
\hline B97090HB & Handelsblatt & $\begin{array}{l}04.09 . \\
1997\end{array}$ & »Berentzen setzt weiter auf Alkopops» \\
\hline B971224HB & Handelsblatt & $\begin{array}{l}24.12 . \\
1997\end{array}$ & $\begin{array}{l}\text { "Ernüchterung bei Spirituosen- } \\
\text { Aktionären" }\end{array}$ \\
\hline B981228NZ & $\begin{array}{l}\text { Neue Osnabrücker } \\
\text { Zeitung }\end{array}$ & $\begin{array}{l}28.12 . \\
1998 \\
\end{array}$ & $\begin{array}{l}\text { "Berentzen-Gruppe übernimmt } \\
\text { Dethleffsen« }\end{array}$ \\
\hline B981229NZ & $\begin{array}{l}\text { Neue Osnabrücker } \\
\text { Zeitung }\end{array}$ & $\begin{array}{l}29.12 . \\
1998\end{array}$ & $\begin{array}{l}\text { »Berentzen übernimmt zum } \\
\text { Jahreswechsel Dethleffsen« }\end{array}$ \\
\hline B000622NZ & $\begin{array}{l}\text { Neue Osnabrücker } \\
\text { Zeitung }\end{array}$ & $\begin{array}{l}22.06 . \\
2000\end{array}$ & $\begin{array}{l}\text { „Dem Standort Haselünne für immer } \\
\text { verpflichtet» }\end{array}$ \\
\hline B010504NZ & $\begin{array}{l}\text { Neue Osnabrücker } \\
\text { Zeitung }\end{array}$ & $\begin{array}{l}04.05 \\
2001\end{array}$ & $\begin{array}{l}\text { „Christian Berentzen wird } \\
\text { Generalbevollmächtigter» }\end{array}$ \\
\hline B020612LZ & $\begin{array}{l}\text { Lebensmittel } \\
\text { Zeitung }\end{array}$ & $\begin{array}{l}12.06 . \\
2002\end{array}$ & $\begin{array}{l}\text { »Berentzen. Der Gründer zieht sich } \\
\text { zurück» }\end{array}$ \\
\hline B030326NZ & $\begin{array}{l}\text { Neue Osnabrücker } \\
\text { Zeitung }\end{array}$ & $\begin{array}{l}26.03 . \\
2003\end{array}$ & »Mariacron zu Berentzen?» \\
\hline В030513HB & Handelsblatt & $\begin{array}{l}13.05 . \\
2003\end{array}$ & $\begin{array}{l}\text { »Eckes bläst den Verkauf seiner } \\
\text { Spirituosen ab» }\end{array}$ \\
\hline B030513NZ & $\begin{array}{l}\text { Neue Osnabrücker } \\
\text { Zeitung }\end{array}$ & $\begin{array}{l}13.05 . \\
2003\end{array}$ & $\begin{array}{l}\text { »Mariacron« bleibt bei Eckes. Bot } \\
\text { Berentzen nicht genug?» }\end{array}$ \\
\hline B030522MM & Manager Magazin & $\begin{array}{l}22.05 . \\
2003\end{array}$ & $\begin{array}{l}\text { »Berentzen. Der Chefdestillator } \\
\text { verflüchtigt sich« }\end{array}$ \\
\hline B040625NZ & $\begin{array}{l}\text { Neue Osnabrücker } \\
\text { Zeitung }\end{array}$ & $\begin{array}{l}06.05 . \\
2004\end{array}$ & $\begin{array}{l}\text { »Berentzens Ziel: Stärker und } \\
\text { internationaler» }\end{array}$ \\
\hline B050524NZ & $\begin{array}{l}\text { Neue Osnabrücker } \\
\text { Zeitung }\end{array}$ & $\begin{array}{l}24.05 . \\
2005\end{array}$ & $\begin{array}{l}\text { „Bei Berentzen keine TV-reife } \\
\text { Familiensaga» }\end{array}$ \\
\hline B050626NW & $\begin{array}{l}\text { Neue } \\
\text { Württembergische }\end{array}$ & $\begin{array}{l}26.06 . \\
2005\end{array}$ & „Berentzen-Chef räumt Sessel vorzeitig“ \\
\hline
\end{tabular}


(Fortsetzung)

\begin{tabular}{|c|c|c|c|}
\hline Kürzel & Quelle & Datum & Titel \\
\hline B050607MM & Manager Magazin & $\begin{array}{l}12.06 . \\
2005\end{array}$ & »Rückzug in den Aufsichtsrat» \\
\hline B050608NW & Nordwest-Zeitung & $\begin{array}{l}08.06 . \\
2005\end{array}$ & »Berentzen-Chef räumt Sessel vorzeitig» \\
\hline B060619HB & Handelsblatt & $\begin{array}{l}19.06 . \\
2006\end{array}$ & »Die Realität entrückt« \\
\hline B060629HB & Handelsblatt & $\begin{array}{l}29.06 . \\
2006\end{array}$ & $\begin{array}{l}\text { „Getränkehaus Berentzen zunächst ohne } \\
\text { Vorstandssprecher« }\end{array}$ \\
\hline B061002FM & Finance Magazin & $\begin{array}{l}02.10 \\
2006\end{array}$ & $\begin{array}{l}\text { „Finanzchef Rost verlässt Berentzen, } \\
\text { Dahm tritt in Vorstand ein« }\end{array}$ \\
\hline B061218FT & Financial Times & $\begin{array}{l}18.12 . \\
2006\end{array}$ & »Flaschenkampf« \\
\hline B070725HB & Handelsblatt & $\begin{array}{l}25.07 \\
2007\end{array}$ & »Berentzen besinnt sich auf alte Werte» \\
\hline B080326HB & Pressemitteilung & $\begin{array}{l}26.03 . \\
2008\end{array}$ & $\begin{array}{l}\text { »Berentzen setzt Innovationsfreude } \\
\text { fort» }\end{array}$ \\
\hline B080627MM & Manager Magazin & $\begin{array}{l}27.06 . \\
2008\end{array}$ & »Verkauf als Notlösung" \\
\hline B080716HB & Handelsblatt & $\begin{array}{l}16.07 . \\
2008\end{array}$ & »Eklat erschüttert Berentzen« \\
\hline B080717KR & $\begin{array}{l}\text { Kölnische } \\
\text { Rundschau }\end{array}$ & $\begin{array}{l}17.07 \\
2008\end{array}$ & $\begin{array}{l}\text { "Führungschaos bei Berentzen. } \\
\text { Kleinkrieg in der Brennerei-Idylle» }\end{array}$ \\
\hline B080717MM & Manager Magazin & $\begin{array}{l}17.07 \\
2008\end{array}$ & "Aufsichtsräte treten zurück» \\
\hline B080720MM & Manager Magazin & $\begin{array}{l}20.07 \\
2008\end{array}$ & »Ex-Aufsichtsräte in Rage» \\
\hline B080721HB & Handelsblatt & $\begin{array}{l}21.07 \\
2008\end{array}$ & $\begin{array}{l}\text { »Berentzen-Chef Axel Dahm steht vor } \\
\text { Rücktritt« }\end{array}$ \\
\hline B080722MM & Manager Magazin & $\begin{array}{l}22.07 \\
2008\end{array}$ & »Investor schluckt Berentzen« \\
\hline B081216HB & Handelsblatt & $\begin{array}{l}16.12 . \\
2008\end{array}$ & »Berentzen mit Kater» \\
\hline B130621IF & Ifo-Institut & $\begin{array}{l}21.06 . \\
2013\end{array}$ & »Pro-Kopf-Verbrauch an Spirituosen» \\
\hline $\mathrm{B} 130614 \mathrm{NZ}$ & $\begin{array}{l}\text { Neue Osnabrücker } \\
\text { Zeitung }\end{array}$ & $\begin{array}{l}14.06 . \\
2013\end{array}$ & $\begin{array}{l}\text { "275 Jahre, acht Generationen - eine } \\
\text { Kaufmannsfamilie» }\end{array}$ \\
\hline $\mathrm{B} 131218 \mathrm{NZ}$ & $\begin{array}{l}\text { Neue Osnabrücker } \\
\text { Zeitung }\end{array}$ & $\begin{array}{l}18.12 . \\
2013\end{array}$ & $\begin{array}{l}\text { "Der Engel von Haselünne - } \\
\text { Bundesverdienstkreuz für Margret } \\
\text { Berentzen« }\end{array}$ \\
\hline B140121HB & Handelsblatt & $\begin{array}{l}21.01 . \\
2014\end{array}$ & »Berentzen tauscht Pepsi gegen Sinalco« \\
\hline
\end{tabular}


(Fortsetzung)

\begin{tabular}{|c|c|c|c|}
\hline Kürzel & Quelle & Datum & Titel \\
\hline B140902HA & $\begin{array}{l}\text { Hamburger } \\
\text { Abendblatt }\end{array}$ & $\begin{array}{l}02.09 . \\
2014\end{array}$ & $\begin{array}{l}\text { »Berentzen übernimmt } \\
\text { Orangensaftproduzenten« }\end{array}$ \\
\hline B160324HB & Handelsblatt & $\begin{array}{l}24.03 \\
2016\end{array}$ & "Orangensaft statt Apfelkorn» \\
\hline E000920MM & Manager Magazin & $\begin{array}{l}20.09 \\
2000\end{array}$ & "Fusion mit Campari gescheitert» \\
\hline E021017LZ & $\begin{array}{l}\text { Lebensmittel } \\
\text { Zeitung }\end{array}$ & $\begin{array}{l}17.10 . \\
2002\end{array}$ & »Käufer und -Verkäufer nervös« \\
\hline E021017LZ & $\begin{array}{l}\text { Lebensmittel } \\
\text { Zeitung }\end{array}$ & $\begin{array}{l}17.10 . \\
2002\end{array}$ & „Großes Echo auf Pläne von Eckes« \\
\hline E021008LZ & $\begin{array}{l}\text { Lebensmittel } \\
\text { Zeitung }\end{array}$ & $\begin{array}{l}08.10 \\
2002\end{array}$ & $\begin{array}{l}\text { "Eckes trennt sich von alkoholischen } \\
\text { Getränken» }\end{array}$ \\
\hline E021008MM & Manager Magazin & $\begin{array}{l}08.10 \\
2002\end{array}$ & »Ein Hohes Lied auf Hohes C» \\
\hline E100825LZ & $\begin{array}{l}\text { Lebensmittel } \\
\text { Zeitung }\end{array}$ & $\begin{array}{l}25.08 \\
2010\end{array}$ & $\begin{array}{l}\text { »Eckes mit neuen } \\
\text { Eigentumsverhältnissen» }\end{array}$ \\
\hline E100825LZ & $\begin{array}{l}\text { Lebensmittel } \\
\text { Zeitung }\end{array}$ & $\begin{array}{l}25.08 . \\
2010\end{array}$ & »Triumph eines Verkannten« \\
\hline R100218HB & Handelsblatt & $\begin{array}{ll}18.02 . \\
2010 \\
\end{array}$ & »Wie Dirk Roßmann Mitarbeiter führt« \\
\hline R120414WE & Die Welt & $\begin{array}{ll}14.04 . \\
2012 \\
\end{array}$ & $\begin{array}{l}\text { „Dirk Roßmanns Söhne haben keinen } \\
\text { Plan B« }\end{array}$ \\
\hline R120602HB & Handelsblatt & $\begin{array}{l}02.06 . \\
2012 \\
\end{array}$ & $\begin{array}{l}\text { "Anton Schlecker hatte mehr als dreißig } \\
\text { Jahre Erfolg" }\end{array}$ \\
\hline R150327LZ & $\begin{array}{l}\text { Lebensmittel } \\
\text { Zeitung }\end{array}$ & $\begin{array}{l}27.03 . \\
2015\end{array}$ & $\begin{array}{l}\text { Drogisten kämpfen um Standorte dm } \\
\text { und Rossmann treiben Expansion weiter } \\
\text { voran }\end{array}$ \\
\hline R150415HB & Handelsblatt & $\begin{array}{l}15.04 . \\
2015\end{array}$ & $\begin{array}{l}\text { „Roßmanns wunderbare Welt des } \\
\text { Wachstums« }\end{array}$ \\
\hline R150416HB & Handelsblatt & $\begin{array}{l}16.04 . \\
2015\end{array}$ & "Er ist wie ich ein harter Knochen" \\
\hline S810522ZE & Die Zeit & $\begin{array}{l}22.05 . \\
1981\end{array}$ & »Bittere Pille für den Drogisten« \\
\hline S930426SP & Der Spiegel & $\begin{array}{l}26.04 . \\
1993\end{array}$ & »Augenmaß verloren« \\
\hline S940101DH & Der Handel & $\begin{array}{l}01.01 . \\
1994\end{array}$ & $\begin{array}{l}\text { "Werner: Schlecker ist das } \\
\text { unproduktivste Unternehmen der } \\
\text { Branche« }\end{array}$ \\
\hline S940415ZE & Die Zeit & $\begin{array}{l}15.04 . \\
1994\end{array}$ & "Hauptsache billig» \\
\hline S991227LZ & $\begin{array}{l}\text { Lebensmittel } \\
\text { Zeitung }\end{array}$ & $\begin{array}{l}27.12 . \\
1999 \\
\end{array}$ & "Schlecker: unverminderte Expansion" \\
\hline
\end{tabular}


(Fortsetzung)

\begin{tabular}{|c|c|c|c|}
\hline Kürzel & Quelle & Datum & Titel \\
\hline S000413LZ & $\begin{array}{l}\text { Lebensmittel } \\
\text { Zeitung }\end{array}$ & $\begin{array}{l}13.04 \\
2000\end{array}$ & "Schlecker bietet mehr» \\
\hline S000824LZ & $\begin{array}{l}\text { Lebensmittel } \\
\text { Zeitung }\end{array}$ & $\begin{array}{l}24.08 \\
2000\end{array}$ & "Schlecker baut Food-Sortiment aus" \\
\hline S010412LZ & $\begin{array}{l}\text { Lebensmittel } \\
\text { Zeitung }\end{array}$ & $\begin{array}{l}12.04 . \\
2001\end{array}$ & "Abstand zu Schlecker bleibt riesig" \\
\hline S010823LZ & $\begin{array}{l}\text { Lebensmittel } \\
\text { Zeitung }\end{array}$ & $\begin{array}{l}23.08 . \\
2001\end{array}$ & »Ungewöhnliche Bilanz» \\
\hline S020110LZ & $\begin{array}{l}\text { Lebensmittel } \\
\text { Zeitung }\end{array}$ & $\begin{array}{l}10.01 \\
2002\end{array}$ & »Nur dm unterbietet Aldis Euro-Preise» \\
\hline S020725LZ & $\begin{array}{l}\text { Lebensmittel } \\
\text { Zeitung }\end{array}$ & $\begin{array}{l}25.07 \\
2002\end{array}$ & $\begin{array}{l}\text { „Schlecker tritt in Deutschland auf der } \\
\text { Stelle» }\end{array}$ \\
\hline S021114LZ & $\begin{array}{l}\text { Lebensmittel } \\
\text { Zeitung }\end{array}$ & $\begin{array}{l}14.11 . \\
2002\end{array}$ & "Handel ist eine Kulturveranstaltung" \\
\hline S030102LZ & $\begin{array}{l}\text { Lebensmittel } \\
\text { Zeitung }\end{array}$ & $\begin{array}{l}02.01 . \\
2003\end{array}$ & „Schlecker weiter mit Expansionsdrang“ \\
\hline S031023LZ & $\begin{array}{l}\text { Lebensmittel } \\
\text { Zeitung }\end{array}$ & $\begin{array}{l}23.10 . \\
2003\end{array}$ & „Schlecker mit leichter Schwächephase» \\
\hline S031204MM & Manager Magazin & $\begin{array}{l}04.12 . \\
2003\end{array}$ & »Knüppeln. Knausern. Kontrollieren« \\
\hline S031218LZ & $\begin{array}{l}\text { Lebensmittel } \\
\text { Zeitung } \\
\end{array}$ & $\begin{array}{l}18.12 . \\
2003\end{array}$ & $\begin{array}{l}\text { "Rossmann geht im Süden in die } \\
\text { Offensive» }\end{array}$ \\
\hline S040819LZ & $\begin{array}{l}\text { Lebensmittel } \\
\text { Zeitung }\end{array}$ & $\begin{array}{l}19.08 . \\
2004\end{array}$ & "Schlecker-Start in Polen" \\
\hline S041021LZ & $\begin{array}{l}\text { Lebensmittel } \\
\text { Zeitung }\end{array}$ & S041021LZ & $\begin{array}{l}\text { »m-drogeriemarkt zieht es in den } \\
\text { Norden« }\end{array}$ \\
\hline S040115LZ & $\begin{array}{l}\text { Lebensmittel } \\
\text { Zeitung }\end{array}$ & $\begin{array}{l}15.01 . \\
2004\end{array}$ & »Rossmann mischt den Markt auf« \\
\hline S040220MM & Manager Magazin & $\begin{array}{l}20.02 . \\
2004\end{array}$ & »Zum Wachsen verdammt» \\
\hline S040520LZ & $\begin{array}{l}\text { Lebensmittel } \\
\text { Zeitung } \\
\end{array}$ & $\begin{array}{l}20.05 . \\
2004 \\
\end{array}$ & "Schlecker drücken Umsatzprobleme» \\
\hline S040617LZ & $\begin{array}{l}\text { Lebensmittel } \\
\text { Zeitung }\end{array}$ & $\begin{array}{l}17.06 . \\
2004\end{array}$ & $\begin{array}{l}\text { „Schleckers Verfolger werden immer } \\
\text { stärker« }\end{array}$ \\
\hline S040805LZ & $\begin{array}{l}\text { Lebensmittel } \\
\text { Zeitung }\end{array}$ & $\begin{array}{l}05.08 . \\
2004\end{array}$ & „Schlecker erhöht die Schlagzahl« \\
\hline S040908MM & Manager Magazin & $\begin{array}{l}08.09 . \\
2004\end{array}$ & $\begin{array}{l}\text { "Wir tauschen offensiv unsere Standorte } \\
\text { aus« }\end{array}$ \\
\hline S041105HB & Handelsblatt & $\begin{array}{l}05.11 . \\
2004\end{array}$ & "Schlecker lernt seine Grenzen kennen« \\
\hline S041026MM & Manager Magazin & $\begin{array}{l}26.10 . \\
2004\end{array}$ & $\begin{array}{l}\text { „Großer Unternehmer mit kleinem } \\
\text { Herz« }\end{array}$ \\
\hline
\end{tabular}


(Fortsetzung)

\begin{tabular}{|c|c|c|c|}
\hline Kürzel & Quelle & Datum & Titel \\
\hline S041028LZ & $\begin{array}{l}\text { Lebensmittel } \\
\text { Zeitung }\end{array}$ & $\begin{array}{l}28.10 . \\
2004\end{array}$ & $\begin{array}{l}\text { „Schwache Konjunktur deckt } \\
\text { Schwächen auf« }\end{array}$ \\
\hline S041105LZ & $\begin{array}{l}\text { Lebensmittel } \\
\text { Zeitung }\end{array}$ & $\begin{array}{l}05.11 . \\
2004\end{array}$ & $\begin{array}{l}\text { »Konzept: Runderneuerung bei } \\
\text { Schlecker« }\end{array}$ \\
\hline S050210LZ & $\begin{array}{l}\text { Lebensmittel } \\
\text { Zeitung }\end{array}$ & $\begin{array}{l}10.02 . \\
2005\end{array}$ & "Lidl geht in die Offensive» \\
\hline S050512LZ & $\begin{array}{l}\text { Lebensmittel } \\
\text { Zeitung }\end{array}$ & $\begin{array}{l}12.05 . \\
2005\end{array}$ & „Schleckers Karten-Coup» \\
\hline S050523LZ & $\begin{array}{l}\text { Lebensmittel } \\
\text { Zeitung }\end{array}$ & $\begin{array}{l}23.05 . \\
2005\end{array}$ & »In dubio pro Schlecker« \\
\hline S050331LZ & $\begin{array}{l}\text { Lebensmittel } \\
\text { Zeitung }\end{array}$ & $\begin{array}{l}31.03 \\
2005\end{array}$ & »Schlecker strafft Filialnetz« \\
\hline S051021HB & Handelsblatt & $\begin{array}{l}21.10 . \\
2005\end{array}$ & $\begin{array}{l}\text { „Drogeriemarkt dm kommt Schlecker } \\
\text { näher« }\end{array}$ \\
\hline S060407LZ & $\begin{array}{l}\text { Lebensmittel } \\
\text { Zeitung }\end{array}$ & $\begin{array}{l}07.04 . \\
2006\end{array}$ & $\begin{array}{l}\text { »Entwicklung: Schlecker legt den } \\
\text { Rückwärtsgang ein« }\end{array}$ \\
\hline S061027LZ & $\begin{array}{l}\text { Lebensmittel } \\
\text { Zeitung }\end{array}$ & $\begin{array}{l}27.10 . \\
2006\end{array}$ & $\begin{array}{l}\text { »Unter Druck: Anton Schlecker greift } \\
\text { durch« }\end{array}$ \\
\hline S070503LZ & $\begin{array}{l}\text { Lebensmittel } \\
\text { Zeitung }\end{array}$ & $\begin{array}{l}03.05 . \\
2007\end{array}$ & „Schleckers Bilanz bleibt grundsolide« \\
\hline S070504LZ & $\begin{array}{l}\text { Lebensmittel } \\
\text { Zeitung }\end{array}$ & $\begin{array}{l}04.05 . \\
2007\end{array}$ & "Schlecker zurück in der Erfolgsspur» \\
\hline S071004FA & $\begin{array}{l}\text { Frankfurter } \\
\text { Allgemeine Zeitung }\end{array}$ & $\begin{array}{l}04.10 . \\
2007\end{array}$ & „Schlecker will die Premium-Kunden« \\
\hline S071004LZ & $\begin{array}{l}\text { Lebensmittel } \\
\text { Zeitung }\end{array}$ & $\begin{array}{l}04.10 . \\
2007\end{array}$ & „Schlecker übernimmt Ihr Platz« \\
\hline S071005HB & Handelsblatt & $\begin{array}{l}05.10 . \\
2007\end{array}$ & $\begin{array}{l}\text { »Der Drogeriekönig erweitert sein } \\
\text { Reich» }\end{array}$ \\
\hline S071296LZ & $\begin{array}{l}\text { Lebensmittel } \\
\text { Zeitung }\end{array}$ & $\begin{array}{l}06.12 . \\
2007\end{array}$ & "Schlecker stellt die Weichen« \\
\hline S080325MM & Manager Magazin & $\begin{array}{l}25.03 . \\
2008\end{array}$ & »Letztes Aufgebot» \\
\hline S080619LZ & $\begin{array}{l}\text { Lebensmittel } \\
\text { Zeitung }\end{array}$ & $\begin{array}{l}19.06 . \\
2008\end{array}$ & »Anton Schlecker handelt» \\
\hline S081030LZ & $\begin{array}{l}\text { Lebensmittel } \\
\text { Zeitung }\end{array}$ & $\begin{array}{l}30.10 . \\
2008\end{array}$ & $\begin{array}{l}\text { "Schlecker wird in die Zange } \\
\text { genommen" }\end{array}$ \\
\hline S090129LZ & $\begin{array}{l}\text { Lebensmittel } \\
\text { Zeitung }\end{array}$ & $\begin{array}{l}29.01 . \\
2009\end{array}$ & »Schlecker räumt auf« \\
\hline S090820LZ & $\begin{array}{l}\text { Lebensmittel } \\
\text { Zeitung }\end{array}$ & $\begin{array}{l}20.08 \\
2009\end{array}$ & "Schleckers neue Welt» \\
\hline S091008LZ & $\begin{array}{l}\text { Lebensmittel } \\
\text { Zeitung }\end{array}$ & $\begin{array}{l}08.10 \\
2009\end{array}$ & "Schlecker bringt sich in XL-Form» \\
\hline
\end{tabular}


(Fortsetzung)

\begin{tabular}{|c|c|c|c|}
\hline Kürzel & Quelle & Datum & Titel \\
\hline$\overline{S 091119 L Z}$ & $\begin{array}{l}\text { Lebensmittel } \\
\text { Zeitung }\end{array}$ & $\begin{array}{l}19.11 . \\
2009\end{array}$ & XL-Filiale in Bad Grönenbach \\
\hline$\overline{S 100517 S U ̈ ~}$ & $\begin{array}{l}\text { Süddeutsche } \\
\text { Zeitung }\end{array}$ & $\begin{array}{l}17.05 \\
2010\end{array}$ & "Die Königin von Ehingen» \\
\hline S100924LZ & $\begin{array}{l}\text { Lebensmittel } \\
\text { Zeitung }\end{array}$ & $\begin{array}{l}24.09 . \\
2010\end{array}$ & "Schlecker schreibt erneut Rot» \\
\hline S101015LZ & $\begin{array}{l}\text { Lebensmittel } \\
\text { Zeitung }\end{array}$ & $\begin{array}{l}15.10 . \\
2010\end{array}$ & "Schlecker gibt sich nicht geschlagen» \\
\hline S100121MM & Manager Magazin & $\begin{array}{l}21.01 \\
2010\end{array}$ & $\begin{array}{l}\text { "Anton Schlecker wehrt sich. »Wir } \\
\text { wollen keinen Ärger«» }\end{array}$ \\
\hline S110118MM & Manager Magazin & $\begin{array}{l}18.01 \\
2011\end{array}$ & "Jugendweihe bei Schlecker» \\
\hline S110120HB & Handelsblatt & $\begin{array}{l}20.01 \\
2011\end{array}$ & $\begin{array}{l}\text { "Schlecker holt familienfremde Manager } \\
\text { in die Spitze« }\end{array}$ \\
\hline S110303LZ & $\begin{array}{l}\text { Lebensmittel } \\
\text { Zeitung }\end{array}$ & $\begin{array}{l}03.03 . \\
2011\end{array}$ & »Schlecker dreht an den Personalkosten« \\
\hline S110309LZ & $\begin{array}{l}\text { Lebensmittel } \\
\text { Zeitung }\end{array}$ & $\begin{array}{l}09.03 . \\
2011\end{array}$ & »Schleckers Testfiliale in Allmendingen \\
\hline S110502LZ & $\begin{array}{l}\text { Lebensmittel } \\
\text { Zeitung }\end{array}$ & $\begin{array}{l}02.05 . \\
2011\end{array}$ & $\begin{array}{l}\text { "Schlecker führt Listen missliebiger } \\
\text { Mitarbeiter« }\end{array}$ \\
\hline$\overline{S 110523 L Z}$ & $\begin{array}{l}\text { Lebensmittel } \\
\text { Zeitung }\end{array}$ & $\begin{array}{l}23.05 . \\
2011\end{array}$ & »Der »sympathische Nachbar Schlecker» \\
\hline S110613FA & $\begin{array}{l}\text { Frankfurter } \\
\text { Allgemeine Zeitung }\end{array}$ & $\begin{array}{l}13.06 . \\
2011\end{array}$ & $\begin{array}{l}\text { "Bei uns muss keine Kassiererin } \\
\text { schuften« }\end{array}$ \\
\hline S110616LZ & $\begin{array}{l}\text { Lebensmittel } \\
\text { Zeitung }\end{array}$ & $\begin{array}{l}16.06 . \\
2011\end{array}$ & "Schrumpfen will gelernt sein» \\
\hline S110624ZE & Die Zeit & $\begin{array}{l}22.06 . \\
2011\end{array}$ & »Die Saulus-Kinder» \\
\hline$\overline{S 111125 L Z}$ & $\begin{array}{l}\text { Lebensmittel } \\
\text { Zeitung }\end{array}$ & $\begin{array}{l}25.11 . \\
2011\end{array}$ & "Die Zukunft der Schleckerwelt» \\
\hline$\overline{S 120126 L Z}$ & $\begin{array}{l}\text { Lebensmittel } \\
\text { Zeitung }\end{array}$ & $\begin{array}{l}26.01 \\
2012\end{array}$ & »Der Fall Schlecker(s)« \\
\hline S120128WW & Wirtschaftswoche & $\begin{array}{l}28.01 \\
2012\end{array}$ & "Die Schlacht um Schlecker» \\
\hline S120204SP & Der Spiegel & $\begin{array}{l}04.02 . \\
2012\end{array}$ & »Endstation Schleckerland» \\
\hline S120313WW & Wirtschaftswoche & $\begin{array}{l}13.03 . \\
2012\end{array}$ & „Schleckers fatale Fehler bei Ihr Platz« \\
\hline S120316SW & $\begin{array}{l}\text { Schwäbische } \\
\text { Zeitung }\end{array}$ & $\begin{array}{l}16.03 . \\
2012\end{array}$ & $\begin{array}{l}\text { „Schlecker schließt Baumarkt, } \\
\text { Tankstelle und Restaurant« }\end{array}$ \\
\hline S120324TA & Die Tageszeitung & $\begin{array}{l}24.03 . \\
2012\end{array}$ & »Die Schlecker-Saga» \\
\hline
\end{tabular}


(Fortsetzung)

\begin{tabular}{llll}
\hline Kürzel & Quelle & Datum & Titel \\
\hline S120401BE & Brand Eins & 01.04. & "Eine Dimension mehr» \\
& & 2012 & \\
\hline S120601FA & Frankfurter & 01.06. & "Logisches Ende» \\
& Allgemeine Zeitung & 2012 & \\
\hline S120601LZ & Lebensmittel & 01.06. & "Schlecker am Ende» \\
& Zeitung & 2012 & \\
\hline S120602HB & Handelsblatt & 02.06. & "Roßmann: Schlecker hatte mehr als \\
& & 2012 & 30 Jahre lang Erfolg» \\
\hline S120813SP & Der Spiegel & 13.08. & "Schlecker soll drohende Pleite ignoriert \\
& & 2012 & haben« \\
\hline S130821LZ & Lebensmittel & 21.08. & "Ehemalige Schlecker-Filialen sind \\
& Zeitung & 2013 & Ladenhüter» \\
\hline
\end{tabular}

Internet

\begin{tabular}{lllll}
\hline Kürzel & Quelle & Titel & URL & Zugriff \\
\hline R140302H1 & H1 & $\begin{array}{l}\text { Dirk Roßmann im } \\
\text { Interview bei h1 }\end{array}$ & $\begin{array}{l}\text { https://www.youtube.com/ } \\
\text { watch?v=vHFGEDvj55I }\end{array}$ & 09.07.15 \\
\hline B160212YT & You Tube & $\begin{array}{l}\text { Berentzen-Werbung } \\
\text { "komm raus auf's Land « }\end{array}$ & $\begin{array}{l}\text { https://www.youtube.com/ } \\
\text { watch?v=GmwttR0tGCM }\end{array}$ & 18.07 .16 \\
\hline B061125YT & You Tube & $\begin{array}{l}\text { Berentzen-Werbung } \\
\text { "das fruchtet!» }\end{array}$ & $\begin{array}{l}\text { https://www.youtube.com/ } \\
\text { watch?v=P_zxvLfQ3dg }\end{array}$ & 05.06.16 \\
\hline B020812YT & You Tube & $\begin{array}{l}\text { Berentzen-Werbung } \\
\text { "auf Eis« }\end{array}$ & $\begin{array}{l}\text { https://www.youtube.com/ } \\
\text { watch?v=AnDv-_XXMh0 }\end{array}$ & 05.06 .16 \\
\hline
\end{tabular}

\section{Sonstiges}

\section{Beispiel der erzählstimulierenden Eingangsfrage der narrativen Interviews}

»Wenn Sie Ihre Zeit bei x noch einmal gedanklich Revue passieren lassen ...

... würde ich Sie nun bitten mir von dieser Zeit bei x zu erzählen. Wie kamen Sie zu x, wie verlief Ihre Zeit dort ... und was waren Ihre persönlichen Eindrücke... die Entscheidungsprozesse, die Organisationskultur, die Familie betreffend ...

Nehmen Sie sich ruhig Zeit um die Ereignisse, Prozesse und beteiligten Akteure in der gebotenen Ausführlichkeit darzustellen. Auch Details können wichtig sein. Ich werde Ihnen erst einmal ganz in Ruhe zuhören und Sie in keinem Falle unterbrechen ..." (Beispiel einer erzählstimulierenden Eingangsfrage zum Einstieg in einen Fall) 


\section{Literaturverzeichnis}

ABRAMS, D. E. \& HOGG, M. A. (1990): Social identity theory: Constructive and critical advances New York: Springer-Verlag Publishing.

ACEMOGLU, D. \& ROBINSON, J. (2012): Why nations fail: the origins of power, prosperity, and poverty New York: Crown Business.

ACKERMANN, R. (2003): Die Pfadabhängigkeitstheorie als Erklärungsansatz unternehmerischer Entwicklungsprozesse, in: Managementforschung 13: 225-255.

ALBACH, H. \& FREUND, W. (1989): Generationswechsel und Unternehmenskontinuität Chancen, Risiken, Maßnahmen: eine empirische Untersuchung bei Mittel- und Großunternehmen Gütersloh: Verlag Bertelsmann-Stiftung.

ALTMAN, E. I. (1968): Financial ratios, discriminant analysis and the prediction of corporate bankruptcy, in: The journal of finance 23(4): 589-609.

ALTMAN, E. I. \& HOTCHKISS, E. (2006): Corporate financial distress and bankruptcy: Predict and avoid bankruptcy, analyze and invest in distressed debt (4. Aufl.). Hoboken, NJ: Wiley.

AMASON, A. (1996): Distinguishing the effects of functional and dysfunctional conflict in strategic decision making: resolving the paradox for top management teams, in: Academy of Management Journal 39(1): 123-148.

AMASON, A. C. \& SAPIENZA, H. J. (1997): The effects of top management team size and interaction norms on cognitive and affective conflict, in: Journal of Management 23(4): 495-516.

AMBURGEY, T. L., KELLY, D. \& BARNETT, W. P. (1990): Resetting the Clock: The Dynamics of Organizational Change and Failure, in: Academy of Management Proceedings 1990(1): 160-164.

ANDERSON, R. C., MANSI, S. A. \& REEB, D. M. (2003): Founding family ownership and the agency cost of debt, in: Journal of Financial Economics 68(2): 263-285.

ANDERSON, R. C. \& REEB, D. M. (2003): Founding-family ownership and firm performance: evidence from the S\&P 500, in: The journal of finance 58(3): 1301-1327.

ANDERSON, R. C. \& REEB, D. M. (2004): Board composition: Balancing family influence in S\& 500 firms, in: Administrative Science Quarterly 49(2): 209-237.

ANGERMANN, H. F. (1970): Wachsen oder Weichen: Überlebenschancen für den wirtschaftlichen Mittelstand: Forkel.

ANSOFF, H. I. (1970): Corporate strategy: An analytic approach to business policy for growth and expansion New York: McGraw-Hill. 
ANSOFF, H. I. (1988): The new corporate strategy New York u. a.: Wiley.

ANTONELLI, C. (1997): The economics of path-dependence in industrial organization, in: International Journal of Industrial Organization 15(6): 643-675.

ARGENTI, J. (1976): Corporate collapse: The causes a symptoms London: McGraw-Hill. ARKES, H. R. \& BLUMER, C. (1985): The psychology of sunk cost, in: Organizational behavior and human decision processes 35(1): 124-140.

AROGYASWAMY, K., BARKER, V. L. \& YASAI-ARKEDIANI, M. (1995): Firm turnarounds: An integrative two stage model, in: Journal of Management Studies 32(4): 493525.

ARREGLE, J. L., HITT, M. A., SIRMON, D. G. \& VERY, P. (2007): The development of organizational social capital: attributes of family firms ${ }^{*}$, in: Journal of Management Studies 44(1): 73-95.

ARROW, K. J. (2003): Path dependence and competitive equilibrium. History Matters. Essays on Economic Growth, Technology, and Demographic Change, in: T. W. GUINNANE (Hrsg.). Stanford, Calfif.: Stanford University Press: 23-35.

ARTHUR, W. (1994): Increasing returns and path dependency in the economy Ann Arbor: University of Michigan Press.

ARTHUR, W. B. (1989): Competing technologies, increasing returns, and lock-in by historical events, in: The Economic Journal 99(394): 116-131.

ASHBY, W. R. (1956): An introduction to cybernetics London: Champman \& Hall.

ASTRACHAN, J. H., KLEIN, S. B. \& SMYRNIOS, K. X. (2002): The F-PEC scale of family influence: A proposal for solving the family business definition problem, in: Family Business Review 15(1): 45-58.

ATHANASSIOU, N., CRITTENDEN, W. F., KELLY, L. M. \& MARQUEZ, P. (2002): Founder centrality effects on the Mexican family firm's top management group: Firm culture, strategic vision and goals, and firm performance, in: Journal of World Business 37(2): 139-150.

BAECKER, D. (2002): Wozu Systeme? Berlin: Kulturverlag Kadmos.

BANTEL, K. \& JACKSON, S. (1989): Top-Management and innovations in banking: Does the composition of the top team make a difference?, in: Strategic Management Journal 10 (Summer 1989): 107-124.

BARKER, V. I. \& PATTERSON, P. J. (1996): Top-Management team tenure and top manager causal attributions in declining firms attempting turnaround, in: Group and Organization Management 21(3): 304-336.

BARNETT, W. P. \& BURGELMAN, R. A. (1996): Evolutionary Perspectives on Strategy, in: Strategic Management Journal 17(51): 5-19.

BARNEY, J. (1991): Firm resources and sustained competitive advantage, in: Journal of Management 17(1): 99-121.

BARNEY, J. B. (2002): Gaining and sustaining competitive advantage (2. Aufl.). Upper Saddle River, NJ: Prentice Hall.

BARON, J. N., HANNAN, M. T. \& BURTON, M. D. (1999): Building the iron cage: Determinants of managerial intensity in the early years of organizations, in: American Sociological Review 64(4): 527-547.

BATHELT, H. \& GLÜCKLER, J. (2012): Wirtschaftsgeographie (3. Aufl.). Stuttgart: UTB. BAUS, K. (2013): Die Familienstrategie (4. Aufl.). Wiesbaden: Gabler. 
BAYSINGER, B. D., KOSNIK, R. D. \& TURK, T. A. (1991): Effects of board and ownership structure on corporate R\&D strategy, in: Academy of Management Journal 34(1): 205214.

BAZERMAN, M. H., GIULIANO, T. \& APPELMAN, A. (1984): Escalation of commitment in individual and group decision making, in: Organizational behavior and human performance 33(2): 141-152.

BECKHARD, R. (1969): Organization development: Strategies and models Reading, Mass.: Addison-Wesley Publ.

BEREKOVEN, L. (1981): Markenartikel und Markenpolitik im deutschen Spirituosenmarkt - insbesondere bei Klaren. Zur Lage der Industrie klarer Spirituosen 1981, in: DOORNKAAT (Hrsg.): Doornkaat: 25-44.

BERENTZEN, J. B. (1981): J. B. Berentzen - Chronik eines Hauses Haselünne: Berentzen. BERLE, A. A. \& MEANS, G. G. C. (1932): The modern corporation and private property New York: McMillian.

BERRONE, P., CRUZ, C. \& GOMEZ-MEJIA, L. R. (2012): Socioemotional wealth in family firms theoretical dimensions, assessment approaches, and agenda for future research, in: Family Business Review 25(3): 258-279.

BERRONE, P., CRUZ, C., GOMEZ-MEJIA, L. R. \& LARRAZA-KINTANA, M. (2010): Socioemotional wealth and corporate responses to institutional pressures: Do familycontrolled firms pollute less?, in: Administrative Science Quarterly 55(1): 82-113.

BETHEL, J. E. \& LIEBESKIND, J. (1993): The effects of ownership structure on corporate restructuring, in: Strategic Management Journal 14(S1): 15-31.

BEYER, J. (2005): Pfadabhängigkeit ist nicht gleich Pfadabhängigkeit! Wider den impliziten Konservatismus eines gängigen Konzepts, in: Zeitschrift für Soziologie 34(1): 521.

BEYER, J. (2010): The same or not the same - on the variety of mechanisms of path dependence, in: International Journal of Social Sciences 5(1): 1-11.

BEYER, J. (2015): Pfadabhängigkeit. Handbuch Policy-Forschung, in: (Hrsg.). Wiesbaden: Springer: $149-171$.

BIBEAULT, D. B. (1982): Corporate Turnaround: How managers turn losers into winners New York: McGraw-Hill.

BIRD, B., WELSH, H., ASTRACHAN, J. H. \& PISTRUI, D. (2002): Family business research: The evolution of an academic field, in: Family Business Review 15(4): 337-350.

BIRLEY, S., NG, D. \& GODFREY, A. (1999): The family and the business, in: Long Range Planning 32(6): 598-608.

BÖCKENFÖRDE, B. (1991): Unternehmenssanierung Stuttgart: Schäffer-Poeschel.

BOEKER, W. (1989): The development and institutionalization of subunit power in organizations, in: Administrative Science Quarterly 34(3): 388-410.

BOEKER, W. (1989): Strategic change: The effects of founding and history, in: Academy of Management Journal 32(3): 489-515.

BOURDIEU, P. (2012): Ökonomisches Kapital, kulturelles Kapital, soziales Kapital. Handbuch Bildungs-und Erziehungssoziologie, in: U. BAUER and BITTLINGMAYER, U. (Hrsg.). Wiesbaden: Springer: 229-242.

BOWER, J. L. (1970): Managing the resource allocation process: A study of corporate planning and investment Boston, Mass.: Harvard University Press. 
BOWERS, D. G. (1977): Systems of organization: Management of the human resource Ann Arbor: University of Michigan Press.

BRANDES, D. (2001): Konsequent einfach: die ALDI-Erfolgsstory (3. Aufl. ). München: Heyne.

BRATSCHITSCH, R. \& SCHNELLINGER, W. (1981): Unternehmenskrisen: Ursachen, Frühwarnung, Bewältigung: Bericht über die Pfingsttagung in Innsbruck, Juni 1979, Verband der Hochschullehrer für Betriebswirtschaft Stuttgart: Poeschel.

BRENDEL, H. \& OBERENDER, P. (1981): Eine markttheoretische Analyse unter besonderer Berücksichtigung der institutionellen Rahmenbedingungen. Zur Lage der Industrie klarer Spirituosen, in: DOORNKAAT (Hrsg.). Uelzen: Becker.

BRESSER, R. K. F. (2010): Strategische Managementtheorie Stuttgart: Kohlhammer.

BROCKHAUS, R. H. (1994): Entrepreneurship and family business research: Comparisons, critique, and lessons, in: Entrepreneurship Theory and Practice 19(1): 25-39.

BRUNNER, O. (1968): Neue Wege der Verfassungs- und Sozialgeschichte Göttingen: Vandenhoeck \& Ruprecht.

BUDE, H. (1985): Der Sozialforscher als Narrationsanimateur, in: Kölner Zeitschrift für Soziologie und Sozialpsychologie 37: 327-336.

BURCH, P. (1972): The managerial revolution reassessed Lexington, MA: DC Heath.

BURGELMAN, R. (2008): Strategic Consequences of Co-Evolution Lock-In: Insights from a Longitudinal Process Study. The hidden dynamics of path dependence: Institutions and organizations, in: G. SCHREYÖGG (Hrsg.). Basingstoke u. a.: Palgrave Macmillan.

BURGELMAN, R. A. (1994): Fading memories: A process theory of strategic business exit in dynamic environments, in: Administrative Science Quarterly 39(1): 24-56.

BURGELMAN, R. A. (2002): Strategy as vector and the inertia of coevolutionary lock-in, in: Administrative Science Quarterly 47(2): 325-357.

BURGELMAN, R. A. (2011): Bridging history and reductionism: a key reolge for longitudinal qualitative research, in: Journal of International Business Studies 42(5): 591601.

BURGELMAN, R. A. \& GROVE, A. S. (1996): Strategie Dissonance, in: California Management Review 38(2): 8-28.

BURKART, G. (2008): Familiensoziologie Konstanz: UVK-Verl.-Ges.

BURNHAM, J. (1948): Das Regime der Manager Stuttgart: Union Dt. Verl.-Ges.

BUSCHMANN, H. (2006): Turnaround-Management - Empirische Untersuchung mit Schwerpunkt auf den Einfluss der Stakeholder im Turnaround Wiesbaden: Deutscher Universitäts Verlag.

CAMERON, K. S., KIM, M. U. \& WHETTEN, D. A. (1987): Organizational effects of decline and turbulence, in: Administrative Science Quarterly 32(2): 222-240.

CAMERON, K. S., WHETTEN, D. A. \& KIM, M. (1987): Organizational dysfunctions of decline, in: Academy of Management Journal 30(1): 126-138.

CAMPBELL-KELLY, M. (2001): Not only Microsoft: The maturing of the personal computer software industry, 1982-1995, in: Business History Review 75(1): 103-145.

CANNELLA, A. A., JONES, C. D. \& WITHERS, M. C. (2015): Family-versus lone-foundercontrolled public corporations: Social identity theory and boards of directors, in: Academy of Management Journal 58(2): 436-459. 
CAPOCCIA, G. \& KELEMEN, R. D. (2007): The study of critical junctures: Theory, narrative, and counterfactuals in historical institutionalism, in: World politics 59(3): 341369.

CARLSSON, G. \& KARLSSON, K. (1970): Age, cohorts and the generation of generations, in: American Sociological Review 35(2): 710-718.

CARNEY, M. (2005): Corporate governance and competitive advantage in family-controlled firms, in: Entrepreneurship Theory and Practice 29(3): 249-266.

CARPENTER, M. A., GELETKANYCZ, M. A. \& SANDERS, W. G. (2004): Upper echelons research revisited: Antecedents, elements, and consequences of top management team composition, in: Journal of Management 30(6): 749-778.

CASILLAS, J. \& ACEDO, F. (2007): Evolution of the intellectual structure of family business literature: A bibliometric study of FBR, in: Family Business Review 20(2): 141-162.

CASSON, M. (1999): The economics of the family firm, in: Scandinavian Economic History Review 47(1): 10-23.

CATER, J. \& SCHWAB, A. (2008): Turnaround Strategies in Established Small Family Firms, in: Family Business Review 21(31): 31-50.

CEZANNE, M. (1999): Krisenmanagement und Komplexität: betriebswirtschaftliche Krisentheorien im Kontext multioptionalen Konsumentenverhaltens Wiesbaden: Gabler.

CHAGANTI, R. \& SAMBHARYA, R. (1987): Strategic orientation and characteristics of upper management, in: Strategic Management Journal 8(4): 393-401.

CHANDLER, A. D. (1962): Strategy and structure: Chapters in the history of the industrial enterprise Cambridge, Mass.: M.I.T. Press.

CHANDLER, A. D. (1980): Managerial hierarchies: Comparative perspectives on the rise of the modern industrial enterprise (9. Aufl.). Cambridge, Mass. u. a.: Harvard University Press.

CHANDLER, A. D. (1990): Strategy and structure: Chapters in the history of the industrial enterprise (16. Aufl.). Cambridge, Mass. u. a.: MIT press.

CHANDLER, A. D. (1994): Scale and scope: The dynamics of industrial capitalism Cambridge, Mass.: Harvard University Press.

CHILD, J. (1972): Organizational structure, environment and performance: The role of strategic choice, in: Sociology 6(1): 1-22.

CHILD, J. (1974): Managerial and organizational factors associated with company performance part I, in: Journal of Management Studies 11(3): 175-189.

CHOWDHURY, S. \& LANG, J. R. (1996): Turnaround in small firms - an assesment of effiency strategies, in: Journal of Business Research 36(2): 169-178.

CHRISMAN, J. J., CHUA, J. H. \& KELLERMANNS, F. (2009): Priorities, resource stocks, and performance in family and nonfamily firms, in: Entrepreneurship Theory and Practice 33(3): 739-760.

CHRISMAN, J. J., CHUA, J. H. \& SHARMA, P. (2005): Trends and directions in the development of a strategic management theory of the family firm, in: Entrepreneurship Theory and Practice 29(5): 555-576.

CHRISTENSEN, C. (2013): The innovator's dilemma: when new technologies cause great firms to fail Boston, Mass.: Harvard Business Review Press.

CHRISTENSEN, C. R., ANDREWS, K. R., BOWER, J. L., HAMERMESH, R. G. \& PORTER, M. E. (1982): Business policy: Text and cases (5. Aufl.). Homewood, Ill.: Irwin. 
CHUA, J. H., CHRISMAN, J. J. \& CHANG, E. P. C. (2004): Are family firms born or made? An exploratory investigation, in: Family Business Review 17(1): 37-54.

CHUA, J. H., CHRISMAN, J. J. \& SHARMA, P. (1999): Defining the family business by behavior, in: Entrepreneurship Theory and Practice 23(4): 19-39.

CHURCHILL, N. C. \& HATTEN, K. J. (1997): Non-market-based transfers of wealth and power: A research framework for family business, in: Family Business Review 10(1): 53-67.

CLAUDE, L.-S. (1981): Die elementaren Strukturen der Verwandtschaft Frankfurt: Suhrkamp.

COLLI, A. (2003): The history of family business, 1850-2000 Cambridge: Cambridge University Press.

COLLI, A. (2013): Risk, uncertainty, and family ownership. The endurance of family businesses: A global overview, in: P. F. PEREZ and COLLI, A. (Hrsg.). New York: Cambridge University Press: 85-110.

COLLI, A., PÉREZ, P. F. \& ROSE, M. B. (2003): National determinants of family firm development? Family firms in Britain, Spain, and Italy in the nineteenth and twentieth centuries, in: Enterprise \& Society 4(1): 28-64.

COLLIER, R. B. \& COLLIER, D. (1991): Shaping the political arena: Critical junctures, the labor movement and regime dynamics in Latin America Princeton, N.J.: Princeton University Press.

CORBETTA, G. \& SALVATO, C. (2004): Self-Serving or self-actualizing? Models of man and agency costs in different types of family firms: A commentary on "Comparing the agency costs of family and non-family firms: Conceptual issues and exploratory evidence«, in: Entrepreneurship Theory and Practice 28(4): 355-362.

COSIER, R. A. \& HARVEY, M. (1998): The Hidden Strengths in Family Business: Functional Conflict, in: Family Business Review 11(1): 75-79.

CROMIE, S., STEPHENSON, B. \& MONTEITH, D. (1995): The management of family firms: an empirical investigation, in: International Small Business Journal 13(4): 1134.

CROSSLAND, C. \& HAMBRICK, D. C. (2007): How national systems differ in their constraints on corporate executives: A study of CEO effects in three countries, in: Strategic Management Journal 28(8): 767-789.

CUSUMANO, M. A., MYLONADIS, Y. \& ROSENBLOOM, R. S. (1992): Strategic maneuvering and mass-market dynamics: The triumph of VHS over Beta, in: Business History Review 66(1): 51-94.

CYERT, R. M. \& MARCH, J. G. (1963): A Behavioural Theory of the Firm Eaglewood Cliffs, NJ: Prentice-Hall.

DAHL, R. A. (1957): The concept of power, in: Behavioral science 2(3): 201-215.

DAILY, C. M. \& DOLLINGER, M. J. (1992): An empirical examination of ownership structure in family and professionally managed firms, in: Family Business Review 5(2): $117-136$.

DAVID, P. A. (1985): Clio and the economics of QWERTY, in: American Economic Review 75(2): 332-337.

DAVID, P. A. (1986): Understanding the economics of QWERTY: The necessity of history. Economic history and the modern economist, in: W. N. PARKER (Hrsg.). Oxford, UK, New York, NY, USA Blackwell. 
DAVIS, J. H., SCHOORMAN, F. D. \& DONALDSON, L. (1997): Toward a stewardship theory of management, in: Academy of Management Review 22(1): 20-47.

DE DREU, C. K. \& WEINGART, L. R. (2003): Task versus relationship conflict, team performance, and team member satisfaction: a meta-analysis, in: Journal of applied Psychology 88(4): 741-749.

DE WIT, F. R., GREER, L. L. \& JEHN, K. A. (2012): The paradox of intragroup conflict: a meta-analysis, in: Journal of applied Psychology 97(2): 360-390.

DEARBORN, D. C. \& SIMON, H. A. (1958): Selective perception: A note on the departmental identifications of executives, in: Sociometry 21(2): 140-144.

DEMSETZ, H. (1967): Toward a theory of property rights, in: The American Economic Review 57(2): 347-359.

DENISON, D., LIEF, C. \& WARD, J. L. (2004): Culture in family-owned enterprises: Recognizing and leveraging unique strengths, in: Family Business Review 17(1): 61-70.

DEUTSCH, M. \& LEWICKI, R. J. (1970): »Locking-in« effects during a game of Chicken, in: Journal of Conflict Resolution 14(3): 367-378.

DICT (2016). »autonomia im Altgriechischen.« Zugriff: 17.07.16, von http://www.dict.gr/ index?s=\%CE\%B1\%CF\%85\%CF\%84\%CE\%BF\%CE\%BD\%CE\%BF\%CE\%BC\%CE\%A $\mathrm{F} \% \mathrm{CE} \% \mathrm{~B} 1$.

DIETRICH, M. (1997): Strategic lock-in as human issue - the role of professional orientation. Evolutionary economics and path dependence, in: S. J. MAGNUSSON and OTTOSON, J. (Hrsg.). Cheltenham: Elgar: 168-185.

DIMAGGIO, P. \& POWELL, W. W. (1983): The iron cage revisited: Collective rationality and institutional isomorphism in organizational fields, in: American Sociological Review 48(2): 147-160.

DISTELBERG, B. \& SORENSON, R. L. (2009): Updating systems concepts in family businesses A focus on values, resource flows, and adaptability, in: Family Business Review 22(1): 65-81.

DOBUSCH, L. \& KAPELLER, J. (2013): Striking new paths: Theory and method in path dependence research, in: Schmalenbach Business Review 65: 288-311.

DOBUSCH, L. \& SCHÜßLER, E. (2013): Theorizing path dependence: a review of positive feedback mechanisms in technology markets, regional clusters, and organizations, in: Industrial and Corporate Change 22(3): 617-647.

DONCKELS, R. \& FRÖHLICH, E. (1991): Are family businesses really different? European experiences from STRATOS, in: Family Business Review 4(2): 149-160.

DONNELLEY, R. G. (1964): The family business, in: Harvard Business Review 42(4): $93-$ 105.

DRUCKER, P. F. (1995): People and performance: The best of Peter Drucker on management London New York: Routledge.

DUDEN (2014). »Krise.« Zugriff: 25.04.2014, von http://www.duden.de/rechtschreibung/ Krise.

DUTTON, J. E. \& DUNCAN, R. B. (1987): The creation of momentum for change through the process of strategic issue diagnosis, in: Strategic Management Journal 8(3): 279295.

DYER JR., W. G. \& DYER, W. J. (2009): Putting the family into family business research, in: Family Business Review 22(3): 216-219.

DYER, J. W. G. (1986): Cultural change in family firms San Fransisco: Jossey Bass. 
DYER, W. G. (1988): Culture and continuity in family firms, in: Family Business Review 1(1): $37-50$.

DYER, W. G. J. (2003): The family: The missing variable in organizational research, in: Entrepreneurship Theory and Practice 27(4): 401-416.

ECKES (2007): 150 Jahre Eckes - 150 Jahre Impulse Bochum: Laupenmühlen Druck.

EDDLESTON, K. A. (2008): Commentary: The prequel to family firm culture and stewardship: The leadership perspective of the founder, in: Entrepreneurship Theory and Practice 32(6): 1055-1061.

EDDLESTON, K. A. \& KELLERMANNS, F. W. (2007): Destructive and productive family relationships: A stewardship theory perspective, in: Journal of Business Venturing 22(4): 545-565.

EISENHARDT, K. M. (1989): Building theories from case study research, in: Academy of Management Review 14(4): 532-550.

EISENHARDT, K. M. \& BOURGEOIS, L. J. (1988): Politics of strategic decision making in high-velocity environments: Toward a midrange theory, in: Academy of Management Journal 31(4): 737-770.

EISENHARDT, K. M. \& GRAEBNER, M. E. (2007): Theory building from cases: Opportunities and challenges, in: Academy of Management Journal 50(1): 25-32.

EISENHARDT, K. M., KAHWAJY, J. L. \& BOURGEOIS III, L. J. (1997): Conflict and strategic choice: How top management teams disagree, in: California Management Review 39(2): 42.

EISENHARDT, K. M., KAHWAJY, J. L. \& BOURGEOIS, L. (1997): How management teams can have a good fight, in: Harvard Business Review 75(4): 77-86.

EISENHARDT, K. M. \& SCHOONHOVEN, C. B. (1990): Organizational growth: Linking founding team, strategy, environment, and growth among US semiconductor ventures, 1978-1988, in: Administrative Science Quarterly 35(3): 504-529.

EISENHARDT, K. M. \& ZBARACKI, M. J. (1992): Strategic decision making, in: Strategic Management Journal 13(S2): 17-37.

ENDRUWEIT, G., Ed. (2014): Wörterbuch der Soziologie. UTB; 8566: Soziologie. Kon$\operatorname{stanz}$, München, Lucius.

ESSER, H. (2000): Die Konstruktion der Gesellschaft Frankfurt: Campus-Verl.

ESSER, H. (2002): Soziologie: Sinn und Kultur (6. Aufl.). Frankfurt: Campus Verlag.

FABIS, F. G. (2007): Gesellschafterkonflikte in Familienunternehmen Berlin: Logos.

FACCIO, M. \& LANG, L. H. (2002): The ultimate ownership of Western European corporations, in: Journal of Financial Economics 65(3): 365-395.

FAMA, E. F. \& JENSEN, M. C. (1983): Agency problems and residual claims, in: The Journal of Law \& Economics 26(2): 327-349.

FAMA, E. F. \& JENSEN, M. C. (1983): Separation of ownership and control, in: The Journal of Law \& Economics 26(2): 301-325.

FBN (2008): Family business international monitor: FBN International.

FELDEN, B. \& HACK, A. (2014): Management von Familienunternehmen: Besonderheiten - Handlungsfelder - Instrumente Wiesbaden: Springer Gabler.

FINKELSTEIN, S. (1992): Power in top management teams: Dimensions, measurement, and validation, in: Academy of Management Journal 35(3): 505-538. 
FINKELSTEIN, S. \& D'AVENI, R. A. (1994): CEO duality as a double-edged sword: How boards of directors balance entrenchment avoidance and unity of command, in: Academy of Management Journal 37(5): 1079-1108.

FINKELSTEIN, S. \& HAMBRICK, D. C. (1989): Chief executive compensation: A study of the intersection of markets and political processes, in: Strategic Management Journal 10(2): 121-134.

FINKELSTEIN, S. \& HAMBRICK, D. C. (1990): Top-management-team tenure and organizational outcomes: The moderating role of managerial discretion, in: Administrative Science Quarterly 35(3): 484-503.

FINKELSTEIN, S., HAMBRICK, D. C. \& CANNELLA, A. A. (2009): Strategic leadership: Theory and research on executives, top management teams, and boards New York: Oxford University Press.

FLEGE-ALTHOFF (1930): Die notleidende Unternehmung. Erster Band: Krankheitserscheinungen und Krankheitsursachen Stuttgart: Schaeffer-Poeschel.

FLETCHER, D., MELIN, L. \& GIMENO, A. (2012): Culture and values in family business A review and suggestions for future research, in: Journal of Family Business Strategy 3(3): 127-131.

FLICK, U. (2002): Qualitative Sozialforschung. Eine Einführung Hamburg: Rowohlt.

FLICK, U. (2007): Qualitative Sozialforschung - eine Einführung Hamburg: rororo Verlag.

FLORIS, M., KAMMERLANDER, N., DESSI, C. \& MURRU, A. (2013): Breaking with or adhering to tradition? Path dependence and innovation in Italian family-owned wineries. FFI. San Diego.

FNP (2011). "Tengelmann-Chef Haub sieht Renaissance der Familienunternehmen.» Zugriff: 03.05. (12:55), 2016, von http://www.fnp.de/nachrichten/wirtschaft/Tengel mann-Chef-Haub-sieht-Renaissance-der-Familienunternehmen;art686,495642.

FORAY, D. (1997): The dynamic implications of increasing returns: Technological change and path dependent inefficiency, in: International Journal of Industrial Organization 15(6): 733-752.

FRANK, H., LUEGER, M., NOSÉ, L. \& SUCHY, D. (2010): The concept of »familiness«: Literature review and systems theory-based reflections, in: Journal of Family Business Strategy 1(3): 119-130.

FREDRICKSON, J. W. (1986): The strategic decision process and organizational structure, in: Academy of Management Review 11(2): 280-297.

FRENCH, J. R., RAVEN, B. \& CARTWRIGHT, D. (1959): The bases of social power, in: Classics of organization theory: 311-320.

FUTUYMA, D. J., Ed. (1983): Coevolution. Sunderland, Mass., Sinauer.

GALLO, M. A. \& PONT, C. G. (1996): Important factors in family business internationalization, in: Family Business Review 9(1): 45-59.

GARCIA-ALVAREZ, E., LOPEZ-SINTAS, J. \& GONZALVO, P. S. (2002): Socialization patterns of successors in first- to second-generation family businesses, in: Family Business Review 15(3): 189-203.

GARFINKEL, H. (1956): Conditions of successful degradation ceremonies, in: American Journal of Sociology: 420-424.

GARFINKEL, H. (1967): Studies in ethnomethodology Englewood Cliffs, N.J.: PrenticeHall. 
GARUD, R., KUMARASWAMY, A. \& KARNØE, P. (2010): Path dependence or path creation?, in: Journal of Management Studies 47(4): 760-774.

GEORGE, A. L. \& BENNETT, A. (2005): Case studies and theory development in the social sciences Cambridge, Mass.: MIT Press.

GERSICK, K. E., DAVIS, J. A., M.M., H. \& LANSBERG, I. (1997): Generation to generation: Life cycles of the family business Boston, Mass.: Harvard Business School Press.

GESTRICH, A. (2010): Geschichte der Familie im 19. und 20. Jahrhundert München: Oldenbourg.

GIMENO, J., FOLTA, T. B., COOPER, A. C. \& WOO, C. Y. (1997): Survival of the fittest? Entrepreneurial human capital and the persistence of underperforming firms, in: Administrative Science Quarterly 42(4): 750-783.

GLASER, B. \& STRAUSS, A. (1967): The discovery of grounded theory. Strategies for qualitative research New York: Aldine.

GLASL, F. (2013): Konfliktmanagement. Ein Handbuch für Führungskräfte, Beraterinnen und Berater (11. Aufl.). Bern u. a.: Haupt.

GLESS, S.-E. (1996): Unternehmenssanierung: Grundlagen - Strategien - Maßnahmen Wiesbaden: Deutscher Universitäts Verlag.

GOEHLER, A. W. (1993): Der Erfolg großer Familienunternehmen im fortgeschrittenen Marktlebenszyklus: dargestellt am Beispiel der deutschen Brauwirtschaft. St. Gallen, Dissertation.

GOFFMAN, E. (1955): On face-work: An analysis of ritual elements in social interaction, in: Psychiatry 18(3): 213-231.

GÓMEZ-MEJÍA, L. R., HYNES, K. T., NÚÑEZ-NICKEL, M. \& MOYANO-FUENTES, H. (2007): Socioemotional wealth and business risk in family-controlled firms: Evidence from Spanish olive oil mills, in: Administrative Science Quarterly 52(1): 106-137.

GOMEZ-MEJIA, L. R., NUNEZ-NICKEL, M. \& GUTIERREZ, I. (2001): The role of family ties in agency contracts, in: Academy of Management Journal 44(1): 81-95.

GRÄFER, H., SCHILLER, B. \& RÖSNER, S. (2014): Finanzierung: Grundlagen, Institutionen, Instrumente und Kapitalmarkttheorie Berlin: Schmidt.

GREINER, L. E. (1972): Evolution and revolution as organizations grow, in: Harvard Business Review 45(3): 37-46.

GRINYER, P. H., MAYES, D. \& MCKIERNAN, P. (1990): The sharpbenders: Achieving a sustained improvement in performance, in: Long Range Planning 23(1): 116-125.

GROßMANN, S. (2014): Konflikte und Krisen in Familienunternehmen. Witten/Herdecke, Dissertation.

GRUNDSTRÖM, C., ÖBERG, C. \& ÖHRWALL RÖNNBÄCK, A. (2012): Family-owned manufacturing SMEs and innovativeness: A comparison between within-family successions and external takeovers, in: Journal of Family Business Strategy 3(3): 162-173.

GUTENBERG, E. (1976): Die Produktion Berlin ; Heidelberg: Springer.

HABBERSHON, T. \& WILLIAMS, M. (1999): A resourcebased framework for assessing the strategic advantages of family firms, in: Family Business Review 12(1): 1-25.

HABBERSHON, T. G., WILLIAMS, M. \& MACMILLAN, I. C. (2003): A unified systems perspective of family firm performance, in: Journal of Business Venturing 18(4): 451465.

HABBERSHON, T. W., M. (1999): A Resourcebased Framework for Assessing the Strategic Advantages of Family Firms., in: Family Business Review 12: 1-25. 
HABERMAS, J. (1981): Theorie des kommunikativen Handelns. 2. Zur Kritik der funktionalistischen Vernunft Frankfurt am Main: Suhrkamp.

HALL, A., MELIN, L. \& NORDQVIST, M. (2001): Entrepreneurship as radical change in the family business: Exploring the role of cultural patterns, in: Family Business Review 14(3): 193-208.

HALL, A. D. \& FAGEN, R. E. (1956): Definition of system, in: General systems 1(1): 18-28.

HALL, D. J. \& SAIAS, M. A. (1980): Strategy follows structure!, in: Strategic Management Journal 1(2): 149-163.

HALL, P. A. (2001): Varieties of capitalism Oxford: Oxford University Press.

HALLER, S. (2008): Handelsmarketing (3. Aufl.). Ludwigshafen: Kiehl.

HAMBRICK, D. (1994): Top management groups: A conceptual integration and reconsideration of the »team « label. Research in Organizational Behavior, in: B. M. STAW and CUMMINGS, L. L. (Hrsg.). Greenwich, CT: JAI Press: 171-214.

HAMBRICK, D. C. (1981): Environment, strategy, and power within top management teams, in: Administrative Science Quarterly 26(2): 253-275.

HAMBRICK, D. C. (1985): Turnaround strategies. Handbook of Business Strategy, in: W. GUTH (Hrsg.). Boston, Mass.: Warren, Gorham \& Lamont.

HAMBRICK, D. C. (2007): Upper echelons theory: An update, in: Academy of Management Review 32(2): 334-343.

HAMBRICK, D. C. \& BRANDON, G. L. (1988): Executive values. The executive effect: Concepts and methods for studying top managers, in: D. HAMBRICK (Hrsg.). Greenwich, Conn. u.a: Elsevier Science/JAI Press: 3-34.

HAMBRICK, D. C. \& D'AVENI, R. (1988): Large corporate failures as downward spirals, in: Administrative Science Quarterly 33(1): 1-23.

HAMBRICK, D. C. \& FINKELSTEIN, S. (1987): Managerial discretion: A bridge between polar views of organizational outcomes, in: Research in Organizational Behavior 9: 369-406.

HAMBRICK, D. C. \& FUKUTOMI, G. D. (1991): The seasons of a CEO's tenure, in: Academy of Management Review 16(4): 719-742.

HAMBRICK, D. C. \& MASON, P. A. (1984): Upper echolons: The organization as a reflection of its top managers, in: Academy of Management Review 9(2): 193-206.

HAMBRICK, D. C. \& SCHECTER, S. (1983): Turnaround strategies in mature industrialproduct business units, in: Academy of Management Journal 26(2): 231-248.

HANDLER, W. C. (1989): Methodological issues and considerations in studying family businesses, in: Family Business Review 2(3): 257-276.

HANDLER, W. C. (1994): Succession in family business: A review of the research, in: Family Business Review 7(2): 133-157.

HANNAN, M. T., BURTON, M. D. \& BARON, J. N. (1996): Inertia and change in the early years: Employment relations in young, high technology firms, in: Industrial and Corporate Change 5(2): 503-536.

HANNAN, M. T. \& FREEMAN, J. (1977): The population ecology of organizations, in: American Journal of Sociology 85(5): 929-964.

HANNAN, M. T. \& FREEMAN, J. (1984): Structutal inertia and organizational change, in: American Sociological Review 49(5): 929-964.

HARVEY, M. \& EVANS, R. E. (1994): Family business and multiple levels of conflict, in: Family Business Review 7(4): 331-348. 
HASENACK, W. (1932): Die Überwindung der Wirtschaftskrise und das deutsche Unternehmertum, in: Zeitschrift für Betriebswirtschaft: $705 \mathrm{ff}$.

HATUM, A., PETTIGREW, A. \& MICHELINI, J. (2010): Building organizational capabilities to adapt under turmoil, in: Journal of Change Management 10(3): 257-274.

HATUM, A. \& PETTIGREW, A. M. (2006): Determinants of organizational flexibility: a study in an emerging economy, in: British Journal of Management 17(2): 115-137.

HEDSTRÖM, P. \& SWEDBERG, R. (1998): Social mechanisms: An analytical approach to social theory Cambridge u. a.: Cambridge University Press.

HEDSTRÖM, P. \& YLIKOSKI, P. (2010): Causal mechanisms in the social sciences, in: Annual Review of Sociology 36(1): 49-67.

HERMANN, C. F. (1963): Some consequences of crisis which limit the viability of organizations, in: Administrative Science Quarterly 8(1): 61-82.

HERMANN, C. F. (1972): International crises: insights from behavioral research New York: Free Press.

HILDENBRAND, B., Ed. (1992): Bauernfamilien im Modernisierungsprozeß. Frankfurt a. M. u.a., Campus.

HILDENBRAND, B. (2002): Familienbetriebe als »Familien eigener Art...«. Die Familie des Familienunternehmens. Ein System zwischen Gefühl und Geschäft, in: F. B. SIMON (Hrsg.). Heidelberg: Carl-Auer-Systeme Verlag.: 115-144.

HITT, M. A. \& TYLER, B. B. (1991): Strategic decision models: Integrating different perspectives, in: Strategic Management Journal 12(5): 327-351.

HOFER, C. W. (1980): Turnaround-Strategies, in: Journal of Business Strategy 3(1): 19-31. HOFFMANN-RIEM, C. (1980): Die Sozialforschung einer interpretativen Soziologie, in: Kölner Zeitschrift für Soziologie und Sozialpsychologie 32: 325-338.

HOLT, D. T., RUTHERFORD, M. W. \& KURATKO, D. F. (2010): Advancing the field of family business research: Further testing the measurement properties of the F-PEC, in: Family Business Review 23(1): 76.

HOLTMANN, J. P. (2008): Pfadabhängigkeit strategischer Entscheidungen: Eine Fallstudie am Beispiel des Bertelsmann Buchclubs Deutschland Köln: Kölner Wiss.-Verlag.

HOPFENBECK, W. (1989): Allgemeine Betriebswirtschafts- und Managementlehre: Unternehmen im Spannungsfeld zwischen ökonomischen sozialen u. ökologischen Interessen Landsberg am Lech: Verl. Moderne Industrie.

HUNGENBERG, H. (2011): Strategisches Management in Unternehmen. Ziele - Prozesse Verfahren. (6. Aufl.). Wiesbaden: Gabler.

HUNGER, D. J. \& WHEELEN, T. L. (2008): Concepts in strategic management and business policy New Jersey: Pearson Prentice Hall.

HWANG, P. \& LICHTENTHAL, J. D. (2000): Anatomy of organizational crises, in: Journal of Contingencies and Crisis Management 8(3): 129-140.

IBRAHIM, N. A., ANGELIDIS, J. P. \& PARSA, F. (2008): Strategic management of family businesses: Current findings and directions for future research, in: International Journal of Management 25(1): 95-110.

IFM (2006). "Anteil der Familienunternehmen an allen Unternehmen in Deutschland.» Zugriff: 08.05. (12:05), 2016, von http://www.ifm-bonn.org/statistiken/unternehmens bestand/\#accordion $=0 \& \operatorname{tab}=5$.

ISAAC, A. (1932): Wirtschaftskrise und Wirtschaftsgesinnung, in: ZfB 1932: $385 \mathrm{ff}$. 
JÄKEL-WURZER, D. (2010): Töchter im Engpass. Eine fallrekonstruktive Studie zur weiblichen Nachfolge in Familienunternehmen Heidelberg: Carl-Auer.

JAMES, A. E., JENNINGS, J. E. \& BREITKREUZ, R. S. (2012): Worlds apart? Rebridging the distance between family science and family business research, in: Family Business Review 25(1): 87-108.

JAMES, H. (2006): Family Capitalism: Wendels, Haniels, Falcks, and the Continental European Model Cambride, Mass.: Harvard University Press.

JANIS, I. L. (1972): Victims of groupthink: A psychological study of foreign-policy decisions and fiascoes Boston: Houghton.

JEHN, K. A. (1994): Enhancing effectiveness: An investigation of advantages and disadvantages of value-based intragroup conflict, in: International Journal of Conflict Management 5(3): 223-238.

JEHN, K. A. (1995): A multimethod examination of the benefits and detriments of intragroup conflict, in: Administrative Science Quarterly 40(2): 256-282.

JEHN, K. A. (1997): A qualitative analysis of conflict types and dimensions in organizational groups, in: Administrative Science Quarterly 42(3): 530-557.

JEHN, K. A. \& BENDERSKY, C. (2003): Intragroup conflict in organizations: A contingency perspective on the conflict-outcome relationship, in: Research in Organizational Behavior 25: 187-242.

JENSEN, M. C. (1993): The modern industrial revolution, exit, and the failure of internal control systems, in: The journal of finance 48(3): 831-880.

JENSEN, M. C. (1994): Self-Interest, altruism, incentives, and agency theory, in: Journal of Applied Corporate Finance 7(2): 40-45.

JENSEN, M. C. \& MECKLING, W. H. (1976): Theory of the firm: Managerial behavior, agency costs and ownership structure, in: Journal of Financial Economics 3(4): 305360.

KAHN, H. (1965): On escalation: Metaphors and scenarios Harmon-on-Hudson: Hudson Inst.

KATZ, M. L. \& SHAPIRO, C. (1985): Network externalities, competition, and compatibility, in: American Economic Review 75(3): 424-440.

KATZ, R. (1982): The effects of group longevity on project communication and performance, in: Administrative Science Quarterly 27(1): 81-104.

KETS DE VRIES, M. F. \& MILLER, D. (1986): Personality, culture, and organization, in: Academy of Management Review 11(2): 266-279.

KETS DE VRIES, M. F. R. (1993): The family firm: The good news and the bad news, in: Organizational Dynamics Winter: 59-71.

KETS DE VRIES, M. F. R. (1996): Family business: Human dilemmas in family firms London: International Thompson Business Press.

KIESER, A. (2006): Max Webers Analyse der Bürokratie, in: Organisationstheorien 6: 6392.

KIESER, A. \& HEGELE-RAIH, C. (1998): Kommunikation im organisatorischen Wandel Stuttgart: Schäffer-Poeschel.

KIESER, A. \& WOYWODE, M. (2006): Evolutionstheoretische Ansätze. Organisationstheorien, in: A. KIESER and EBERS, M. (Hrsg.). Stuttgart: Kohlhammer: 309-352.

KIMBERLY, J. R. (1979): Issues in the creation of organizations: Initiation, innovation, and institutionalization, in: Academy of Management Journal 22(3): 437-457. 
KIMBERLY, J. R. (1987): The study of organization: Toward a biographical perspective, in: Handbook of organizational behavior: 223-237.

KIMBERLY, J. R. \& BOUCHIKHI, H. (1995): The dynamics of organizational development and change: How the past shapes the present and constrains the future, in: Organization Science 6(1): 9-18.

KIRSCH, W. (1992): Kommunikatives Handeln, Autopoiese, Rationalität: Sondierungen zu einer evolutionären Führungslehre München: Kirsch.

KLEIN, S. (1991): Der Einfluss von Werten auf die Gestaltung von Organisationen Berlin: Duncker \& Humblodt.

KLEIN, S. B. (2010): Familienunternehmen: Theoretische und empirische Grundlagen Lohmar, Köln: Eul.

KLEIN, S. B., ASTRACHAN, J. H. \& SMYRNIOS, K. X. (2005): The F-PEC scale of family influence: Construction, validation, and further implication for theory, in: Entrepreneurship Theory and Practice 29(3): 321-339.

KOCH, J. (2011): Inscribed Strategies: Exploring the Organizational Nature of Strategic Lock-in, in: Organization Studies 32(3): 337-363.

KOCH, J., EISEND, M. \& PETERMANN, A. (2009): Path dependence in decision-making processes: Exploring the impact of complexity under increasing returns, in: Business Research Journal 2(1): 67-84.

KOCKA, J. (1975): Unternehmer in der deutschen Industrialisierung Göttingen: Vandenhoeck und Ruprecht.

KOCKA, J. (1979): Familie, Unternehmer und Kapitalismus. An Beispielen aus der frühen deutschen Industrialisierung., in: Zeitschrift für Unternehmensgeschichte / Journal of Business History: 99-135.

KOEBERLE-SCHMIDT, A. (2008): Family Business Governance: Aufsichtsgremium und Familienrepräsentanz Wiesbaden: Gabler.

KÖNIG, A., KAMMERLANDER, N. \& ENDERS, A. (2013): The family innovator's dilemma: How family influence affects the adoption of discontinuous technologies by incumbent firms, in: Academy of Management Review 38(3): 418-441.

KOVOOR-MISRA, S., CLAIR, J. A. \& BETTENHAUSEN, K. L. (2001): Clarifying the attributes of organizational crises, in: Technological Forecasting and Social Change 67(1): 77-91.

KRAUSE, R., SEMADENI, M. \& CANNELLA, A. A. (2014): CEO duality: A review and research agenda, in: Journal of Management 40(1): 256-286.

KRUGMAN, P. (1991): History and industry location: the case of the manufacturing belt, in: The American Economic Review 81(2): 80-83.

KRUMMENACHER, A. (1981): Krisenmanagement. Leitfaden zum Verhindern und Bewältigen von Unternehmungskrisen Zürich: Verlag Industrielle Organisation.

KRYSTEK, U. (1987): Unternehmungskrisen. Beschreibung, Vermeidung, und Bewältigung überlebenskritischer Prozesse in Unternehmungen Wiesbaden: Gabler.

KRYSTEK, U. \& MOLDENHAUER, R. (2007): Handbuch Krisen- und Restrukturierungsmanagement Stuttgart: W. Kohlhammer.

KUNOW, K. (2013): and the hits just keep on coming...: adaptive Erwartungen, Pfadabhängigkeit und-bruch am Beispiel der Vermarktung von Musik durch große Tonträgerunternehmen. Berlin, Freie Universität Berlin, Diss., 2012. 
LA PORTA, R., LOPEZ-DE-SILANES, F. \& SHLEIFER, A. (1999): Corporate ownership around the world, in: The journal of finance 54(2): 471-517.

LAMNEK, S. (2005): Qualitative Sozialforschung: Lehrbuch Weinheim; Basel: Beltz.

LANSBERG, I. (1988): The succession conspiracy in: Family Business Review 1(1): 119143.

LANSBERG, I. (1999): Succeeding generations: Realizing the dream of families in business Boston, Mass.: Harvard Business Press.

LAWRENCE, B. S. (1997): Perspective-the black box of organizational demography, in: Organization Science 8(1): 1-22.

LAWRENCE, P. R. \& LORSCH, J. W. (1967): Differentiation and integration in complex organizations, in: Administrative Science Quarterly 12(1): 1-47.

LE'COUTRE (1926): Krisenlehren für die Unternehmensführung, in: Das Geschäft 1926: $63 \mathrm{ff}$.

LEE, K. S., LIM, G. H. \& LIM, W. S. (2003): Family business succession: Appropriation risk and choice of successor, in: Academy of Management Review 28(4): 657-666.

LEONARD-BARTON, D. (1992): Core capabilities and core rigidities: a paradox in managing new product development in: Strategic Management Journal 13(S1): 111-125.

LEVINSON, H. (1971): Conflicts that plague family businesses, in: Harvard Business Review 49(2): 90-98.

LEWIN, K. (1947): Group decision and social change, in: Readings in social psychology 3: 197-211.

LEWIN, K. (1951): Field theory in social science: selected theoretical papers New York: Harper.

LIEBERSON, S. \& O'CONNOR, J. F. (1972): Leadership and organizational performance: A study of large corporations, in: American Sociological Review 37(2): 117-130.

LIEBOWITZ, S. J. \& MARGOLIS, S. E. (1995): Path dependence, lock-in, and history, in: The Journal of Law, Economics, and Organization 11(1): 205-226.

LITTUNEN, H. \& HYRSKY, K. (2000): The early entrepreneurial stage in Finnish family and nonfamily firms, in: Family Business Review 13(1): 41-53.

LITZ, R. A. (1995): The family business: Toward definitional clarity, in: Family Business Review 8(2): 71-81.

LITZ, R. A. (1997): The family firm's exclusion from business school research: Explaining the void, in: Entrepreneurship Theory and Practice 21(3): 55-72.

LITZ, R. A., PEARSON, A. W. \& LITCHFIELD, S. (2012): Charting the future of family business research: Perspectives from the field, in: Family Business Review 25(1): 16-32.

LOHRKE, F. T., BEDEIAN, A. G. \& PALMER, T. B. (2004): The role of top management teams in formulating and implementing turnaround strategies: a review and research agenda, in: INTERNATIONAL JOURNAL OF MANAGEMENT REVIEWS 5-6(2): 6390.

LUBINSKI, C. (2010): Familienunternehmen in Westdeutschland: Corporate Governance und Gesellschafterkultur seit den 1960er Jahren München: Beck.

LUHMANN, N. (1975): Legitimation durch Verfahren Darmstadt: Luchterhand.

LUHMANN, N. (1977): Differentiation of society, in: The Canadian Journal of Sociology / Cahiers canadiens de sociologie 2(1): 29-53.

LUHMANN, N. (1984): Soziale Systeme: Grundriss einer allgemeinen Theorie Frankfurt am Main: Suhrkamp. 
LUHMANN, N. (1990): Soziologische Aufklärung 5 - Konstruktivistische Perspektiven Opladen: Westdt. Verl.

LUHMANN, N. (1991 (1968)): Zweckbegriff und Systemrationalität (5. Aufl.). Frankfurt am Main: Suhrkamp.

LUHMANN, N. (1993): Das Recht der Gesellschaft Frankfurt am Main: Suhrkamp.

LUHMANN, N. (1995): Soziologische Aufklärung 6. Die Soziologie und der Mensch Opladen: VS, Verlag für Sozialwissenschaften.

LUHMANN, N. (2000): Organisation und Entscheidung Opladen: VS, Verlag für Sozialwissenschaften.

LUHMANN, N. (2002): Einführung in die Systemtheorie Heidelberg: Carl-Auer-SystemeVerl.

MACK, R. W. \& SNYDER, R. C. (1957): The analysis of social conflict-toward an overview and synthesis, in: Conflict Resolution 1(2): 212-248.

MAHONEY, J. (2000): Path dependence in historical sociology, in: Theory and Society 29(4): 507-548.

MAINDOK, H., Ed. (1996): Qualitative Sozialforschung: Das narrative Interview. Professionelle Interviewführung in der Sozialforschung. Pfaffenweiler, Centaurus.

MARCH, J. G. (1962): The business firm as a political coalition, in: The Journal of politics 24(4): 662-678.

MARCH, J. G. \& SIMON, H. A. (1958): Organizations New York: Wiley.

MARQUIS, C. \& TILCSIK, A. (2013): Imprinting: Toward a multilevel theory, in: The Academy of Management Annals 7(1): 195-245.

MARTENS, W. \& ORTMANN, G. (2006): Organisationen in Luhmanns Systemtheorie. Organisationstheorien, in: A. KIESER and EBERS, M. (Hrsg.). Stuttgart: Kohlhammer: 427-461.

MASLOW, A. H. (1954): Motivation and personality New York, NY u. a.: Harper \& Row.

MASUCH, M. (1985): Vicious circles in organizations, in: Administrative Science Quarterly 30(1): 14-33.

MATTMÜLLER, R. \& TUNDER, R. (2004): Strategisches Handelsmarketing München: Vahlen.

MATURANA, H. R. (1982): Erkennen: Die Organisation und Verkörperung von Wirklichkeit: ausgewählte Arbeiten zur biologischen Epistemologie, Wissenschaftstheorie, Wissenschaft und Philosophie Vieweg.

MATURANA, H. R. \& VARELA, F. J. (1987): Der Baum der Erkenntnis: die biologischen Wurzeln des menschlichen Erkennens München u. a.: Scherz.

MAYNTZ, R. (2005): Soziale Mechanismen in der Analyse gesellschaftlicher Makro-Phänomene. Was erklärt die Soziologie? Methoden, Modelle, Perspektiven, in: U. SCHIMANK and GESOFF, R. (Hrsg.). Berlin u. a.: LIT Verlag: 204-227.

MAYRING, P. (2002): Einführung in die qualitative Sozialforschung: eine Anleitung zu qualitativem Denken Weinheim, Basel: Beltz.

MAYRING, P. (2010): Qualitative Inhaltsanalyse: Grundlagen und Techniken Weinheim, Basel: Beltz.

MCCONAUGHY, D. L. (2000): Family CEOs vs. nonfamily CEOs in the family-controlled firm: An examination of the level and sensitivity of pay to performance, in: Family Business Review 13(2): 121-131.

MCGREGOR, D. (1960): The human side of enterprise New York: McGraw-Hill. 
MEFFERT, H. (1994): Herausforderungen und Anspruchsspektrum der marktorientierten Unternehmensführung Wiesbaden: Gabler.

MEFFERT, H., BURMANN, C. \& KIRCHGEORG, M. (2009): Marketing Arbeitsbuch Wiesbaden: Springer Gabler.

MEFFERT, H., BURMANN, C. \& KIRCHGEORG, M. (2015): Marketing: Grundlagen marktorientierter Unternehmensführung Konzepte - Instrumente - Praxisbeispiele Wiesbaden: Springer Gabler.

MEYER, J. W. \& ROWAN, B. (1977): Institutionalized organizations: Formal structure as myth and ceremony, in: American Journal of Sociology 83(2): 340-363.

MICELOTTA, E. R. \& RAYNARD, M. (2011): Concealing or revealing the family? Corporate brand identity strategies in family firms, in: Family Business Review 24(3): 3754.

MICHLER, I. (2009): Wirtschaftswunder 2010: Deutschlands Familienunternehmer erobern die Weltmärkte Frankfurt a. M.: Campus Verlag.

MILES, R. E., SNOW, C. C., MEYER, A. D. \& COLEMAN, H. J. (1978): Organizational strategy, structure, and process, in: Academy of Management Review 3(3): 546-562.

MILLER, D. (1991): Stale in the saddle: CEO tenure and the match between organization and environment, in: Management Science 37(1): 34-52.

MILLER, D. (1992): The Icarus paradox: How exceptional companies bring about their own downfall, in: Business Horizons 35(1): 24-35.

MILLER, D. (1993): The architecture of simplicity, in: Academy of Management Review 18(1): 116-138.

MILLER, D. \& BRETON-MILLER, L. (2006): Family governance and firm performance: Agency, stewardship, and capabilities, in: Family Business Review 19(1): 73-87.

MILLER, D., DE VRIES, M. F. K. \& TOULOUSE, J.-M. (1982): Top executive locus of control and its relationship to strategy-making, structure, and environment, in: Academy of Management Journal 25(2): 237-253.

MILLER, D. \& FRIESEN, P. H. (1980): Momentum and revolution in organizational adaptation, in: Academy of Management Journal 23(4): 591-614.

MILLER, D. \& LEBRETON-MILLER, I. (2005): Managing for the long-run: Lessons in competitive advantage from great family businesses. Boston, MA Harvard Business School Press.

MILLIKEN, F. J. \& MARTINS, L. L. (1996): Searching for common threads: Understanding the multiple effects of diversity in organizational groups, in: Academy of Management Review 21(2): 402-433.

MINTZBERG, H. (1979): The structuring of organizations: A synthesis of the research Englewood Cliffs, NJ: Prentice-Hall.

MINTZBERG, H. (1983): Power in and around organizations Englewood Cliffs, NJ: Prentice-Hall.

MINTZBERG, H. (1984): Power and organization life cycles, in: Academy of Management Review 9(2): 207-224.

MINTZBERG, H. (1987): The strategy concept 1: five p's for strategy, in: California Management Review 30(1): 11-24.

MINTZBERG, H. (2005): Developing theory about the development of theory, in: M. A. HITT and SMITH, K. (Hrsg.): Great minds in management: The process of theory development (2005). Oxford University Press: 355-372. 
MINTZBERG, H., RAISINGHANI, D. \& THEORET, A. (1976): The structure of "unstructured « decision processes, in: Administrative Science Quarterly 21(2): 246-275.

MINTZBERG, H. \& WATERS, J. A. (1982): Tracking strategy in an entrepreneurial firm, in: Academy of Management Journal 25(3): 465-499.

MINTZBERG, H. \& WATERS, J. A. (1985): Of strategies, deliberate and emergent, in: Strategic Management Journal 6(3): 257-272.

MINTZBERG, H. \& WATERS, J. A. (1990): Tracking strategy in an entrepreneurial firm, in: Family Business Review 3(3): 285-315.

MITTERAUER, M. \& SIEDER, R. (1977): Vom Patriarchat zur Partnerschaft: zum Strukturwandel der Familie München: Beck.

MORCK, R., SHLEIFER, A. \& VISHNY, R. W. (1989): Alternative mechanisms for corporate control, in: The American Economic Review 79(4): 842-852.

MORIKAWA, H. (2001): A history of top management in Japan: Managerial enterprises and family enterprises New York: Oxford University Press.

MORRIS, M. H., WILLIAMS, R. O., ALLEN, J. A. \& AVILA, R. A. (1997): Correlates of success in family business transitions, in: Journal of Business Venturing 12(5): 385401.

MORRISON, E. W. \& MILLIKEN, F. J. (2000): Organizational silence: A barrier to change and development in a pluralistic world, in: Academy of Management Review 25(4): 706-725.

MÜLLER, R. (1982): Krisenmanagement in der Unternehmung - ein Beitrag zur organisatorischen Gestaltung des Prozesses der Krisenbewältigung Frankfurt a.M.: Lang.

MÜLLER, R. (1986): Krisenmanagement in der Unternehmung - Vorgehen, Maßnahmen, Organisation Frankfurt am Main: Lang.

MURRAY, A. I. (1989): Top management group heterogeneity and firm performance, in: Strategic Management Journal 10(1): 125-141.

NASSEHI, A. \& SAAKE, I. (2002): Kontingenz: Methodisch verhindert oder beobachtet? Ein Beitrag zur Methodologie der qualitativen Sozialforschung, in: ZfS 31(1): 66-86.

NAVE-HERZ, R., Ed. (2014): Familiensoziologie: ein Lehr- und Studienbuch. München, De Gruyter Oldenbourg.

NELSON, T. (2003): The persistence of founder influence: Management, ownership, and performance effects at initial public offering, in: Strategic Management Journal 24(8): 707-724.

NIELSEN, S. (2010): Top management team diversity: A review of theories and methodologies, in: INTERNATIONAL JOURNAL OF MANAGEMENT REVIEWS 12(3): 301316.

NORDQVIST, M., HALL, A. \& MELIN, L. (2009): Qualitative research on family businesses: The relevance and usefulness of the interpretive approach, in: Journal of Management \& Organization 15(3): 294-308.

NORTH, D. C. (1990): Institutions, institutional change and economic performance Cambridge: Cambridge University Press.

NORTH, D. C. (1993): Institutions, and credible commitment, in: Journal of Institutional and Theoretical Economics 149(1): 11-23.

NYSTROM, P. C. \& STARBUCK, W. H. (1984): To avoid organizational crises, unlearn, in: Organizational Dynamics 12(4): 53-65. 
O'NEILL, H. M. (1986): Turnaround and recovery: what strategy do you need?, in: Long Range Planning 19(1): 80-88.

OMER, Ḥ., ALLÔN, N. \& SCHLIPPE, A. V. (2007): Feindbilder: Psychologie der Dämonisierung Göttingen: Vandenhoeck \& Ruprecht.

OUCHI, W. G. \& JAEGER, A. M. (1978): Type Z organization: Stability in the midst of mobility, in: Academy of Management Review 3(2): 305-314.

PAJUNEN, K. (2006): Stakeholder influences in organizational survival, in: Journal of Management Studies 43(6): 1261-1288.

PALAZZOLI, M. S. (1984): Hinter den Kulissen der Organisation Stuttgart: Klett-Cotta.

PANDIT, N. R. (2000): Some Recommendations for Improved Research on Corporate Turnaround, in: Management 3(2): 31-56.

PARSONS, T. (1952): The Social system (2. Aufl.). Glencoe, Ill.: Free Press.

PATTON, M. Q. (2001): Qualitative evaluation and research methods (3. Aufl.). London: Sage.

PEARCE, J. A. I. \& ROBBINS, D. K. (1993): Toward improved theory and research on business turnaround in: Journal of Management 19(3): 613-636.

PEARCE, J. A. I. \& ROBBINS, D. K. (2008): Strategic transformation as the essential last step in the process of business turnaround in: Business Horizons 51(2): 121-130.

PEARSON, C. M. \& CLAIR, J. A. (1998): Reframing crisis management, in: Academy of Management Review 23(1): 59-76.

PELLED, L. H., EISENHARDT, K. M. \& XIN, K. R. (1999): Exploring the black box: An analysis of work group diversity, conflict and performance, in: Administrative Science Quarterly 44(1): 1-28.

PENROESE, E. (1959): The theory of the growth of the firm Oxford: Blackwell.

PENROSE, J. M. (2000): The role of perception in crisis planning, in: Public Relations Review 26(2): 155-171.

PEREZ, P. F. \& COLLI, A. (2013): A global revolution: The endurance of large family businesses around the world. The Endurance of Family Businesses: A Global Overview, in: P. F. PEREZ and COLLI, A. (Hrsg.). New York: Cambridge University Press.

PERROW, C. (1972): Complex organizations. Glenview, Illinois: Scott, Foresman, and Company.

PETERMANN, A. (2010): Pfadabhängigkeit und Hierarchie: Zur Durchsetzungskraft von selbstverstärkenden Effekten in hierarchischen Organisationen. Freie Universität Berlin, Dissertation.

PETERSON, R. S., SMITH, D. B., MARTORANA, P. V. \& OWENS, P. D. (2003): The impact of chief executive officer personality on top management team dynamics: one mechanism by which leadership affects organizational performance, in: Journal of applied Psychology 88(5): 795.

PETTIGREW, A. M. (1973): The politics of organizational decision-making London: Tavistock.

PETTIGREW, A. M. (1977): Strategy formulation as a political process, in: International studies of Management \& Organization 7(2): 78-87.

PETTIGREW, A. M. (1987): Context and action in the transformation of the firm, in: Journal of Management sStudies 24(6): 649-670.

PETTIGREW, A. M. (1987): Context and action in the transformation of the firm, in: Journal of Management Studies 24(6): 649-670. 
PETTIGREW, A. M. (1990): Longitudinal field research on change: Theory and practice, in: Organization Science 1(3): 267-292.

PETTIGREW, A. M. (2012): Context and action in the transformation of the firm: A reprise, in: Journal of Management Studies 49(7): 1304-1328.

PFEFFER, J. (1981): Power in organizations Marshfield, Mass.: Pitman.

PFEFFER, J. (1992): Managing with power: Politics and influence in organizations Boston, Mass.: Harvard Business Press.

PFEFFER, J. \& SALANCIK, G. R. (2003 (1978)): The external control of organizations: A resource dependence perspective Stanford, Calif.: Stanford University Press.

PIEPER, T. M. \& KLEIN, S. B. (2007): The bulleye: a systems approach to modeling family firms, in: Family Business Review 20(4): 301-319.

PIEPER, T. M., SMITH, A. D., KUDLATS, J. \& ASTRACHAN, J. H. (2015): The persistence of multifamily firms: Founder imprinting, simple rules, and monitoring processes, in: Entrepreneurship Theory and Practice 39(6): 1313-1337.

PIERCE, J. L., KOSTOVA, T. \& DIRKS, K. T. (2001): Toward a theory of psychological ownership in organizations, in: Academy of Management Review 26(2): 298-310.

PIERSON, P. (2000): Increasing returns, path dependence, and the study of politics, in: The American Political Science Review 94(2): 251-267.

PIERSON, P. (2004): Politics in time: History, institutions, and social analysis Princeton, NJ: Princeton University Press.

PLATT, H. D. \& PLATT, M. B. (2002): Predicting corporate financial distress: Reflections on choice-based sample bias in: Journal of Economics and Finance 26(2): 184-199.

PONS (2016). »famulus." von http://de.pons.com/\%C3\%BCbersetzung? $\mathrm{q}=$ famulus\&l= dela\&in $=\& l f=$ la.

PONS (2016). »Krise im Chinesischen.«Zugriff: 25. 04. 2016, von http://de.pons.com/\%C3 $\%$ BCbersetzung $? \mathrm{q}=$ Krise $\& \mathrm{l}=$ dezh $\&$ in $=\mathrm{ac} \_$de $\& \mathrm{lf}=\mathrm{de}$.

PORTER, M. E. (1980): Competitive strategy: techniques for analyzing industries and competitors New York: Free Press.

PRAHALAD, C. K. \& BETTIS, R. A. (1986): The dominant logic: A new linkage between diversity and performance, in: Strategic Management Journal 7(6): 485-501.

PRIEM, R. L., LYON, D. W. \& DESS, G. G. (1999): Inherent limitations of demographic proxies in top management team heterogeneity research, in: Journal of Management 25(6): 935-953.

PRZYBORSKI, A. \& WOHLRAB-SAHR, M. (2008): Qualitative Sozialforschung - ein Arbeitsbuch. München: Oldenbourg.

QUINN, J. B. (1980): Strategies for change: Logical incrementalism Homewood, Ill.: Irwin

QUINN, R. E. \& CAMERON, K. (1983): Organizational life cycles and shifting criteria of effectiveness: Some preliminary evidence, in: Management Science 29(1): 33-51.

RAYNARD, M., LOUNSBURY, M. \& GREENWOOD, R. (2013): Legacies of logics: Sources of community variation in CSR implementation in China. Institutional Logics in Action, in: M. LOUNSBURY and BOXENBAUM, E. (Hrsg.). Bingley: Emerald: 243-276.

REDLEFSEN, M. (2004): Der Ausstieg von Gesellschaftern aus großen Familienunternehmen: eine praxisnahe Untersuchung der Corporate Governance-Faktoren Wiesbaden: Deutscher Uni.-Verlag.

RICHARDSON, L. F. (1960): Arms and insecurity: A mathematical study of the causes and origins of war Pittsburgh: Boxwood Pr. 
RINDFLEISCH, H. (2011): Insolvenz und Rigidität. Eine theoretische und empirische Ursachenanalyse auf Basis von Insolvenzplänen Wiesbaden: Gabler.

ROBBINS, D. K. \& PEARCE, J. A., II (1992): Turnaround: Retrenchement and recovery, in: Strategic Management Journal 13(4): 287-309.

ROBBINS, D. K. \& PEARCE, J. A., II (1993): Entrepreneurial retrenchment among small manufacturing firms, in: Journal of Business Venturing 8(4): 301-318.

ROEDENBECK, M. R. (2011): Individual path dependency and social inertia: Facing the crudeness of sociology, in: Journal of Futures Studies 15(4): 25-44.

ROKEACH, M. (1973): The nature of human values New York: Free Press.

ROSENBAUER, C. (1994): Strategische Erfolgsfaktoren des Familienunternehmens im Rahmen seines Lebenszyklus - Ein eignerorientiertes Konzept zur Steigerung der Lebens- und Entwicklungsfähigkeit des Familienunternehmens Hallstadt: Rosch-Buch.

ROSENTHAL, G. \& FISCHER-ROSENTHAL, W. (2012): Anaylse narrativ-biografischer Interviews. Qualitative Forschung. Ein Handbuch., in: U. FLICK, VON KARDORFF, E. and STEINKE, I. (Hrsg.). Reinbek bei Hamburg: Rowohlt Enzyklopädie. 9. Aufl.

ROSS, J. \& STAW, B. M. (1993): Organizational escalation and exit: Lessons from the Shoreham nuclear power plant, in: Academy of Management Journal 36(4): 701-732.

ROTTER, J. B. (1966): Generalized expectancies for internal versus external control of reinforcement, in: Psychological monographs: General and applied 80(1): 1.

ROUX-DUFORT, C. (2007): Is crisis management (only) a management of exceptions?, in: Journal of Contingencies and Crisis Management 15(2): 105-114.

RÜSEN, T. A. (2009): Krisen und Krisenmanagement in Familienunternehmen Schwachstellen erkennen, Lösungen erarbeiten, Existenzbedrohungen meistern Wiesbaden: Gabler.

RÜSEN, T. A. \& VON SCHLIPPE, A. (2007): Krisen in Familienunternehmen und Unternehmensfamilien. Über parallele, interdependente Dynamiken in Familie und Unternehmen, in: Organisation, Supervision, Beratung 14(4): 309-330.

RUTHERFORD, M. W., KURATKO, D. F. \& HOLT, D. T. (2008): Examining the link between "familiness" and performance: Can the F-PEC untangle the family business theory jungle?, in: Entrepreneurship Theory and Practice 32(6): 1089-1109.

SARTORI, G. (1970): Concept misformation in comparative politics, in: The American Political Science Review 64(4): 1033-1053.

SCHEIN, E. H. (1983): The role of the founder in creating organizational culture, in: Organizational Dynamics 12(1): 13-28.

SCHEIN, E. H. (1985): Organisational culture and leadership: A dynamic view, in: San Francisco.

SCHEIN, E. H. (2010): Organisational culture and leadership: A dynamic view San Francisco: Wiley.

SCHELLING, T. C. (1958): The strategy of conflict prospectus for a reorientation of game theory, in: Journal of Conflict Resolution 2(3): 203-264.

SCHENDEL, D. E. \& HOFER, C. W. (1979): A new view of business policy and planning Boston: Little, Brown and Company.

SCHENDEL, D. E., PATTON, G. R. \& RIGGS, J. (1976): Corporate turnaround strategies: A study of profit decline and recovery, in: Journal of General Management 3(3): 3-11.

SCHENDEL, D. E. P., G.R. (1976): Corporate Stagnation and Turnaround, in: Journal of Econmics and Business 28(3): 236-241. 
SCHOLLE, S. (2013): Die deutschen Druckmaschinenhersteller im Spannungsfeld von Digitalisierung, strategischer Anpassungsnotwendigkeit und organisatorischer Pfadabhängigkeit. Freie Universität Berlin.

SCHREYÖGG, G. (1984): Unternehmensstrategie: Grundfragen einer Theorie strategischer Unternehmensführung Berlin: Walter de Gruyter.

SCHREYÖGG, G. (1999): Strategisches Management - Entwicklungstendenzen und Zukunftsperspektiven, in: Die Unternehmung 53(6): 387-407.

SCHREYÖGG, G. (2006): Organisation: Grundlagen moderner Organisationsgestaltung (4. Auflage). Wiesbaden: Gabler.

SCHREYÖGG, G. \& OSTERMANN, S. M. (2014): Krisenwahrnehmung und Krisenbewältigung. Handbuch Krisenmanagement, in: A. THIEßEN (Hrsg.). Wiesbaden: Springer: 119-139.

SCHREYÖGG, G. \& STEINMANN, H. (1987): Strategic control: a new perspective, in: Academy of Management Review 12(1): 91-103.

SCHREYÖGG, G., SYDOW, J. \& HOLTMANN, P. (2011): How history matters in organisations: The case of path dependence, in: Management \& Organizational History 6(1): 81-100.

SCHREYÖGG, G., SYDOW, J. \& KOCH, J. (2003): Organisatorische Pfade - Von der Pfadabhängigkeit zur Pfadkreation?, in: Managementforschung 13: 257-294.

SCHULENBURG, N. (2009): Entstehung von Unternehmenskrisen: Eine evolutionstheoretische Erklärung Wiesbaden: Gabler.

SCHULZE, W., LUBATKIN, M., DINO, R. \& BUCHHOLTZ, A. (2001): Agency relationships in family firms: theory and evidence in: Organization Science 12(2): 99-116.

SCHULZE, W. S., LUBATKIN, M. H. \& DINO, R. N. (2003): Exploring the agency consequences of ownership dispersion among the directors of private family firms, in: Academy of Management Journal 46(2): 179-194.

SCHUMPETER, J. A. (1950): Capitalism, socialism, and democracy (3. Aufl.). New York: Harper.

SCHÜßLER, E. (2008): Strategische Prozesse und Persistenzen - Pfadabhängige Organisation der Wertschöpfungskette in der Bekleidungsindustrie Stuttgart: Kohlhammer.

SCHÜTZE, F. (1978): Die Technik des narrativen Interviews in Interaktionsfeldstudiendargestellt an einem Projekt zur Erforschung von kommunalen Machtstrukturen: Arbeitsberichte und Forschungsmaterialien Bielefeld: Universität Bielefeld.

SCHÜTZE, F. (1983): Biographieforschung und narratives Interview, in: Neue Praxis 13(3): 283-293.

SCHWEDLER, E. (2013): Kompaktwissen Marketing: Grundlagen für Studium und Praxis Wiesbaden: Springer Gabler.

SCHWEIGER, D. M., SANDBERG, W. R. \& RAGAN, J. W. (1986): Group approaches for improving strategic decision making: A comparative analysis of dialectical inquiry, devil's advocacy, and consensus, in: Academy of Management Journal 29(1): 51-71.

SCHWEIGER, D. M., SANDBERG, W. R. \& RECHNER, P. L. (1989): Experiential effects of dialectical inquiry, devil's advocacy and consensus approaches to strategic decision making, in: Academy of Management Journal 32(4): 745-772.

SEWELL, W. H. (1996): Three temporalities: Toward an eventful sociology, in: The historic turn in the human sciences: $245-280$.

SHARMA (2013): 2012: A Year in Review, in: Family Business Review 25(1): 5-15. 
SHARMA, P. (2004): An overview over the field of family business studies: Current status and directions for the future, in: Family Business Review 17(1): 1-36.

SHARMA, P., CHRISMAN, J. J. \& CHUA, J. H. (1997): Strategic management of the family business: Past research and future challenges in: Family Business Review 10: 1-36.

SHARMA, P., CHRISMAN, J. J. \& GERSICK, K. E. (2012): 25 years of family business review: Reflections on the past and perspectives for the future, in: Family Business Review 25(1): 5.

SHARMA, P., HOY, F., ASTRACHAN, J. H. \& KOIRANEN, M. (2007): The practice-driven evolution of family business education, in: Journal of Business Research 60(10): 10121021.

SHARMA, P. \& MANIKUTTY, S. (2005): Strategic divestments in family firms: Role of family structure and community culture, in: Entrepreneurship Theory and Practice 29(3): 293-311.

SHARMA, P. \& SALVATO, C. (2013): Family firm longevity: A balancing act between continuity and change. The Endurance of Family Businesses: A Global Overview, in: P. F. PEREZ and COLLI, A. (Hrsg.). New York: Cambridge University Press.

SHLEIFER, A. \& VISHNY, R. W. (1986): Large shareholders and corporate control, in: The Journal of Political Economy 94(3): 461-488.

SHLEIFER, A. \& VISHNY, R. W. (1997): A survey of corporate governance, in: The journal of finance 52(2): 737-783.

SIEBELS, J. F. \& ZU KNYPHAUSEN-AUFSEß, D. (2012): A review of theory in family business research: The implications for corporate governance, in: INTERNATIONAL JOURNAL OF MANAGEMENT REVIEWS 14(3): 280-304.

SIMON, A., MARQUES, P., BIKFALVI, A. \& MUNÓZ, M. D. (2012): Exploring value differences across family firms: The influence of choosing and managing complexity, in: Journal of Family Business Strategy 3(2012): 132-146.

SIMON, F. B. (2002): Die Familie des Familienunternehmens - Besonderheiten der Familiendynamik. Die Familie des Familieunternehmens - ein System zwischen Gefühl und Geschäft, in: F. B. SIMON (Hrsg.). Heidelberg: Carl-Auer: 35-54.

SIMON, F. B. (2002): Familien und Unternehmen - Überlegungen zu Unterschieden, Gemeinsamkeiten und den Folgen. Die Familie des Familienunternehmens. Ein System zwischen Gefühl und Geschäft, in: F. B. SIMON (Hrsg.). Heidelberg: Carl-Auer: 17-34.

SIMON, F. B. (2002): Zwischen Gefühl und Geschäft - Familien und ihre Unternehmen. Die Familie des Familienunternehmens. Ein System zwischen Gefühl und Geschäft., in: F. B. SIMON (Hrsg.). Heidelberg: Carl-Auer: 7-15.

SIMON, F. B. (2011): Einführung in die systemische Organisationstheorie (3. Aufl.). Heidelberg: Carl-Auer.

SIMON, F. B. (2012): Einführung in die Systemtheorie des Konflikts Heidelberg: CarlAuer.

SIMON, F. B. (2012): Einführung in die Theorie des Familienunternehmens Heidelberg: Carl-Auer.

SIMON, F. B., WIMMER, R. \& GROTH, T. (2005): Mehrgenerationen-Familienunternehmen. Erfolgsgeheimnisse von Oetker, Merck, Haniel und anderen Heidelberg: CarlAuer.

SIMON, H. (2007): Hidden Champions des 21. Jahrhunderts: Die Erfolgsstrategien unbekannter Weltmarktführer Frankfurt a. M.: Campus. 
SIMON, H. A. (1957): Models of man. Social and rational New York: Wiley.

SIMONS, T., PELLED, L. H. \& SMITH, K. A. (1999): Making use of difference: Diversity, debate, and decision comprehensiveness in top management teams, in: Academy of Management Journal 42(6): 662-673.

SINGAL, M. \& GERDE, V. W. (2015): Is diversity management related to financial performance in family firms?, in: Family Business Review 28(3): 243-259.

SIRMON, D. G., ARREGLE, J. L., HITT, M. A. \& WEBB, J. W. (2008): The role of family influence in firms' strategic responses to threat of imitation, in: Entrepreneurship Theory and Practice 32(6): 979-998.

SIRMON, D. G. \& HITT, M. A. (2003): Managing resources: Linking unique resources, management, and wealth creation in family firms, in: Entrepreneurship Theory and Practice 27(4): 339-358.

SLATTER, S. (1984): Corporate recovery - Succesful turnaround strategies and their implementation London: Penguin.

SLATTER, S. \& LOVETT, D. (1999): Corporate turnaround: Managing companies in distress London: Penguin.

SLOAN, A. P. (1964): My years with general motors Garden City, NY: Doubleday.

SMITH, K. G., SMITH, K. A., OLIAN, J. D., SIMS JR, H. P., O’BANNON, D. P. \& SCULLY, J. A. (1994): Top management team demography and process: The role of social integration and communication, in: Administrative Science Quarterly 39(3): 412-438.

SMITH, N. R. \& MINER, J. B. (1983): Type of entrepreneur, type of firm, and managerial motivation: Implications for organizational life cycle theory, in: Strategic Management Journal 4(4): 325-340.

STAEHLE, W. H. (1980): Management: eine verhaltenswissenschaftliche Perspektive München: Vahlen.

STAEHLE, W. H. \& ALBACH, H., Eds. (1984): Betriebswirtschaftslehre und ökonomische Krise: kontroverse Beiträge zur betriebswirtschaftlichen Krisenbewältigung. Wiesbaden, Gabler.

STAFFORD, K., DUNCAN, K. A., DANE, S. \& WINTER, M. (1999): A research model of sustainable family businesses, in: Family Business Review 12(3): 197-208.

STAMM, I. (2013): Unternehmerfamilien. Über den Einfluss des Unternehmens auf Lebenslauf, Generationenbeziehungen und soziale Identität. Opladen, Berlin, Toronto: Verlag Barbara Budrich.

STARBUCK, W. H., GREVE, A. \& HEDBERG, B. L. T. (1978): Responding to crisis, in: Journal of Business Administration 9(2): 111-137.

STATISTA (2016). »Deutsches Weininstitut. (n.d.). Pro-Kopf-Konsum von Getränken in Deutschland nach Segmenten in den Jahren 2003 bis 2014 (in Liter). In Statista - Das Statistik-Portal. Zugriff am 14. Mai 2016, von http://de.statista.com/statistik/daten/ studie/219408/umfrage/pro-kopf-verbrauch-von-getraenken-in-deutschland/.»

STATISTA (2016). „Durchschnittlicher Umsatz je Quadratmeter Verkaufsfläche der dmdrogerie markt GmbH \& Co. KG in Deutschland in Jahren 2011 bis 2015 (in Euro)«. Zugriff: 17.05. (21:07), 2016, von http://de.statista.com/statistik/daten/studie/277457/ umfrage/umsatz-je-quadratmeter-von-dm-in-deutschland/.

STAW, B. M. (1976): Knee-deep in the big muddy: A study of escalating commitment to a chosen course of action, in: Organizational behavior and human performance 16(1): $27-44$. 
STAW, B. M., SANDELANDS, L. E. \& DUTTON, J. E. (1981): Threat rigidity effects in organizational behavior: A multilevel analysis, in: Administrative Science Quarterly 26(4): 501-524.

STEINMANN, H. \& SCHREYÖGG, G. (2005): Management: Grundlagen der Unternehmensführung. (6. Aufl.). Wiesbaden: Gabler.

STINCHCOMBE, A. (1965): Social structures and organizations. Handbook of organizations, in: J. G. MARCH (Hrsg.). Chigago: Rand McNally: 142-193.

STRAUSS, A. L. (1991): Grundlagen qualitativer Sozialforschung: Datenanalyse und Theoriebildung in der empirischen soziologischen Forschung München: Fink.

SUDARSANAM, S. \& LAI, J. (2001): Corporate financial distress and turnaround strategies: An empirical analysis, in: British Journal of Management 12(3): $183 \mathrm{ff}$.

SYDOW, J., SCHREYÖGG, G. \& KOCH, J. (2009): Organizational path dependence: Opening the black box, in: Academy of Management Review 34(4): 689-709.

TAGIURI, R. \& DAVIS, J. (1996): Bivalent attributes of the family firm, in: Family Business Review 9(2 Summer 1996): 199-208.

TAGIURI, R. \& DAVIS, J. A. (1992): On the goals of successful family companies, in: Family Business Review 5(1): 43-62.

TAJFEL, H. (2010): Social identity and intergroup relations Cambrige u.a.: Cambridge University Press.

TAJFEL, H. \& STROEBE, W. (1982): Gruppenkonflikt und Vorurteil: Entstehung und Funktion sozialer Stereotypen. Human groups and social categories Bern [u. a.]: Huber.

TAN, W.-L. \& FOCK, S. T. (2001): Coping with growth transitions: The case of Chinese family businesses in Singapore, in: Family Business Review 14(2): 123-139.

TEECE, D. J., PISANO, G. \& SHUEN, A. (1997): Dynamic capabilities and strategic management, in: Strategic Management Journal 18(7): 509-533.

THALER, R. H. \& SHEFRIN, H. M. (1981): An economic theory of self-control, in: Journal of Political Economy 89(2): 392-406.

THELEN, K. (1999): Historical institutionalism in comparative politics, in: Annual review of political science 2(1): 369-404.

THOMAS, A. S., LITSCHERT, R. J. \& RAMASWAMY, K. (1991): The performance impact of strategy-manager coalignment: An empirical examination, in: Strategic Management Journal 12(7): 509-522.

THOMPSON, J. (1967): Organizations in action: Social science bases of administrative theory New York: McGraw-Hill.

TICHY, N. M. \& DEVANNA, M. A. (1986): The transformational leader New York: Wiley.

TJOSVOLD, D. (1991): The conflict-positive organization: Stimulate diversity and create unity Reading, Mass.: Addison-Wesley.

TOBIAS, R. (2012): Pfadbruch durch Insolvenz? Eine Fallstudie zur Wirkung von Insolvenzverfahren auf betriebliche Pfadabhängigkeiten bei zwei mittelständischen Unternehmen Marburg: Tectum-Verlag.

TRAHMS, C. A., NDOFOR, H. A. \& SIRMON, D. G. (2013): Organizational decline and turnaround a review and agenda for future research, in: Journal of Management 39(5): 1277-1307.

TUSHMAN, M. L. \& ANDERSON, P. (1986): Technological discontinuities and organizational environments, in: Administrative Science Quarterly 31(3): 439-465. 
ULRICH, H. (1968): Die Unternehmung als produktives soziales System: Grundlagen der allgemeinen Unternehmungslehre Bern; Stuttgart: Haupt.

ULRICH, P. \& FLURI, E. (1992 (1975)): Management: eine konzentrierte Einführung Bern ; Stuttgart: Haupt.

VAN DE VEN, A. H. \& POOLE, M. S. (1995): Explaining development and change in organizations, in: Academy of Management Review 20(3): 510-540.

VERGNE, J.-P. \& DURAND, R. (2010): The missing link between the theory and empirics of path dependence: Conceptual clarification, testability issue, and methodological implications, in: Journal of Management Studies 47(4): 736-759.

VOIGT, J. F. (1990): Familienunternehmen. Im Spannungsfeld zwischen Eigentum und Fremdmanagement Wiesbaden: Gabler.

VON FOERSTER, H. (1984): Priciples of self-organization - in a socio-managerial context. Self-organization and management of social systems, in: H. ULRICH and PROBST, G. J. B. (Hrsg.). Heidelberg: Springer: 2-24.

VON SCHLIPPE, A. (1995): Familientherapie im Überblick: Basiskonzepte, Formen, Anwendungsmöglichkeiten (6. Aufl.). Paderborn: Junfermann.

VON SCHLIPPE, A. (2009): Beiträge zur Theorie des Familienunternehmens Lohmar: Eul.

VON SCHLIPPE, A. \& FRANK, H. (2013): The theory of social systems as a framework for understanding family businesses, in: Family Relations 62(3): 384-398.

VON SCHLIPPE, A. \& KELLERMANNS, F. W. (2008): Emotionale Konflikte in Familienunternehmen, in: ZEITSCHRIFT FUR KMU UND ENTREPRENEURSHIP 56(1): 4058.

VROOM, V. H. \& PAHL, B. (1971): Relationship between age and risk taking among managers, in: Journal of applied Psychology 55(5): 399.

WANOUS, J. P. \& YOUTZ, M. A. (1986): Solution diversity and the quality of groups decisions, in: Academy of Management Journal 29(1): 149-159.

WARD, J. L. (1987): Keeping the family business healthy: How to plan for continuing growth profitability and family leadership San Francisco, CA Jossey-Bass.

WARD, J. L. (1988): The special role of strategic planning for family businesses, in: Family Business Review 1(2): 105-117.

WARD, J. L. (2004): Perpetuating the family business: 50 lessons learned from long-lasting, successful families in business Palgrave: Macmillan.

WATZLAWICK, P., BAVELAS, J. B. \& JACKSON, D. D. (2007): Menschliche Kommunikation: Formen, Störungen, Paradoxien. Pragmatics of human communication Bern [u. a.]: Huber.

WATZLAWICK, P., BEAVIN, J. H. \& JACKSON, D. D. (1974): Menschliche Kommunikation: Formen, Störungen, Paradoxien: H. Huber.

WEBER, M. (1972): Wirtschaft und Gesellschaft: Grundriß der verstehenden Soziologie Tübingen: Mohr.

WEICK, K. E. (1988): Enacted sensemaking in crisis situations, in: Journal of Management Studies 25(4): 305-317.

WEITZEL, W. \& JONSSON, E. (1989): Decline in organizations: A literature integration and extension, in: Administrative Science Quarterly 34(1): 91-109.

WELGE, M. K. \& AL-LAHAM, A. (2012): Strategisches Management: Grundlagen Prozess - Implementierung. (6. Aufl.). Wiesbaden: Gabler. 
WELSCH, C. (2010): Organisationale Trägheit und ihre Wirkung auf die strategische Früherkennung von Unternehmenskrisen Wiesbaden: Gabler.

WERNER, G. (2013): Womit ich nie gerechnet habe: Die Autobiographie Berlin: Econ. WESTHEAD, P. \& COWLING, M. (1998): Family firm research: The need for a methodological rethink, in: Entrepreneurship Theory and Practice 23(1): 31-33.

WESTHEAD, P., COWLING, M. \& HOWORTH, C. (2001): The development of family companies: Management and ownership imperatives, in: Family Business Review 14(4): 369-385.

WESTPHAL, J. D. \& ZAJAC, E. J. (1995): Who shall govern? CEO/board power, demographic similarity, and new director selection, in: Administrative Science Quarterly 40(1): 60-83.

WHETTEN, D. A. (1980): Organizational decline: A neglected topic in organizational science, in: Academy of Management Review 5(4): 577-588.

WHETTEN, D. A. (1987): Organizational growth and decline processes, in: Annual Review of Sociology 131(1): 335-358.

WHITESIDE, M. F. \& BROWN, F. H. (1991): Drawbacks of a dual systems approach to family firms: Can we expand our thinking?, in: Family Business Review 4(4): 383-395.

WIECHERS, R. (2006): Familienmanagement zwischen Unternehmen und Familie - Zur Handhabung typischer Eigenarten von Unternehmensfamilien und Familienunternehmen Heidelberg: Carl-Auer.

WIERSEMA, M. F. \& BANTEL, K. A. (1992): Top management team demography and corporate strategic change, in: Academy of Management Journal 35(1): 91-121.

WILLIAMS, K. Y. \& O'REILLY III, C. A. (1998): A review of 40 years of research, in: Research in Organizational Behavior 20: 77-140.

WILLIAMSON, O. E. (1975): Markets and hierarchies, in: New York: 26-30.

WIMMER, R. (2011): Typische Schwächen und Potenziale der Selbstgefährdung von Familienunternehmen. Familienunternehmen erfolgreich sanieren. Der Einfluss des Familienfaktors bei Restrukturierungen, in: T. A. RÜSEN (Hrsg.). Berlin: Erich Schmidt Verlag.

WIMMER, R., DOMEYER, E., OSWALD, M. \& VATER, G. (2005): Familienunternehmen Auslaufmodell oder Erfolgstyp? (2. Aufl.). Wiesbaden: Gabler.

WITTE (1981): Die Unternehmenskrise? Unternehmskrisen - Ursachen, Frühwarnung, Bewältigung, in: V. B. SCHNELLINGER (Hrsg.). Stuttgart: Schäffer-Poeschel.

WÖHE, G. \& DÖRING, U. (2005): Einführung in die allgemeine Betriebswirtschaftslehre München: Vahlen.

WORTMAN JR., M. S. (1994): Theoretical foundations for family-owned businesses: A conceptual and research based paradigm, in: Family Business Review 7(1): 3-27.

YIN, R. K. (2009): Case study research: Design and methods Los Angeles, Calif. [u. a.]: Los Angeles, Calif. [u. a.]: Sage.

ZAHRA, S. A. (2005): Entrepreneurial risk taking in family firms, in: Family Business Review 18(1): 23-40.

ZAHRA, S. A., HAYTON, J. C. \& SALVATO, C. (2004): Entrepreneurship in family vs. nonfamily firms: A resource-based analysis of the effect of organizational culture, in: Entrepreneurship Theory and Practice 28(4): 363-381.

ZAHRA, S. A. \& SHARMA, P. (2004): Family business research: A strategic reflection in: Family Business Review 17(4): 331-346. 
ZAJAC, E. J., KRAATZ, M. S. \& BRESSER, R. K. (2000): Modeling the dynamics of strategic fit: A normative approach to strategic change, in: Strategic Management Journal 21(4): 429-453.

ZATTONI, A., GNAN, L. \& HUSE, M. (2015): Does family involvement influence firm performance? Exploring the mediating effects of board processes and tasks, in: Journal of Management 41(4): 1214-1243.

ZELLWEGER, T. \& ASTRACHAN, J. (2008): On the emotional value of owning a firm in: Family Business Review 21(4): 347-363. 